University of Louisville

ThinkIR: The University of Louisville's Institutional Repository

Electronic Theses and Dissertations

$12-2013$

\title{
Effect of slope and paver characteristics on performance of permeable pavement $\mathrm{GI}$.
}

Amirhossein Ehsaei 1986-

University of Louisville

Follow this and additional works at: https://ir.library.louisville.edu/etd

\section{Recommended Citation}

Ehsaei, Amirhossein 1986-, "Effect of slope and paver characteristics on performance of permeable pavement GI." (2013). Electronic Theses and Dissertations. Paper 393.

https://doi.org/10.18297/etd/393

This Doctoral Dissertation is brought to you for free and open access by ThinkIR: The University of Louisville's Institutional Repository. It has been accepted for inclusion in Electronic Theses and Dissertations by an authorized administrator of ThinkIR: The University of Louisville's Institutional Repository. This title appears here courtesy of the author, who has retained all other copyrights. For more information, please contact thinkir@louisville.edu. 


\title{
EFFECT OF SLOPE AND PAVER CHARACTERISTICS ON PERFORMANCE OF PERMEABLE PAVEMENT GI
}

\author{
by \\ Amirhossein Ehsaei \\ B.A., Ferdowsi University of Mashhad, 2009 \\ M.S., University of Birmingham, UK, 2010

\begin{abstract}
A Dissertation
Submitted to the Faculty of the

Speed School of Engineering of the University of Louisville

in Partial Fulfillment of the Requirements

for the Degree of
\end{abstract} \\ Doctor of Philosophy \\ Department of Civil and Environmental Engineering \\ University of Louisville \\ Louisville, Kentucky
}

December 2013 
Copyright 2013 by Amirhossein Ehsaei

All rights reserved 



\title{
EFFECT OF SLOPE AND PAVER CHARACTERISTICS ON PERFORMANCE OF
} PERMEABLE PAVEMENT GI

By

\author{
Amirhossein Ehsaei \\ B.S., Ferdowsi University of Mashhad, 2009 \\ M.S., University of Birmingham, UK, 2010
}

A Dissertation Approved on

August 30, 2013

by the following Dissertation Committee:

Dissertation Director: Dr. Thomas D. Rockaway

Dr. William E. Biles

Dr. Mark N. French

Dr. W. Mark McGinley

Dr. J. P. Mohsen 


\section{DEDICATION}

I dedicate my dissertation to my family and many friends. A special feeling of gratitude to my loving parents, whose words of encouragement and push got me this far. I also dedicate this dissertation to my wife, who stood by me, and I will always appreciate all her support throughout the process. 


\section{ACKNOWLEDGMENTS}

First and foremost I would like to thank my PhD advisor, Dr. Thomas Rockaway, for supporting me during all the stages of my education for the degree through the past three years. The project itself and this final report would not have been possible without his supervision and support. I am very grateful to him for his scientific advice and knowledge and many insightful discussions and suggestions. I am also very grateful to him for the opportunities he provided for me to be a part and a member of the Center for Infrastructure Research (CIR) and to become involved and participate in the project that led to this research. It was his support that gave me with the opportunity to spend so much time in the U.S. Environmental Protection Agency National Laboratory in Edison, New Jersey, where I was able to conduct a research project on a scale that I had never attempted before. I would also like to thank the other members of my defense committee, Dr. William E. Biles, Dr. Mark N. French, Dr. W. Mark McGinley, and Dr. J. P. Mohsen.

I also want to thank my mentor in the USEPA Office of Research and Development (ORD), Michael Borst, who made this research project happen. He oversaw the logistics of this project and many other aspects of conducting such work in a federal government agency, in addition to day to day feedback at all the stages of the research conducted in New Jersey without any of the members and staff of ORD. 


\section{ABSTRACT \\ EFFECT OF SLOPE AND PAVER CHARACTERISTICS ON PERFORMANCE OF PERMEABLE PAVEMENT GI \\ Amirhossein Ehsaei}

August 30, 2013

This dissertation is an experimental study based on the findings of two Green Infrastructure (GI) stormwater control measures (SCMs) in Louisville, KY, which focused on the effects of the physical environment on the performance of GI. The GI installed in Louisville are suffering from extensive and rapid surface clogging and in order to optimize current and future GI, an understanding of the factors affecting the performance of the system is required. The study used the current literature to determine the surrounding factors and those of the permeable surface that had not been investigated enough, and used several configurations of GI to determine the effects of these variables on the surface clogging.

The module used to test these variables was a wooden flume, specifically designed and constructed from plywood so that the variables of the experiment could be incorporated in testing. The flume simulates a permeable pavement system with storage gallery and a bedding layer, and is paved with three different interlocking concrete pavements that provide gaps of three different sizes. The flume's longitudinal slope can be adjusted, and the permeable joint material can be included. The performance of the 
flume was measured using 7 time domain reflectometer (TDRs) instruments, manufactured by Campbell Scientific, which are located inside the storage gallery.

After conducting 21 experiments with various configurations, the data was analyzed to reveal meaningful information. As expected, the experiments with permeable joint material show a clear separation for the sediment deposited on the surface, where empty gaps resulted in inorganics being deposited on the up gradient and organics on the down gradient.

The Analysis of Variance (ANOVA) of the progression of clogging and progression of infiltration edge on the permeable surface showed that the increase of gap size from $6 \mathrm{~mm}$ to $9 \mathrm{~mm}$ did not result in a significant change, but the change to $12 \mathrm{~mm}$ gaps resulted in a significantly different rate for the progression of the first rate. The presence of \#8 aggregate in the gaps resulted in significant changes in both rates and finally the change of slope from $1 \%$ to $3 \%$ created a significant change in the rate at which surface clogging progressed. 


\section{TABLE OF CONTENTS}

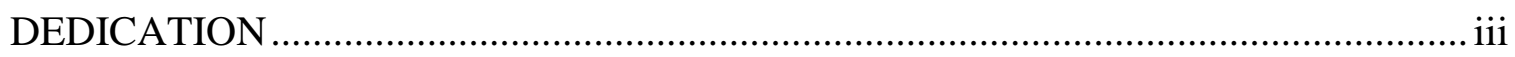

ACKNOWLEDGMENTS ................................................................................... iv

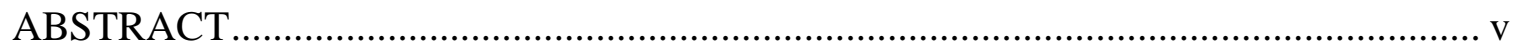

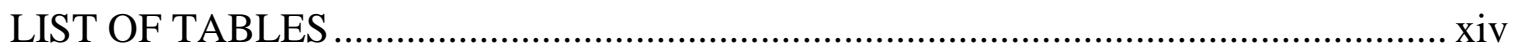

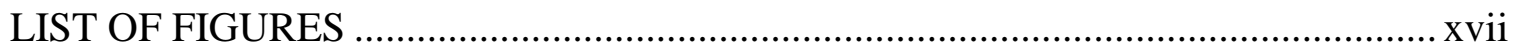

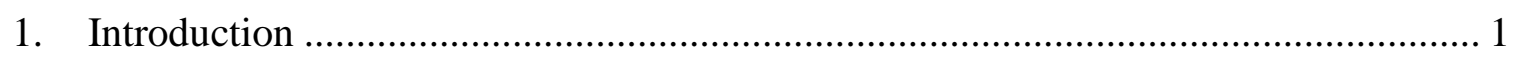

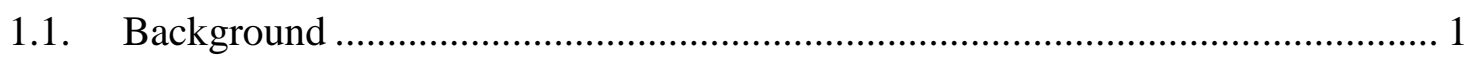

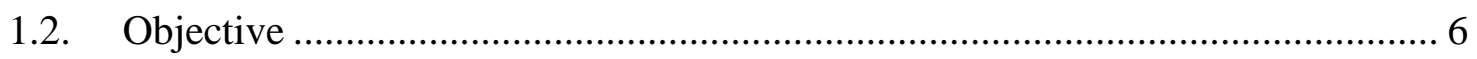

1.3. Statement of the Problem ..................................................................... 7

1.4. Potential Contributions of this Research .................................................... 8

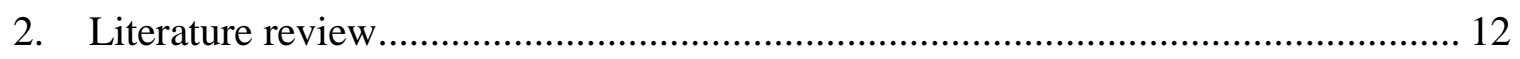

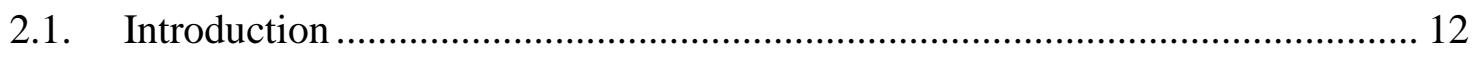

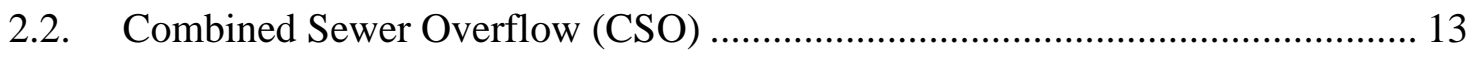

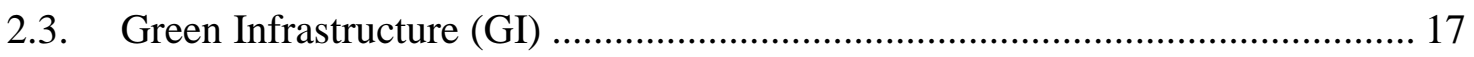

2.4. Permeable Pavement Systems .................................................................. 21

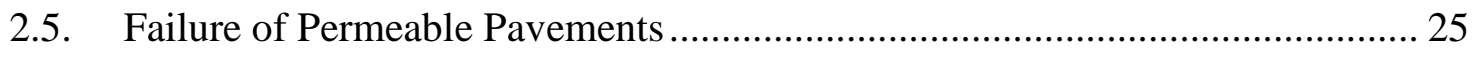

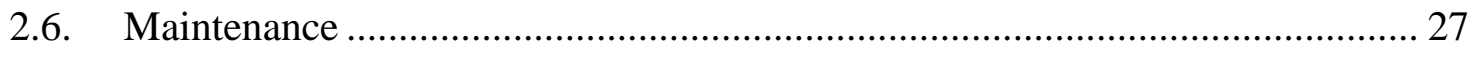

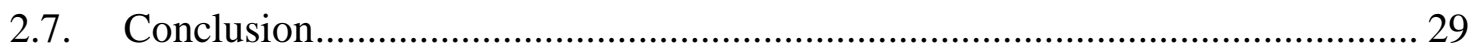


3. Characterization of the sediment found in urban stormwater runoff......

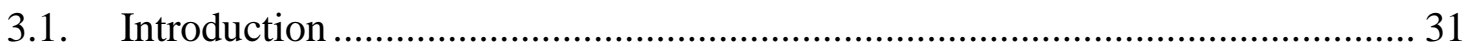

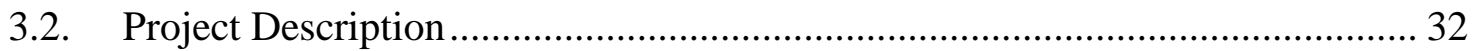

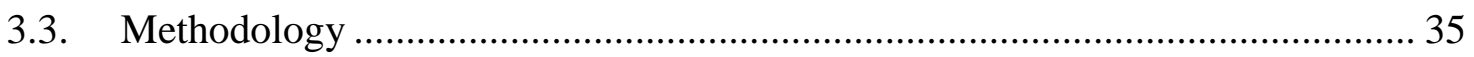

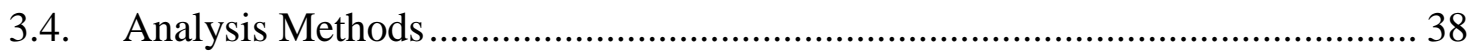

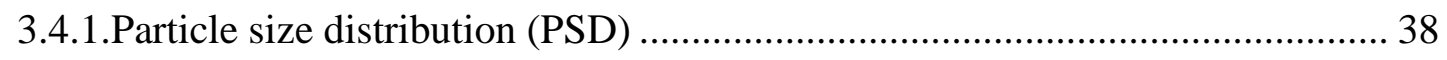

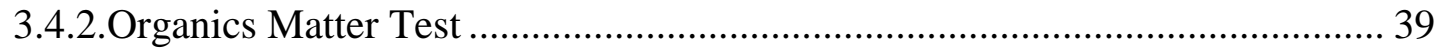

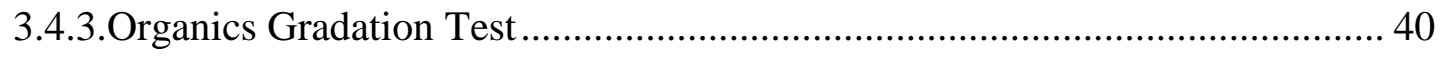

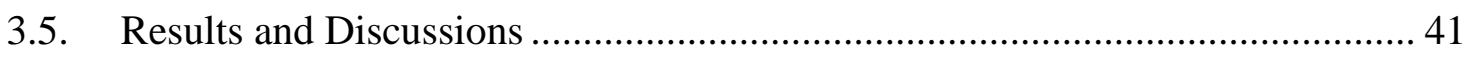

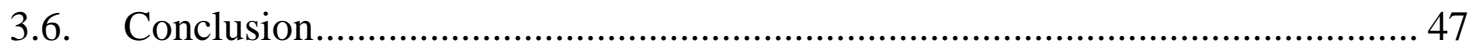

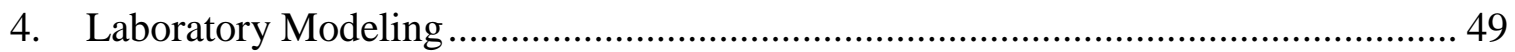

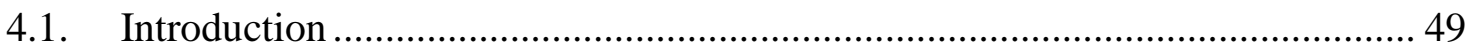

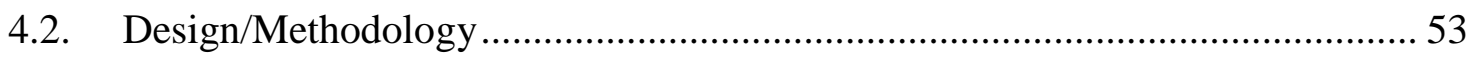

4.3. Construction ....................................................................................... 55

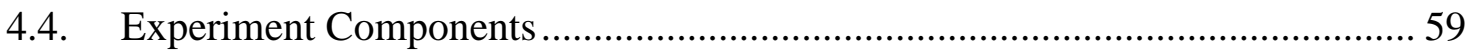

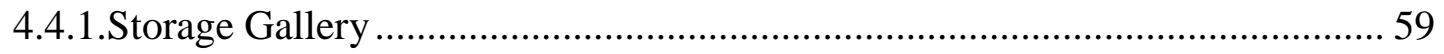

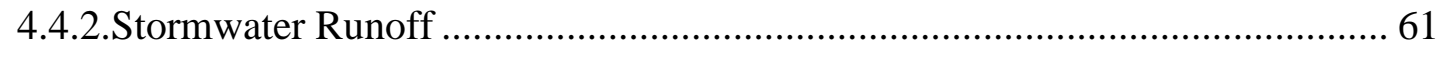

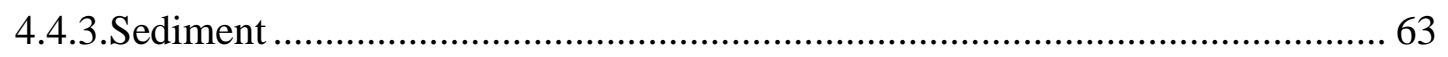

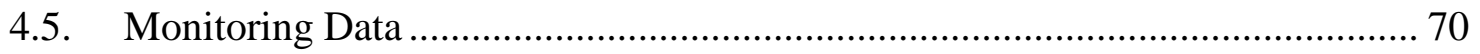

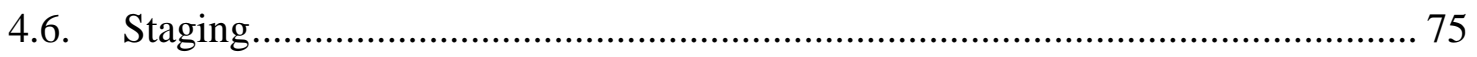




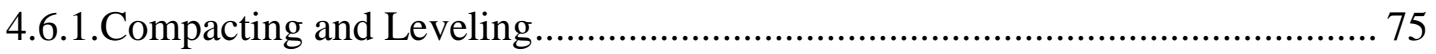

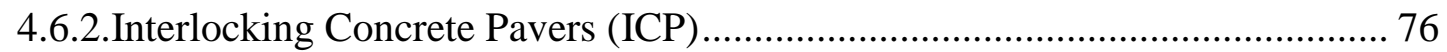

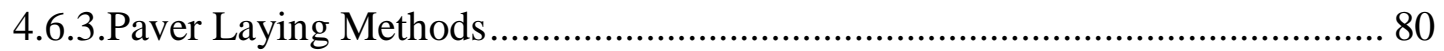

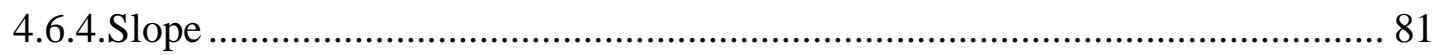

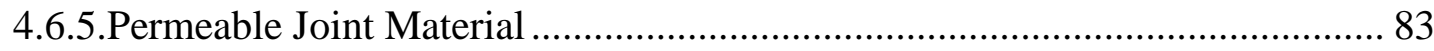

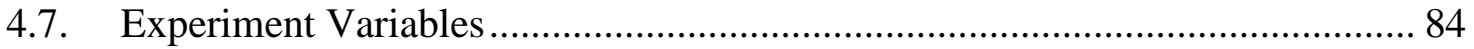

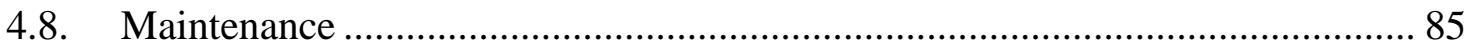

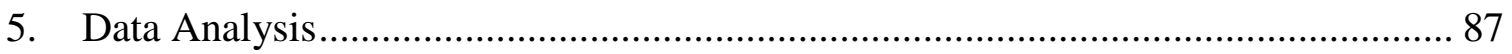

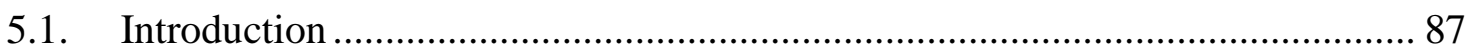

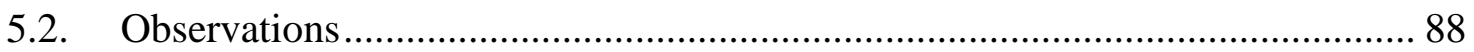

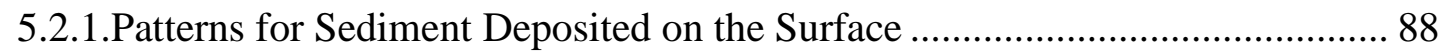

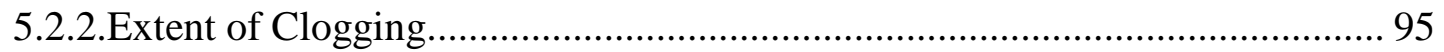

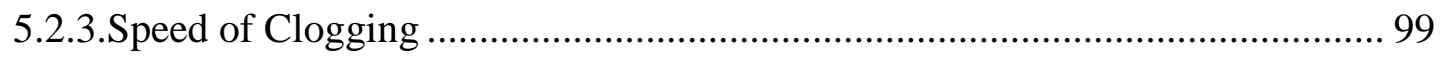

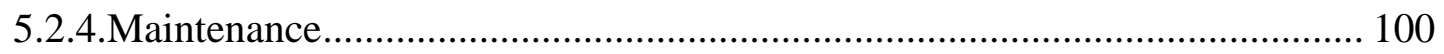

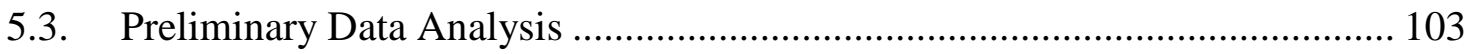

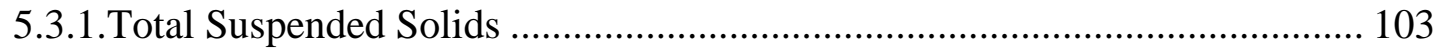

5.3.2.Effluent Flow Rate................................................................................ 104

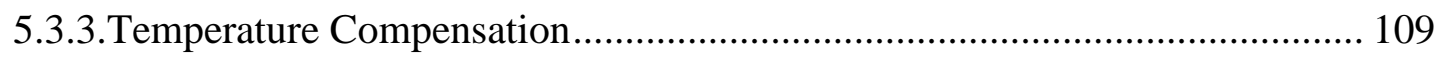

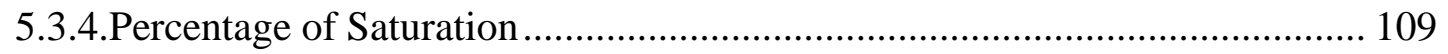

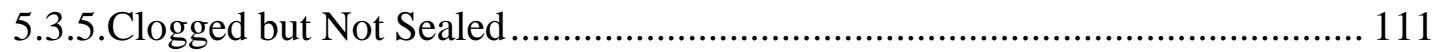


5.4. In-depth Data Analysis ............................................................................ 118

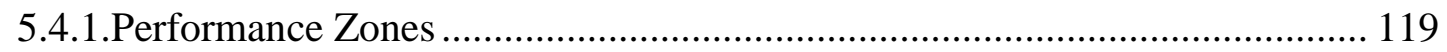

5.4.2.Progression of Clogging ................................................................................ 125

5.4.3.Progression of the Infiltration Edge............................................................... 129

5.5. Experimental Approach............................................................................... 133

5.5.1.Data Quality Considerations.......................................................................... 135

5.5.1.1. Qualitative Accuracy ……………………........................................ 136

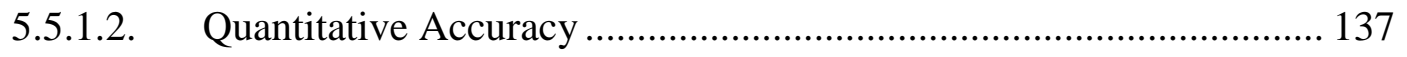

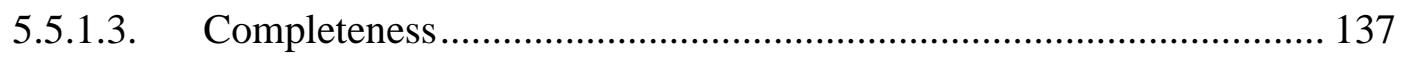

5.5.2.Repeatability and Reproducibility of Results ................................................ 138

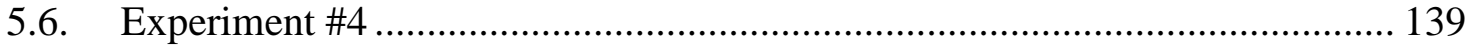

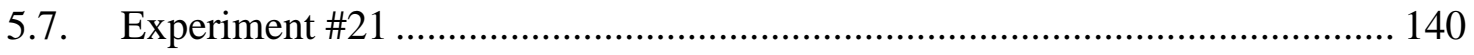

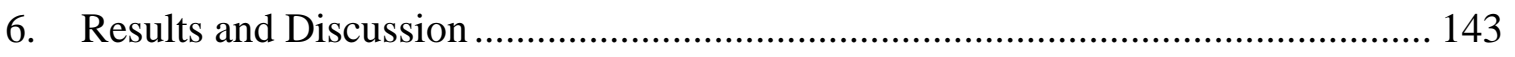

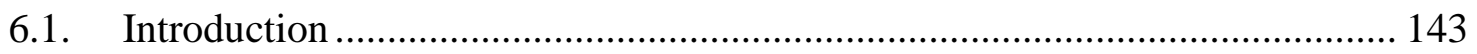

6.2. Observations and Preliminary Analyses ........................................................ 145

6.3. Progression of Clogging and Infiltration Edge ............................................. 147

6.3.1. Paver Gap Size....................................................................................... 150

6.3.1.1. Gap Size with 1\% Longitudinal Slope without Aggregate.................. 151

6.3.1.2. Gap Size with Base 1\% Longitudinal Slope with Aggregate ............. 154

6.3.1.3. Gap Size with Base 3\% Longitudinal Slope without Aggregate....... 157 
6.3.1.4. Gap Size with Base 3\% Longitudinal Slope with Aggregate. 159

6.3.1.5. Gap Size with Base 5\% Longitudinal Slope without Aggregate....... 162

6.3.1.6. Gap Size with Base 5\% Longitudinal Slope with Aggregate 165

6.3.2. Permeable Joint Material ........................................................................... 168

6.3.2.1. Permeable Joint Material for $6 \mathrm{~mm}$ Gap at 1\% Slope ......................... 169

6.3.2.2. Permeable Joint Material for $9 \mathrm{~mm}$ Gap at 1\% Slope ........................ 171

6.3.2.3. Permeable Joint Material for $12 \mathrm{~mm}$ Gap at 1\% Slope ...................... 173

6.3.2.4. Permeable Joint Material for $6 \mathrm{~mm}$ Gap at 3\% Slope ......................... 175

6.3.2.5. Permeable Joint Material for 9 mm Gap at 3\% Slope ........................ 177

6.3.2.6. Permeable Joint Material for $12 \mathrm{~mm}$ Gap at 3\% Slope ...................... 179

6.3.2.7. Permeable Joint Material for $6 \mathrm{~mm}$ Gap at 5\% Slope ........................ 181

6.3.2.8. Permeable Joint Material for $9 \mathrm{~mm}$ Gap at 5\% Slope ......................... 183

6.3.2.9. Permeable Joint Material for $12 \mathrm{~mm}$ Gap at 5\% Slope ....................... 185

6.3.3. Longitudinal Slope ................................................................................ 187

6.3.3.1. Slope for $6 \mathrm{~mm}$ Gap without Aggregate.............................................. 188

6.3.3.2. Slope for $6 \mathrm{~mm}$ Gap and \#8 Aggregate ............................................. 190

6.3.3.3. Slope for $9 \mathrm{~mm}$ Gap without Aggregate.............................................. 193

6.3.3.4. Slope for $9 \mathrm{~mm}$ Gap and \#8 Aggregate .............................................. 195

6.3.3.5. Slope for $12 \mathrm{~mm}$ Gap without Aggregate........................................... 197

6.3.3.6. Slope for $12 \mathrm{~mm}$ Gap and \#8 Aggregate ........................................... 200 


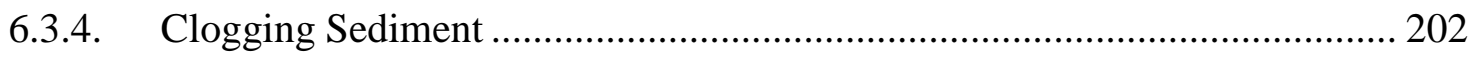

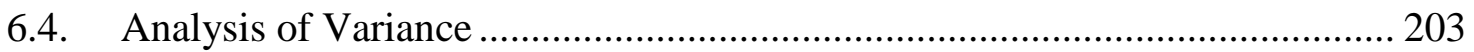

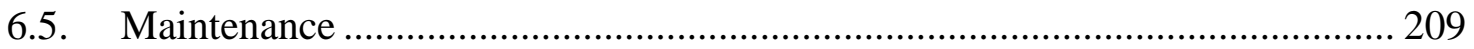

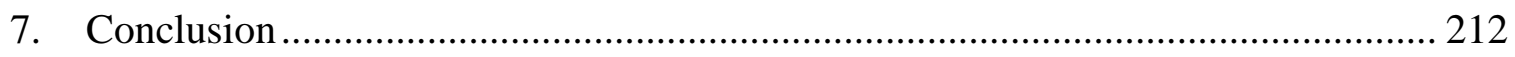

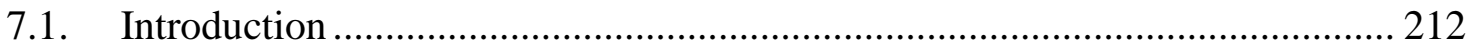

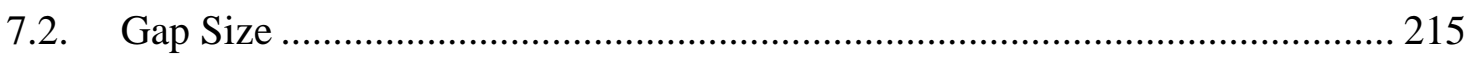

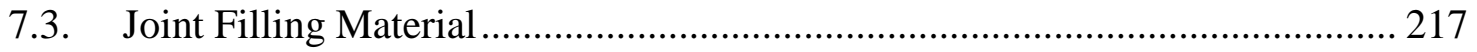

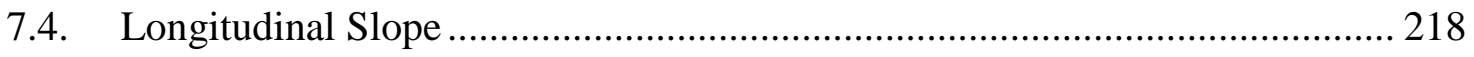

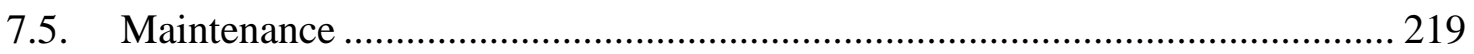

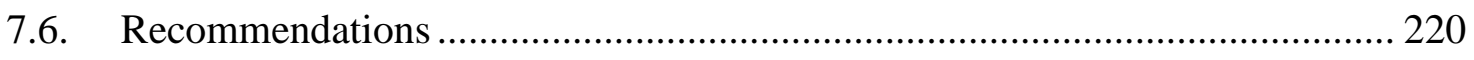

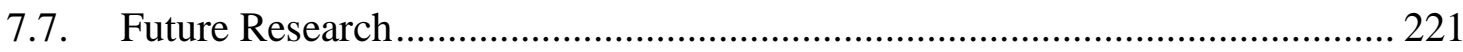

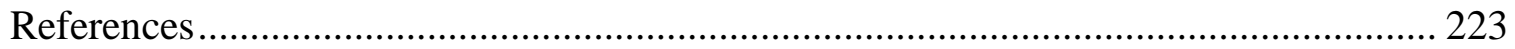

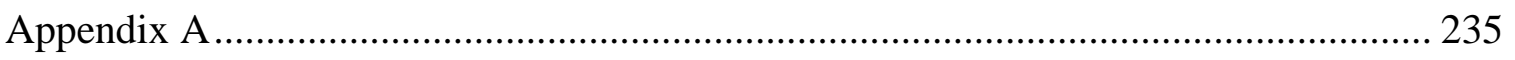

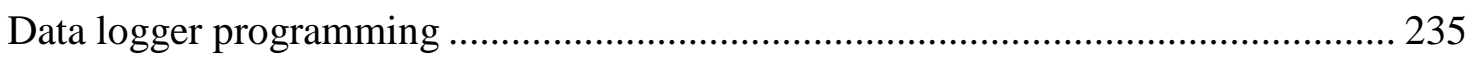

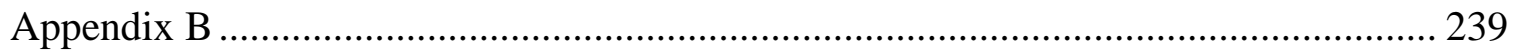

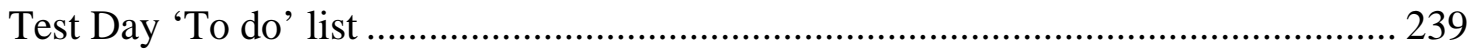

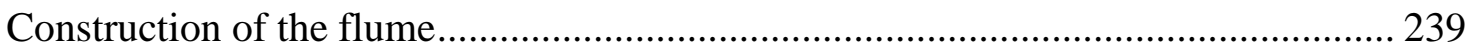

Schedule and order of work for the test day...................................................... 242

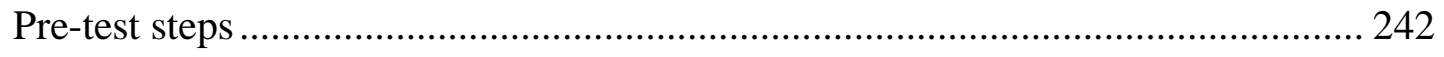

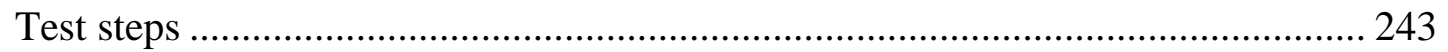


After the test steps

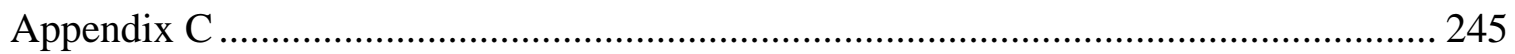

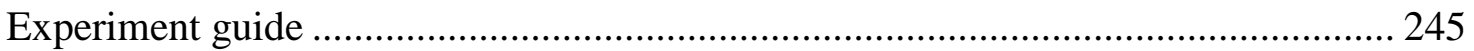

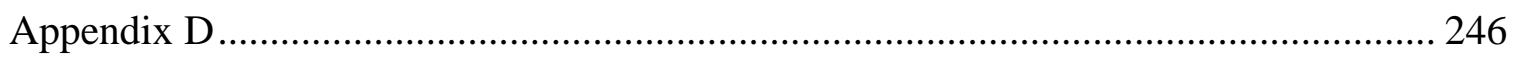

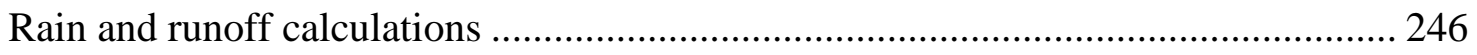

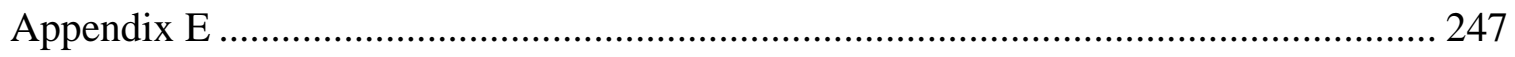

“Percentage of saturation vs. Time” graphs ..................................................... 247

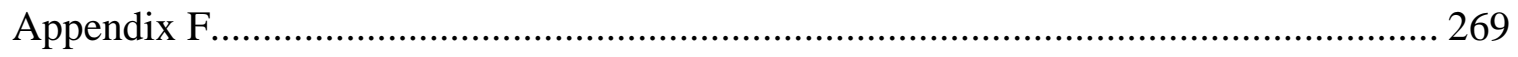

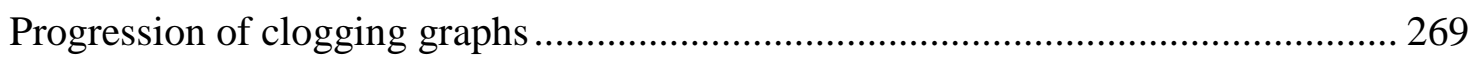

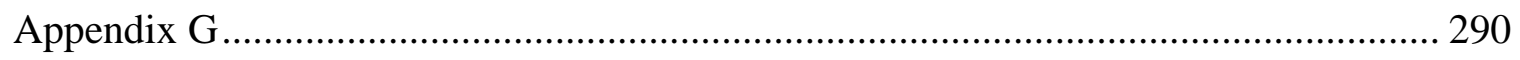

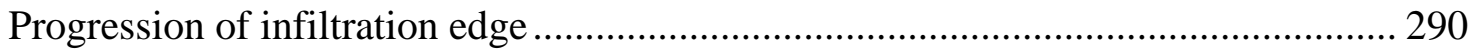

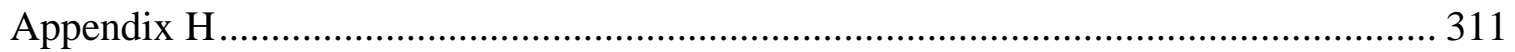

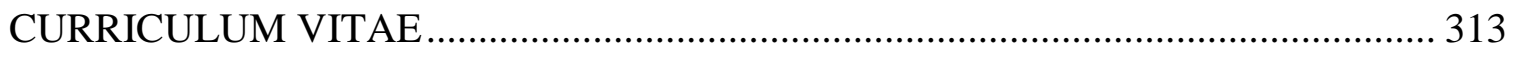




\section{LIST OF TABLES}

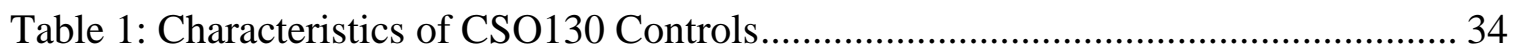

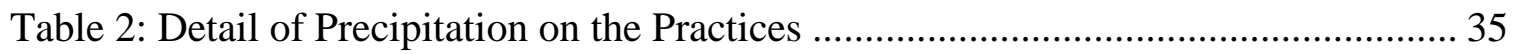

Table 3: Coefficients of Uniformity and Curvature for Sampels ................................... 39

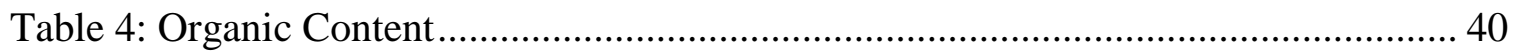

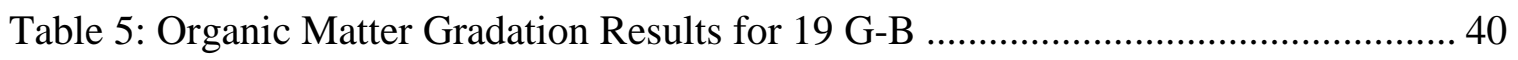

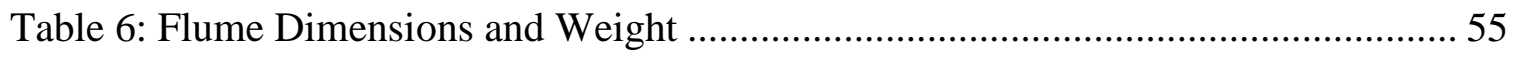

Table 7: TSS Concentrations, mg/L, for Different Land Uses and Geographical Areas

(EPA Rain Zones), Average (Number of Observatiosn) (Pitt et al., 2011) ...................... 65

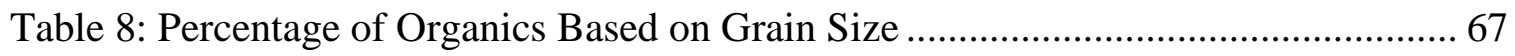

Table 9: Particle Size Distribution of the Material Used as Runoff Sediment, Based on

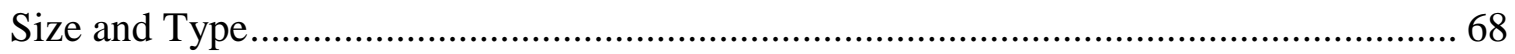

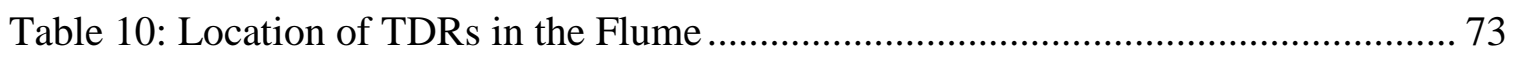

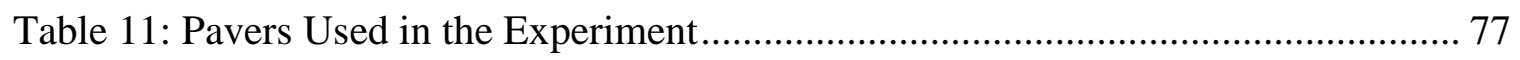

Table 12: Experiment Variables ......................................................................... 85

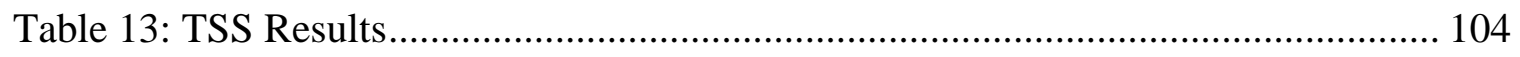

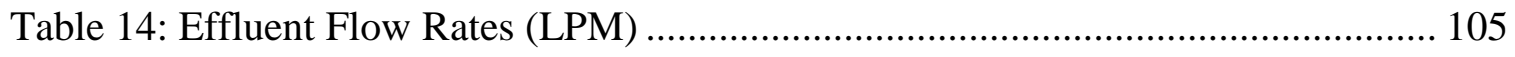

Table 15: Volume of Stormwater Runoff Used in the Experiment .............................. 108

Table 16: Slope of Incline of TDRs Measurements in Experiment \#1 _......................... 113

Table 17: Slope of Incline in TDRs Measurements in Experiment \#5 ......................... 116

Table 18: Slope of Incline in TDRs Measurements in Experiment \#7 .......................... 117

Table 19: Sediment Used in Experiment \#21 ............................................................ 141 
Table 20: Additional Sampling in Louisville CSO130................................................. 146

Table 21: Characteristics of Progression of Clogging for All Experiments .................... 148

Table 22: Characteristics of Progression of Infiltration Edge for All Experiments ....... 149

Table 23: Comparing the Progression of Clogging in Experiments \#2, \#5, And \#7 ...... 153

Table 24: Comparing the Progression of Clogging in Experiments \#2, \#5, And \#7 ...... 153

Table 25: Comparing the Progression of Clogging in Experiments \#4, \#6, and \#8 ....... 156

Table 26: Comparing the Progression of Infiltration Edge in Experiments \#4, \#6, and \#8 156

Table 27: Comparing the Progression of Clogging in Experiments \#13, \#11, and \#10 . 159 Table 28: Comparing the Progression of Infiltration Edge in Experiments \#13, \#11, and $\# 10$. 159

Table 29: Comparing the Progression of Clogging in Experiments \#14, \#12, and \#9 ... 162 Table 30: Comparing the Progression of Infiltration Edge in Experiments \#14, \#12, and \#9. 162

Table 31: Comparing the Progression of Clogging in Experiments \#19, \#17, and \#15 . 165 Table 32: Comparing the Progression of Infiltration Edge in Experiments \#19, \#17, and $\# 1$ 165

Table 33: Comparing the Progression of Clogging in Experiments \#20, \#18, and \#16. 168 Table 34: Comparing the Progression of Infiltration Edge in Experiments \#20, \#18, and $\# 16$. 168 Table 35: Comparison of the Effects of Slope in Progression of Clogging in Experiments with $6 \mathrm{~mm}$ Gap Size and No Permeable Joint Material 189 
Table 36: Comparison of the Effects of Slope in Progression of Infiltration Edge in Experiments with 6 mm Gap Size and No Permeable Joint Material............................... 190

Table 37: Comparison of the Effects of Slope in Progression of Clogging in Experiments with 6 mm Gap Size and Permeable Joint Material 191

Table 38: Comparison of the Effects of Slope in Progression of Infiltration Edge in Experiments with 6 mm Gap Size and Permeable Joint Material 192 Table 39: Comparison of the Effects of Slope in Progression of Clogging in Experiments with 9 mm Gap Size and no Permeable Joint Material. 194

Table 40: Comparison of the Effects of Slope in Progression of Infiltration Edge in Experiments with 9 mm Gap Size and No Permeable Joint Material

Table 41: Comparison of the Effects of Slope in Progression of Clogging in Experiments with 9 mm Gap Size and Permeable Joint Material 196

Table 42: Comparison of the Effects of Slope in Progression of Infiltration Edge in Experiments with $9 \mathrm{~mm}$ Gap Size and Permeable Joint Material 197

Table 43: Comparison of the Effects of Slope in Progression of Clogging in Experiments with 12 mm Gap Size and No Permeable Joint Material 199

Table 44: Comparison of the Effects of Slope in Progression of Infiltration Edge in Experiments with 12 mm Gap Size and No Permeable Joint Material 199 Table 45: Comparison of the Effects of Slope in Progression of Clogging In Experiments with 12 mm Gap Size and Permeable Joint Material. 201 Table 46: Comparison of the Effects of Slope in Progression of Infiltration Edge in Experiments with $12 \mathrm{~mm}$ Gap Size and Permeable Joint Material 202 


\section{LIST OF FIGURES}

Figure 1: Comparison of pre-development and post-development of watershed (USEPA, 2003a) 18

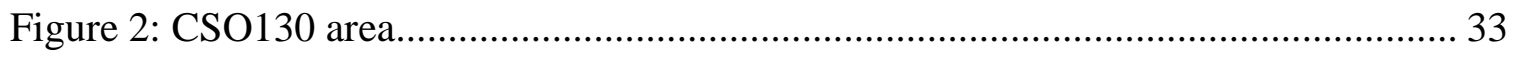

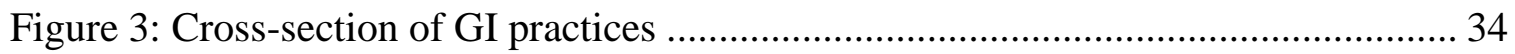

Figure 4: Regenerative sweeper truck with NQR 435 vaccum chamber (53 hp) ............ 36

Figure 5: Visuals of practice $19 \mathrm{G}$ before (above) and after (below) the maintenance .... 37

Figure 6: Site plan of CSO130 permeable pavement practices .................................... 38

Figure 7: Grain size distribution curvature and percentages passing the paver gaps for 19

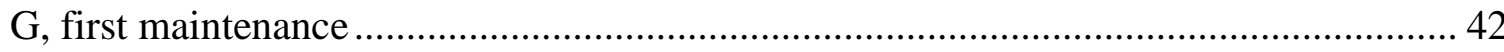

Figure 8: Grain size distribution curvature and percentages passing the paver gaps for 19

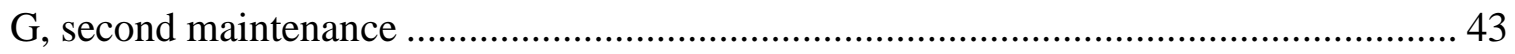

Figure 9: Grain size distribution curvature and percentages passing the paver gaps for 19

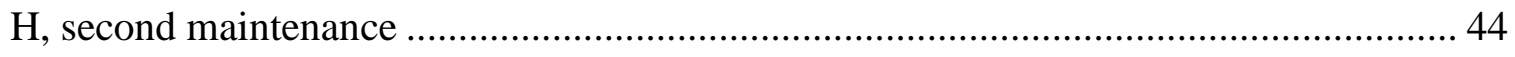

Figure 10: Percentage of organic contents of samples 19 G-B vs. passed sieve diameter 45

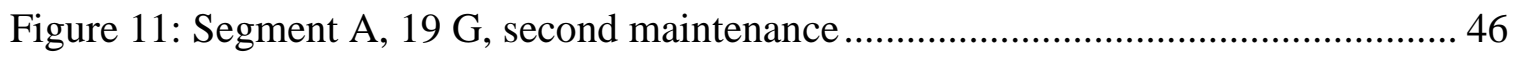

Figure 12: Construction of the flume's panels....................................................... 56

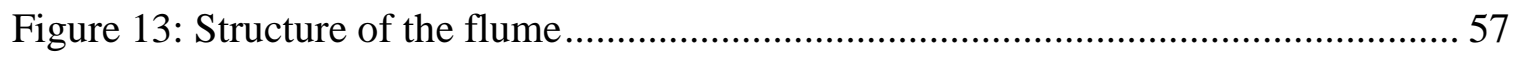

Figure 14: Inside of the flume with the liners and sealed joints .................................. 58

Figure 15: Flume’s feeder with the up gradient stormwater tank ................................ 59

Figure 16: Washing the aggregate using a pressure washer ...................................... 61

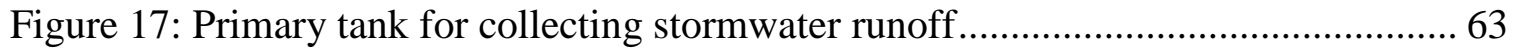


Figure 18: EPA rain zones, Source: NPDES Phase I Regulations, 40 CFR part 122

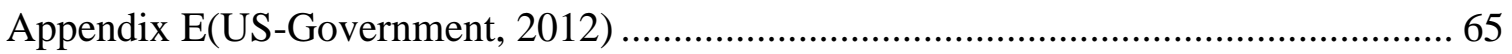

Figure 19: Dumping sediment during the experiment ............................................ 70

Figure 20: Time domain reflectometer (TDR) ..................................................... 71

Figure 21: Arrangement of TDRs in the storage gallery ......................................... 72

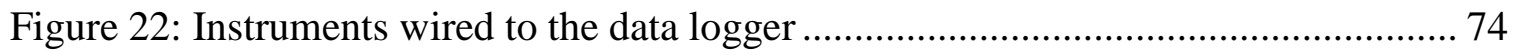

Figure 23: Screenshot of CR Basic Editor ............................................................ 75

Figure 24: Compacted and leveled bedding layer................................................. 76

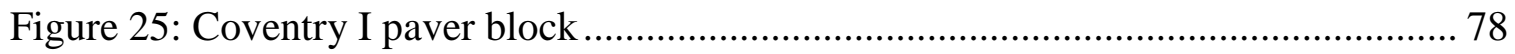

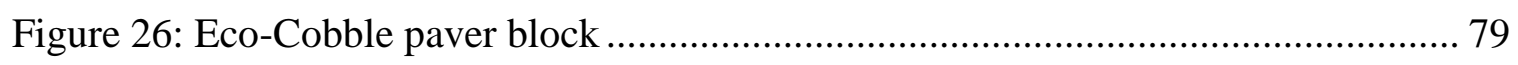

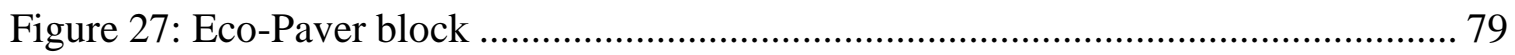

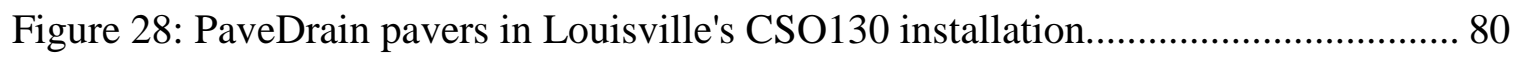

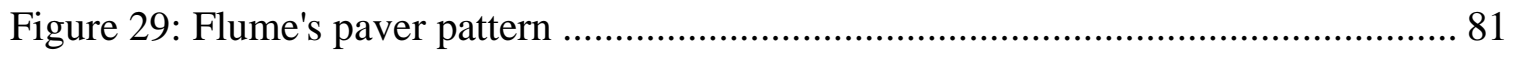

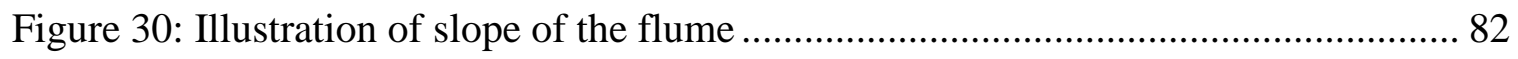

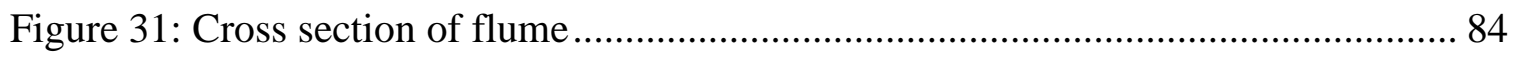

Figure 32: Experiment \#1, 1\% slope, 6 mm gap size, no aggregate between the pavers . 89 Figure 33: Experiment \#5, 1\% slope, 9 mm gap size, no aggregate between the pavers . 89 Figure 34: Experiment \#7, 1\% slope, $12 \mathrm{~mm}$ gap size, no aggregate between the pavers 90 Figure 35: Experiment \#4, 1\% slope, 6 mm gap size, no aggregate between the pavers . 91 Figure 36: Experiment \#6, 1\% slope, 9 mm gap size, no aggregate between the pavers . 91 Figure 37: Experiment \#8, 1\% slope, $12 \mathrm{~mm}$ gap size, no aggregate between the pavers 92 Figure 38: Stormwater runoff flow unpredicted pattern in experiment \#5 ...................... 94 
Figure 39: Clogged but not sealed, experiment \#14, 3\% slope, 6 mm gap size, \#8 aggregate between the pavers 96

Figure 40: Clogged but not sealed, experiment \#15, 5\% slope, $12 \mathrm{~mm}$ gap size, \#8 aggregate between the pavers 96

Figure 41: Clogged but not sealed, experiment \#18, 5\% slope, 9 mm gap size, \#8

aggregate between the pavers 97

Figure 42: Clogged but not sealed, experiment \#20, 5\% slope, 6 mm gap size, \#8

aggregate between the pavers 98

Figure 43: Ponding in the most down gradient segment of flume in experiment \#20...... 98

Figure 44: Experiment \#1, 1\% slope, 6 mm gap size, no aggregate between the pavers . 99 Figure 45: Experiment \#19, 5\% slope, $6 \mathrm{~mm}$ gap size, no aggregate between the pavers

Figure 46: Experiment \#19, 5\% slope, 6 mm gap size, no aggregate between the pavers, after removing the pavers. 101

Figure 47: Experiment \#11, 3\% slope, 9 mm gap size, no aggregate between the pavers, after removing the pavers 102 Figure 48: Experiment \#9, 3\% slope, $12 \mathrm{~mm}$ gap size, \#8 aggregate between the pavers,

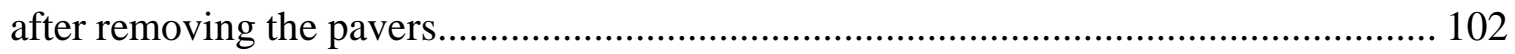

Figure 49: Discharge flow rates of all experiments ............................................... 106

Figure 50: Standard deviation of the discharge flow rates ...................................... 107

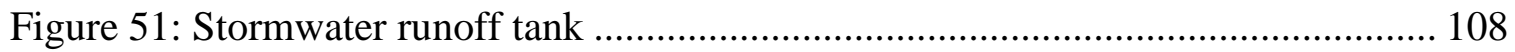

Figure 52: Experiment \#0, failure mechanism of the flume, TC_VWC........................ 110

Figure 53: Experiment \#0, failure mechanism of the flume, percentage of saturation... 111 
Figure 54: Experiment \#1, illustration of rate of increase

Figure 55: TDRs in the flume

Figure 56: Incline in the measurements of TDRs in experiment \#1 ............................ 115

Figure 57: Experiment \#5, illustration of rate of increase ......................................... 117

Figure 58: Experiment \#7, illustration of rate of increase ...................................... 118

Figure 59: Constant decrease in the TDRs' measurement after the experiment over 4 days

Figure 60: Experiment \#14, different zones in the measurements by TDRs ................. 123

Figure 61: Graph for progression of clogging for experiment \#1................................ 126

Figure 62: Flume’s overview for progression of clogging in experiment \#1 ................ 127

Figure 63: Corrected graph for progression of clogging for experiment \#1 .................. 128

Figure 64: Comparison of progression of clogging in experiments \#1, \#5, and \#7....... 129

Figure 65: Progression of infiltration edge, experiment \#1, graph .............................. 131

Figure 66: Comparison of progression of infiltration edge in experiments \#1, \#5 and \#7

Figure 67: Experiment \#4, illustration of the interruption in the experiment ................. 140

Figure 68: Experiment \#21, 1\% slope, 6 mm gap size, no aggregate between the pavers

Figure 69: Comparison of clogging debris between the pavers gaps in experiments

conducted with no permeable joint material ......................................................... 146

Figure 70: Comparison of progression of clogging in experiments \#2, \#5, and \#7........ 151

Figure 71: Comparison of progression of infiltration edge in experiments \#2, \#5, and \#7 
Figure 72: Comparison of progression of clogging in experiments \#4, \#6, and \#8....... 154

Figure 73: Comparison of progression of infiltration edge in experiments \#4, \#6, and \#8 155

Figure 74: Comparison of progression of clogging in experiments \#13, \#11, and \#10.. 157 Figure 75: Comparison of progression of infiltration edge in experiments \#13, \#11, and \#10 158

Figure 76: Comparison of progression of clogging in experiments \#14, \#12, and \#9.... 160 Figure 77: Comparison of progression of infiltration edge in experiments \#14, \#12, and \#9. 161

Figure 78: Comparison of progression of clogging in experiments \#19, \#17, and \#15.. 163 Figure 79: Comparison of progression of infiltration edge in experiments\#19, \#17, and $\# 15$ 164

Figure 80: Comparison of progression of clogging in experiments \#20, \#18, and \#16.. 166 Figure 81: Comparison of progression of infiltration edge in experiments \#20, \#18, and $\# 16$. 167

Figure 82: Comparison of progression of clogging in experiments \#2 and \#4..... 170

Figure 83: Comparison of progression of infiltration edge in experiments \#2 and \#4 ... 171

Figure 84: Comparison of progression of clogging in experiments \#5 and \#6. 172 Figure 85: Comparison of progression of infiltration edge in experiments \#5 and \#6 ... 173 Figure 86: Comparison of progression of clogging in experiments \#7 and \#8. 174 Figure 87: Comparison of progression of infiltration edge in experiments \#7 and \#8 ... 175 Figure 88: Comparison of progression of clogging in experiments \#13 and \#14.......... 176 Figure 89: Comparison of progression of infiltration edge in experiments \#13 and \#14177 
Figure 90: Comparison of progression of clogging in experiments \#11 and \#12.......... 178

Figure 91: Comparison of progression of infiltration edge in experiments \#11 and \#12179

Figure 92: Comparison of progression of clogging in experiments \#9 and \#10............ 180

Figure 93: Comparison of progression of infiltration edge in experiments \#9 and \#10. 181

Figure 94: Comparison of progression of clogging in experiments \#19 and \#20.......... 182

Figure 95: Comparison of progression of infiltration edge in experiments \#19 and \#20183

Figure 96: Comparison of progression of clogging in experiments \#17 and \#18........... 184

Figure 97: Comparison of progression of infiltration edge in experiments \#17 and \#18185

Figure 98: Comparison of progression of clogging in experiments \#15 and \#16.......... 186

Figure 99: Comparison of progression of infiltration edge in experiments \#15 and \#16187

Figure 100: Comparison of progression of clogging in experiments \#2, \#13, and \#19.. 188

Figure 101: Comparison of progression of infiltration edge in experiments \#2, \#13, and

\#19 189

Figure 102: Comparison of progression of clogging in experiments \#4, \#14, and \#20.. 191 Figure 103: Comparison of progression of infiltration edge in experiments \#4, \#14, and \#20. 192

Figure 104: Comparison of progression of clogging in experiments \#5, \#11, and \#17.. 193 Figure 105: Comparison of progression of infiltration edge in experiments \#5, \#11, and \#17 194

Figure 106: Comparison of progression of clogging in experiments \#6, \#12, and \#18.. 196 Figure 107: Comparison of progression of infiltration edge in experiments \#6, \#12, and \#18. 197

Figure 108: Comparison of progression of clogging in experiments \#7, \#10, and \#15.. 198 
Figure 109: Comparison of progression of infiltration edge in experiments \#7, \#10, and \#15. 199

Figure 110: Comparison of progression of clogging in experiments \#8, \#9, and \#16.... 200 Figure 111: Comparison of progression of infiltration edge in experiments \#8, \#9, and \#16 201

Figure 112: Comparison of progression of clogging in experiments \#2 and \#21.......... 203

Figure 113: Analysis of variance of paver gap size for progression of clogging ........... 204 Figure 114: Analysis of variance of permeable joint material for progression of clogging 205

Figure 115: Analysis of variance of longitudinal slope for progression of clogging ..... 206 Figure 116: Analysis of variance of paver gap size for progression of infiltration edge 207 Figure 117: Analysis of variance of permeable joint material for progression of infiltration edge. 208 Figure 118: Analysis of variance of longitudinal slope for progression of infiltration edge 209 


\section{INTRODUCTION}

\subsection{Background}

The hydrologic cycle, is the natural movement of water on the ground, in the atmosphere and below the surface of the earth (USGS, 2012). In the natural state of the hydrologic cycle, $40 \%$ of stormwater is turned into evapotranspiration, $25 \%$ shallow infiltration, and 25\% deep infiltration, while $10 \%$ is contained as surface runoff (USEPA, 2003b). The relatively high infiltration and evapotranspiration rate percentages result from undeveloped areas being typically porous; they trap rainwater; letting it infiltrate slowly into the ground or evaporate. Developed areas, however, have many more impervious surfaces such as rooftops and roads that do not allow water into the ground, thus disrupting the hydrologic cycle. In urban areas the evapotranspiration is reduced to $30 \%$ of the rainfall, shallow infiltration is reduced to $10 \%$ and deep infiltration is reduced to $5 \%$. Thus, the greater part of the rainfall, approximately $55 \%$, is transformed into surface runoff (USEPA, 2003b). These changes to the natural hydrologic cycle within an urban environment result in intense groundwater changes, greater risks of flooding and less water in streams during dry seasons (USEPA, 2003b).

As urban communities developed, Combined Sewer Systems (CSS) were among the earliest infrastructure systems incorporated into city planning efforts. In dry conditions, a CSS system will collect sanitary sewer water from residential, commercial 
and industrial users. In wet conditions, the same piping network will continue to collect the sanitary sewage, but will also collect stormwater runoff. Most of the time, the CSS system is able to capture the flow from both sources and convey it to a treatment facility prior to release into a water body or stream. During significant wet weather however, the stormwater runoff or snow melt combined with the sanitary sewage exceeds the capacity of treatment facilities. The excess flow (combined stormwater and sanitary), therefore passes the interceptor and enters rivers, lakes, creeks or local water sources with little or no treatment.

Combined Sewer Overflows (CSO), which are a common side effect of using CSS in urban areas are among the major sources of pollution for waterways (ASCE, 1992). Throughout the United States, these systems are in widespread use and serve about 40 million people in 772 communities (USEPA, 2008). CSOs disrupt the adjacent waterways by rapid runoff, the transportation of heavy metals, and the depletion of dissolved oxygen which also endangers the aquatic life (Hamilton, Revitt, \& Warren, 1984; Hvitved-Jacobsen, 1982; Shuster, Bonta, Thurston, Warnemuende, \& Smith, 2005).

A direct result of urbanization is the increase of impervious surfaces, which will result in an increase in the volume of stormwater runoff (Dunne \& Leopold, 1978). When $10 \%-20 \%$ of a catchment is covered with impervious surfaces, the volume of stormwater runoff increases twofold; with 35\%-50\% of a catchment covered with impervious surfaces, the volume of runoff increases threefold; and a 75\%-100\% impervious cover results in an increase of stormwater runoff more than fivefold of natural conditions (Arnold \& Gibbons, 1996). Therefore, it is evident that CSOs are the direct outcomes of 
increasing impervious areas and disrupting the natural hydrologic cycle. Any attempt to resolve the CSO problem will have to address the increasing imperviousness in urban settings.

The Clean Water Act (CWA) was passed by Congress to establish the environmental oversight necessary to protect the nation’s waters and to direct U.S. Environmental Protection Agency (USEPA) to develop, implement, and enforce appropriate rules and regulations. As a result, the USEPA in 1994 and the Congress in 2000 issued policies that require the municipalities to reduce their CSO related pollution problems (US-Government, 2000; USEPA, 1994). USEPA's CSO Control Policy, which is a framework for the national control of CSOs through a discharge elimination system, has set a deadline for communities dealing with CSOs to firstly meet technology based limitations, then develop long term CSO control plans and ultimately comply with all the requirements of the CWA (USEPA, 2012).

The city of Louisville, Kentucky, is dealing with an increasing number of CSOs that are a result of several issues. During heavy rainstorms, the sewer capacity is exceeded and the untreated combined stormwater runoff and sanitary sewage overflows into local streams and the Ohio River. However the Louisville and Jefferson County Metropolitan Sewer District (MSD) is committed to a consent decree with the State Department for Environmental Protection (KDEP), the Department of Justice and the USEPA to take the necessary measures to control the overflows (MSD, 2010a).

MSD has completed a comprehensive study of different methods that can be used to meet the requirements of the consent decree, eliminate SSOs and reduce CSOs. MSD's 
comprehensive plan is known as the Integrated Overflow Abatement Plan (IOAP) and consists of two steps. The first is to construct and/or maintain conventional methods such as large storage basins, pipelines, and sewage treatment facilities. Below are some of the conventional solutions that are typically used to address the problems caused by CSOs.

- Construction of relief structures, also known as CSO chambers which will divert any excess flow out of the combined sewer system either to the nearest water course or to storage for subsequent discharge to the treatment works (Harwood \& Saul, 2001).

- Optimization of the treatment facilities and employment of methods such as coagulation of CSOs and removing particulate sedimentation, a more suitable method for the cities where the current systems cannot be easily upgraded (El Samrani, Lartiges, \& Villiéras, 2008).

The second step is to use Low Impact Development (LID), which is a set of green approaches to reduce the source of stormwater runoff where it is generated, by infiltration, evapotranspiration, and reuse of stormwater runoff (USEPA, 2007). The idea of using LID is to mimic the conditions found in undeveloped areas. Below are some examples of the green infrastructures (GI) that MSD has reviewed (MSD, 2010b).

- Rain gardens and bio-swales

- Pervious pavements

- Green roofs

- Infiltration drains 
Choosing the right type of GI for a location depends on many factors, such as the characteristics of the project site, the space available, available project funds, etc. Although all types of GI share the same goal, they behave differently and each type needs to be designed, operated and maintained with specific methods and tools.

As a part of MSD's comprehensive plan, green stormwater mitigation programs are built to address the overflow events of the combined sewer watersheds located within the urban core of Louisville. The green stormwater infrastructure is designed to reduce the demand placed on the ageing sewer infrastructure system by diverting stormwater before it enters the collection system so as to eliminate SSOs and reduce the volume of CSOs. The first phase of the project, which was constructed in December 2011, consists of installing permeable pavement systems within watershed CSO130, encompassing 11.3 hectares, is located in the Butchertown neighborhood. Other phases of the project are still in progress, with the second phase installed in the winter and spring of 2013.

Once the green infrastructure systems are constructed within CSO130 it will be necessary to instrument and monitor them to determine if they are performing effectively. The instrumentation and monitoring plan was developed by first determining the factors which could be used to assess the effectiveness of the green infrastructure. Once these factors were identified, a list of commercially available instruments and pieces of equipment was prepared.

The data collected from the CSO130 GI will be used to assess the effectiveness of the current design and installation protocols and to identify the usage characteristics of the GI in place. The limited knowledge, and in some cases the ignorance about the long- 
term performance of the systems can be a factor for decision makers that slows down, or even halts, that adoption of green infrastructure technologies. In order to overcome current limits, a thorough investigation on monitoring methods for determining a permeable pavement system's conditions is required. An optimum monitoring plan would require minimum equipment, as well as providing enhanced design and construction suggestions to minimize the need of maintenance, and a maintenance protocol to efficiently recover and restore the infiltration capacity when needed.

\subsection{Objective}

The objective of this study is to determine the factors that affect the performance of the permeable pavement systems and use the obtained knowledge to provide siting, design and maintenance suggestions for both present and future GI. The defined objective of this research project is to conduct a thorough investigation of the permeable pavements installed in the Louisville CSO130 project and identify the factors that affect the surface clogging and maintenance performance of the installed GI. After a thorough investigation on the performance of the systems in Louisville CSO130 project and investigation on their surface clogging mechanisms, the field performance is used as the basis of laboratory experiments. Based upon this work and a review of the current knowledge of the factors affecting the performance and maintenance needs of permeable pavement systems, a methodology to investigate those missing factors was developed. A series of experiments in line with the defined methodology are then designed, set up and constructed. Using remote data collection techniques and other tools, the experiments were closely monitored and performance data collected. Finally the data was analyzed 
and turned into meaningful information and siting, design, operation and maintenance suggestions for both current and future permeable pavement systems were offered.

\subsection{Statement of the Problem}

Two interlocking concrete permeable pavement systems were installed in the Louisville, KY CSO130 demonstration project in December 2011. During the time they have been performing, they had to be maintained more often than the standard suggestions from the vendor. The biggest issue has been the surface clogging caused by the debris carried by stormwater runoff which adversely affects the systems' performance. Frequent maintenance does not only increase the operation and maintenance (OM) cost of the project; it causes traffic disruption and it can affect future GI projects.

Having a full and deep understanding of the characteristics of the clogging debris can help to determine the factors that do most damage to GI performance. The surface clogging debris of the Louisville CSO130 project was sampled and analyzed in five separate events. Using common analysis methods, such as testing for particle size distribution and organic content, the characteristics of the clogging debris were ascertained. However it was found that many other factors derived from the surrounding environment may affect performance.

Lessons learned from the Louisville CSO130 project have shown that the physical environment of the GI can significantly influence the performance, effectiveness, and maintenance needs of the system. The surrounding area, however, is not the only factor affecting the performance of the GI; the characteristics of the paver blocks and the 
amount and type of sediment carried by stormwater runoff also affect performance. An optimum outcome can be expected only from a full and in depth analysis of the effects on performance of the physical environment and the characteristics of the GI system.

Since there has been little previous work on the effects of the physical environment and other factors mentioned above, the work must start by determining the effects on performance of a basic set of variables. In order to determine these variables, the existing GI systems in the Louisville CSO130 project and the Edison permeable parking lot in the USEPA facility were used as the basis and their specifications were compared with those recommended by the Interlocking Concrete Pavement Institute (ICPI).

\subsection{Potential Contributions of this Research}

Green infrastructure systems have been used to reduce stormwater runoff for many years and significant research work to understand their behavior has been completed. Much of the initial work, however, has focused on water quality (Berndtsson, Bengtsson, \& Jinno, 2009; Boucher, Tremwel, \& Campbell, 1995; Scholz \& Grabowiecki, 2007; Urbonas, 2003) and on the application of different types of GI, their effectiveness with or without comparison to other types of GI, and their failure models (Dreelin, Fowler, \& Ronald Carroll, 2006; Haselbach, Valavala, \& Montes, 2006; Scholz \& Grabowiecki, 2007; Yang \& Jiang, 2003). Another limitation in the current literature is that the research is focused on specific climates and the results obtained thus may not be as useful for others. 
One very similar study that had the greatest influence on the present work and the Louisville project GI is a study comparing three different types of GI (interlocking concrete pavers or ICP, permeable asphalt or PA and porous concrete or PC) that has been modeled on a small scale in a parking lot in the USEPA facility in Edison, New Jersey. This study has focused on water quality studies, the failure of different types of permeable pavement, and the instrumentation of the permeable pavement systems to monitor the performance, type and frequency of tests that indicate current performance, etc.

Although the installations in Edison have immense research value, the scope of such work is somewhat limited. EPA's study uses only a predefined design and configuration and seeks to monitor its effectiveness, while the experiments conducted for this research project promote a broader range of research, where the aim is to come up with an innovative approach to adjust or re-design a permeable pavement system to reach maximum efficiency with minimum maintenance. Some of the other aspects that are not included in the study conducted in Edison use different design patterns for the interlocking concrete pavements and their storage galleries, experiments on the installation of the monitoring instrument and investigate on different layout designs and their effects on the results.

As discussed above, this study focuses on new aspects of the work and uses the Louisville project to investigate the patterns that contribute to the performance or failure of a GI and use the data that is collected to validate or enhance the current designs, and compare the maintenance methods which are practiced periodically on the permeable pavement systems to determine new maintenance procedures. By its further research into 
the existing literature and by describing the work done, the significance of this research project becomes more evident.

In order to fully understand the design, construction methods, operation and maintenance needs of any GI that is to perform effectively for long periods with minimum maintenance, ineffectual methods must first be identified and eliminated. Methods that do work must be carefully investigated and their issues must be addressed.

Not enough is known about the effects of the physical environment on performance. The experience gained by the Louisville CSO130 case study has shown that environmental factors combined with product specifications can significantly influence the operating performance of the structures. These factors must be investigated in a comparable environment so that researchers are able to draw meaningful conclusions about their effects on GI performance.

To appropriately assess the environmental factors that contribute to GI performance, a laboratory test bed was constructed in Edison. The laboratory setup worked to recreate the physical condition of an installed permeable pavement system, but also incorporate an extensive instrumentation system. The physical model in the laboratory would enable the research to determine the effectiveness of specific design and operating configurations.

The physical environment of permeable pavement systems, such as the installation slope or pitch, and the characteristics of permeable paver blocks such as the gaps and the permeable joint filling material, will affect the performance, maintenance needs and the clogging patterns. Determining the effect of these factors will help advance 
tour abilities to predict the progression of surface clogging. As such, these factors were selected as the variables for the experiment so that their influence on the performance of the experimental system can be measured.

The physical model must appropriately replicate all of the significant aspects that are typical of full-scale GI system. Prior to conducting any type of laboratory experimentation, there are many aspects to decide and re-create in the lab, from the size of the physical experiment module to the type of materials used, monitoring instruments needed to collect performance data, type of products used, duration of experiments, methods used to simulate urban stormwater runoff, and many other factors that happen in real time. In order to fully replicate the real world scenario in a lab experiment, the sequence of events in the real world must all be logged and the factors thought to be affecting the performance of the GI system must be understood and re-created within practical limits.

After understading the weaknesses of the current systems and using a predefined hypothesis, a laboratory physical experiment was designed and constructed. The methods used with the laboratory model had to be repeatable and the recorded data had to be feasibly close to the real word data. The solution was intended to create a path that not only followed the state of the art at present, but also created a smoother path for other researchers. It was intended that the results of this research could be used to further develop academic knowledge about the performance and failure mechanism of permeable pavement systems. Finally the data collected during the experiments was used to investigate the effects of the variables on a performance experimental module. 


\section{LITERATURE REVIEW}

\subsection{Introduction}

The increasing impervious areas in urban environments and growing CSO related problems has created an enthusiasm in using different types of GI, including permeable pavement systems. However proper siting, design, construction, and maintenance are essential tools to optimize any existing and future GI and to and help achieve stormwater control goals.

The CSO130 GI installed in Louisville, KY, is an example of unknown factors and the extent of their effects on performance of permeable pavement systems. Lessons learned from the Louisville CSO130 project have shown that the physical environment of the GI can significantly influence the performance, effectiveness, and maintenance needs of the system. The surrounding area, however, is not the only factor affecting the performance of the GI; the characteristics of the paver blocks and the amount and type of sediment carried by stormwater runoff also affect performance. An optimum outcome can be expected only from a full and in depth analysis of the effects on performance of the physical environment and the characteristics of the GI system.

In order to conduct a full and in depth study of the effects of the physical environment on the performance of a permeable pavement system, all known factors affecting the performance must be fully analyzed and the extent of their effects studied. 
However in order to fully understand the factors affecting a system, the cause and effects leading to implementation of GI in urban environment must be analyzed.

In this chapter, research begins from early stages of the work and by studying combined sewer systems and how they have raised a need to eliminate sanitary sewer overflows and reduce combined sewer overflows. After understanding the source of the problem, common GI practices designed to address such issues are studies and their advantages are investigated. Since Louisville CSO130 project uses permeable pavement systems to address CSO related problem, the focus of Literature Review is on this type of GI, with thorough investigation of their mechanisms, failure modes and maintenance needs.

After a complete review of the current literature, it is possible to continue the path of investigating on performance of permeable pavement systems and contribute to this field of knowledge with minimum redundant research.

\subsection{Combined Sewer Overflow (CSO)}

A variety of approaches are used to convey stormwater and sanitary flows from urbanized areas. Many municipal areas in the United States use a combination of sanitary sewage systems, separate stormwater drainage systems, and combined sanitary and stormwater sewage systems. The sanitary sewer systems are designed to collect and convey the sewage from residential, industrial and commercial areas to a treatment facility where it is treated and then discharged to the water bodies (Moffa, 1997). The current combined sewer systems (CSS) are designed to collect sanitary sewage from residential, industrial and commercial buildings, which in normal conditions is called dry 
flow, and storm sewage, in addition to all named in the event of precipitation (wet flow). CSS are designed to provide enough conveyance capacity for sizeable storms whose return frequencies are as rare as 10 years (Field, Sullivan, \& Tafuri, 2003).

In typical designs the CSS is capable of handling 3 to 4 times the volume of the dry flow. However, the stormwater flow entering the systems may be significantly higher in extremely rainy conditions. To accommodate the excess flow at such times, there is a need for temporary storage and/or diversion of the flow to receiving streams as a relief system for the sewer network. These interceptors are known as combined sewer overflows (CSOs) (DECNY, 2012; Moffa, 1997).

Increasing urbanization and the associated increase in impervious surfaces are the main causes of the increasing volumes of stormwater runoff. With the development of urban and suburban areas, the proportion of the landscape associated with roofs, roads, sidewalks, etc. increases significantly. Increasing impervious surfaces disturb the balance of the hydrologic cycle. With more impervious surfaces, the time between the precipitation and accumulation of runoff decreases (Shuster et al., 2005). The consequences of this phenomenon are a reduction of infiltration into native soil and slower rate of ground water recharge into the water table. Other effects of the increase in impervious areas are a decrease in the time needed to reach peak runoff flow and an increase in the "flashiness" of the peak discharge flow (Arnold \& Gibbons, 1996).

Urban surface water runoff and storm sewer overflows are listed as primary sources of pollution by the Environmental Protection Agency. While the percentage of stream miles affected by the municipal discharges of sewage through combined sewer 
overflows and sewage treatment plant discharges reduced from around $14 \%$ to less than 10\% between 1994 and 2004, there are still near 23,000 miles of waterways which are heavily affected by this pollution source (USEPA, 1998, 2004). Similar studies have indicated a deterioration of the water quality of streams in areas where more than $10 \%$ of the watershed surfaces are impervious and a severe degradation of quality indicators where the ratio exceeded 25\% (CWP, 2003).

Moreover, the existing problem of CSOs which contain urban runoff generated from impervious surfaces and carrying pollutants, has caused deterioration in the condition of streams and rivers. When CSO that is untreated or has had minimum treatment enters waterways it damages the environment. U.S. EPA has recognized the consequences of CSOs on receiving waterways (USEPA, 1994), and states:

“CSOs consist of mixtures of domestic sewage, industrial and commercial wastewater and stormwater runoff. CSOs often contain high levels of suspended solids, pathogenic microorganisms, toxic pollutants, floatables, nutrients, oxygendemanding compounds, oil and grease and other pollutants. CSOs can cause exceedances of water quality standards. Such exceedances may pose risk to human health, threaten aquatic life and its habitat and impair the use and enjoyment of the Nation's waterways."

Deterioration of the quality of receiving water bodies and streams has been a common topic for many studies. However there are many CSO related problems and each study has focused on one or a few specific issues. Studies have shown that overflows of combined sewage into rivers and streams depletes the immediate dissolved oxygen within 
4 kilometers of the discharge point and delayed effects at the depth of the stream which usually last between 12 to 24 hours (Hvitved-Jacobsen, 1982). Another study focused on the presence of heavy metals found that storm runoff over transport pathways washes heavy metals off the road surface which eventually appear in waterways. This study finds that about $50 \%$ by mass of $\mathrm{Cd}, \mathrm{Cu}, \mathrm{Pb}$ and $\mathrm{Zn}$ in the roadway environment are smaller than $500-\mu \mathrm{m}$ and street sweeping is effective for collecting particles only if they are larger than $250-\mu \mathrm{m}$; consequently the other $50 \%$ of metals are flushed with the stormwater runoff. The study shows that the concentration of heavy metals has a seasonal pattern with the lowest percentages in August to December and the highest in March; it also has a direct relation to the traffic density of the road environment (Hamilton et al., 1984). Another study conducted in Ontario, Canada discovered that the CSO toxicity of highway runoff with $24 \%$ was noticeably higher than other locations. The study used the following procedures to test toxicity: enzyme activity in sediments, the effect of water on fish and mussels' feeding and growth rates, ammonium, respiration rates in rainbow trout, and the presence of heavy metals in water (Marsalek et al., 1999). Pollution by CSO is fed from such sources as soil surfaces, urban surfaces and sewer sediments and CSO sampling and analysis reveal that Zn, phosphates, clay and sulfide species are the major sources of pollution caused by all feeders (El Samrani, Lartiges, Ghanbaja, Yvon, \& Kohler, 2004). Other studies have shown that CSO increases the presence of heavy metals, nutrients, organic matter, contaminants, pathogens, debris, etc. in waterways (El Samrani et al., 2004; El Samrani et al., 2008; Grout, Wiesner, \& Bottero, 1999; Hamilton et al., 1984; Marsalek et al., 1999). 


\subsection{Green Infrastructure (GI)}

The traditional approach to mitigating of stormwater flow and flooding has been to drain impervious surfaces as quickly and efficiently as possible. As urbanization increased and combined sewer overflows became a problem, it was solved by increasing the capacity of the sewer system to accommodate the excess runoff and improve the treatment facilities so that they could handle the increasing capacity of the sewer (a "grey solution”). While these remedies are effective, they are very costly to construct and maintain (Gunderson, Roseen, Janeski, Houle, \& Simpson, 2011). A consequence of this strategy was to increase erosion, degrade downstream ecological conditions and increase pollution within the waterways. Thus, the design hypothesis of these drainage systems was based on an incomplete understanding of the effects of the system as a whole.

The new thinking with respect to effective stormwater control considers flood reduction, but also includes other factors associated with the environment and sustainability (Roseen et al., 2012). With the capture of stormwater runoff in small quantities from frequent storms, and the environment being kept close to the predevelopment conditions, the need to construct additional treatment facilities would cease to exist. Solutions based on the new thinking of stormwater control are called Low Impact Development (LID).

Green stormwater infrastructure is an alternative design solution to mitigate CSO. A GI practice can be any design feature that aims to delay the peak flow of stormwater runoff or to collect stormwater runoff in small volumes and infiltrate them into the native soil. The concept of GI is based on recreating pre-development conditions in which around half the precipitation infiltrates the ground. 
Figure 1 illustrates a comparison of the pre and post-development condition of precipitation over a watershed. With an increasing ratio of pervious to impervious areas by means of rain gardens, green roofs, bioretention cells, permeable pavements, and other practices, GI assists a natural process that results in less volume for the peak flow of stormwater runoff, a recharge of groundwater, and protection of hydrological stability of the environment (Wastewater-Treatment-Division, 2011).
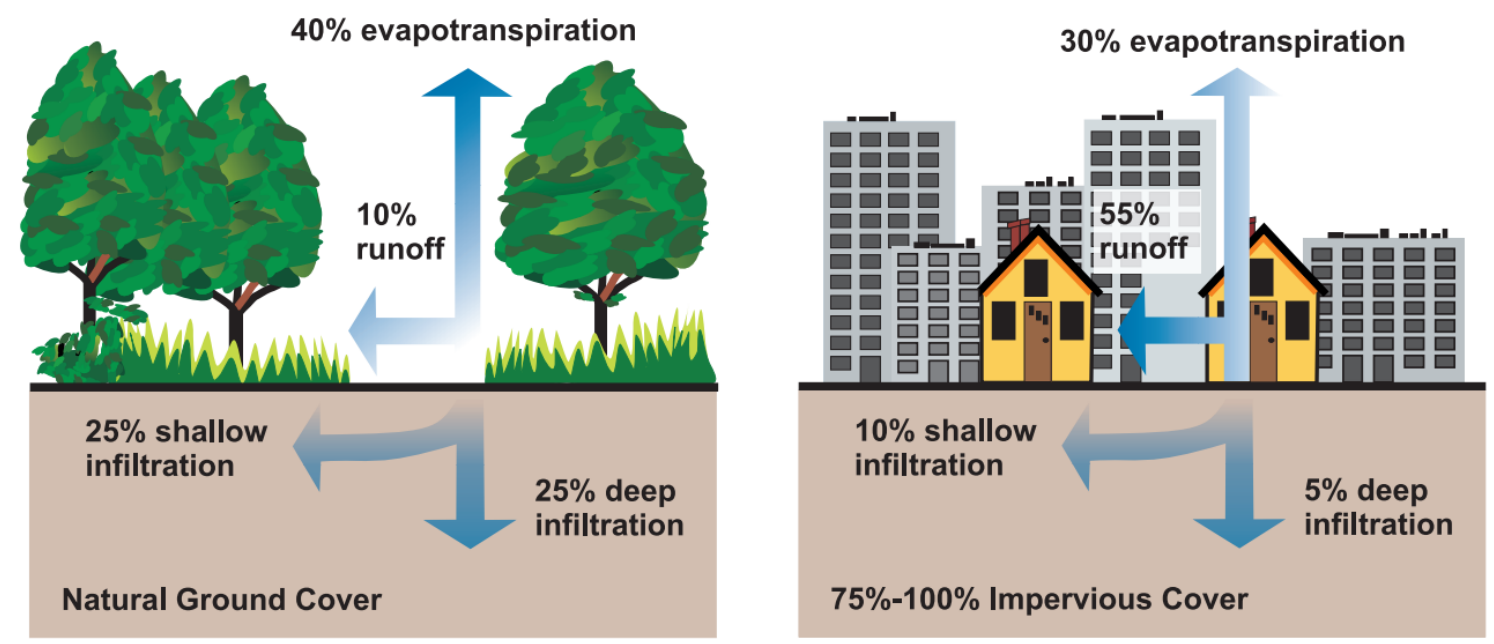

Figure 1: Comparison of pre-development and post-development of watershed (USEPA, 2003a)

Many studies have focused on determining the effectiveness of GI, and in order to attain this goal, basic criteria for what is effective must be set up. Effectiveness can be described as the ability to achieve the design goals and objectives within budget and practicality constraints. A study has concluded that GI's reduce total suspended solids, total nitrogen, and the volume of peak flow of stormwater runoff (Jaffe et al., 2010). Green roofs have been proven to reduce stormwater runoff and help in increase the energy efficiency of buildings, with absorption of close to $70 \%$ of the rainfall on buildings, also helping to reduce ground level ozone in urban areas (Clark, Adriaens, \& Talbot, 2008). The results of the latter study suggested that replacing a conventional roof 
of $2000 \mathrm{~m}^{2}$ by a green roof in Ann Arbor, Michigan cut the stormwater charge of $\$ 520$ per year and would cost nothing. The mean stormwater fee for the area was declared to be $\$ 0.17 / \mathrm{m}^{2}$ and with such reductions it decreased to $\$ 0.08 / \mathrm{m}^{2}$, therefore the cost incentives justified the project.

Other studies have focused on specific types of GI. For instance Shammaa, Zhuet et al. worked to retrofit and enhance the existing dry detention ponds to remove the total suspended solids (TSS) from stormwater. The goal of a dry pond is to maximize the settling of sediments, based on the size, specific gravity, and shape of the particle. It can be seen that multi-level design is crucial to obtaining the desired results with an optimal detention time of 24 to 40 hours and an efficiency rate of $60 \%$ for TSS removal at 24 hours of draw down time (Shammaa, Zhu, \& Labatiuk, 2002).

Bioretention cells are a common green infrastructure practice incorporated by cities to reduce stormwater flow as they have proved effective in reducing flooding and are aesthetically pleasing. In addition, studies in an urban area in North Carolina have found significant reductions in the concentration of TN, TKN, $\mathrm{NH}_{4}-\mathrm{N}, \mathrm{BOD}-5$, fecal coliform, E-coli, TSS, Cu, Zn, and Pb. However, while the concentration of many pollutants did decrease after infiltration through the bioretention cell, the concentration of iron significantly increased, and some pollutants such as $\mathrm{NO}_{2-3}-\mathrm{N}$ remained intact. Apart from water quality aspects, using bioretention cells proved to be effective in mitigating peak runoff generated by small and midsize storm events (Davis, Shokouhian, Sharma, Minami, \& Winogradoff, 2003; Hsieh \& Davis, 2005; Hunt, Smith, Jadlocki, Hathaway, \& Eubanks, 2008). Other studies have also verified that bioretention cells can reduce the 
volume of stormwater runoff, minimize peak flows, and recharge ground water while increasing evapotranspiration (Wossink \& Hunt, 2003).

Rain gardens are another application of GI, intended to reduce stormwater runoff and improve runoff quality. They are shallow depressions in the landscape that are planted with trees and/or shrubs. The surfaces of rain gardens are usually covered with a mulch layer. Rain gardens provide similar advantages in reducing the volume of stormwater runoff, removing pollutants such as nitrogen and phosphorous (M. E. Dietz \& Clausen, 2005a; MichaelE Dietz \& Clausen, 2005b).

Other types of GI have been studied and their effectiveness in achieving design goals has been assessed. Permeable pavements have always been considered an alternative to traditional impervious pavement systems. In an investigation of the effectiveness of four different types of permeable pavement systems constructed in a parking lot, no major signs of wear were found and the system was capable of removing significant amounts of copper and zinc. Motor oil that was observed in the surface runoff was also successfully removed after infiltration through the permeable surface (Brattebo \& Booth, 2003). The study used Grass-pave ${ }^{\circledR}$, Gravel-pave ${ }^{\circledR}$, Turf-stone ${ }^{\circledR}$ and Uni EcoStone ${ }^{\circledR}$, which are commercially available pavers and they all proved to be effective in virtually infiltrating all precipitation caused by the low intensity rainfalls of the Pacific Northwest. Although the results of this study are promising, it should be noted that the same good performance cannot be guaranteed everywhere (Brattebo \& Booth, 2003).

Many studies have focused attention on assessing the ability of GI in treating stormwater and reducing pollutant concentration and loadings in stormwater system 
discharge. According to one study researching the performance of wet ponds, grass swales, and stormwater wetlands (Strecker, Quigley, Urbonas, Jones, \& Clary, 2001) the inconsistencies of study methods and lack of information on the design methods for each GI resulted in different assessment results from each individual GI. The effectiveness of each GI has been reported in a specific way and therefore it is hard to compare the effectiveness of different installations. Studies based on a vegetated storage-infiltration GI; using a mathematical model of an idealized GI have shown that the hydrologic and pollutant removal performance of the GI can be highly variable (Wild \& Davis, 2009).

\subsection{Permeable Pavement Systems}

Permeable pavement GI are among most common practices constructed as an alternative to traditional impermeable pavements in urban and suburban areas. These systems are most suitable in areas with minimal traffic, such as parking lanes, parking lots, highway shoulders, and driveways (Brattebo \& Booth, 2003). Permeable pavement systems work by conveying stormwater runoff into an underground storage gallery and then infiltrating it into the native soil. In addition to effectively capturing stormwater runoff, permeable types of paver have also been shown to be providing non stormwater related advantages; for instance they are proven to be more functional in cold climates due to reduced salting needs in winter (Houle, 2008; Tennis, Leming, \& Akers, 2004).

Although permeable pavement systems provide several stormwater control management advantages, these types of GI cannot be used everywhere and there are numerous limiting factors on their applications. Vehicular traffic in the area, physical environment, and ongoing and proposed development plans for the site are among those limits. Permeable pavement systems are not suitable for locations with high traffic loads 
and volumes (Eban Z. Bean, William F. Hunt, \& David A. Bidelspach, 2007; Brattebo \& Booth, 2003). These systems may also require more careful winter maintenance (Michael Dietz, 2011) in order to avoid damage to the surface and the snow plow. To sum up their surface infiltration properties can be damaged due to surface clogging from the debris carried by stormwater runoff (Abbott \& Comino-Mateos, 2003; Amirjani, 2010; R. Brown \& Borst, 2013; González-Angullo, Castro, Rodríguez-Hernández, \& Davies, 2008; Haselbach et al., 2006; Siriwardene, Deletic, \& Fletcher, 2007).

Surface clogging along the gutter is believed to be the most frequently discussed deficiency of permeable pavement systems. The surface clogging debris, which is known also to carry the pollutants, causes the most damage to the top layer of the surface, 20 mm-to-25 mm (Krein \& Schorer, 2000; PICP, 2007; Roesner \& Kidner, 2007). The major contributors to the clogging are fine particles that accumulate in the void spaces of permeable surface and trap other particles (Pratt, Mantle, \& Schofield, 1995). The performance deficiencies caused by surface clogging has imposed some limitations on the use of permeable pavement systems. For instance, in 2003 the state of North Carolina did not give recognize the permeable pavement systems as a GI that would qualify owners to gain stormwater credits, yet they were identified as innovative approaches towards stormwater control (Eban Z. Bean et al., 2007).

When replacing conventional impervious pavement systems with permeable surfaces, a variety of options are available. Commonly used permeable pavers include: porous asphalt (PA), porous concrete (PC), and interlocking concrete pavers (ICP) (Eban Z. Bean et al., 2007; Borst, Rowe, Stander, \& O'Connor, 2010). PA is very similar to conventional hot mixed asphalt (HMA) and is a mix of bituminous materials which, due 
to its composition, contains about $22 \%$ void space. Historically, PA has been used to construct a paved surface that would provide more skid resistance in wet weather, reduce the splash and spray of traffic movement on wet pavements, and reduce rolling noise levels., With recent developments in the use of permeable surfaces to meet stormwater requirements, PA surfaces have grown in popularity and been used to mitigate stormwater runoff (Van Heystraeten \& Moraux, 1990). PA has also proven effective in attaining such goals as remediating the quality of stormwater runoff and removing some of the pollutants from stormwater (Legret, Colandini, \& Le Marc, 1996). Although PA provides a surface infiltration bed for stormwater runoff, it must be located over permeable soil to effectively transfer the infiltrated stormwater runoff to the surrounding soils and ultimately to the aquifers (D. C. Brown, 2003).

PC has been used to meet stormwater requirements and to allow stormwater runoff to infiltrate to ground, instead of running on an impermeable surface where it can absorb pollutants. This type of pavement, which is constructed using a carefully selected mix of cementitious material, water and aggregate, provides between $15 \%$ and $25 \%$ void space, allowing for surface infiltration rates as high as $200 \mathrm{~L} / \mathrm{m}^{2} / \mathrm{min}$. Like PA, this type of permeable pavement is intended for areas with low volumes of traffic such as parking los, residential roads, driveways, patios, sidewalks and pathways. PC has been particularly recommended to improve the durability of concrete in freeze-thaw cycles (Tyner, Wright, \& Dobbs, 2009). It has proven effective in reducing the pollutant loads in stormwater runoff, while meeting EPA stormwater requirements for tools to manage stormwater runoff (Tennis et al., 2004). 
ICP has been recognized as a tool to mitigate stormwater runoff in urban areas and reduce the effects of urban heat islands. It provides the same advantages as other types of BMP and a paver product is selected according to the needs of each project. Thick paver blocks can be used to accommodate areas with vehicular traffic, while thinner paver blocks can be used for sidewalks and pathways. The open area provided by the ICP is between $5 \%$ and $15 \%$ and in some configurations this is filled with highly permeable small aggregates (USEPA, 2010). Although the open surface area of ICP seems to be lower than the other two types, DR Smith, 2011, states that the surface infiltration rate is a better tool to assess and define the characteristics of permeable surfaces (Smith, 2011). With regard to the permeable joint material, some authors have recommended the use of a small size aggregate such as AASHTO No. 8, No. 89 or No. 9 stone, which helps to retain the pollutants in the top 20 to $25 \mathrm{~mm}$ of the surface (Smith, 2011).

All three types of permeable surface can suffer from surface clogging, where the openings and joints of the permeable surface become clogged. The clogging is caused by the fine particles carried by stormwater runoff and can increase with the age and use of the permeable pavement system. The USEPA interlocking concrete pavement factsheet suggests that when clogging increases, the surface infiltration rate decreases at first, but then levels off with time. This means that the permeable surface never completely loses its permeability and over long periods of time, a surface that started with infiltration rates of several hundreds of centimeters per hour will retain an infiltration rate of well over 2-3 centimeters per hour (USEPA, 2010). 


\subsection{Failure of Permeable Pavements}

As discussed above, the common issue with all types of permeable pavements is surface clogging due to the sediment carried by urban stormwater runoff (Elizabeth A. Fassman \& Blackbourn, 2011). The extent of damage caused by this sediment depends on the quantity or volume contained within the stormwater flow. Predicting sediment yields in urban stormwater runoff has been studied previously. Haster et al, 1994, investigated the sediment yields in stormwater runoff from urban areas and concluded that the rate and volume at which runoff occurs during storm events affects the amount of sediment carried. This study focuses on bare soil areas and indicates that by separating watersheds into smaller components, each of which have a unique land surface; it is easier to get a more accurate estimate of the amount of sediment carried by stormwater runoff (Haster \& James, 1994).

In a study conducted by Dr. Robert Pitt in the University of Alabama and the Center for Watershed Protection, stormwater data was collected from a representative number of permit holders under the National Pollutant Discharge Elimination System (NPDES) and municipal separate storm sewer system (MS4) and gathered in the National stormwater quality database (NSQD) (Pitt, 2004). This system divides different regions of the country into EPA rain zones (US-Government, 2012) and a median of sediment load for all the rain events in multiple locations in each zone is used to determine the total of suspended solids carried in that zone. For instance, the database has close to 4000 data points determining the $97 \mathrm{mg} / \mathrm{l}$ of sediment in region 2, which includes Kentucky (Pitt, Maestre, \& Morquecho, 2011). 
Although knowledge of the amount of sediment carried by urban stormwater runoff may help to predict the long term effectiveness of permeable pavement systems in a location, prediction of the time of failure is difficult, due to the fact that failure can be affected by a wide range of the factors contributing to the sediment supply (Pratt et al., 1995).

It is understood that surface clogging of the permeable pavement systems originates from the fine particles carried by the runoff water. Multiple studies have used a comparison between different permeable pavement types’ surface infiltration rates to study effects of surface clogging. These comparisons include surface infiltration rates before and after surface clogging (Eban Z. Bean et al., 2007; Scholz \& Grabowiecki, 2007). Some have gone as far as declaring that surface clogging is essentially a phenomenon restricted to the surface and no sign of sediment accumulation on any other level of the storage galleries has been noticed (Balades, Legret, \& Madiec, 1995).

The characteristics of the clogging debris are also though to affect the mechanism of surface clogging. Prior to responding to a specific type of debris the defects caused by that debris must be identified. There has been some effort to identify the clogging debris by understanding the characteristics of the sediment carried by urban stormwater runoff. Kayhanian et al have examined the characteristics of the suspended solids in urban runoff, concluding that the density of the particles in the runoff has a close relationship with particle size distribution. They add that a smaller range of particles carried contain more organic matter, which justifies their lighter densities. At the same time, the density of runoff in their investigation was about 1.5 to $1.8 \mathrm{gr} / \mathrm{cm}^{3}$ (Kayhanian, Rasa, Vichare, \& Leatherbarrow, 2008). 
Other factors that are thought to contribute to surface clogging include the slope and orientation of the installation. Fassman et al 2010 predict that on steep slopes during intense or frequent rainfalls the surface may fail to absorb all the stormwater (Elizabeth A Fassman \& Blackbourn, 2010). In a research study conducted by the same authors, they conclude that the steep slope of GI has also contributed to the displacement of the permeable joint material. Thus, in order to design a system that is less susceptible to surface clogging, it is also important to understand the influence of structure orientation, surface pores and open gaps and the corresponding resistance to clogging (Deo, Sumanasooriya, \& Neithalath, 2010).

\subsection{Maintenance}

Clogging, which can lead to the failure of permeable pavement systems, is a constant threat to the performance of permeable pavement systems. Acknowledging this threat and planning to prevent surface clogging from advancing on the surface as well as having remedial maintenance plans to restore the system is an essential tool for keeping the GI in a good working condition (Sansalone, Kuang, Ying, \& Ranieri, 2011).

Most of the installed permeable pavement systems do not consider the costs and labor required to maintain the systems at the planning stage. For the few projects that do acknowledge these costs, the appropriate maintenance method is selected by the availability of the methods chosen, rather than the most effective methods to restore and maintain the system in good working condition (Vancura, MacDonald, \& Khazanovich, 2012). Among the ways to maintain and restore surface infiltration to the permeable surface are using items of equipment, such as a vacuum truck street sweeper, regenerative air street sweeper, vacuum truck with a suction hose, or pressure washing. Combining 
these methods may also be effective (Chopra, Kakuturu, Ballock, Spence, \& Wanielista, 2010).

The studies of the effects of rejuvenation methods for restoring surface infiltration to permeable pavements offer different suggestions. For instance Chopra et al 2010 conclude that pressure washing the permeable surface is a more effective method than vacuum sweeping. However an excessive use of pressure washing may cause the pollutants to be pushed to the lower layers of the storage gallery and ultimately into the groundwater (Chopra et al., 2010).

ICPI suggests that surface clogging occurs in the top 20 to $25 \mathrm{~mm}$ of the permeable surface (Smith, 2011). Studies conducted on the density of surface clogging, using gamma rays and visual examinations using scanners have also confirmed that the clogging is limited to the top $20 \mathrm{~mm}$ of the surface structure (Balades et al., 1995; Kevern, 2010). With this in mind a combination of remedial maintenance methods and preventive maintenance using suction tools such as regenerative sweeper trucks and sediment traps can be used to maintain and unclog the permeable surface (Balades et al., 1995).

Maintenance can be divided into preventive and remedial treatments. Another categorization for maintenance treatments of a permeable surface can be made by dividing them into maintenance treatments for permeability and those for pavement serviceability. From the first category, Kevern 2010 suggests that the amount of sediment carried by stormwater and the slope of the pavement must be taken into consideration, as maintenance is very site dependent. Clogging, he claims, most often occurs when erosion 
control techniques in adjacent construction sites are poorly implemented or not used and with routine cleaning the effects of such treatment can be controlled. Other maintenance methods including pavement distresses, raveling, and rutting have also proven to help the permeability of the surface (Kevern, 2010).

The effect of different materials on surface clogging must also be taken into consideration and maintenance should be planned accordingly. For instance a study has concluded that silt and clay particles migrate to lower layers of the storage gallery, while sand particles clog the surface (Kevern, 2010). Another study focusing on construction debris as the clogging material has determined that even with a fully clogged surface, one fifth of the runoff arising from intense rain is infiltrated through the permeable surface and planning maintenance according to the intensity of rainfall for the specific area and the conditions of clogging can improve the efficiency of the maintenance treatments (González-Angullo et al., 2008).

\subsection{Conclusion}

There have been many applications of GI across the US and around the globe; however, a review of the current literature has revealed a gap in the understanding and knowledge of performance of permeable pavement GI in different conditions. What is missing includes the assessment of different application of a certain GI by comparing them in different locations. Cities, municipalities, and private entities have been using different types of GI to address their stormwater needs; however, there has been no coordination between them. Moreover, the current understanding of the factors that affect the performance of each system is somewhat limited. The current GI systems are not designed precisely and show limited understanding of the factors that affect their 
performance, thus creating a GI system that is susceptible to many environmental factors which reduce their efficiency and may reveal weaknesses.

The current literature fails to consider some of the aspects of permeable pavement systems and their possible effect on the surface clogging, maintenance of the systems, and ultimately on the performance of GI. Different suggestions have been provided and each study has used a different set of tools and equipment to investigate the effectiveness of maintenance treatments and performance of the systems. The characteristics of the paver product used, the amount and characteristics of the sediment carried by urban stormwater runoff, and the characteristics of the location where the GI is installed are among those factors commonly neglected in the current literature. Research in this field is relatively new and is evolving. Although using GI to mitigate the impact of increasing impervious urban development is promising, the GI systems still suffer from inaccurate understanding, which may lead to poor siting of the systems (Michael Dietz, 2007). 


\section{CHARACTERIZATION OF THE SEDIMENT FOUND IN URBAN STORMWATER RUNOFF}

\subsection{Introduction}

Permeable pavement systems have been shown to be very effective at infiltrating stormwater runoff and reducing the transfer of pollutants into waterways (Brattebo \& Booth, 2003), but the efficiency of these systems decreases with age and surface clogging. During the life of a permeable pavement system, fine particles accumulate in the openings on the surface. As the clogging proceeds, a trend emerges of more and more progressively smaller particles being trapped by the incumbent particles, (Pratt et al., 1995). Thus, once a system starts to clog, it progresses fairly rapidly towards the complete failure of the system.

The rate and extent to which permeable pavements clog are a function of the physical environment (Gerrits \& James, 2002). The sizes of clogging particles are among the environmental factors that affect the rate of clogging of permeable pavers. The presence of sandy fines in the clogging material reduces the surface infiltration rates dramatically (E. Bean, W. Hunt, \& D. Bidelspach, 2007). Pavement systems clogged with clean sand, however, may still infiltrate $81 \%$ of runoff generated from a $50 \mathrm{~mm} / \mathrm{h}$ simulated rain event (González-Angullo et al., 2008) . 
When clogging lowers the efficiency of GI, many communities restore the infiltration capacity by maintenance activities. Some functionality of these systems can indeed be recovered through routine maintenance operations, which include street sweeping or air blasting. Although much effort has gone into exploring the mechanisms of clogging and the characteristics of clogging material (R. Brown \& Borst, 2013; Haselbach et al., 2006; Welker, Jenkins, McCarthy, \& Nemirovsky, 2012), the current maintenance practices for permeable pavements are based on little understanding of such mechanisms and thus are vague. In order to provide a better maintenance method, the factors influencing the clogging must be identified.

Clogging may be blamed for the failure of some permeable pavement systems to provide expected long-term performance despite being designed appropriately. The extent and depth of clogging depends upon a number of environmental factors that cannot be assessed from a theoretical study. Only by examining the clogging material from the surface of the GI can the effects of clogging on the performance of permeable pavements be fully understood and appropriate maintenance techniques and schedules be identified. In order to provide better maintenance techniques for the GI, this research has analyzed the material recovered from the two different maintenance treatments in the GI project in Louisville, KY.

\subsection{Project Description}

The Louisville and Jefferson County Metropolitan Sewer District (MSD) is currently working to reduce the demands placed on its ageing infrastructure system by implementing green stormwater infrastructure. The objective of the program is to divert stormwater before it enters the collection system so as to eliminate SSOs and reduce 
CSOs. As it was explained in the Introduction, the first phase of the project consists of installing permeable pavement systems within watershed CSO130. CSO 130, extending over 11.3 hectares, is located in the Butchertown neighborhood. When the project began, the area was experiencing an average of 16 overflows per year as a result of stormwater runoff.

Within CSO 130 a series of green infrastructure practices were devised to infiltrate, retain and exfiltrate stormwater runoff during and after rain. The first phase of the GI installations consisted of two strips of permeable pavement, identified as $19 \mathrm{G}$ and $19 \mathrm{H}$ on Figure 2. These GI were designed to accommodate the runoff associated with the $9^{\text {th }}$ largest downpours of a typical year. In general, each GI is composed of a $60 \mathrm{~cm}$ deep storage gallery and a $3 \mathrm{~m}$ deep trench, as shown in Figure 3. The length of the trench is based on the quantity of flow from its tributary area. Table 1 provides more details on the dimensions of $19 \mathrm{G}$ and $19 \mathrm{H}$.

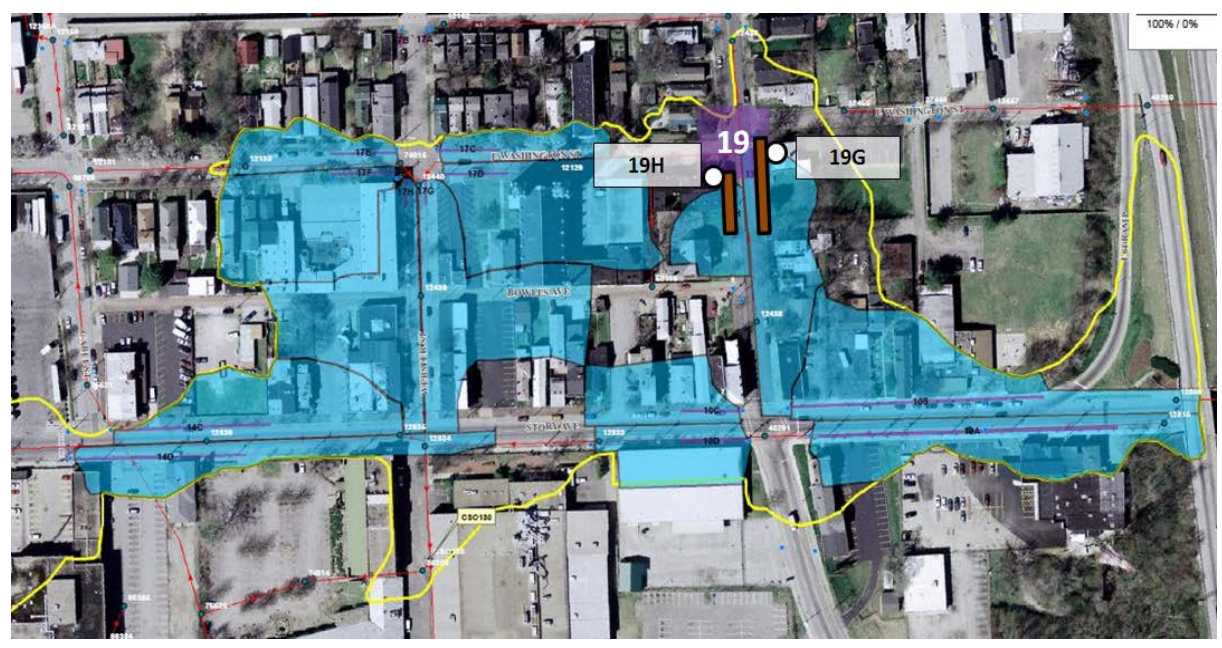

Figure 2: CSO130 area 
Table 1: Characteristics of CSO130 Controls

\begin{tabular}{|l|c|c|}
\hline Characteristics & $\mathbf{1 9}$ G & $\mathbf{1 9 ~ H}$ \\
\hline Drainage Area & $0.29 \mathrm{ha}$ & $0.11 \mathrm{ha}$ \\
\hline Percent Impervious Area & $61 \%$ & $59 \%$ \\
\hline Impervious Area: Control Area & $20: 1$ & $16: 1$ \\
\hline Length of Controls & $36.57 \mathrm{~m}$ & $16.76 \mathrm{~m}$ \\
\hline Width of Controls & $2.43 \mathrm{~m}$ & $2.43 \mathrm{~m}$ \\
\hline Storage Volume & $119 \mathrm{~m}^{3}$ & $55 \mathrm{~m}^{3}$ \\
\hline
\end{tabular}

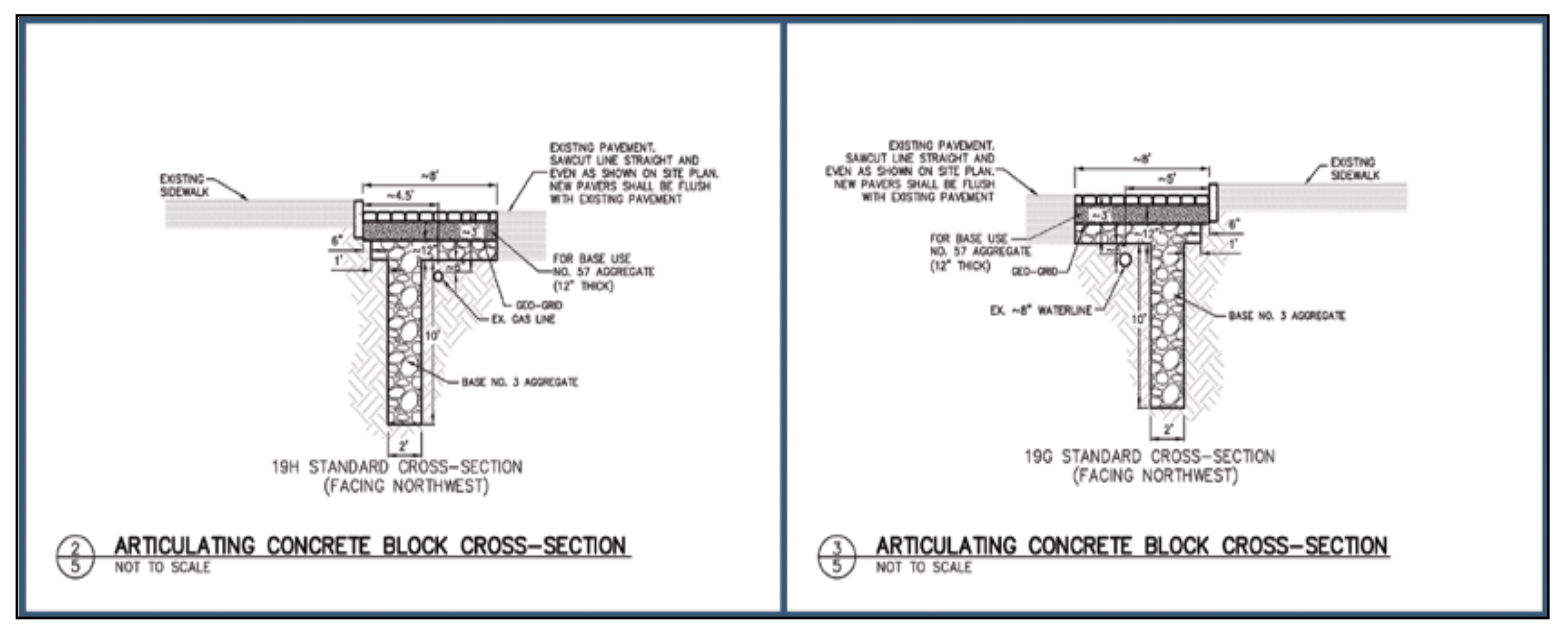

Figure 3: Cross-section of GI practices

During the normal operation of $19 \mathrm{G}$ and $19 \mathrm{H}$ it was expected that these permeable pavement systems would experience clogging due to debris accumulated in their drainage areas due to the traffic loading. Prior to their construction, a maintenance plan specified that $19 \mathrm{G}$ was to be maintained quarterly and $19 \mathrm{H}$ was to be maintained upon request. The maintenance specifications, however did not define a particular maintenance method for any of the GI (vacuum, sweeping, washing, air blowing, etc.). 
Table 2 lists the actual maintenance activities that were completed, with their methods.

Table 2: Detail of Precipitation on the Practices

\begin{tabular}{|l|c|c|c|}
\hline & $\begin{array}{c}\text { Vacuum } \\
\text { Maintenance for 19 } \\
\text { G on 3/20/2012 }\end{array}$ & $\begin{array}{c}\text { Air Pressure } \\
\text { Maintenance for 19 G } \\
\text { on 5/9/2012 }\end{array}$ & $\begin{array}{c}\text { Air Pressure } \\
\text { Maintenance for 19 H } \\
\text { on 5/9/2012 }\end{array}$ \\
\hline $\begin{array}{l}\text { Total rainfall } \\
(\mathrm{cm})\end{array}$ & 24.2 & 22 & 46.2 \\
\hline $\begin{array}{l}\text { Average rainfall } \\
\text { per event (cm) }\end{array}$ & 0.27 & 0.39 & 0.31 \\
\hline $\begin{array}{l}\text { Max rainfall } \\
\text { event (cm) }\end{array}$ & 2.2 & 3.9 & 3.9 \\
\hline $\begin{array}{l}\text { Days between } \\
\text { maintenance }\end{array}$ & 91 & 56 & 147 \\
\hline $\begin{array}{l}\text { Number of } \\
\text { rainfall events }\end{array}$ & 33 & 22 & 55 \\
\hline
\end{tabular}

\subsection{Methodology}

To assess the initial and long-term performance on the project of the permeable pavement systems $19 \mathrm{G}$ and $19 \mathrm{H}$, a series of surface infiltration tests were performed upon its completion (December 2011) and periodically thereafter. The surface infiltration tests were based on modified ASTM C1701 (Borst et al., 2010).

The pavement system maintenance methods evaluated included pressurized air blasts and a regenerative sweeper truck, ISUZU model NQR 435, as shown in Figure 4. The sweeper truck arrived with a clean and empty container. The sweeper truck covered a width of $330 \mathrm{~cm}$ using gutter brooms. The truck’s first run over the permeable pavement system was from the down gradient towards the up gradient, covering the whole width of the pavers and using only the vacuum chambers. The direction of sweeping was chosen 
after considering the water flow (towards the GI) and with the aim of limiting debris movement on the GI. After a visual inspection, the process was repeated using both brushes as well as the vacuum chamber. The entire content of the container was collected as a single sample.

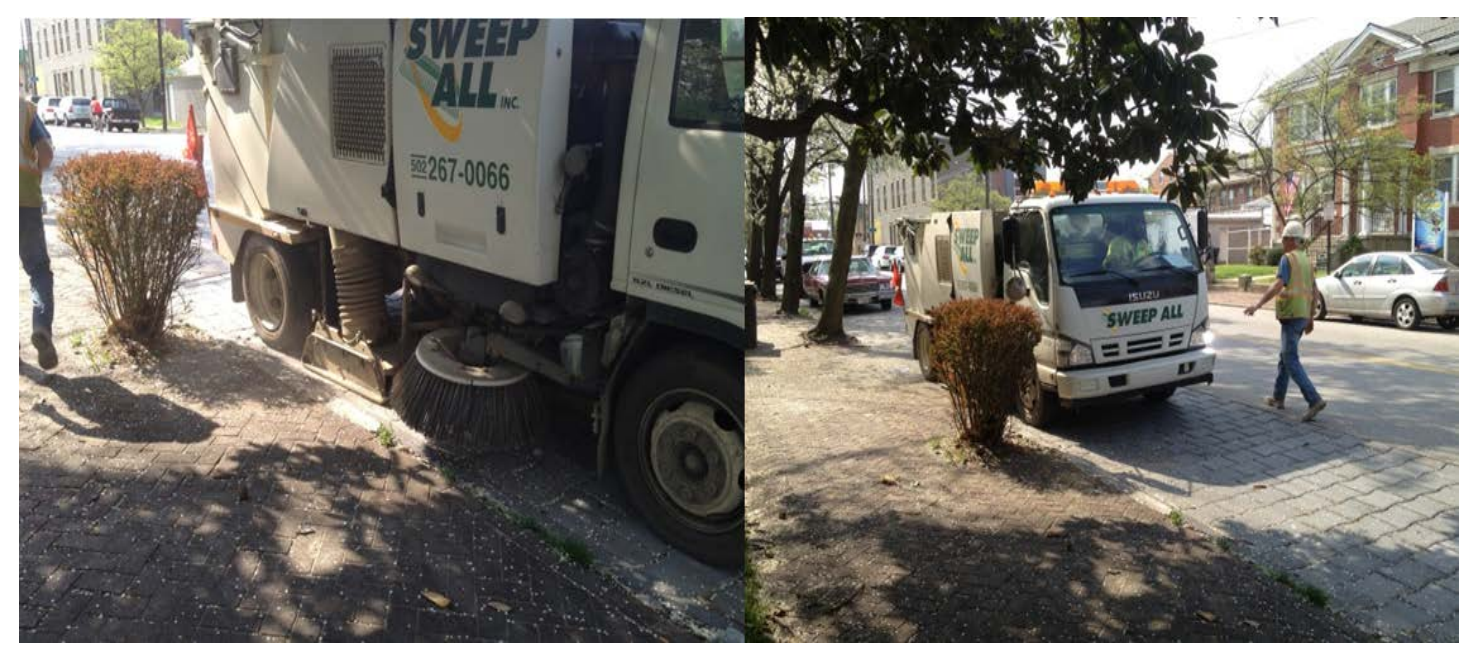

Figure 4: Regenerative sweeper truck with NQR 435 vaccum chamber (53 hp)

During the second round of maintenance for both permeable pavement systems, the effectiveness of pressurized air blasts for pavement cleaning was assessed. The objective of the pressurized air blasts was to blow out the debris from the paver gaps, working from the down gradient towards the gradient. To more accurately capture the spatial variation associated with the clogging debris, each practice was divided into smaller segments, as shown in Figure 6, and was cleaned independently. The debris that was extracted from each segment was swept to one corner and collected using a dry vacuum. The material recovered from each segment was collected as a separate sample; resulting in four samples for the longer practice and in two for the smaller one. Figure 5 is an image of the $19 \mathrm{G}$ surface before and after using the air blast tool for maintenance. 
Samples collected from both the sweeper and air blast practices were labeled and stored according to ASTM D4220 (ASTM, 2007a). Although the samples were stored in air-tight containers some organic decomposition was observed between the time of their retrieval and their analysis.

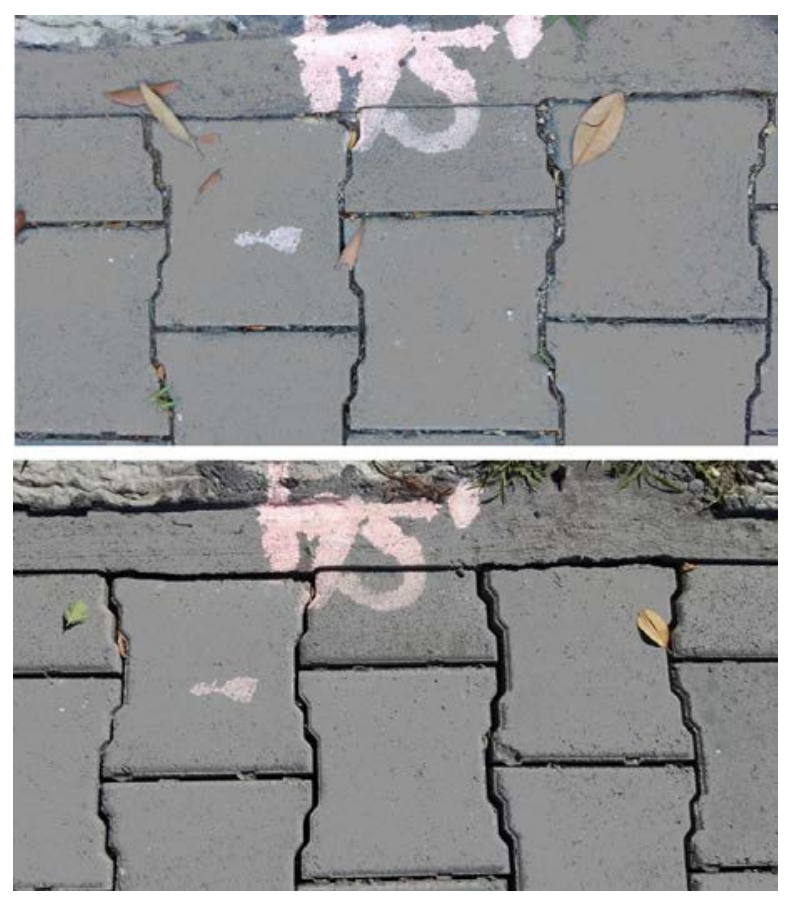

Figure 5: Visuals of practice $19 \mathrm{G}$ before (above) and after (below) the maintenance 


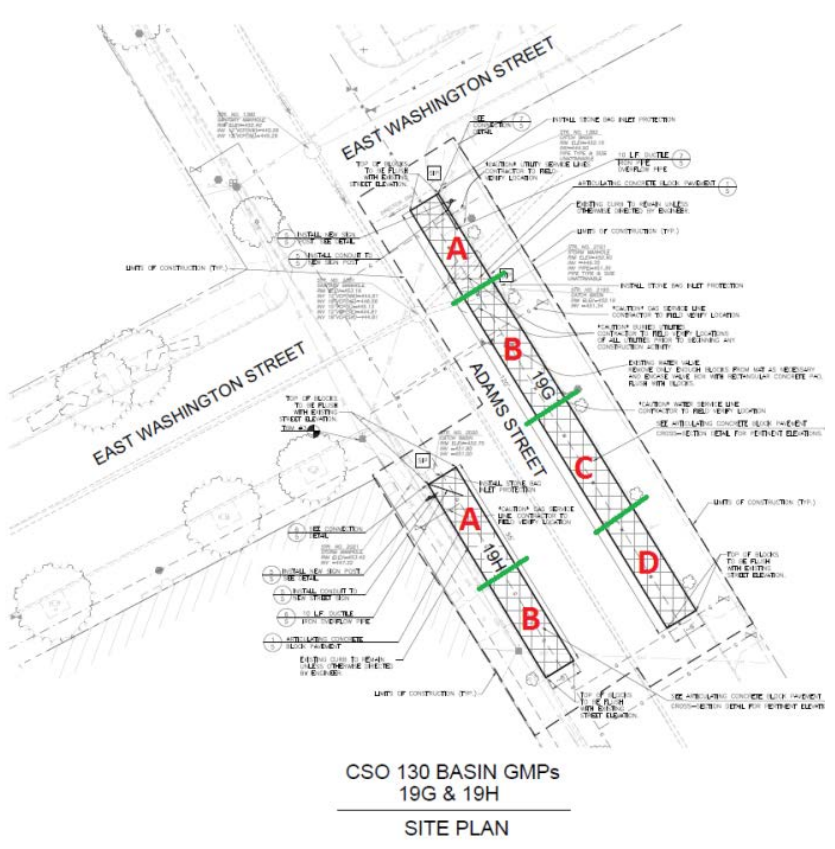

Figure 6: Site plan of CSO130 permeable pavement practices

\subsection{Analysis Methods}

\subsubsection{Particle size distribution (PSD)}

A PSD test was conducted to determine the particle size distribution of the collected samples (ASTM D6913 - 04). Accumulation of particles with various sizes can significantly decrease the surface infiltration rates of the permeable pavement systems and determining PSD is an initial step in analyzing the clogging debris. The concentration of sediments of particular size can affect the performance of the permeable pavement in specific spots by reducing surface infiltration rates; hence, any data on distribution of the fines is a necessary input for developing a maintenance plan.

The PSD of the clogging debris has direct effects on the depth of penetration and ultimately on the migration of sediments into the storage gallery of GI (Haselbach et al., 2006; Mata, 2008). Migration of sediments to the lower layers of the storage gallery, if 
not prevented or left untreated, will eventually create a layer with relatively lower infiltration rates on the base of the infiltration trench. Therefore a PSD test was carried out to find the location where most of the fines were concentrated.

In the PSD test, samples of both $19 \mathrm{G}$ and $19 \mathrm{H}$ permeable pavement systems obtained by both maintenance techniques were analyzed The results of this test were used to plot PSD curves and calculate the Cu (coefficient of uniformity) and Cc (coefficient of curvature). Table 3 lists the calculated coefficients for each sample, showing that the $\mathrm{Cu}$ is greater than 4 and the Cc between 1 and 3, indicating that the clogging debris was a well graded material.

Table 3: Coefficients of Uniformity and Curvature for Sampels

\begin{tabular}{|l|c|c|}
\hline Sample ID & $\mathrm{Cu}$ & $\mathrm{Cc}$ \\
\hline $19 \mathrm{G}-\mathrm{A}$ & 5.40 & 1.41 \\
\hline $19 \mathrm{G}-\mathrm{B}$ & 7.39 & 1.64 \\
\hline $19 \mathrm{G}-\mathrm{C}$ & 6.68 & 1.39 \\
\hline $19 \mathrm{G}-\mathrm{D}$ & 5.97 & 1.28 \\
\hline $19 \mathrm{H}-\mathrm{A}$ & 10.95 & 1.22 \\
\hline $19 \mathrm{H}-\mathrm{B}$ & 6.74 & 1.38 \\
\hline Vacuum Material 19 G & 10.43 & 1.90 \\
\hline
\end{tabular}

\subsubsection{Organics Matter Test}

Organics and their effects on the performance of the GI are unknown.

Determining the amount of organics in the clogging debris is essential for understanding the clogging mechanism, because the material is typically less dense and more likely to decay. In order to investigate the effects of organics, organic matter tests were conducted according to ASTM D 2974 - 07a (ASTM, 2007b). Since the samples were collected from a trafficked street, the litter mixed with the samples (i.e., plastic shreds) 
was manually removed in the preparation stage. Other foreign objects such as cigarette butts were left untouched. Table 4 lists the results of the organic matter test on the samples by the percentage of organics by mass in each sample.

Table 4: Organic Content

\begin{tabular}{|l|c|}
\hline Location & Percent of organics in the collected material \\
\hline $19 \mathrm{G}-\mathrm{A}$ & $\mathbf{4 7 \%}$ \\
\hline $19 \mathrm{G}-\mathrm{B}$ & $\mathbf{2 1 \%}$ \\
\hline $19 \mathrm{G}-\mathrm{C}$ & $\mathbf{2 1 \%}$ \\
\hline $19 \mathrm{G}-\mathrm{D}$ & $\mathbf{1 8 \%}$ \\
\hline $19 \mathrm{H}-\mathrm{A}$ & $\mathbf{1 9 \%}$ \\
\hline $19 \mathrm{H}-\mathrm{B}$ & $\mathbf{1 1 \%}$ \\
\hline Vacuum Material 19 G & $\mathbf{8 \%}$ \\
\hline
\end{tabular}

\subsubsection{Organics Gradation Test}

The PSD test on the collected samples of sediment collected from the surface and between the gaps of the permeable pavement systems in Louisville showed patterns in the material. The material retained on each sieve after testing showed that the composition of organic sediment versus the inorganic sediment varied by particle size. In order to further investigate this pattern, one sample was randomly selected, 19 G-B, and the material retained on each sieve was treated as a separate sample. The organic content of each of the samples was then determined using the same method described in ASTM D 2974 07a. The percentages of organics by mass on each sieve are presented in Table 5.

Table 5: Organic Matter Gradation Results for 19 G-B

\begin{tabular}{|l|r|}
\hline Sieve & Percent of Organics \\
\hline $3 / 8$ & $83 \%$ \\
\hline No. 4 & $36 \%$ \\
\hline No. 10 & $27 \%$ \\
\hline No. 20 & $35 \%$ \\
\hline No. 40 & $32 \%$ \\
\hline No. 80 & $22 \%$ \\
\hline
\end{tabular}




\begin{tabular}{|l|r|}
\hline No. 100 & $21 \%$ \\
\hline No. 200 & $13 \%$ \\
\hline Pan & $8 \%$ \\
\hline
\end{tabular}

\subsection{Results and Discussions}

Comparing the particle size distribution of the clogging material collected during maintenance with the gap size and other characteristics of the Interlocking Concrete Pavers (ICPs) can help to determine the performance of the ICP system and calculate its maintenance needs. The $6 \mathrm{~mm}$ gap in the permeable pavement systems in the Louisville test site provides stormwater runoff with an entrance into the storage gallery. This entrance, however, also makes the system vulnerable. Any object smaller than the paver's gap size can reduce the system's infiltration efficiency and contribute to clogging. Some objects bigger than the paver gaps may also reduce the surface infiltration rate. In order to quantify the surface clogging, the PSD test results are compared with the size of this gap. Three separate samples are used for this purpose and the plots are presented in Figure 7 through Figure 9.

Figure 7 illustrates the particle size distribution of the composite sample collected during the first maintenance of $19 \mathrm{G}$ using the regenerative sweeper truck. The $6 \mathrm{~mm}$ gap between the pavers is shown by a black vertical line on the graph. Most of the particles retrieved during this part of the maintenance were smaller than the $6 \mathrm{~mm}$ gap size and probably originated from between the paver gaps. Samples with a particle diameter greater than the paver gaps were also collected during the maintenance operations. Thus, clogging is also likely to result from materials that remain on the surface of the system, cover the infiltration gaps and restrict inflow. As this sample was a conglomerate 
obtained from material recovered during the entire cleaning operation, no further spatial variability or other defining characteristics could be discerned.

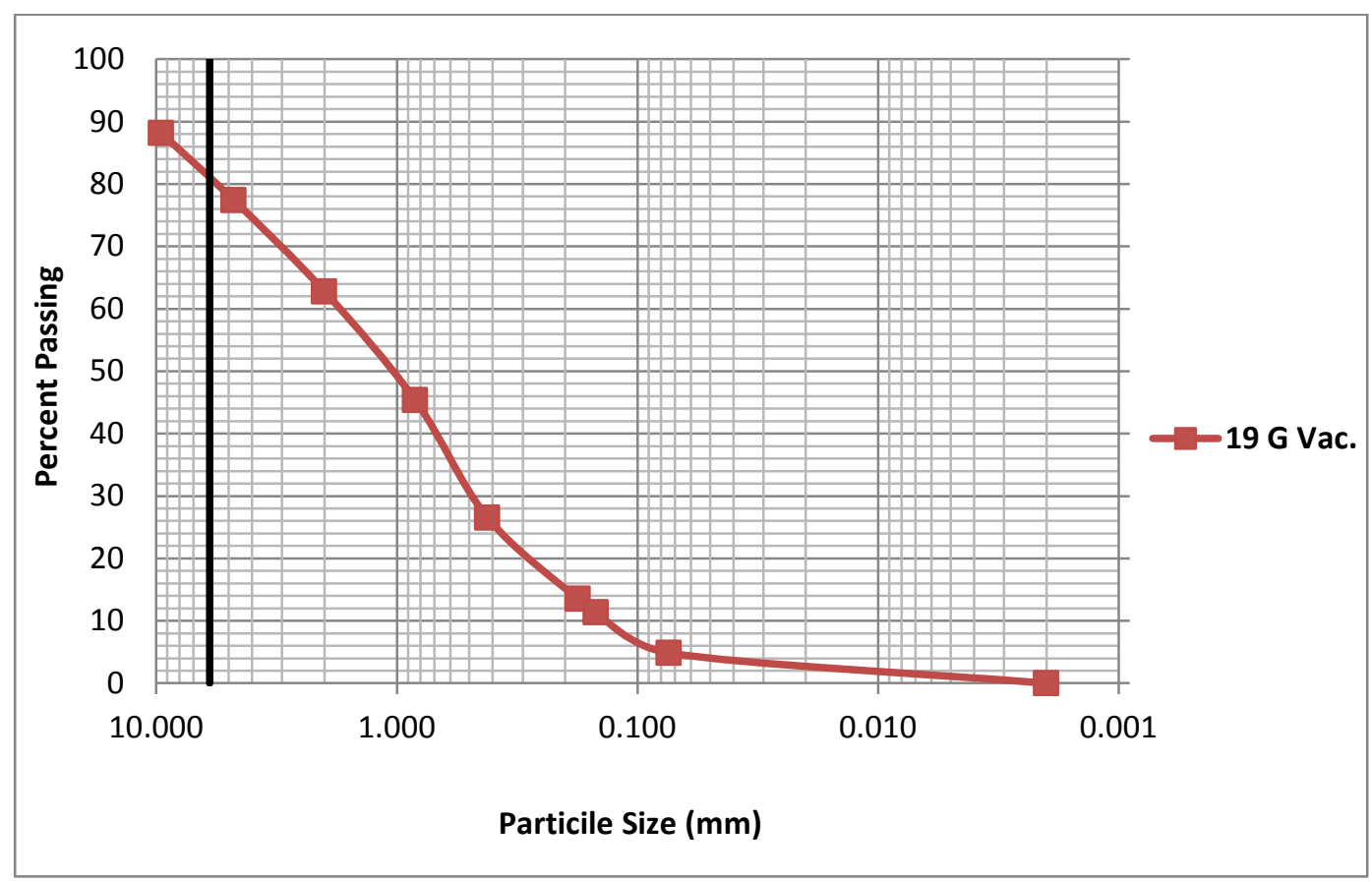

Figure 7: Grain size distribution curvature and percentages passing the paver gaps for 19 G, first maintenance

Figure 8 is an illustration of the particle size distribution of the samples taken

from $19 \mathrm{G}$, during the second maintenance. As indicated, the air blasting was conducted in stages such that materials were recovered from four distinct areas. As in the previous figure, the black line marks the $6 \mathrm{~mm}$ gap size of the pavers. The graph shows that the particles trapped in segment $\mathrm{D}$ are considerably finer than those trapped in segment $\mathrm{A}$. Segment D is the furthest up gradient segment. Thus, fine particles appear to be trapped by the system in the upper segments as the infiltration gaps become progressively more clogged by the larger particles.

On average - for $19 \mathrm{G}$ between $77 \%$ and $80 \%$ and for $19 \mathrm{H} 93 \%$ - the samples recovered using both maintenance methods are equal to or smaller than the $6 \mathrm{~mm}$ gap. A 
portion of the sample was determined to be larger than the gap size. This maybe occurred because of the oblong shape of some of the particles and the limited openings in the sieve. As described above, plastic shreds and other foreign objects that were included in the samples would not pass the sieves either.

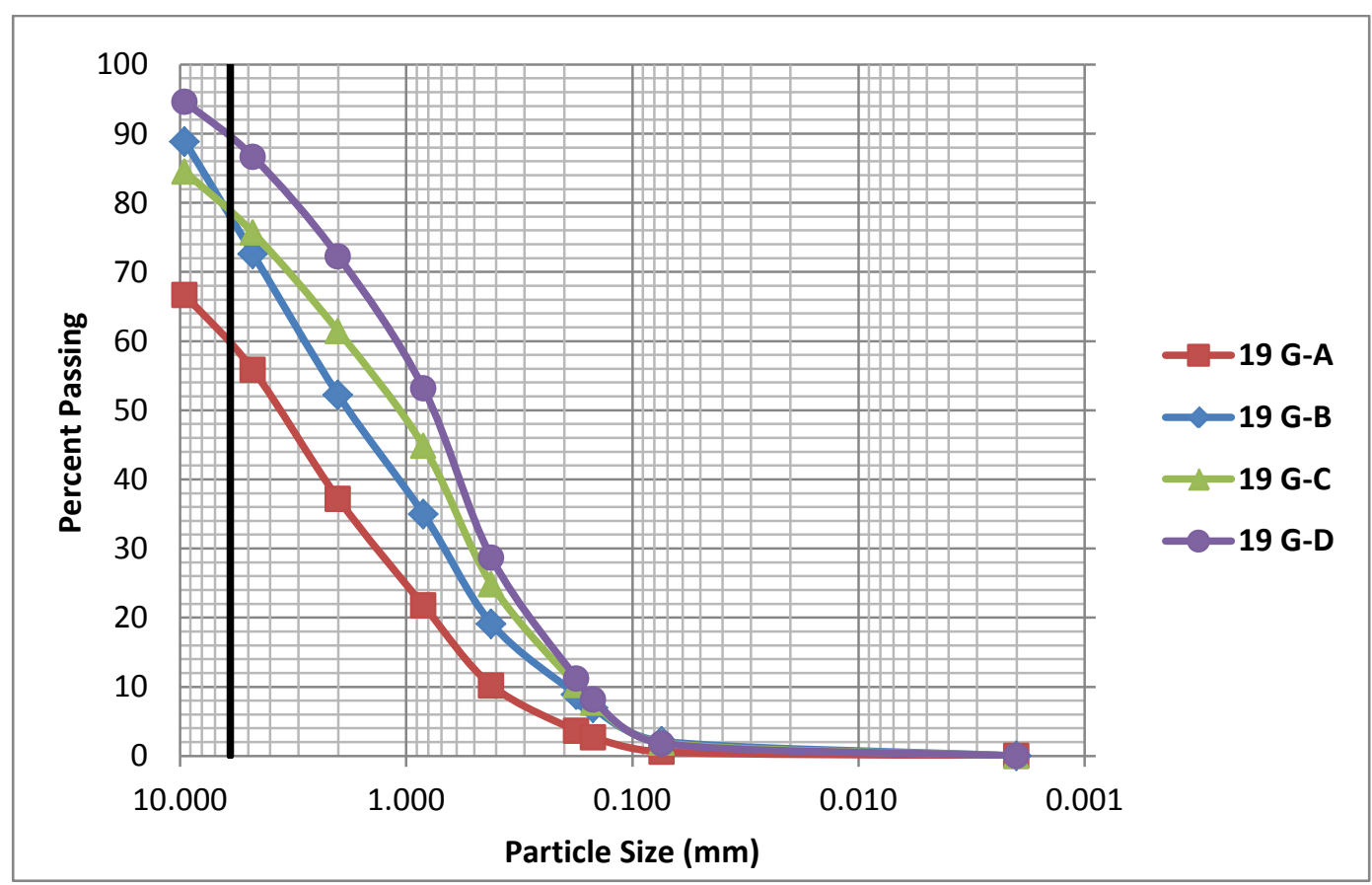

Figure 8: Grain size distribution curvature and percentages passing the paver gaps for $19 \mathrm{G}$, second maintenance

Visual observation of $19 \mathrm{H}$ prior to the second maintenance operations (the first for 19H) suggested that extensive clogging had occurred throughout its entire length. Figure 9 is a plot of the results of PSD on the samples obtained from $19 \mathrm{H}$ during the air blasting activities. The size distribution of samples obtained from both segments of this permeable pavement system was somewhat similar. Both the up gradient and down gradient segments of $19 \mathrm{H}$ experienced extensive clogging and the size of particles penetrating into the gaps were similar. It is evident that the PSD of the samples retrieved from $19 \mathrm{H}$ were noticeably different from those retrieved from $19 \mathrm{G}$. With the longer 
period of service for $19 \mathrm{H}, 147$ days, the results suggest that clogging caused by fine particles extended to the whole length of $19 \mathrm{H}$. However, $19 \mathrm{G}$, which had been in service for only 50 days, experienced clogging by fine particles in the up gradient segment only.

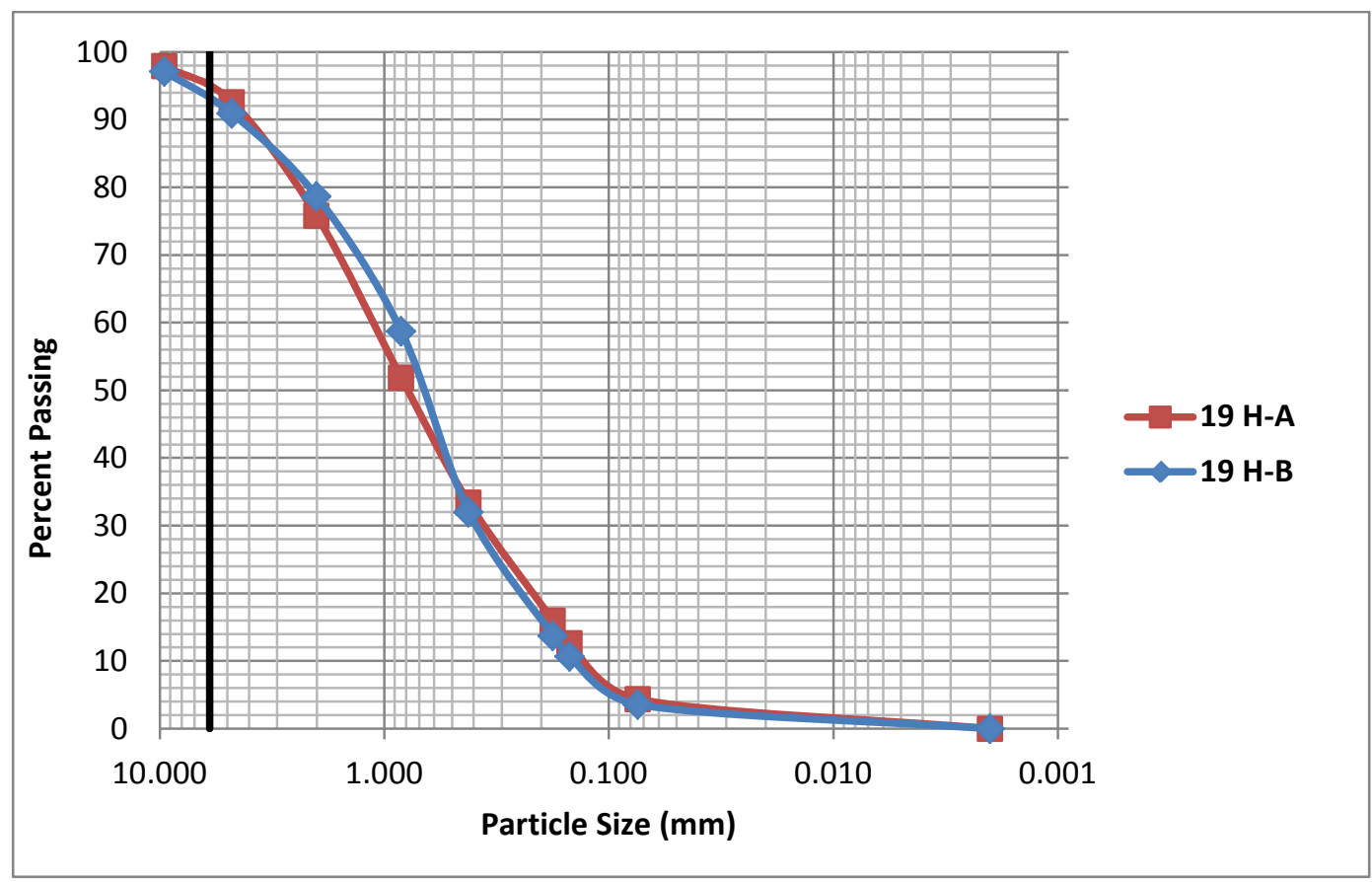

Figure 9: Grain size distribution curvature and percentages passing the paver gaps for $19 \mathrm{H}$, second maintenance

Figure 10 illustrates the organics particle distribution for 19 G-B from the second maintenance and also shows that materials smaller than the pavers gap sizes contain only between 10 and 30 per cent of organic matter. The mass percentage for inorganics capable of passing along the paver gaps is $98 \%$. In other words, in segment $19 \mathrm{G}-\mathrm{B}$, most of the organic particles were collected from the surface and most of the collected inorganics were collected from the gaps. 


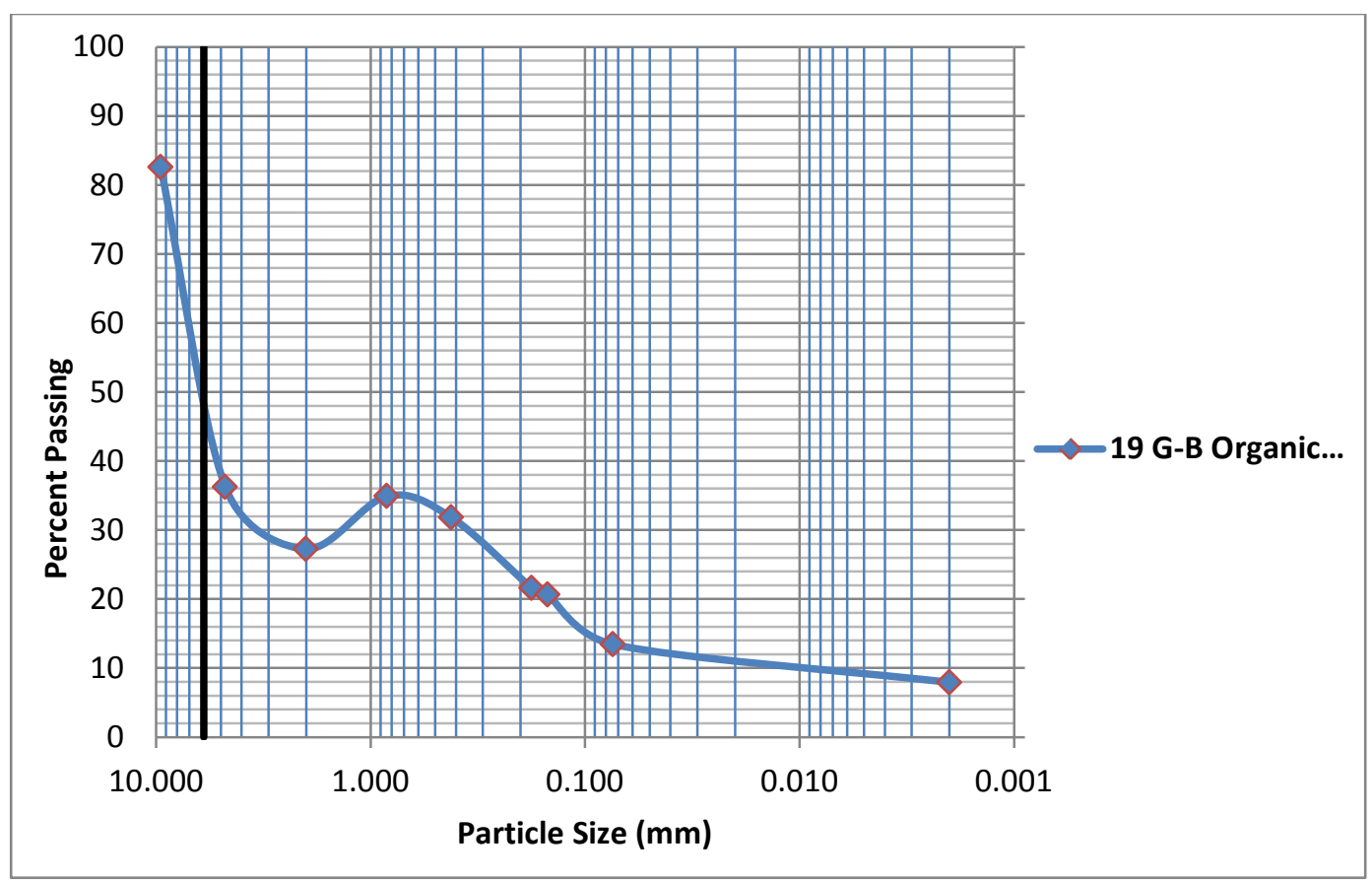

Figure 10: Percentage of organic contents of samples 19 G-B vs. passed sieve diameter

Samples to assess the characteristics of the clogging debris were retrieved from both the air blasting and the regenerative sweeper truck maintenance operations performed on the ICP blocks. The test results suggest that the fine particles cause clogging of the up gradient segments and they have the greatest effect on reducing the infiltration performance within these segments. The results obtained by the PSD test, compared to the reports in the current literature, indicate that well graded sediments carried by the stormwater flow enter the infiltration channels, the larger particles are initially captured and a trapping filter is created. As the filter develops, the surface infiltration rates decrease (AGF, 2000).

Within the service period of $19 \mathrm{G}$, the down gradient of the permeable pavement is mostly covered with organics which do not affect the performance of the permeable pavements as significantly as do the fine particles. The segments located adjacent to planting on the sidewalk on $19 \mathrm{G}$ have caused mulch to migrate towards the surface 
(Figure 10). Settlement in the structure of the pavement has also created an uneven surface that traps leaves and other organics. Results of similar studies have shown that the highest concentration of organic material recovered from a permeable pavement systems is located in the down gradient segments (Welker et al., 2012). The velocity of water running over the permeable pavement and carrying less dense particles towards down gradient segments may explain the concentration of organics at this location. Studies have also shown that the presence of a sidewalk will have a significant effect on the amount of sediment accumulated on the pavement (Viklander, 1998). The geometry of the sidewalk and height of the curb, as well as boundaries around the planting on the sidewalk can minimize the migration of sediment to the permeable pavement systems.

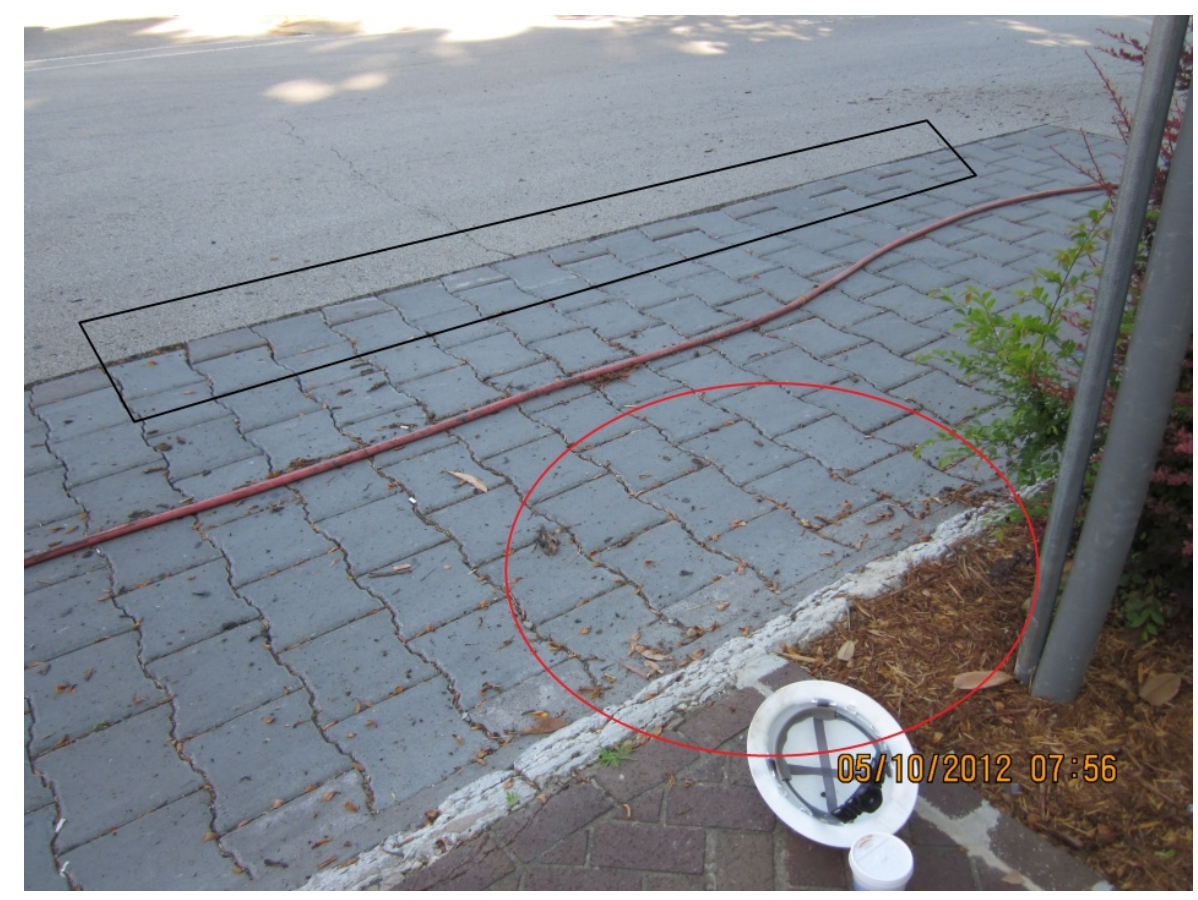

Figure 11: Segment A, 19 G, second maintenance 


\subsection{Conclusion}

The City of Louisville is currently installing numerous green infrastructure systems to help mitigate stormwater flows. As this work continues, planners want to understand how these systems clog, and the effectiveness of the maintenance methods. Within the Butchertown neighborhood of Louisville, two permeable pavement systems were installed, serving as pilot projects. One was first maintained using a regenerative sweeper and both were subsequently maintained using an air blasting tool. Both maintenance methods were effective in terms of regenerating the infiltration capability of the GI.

During the maintenance operations, debris samples were retrieved and used to assess the clogging characteristics of the GI. A clear assessment of the physical characteristics of the clogging material can help to create a better understanding of the clogging mechanism. The retrieved material was assessed on the basis of particle size distribution, organic content, and by percentage mass of organics.

The PSD assessment shows that particle clogging is a spatially progressive action. Particles are initially trapped in the up gradient segments and the reduced pore size works to trap even smaller particles. As the clogging progresses, a bypass is created for larger particles so that they are transported farther along the down gradient before being trapped. The organic content testing suggests that the up gradient segments accumulate fewer organics than the down gradient segments. Thus, the organic materials appear to be preferentially transported farther down the GI. The finding of a larger percentage of organic matter in the down gradient segments is reasonable, given that organic debris has 
a lower density than inorganic material and that larger items of debris may bypass the infiltration channels if partially obstructed.

To conclude, the use of an air pressure tool as a maintenance technique resulted in acceptable results. This method also enables researchers to divide the GI into smaller segments, facilitating the sampling process and raising its accuracy, compared to the use of a regenerative sweeper truck. In locations where the sediment has high clay content, or where the ability to perform quarterly maintenances is limited, it is suggested that the up gradient segments of the GI be maintained more frequently than the down gradient segments.

The maintenance needs of the pavers used in Louisville suggested by the paver vendor not only clearly underestimates the defects caused by surface clogging, but also fails to consider the effects on the progression of clogging of sediment characteristics which resemble those seen in Louisville (PaveDrain, 2013). Typically, it is thought that this surface clogging is affected by the amount of runoff and vehicular traffic at the site; however, various effects of other factors such as the characteristics of the pavers and slope of installation have been neglected. Using a well defined problem and investigating all aspects of the problem, in the current state of knowledge, it may be concluded that the GI permeable pavements systems installed in Louisville and the results of investigations made on them should form the backbone of this research and are key components for determining its next steps. Therefore the results of this Chapter are used to create a series of laboratory experiments to investigate on the effects of those neglected factors on the performance of the permeable pavement systems, their failure modes and ultimately their maintenance needs, which are explained in Chapter 4. 


\section{LABORATORY MODELING}

\subsection{Introduction}

Permeable pavement systems have been used as an alternative to offset the environmental effects of the increase in impervious urban surfaces. Many locations throughout the US and other countries have been using these systems to meet their stormwater reduction requirements, recharge groundwater basins, and improve their water quality by reducing nutrients, removing pollutants, etc. (Eban Z. Bean et al., 2007; Borst et al., 2010; Gerrits \& James, 2002; MSD, 2011; Scholz \& Grabowiecki, 2007; Urbonas, 2003). The installed GI practices share the same goal, but they also represent a significant geographical diversity. The performance of these systems can be greatly affected by the characteristics of the sediment in the urban stormwater runoff and Pitt et al. have shown that variation in the quantity and quality of the sediments carried by stormwater runoff in different locations can be significant (Pitt et al., 2011).

One of the challenges caused by the geographical diversity of the GI practices is that the location of each permeable pavement system is unique; therefore, it may be misleading to compare two systems installed in different locations and expect useful conclusions on their performance. Some researchers have argued that reduction in the volume of stormwater runoff can be used as a measure to compare different permeable 
pavement systems (Eban Zachary Bean, William Frederick Hunt, \& David Alan Bidelspach, 2007; Booth \& Leavitt, 1999; Collins, Hunt, \& Hathaway, 2008; Grote, Hubbard, Harvey, \& Rubin, 2005) but this method of comparison fails to consider the effect of such deficiencies as are not represented nor driven by the captured runoff volume, for example, the effects of the characteristics of the permeable surface, the amount of sediment carried by the stormwater runoff, and the characteristics of the carried sediment.

With the geographical diversity of the installed permeable pavement systems, it is harder to compare the effects of the physical environment and draw conclusions from them. Understanding the effects of physical environment on the performance of a permeable pavement system can help in creating a better and more effective maintenance plan based on the needs of the GI system (Ehsaei \& Rockaway, under review). Having a stable and consistent configuration and environment of for the permeable pavement surface, and studying the performance of this system, would enable researchers to assess the changes caused by various with respect to configuration and the physical environment. The physical environment used as the basis must include probable conditions and the changes in those conditions must be closely monitored.

In order to understand the factors that affect the performance of a GI system, and the extent of their effect on it, first a full scale system must be examined thoroughly and the results of this examination must be analyzed. There are many factors affecting the performance of permeable pavement surfaces; however, some factors have a greater effect on the performance than others. Observing constructed GI systems, such as those 
installed in the Louisville CSO130 project, and comparing their physical surroundings is very enlightening.

As the basis of this work, two installed GI systems were chosen for a preliminary investigation. The permeable pavement strips $19 \mathrm{G}$ and $19 \mathrm{H}$, installed on Adams Street, Louisville, KY, as the first phase of the CSO130 project to incorporate green infrastructure to reduce stormwater runoff, represent a typical urban application of GI. A semi-residential neighborhood was chosen for its installation; it had occasional heavy traffic, tree pits and plantings on the sidewalks. This GI uses articulated concrete mats with $6 \mathrm{~mm}$ gaps between the paver blocks, no joint filling material, and a 1\% longitudinal slope. The installed permeable pavements cover the entire width of the parking lane, which is $2.43 \mathrm{~m}$ (8 feet) (MSD, 2012).

The second project for the comparison is a permeable pavement parking lot in the EPA region 2 facility in Edison, NJ. The project installed porous asphalt, porous concrete and ICP as a selection of permeable surfaces. The ICP installation is located at an officetype parking lot, where most of the traffic consists of passenger vehicles, parking in the morning and leaving in the afternoon. There is very little planting around the parking lot and plants are well isolated by concrete curbs. This GI uses ICP with $12 \mathrm{~mm}$ gaps between the paver blocks, \#8 as the joint filling material and a 1\% longitudinal slope. The width of the GI covers two cars parked head to head and it runs for the entire length of the parking lot (Borst et al., 2010; R. Brown \& Borst, 2013).

The performance of permeable pavement systems, regardless of their goal, can be jeopardized by some of the surrounding physical features or the setting chosen for the 
system. The observations made of these two installations (Borst et al., 2010; R. Brown \& Borst, 2013; MSD, 2011) at two different locations show that they behave differently and much of this difference may result from the physical surroundings and the configurations of each system. For instance, the clogging seen in the permeable pavement system installed in Louisville was greatly affected by the characteristics of the sediment and the gap size, while a different installation environment and bigger gap size, along with the presence of joint filling material in the Edison installation enabled the system to last much longer. Obviously these claims are all speculative and an in depth analysis would be needed to fully understand the effects.

After reviewing the current literature, an experiment was designed to determine the characteristics of the surface clogging and how it affected permeable pavement systems. In order to research these characteristics, a series of hypotheses were written and used as the basis of this work. The hypotheses that led to the choice of experiments were:

1. The performance and surface clogging in permeable pavements is heavily affected by the physical environment and the characteristics of the ICP blocks.

2. The physical environment factors affecting the permeable pavers include the longitudinal slope of the installation.

3. The characteristics of ICP blocks include the size of the gaps between the pavers and the joint filling material in the pavers’ gaps.

The method used here is in essence based on the experiences gained by studying the projects in Louisville and Edison, The object of the experiment is to determine the different clogging patterns caused by having different paver products, different slopes 
and the joint filling material in between the pavers, using monitoring instruments to measure surface clogging.

These hypotheses were investigated using several tools. Firstly observations were used as an important and reliable tool for assessing the experiments and comparing them to conditions and observations elsewhere. Secondly, monitoring instruments resembling those installed in both the Louisville and Edison projects were used to measure performance data collected during the experiments. This data was then analyzed to find significant patterns. Finally the process of the experiments and secondary measurements taken during and after the experiments was used to assess other factors.

\subsection{Design/Methodology}

Creating a physical model of a real system is an effective tool to assess multiple scenarios in a controlled environment. The model was constructed and used to determine the effect of changes to the surface of a permeable pavement system on the progression of surface clogging. As discussed in the hypotheses of the work, these changes include the longitudinal slope of the installation, the gap size between the pavers and the permeable joint material. The flume constructed for the experiment was designed to accommodate conditions where changing configurations were possible.

The aim of the experiment was to mimic the conditions of a GI during and after rain events. During the operation of a permeable pavement system, a portion of the stormwater runoff, which is generated from the impervious grounds, runs toward the permeable surface. The runoff carries a load of sediments, which varies according to geographic location and the dominant land use in the watershed (Pitt et al., 2011). 
Depending on the amount of sediment carried, the stormwater runoff causes the performance of the permeable surface to deteriorate and eventually it becomes clogged. Pitt et al. 2004 conducted a national survey for the median concentrations reported in individual studies, showing that rain zone \#2 of the study (Figure 18), which includes Kentucky, has a median concentration of $97 \mathrm{mg} / \mathrm{l}$ of sediment load in mixed land uses (Pitt et al., 2011).

The present experiment was conducted in a wooden flume. The idea of using a flume was based on an existing HDPE flume in the EPA ORD facilities in Edison, NJ. However the dimensions of this flume were inappropriate for the present study and adjusting its dimensions, while keeping its structural integrity was not feasible. As an alternative, pressure treated plywood was chosen as the construction material.

The flume's permeable surface was created using ICP blocks. The dimensions of the flume were calculated with the aim of minimizing the half paver blocks to be used. The flume was set up under cover to allow testing in different weather conditions. The flume’s inner dimensions were $228.6 \mathrm{~cm}$ (90 inches) long, $55.88 \mathrm{~cm}$ (22 inches) wide and $60.96 \mathrm{~cm}$ (24 inches) deep. The dimensions and the approximate weight of the flume and the material, once filled, are listed in Table 6 .

The flume's weight is approximated from the construction material used, the aggregate, paver blocks and also the water flowing in the flume at any given time. The flume was set on three cinderblock supports, which enabled the slopes to be accurately adjusted. 
Table 6: Flume Dimensions and Weight

\begin{tabular}{|c|c|c|c|}
\hline \multicolumn{2}{|c|}{ Flume dimension (cm) } & \multirow{2}{*}{$\begin{array}{c}\text { Approximate } \\
\text { flume’s weight (kg) }\end{array}$} \\
\cline { 1 - 3 } Length & Width & Depth & 2000 \\
\hline 229 & 56 & 61 & 2000 \\
\hline
\end{tabular}

The flume's surface provides $1.28 \mathrm{~m}^{2}$ (13.75 sq. ft.) of pervious area. Industry suggestions indicate a maximum 5:1 ratio of impervious surface to pervious (Smith, 2011); however, sites like those in Louisville have ratios as high as 20:1. Because this study sought to determine the characteristics of the best management practices for stormwater control in worst case scenarios, conditions like those in the permeable pavement systems in Louisville were used in the simulation. The total area of the watershed feeding in to the flume was considered to be 21 times the area of the permeable surface or the equivalent of $26.83 \mathrm{~m}^{2}$ (288.75 sq. ft.).

\subsection{Construction}

Prior to construction, a static analysis was made. It had a $25 \%$ margin of safety in all numbers, to ensure the stability of the flume's structure, using the dead loads of the aggregate, pavers, stormwater, and the construction material of the flume. The construction material was chosen to bear the calculated loads on the structure. The main goal for the flume was not only to withstand the extreme weights and forces, but also to tolerate the repetitive loading and unloading of the pavers and at the same time be at an accessible height.

With the considerations mentioned, the flume was constructed by attaching together two layers of pressure treated plywood. The maximum thickness of the available 
plywood was $1.90 \mathrm{~cm}$ and therefore the flume was constructed using custom structure with the two layers of plywood glued and screwed together (Figure 12).

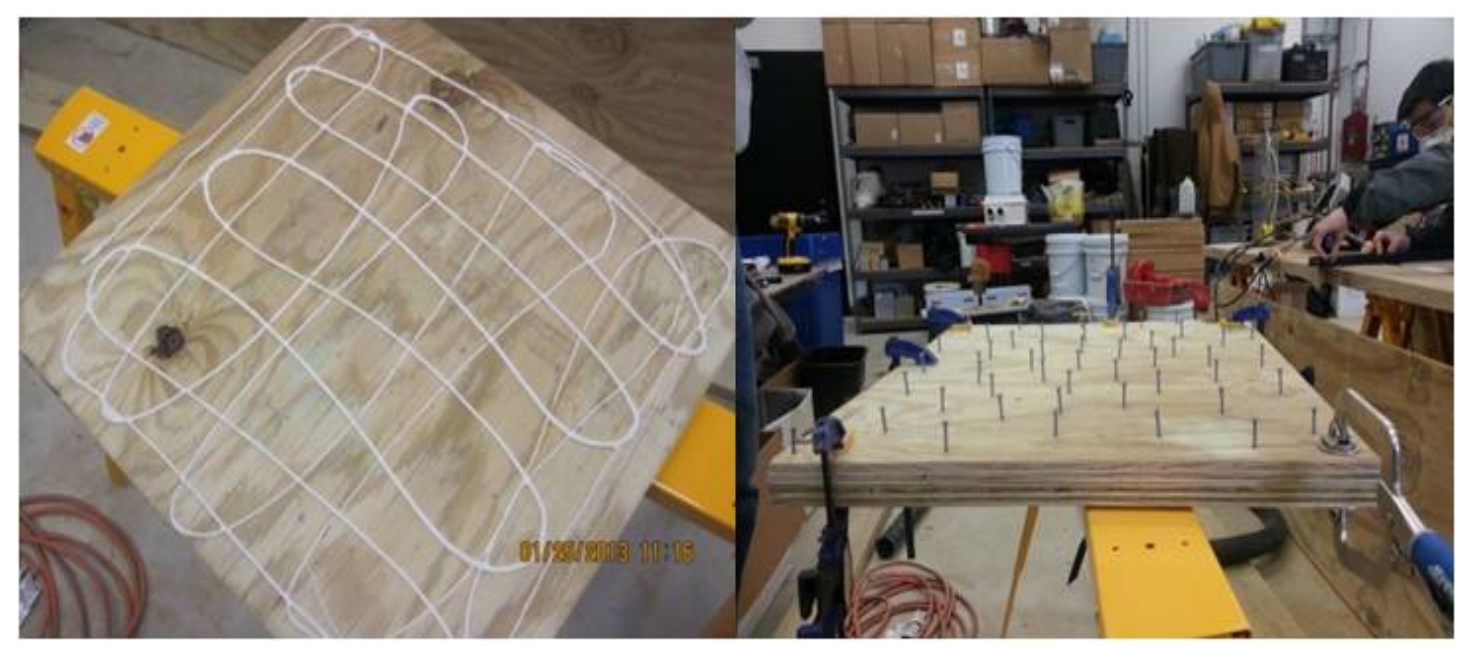

Figure 12: Construction of the flume's panels

The walls were constructed with additional wooden beams and steel L brackets to support the lateral forces. The supports of the flume were also reinforced using additional wooden beams (Figure 13). 


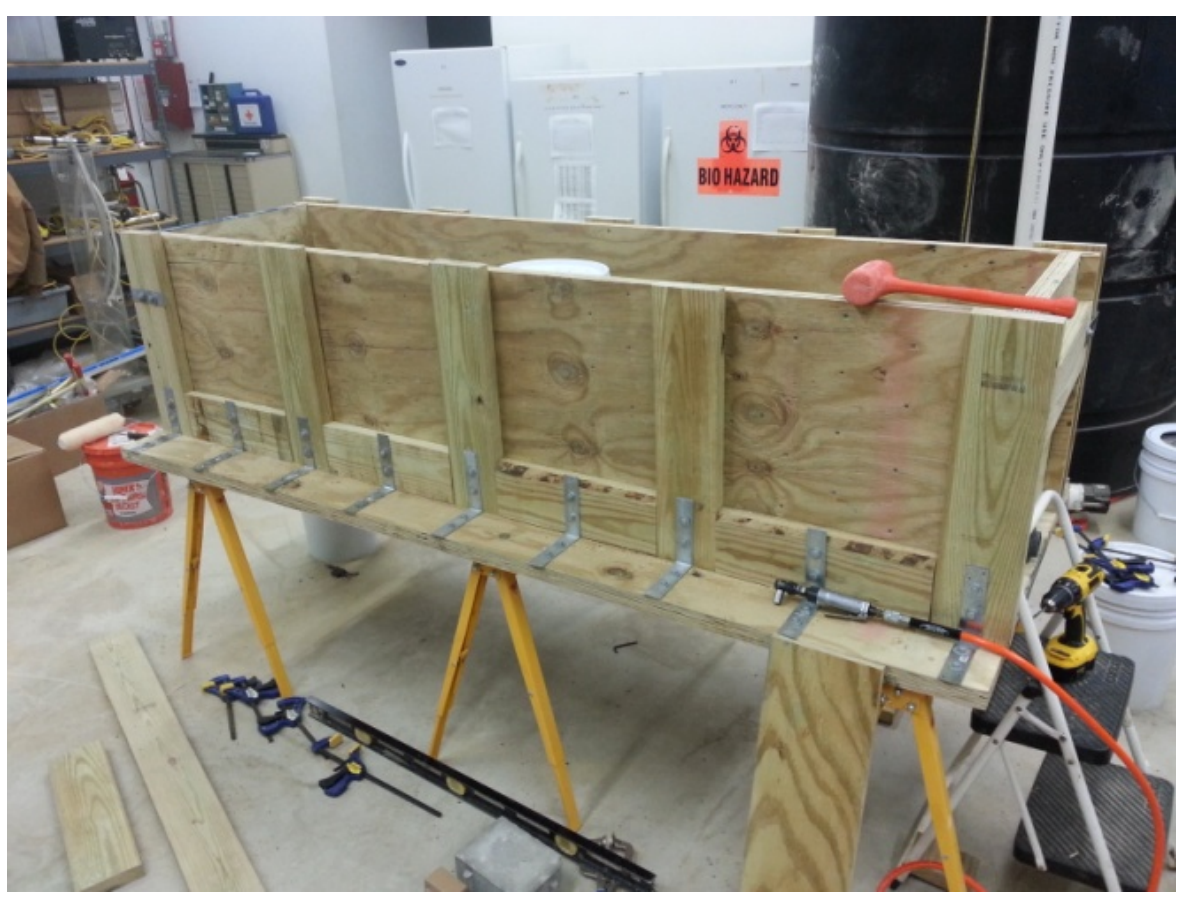

Figure 13: Structure of the flume

The bottom section of the flume was equipped with three $10 \mathrm{~cm}$ high check dams, which were spaced $57.15 \mathrm{~cm}$ (22.5 inches) from both each other and the end walls. Each check dam had a valve located $5 \mathrm{~cm}$ along of its up gradient and designed to be used as an optional access port for sampling water and making visual assessments of the conditions during the experiments.

In the end and in order to ensure that the flume would withstand the extreme forces during the period of the experiments, which was expected to take months, and to avoid damage and structural weakness caused by standing water, a truck bed liner material was used to waterproof the inside of the flume. The bed liner was applied after applying the primer material. After letting the bed liner cure in moist and warm conditions, the joints were sealed using a marine sealant product (Figure 14). 


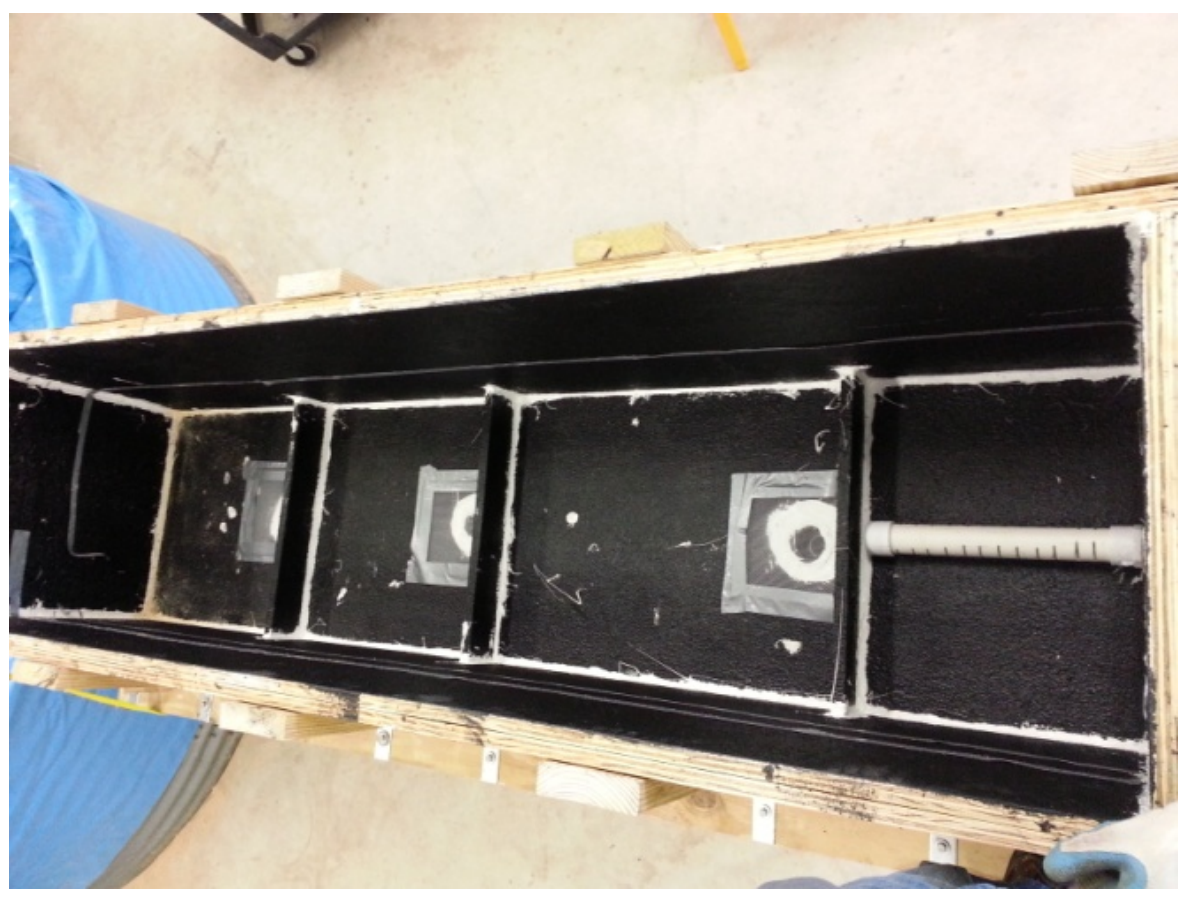

Figure 14: Inside of the flume with the liners and sealed joints

The flume's two key components were the feeder section and the effluent. The feeder was constructed within practical limits and with the goal of delivering stormwater runoff to the entire width of the surface, while keeping all the sediment in the runoff. The original plans were to mix the sediment with the stormwater runoff in the tank and use stirrers to keep the solids suspended; however, after experimenting with the sediment sizes used for the test, it was found that the method might result in some portion of the sediments settling in the stormwater tank and creating inconsistencies in the sediment loading.

As a substitute for the sediment delivery system, and after extensive research, it was concluded that the best method of delivering the sediment to the flume was to custom-make a funnel shape feeder inspired by the working mechanism of an eductor. Figure 15 illustrates the feeder section of the flume, where stormwater runoff was 
pumped from the experiment tank located on the left hand side and was then delivered to the flume on the right hand side. The feeder funnel, which was located in the middle, was the place where sediment entered the flow of the stormwater runoff.

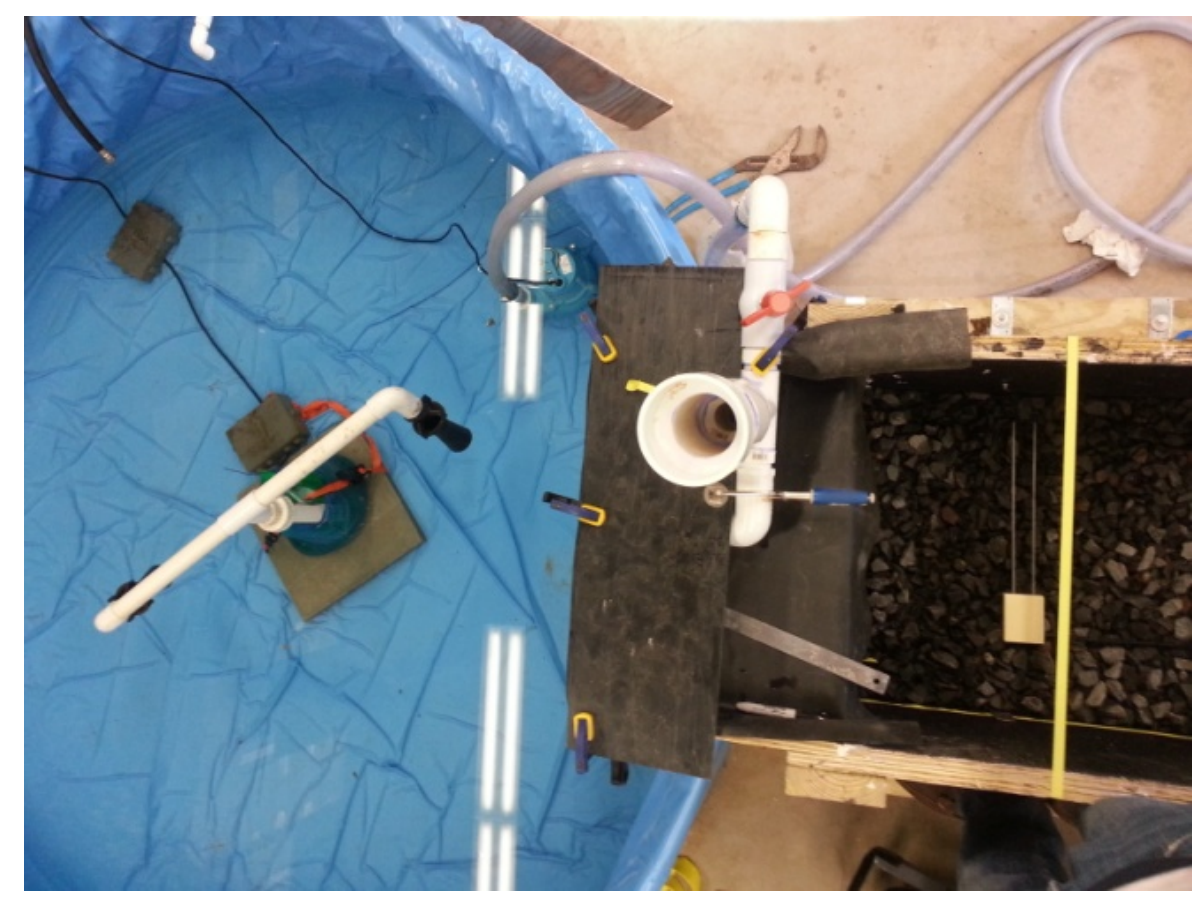

Figure 15: Flume's feeder with the up gradient stormwater tank

The effluent, which was a $5 \mathrm{~cm}$ (2 inches) slotted pipe was installed in the down gradient bottom section to drain the flume during the test. No decision on recycling the used stormwater was made in the process, since it was anticipated that some solids might remain in the runoff from the effluent. A series of pipes therefore directed the flow of effluent to the outside of the laboratory.

\subsection{Experiment Components}

\subsubsection{Storage Gallery}

A GI that uses permeable paver blocks to infiltrate water usually has a storage gallery, which is formed from a bedding layer, a base layer and a sub base layer. Studies 
have shown that the evaporation, infiltration and retention of runoff within the permeable structure are greatly influenced by the particle size distribution of the aggregates in the storage gallery (Scholz \& Grabowiecki, 2007). Each layer is designed to certain specifications; however, the common goal for the storage gallery is to provide capacity for the runoff to exfiltrate to the underlying soil. The depth of each layer depends on the design specifications and guidelines provided by each separate paver vendor. The Interlocking Concrete Paver Institute (ICPI) has issued design and installation guidelines that can be used for a variety of paver blocks (Smith, 2011). The recommended bedding layer's depth must generally be at least $5 \mathrm{~cm}$ underneath the pavers. The recommended material for the bedding layer is \#8 AASHTO aggregate. For the layer underneath the bedding layer, a base layer of \#57 aggregate is recommended. Since the present experiment was designed to investigate the behavior of the surface, these two layers met the requirements of the study.

The first $35.5 \mathrm{~cm}$ (14 inches) of the flume was filled with aggregate \#57 to create the base layer. The TDRs were buried at the $25.4 \mathrm{~cm}$ (10 inches) mark from the bottom of the flume. On top of the base layer, a bedding layer of $5.08 \mathrm{~cm}$ (2 inches) with aggregate \#8 is placed and pavers were set. The depth of the installation was designed to accommodate pavers of $8.25 \mathrm{~cm}$ (3.25 inches) plus an additional $10.16 \mathrm{~cm}$ (4 inches) of free space on the surface used to accommodate the runoff. The depth of each layer was derived from the recommendations of ICPI.

The AASHTO aggregates used as the storage gallery and the bedding layer of the flume were made of \#57 aggregate and \#8 aggregate, respectively. Based on the Louisville CSO130 project and observations made during the first and second phases of 
the project, it was determined that the "double washed aggregate" still carried about 3\%5\% (by mass) attached solids. This number was determined from a series of attached solids tests on multiple samples taken from different truckloads of each type of aggregate delivered to the construction site. In order to minimize what effect the attached solids being washed off the aggregate would have on the clogging of the permeable pavement, all the stones used in the experiment were washed using a 3000 psi pressure washer (Rigid, 2013). They were washed in a perforated bucket until the water coming out of the perforations was visibly clear. Figure 16 illustrates the washing process.

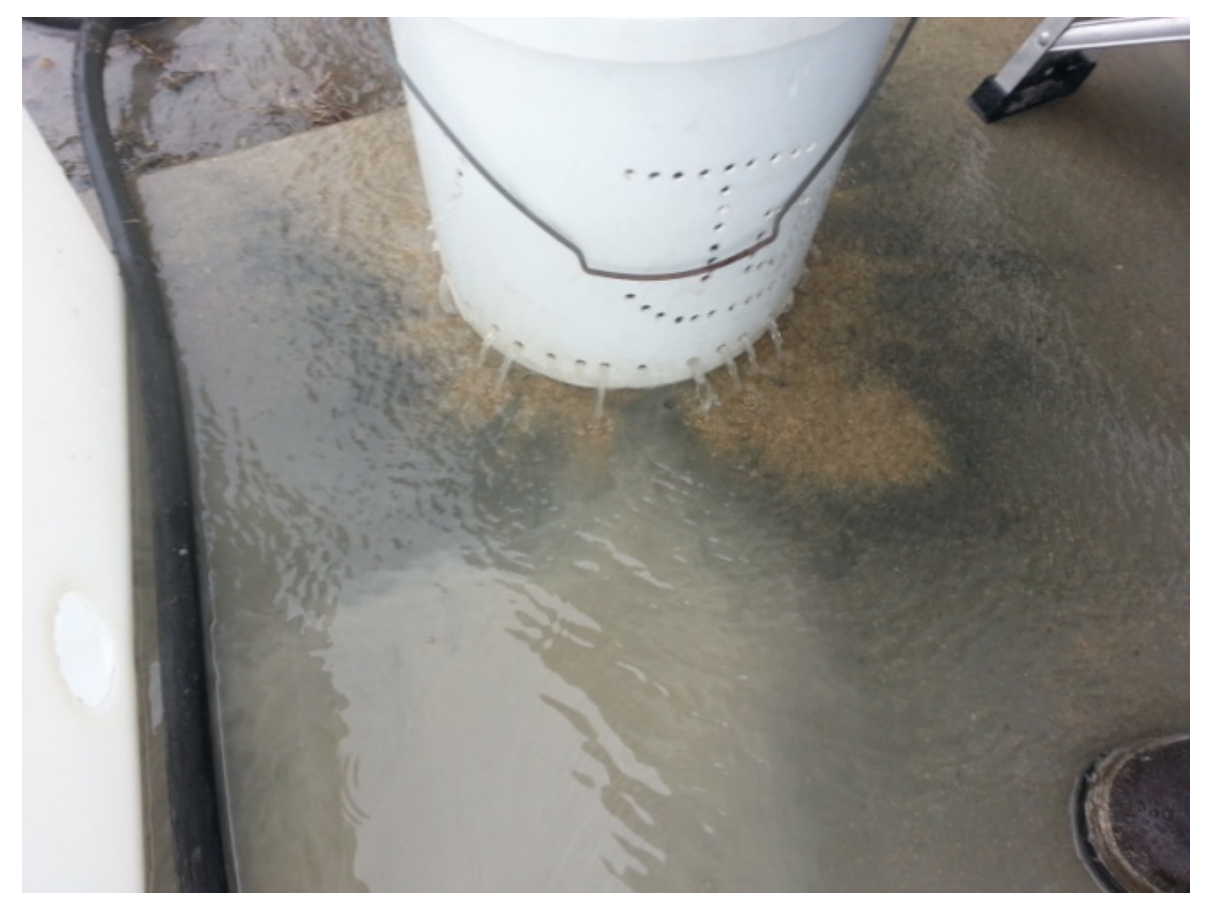

Figure 16: Washing the aggregate using a pressure washer

\subsubsection{Stormwater Runoff}

In the process of simulating rain over the watershed of the permeable pavers, the runoff which would be generated by a specific amount of rain over a period of months would be made to flow on the flume's surface. The stormwater runoff would go over the 
void spaces in between the pavers and the sediment suspended in the runoff would cause the gaps to clog, resulting in impaired performance. The runoff that passed through the pavers' gaps and was stored inside the storage gallery would be drained from the bottom In order to mimic the $2.54 \mathrm{~cm}$ (1 inch) of rain over the watershed which results in 684 liters of runoff over this area, the water had to flow over the flume's permeable surface. Given that each test scenario was simulating cumulative rainfall over a period of 6 months, which is 50.8 cms' (20 inches') worth of rain, the total volume of stormwater runoff needed to run an experiment was 14364 liters. In order to facilitate the work this volume of water was reduced to approximately $25 \%$ of the design volume or 3785.41 liters (1000 gallons). Reducing the volume of water used in each test, while having the same amount of sediment carried, is practical and eliminates the need to refill the stormwater tank during the experiment, which may cause disruptions.

The stormwater used as the runoff in the test was actual stormwater runoff that had been collected from a nearby residential complex and community college. The current infrastructure at the EPA facilities in Edison, NJ collects this stormwater runoff and transfers it into an existing 80,000 liter primary tank. The area of the watershed feeding into the stormwater collected runoff is enough to generate a substantial volume after only a few millimeters of rainfall, making the collection of stormwater easy and practical.

Prior to the experiments, the primary tank was emptied, thoroughly cleaned to avoid any remaining debris, and the tears in the primary tank's lining were patched. The primary tank was then connected to the stormwater runoff source and the tank was filled over a wet weekend. In order to minimize the effect of existing suspended solids in the 
tank, the water remained in the tank for a month before it was transferred to the smaller experiment tank.

The collected stormwater runoff in the primary tank, Figure 17, was expected to have an initial load of suspended solids. In order to monitor, understand and account for the effects on the experiment of the initial suspended solids in the stormwater runoff, after transferring the water to the test tank a 2 liter sample of the stormwater was collected and analyzed for total suspended solids (TSS).

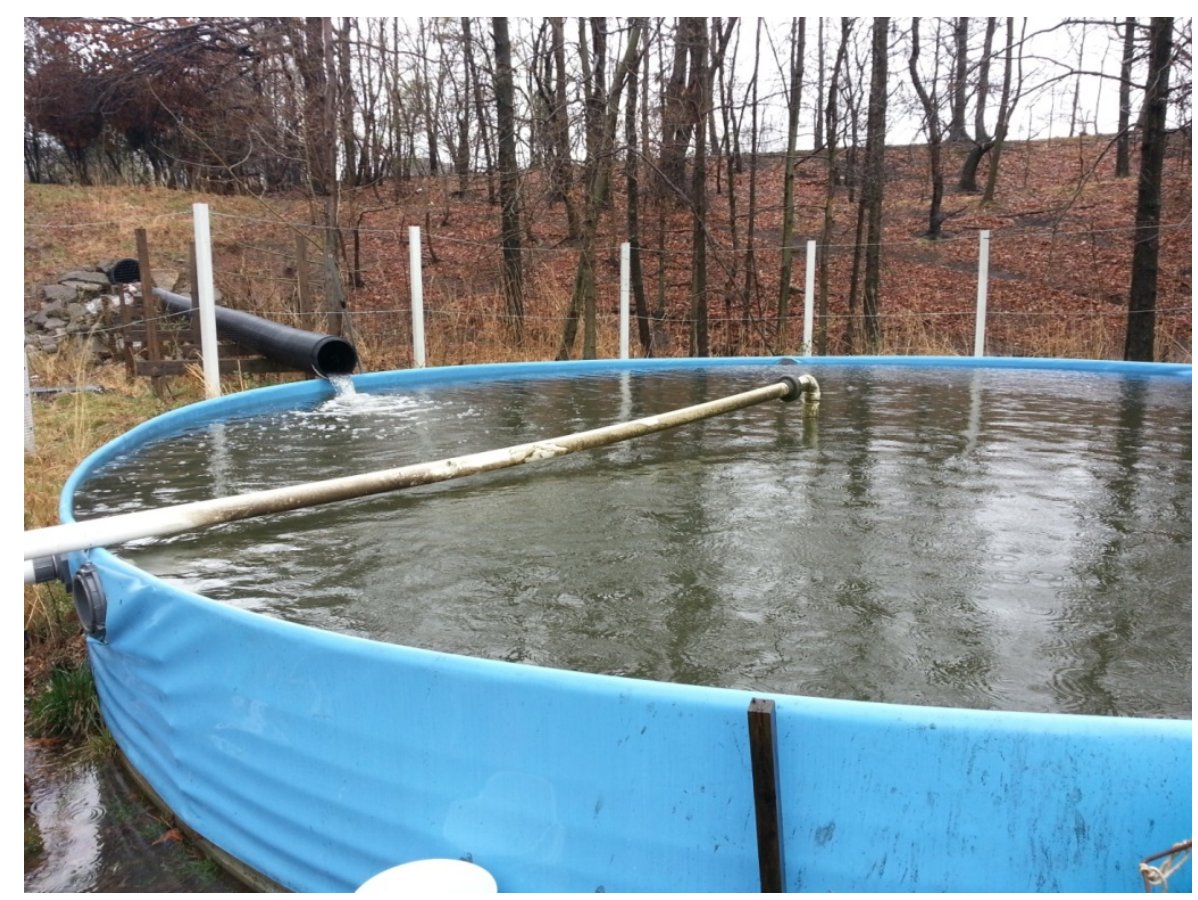

Figure 17: Primary tank for collecting stormwater runoff

\subsubsection{Sediment}

The sediment present in urban stormwater runoff may be generated from different sources including construction sites, landscapes, sanding and salting roadways during cold weather, decaying leaves, plant debris and other organic matter, metallic dust 
generated from vehicular activities, and other sources (Leisenring, Clary, Lawler, \& Hobson, 2011).

The amount of sediment carried by urban stormwater runoff is significantly different in quantity and quality from the runoff generated in rural and undeveloped areas. The different size of the particles in urban runoffs is one of the specific characteristics not seen in the runoff generated from rural areas (Vaze \& Chiew, 2004). In order to fully understand the nature and characteristics of the sediment carried by urban runoff, the PSD test is sometimes very informative and helpful (Osei, Andoh, Brown, \& Gwinn).

The characteristics and the amount of sediment in stormwater discharges vary considerably in line with the geographical area and its dominant land use. The National Stormwater Quality Database (NSQD) is a compilation of the runoff characteristics from different sources. A total of 8000 events from various locations in the US are used to create this database. The events are recorded as per the EPA rain zones in the US, shown in Figure 18 (Pitt et al., 2011; US-Government, 2012; USEPA, 1983). 


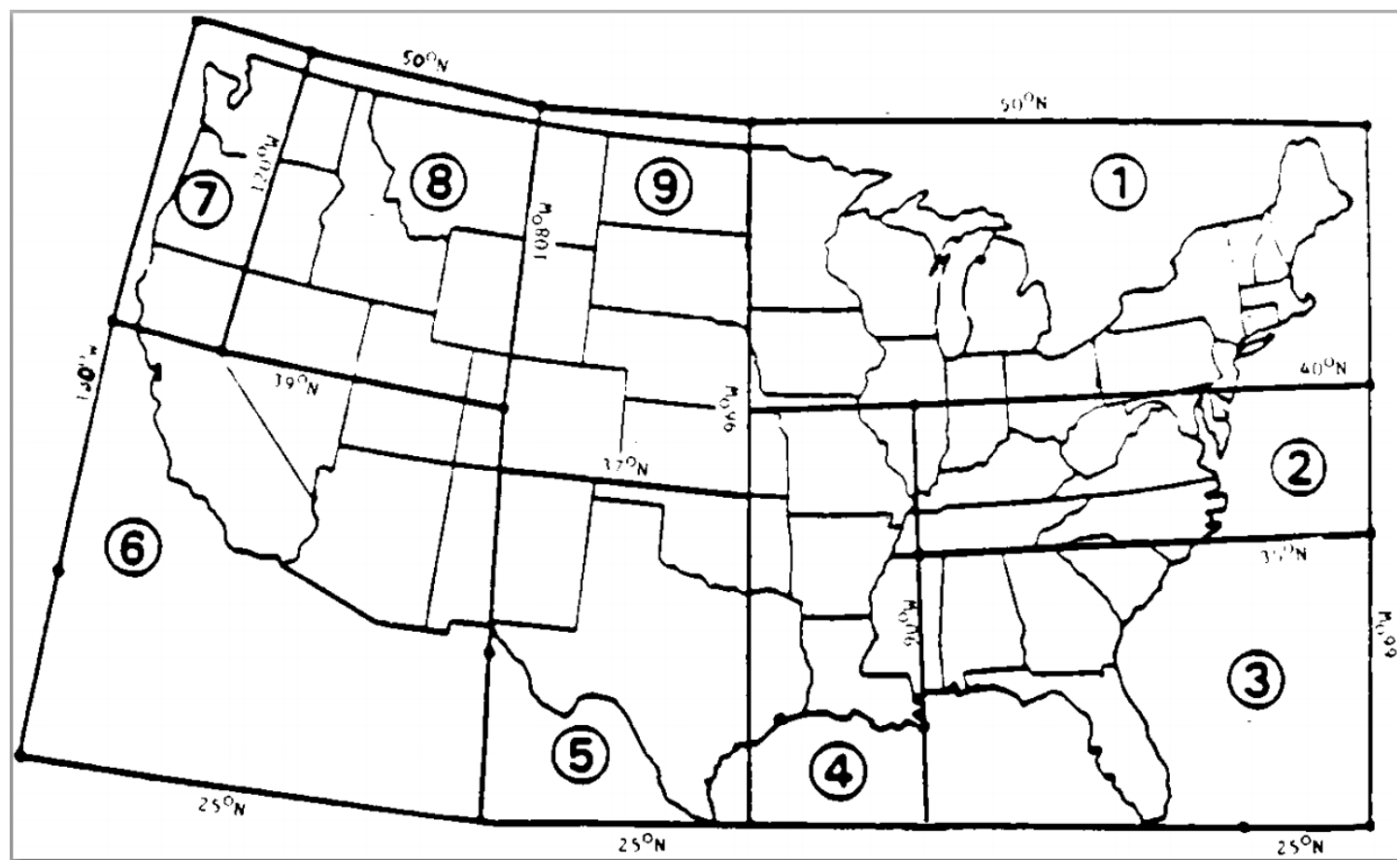

Figure 18: EPA rain zones, Source: NPDES Phase I Regulations, 40 CFR part 122 Appendix E(US-Government, 2012)

NSQD version 3.1 categorizes the observations made from the outfall locations

without significant snowmelts or erosions. This database includes many of the characteristics of the stormwater runoff in each zone and uses different categories including volumetric runoff coefficient and total suspended solids. Table 7 lists the total suspended solids for each of the rain zones and based on land use (Pitt et al., 2011):

Table 7: TSS Concentrations, mg/L, for Different Land Uses and Geographical Areas (EPA Rain Zones), Average (Number of Observatiosn) (Pitt et al., 2011)

\begin{tabular}{|l|c|c|c|c|c|c|c|c|c|c|}
\hline Land Use & RZ1 & RZ2 & RZ3 & RZ4 & RZ5 & RZ6 & RZ7 & $\begin{array}{c}\text { RZ } \\
8\end{array}$ & RZ9 & $\begin{array}{c}\text { ALL } \\
\text { RZ }\end{array}$ \\
\hline $\begin{array}{l}\text { Commercia } \\
\text { l }\end{array}$ & $\begin{array}{c}201 \\
(310)\end{array}$ & $\begin{array}{c}101 \\
(669)\end{array}$ & $\begin{array}{c}56 \\
(55)\end{array}$ & $\begin{array}{c}232 \\
(67)\end{array}$ & $\begin{array}{c}108 \\
(100 \\
)\end{array}$ & $\begin{array}{c}132 \\
(41)\end{array}$ & $\begin{array}{c}87 \\
(61)\end{array}$ & $\begin{array}{c}98 \\
(7)\end{array}$ & $\begin{array}{c}247 \\
(32)\end{array}$ & $\begin{array}{c}133 \\
(1342 \\
)\end{array}$ \\
\hline Freeways & $24(3)$ & $\begin{array}{c}80 \\
(225)\end{array}$ & $\begin{array}{c}36 \\
(13)\end{array}$ & $\mathrm{n} / \mathrm{a}$ & $\begin{array}{c}144 \\
(12)\end{array}$ & $\begin{array}{c}183 \\
(105 \\
)\end{array}$ & $\mathrm{n} / \mathrm{a}$ & $\mathrm{n} / \mathrm{a}$ & $\mathrm{n} / \mathrm{a}$ & $\begin{array}{c}114 \\
(381)\end{array}$ \\
\hline Industrial & $\begin{array}{c}177 \\
(100)\end{array}$ & $\begin{array}{c}97 \\
(375)\end{array}$ & $\begin{array}{c}105 \\
(105 \\
)\end{array}$ & $\begin{array}{c}164 \\
(64)\end{array}$ & $\begin{array}{c}155 \\
(106\end{array}$ & $\begin{array}{c}385 \\
(95)\end{array}$ & $\begin{array}{c}164 \\
(30)\end{array}$ & $\mathrm{n} / \mathrm{a}$ & $\begin{array}{c}360 \\
(39)\end{array}$ & $\begin{array}{c}160 \\
(918)\end{array}$ \\
\hline
\end{tabular}




\begin{tabular}{|c|c|c|c|c|c|c|c|c|c|c|}
\hline $\begin{array}{l}\text { Institutiona } \\
\text { l }\end{array}$ & $91(8)$ & $\begin{array}{c}86 \\
(46)\end{array}$ & $\begin{array}{c}68 \\
(15)\end{array}$ & n/a & $\mathrm{n} / \mathrm{a}$ & $\mathrm{n} / \mathrm{a}$ & $\mathrm{n} / \mathrm{a}$ & $\mathrm{n} / \mathrm{a}$ & $\mathrm{n} / \mathrm{a}$ & $\begin{array}{c}83 \\
(69)\end{array}$ \\
\hline Open Space & $\begin{array}{c}176 \\
(128)\end{array}$ & $\begin{array}{c}98 \\
(107)\end{array}$ & n/a & $\begin{array}{l}370 \\
(18)\end{array}$ & $\begin{array}{l}202 \\
(67)\end{array}$ & $\begin{array}{l}330 \\
(1)\end{array}$ & $\mathrm{n} / \mathrm{a}$ & $\mathrm{n} / \mathrm{a}$ & $\begin{array}{l}846 \\
(7)\end{array}$ & $\begin{array}{c}182 \\
(329)\end{array}$ \\
\hline Residential & $\begin{array}{l}135 \\
(507)\end{array}$ & $\begin{array}{c}102 \\
(1893 \\
)\end{array}$ & $\begin{array}{c}102 \\
(207 \\
)\end{array}$ & $\begin{array}{c}374 \\
(140 \\
)\end{array}$ & $\begin{array}{c}129 \\
(203 \\
)\end{array}$ & $\begin{array}{l}162 \\
(75)\end{array}$ & $\begin{array}{c}130 \\
(315 \\
)\end{array}$ & $\begin{array}{l}140 \\
(16)\end{array}$ & $\begin{array}{c}528 \\
(116 \\
)\end{array}$ & $\begin{array}{c}137 \\
(3472 \\
)\end{array}$ \\
\hline $\begin{array}{l}\text { All Land } \\
\text { uses }\end{array}$ & $\begin{array}{c}156 \\
(1132 \\
)\end{array}$ & $\begin{array}{c}97 \\
(3468 \\
)\end{array}$ & $\begin{array}{c}93 \\
(395 \\
)\end{array}$ & $\begin{array}{c}293 \\
(293 \\
)\end{array}$ & $\begin{array}{c}141 \\
(488 \\
)\end{array}$ & $\begin{array}{c}235 \\
(318 \\
)\end{array}$ & $\begin{array}{c}126 \\
(443 \\
)\end{array}$ & $\begin{array}{l}140 \\
(24)\end{array}$ & $\begin{array}{c}460 \\
(194 \\
)\end{array}$ & $\begin{array}{c}135 \\
(6682 \\
)\end{array}$ \\
\hline
\end{tabular}

With the total sediment carried by urban stormwater runoff can be approximated for the Kentucky (region 2 on Figure 18), the characteristic of the sediment had to be determined. Since the experiments were designed to replicate conditions similar to those in Louisville, the clogging debris was also similar to that seen in Louisville.

In order to determine the sediment characteristics for Kentucky, the samples collected during the two different maintenance operations were analyzed for particle size distribution and for organic content using ASTM standards (ASTM, 2007b, 2009). During the maintenance conducted on the permeable pavement strips in Louisville, the clogging material from the surface and from the gaps between the paver blocks was recovered and analyzed. Five separate samplings were made of the material in the paving gaps and underneath the arch in CSO130 installation. The complete results of the tests on the recovered samples are presented in Chapter 3.

After measuring and analyzing the sediment samples taken from Louisville, the sediment was divided into two portions. The first portion, forming $80 \%$ by mass of the total sediment, was inorganic sediment such as is commonly found in urban environments. The other $20 \%$ was composed of blended mulch and leaf shreds, which 
represented the organics found in urban stormwater runoff sediment. The clogging debris used in the experiments included both organic and inorganic material and the amount different particle sizes of each type matched the particle size distribution of the material seen in Louisville. As examination of the sediment recovered from the Louisville GI practices showed that the particle size distribution of the organics and inorganics was different including all sizes of particles in the experiment was necessary to create conditions similar to those seen in Louisville. The distribution of different organic particle sizes in the clogging debris was not constant and was designed to match Louisville samples. The percentages of organics for each particle size are listed in Table 8.

Table 8: Percentage of Organics Based on Grain Size

\begin{tabular}{|l|c|c|}
\hline Sieve & Percent Organics & Organic Sediment (gr) \\
\hline $1 / 4 ”$ & $20 \%$ & 16.4 \\
\hline$\# 4$ & $30 \%$ & 30.8 \\
\hline$\# 10$ & $40 \%$ & 78.2 \\
\hline$\# 20$ & $45 \%$ & 111.6 \\
\hline$\# 40$ & $15 \%$ & 45.3 \\
\hline$\# 60$ & $5 \%$ & 12.6 \\
\hline$\# 100$ & $5 \%$ & 2.0 \\
\hline$\# 200$ & $5 \%$ & 3.5 \\
\hline Pan & $0 \%$ & 0.0 \\
\hline
\end{tabular}

As mentioned in section 4.4.2, the collected stormwater runoff in the primary tank was expected to have an initial load of suspended solids. In order to monitor and understand the effects on the experiment of the initial suspended solids load in the stormwater runoff, a 2 liter sample of the stormwater was collected after transferring the water to the test tank. With the observations made during the water transfer and testing for total suspended solids, it was concluded that the suspended solids in the collected 
stormwater runoff were insignificant; being smaller than the smallest particles found and therefore could not create any inaccuracies in the range of particles used as the clogging material.

The amount of sediment carried by stormwater runoff was calculated based on the $97 \mathrm{mg} / \mathrm{l}$ load of sediment, as seen in NSQD for RZ2. The amount of total sediment carried was determined based on the volume of stormwater runoff generated after an accumulation of $50.8 \mathrm{~cm}$ (20 inches) of rainfall. The area of the watershed receiving this amount of rainfall equals to an area 21 times the area of the flume. The area was derived from a ratio of 20:1 between impervious to pervious, which indicates that the watershed consisted of 20 units' area of impervious surfaces and 1 unit area of permeable surfaces. The result was that the weight of sediment carried by $50.8 \mathrm{~cm}$ worth of rain over the area of the watershed was 1321 grams, which comprised 300.4 grams of organics and 1015.5 grams of inorganics. Table 9 shows the breakdown of the clogging material by size and type.

Table 9: Particle Size Distribution of the Material Used as Runoff Sediment, Based on Size and Type

\begin{tabular}{|c|c|c|c|}
\hline Sieve & $\begin{array}{l}\text { Organic Sediment } \\
\text { (gr) }\end{array}$ & $\begin{array}{l}\text { Inorganic Sediment } \\
\text { (gr) }\end{array}$ & $\begin{array}{l}\text { Total Sediment } \\
\text { (gr) }\end{array}$ \\
\hline $1 / 4 ”$ & 16.4 & 65.8 & 82.2 \\
\hline$\# 4$ & 30.8 & 71.8 & 102.6 \\
\hline$\# 10$ & 78.2 & 117.3 & 195.5 \\
\hline$\# 20$ & 111.6 & 136.4 & 247.9 \\
\hline$\# 40$ & 45.3 & 257 & 302.3 \\
\hline$\# 60$ & 12.6 & 239.4 & 252 \\
\hline$\# 100$ & 2 & 37.5 & 39.5 \\
\hline$\# 200$ & 3.5 & 67.2 & 70.8 \\
\hline
\end{tabular}




\begin{tabular}{|l|l|l|l|}
\hline Pan & 0 & 23.1 & 23.1 \\
\hline
\end{tabular}

The sediment load was introduced to the flow with a feeder funnel, designed on the lines of the eductor working mechanism attached to the runoff hose. The sediment for each experiment was prepared and mixed thoroughly and then was equally divided into 20 smaller sample cups. The sample cups were then dumped into the eductor every 5 minutes during the test, the first one starting after 30 seconds (Figure 19). 


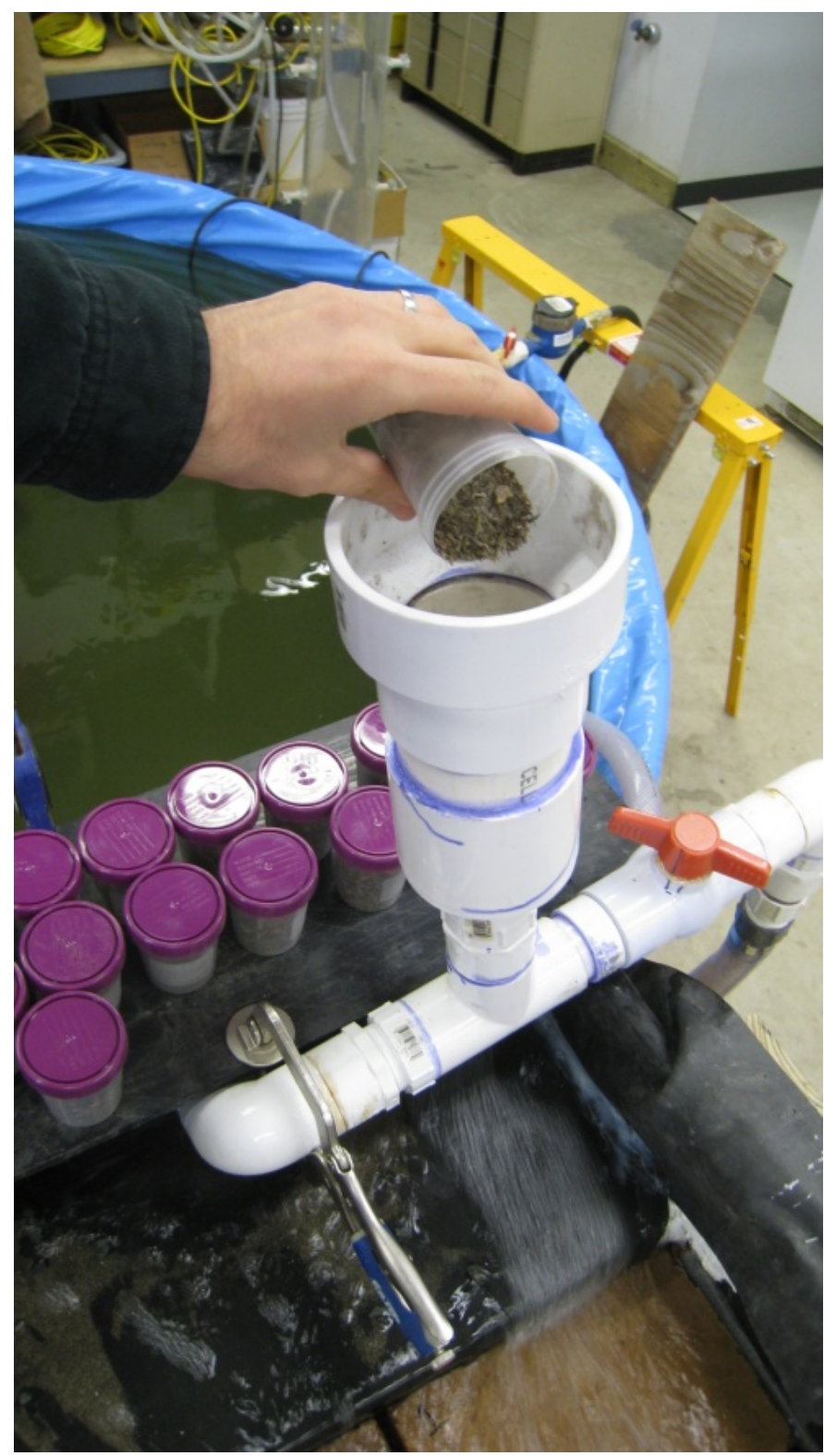

Figure 19: Dumping sediment during the experiment

\subsection{Monitoring Data}

Monitoring the performance of the flume during and after the experiment is essential for providing assessment data and investigating the hypotheses of this research. Monitoring methods were essentially focused on using sensors (i.e., soil moisture, 
temperature, water level, etc.) to assess the conditions of GI and understand its behavioral mechanisms.

The main use of TDRs, (Figure 20) is to measure the soil moisture content in agricultural and turf grass applications to determine their irrigation needs. At first the time domain reflectometry technique measured a dielectric constant to determine the volumetric water content (VWC) of different types of soil (Topp, Davis, \& Annan, 1980). TDRs can be and have been used in GI applications and with the goal of obtaining meaningful performance data, for instance, they have previously been used in determining the wetting front of green infrastructure practices and measuring soil water content to verify predictions made by computer models for other types of GI (Aravena \& Dussaillant, 2009). Another study that has focused on the application of TDRs in both the Louisville and Edison GI projects has shown that time domain reflectometry records reliable results when used in gravel and has proven to be a successful assessment tool for GI (R. Brown \& Borst, 2013).

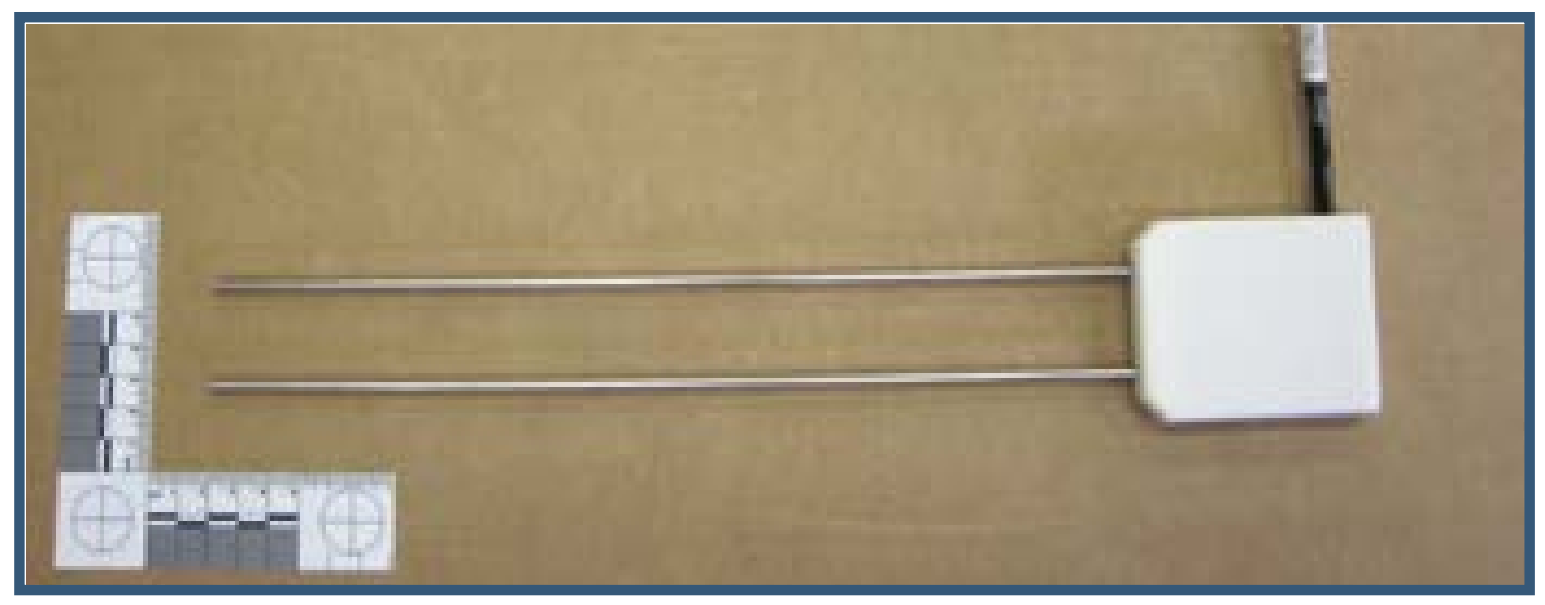

Figure 20: Time domain reflectometer (TDR) 
Performance of the flume in this study was measured using a TDR, (CampbellScientific, 2012). TDRs are used in the Adam's Street project in Louisville to measure the presence of moisture caused by running water in the storage gallery. As mentioned before, the use of TDRs in this experiment is not only a sound method, but would also help to verify the results of other studies that have looked at the application of time domain reflectometry and measuring VWC as an automated way of investigating the performance of GI and determining its maintenance needs. A total of 7 CS616 TDRs were located inside the storage gallery, $25.4 \mathrm{~cm}$ (10 inches) from the bottom. After the instruments were placed in the storage gallery, their communication wires were run through the effluent pipe and out of the flume. The TDRs were buried under another 10 cm (4 inches) of \#57 aggregate and a 5 cm layer (2 inches) of \#8 aggregate. Figure 21 shows the arrangement of the TDRs in the storage gallery of the flume.

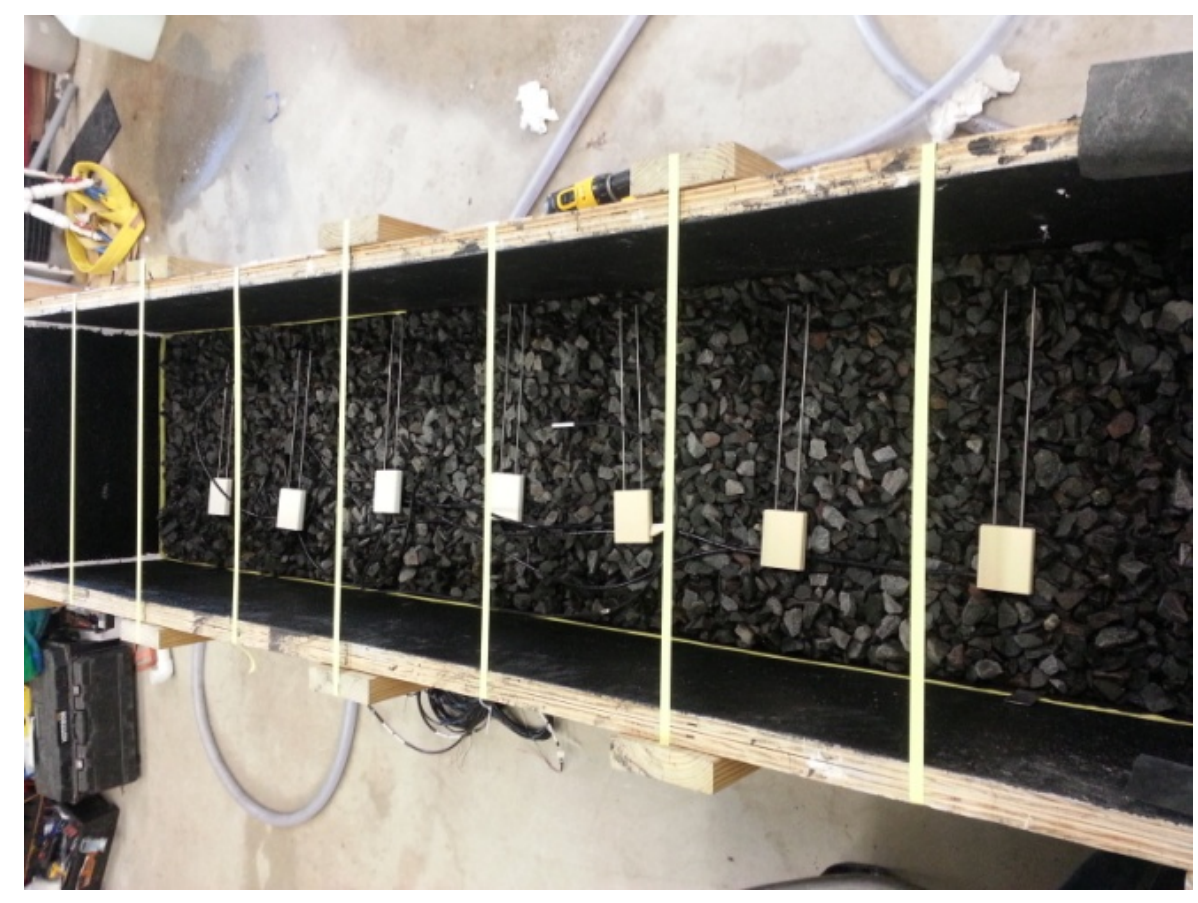

Figure 21: Arrangement of TDRs in the storage gallery 
TDRS located in the storage gallery of the flume constantly monitored the volumetric water content (VWC) of their surroundings. The locations of the TDRs in relation to the up gradient edge of the flume are listed in Table 10. Since the measurements made by the CD616 TDRs are not temperature compensated, a soil temperature sensor (L107) was also located at the midpoint and adjacent to the TDR4. The TDRs were directly connected to a CR1000 data logger (Campbell-Scientific, 2013a), which was powered with a battery and collected the measurement from each instrument. The placing of the TDRs was a function of the number of instruments available for the testing.

Table 10: Location of TDRs in the Flume

\begin{tabular}{|c|c|l|}
\hline Number & $\begin{array}{l}\text { Location from gradient } \\
(\mathrm{cm})\end{array}$ & \multicolumn{1}{|c|}{ Notes } \\
\hline 1 & 28.58 & \\
\hline 2 & 57.15 & \\
\hline 3 & 85.73 & $\begin{array}{l}\text { thermistor in place at this } \\
\text { location }\end{array}$ \\
\hline 4 & 114.3 & \\
\hline 5 & 142.88 & \\
\hline 6 & 171.45 & \\
\hline 7 & 200.03 & \\
\hline
\end{tabular}

The data logger was programmed to read the measurements of the TDR every 10 seconds. The measurements were stored on the data logger and then downloaded into a computer after each experiment. Figure 22 shows the wiring of the instruments and the enclosure with the data logger. 


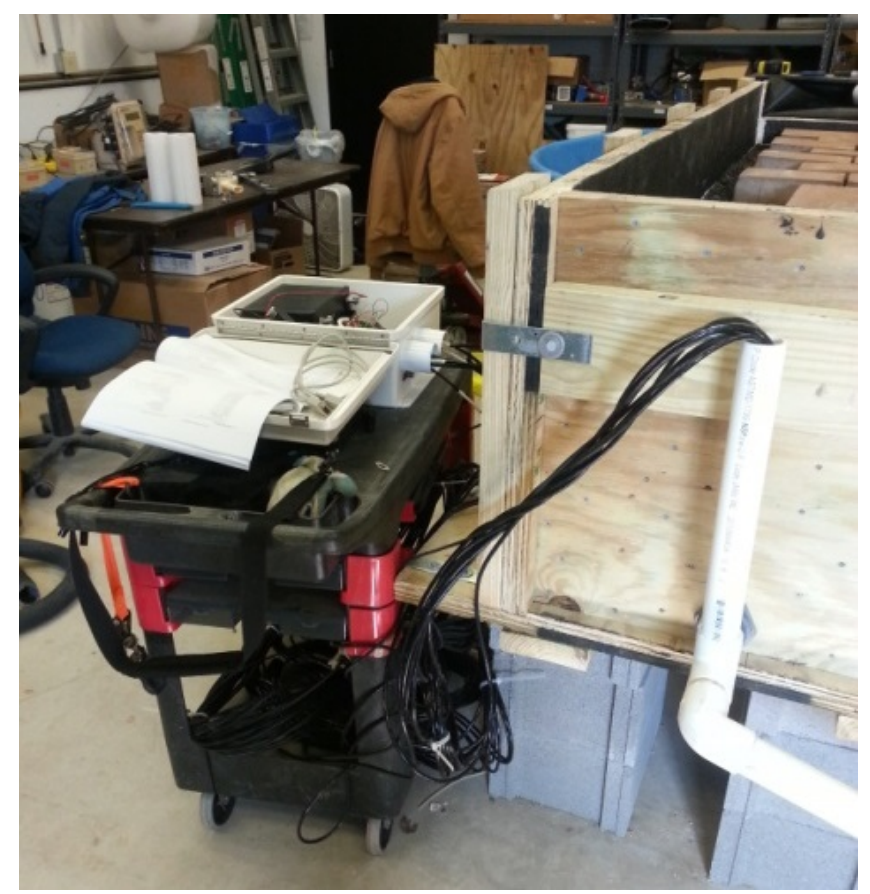

Figure 22: Instruments wired to the data logger

After installing the monitoring instruments in the flume and wiring them to the data logger, the data logger had to be programmed to get the data from specific probes at specific times and record it on a table, which could then be downloaded. The data logger was programmed as described in the manual. The program had to be written in the software provided by the data logger vendor, called the CR Basic Editor. The program included declaring the variables that were to be recorded, declaring the units for those variables, defining the data tables to be generated while the data was being recorded, and the main program. The purpose of the main program was to give the correct address of each instrument to the data logger, according to the way in which they were wired to the data logger, and the frequency of measuring the data for each instrument. The main program ended by defining these criteria for all the installed instruments. Figure 23 is a screenshot of the CR Basic Editor illustrating the data logger program lines. 


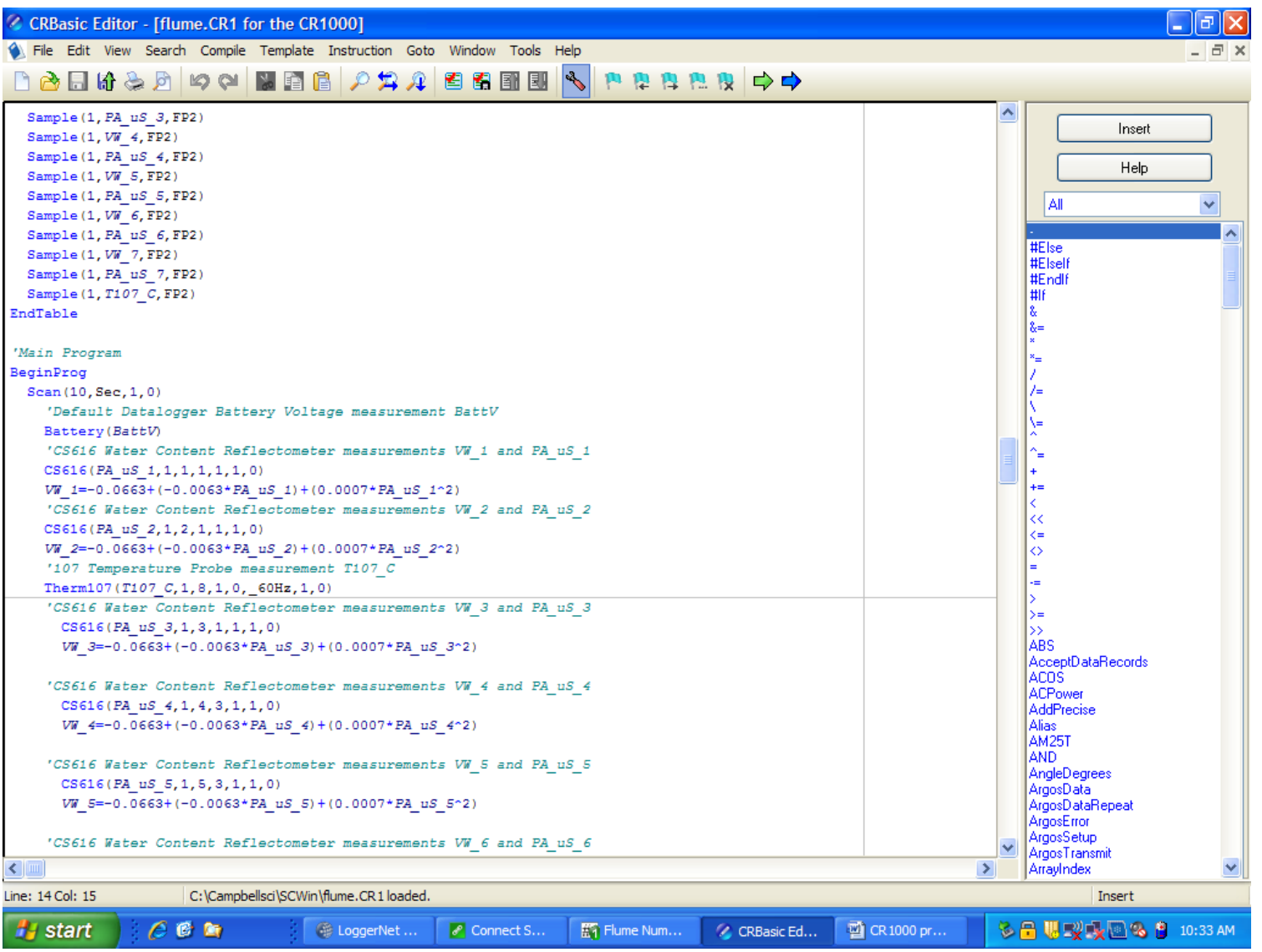

Figure 23: Screenshot of CR Basic Editor

The complete program written for the data logger used in this research is available from Appendix A.

\subsection{Staging}

\subsubsection{Compacting and Leveling}

When the instruments had been wired and the flume filled up with aggregate, the flume had to be staged properly before the first experiment. The first step in staging, which must be taken before placing the pavers in the flume in all the experiments, was to compact the aggregate. ICPI suggests that all layers of storage gallery must be compacted. In order to comply with the requirements stated in the ICPI guidebook, and 
within practical laboratory limits, the top of the storage gallery was compacted by placing a wooden board on the aggregate and pounding it heavily. This method was also used to level the surface before placing the paver blocks in the flume.

Figure 24 is an image of the surface of the bedding layer after compacting and leveling the aggregate.

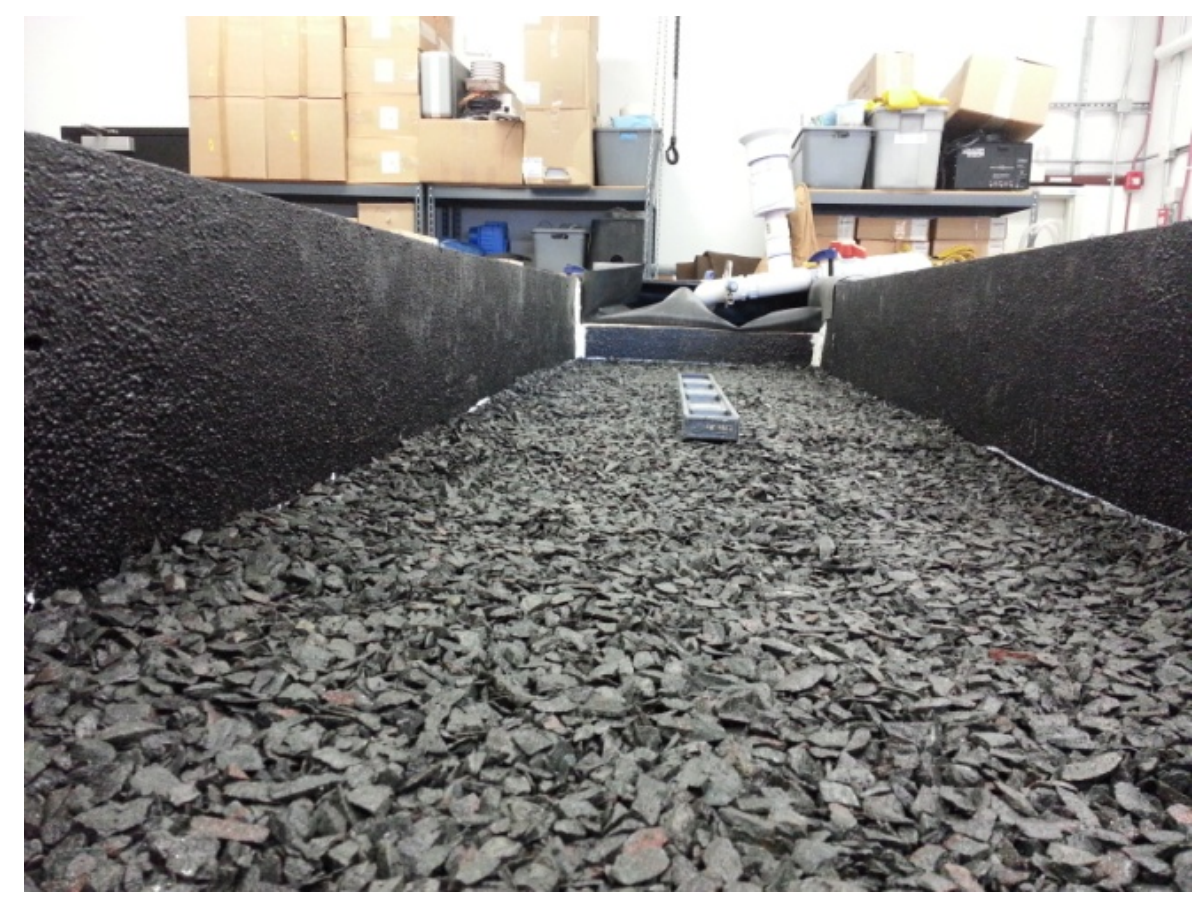

Figure 24: Compacted and leveled bedding layer

\subsubsection{Interlocking Concrete Pavers (ICP)}

Three different paver products were used for the experiments. The products were chosen after consulting with multiple paver vendors and an industry expert (Antunes, 2013) on the available products that would meet the requirements of this research. Three products were selected on the basis of the gaps required between them. The gaps selected for this study were $6 \mathrm{~mm}, 9 \mathrm{~mm}$ and $12 \mathrm{~mm}$. The smallest gap size was selected to mimic the conditions in Louisville. The biggest gap size was bound to the requirements of the 
Americans with Disabilities Act (ADA) for interlocking concrete pavers (DOJ, 1994).

Table 11 lists the details of the pavers used in the experiment.

Table 11: Pavers Used in the Experiment

\begin{tabular}{|l|l|l|l|}
\hline $\begin{array}{l}\text { Paver gap size } \\
(\mathrm{mm})\end{array}$ & Paver name & Paver manufacturer & Dimensions (mm) \\
\hline 6 & Coventry I & EPHenry & $\mathrm{L}: 240, \mathrm{~W}: 159, \mathrm{H}: 60$ \\
\hline 9 & Eco-Cobble & EPHenry & $\mathrm{L}: 240, \mathrm{~W}: 159, \mathrm{H}: 60$ \\
\hline 12 & Eco-Paver & EPHenry & $\mathrm{L}: 240, \mathrm{~W}: 157, \mathrm{H}: 82$ \\
\hline
\end{tabular}

The shape of each paver block is unique. They are designed to provide the required space by spacer lugs molded into their frame, or by plastic spacers between them. Spacer lugs vary in each paver product. In the pavers used in this experiment, EcoCobble and Eco-Paver blocks bring in spacer lugs designed in their structure at the time of the concrete is molded. Coventry I paver blocks lack the molded spacer lugs and the vendor suggests the use of plastic spacers. All the suggestions of the vendor for product handling and installation guides were followed in the course of the experiment.

Figure 25 shows Coventry I paver blocks, which provide $6 \mathrm{~mm}$ gap between the pavers using plastic spacers. 


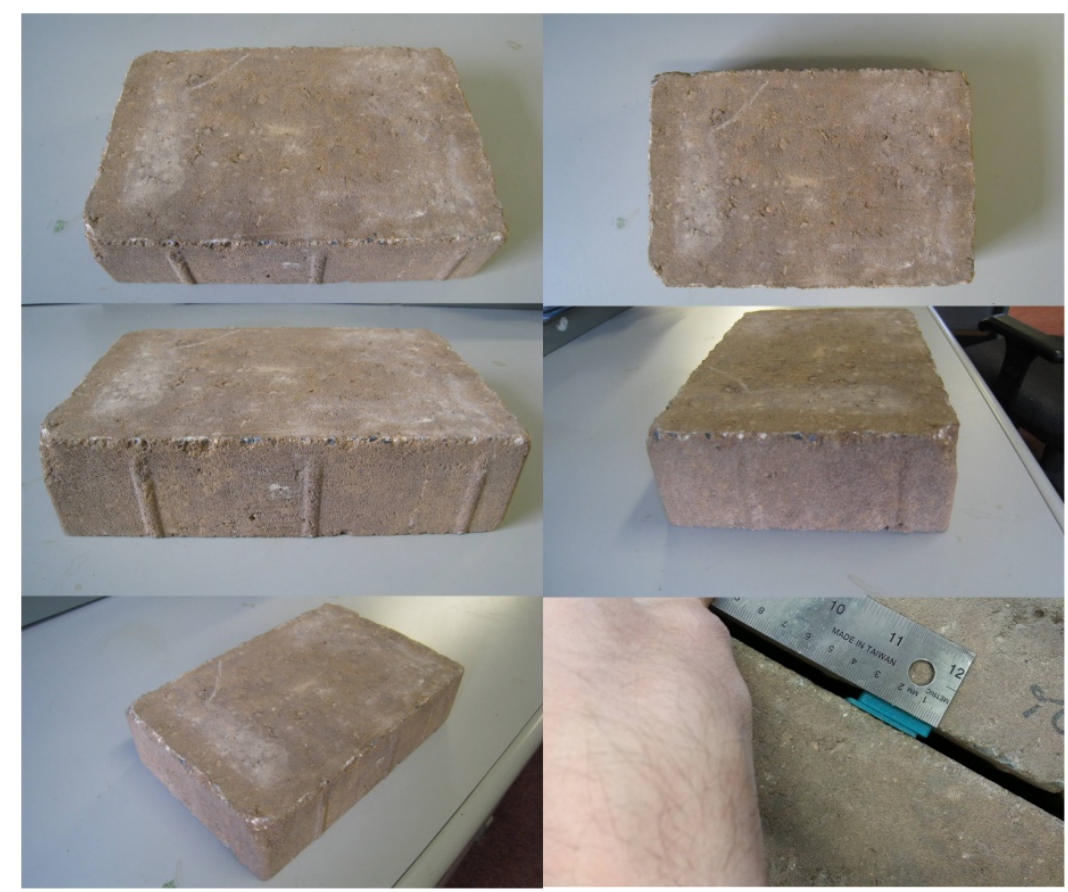

Figure 25: Coventry I paver block

Figure 26 shows Eco-Cobble paver block, which provides a 9 mm gap between the pavers using the molded spacer lugs in the structure of the paver. Once arranged in a mat, the spacer lugs of this paver type touch each other and the gaps between the spacer lugs face each other, providing an exact $9 \mathrm{~mm}$ gap size. 


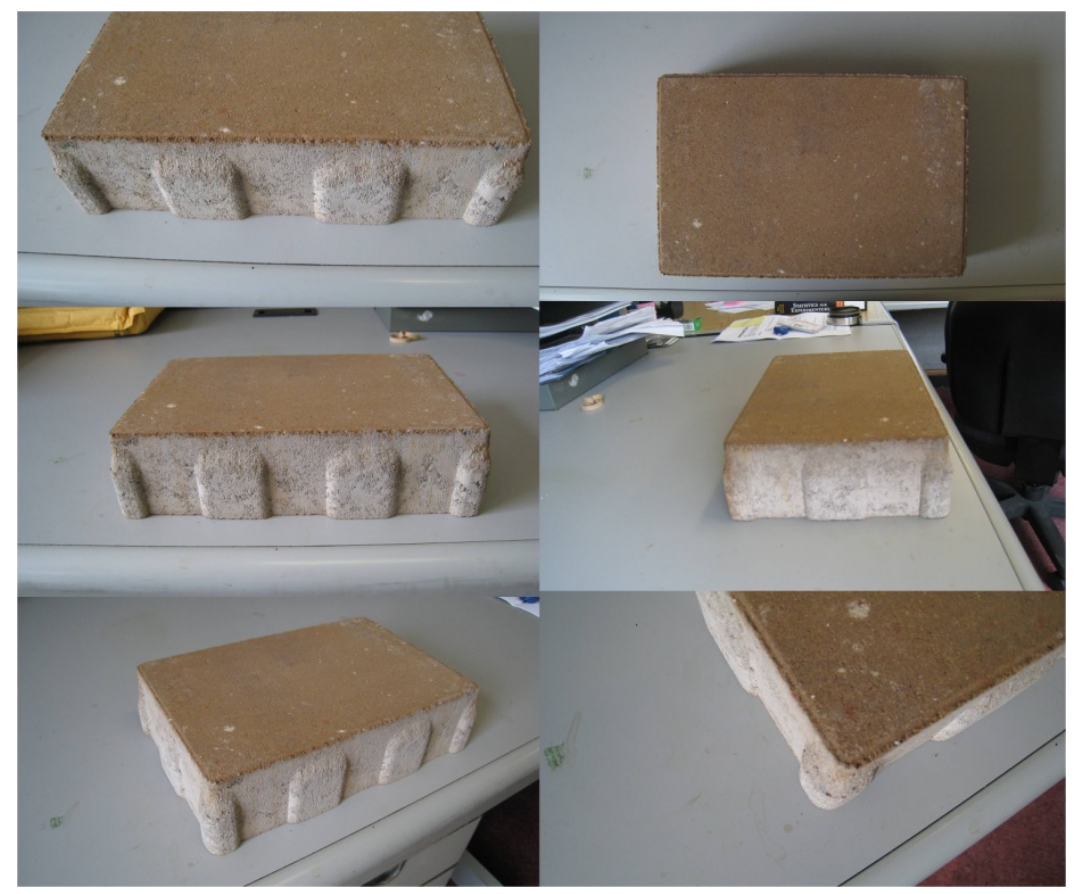

Figure 26: Eco-Cobble paver block

Figure 27 shows Eco-Paver block, which provides a $12 \mathrm{~mm}$ gap between with built in spacer lugs. The lugs also provide the interlocking mechanism for the pavers.

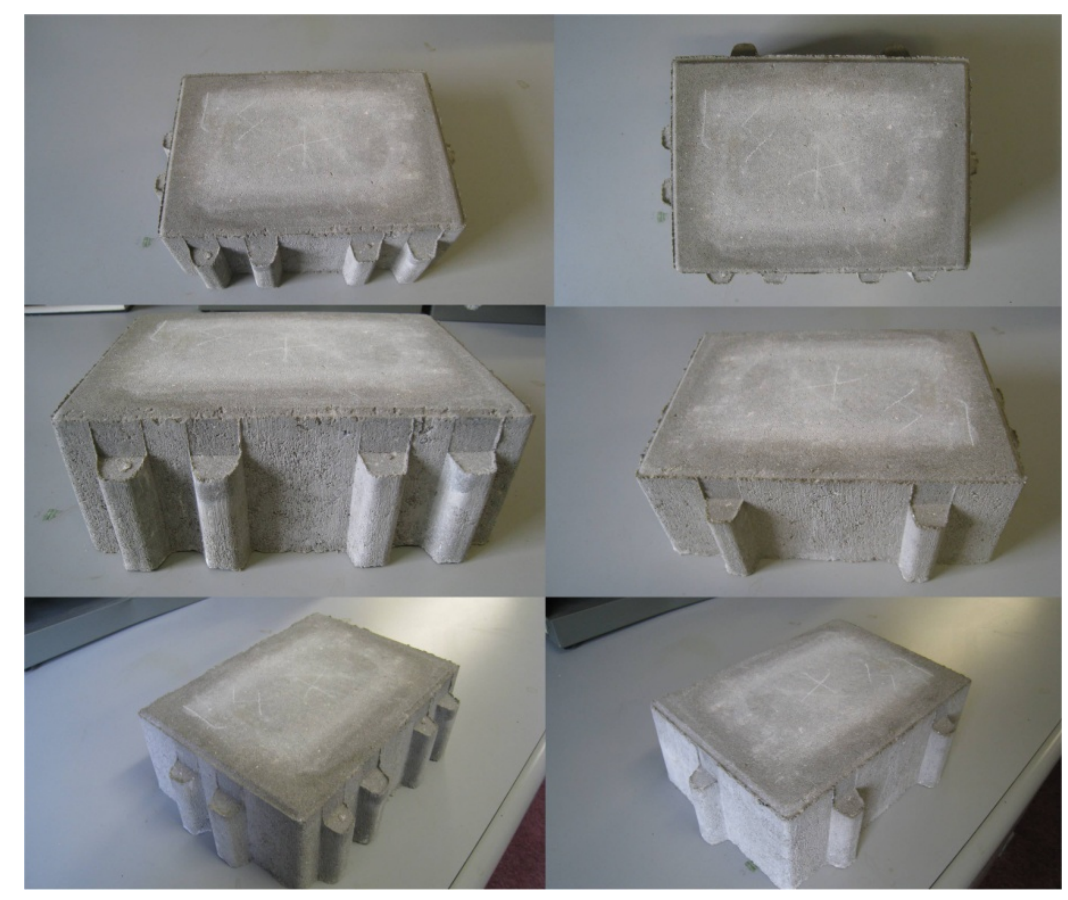

Figure 27: Eco-Paver block 


\subsection{3. $\quad$ Paver Laying Methods}

Various methods and patterns could be used for laying the pavers in the flume. The pattern of installation is a product specific detail and all vendors provide a list of patterns compatible with the shape and design of their paver blocks. There has been little previous work on the effect of patterns on the performance of GI (Margaret Mackisack \& Pywell, 1994; MS Mackisack, 1996), where the focus has been on the effects of traffic on the structure of the pavers and the determining shape and laying strategies which take account of the needs of the pavers.

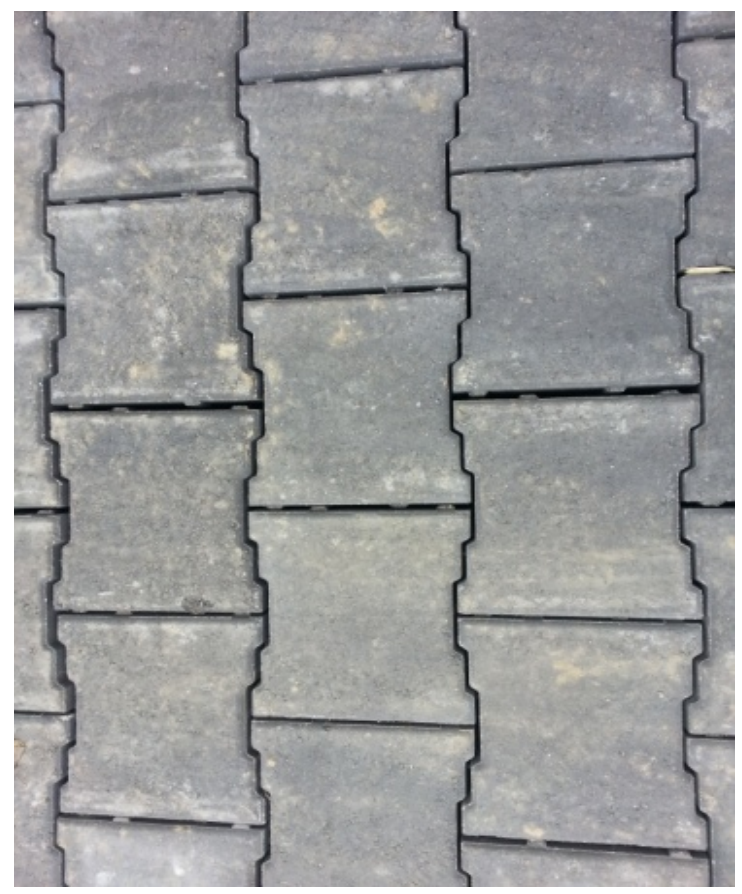

Figure 28: PaveDrain pavers in Louisville's CSO130 installation

After consulting with industry experts (Antunes, 2013), it was determined that the running bond was the most commonly used pattern in permeable pavement systems. As an example, the permeable pavement strip on Adams Street in Louisville, KY was arranged with a running bond pattern (Figure 28).; hence this pattern was selected for all 
configurations of the experiment. The arrangement of pavers with the selected running bond pattern is shown in Figure 28.

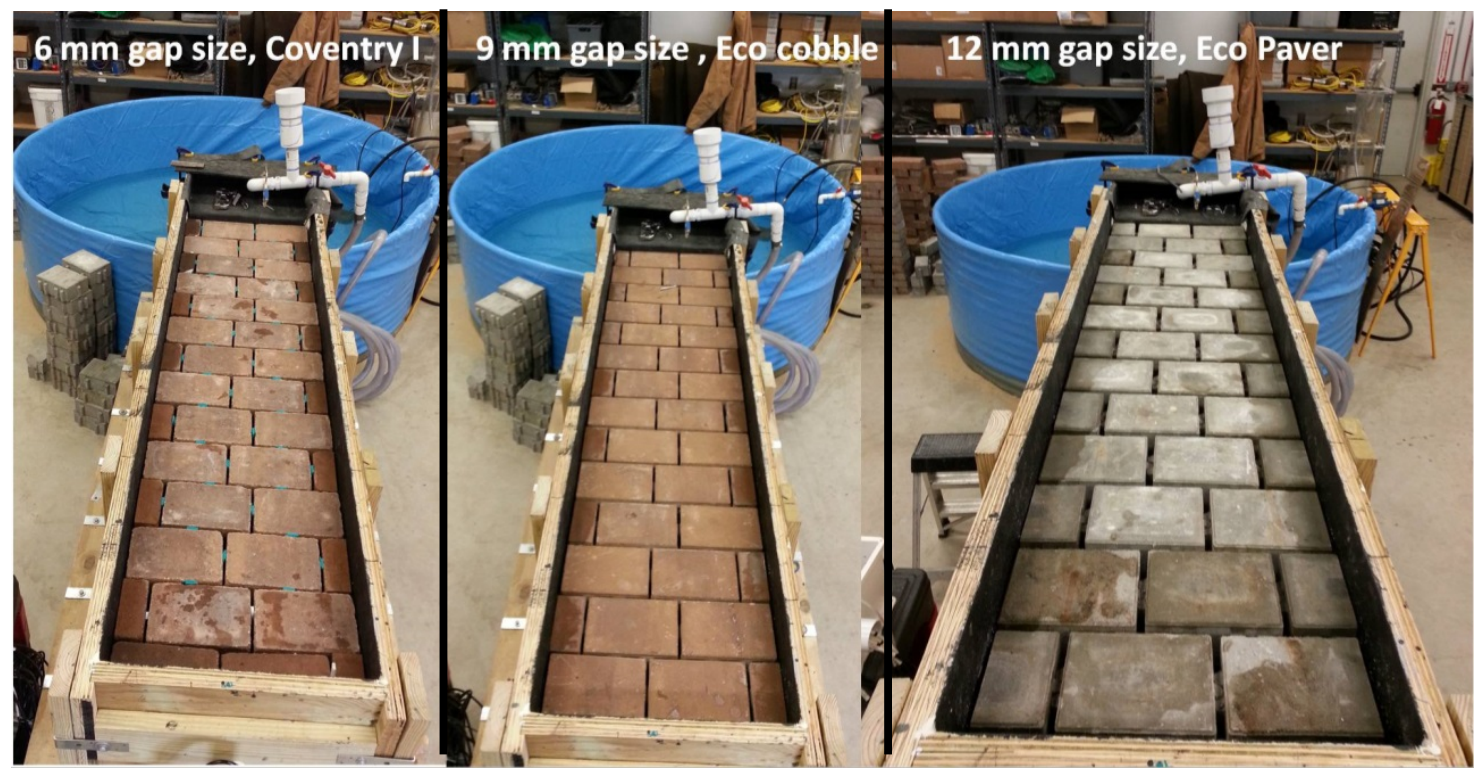

Figure 29: Flume's paver pattern

With the running bond pattern for all three types of pavements used, the number of permeable gaps in each row and in total is the same for all the experiments. The gaps between the pavers and the sides of the flume were sealed using a neoprene to avoid any inconsistencies between experiments.

\subsubsection{Slope}

Adjusting the slope of the flume is essential as a variable to create multiple scenarios for a GI installation in different types of road environment and the effect of different slopes has been acknowledged by previous writers (Elizabeth A. Fassman \& Blackbourn, 2011). An experiment on clogging pervious concrete with sand particles on two different slopes has shown that a $10 \%$ longitudinal slope would result in less surface infiltration after clogging than a 2\% longitudinal slope (Haselbach et al., 2006). The 
minimum longitudinal slope recommended by the ICPI to facilitate the drainage of stormwater runoff on the surface is $1 \%$ (Smith, 2011). Steep slopes are a limiting factor for ICP as the reduce the storage capacity available in the storage gallery of the GI (Virginia-DCR, 2011). The maximum slope used in urban areas varies from state to state. Common suggestions for installing permeable pavement strips in urban areas are recommended not to exceed a 5\% longitudinal slopes (NJ-DEP, 2004).

In order to investigate the effect of the slopes recommended, the slopes used in this experiment changed from $1 \%$ to $3 \%$ and then to $5 \%$, the 3 slopes selected for the experiment. The slope is illustrated in Figure 30.

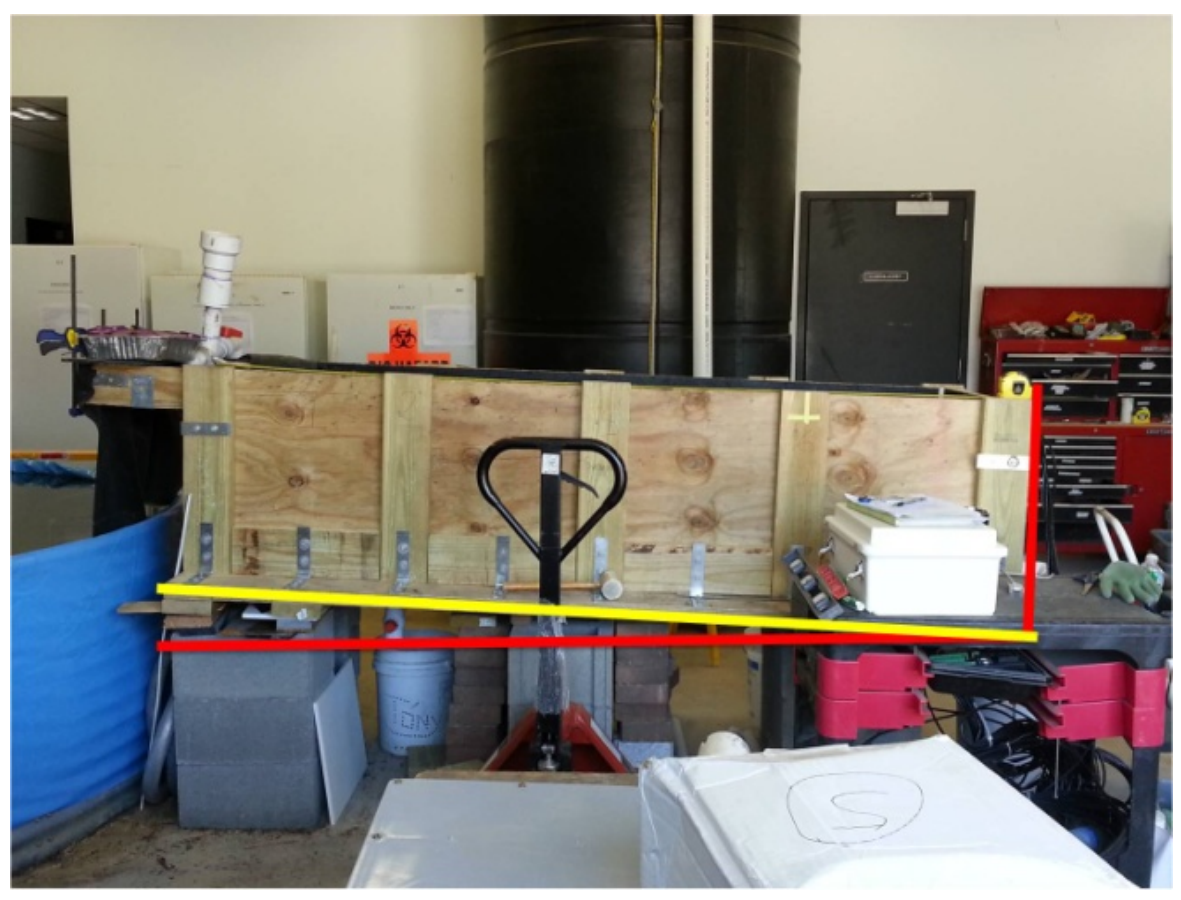

Figure 30: Illustration of slope of the flume

The slope was adjusted by lifting the entire experiment module using a pallet jack, and fitting wooden boards under the up gradient segment of the flume. After each adjustment of the flume, the cross slope was checked at the location of each support. 


\subsubsection{Permeable Joint Material}

Surface clogging in permeable pavements has always been an issue. With time and rainfall, materials will accumulate on the surface and in the joints of any type of permeable surface (R. Brown \& Borst, 2013; Deo et al., 2010; Haselbach et al., 2006; Siriwardene et al., 2007). ICPI suggests that using a joint filling material will limit the surface clogging to the top 20-25 mm layer of the surface and would avoid the progression of clogging to deeper layer and the storage gallery. ICPI also states that during high intensity rainfalls, and when the joint filling material is partially filled with debris, the permeable surface will continue to infiltrate the runoff (PICP, 2007). The permeable joint material can also affect the infiltration and retention of stormwater runoff through influencing the retention of water in the surface blocks (Scholz \& Grabowiecki, 2007).

In order to investigate the effects of the permeable joint material on the progress of clogging and performance of the GI, each configuration was made with and without the \#8 AASHTO aggregate used as the joint filling material.

Figure 31 shows a cross section of the flume with all the components that have been described. 


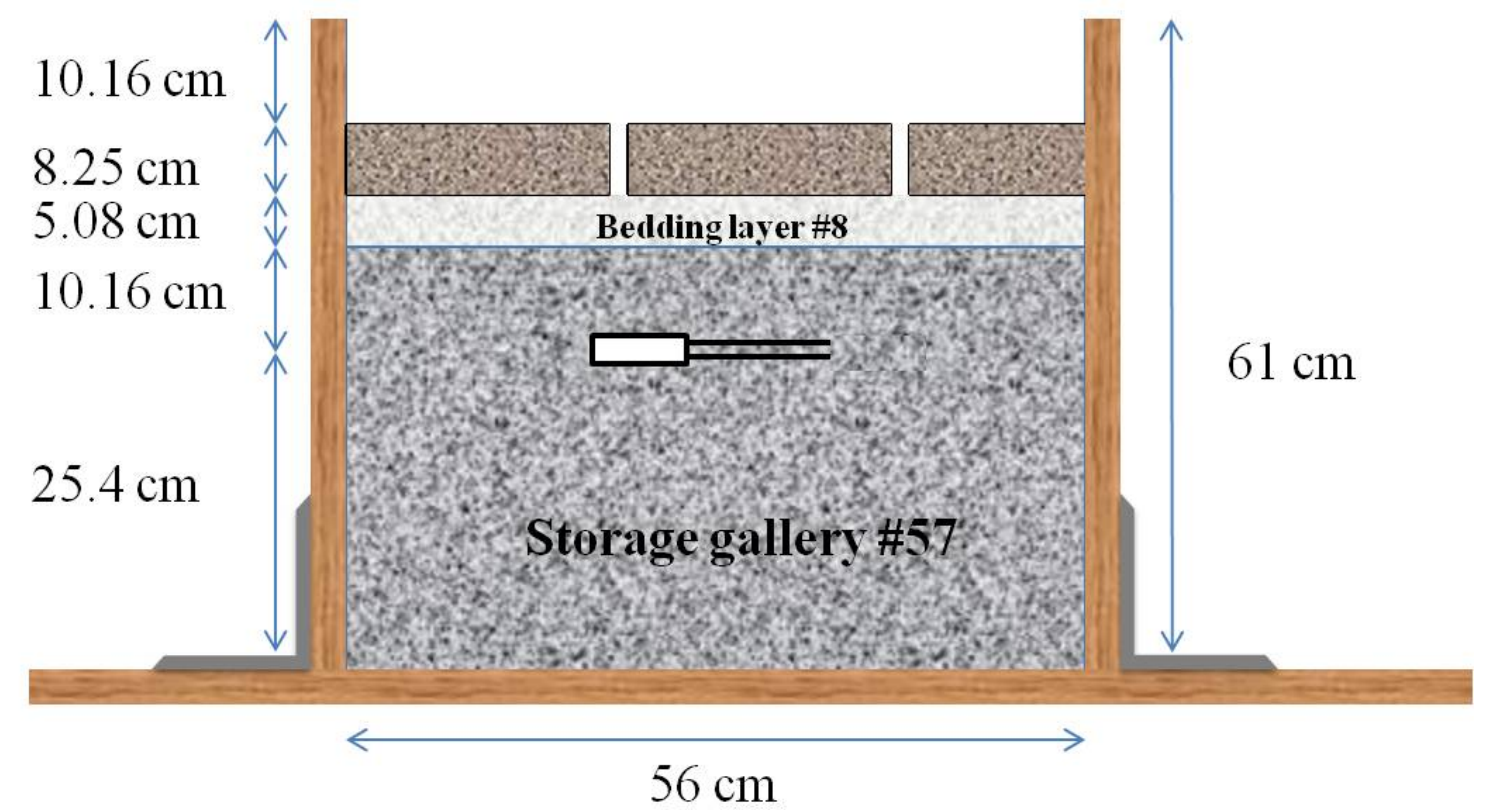

Figure 31: Cross section of flume

\subsection{Experiment Variables}

In order to investigate the effects on clogging of different settings of the physical environment of the GI, and based on the components of the experiment, different variables were designed and changed to create different scenarios as the experiment proceeded. The gap between the pavers, presence of permeable joint material and longitudinal slope of the installation are the variables investigated in this experiment. With all the variables described, a total of 18 different combinations were designed for the experiments, which tested the effects in the physical environment of one change at a time. Table 12 lists the different combinations of the proposed experiment with their respective variables. 
Table 12: Experiment Variables

\begin{tabular}{|c|c|c|c|}
\hline Experiment No. & Slope (\%) & Paver Gap (mm) & Gap Filling \\
\hline 1 & 1 & 6 & None \\
\hline 2 & 1 & 6 & $\# 8$ \\
\hline 3 & 1 & 9 & None \\
\hline 4 & 1 & 9 & $\# 8$ \\
\hline 5 & 1 & 12 & None \\
\hline 6 & 1 & 12 & $\# 8$ \\
\hline 7 & 3 & 12 & $\# 8$ \\
\hline 8 & 3 & 12 & None \\
\hline 9 & 3 & 9 & None \\
\hline 10 & 3 & 9 & $\# 8$ \\
\hline 11 & 3 & 6 & None \\
\hline 12 & 3 & 6 & $\# 8$ \\
\hline 13 & 5 & 12 & None \\
\hline 14 & 5 & 12 & $\# 8$ \\
\hline 15 & 5 & 9 & None \\
\hline 16 & 5 & 9 & $\# 8$ \\
\hline 17 & 5 & 6 & None \\
\hline 18 & 5 & 6 & $\# 8$ \\
\hline
\end{tabular}

\subsection{Maintenance}

After conducting an experiment, which is designed to intentionally clog the permeable surface of the permeable pavers, and in order to keep the flume in working condition by restoring the infiltration capability of permeable pavers, the surface had to be maintained. Maintenance had to remove all surface clogging debris and residue. In addition, since some portion of the sediment might migrate to the lower layers of the flume, the maintenance had to address the issue by replacing the parts of the bedding layer where there was any sign of the migration of fines, to avoid deterioration. However, the TDRs that were being used as a primary tool for monitoring the experiments could not be moved. A constant location for the TDR is required, to limit the noise and inconsistencies in the data. With these factors in mind the maintenance process included: 
- Cleaning the surface and the gaps with an industrial vacuum cleaner

- Removing the first paver and then the remaining pavers from the flume and washing them

- Replacing the bedding layer aggregate with clean \#8 AASHTO aggregate

- Replacing the parts of the storage gallery that showed signs of the migration of fine sediment

- Laying the pavers of the next experiment in the flume

During the maintenance of the flume, observations on the depth of penetration of clogging sediment, the patterns of both organics and inorganics deposited on the surface, ease of removing the material, and any other information that could be utilized as an assessment tool, had to be carefully recorded. 


\section{DATA ANALYSIS}

\subsection{Introduction}

The laboratory experiments were designed to assess the influence of the physical environment on the permeable pavement systems. Three different permeable paving schemes were analyzed which provided the opportunity to assess differing characteristics of the pavers and corresponding maintenance needs. The clogging and maintenance requirements depended on multiple variables, including the longitudinal slope of the system, the specification of the paver product used, and the type/size of permeable joint material (if any). Having a clear and well-structured understanding of the effects of these variables on performance and their contributions to system failure will help to optimize the current designs and choose the best locations and settings for future applications

The analyses are split into groups according to type and data collection methods. Three different levels of analysis were made of the collected data. Each level of analysis answered a specific range of questions and each was essential for optimizing a project based on the design goals of any given permeable pavement system.

The first category of results is mostly based on the observations made during the experiments; therefore it is mostly a qualitative analysis. The use of remote monitoring instruments in the Louisville CSO130 project helped to demonstrate the importance of observing real life events. The opportunity to observe the numerous scenarios of 
permeable pavement systems in action in the laboratory environment and to match the observations with expectations is one of the important benefits of this study. The observations also helped to explain some of the unusual patterns seen in the data collected by means of instruments.

The second category includes a preliminary analysis of the collected data using the TDRs. A dataset was prepared and the raw data converted into readable data sets. This step of the work was an essential tool which gives valuable performance results. It could be used to verify the application of the instruments and the quality of the data collected by this means. This category of analyses is semi-qualitative with some quantitative analysis.

The third step of the analysis is an in-depth analysis, which is mostly quantitative, using statistical tools to interpret the transformed raw data into meaningful information and draw conclusions from it on many aspects of the work, such as design suggestions, maintenance needs, and BMP optimization.

\subsection{Observations}

\subsubsection{Patterns for Sediment Deposited on the Surface}

\subsubsection{1. $\quad$ Predicted Patterns}

Analysis of the sediment collected from the paver gaps in Louisville's installation showed an obvious separation between the organic and inorganic sediments. With the 6 mm gap size and the empty permeable paver joints, the inorganics were trapped at the up gradient segments, while the organic debris flowed to the more down gradient segments. During the flume experiments, and in the scenarios where there was no permeable joint 
material present, the same pattern was predicted and observed. In these experiments the separation between the organic material and the inorganics was quite obvious. This phenomenon is shown in Figure 32 through Figure 34.

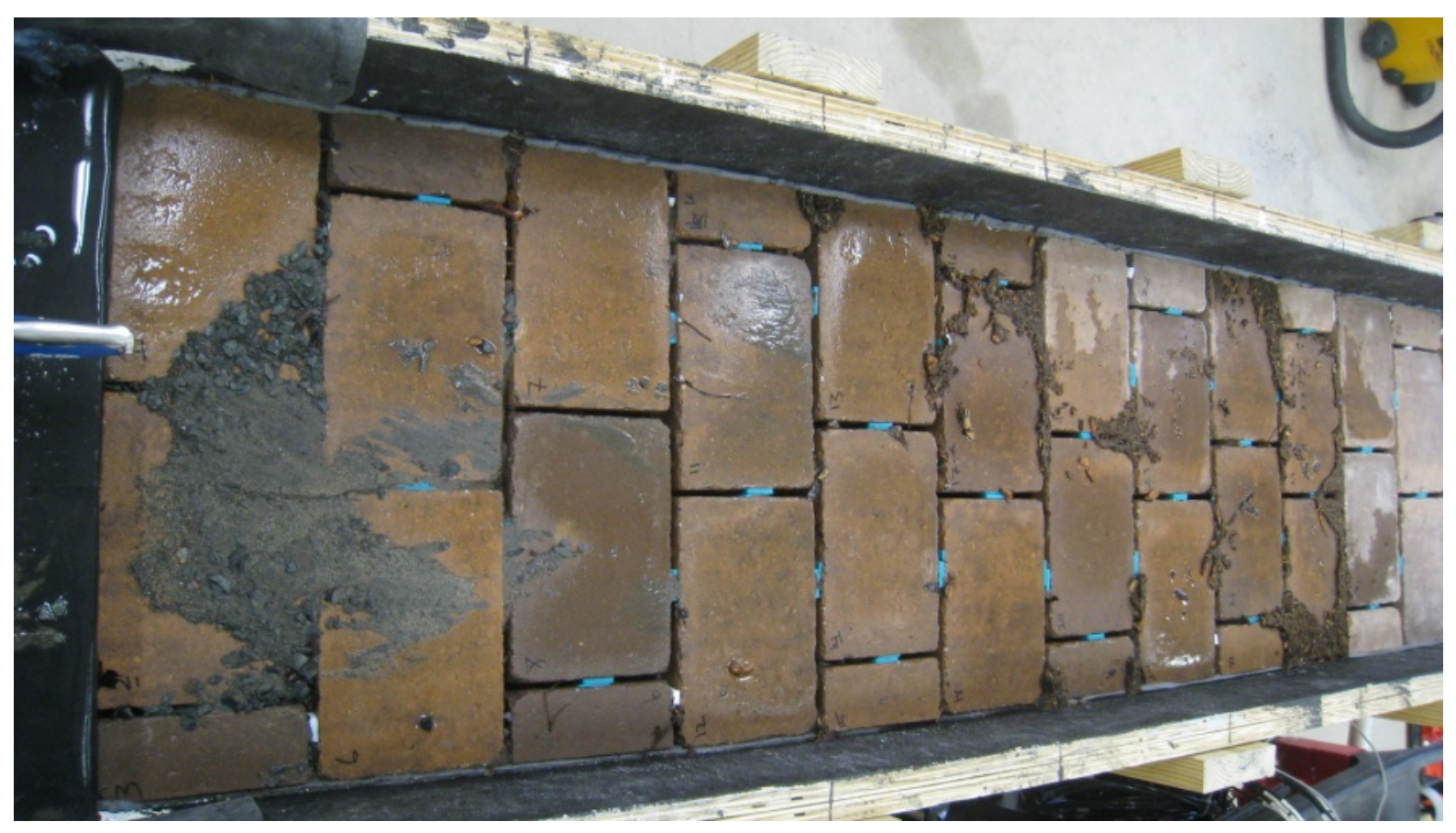

Figure 32: Experiment \#1, 1\% slope, $6 \mathrm{~mm}$ gap size, no aggregate between the pavers

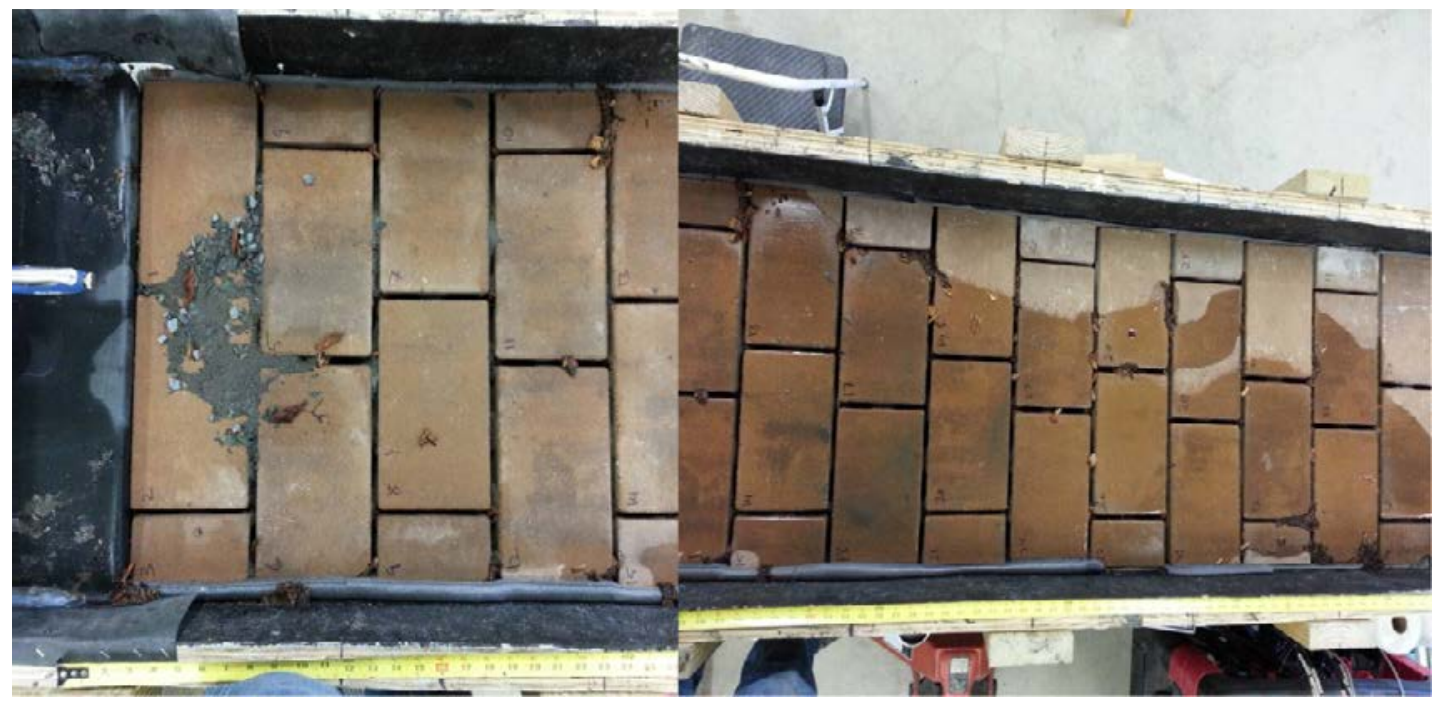

Figure 33: Experiment \#5, $1 \%$ slope, $9 \mathrm{~mm}$ gap size, no aggregate between the pavers 


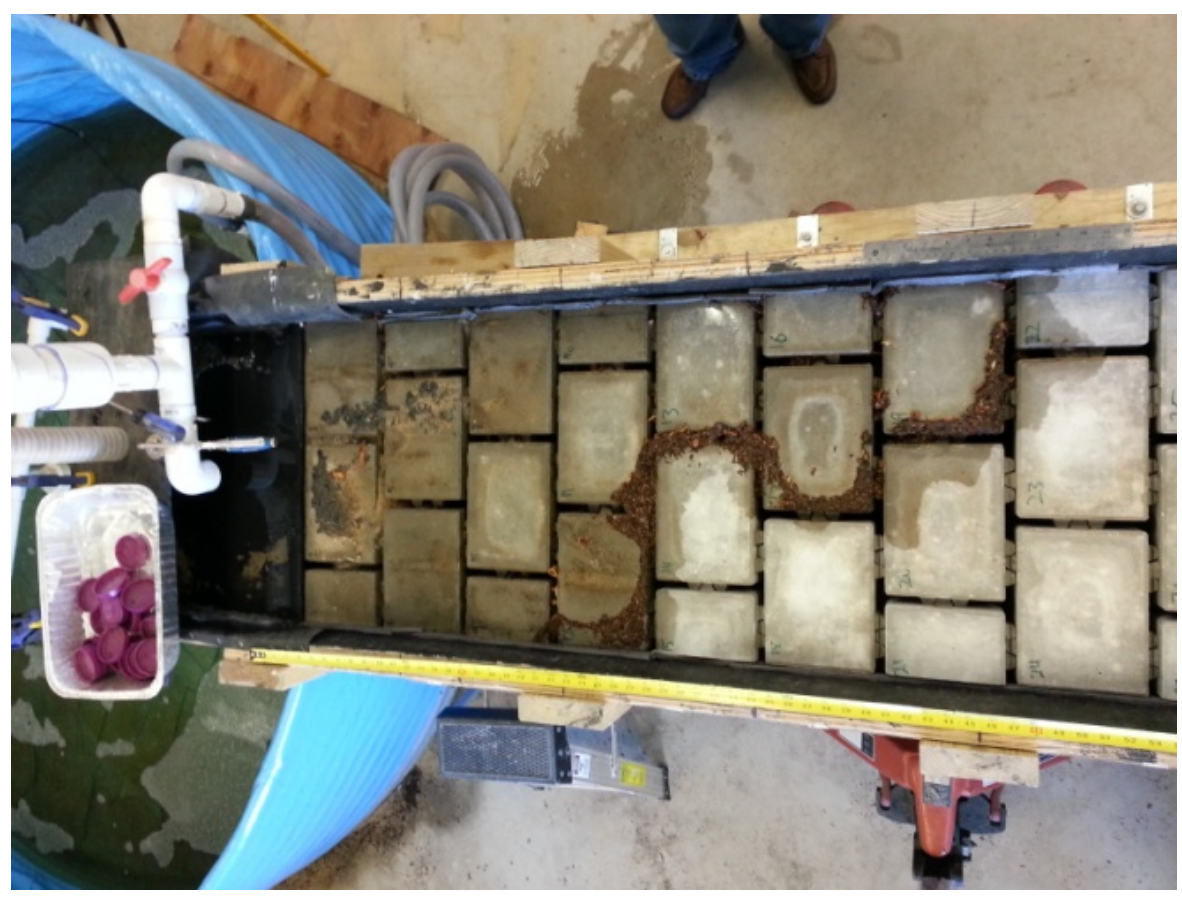

Figure 34: Experiment \#7, 1\% slope, $12 \mathrm{~mm}$ gap size, no aggregate between the pavers In the experiments where the permeable joint material was present in the gaps, however, a different pattern was observed. In these experiments, and depending on the length of the progression of clogging, both the organic and the inorganic sediment reached the down gradient segments of the flume. In these cases, there was little or no separation between the places where the different kinds of sediment were being deposited. Figure 35 through Figure 37 show the pavers of three different gas sizes, all set at a $1 \%$ longitudinal slope, and after the 100-minute period of the experiment. 


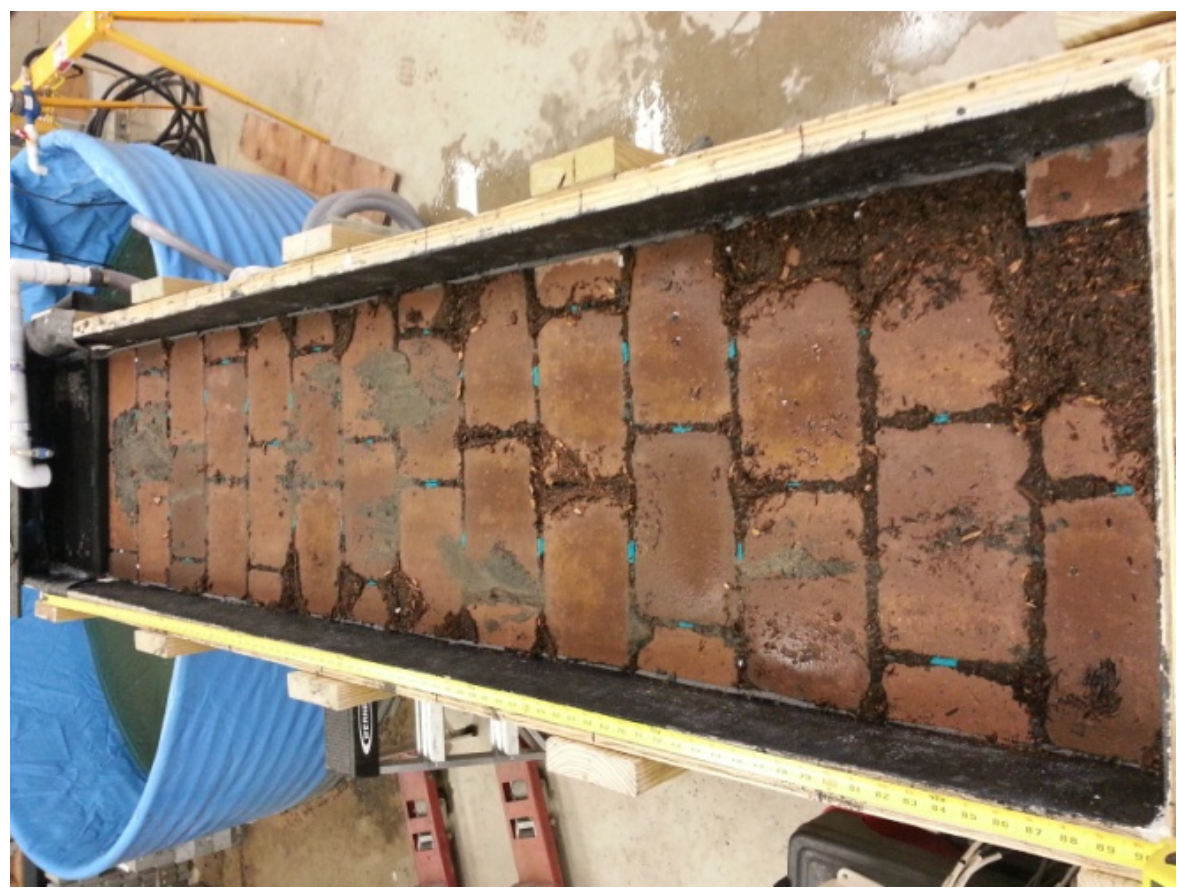

Figure 35: Experiment \#4, 1\% slope, $6 \mathrm{~mm}$ gap size, no aggregate between the pavers

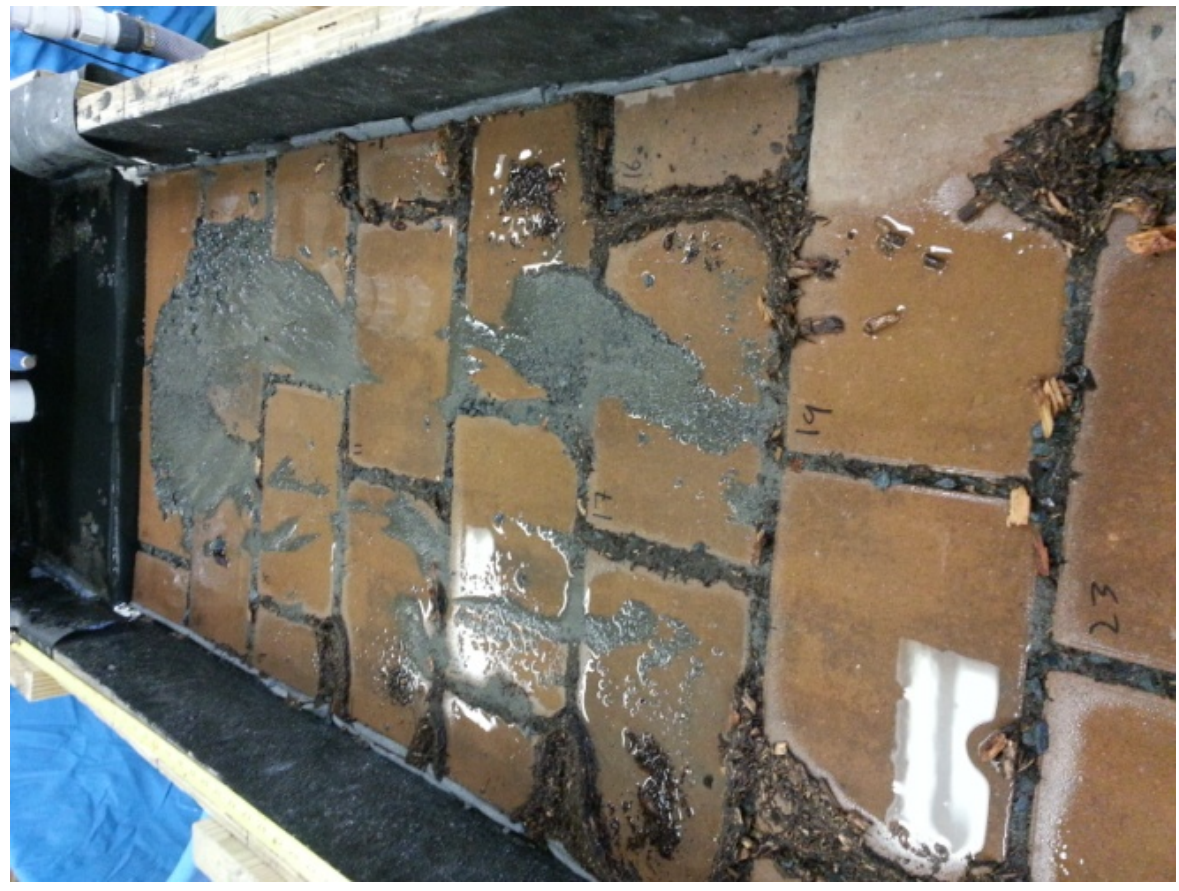

Figure 36: Experiment \#6, 1\% slope, 9 mm gap size, no aggregate between the pavers 


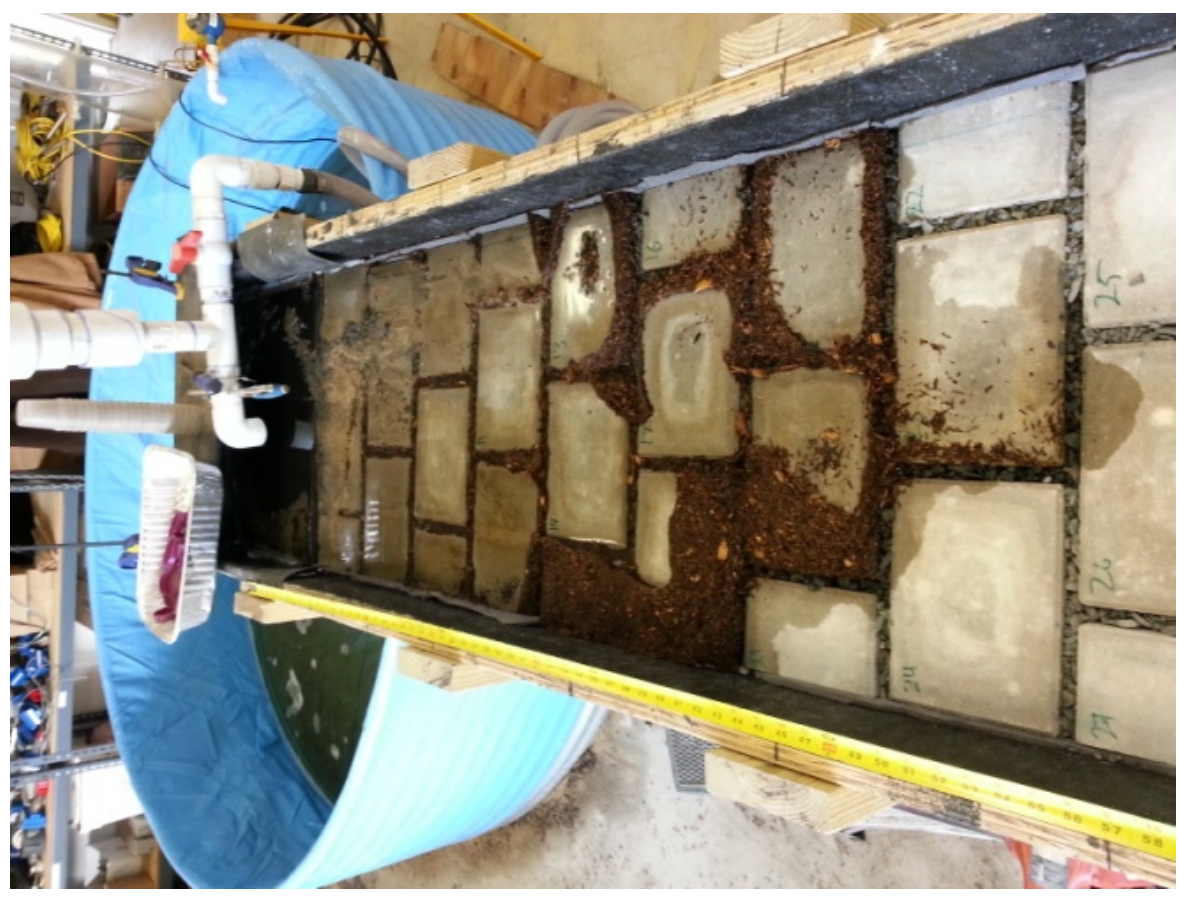

Figure 37: Experiment \#8, 1\% slope, $12 \mathrm{~mm}$ gap size, no aggregate between the pavers

The explained patterns were almost similar in steeper longitudinal slope, but where there was no permeable joint material available, the inorganic sediment traveled further along the surface.

\subsubsection{2. $\quad$ Unpredicted Patterns}

Apart from the patterns that were predicted and expected during the experiment, and matched the previous work, some behavior was observed that was not expected. The first of these patterns included changes in the stormwater runoff flow patterns on the surface of the flume. The flow was greatly affected by the mulch deposited in the gaps, where it caused the flow to be diverted and bypass some of the gaps. Figure 38 gives an example of this unpredicted pattern on the surface, seen in experiment \#5. The top left highlighted area shows a piece of mulch that is stuck in the gaps, causing the water to flow towards the right side of the flume. As the clogging proceeds, the flow direction 
causes more mulch to clog the gaps in the same pattern until the clogging reaches the other end along the width. At this point, the runoff bounces off the wall and flows in the opposite direction. 


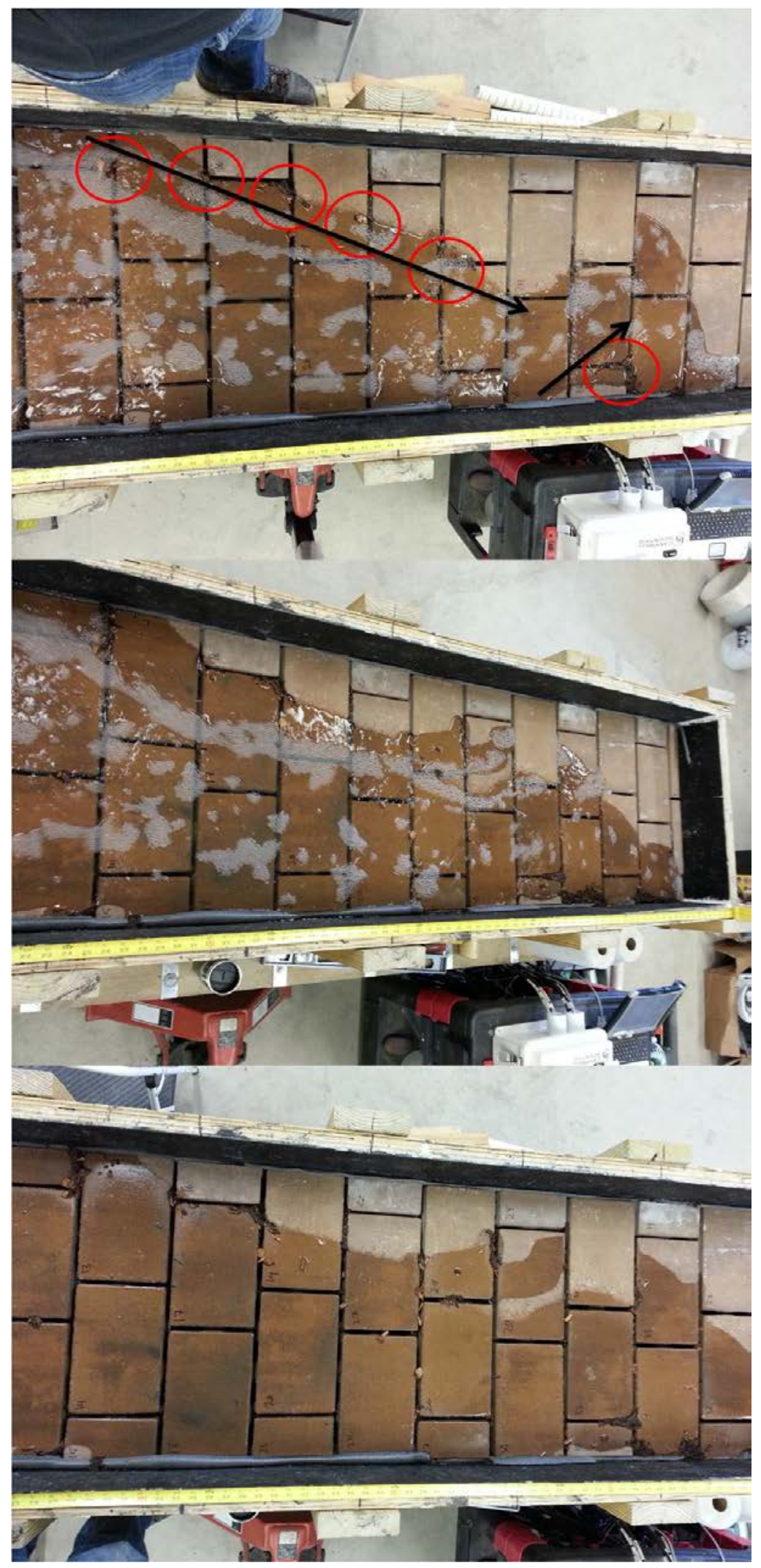

Figure 38: Stormwater runoff flow unpredicted pattern in experiment \#5 
The effect of this unpredicted flow pattern on the measurements is discussed in the preliminary analysis of results.

\subsubsection{Extent of Clogging}

The experiments began by starting the runoff flow, and dumping the first sediment cup 30 seconds after beginning the test. As the experiment went on, in almost all the different configurations, it was observed that the speed of clogging decelerated. This was obvious in cases where the steeper longitudinal slope (3\% and 5\%) and presence of permeable joint material caused the clogging to reach furthest in the down gradient section of the flume. In the tests where this happened, there was no indication of the rapid ponding of water or failure in the flume. Although some ponding was observed, the surface infiltration rate from the clogged pavers was enough to tolerate and infiltrate the runoff flow rate. The condition where the flume's surface is clogged, yet it is still capable of infiltrating some stormwater runoff is referred to as "clogged, but not sealed". 


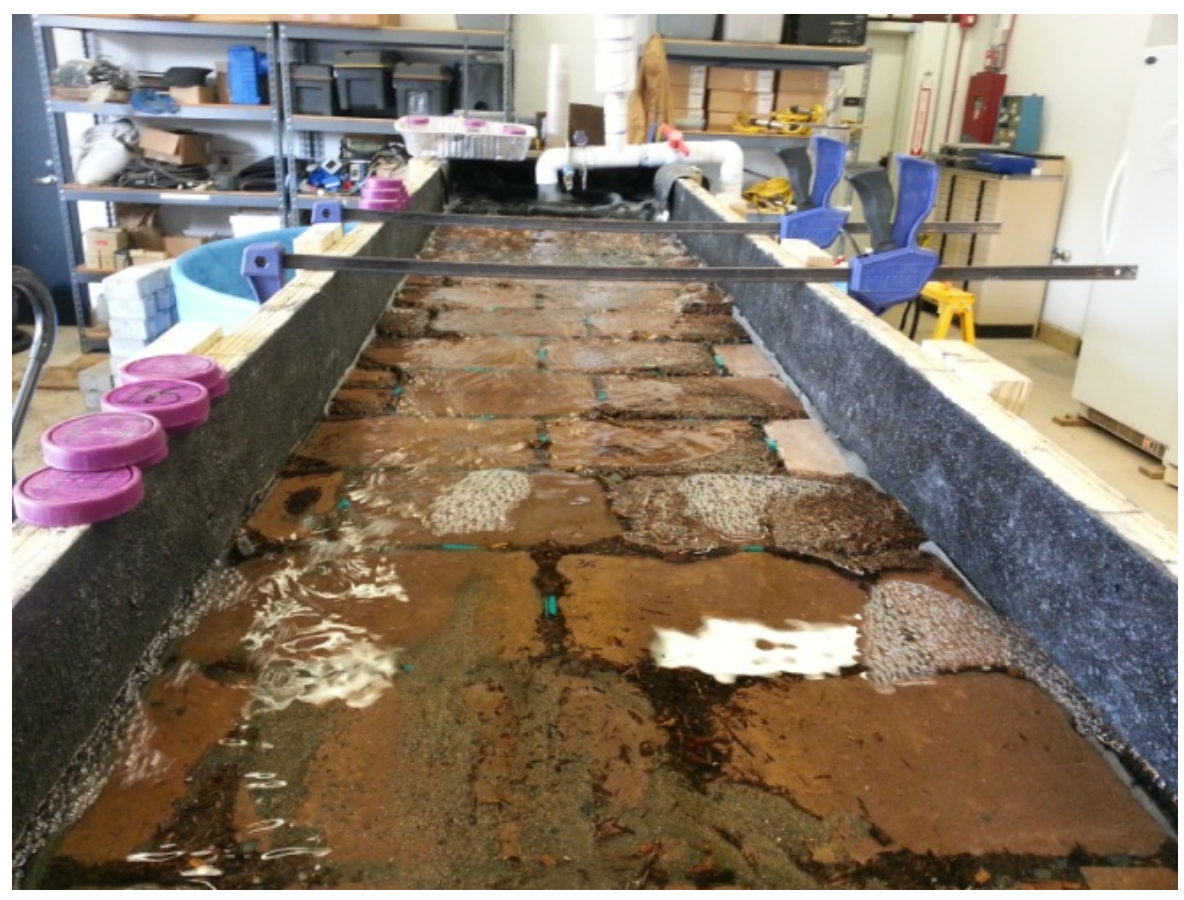

Figure 39: Clogged but not sealed, experiment \#14, 3\% slope, $6 \mathrm{~mm}$ gap size, \#8 aggregate between the pavers

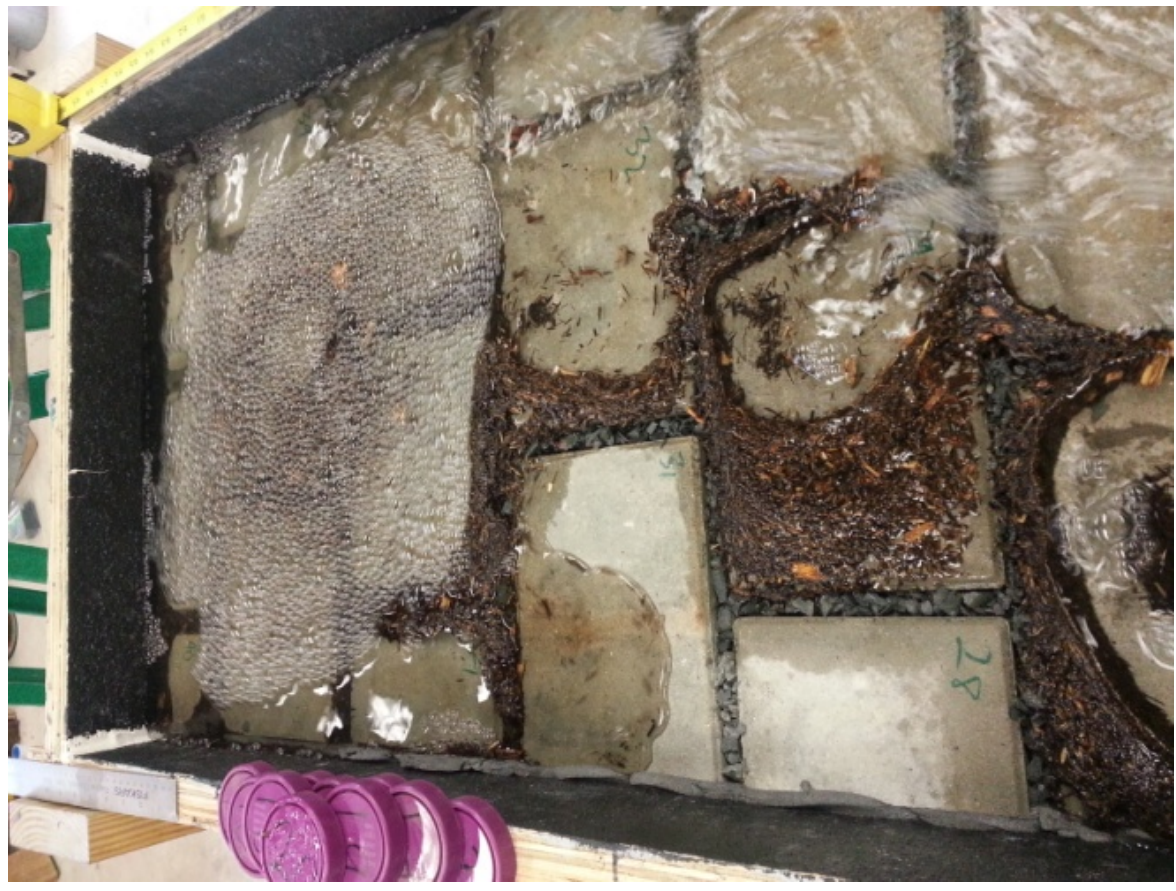

Figure 40: Clogged but not sealed, experiment \#15, 5\% slope, $12 \mathrm{~mm}$ gap size, \#8 aggregate between the pavers 


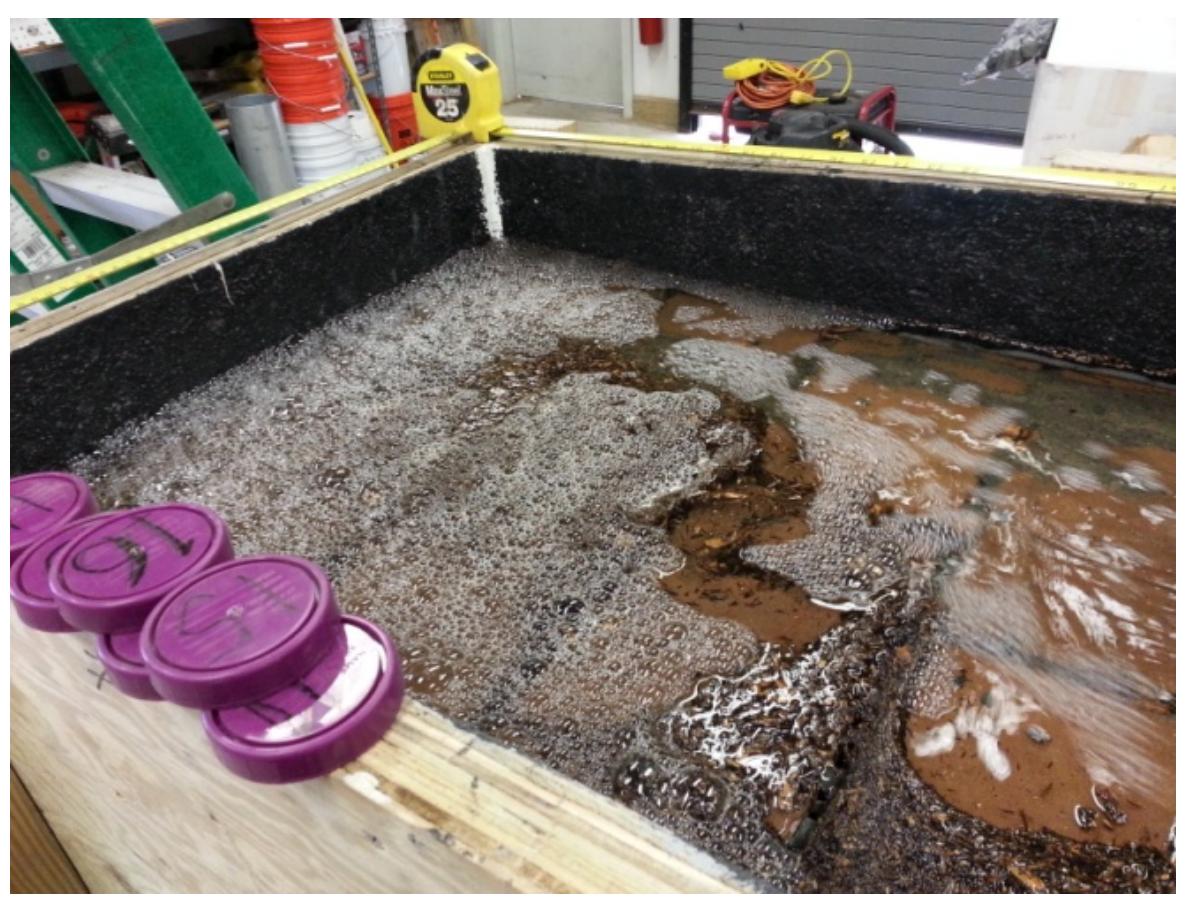

Figure 41: Clogged but not sealed, experiment \#18, 5\% slope, $9 \mathrm{~mm}$ gap size, \#8 aggregate between the pavers

Although in all instances, the system was clogged but not sealed, with the observations and measurements made on the depth of ponding at the furthest down gradient segment of the flume, it was observed that with the increase of longitudinal slope and a reduced gap size, the condition of the system would deteriorate very soon. For instance, in experiment \#20, with a 5\% longitudinal slope, $6 \mathrm{~mm}$ gap size, and \#8 aggregate as permeable joint material, at the 100-minute mark, half the length of the flume was submerged (Figure 42) and the depth of ponding was about $8 \mathrm{~cm}$ (Figure 43). 


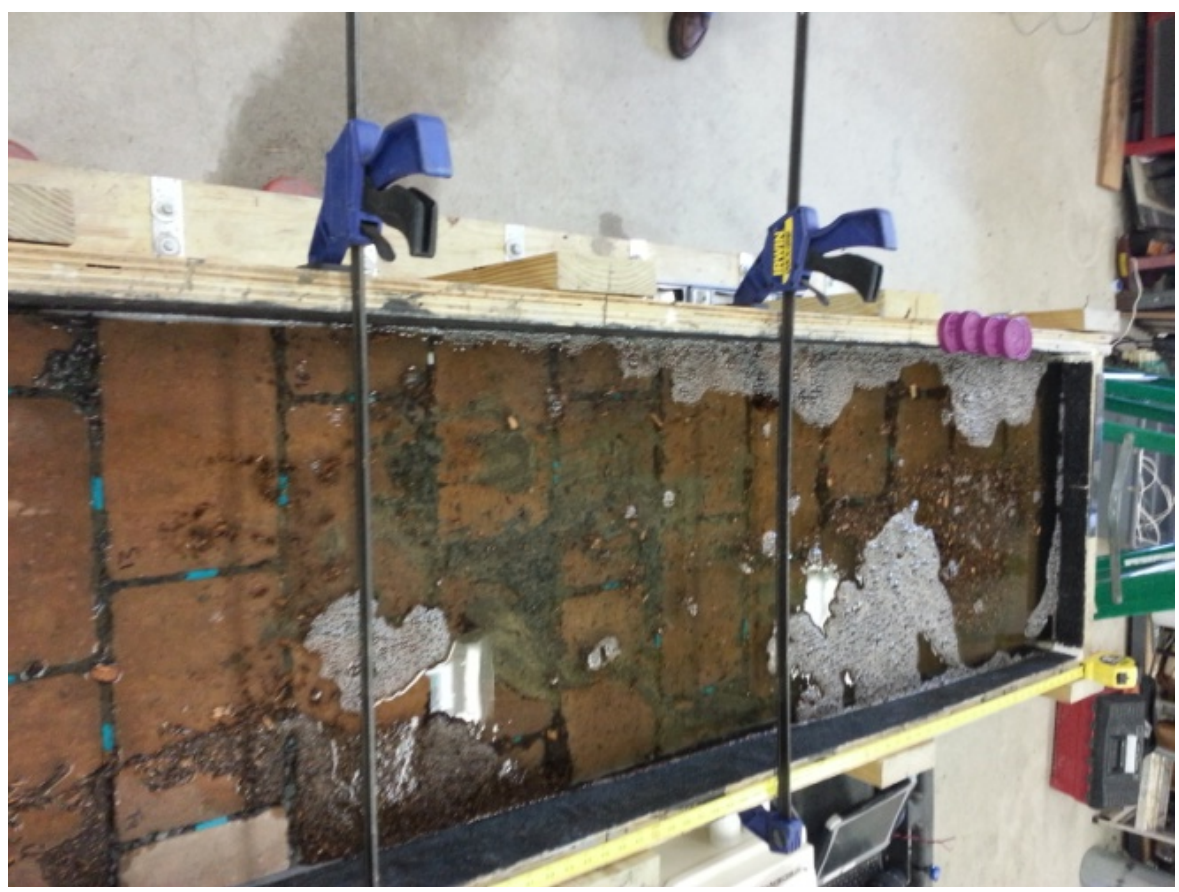

Figure 42: Clogged but not sealed, experiment \#20, 5\% slope, $6 \mathrm{~mm}$ gap size, \#8 aggregate between the pavers

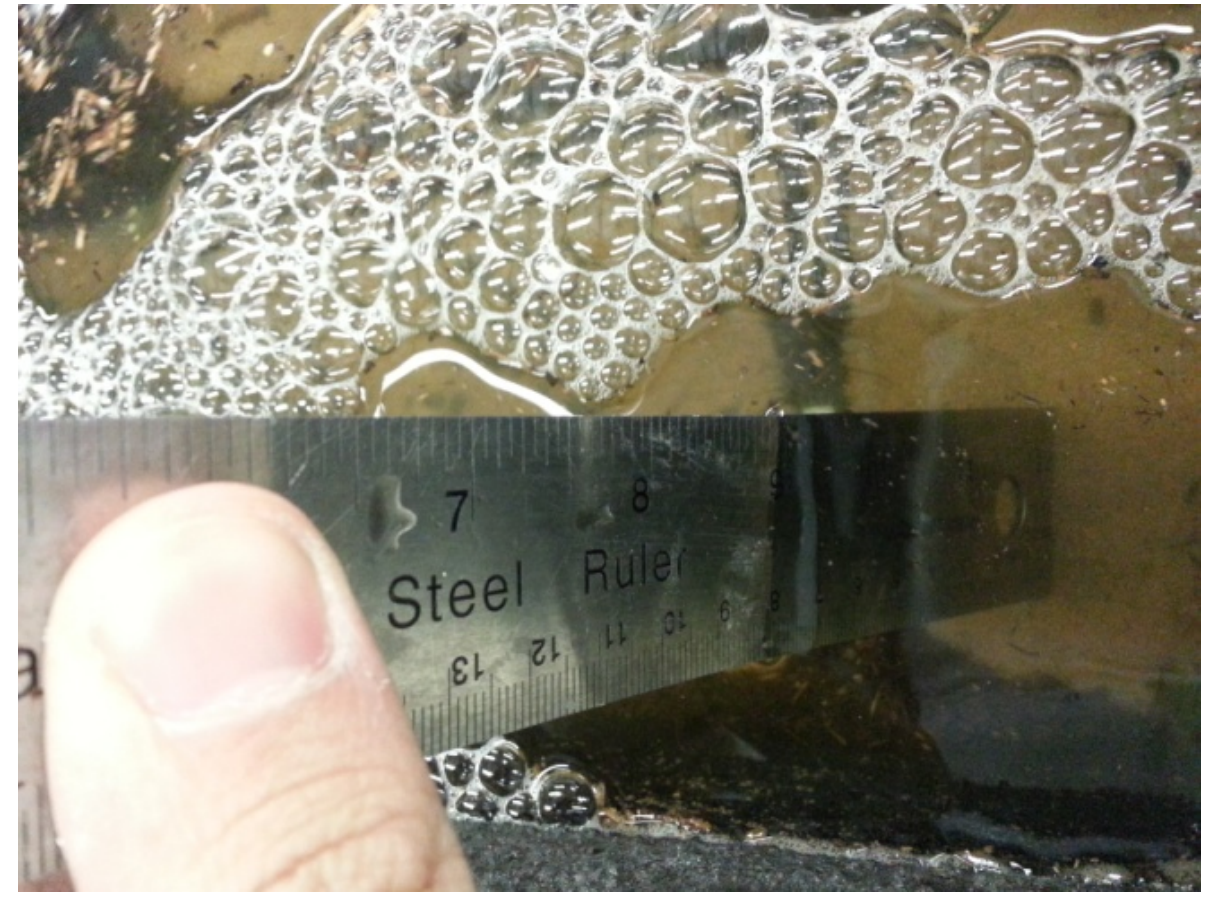

Figure 43: Ponding in the most down gradient segment of flume in experiment \#20 


\subsubsection{Speed of Clogging}

With an increased longitudinal slope, it was observed that the clogging was progressing much faster and in cases where the paver gap sizes were $6 \mathrm{~mm}$ and $9 \mathrm{~mm}$ and the gaps were filled with permeable joint material, in particular at a 3\% and 5\% longitudinal slope, the length of clogging in the flume reached the furthest down gradient segment after about a quarter of the sediment cups. Figure 44 shows the start of the experiment after 4 cups of sediment, which illustrates the clogged gaps, while Figure 45, in a photograph taken after 3 cups of sediment, shows that, compared to the previous figure, the progression of clogging reached further down. It also illustrates that the gaps are not completely clogged, for obvious void space is still available. The extent of this behavior is more significant in the experiments where the permeable joint material is present.

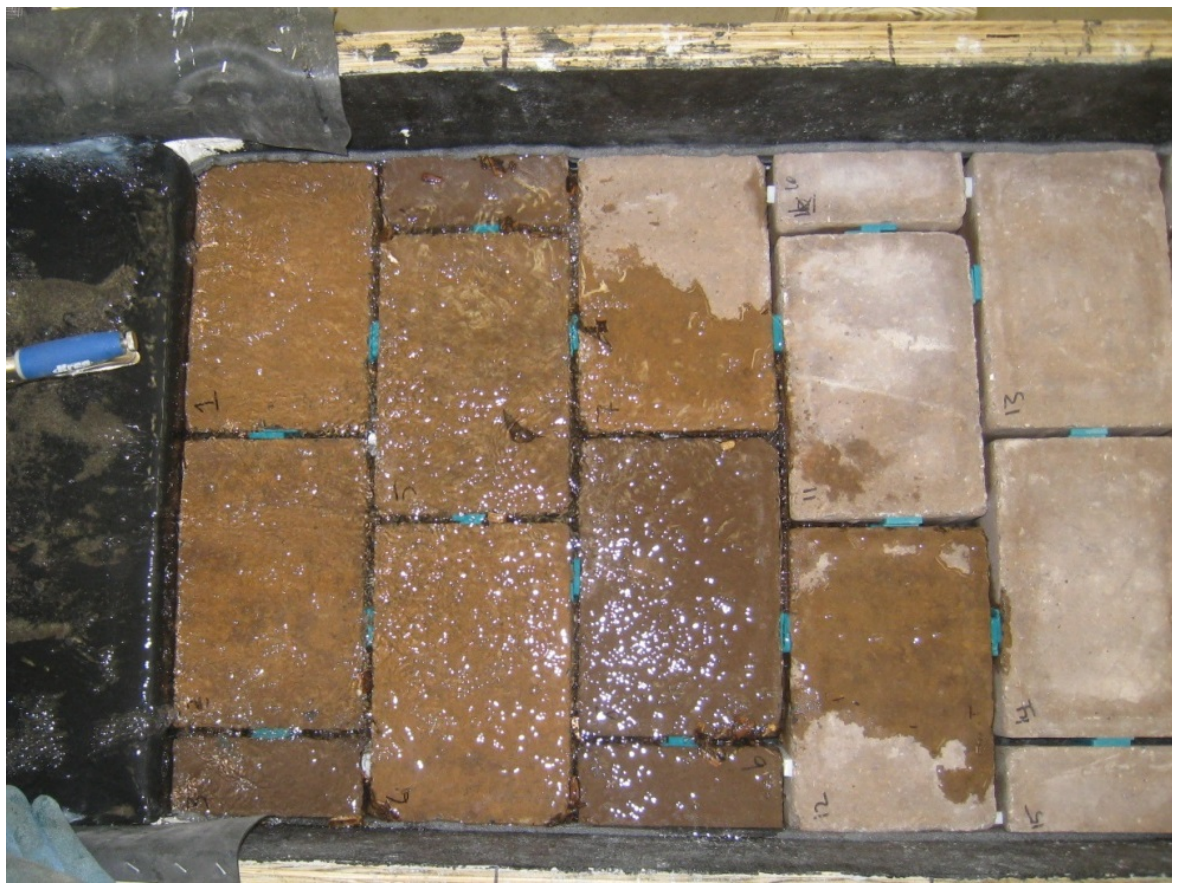

Figure 44: Experiment \#1, 1\% slope, $6 \mathrm{~mm}$ gap size, no aggregate between the pavers 


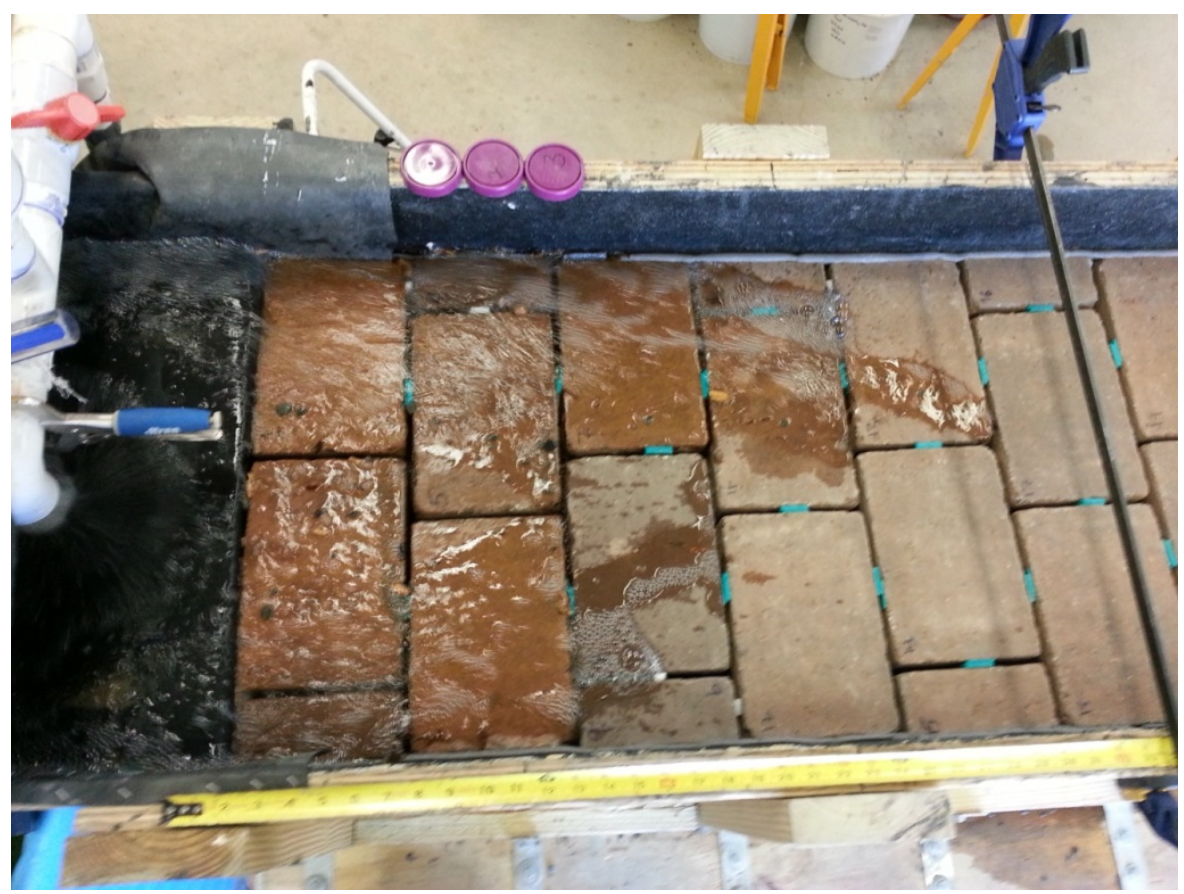

Figure 45: Experiment \#19, 5\% slope, $6 \mathrm{~mm}$ gap size, no aggregate between the pavers

\subsubsection{Maintenance}

After each flume experiment run, the flume was maintained and prepared for the next experiment run. The maintenance included removing and washing the paver blocks, using a Shop Vac. to clean the debris from the surface and the gaps, and replacing the bedding layer with clean stone.

During the maintenance process it was observed that removing the material using the vacuum cleaner was easier when the gaps were bigger and when the material was left to dry overnight. Using a Shop Vac. on wet sediment would have required more suction power, since the sediment would have been intact and therefore heavier. It was also observed that the depth of the paver block had the direct impact on the efficiency of using the Shop Vac to clean the gaps, but when the paver blocks were deeper, the vacuum cleaner was less efficient at removing the debris from the gaps. The paver's spacer lugs 
also had an influence on the process: where the spacer lugs created a more confined space the Shop Vac was not very effective.

When the pavers were removed, there were minimal or no signs of fine particles underneath them and almost all the clogging material was intact (Figure 47), located in the gaps. Figure 46 shows the sediment on the bedding layer after removing the paver from an experiment where no permeable joint material was present. A different pattern was observed in the experiments, where there was \#8 in the pavers’ gaps. Figure 48 shows the surface of the bedding layer directly after removing the paver blocks. It is obvious that the permeable joint material has reduced the extent of migration of the sediment to the storage gallery.

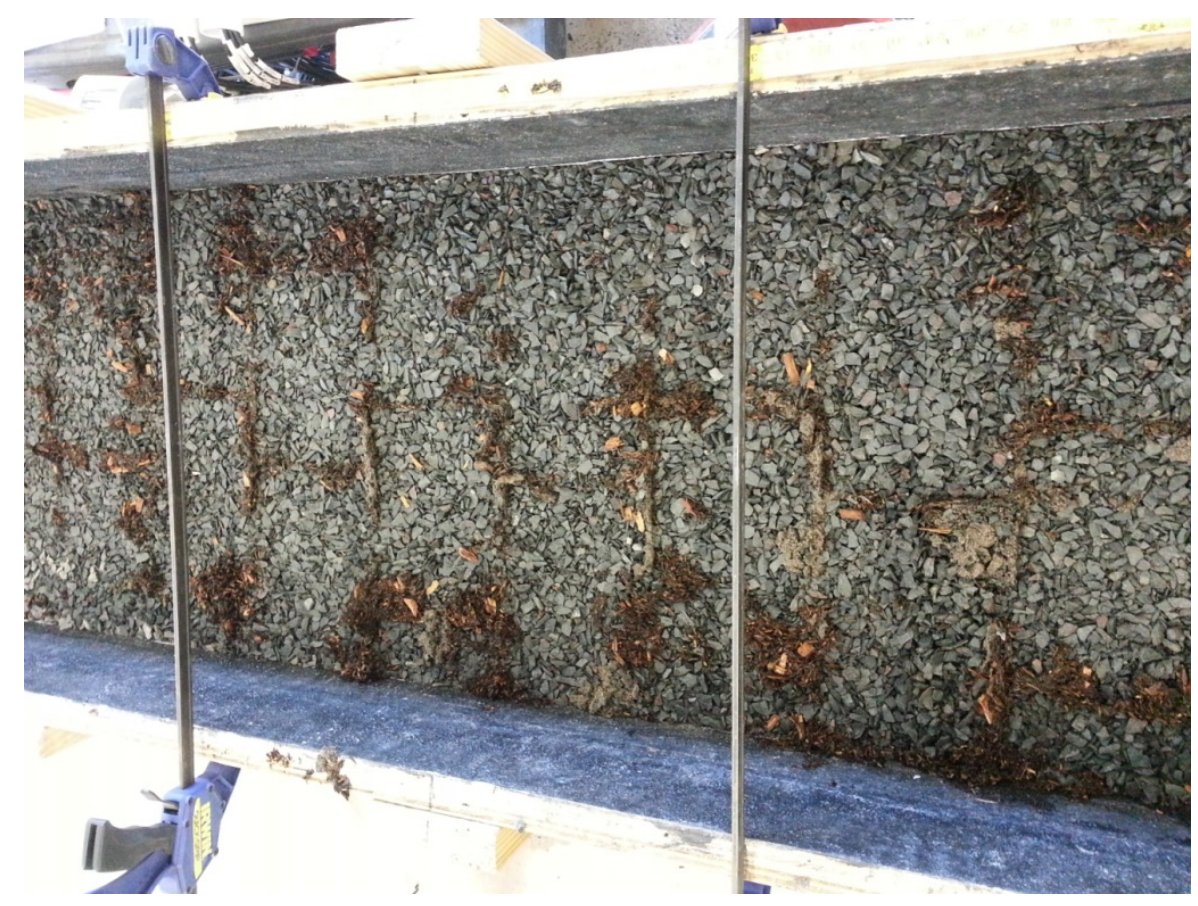

Figure 46: Experiment \#19, 5\% slope, $6 \mathrm{~mm}$ gap size, no aggregate between the pavers, after removing the pavers 


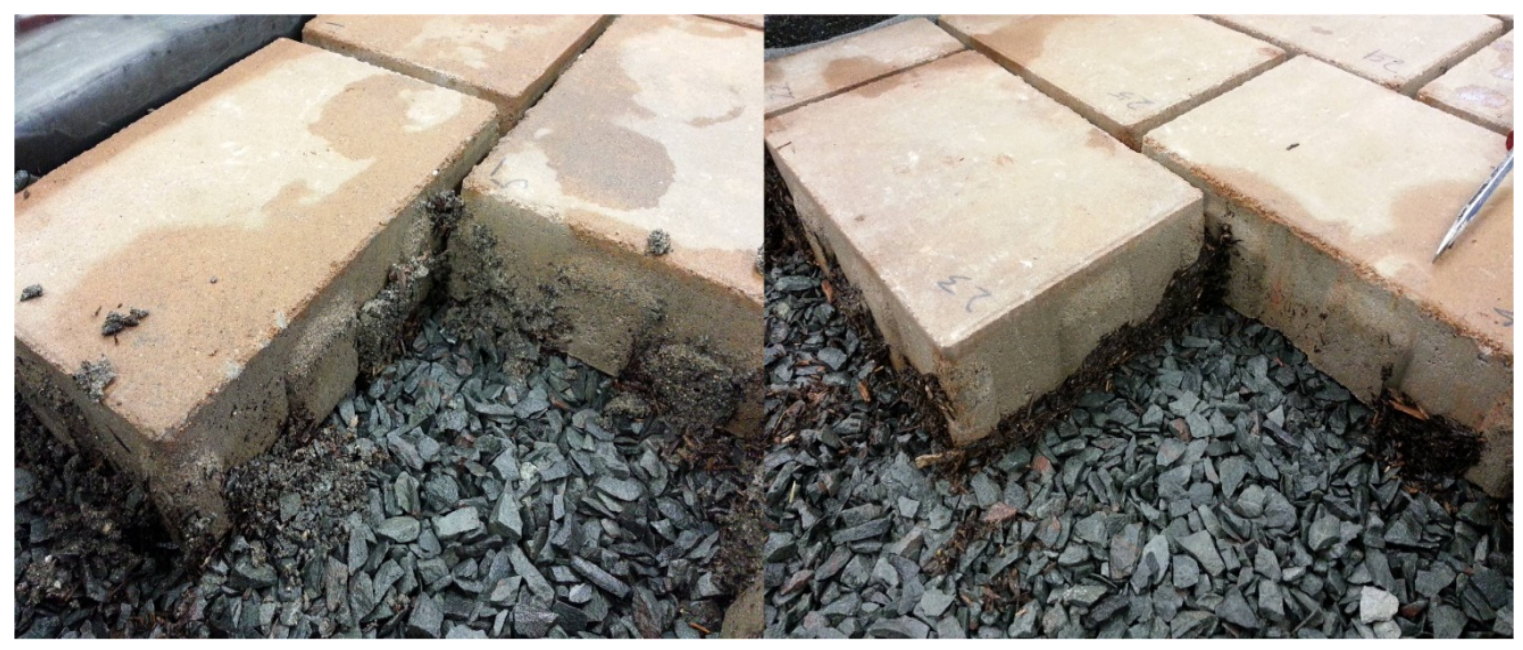

Figure 47: Experiment \#11, 3\% slope, $9 \mathrm{~mm}$ gap size, no aggregate between the pavers, after removing the pavers

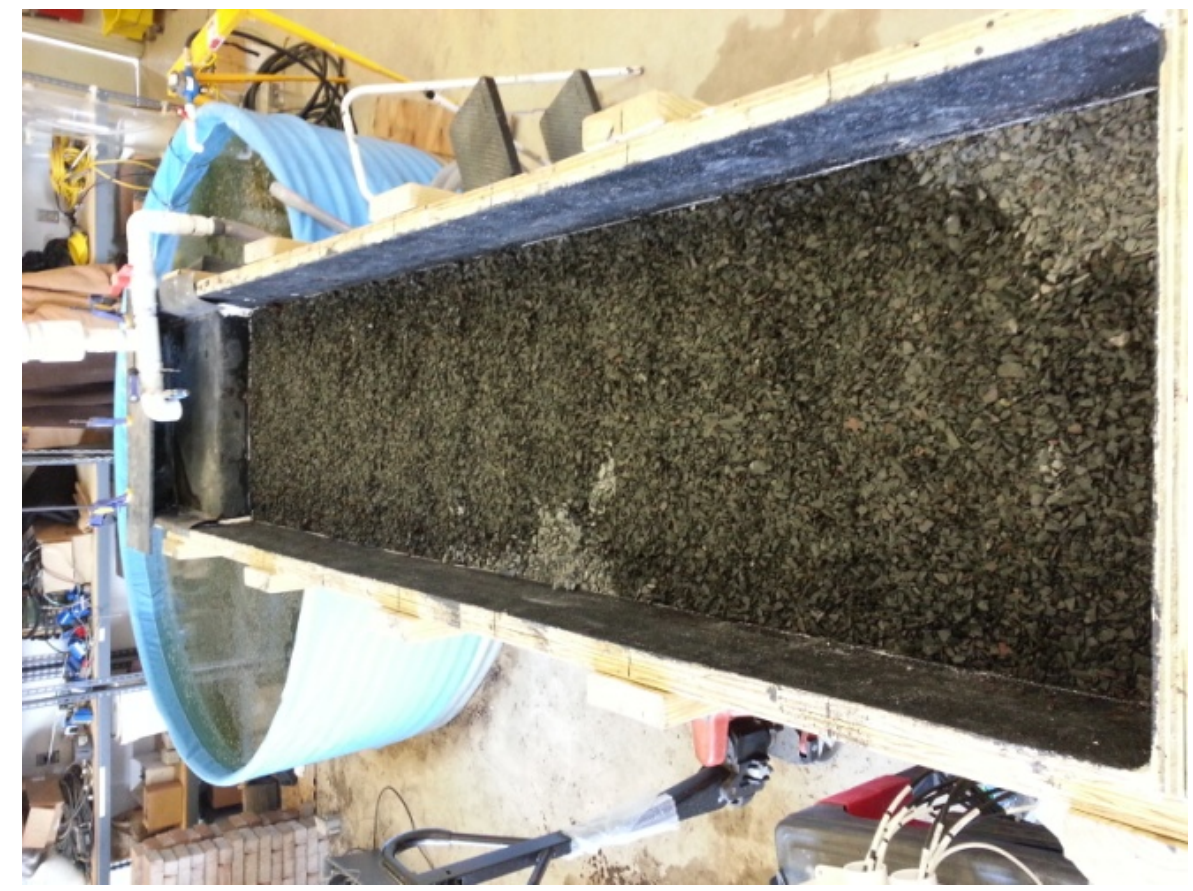

Figure 48: Experiment \#9, 3\% slope, $12 \mathrm{~mm}$ gap size, \#8 aggregate between the pavers, after removing the pavers

Maintenance and the assessment of maintenance methods is not a main focus of this research, but it is worth noting that the observations made during the maintenance show that the efficiency of the maintenance method is a direct result of the power of the vacuum cleaner used. Comparing the results of this section with the two maintenance 
treatments conducted on Louisville’s CSO130 installations makes the need for refining Louisville’s maintenance plan inescapable.

\subsection{Preliminary Data Analysis}

\subsubsection{Total Suspended Solids}

During each experiment and before pumping the stormwater runoff to the flume, a 2 liter sample of the runoff was taken and analyzed for TSS. This was done to determine the effects (if any) of the initial TSS loads in the stormwater runoff. The results of the analyses of the stormwater runoff are presented in Table 13. The TSS results of the initial 13 experiments show a relatively low load of suspended solids, which does not have a significant effect on the sediment load carried by the runoff. Prior to TEST 13 and on 4/12/2013 rain fell in New Jersey and the runoff generated by this rain refilled the primary stormwater runoff tank with relatively turbid stormwater. It is thought that the turbidity was caused by the algae that had grown in the tank as a result of rising temperatures and caused the TSS loads to rise. Although the TSS of the collected stormwater runoff increased from that on 4/15/2013, testing the stormwater showed that the suspended solids were smaller than the smallest sediment size (retained on sieve \#200) and therefore did not affect the surface clogging of the permeable pavers. After determining that the source of TSS load in the stormwater did not affect the result, the source of turbidity in the primary tank was not investigated. 


\begin{tabular}{|c|c|c|}
\hline Test Number & Test Date & TSS (mg/L) \\
\hline 1 & $2 / 26 / 2013$ & 2.1 \\
\hline 2 & $2 / 27 / 2013$ & 3.1 \\
\hline 3 & 2/28/2013 & 3.9 \\
\hline 4 & $4 / 2 / 2013$ & 2.8 \\
\hline 5 & $4 / 3 / 2013$ & 2.9 \\
\hline 6 & $4 / 4 / 2013$ & 2.2 \\
\hline 7 & $4 / 8 / 2013$ & 1.5 \\
\hline 8 & $4 / 8 / 2013$ & 1.3 \\
\hline 9 & $4 / 9 / 2013$ & 4.0 \\
\hline 10 & 4/10/2013 & 2.90 \\
\hline 11 & 4/11/2013 & 1.70 \\
\hline 12 & $4 / 12 / 2013$ & 3.20 \\
\hline 13 & $4 / 15 / 2013$ & 6.00 \\
\hline 14 & $4 / 16 / 2013$ & 11.20 \\
\hline 15 & $4 / 17 / 2013$ & 28.60 \\
\hline 16 & $4 / 18 / 2013$ & 30.90 \\
\hline 17 & $4 / 22 / 2013$ & 29.50 \\
\hline 18 & 4/23/2013 & 23.9 \\
\hline 19 & $4 / 24 / 2013$ & 12.9 \\
\hline 20 & 4/25/2013 & 15.1 \\
\hline
\end{tabular}

\subsection{2. $\quad$ Effluent Flow Rate}

Measuring the flow rate at the effluent was used as a tool to determine the consistency of the runoff flow to the flume. During the experiments, the flow rate of the effluent was measured. This process was started by measuring the two flow rates for the first experiment, in which one flow rate had been measured after dumping the first sediment cup and the other after dumping the $20^{\text {th }}$ sediment cup. Starting from the second experiment, the number of measurements was increased to three readings, i.e., after the first, tenth, and twentieth sediment cups. The only test without three measurements of discharge flow rate is test 3 , where the last flow rate was not recorded. 
The measurements were taken using a graduated cylinder and a timer, and the results are listed in Table 14 . Note that after the $4^{\text {th }}$ and $5^{\text {th }}$ experiments, it was discovered that the section of the effluent that was outside the lab had been dismantled, causing an inconsistent flow rate in the measurements for these experiments. Later in this chapter, the inconsistency of the flow rates at these two experiments is compared to the volume of water used during the experiment to provide more accurate data.

Table 14: Effluent Flow Rates (LPM)

\begin{tabular}{|c|c|c|c|c|c|c|c|c|c|c|c|}
\hline \multirow[b]{2}{*}{$\begin{array}{c}\text { Experimen } \\
\mathrm{t}\end{array}$} & \multicolumn{3}{|c|}{ Discharge 1} & \multicolumn{3}{|c|}{ Discharge 2} & \multicolumn{3}{|c|}{ Discharge 3} & \multirow[b]{2}{*}{$\begin{array}{c}\text { Avg } \\
.\end{array}$} & \multirow{2}{*}{$\begin{array}{l}\text { Std. } \\
\text { Dev }\end{array}$} \\
\hline & $\begin{array}{l}\text { Vol } \\
(\mathrm{ml})\end{array}$ & $\begin{array}{l}\text { Tim } \\
\text { e (s) }\end{array}$ & LPM & $\begin{array}{l}\text { Vol } \\
(\mathrm{ml})\end{array}$ & $\begin{array}{l}\text { Tim } \\
\text { e (s) }\end{array}$ & LPM & $\begin{array}{l}\text { Vol } \\
\text { (ml) }\end{array}$ & $\begin{array}{l}\text { Tim } \\
\text { e (s) }\end{array}$ & LPM & & \\
\hline 1 & $\begin{array}{l}164 \\
0\end{array}$ & 3.21 & $\begin{array}{l}30.6 \\
5\end{array}$ & N/A & N/A & N/A & $\begin{array}{l}158 \\
0\end{array}$ & 2.66 & $\begin{array}{l}35.6 \\
4\end{array}$ & 33.1 & 3.5 \\
\hline 2 & $\begin{array}{l}154 \\
0\end{array}$ & 2.69 & $\begin{array}{l}34.3 \\
5\end{array}$ & $\begin{array}{l}154 \\
0\end{array}$ & 2.56 & $\begin{array}{l}36.0 \\
9\end{array}$ & $\begin{array}{l}150 \\
0\end{array}$ & 2.50 & $\begin{array}{l}36.0 \\
0\end{array}$ & 35.5 & 1.0 \\
\hline 3 & $\begin{array}{l}140 \\
0\end{array}$ & 2.25 & $\begin{array}{l}37.3 \\
3\end{array}$ & $\begin{array}{l}184 \\
0\end{array}$ & 3.06 & $\begin{array}{l}36.0 \\
8\end{array}$ & N/A & N/A & N/A & 36.7 & 0.9 \\
\hline $4^{1}$ & 380 & 1.03 & $\begin{array}{l}22.1 \\
4\end{array}$ & $\begin{array}{l}117 \\
5\end{array}$ & 4.00 & $\begin{array}{l}17.6 \\
3\end{array}$ & $\begin{array}{l}190 \\
0\end{array}$ & 6.35 & $\begin{array}{l}17.9 \\
5\end{array}$ & 20.0 & 2.5 \\
\hline $5^{2}$ & $\begin{array}{l}115 \\
0\end{array}$ & 1.63 & $\begin{array}{l}42.3 \\
3\end{array}$ & $\begin{array}{l}140 \\
0\end{array}$ & 2.86 & $\begin{array}{l}29.3 \\
7\end{array}$ & $\begin{array}{l}140 \\
0\end{array}$ & 3.12 & $\begin{array}{l}26.9 \\
2\end{array}$ & 32.9 & 8.3 \\
\hline 6 & $\begin{array}{l}285 \\
0\end{array}$ & 5.38 & $\begin{array}{l}31.7 \\
8\end{array}$ & $\begin{array}{l}290 \\
0\end{array}$ & 4.94 & $\begin{array}{l}35.2 \\
2\end{array}$ & $\begin{array}{l}290 \\
0\end{array}$ & 5.12 & $\begin{array}{l}33.9 \\
8\end{array}$ & 33.5 & 1.7 \\
\hline 7 & $\begin{array}{l}283 \\
0\end{array}$ & 4.87 & $\begin{array}{l}34.8 \\
7\end{array}$ & $\begin{array}{l}356 \\
0\end{array}$ & 6.06 & $\begin{array}{l}35.2 \\
5\end{array}$ & $\begin{array}{l}292 \\
5\end{array}$ & 5.28 & $\begin{array}{l}33.2 \\
4\end{array}$ & 34.5 & 1.1 \\
\hline 8 & $\begin{array}{l}322 \\
5\end{array}$ & 5.97 & $\begin{array}{l}32.4 \\
1\end{array}$ & $\begin{array}{l}335 \\
0\end{array}$ & 5.72 & $\begin{array}{l}35.1 \\
4\end{array}$ & $\begin{array}{l}305 \\
0\end{array}$ & 5.37 & $\begin{array}{l}34.0 \\
8\end{array}$ & 33.9 & 1.4 \\
\hline 9 & $\begin{array}{l}298 \\
0\end{array}$ & 5.31 & $\begin{array}{l}33.6 \\
7\end{array}$ & $\begin{array}{l}340 \\
0\end{array}$ & 5.87 & $\begin{array}{l}34.7 \\
5\end{array}$ & $\begin{array}{l}350 \\
0\end{array}$ & 5.93 & $\begin{array}{l}35.4 \\
1\end{array}$ & 34.6 & 0.9 \\
\hline 10 & $\begin{array}{l}345 \\
0\end{array}$ & 5.94 & $\begin{array}{l}34.8 \\
5\end{array}$ & $\begin{array}{l}350 \\
0\end{array}$ & 6.00 & $\begin{array}{l}35.0 \\
0\end{array}$ & $\begin{array}{l}310 \\
0\end{array}$ & 5.50 & $\begin{array}{l}33.8 \\
2\end{array}$ & 34.6 & 0.6 \\
\hline 11 & $\begin{array}{l}295 \\
0\end{array}$ & 5.40 & $\begin{array}{l}32.7 \\
8\end{array}$ & $\begin{array}{l}265 \\
0\end{array}$ & 4.38 & $\begin{array}{l}36.3 \\
0\end{array}$ & $\begin{array}{l}345 \\
0\end{array}$ & 6.06 & $\begin{array}{l}34.1 \\
6\end{array}$ & 34.4 & 1.8 \\
\hline 12 & $\begin{array}{l}310 \\
0\end{array}$ & 5.40 & $\begin{array}{l}34.4 \\
4\end{array}$ & $\begin{array}{l}345 \\
0\end{array}$ & 5.97 & $\begin{array}{l}34.6 \\
7\end{array}$ & $\begin{array}{l}328 \\
0\end{array}$ & 5.81 & $\begin{array}{l}33.8 \\
7\end{array}$ & 34.3 & 0.4 \\
\hline 13 & 318 & 5.32 & 35.8 & 362 & 6.22 & 34.9 & 360 & 6.35 & 34.0 & 34.9 & 0.9 \\
\hline
\end{tabular}

\footnotetext{
${ }^{1}$ Experiments \#4 had an incorrect effluent structure, where the flow was inconsistent.

${ }^{2}$ Experiments \#5 had an incorrect effluent structure, where the flow was inconsistent.
} 


\begin{tabular}{|c|l|l|l|l|l|l|l|l|l|l|l|}
\hline & 0 & & 6 & 0 & & 2 & 0 & & 2 & & \\
\hline & 325 & & 29.1 & 340 & & 33.6 & 340 & & 34.0 & & \\
14 & 0 & 6.69 & 5 & 0 & 6.07 & 1 & 0 & 6.00 & 0 & 32.3 & 2.7 \\
\hline & 280 & & 34.9 & 345 & & 35.3 & 345 & & 33.6 & & \\
15 & 0 & 4.81 & 3 & 0 & 5.85 & 8 & 0 & 6.16 & 0 & 34.6 & 0.9 \\
\hline & 320 & & 32.5 & 345 & & 34.6 & 355 & & 33.4 & & \\
16 & 0 & 5.90 & 4 & 0 & 5.97 & 7 & 0 & 6.37 & 4 & 33.6 & 1.1 \\
\hline & 347 & & 30.4 & 355 & & 34.0 & 345 & & 31.5 & & \\
17 & 0 & 6.84 & 4 & 0 & 6.25 & 8 & 0 & 6.56 & 5 & 32.0 & 0.8 \\
\hline & 280 & & 29.0 & 330 & & 37.2 & 335 & & 31.6 & & \\
18 & 0 & 5.79 & 2 & 0 & 5.31 & 9 & 0 & 6.35 & 5 & 32.7 & 4.2 \\
\hline & 330 & & 31.8 & 350 & & 33.6 & 332 & & 33.0 & & \\
19 & 0 & 6.22 & 3 & 0 & 6.25 & 0 & 5 & 6.03 & 8 & 32.8 & 1.2 \\
\hline & 335 & & 24.7 & 347 & & 33.9 & 339 & & 32.5 & & \\
& 0 & 8.11 & 8 & 0 & 6.13 & 6 & 0 & 6.25 & 4 & 30.4 & 6.5 \\
\hline
\end{tabular}

Figure 49, which presents the discharge flow rates of the experiments, is plotted using the recorded flow rates.

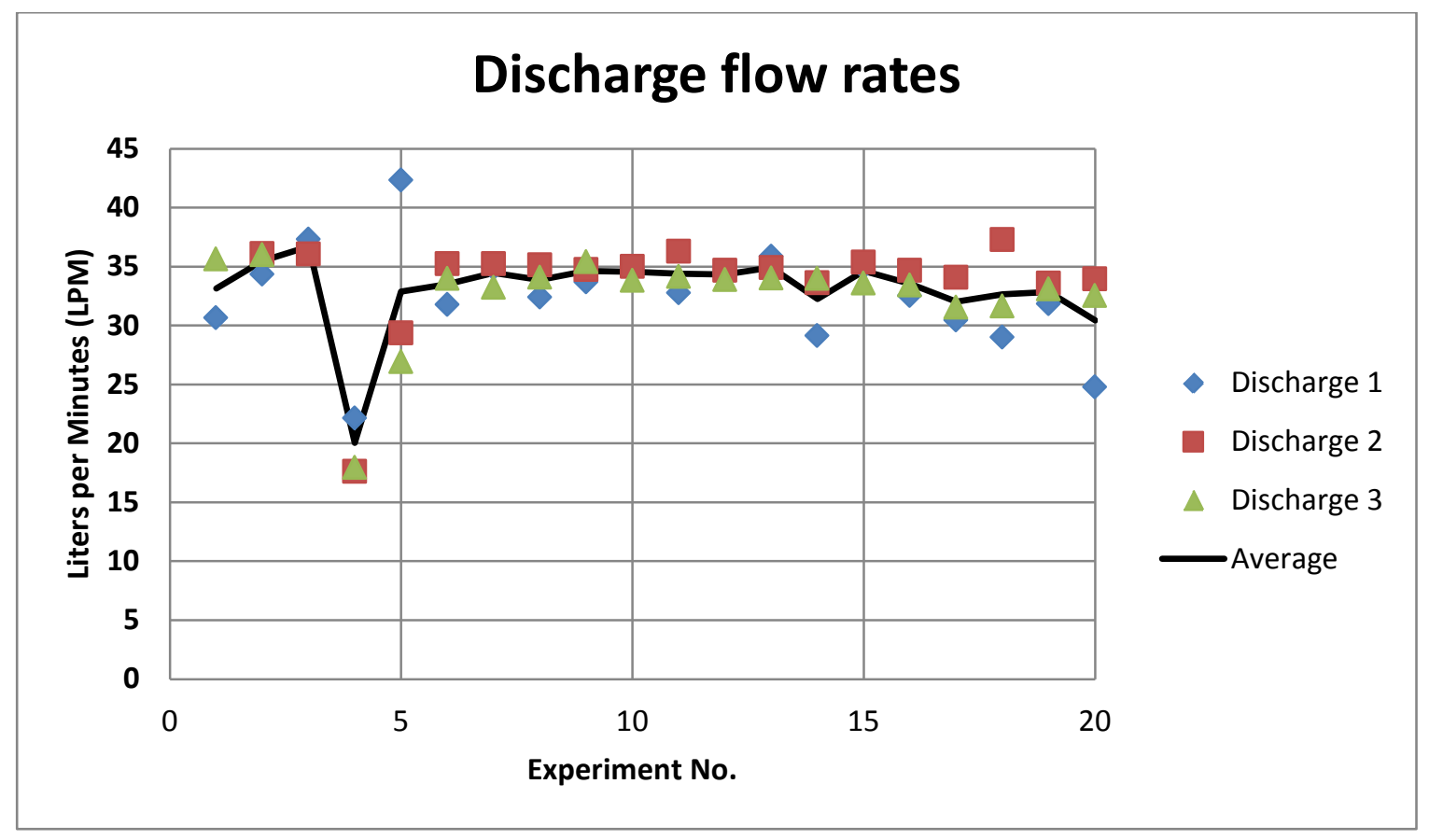

Figure 49: Discharge flow rates of all experiments 
Based on the discharge flow rates and their average, the standard deviation of the measures is also calculated and plotted (see Figure 50). In most cases the standard deviation shows consistent data for the discharge flow rate, but the $5^{\text {th }}$ test and the $20^{\text {th }}$ test have higher standard deviations. As explained, the inconsistency in the $5^{\text {th }}$ test is a result of a dismantled effluent structure that led to incorrect measurements for the flow rate. The reason for the inconsistency seen in the first measurement of discharge flow rate in the $20^{\text {th }}$ test is unknown; however the second and third measurements match the others. The observed inconsistencies stated here can be judged by comparing the volumes of water used in the experiment, as explained in the next paragraph.

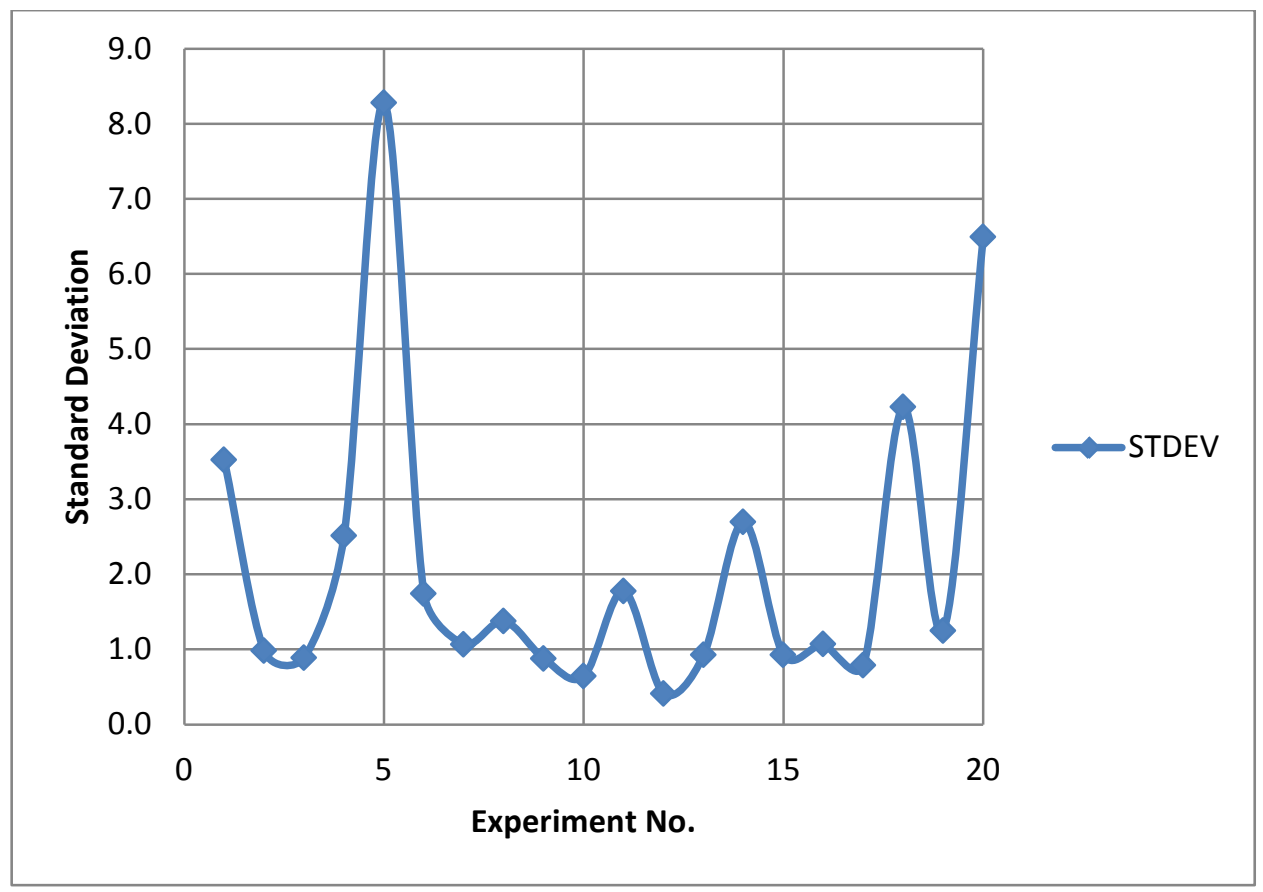

Figure 50: Standard deviation of the discharge flow rates

An approximate volume of the stormwater runoff used during the experiment is among the measurements taken during the experiment, which is calculated using the level of stormwater in the tank before and after the experiment, and the temperature of the 
stormwater in the experiment tank. The diameter of the tank used to store and convey stormwater, shown in Figure 51, is $274.32 \mathrm{~cm}$ (9 feet). The results of calculations made to discover the volume of stormwater used for each experiment is presented in Table 15.

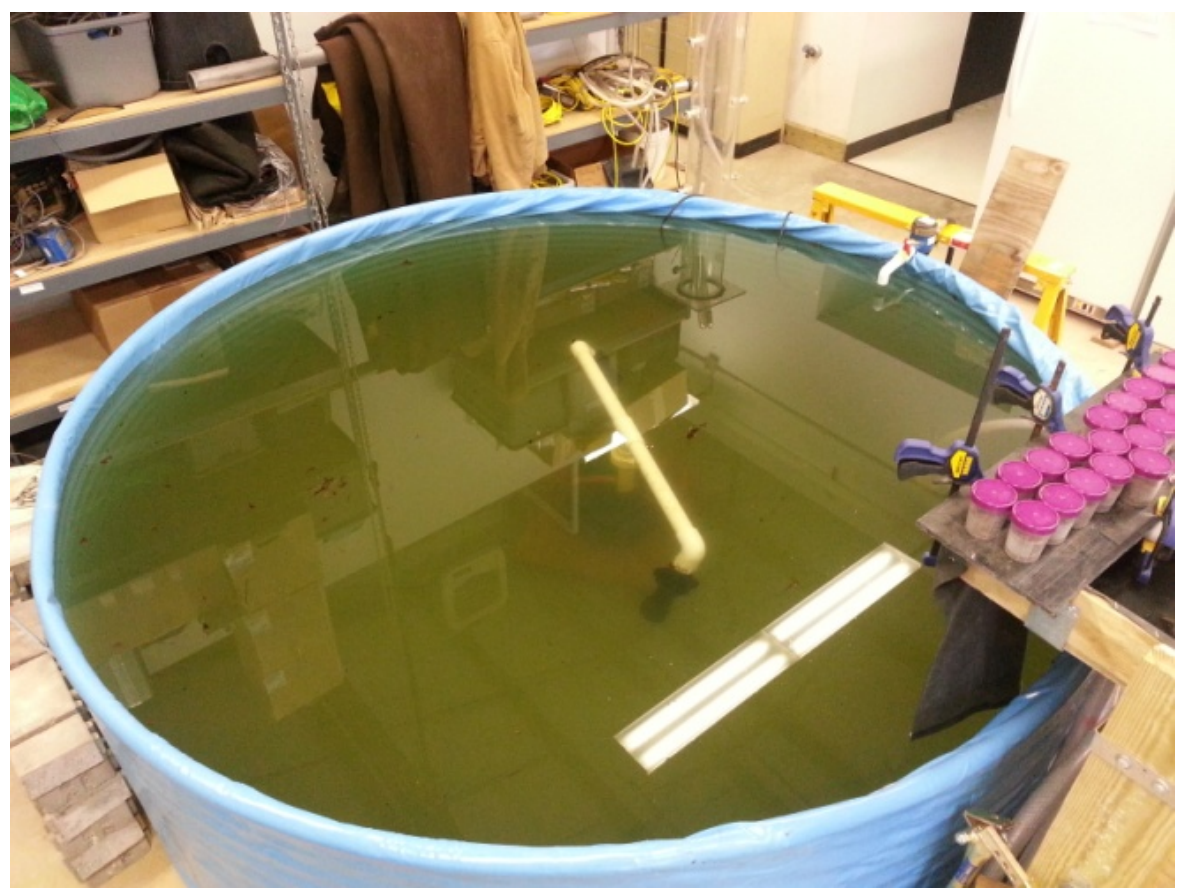

Figure 51: Stormwater runoff tank

Table 15: Volume of Stormwater Runoff Used in the Experiment

\begin{tabular}{|c|c|c|c|c|}
\hline \multirow{2}{*}{ Experiment } & \multicolumn{2}{|c|}{$\begin{array}{c}\text { Water depth in the tank } \\
(\mathrm{cm})\end{array}$} & $\begin{array}{c}\text { Volume of stormwater } \\
\text { used (l) }\end{array}$ & $\begin{array}{c}\text { Stormwater temp } \\
\left({ }^{\circ} \mathrm{c}\right)\end{array}$ \\
\cline { 2 - 3 } & Before & After & N/A \\
\hline 1 & 78.7 & 18 & 3587.5 & N/A \\
\hline 2 & 77.5 & 17 & 3575.7 & 9.2 \\
\hline 3 & N/A & N/A & N/A & 14.1 \\
\hline $4^{*}$ & 83 & 27.5 & 3280.2 & 13.1 \\
\hline $5^{*}$ & 81 & N/A & N/A & 12 \\
\hline 6 & 77 & 17 & 3546.1 & 13.3 \\
\hline 7 & 82 & 22 & 3546.1 & 15.6 \\
\hline 8 & 82.5 & 20.5 & 3664.3 & 17.6 \\
\hline 9 & 81 & 21 & 3546.1 & 19.3 \\
\hline 10 & 75.5 & 15.5 & 3546.1 & 19.5 \\
\hline 11 & 84 & 23 & 3605.2 & 16.7 \\
\hline 12 & 78.5 & 18 & 3575.7 & 13.2 \\
\hline 13 & 79 & 19 & 3546.1 & \\
\hline
\end{tabular}




\begin{tabular}{|l|c|c|c|c|}
\hline 14 & 76 & 16 & 3546.1 & 15.4 \\
\hline 15 & 78 & 17 & 3605.2 & 16.1 \\
\hline 16 & 80.5 & 20 & 3575.7 & 16.4 \\
\hline 17 & 81.5 & 21 & 3575.7 & 15.7 \\
\hline 18 & 78.5 & 18 & 3575.7 & 15.7 \\
\hline 19 & 80 & 19.5 & 3575.7 & 14.1 \\
\hline 20 & 75.5 & 15 & 3575.7 & 16.7 \\
\hline
\end{tabular}

\subsubsection{Temperature Compensation}

The measurements provided by the Campbell Scientific CS616 TDRs are of the raw volumetric water content (Campbell-Scientific, 2012), which are calculated from the recorded periods using a formula built in to the data logger program (CampbellScientific, 2012). These periods are turned into VWC using a quadratic equation that is supplied by the Campbell Scientific soils laboratory and is a result of calibrating the of TDR in different types of soil.

Equation 1: Conversion of period to VWC

$$
\text { VWC }=-0.0663-0.0063 * \text { period }+0.0007 * \text { period } 2
$$

This particular model of TDR records the raw VWC and the recorded numbers must be compensated on the basis of the temperature of the area surrounding the TDRs. A thermistor, the Campbell Scientific L107 temperature sensor, is located along the $4^{\text {th }}$ TDR in the flume and the numbers measured are used to compensate the VWC measurements (Campbell-Scientific, 2013b). Using the VWC, temperature compensated VWC or TC_VWC is measured using:

Equation 2: Temperature compensation for VWC

$\mathrm{TC}_{\text {_ }} \mathrm{VWC}\left(\mathrm{T}_{\text {soil }}\right)=\mathrm{VWC}_{\text {uncorrected }}{ }^{+}\left(20-\mathrm{T}_{\text {soil }}\right) *\left(0.526-0.052 * V W C_{\text {uncorrected }^{*}}+0.00136 * \mathrm{VWC}_{\text {uncorrected }^{2}}\right)$

\subsubsection{Percentage of Saturation}

As mentioned in previous chapters, the primary uses of TDRs are agricultural. In soil, the measurement of volumetric water content ranges from $0 \%$ to $100 \%$, but in 
aggregate, their measurements hardly ever exceed $25 \%$. In order to create a customized range for the TDR measurements in this experiment, an initial experiment called experiment \#0 was created, where the flow of stormwater runoff to the flume was increased up to the point where the flume merely failed. The failure involved a rapid increase in the ponding of the stormwater runoff. The TDR measurements in experiment \#0 indicated a case where the surroundings of the TDR were flooded. The TC_VWC measured with TDR1 through TDR7 in experiment \#0 are plotted in Figure 52.The flooded measurements of each TDR were then used in a saturated condition and all the other measurements were adjusted on the basis of the saturated condition. The converted measurement is called the "percentage of saturation". Figure 53 illustrates the measurements collected during experiment $\# 0$ and in the new format. This format could then be used as the basis for all the other graphs and analyses.

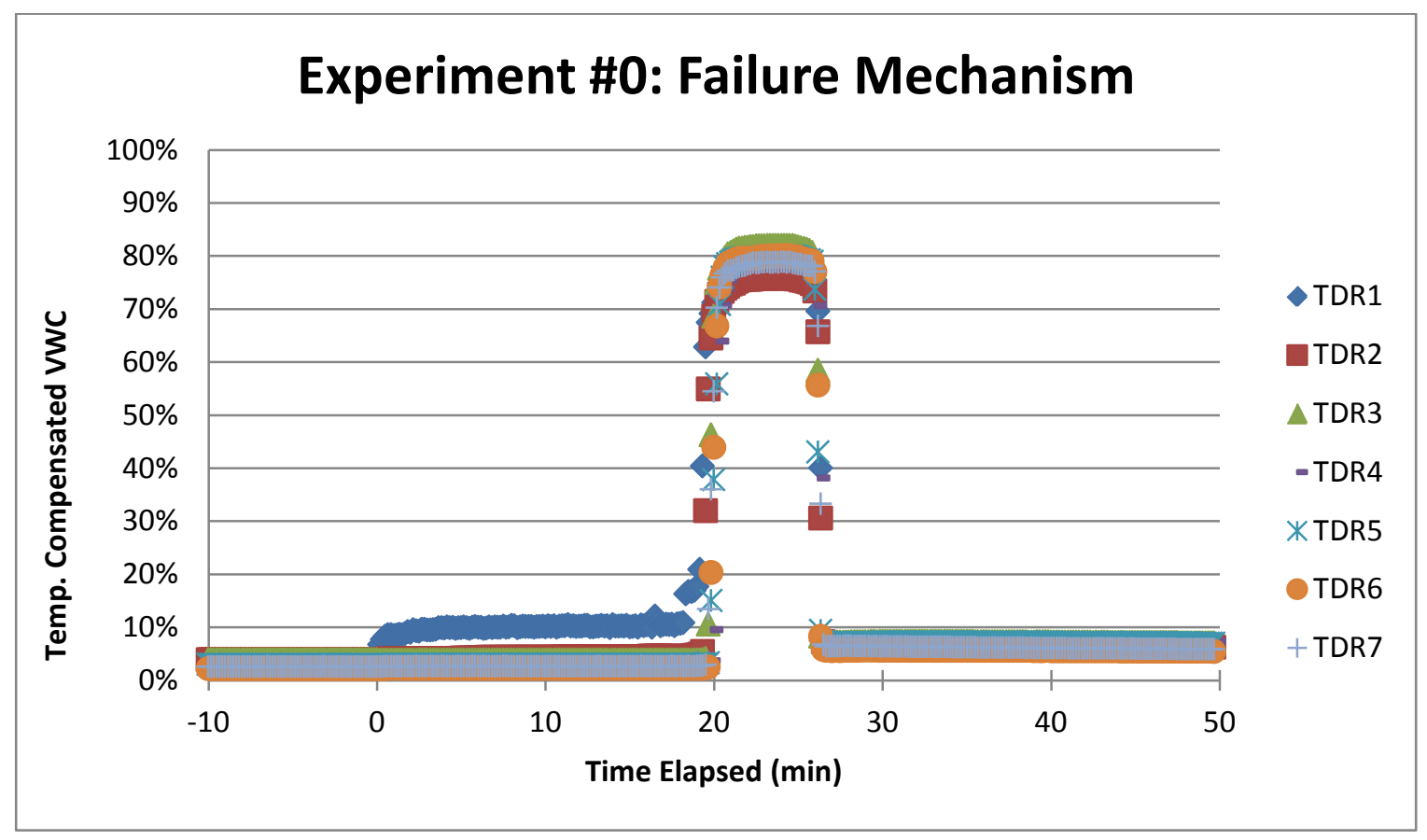

Figure 52: Experiment \#0, failure mechanism of the flume, TC_VWC 


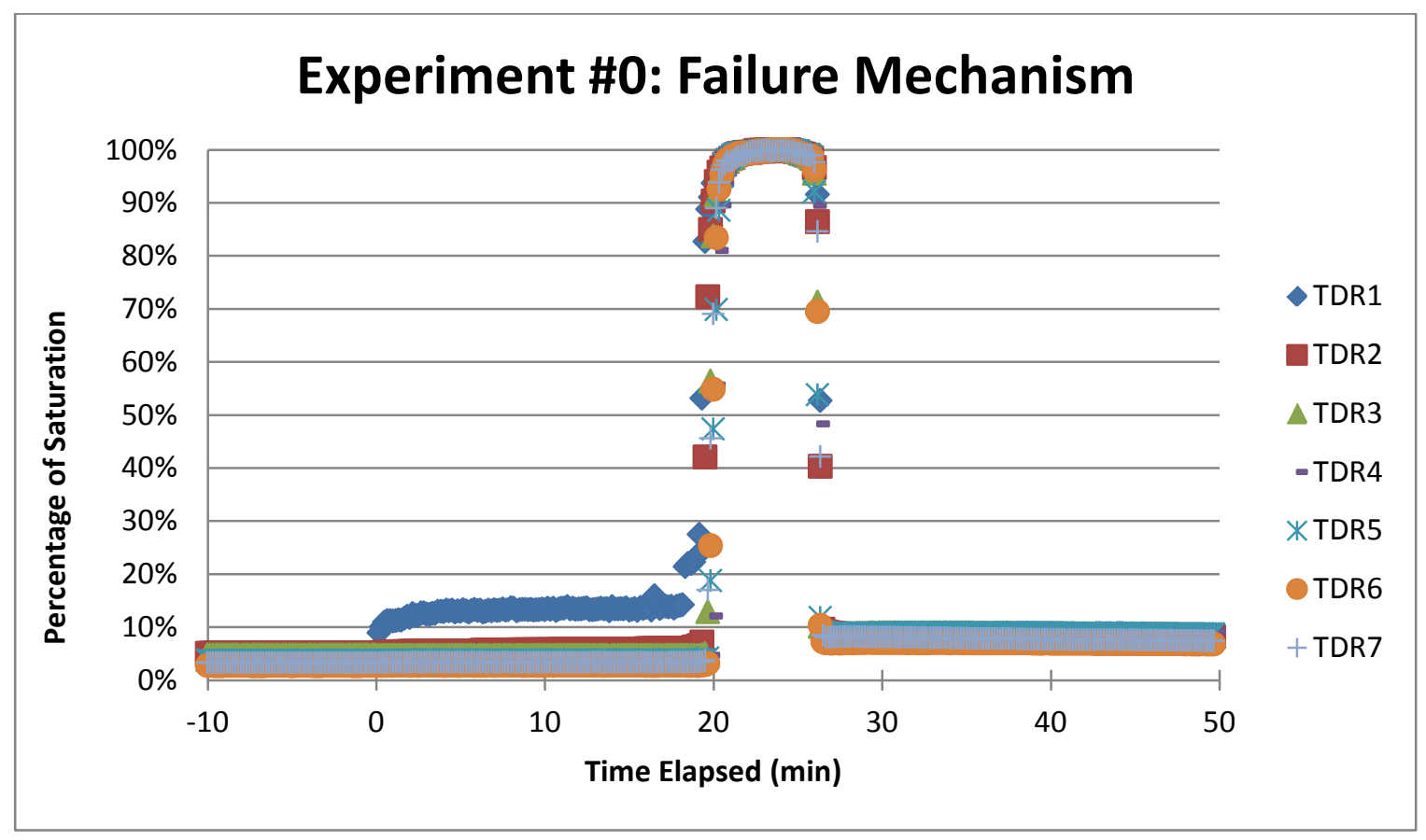

Figure 53: Experiment \#0, failure mechanism of the flume, percentage of saturation

The maximum number measured by each TDR was slightly different from the others and varied between $75 \%$ and $85 \%$. As explained in earlier chapters, since the aggregates surrounding the TDRs were coarse, they might create a slightly different environment for the measurements, thus creating numbers which were somewhat different. This phenomenon is magnified whenever the TDRs are not in the saturated storage gallery. The conversion from TC_VWC to a percentage of saturation created something to offset this effect.

\subsubsection{Clogged but Not Sealed}

As mentioned in the observations made during the experiments, the progression of clogging was slower in the later stages than at first. The steep slope of the incline in the measurements by TDR1 through TDR3 indicated a rapid increase in the percentage of 
saturation, while TDR4 through the last TDR experienced a slower incline. This behavior shown by the TDRs proves that the "clogged but not sealed" sections of the permeable pavers in the GI simulations can bear a considerable load. This amount is may not be easily quantified, however in those experiments where progression of surface clogging reaches the most down gradient sections of the flume, the up gradient "clogged but not sealed" sections provide enough infiltration capacity to avoid complete failure of the experiment module.

Figure 54 is a plot of the percentage of saturation in experiment \#1 vs. the time elapsed of the experiment. It is seen that the first three TDRs measured an almost immediate increase in the percentage of saturation, but when the progression of clogging reached the mid-point in the flume (TDR4) the increase of the percentage of saturation recorded by TDR4 through TDR6 was much slower. The $7^{\text {th }}$ TDR in the flume did not show any change in the percentage of saturation, which means that surface clogging did not reach this point of the flume during the 100 minutes of the experiment. 


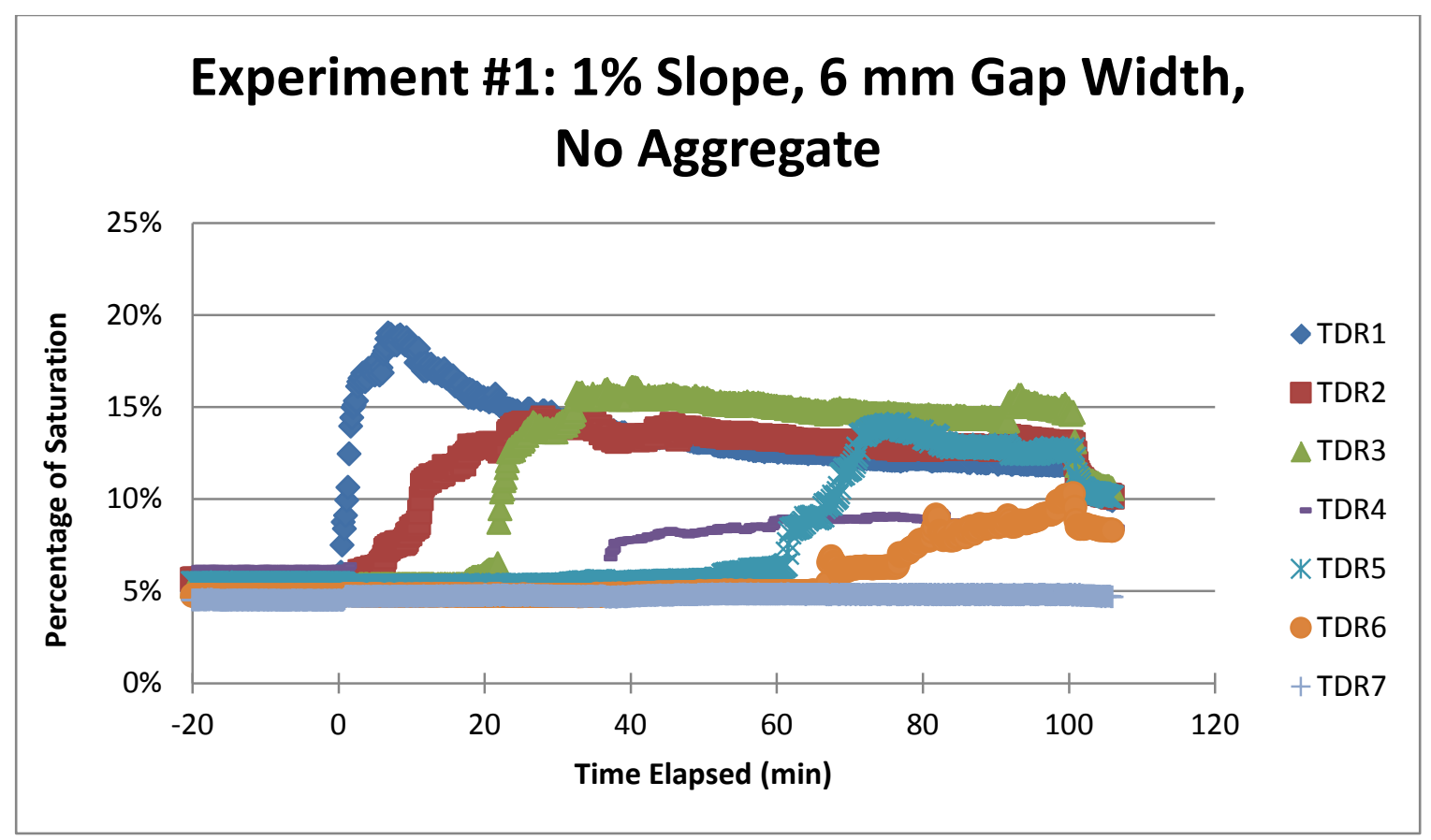

Figure 54: Experiment \#1, illustration of rate of increase

The slopes of the inclines in the TDRs' measurements in experiment \#1, listed in Table 16, support this claim. The numbers represent the slope or the rate of incline from the time that the TDR recorded the first response, which was determined manually, based on the starting time of the experiment and the notes made during the experiment, and the time that the particular TDR reached the maximum measurement for that experiment.

Table 16: Slope of Incline of TDRs Measurements in Experiment \#1

\begin{tabular}{|l|l|l|l|l|l|l|l|}
\hline Rate of increase & TDR1 & TDR2 & TDR3 & TDR4 & TDR5 & TDR6 & TDR7 \\
\hline Slope per minute & 0.01656 & 0.00365 & 0.00471 & 0.00033 & 0.00201 & 0.00089 & N/A \\
\hline $\begin{array}{l}\text { Slope per } 100 \\
\text { minutes }\end{array}$ & 1.656 & 0.365 & 0.471 & 0.033 & 0.201 & 0.089 & N/A \\
\hline
\end{tabular}

Figure 56 shows the inclined section of the measurements of the TDRs in experiment \#1. This experiment was conducted with a 1\% longitudinal slope, $6 \mathrm{~mm}$ gap size, and no permeable joint material. The incline of the measurements followed 
predictable patterns except in some instances. The slope of the incline measured by TDR 3 was more than the one recorded by TDR2. Many cases like this are seen in the recorded data, and were caused by the unpredicted flow patterns on the surface (described in the observations during the test). In more detail, the deposited sediment on the surface may have directed the water towards one of the sides of the flume and caused the water to bypass a TDR at a given time. As the clogging progressed the temporary diversion of stormwater runoff would have caused the TDR at a gradient segment further down to reach the maximum measurement before the previous TDR. The extent of behavior and its effect on the measurements may be increased or decreased according to the orientation of the TDRs. Figure 55 is taken from the down gradient segment of the flume. If the flow of water is concentrated more towards the left side of the flume (viewed from the down gradient), then infiltration may mostly have been passing through the white enclosure of the TDR with the electronics of the instrument, while if the flow is concentrated on the right side, it may have caused a greater increase of the percentage of saturation. 


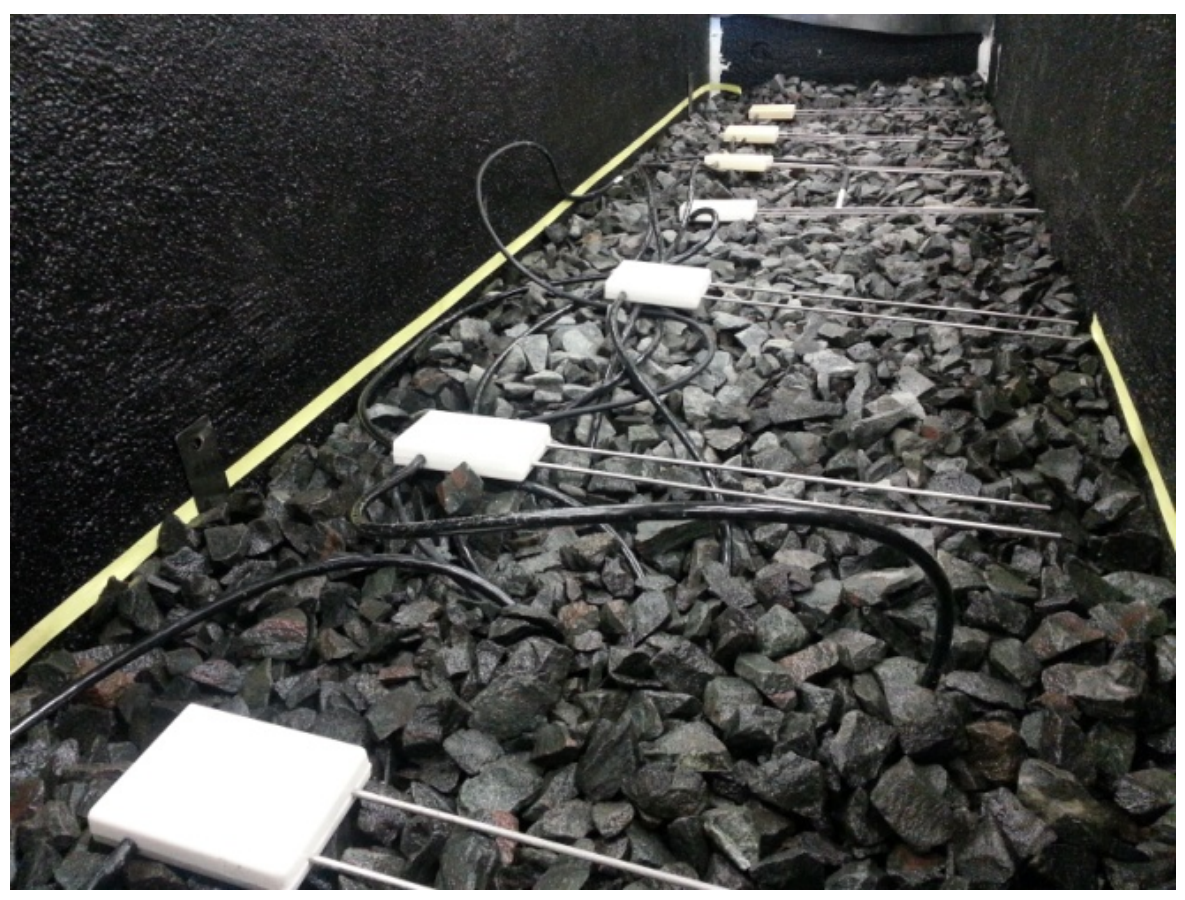

Figure 55: TDRs in the flume

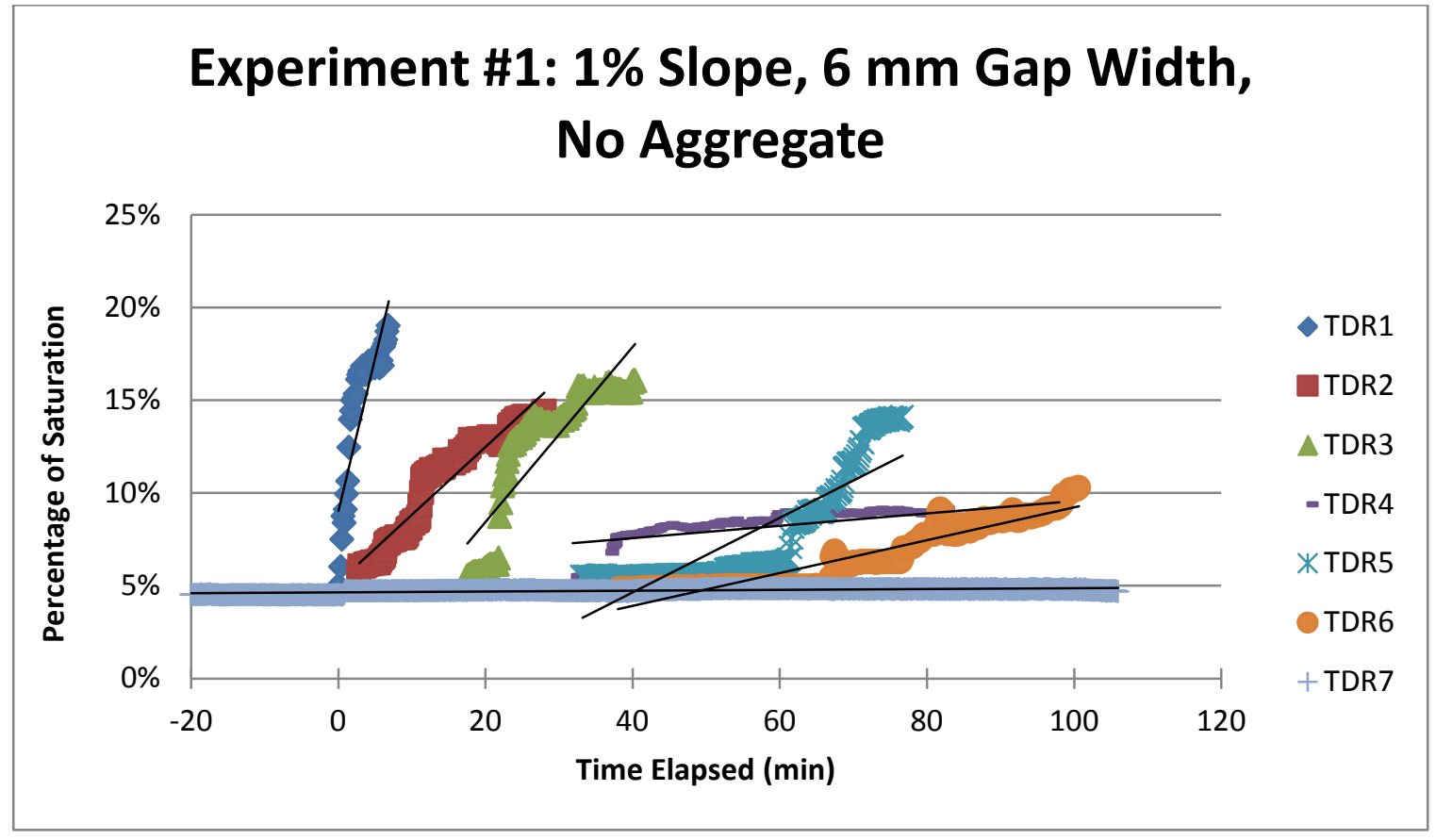

Figure 56: Incline in the measurements of TDRs in experiment \#1

In an experiment with similar configurations and using pavers with bigger gaps, the same patterns were spotted. Figure 57 illustrates the percentage of saturation in the 
experiment \#5 vs. the time elapsed. This experiment was conducted using a $1 \%$ longitudinal slope, $9 \mathrm{~mm}$ gap size and no aggregate between the pavers. As in the first experiment, the rate of increase measured by the TDRs along the length of the flume showed a declining pattern and as the clogging progressed, the TDRs recorded a slower incline, which indicates that the up gradient segments were clogged but not sealed. The slopes of the increases measured by the TDRs in the $5^{\text {th }}$ experiment are presented in Table 17:

Table 17: Slope of Incline in TDRs Measurements in Experiment \#5

\begin{tabular}{|l|l|l|l|l|l|l|l|}
\hline Rate of increase & TDR1 & TDR2 & TDR3 & TDR4 & TDR5 & TDR6 & TDR7 \\
\hline Slope per minute & 0.01480 & 0.00691 & 0.00742 & 0.00388 & 0.00136 & 0.00140 & 0.00095 \\
\hline $\begin{array}{l}\text { Slope per 100 } \\
\text { minutes }\end{array}$ & 1.480 & 0.691 & 0.742 & 0.388 & 0.136 & 0.140 & 0.095 \\
\hline
\end{tabular}

Unlike experiment \#1, where a smaller paver gap size was used and the $7^{\text {th }}$ TDR did not record any change from the baseline measurement, experiment \#5 indicates a change at the location of the $7^{\text {th }}$ TDR, which is a result of the unpredicted flow patterns on the surface, as shown in Figure 38. The overall trend of the changes seen in this experiment, however, matches expectations and the rate of increase in the recorded measurements has a consistently declining pattern. 


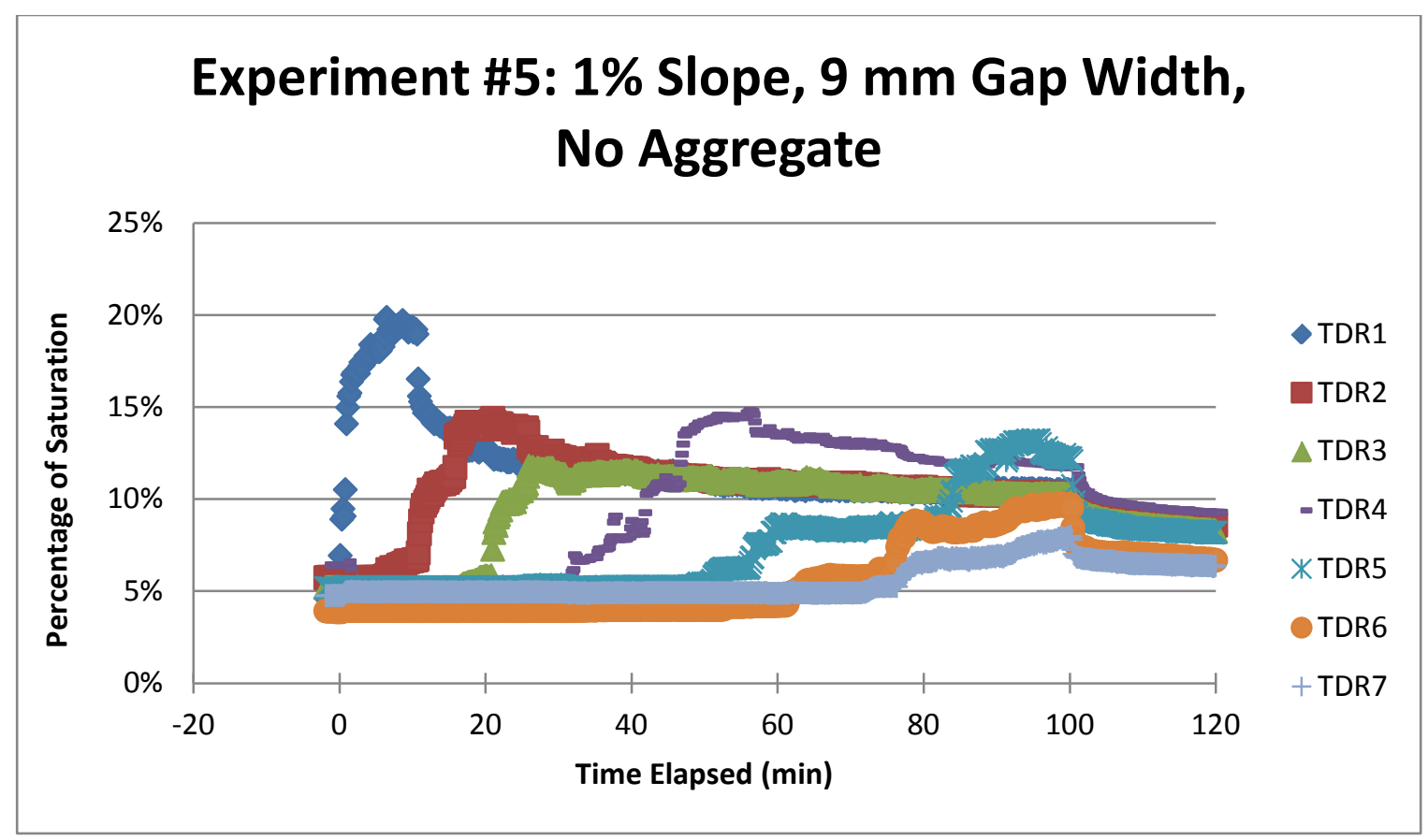

Figure 57: Experiment \#5, illustration of rate of increase

Similarly, Figure 58 shows the same patterns in experiment \#7 as were seen in previous experiments. This experiment, which was staged with a $1 \%$ longitudinal slope, $12 \mathrm{~mm}$ gap size, and no permeable joint material, showed a constant decrease in the incline measured by the TDRs, which means that the big gap width of the pavers created more consistency in the infiltration. The bigger gap size has provided more infiltration capacity for the system; therefore the same amount of sediment in the runoff has only clogged the flume up to the $4^{\text {th }}$ TDR, which is located at the mid-point of the flume.

Table 18: Slope of Incline in TDRs Measurements in Experiment \#7

\begin{tabular}{|l|l|l|l|l|l|l|l|}
\hline Rate of increase & TDR1 & TDR2 & TDR3 & TDR4 & TDR5 & TDR6 & TDR7 \\
\hline Slope per minute & 0.00949 & 0.00853 & 0.00619 & 0.00101 & N/A & N/A & N/A \\
\hline Slope per 100 minutes & 0.949 & 0.853 & 0.619 & 0.101 & N/A & N/A & N/A \\
\hline
\end{tabular}




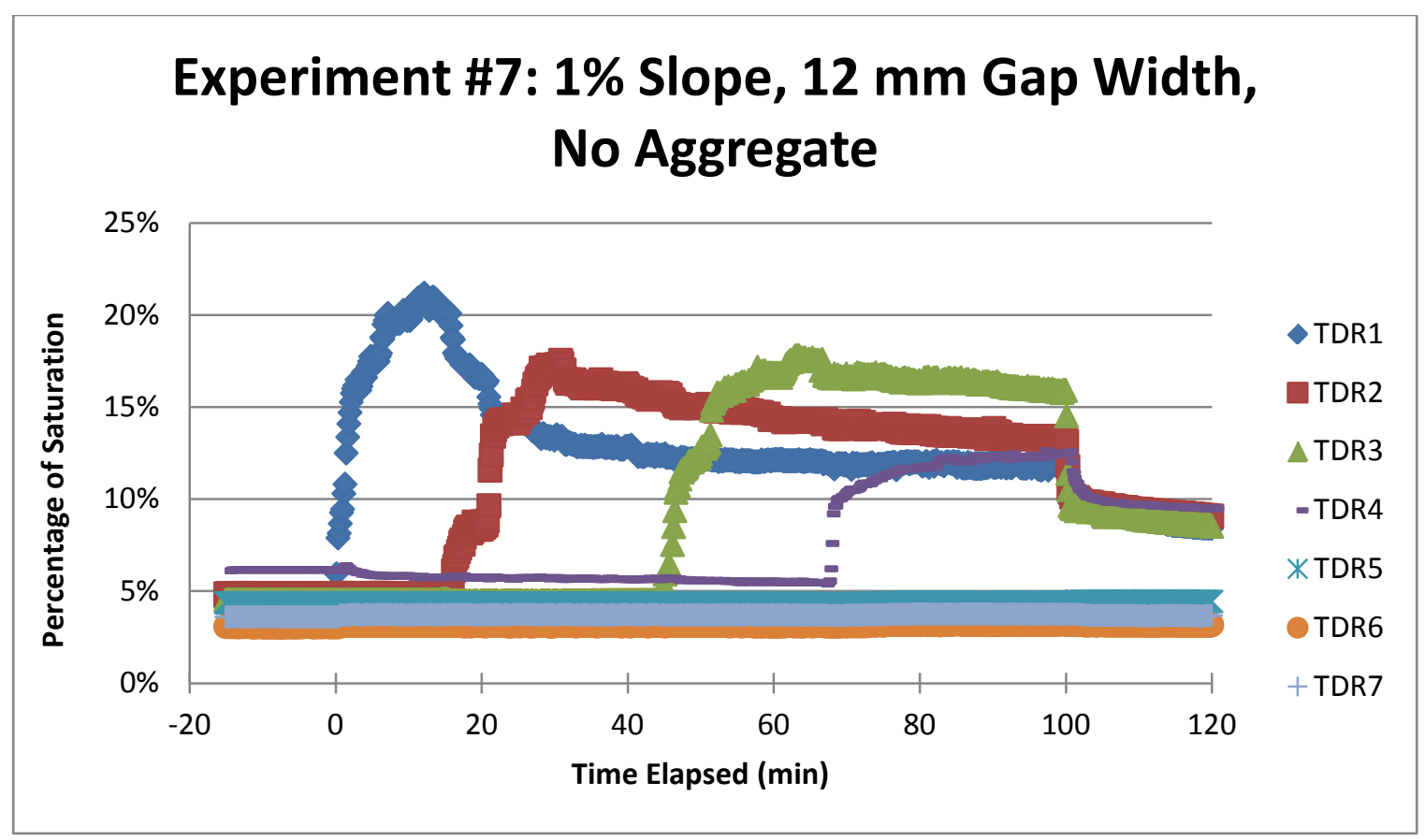

Figure 58: Experiment \#7, illustration of rate of increase

Similar patterns for a constant decrease in the measured percentage of saturation were seen in most of the experiments. Since the intention of this chapter is to shed light on the analytical methods used, because the analysis of the data and the results are inseparable, the same analytical tools are used in the remaining tests and the results and their true meaning are discussed in the next two chapters.

\subsection{In-depth Data Analysis}

In the previous steps of data analysis, the following calculations and modifications were made to transform the raw data into more meaningful processed data:

- Changing time format in the spreadsheet produced by the data logger from portions of a day starting at 01/01/01 to time elapsed in minutes for the duration of the experiment. 
- Measuring the temperature compensation for the VWC by TDRs, based on the temperature recorded using the thermistor (TC-VWC).

- Changing the TC-VWC to a percentage of saturation, based on the measurements and findings of experiment \#0.

In-depth analysis of the processed data constitutes quantitative analysis of the results using statistical tools to investigate the patterns that may exist in the data. The difference between this section and preliminary analysis of results is that the most of the preliminary analysis focuses on individual experiments and even individual TDRs within an experiment; however, the in-depth analysis is focused more on comparing the behaviors seen in different experiments.

\subsubsection{Performance Zones}

Observations made during the tests showed that during the test, which is a simulation of the full scale GI behavior, the performance exhibited major shifts. Based on these transitions, which are obvious in both the observations and the preliminary analysis of the data, separate zones have been defined for the performance data. Dividing the performance into zones also helps to determine the most valuable patterns and also to understand the maintenance needs for each zone.

Before the beginning of the experiment, the TDRs were recording a baseline measurement, which in view of the gap between the tests had a constant declining trend. For the TDR at the up gradient of the flume, this period may constitute the short interval between plugging in the battery to the data logger and the time when the test started and runoff reached to the location of the TDR. However, for the down gradient TDR, this 
time was possibly longer, since the clogging had to continue long enough to direct stormwater runoff over the segments. In some experiments, based on the configuration of the test, some TDRs may stay at this point for the entire time, which means that the down gradient segments of the flume would not have infiltrated any runoff.

During the experiments and directly after starting the tests, the stormwater runoff flow on the permeable pavers tends to find the first available gap and infiltrate through it. With the stormwater the sediment will also flow into the gap, which will result in less infiltration capacity for the gap in question. As this process continues, the slow stormwater will eventually exceed the infiltration of the first gap; in consequence the runoff will start to penetrate from a second gap. During this process there is a period when the flow passing through a gap increases, and, as it does so, the TDRs' measurements show an increase. After this there is a second period when the clogging starts and the infiltration through this gap starts to slow down.

After the 100 minute period of the experiment, and as soon as the flow of runoff to the flume is shut off, all the TDR measurements start to decline. The decline, which is due to the fact that most of the infiltrated runoff is draining through the effluent, will create a much steeper incline in the measurements than in the previous period, and will continue until the TDRs reach $0 \%$ of saturation (if the storage gallery of the flume becomes absolutely moisture free). Figure 59 illustrates the decreasing pattern in the percentage of saturation recorded by all 7 TDRs in experiment 12, which was one of the experiments that took place on a Friday, leaving 3 days for draining and drying. 


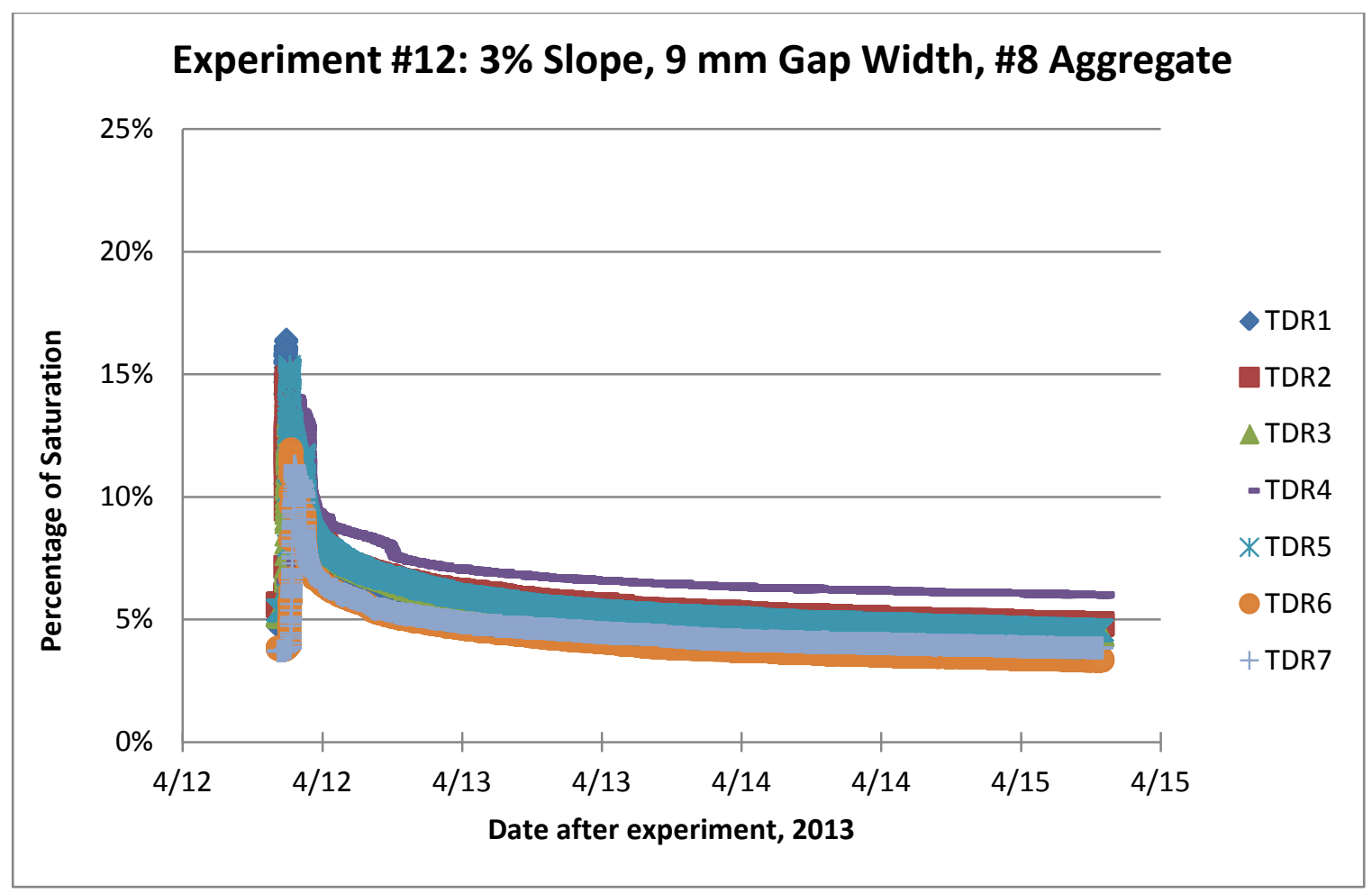

Figure 59: Constant decrease in the TDRs' measurement after the experiment over 4 days

During the time that the TDRs are measuring their baseline percentage of saturation, also called "zone 0", their measurements are constantly decreasing. During the first period, or "zone 1", the measurements are expected to show a steady increase in the percentage of saturation, which would end with the maximum percentage of saturation value measured by a given TDR. During the second phase, or “zone 2”, the infiltration rate of the point has already peaked and is now experiencing a decrease, where it will not go any higher. The last segment of the measurements, also called "zone 3", is reached when the runoff flow from the flume has been cut and all the TDRs' measurements start or continue to decrease at a noticeably rapid rate.

Based on the variations in the recorded behavior of the flume's permeable surface, and since zones zero and three are $\mathrm{n}$ all experiments following the same patterns, 
a constant decrease, they are excluded from further investigations. However, zones one and two, which represent the time when the permeable surface is going through a change in terms of performance and surface infiltration rates, can provide valuable information on the condition of the flume. Figure 60 illustrates the defined zones measured by each TDR in a randomly selected experiment, experiment 14. 

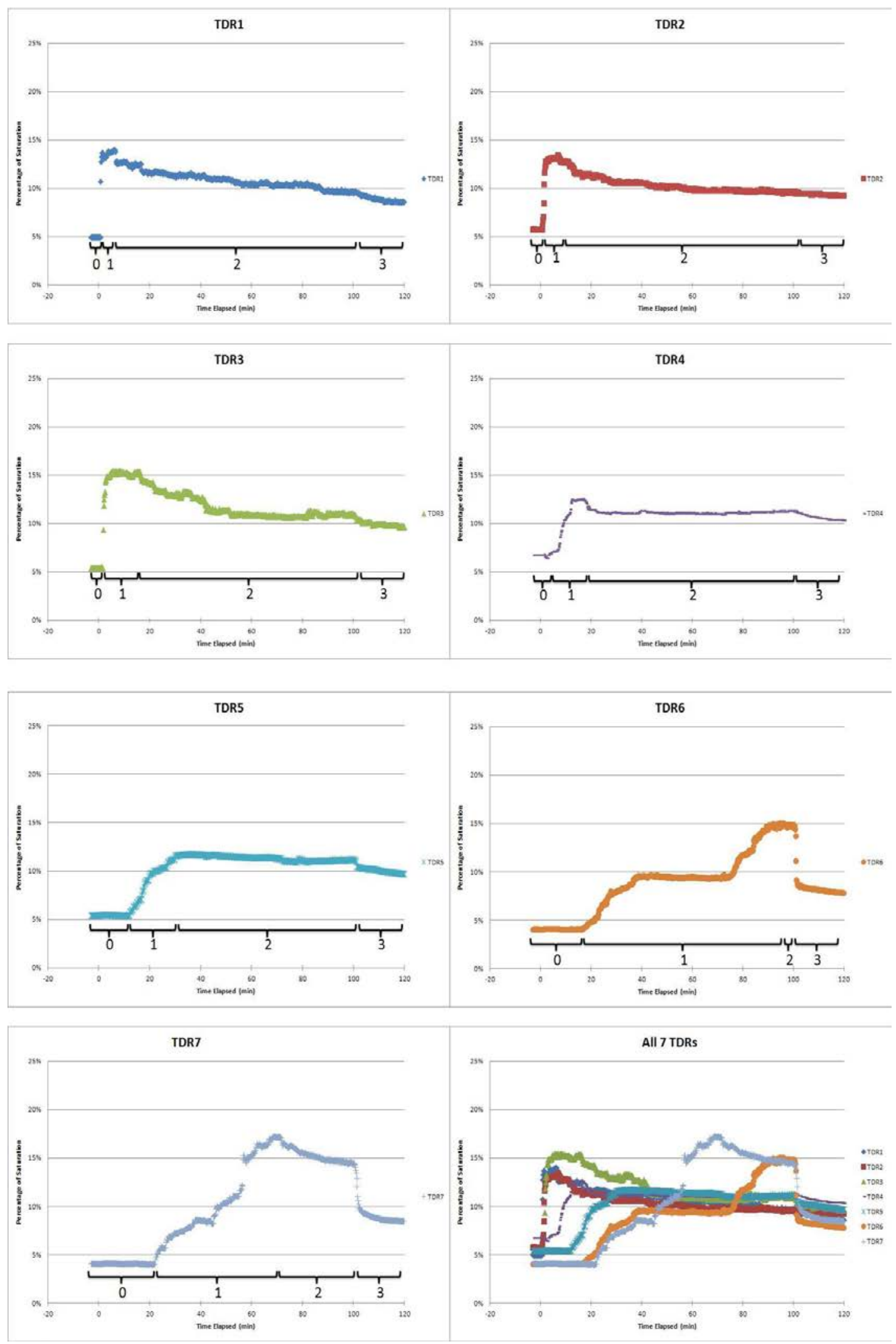

Figure 60: Experiment \#14, different zones in the measurements by TDRs 
Since zone one includes the period that the permeable paver surface starts performing for the first time and uses the pristine conditions to allow a maximum percentage of saturation in the storage gallery, it is referred to as the "infiltration zone". In the light of the previous discussion on the condition of clogging and how the clogged surface of the flume is not sealed and still allows for some runoff to infiltrate, the second zone is referred to as the "decreased infiltration zone". The separation point between the two zones is where the measurement of the TDR reaches its maximum recorded value.

The infiltration zone usually starts with a rapid increase in the percentage of saturation, which is observed after the baseline measurement and is a result of runoff reaching the surrounding area of the TDR. Based on the location of the TDR and the time in the experiment, the trend of increase may have a high slope or a relatively low slope. In other words, the duration of this zone is a quantitative measurement of how long it will take the permeable pavers to move from the initial wetting to their maximum performance.

The decreased infiltration zone starts with the peak value recorded by the TDRs, and constitutes a decreasing trend where the trend of the measurements has a negative slope. The decreased infiltration slope ends when the experiment stops. At the time when the experiment ends, the percentage of saturation plunges. The duration of the decreased infiltration zone and the rate of decline in this zone is a quantitative measurement of how long the clogged but not sealed state can last before reaching a point where the surface infiltration rate is less than the design specifications require. 


\subsubsection{Progression of Clogging}

Surface clogging is a major factor in reducing the performance of any GI that uses permeable pavements. From the early stages of this research project, one of the main hypotheses of the work has concerned the speed at which surface clogging progresses. In order to determine the progression of clogging, the TDRs must be associated with their locations in the flume with respect to the up gradient point. The time elapsed in the experiment is also a representation of an amount of rain over the area of the flume's watershed, with every minute of the test simulating an additional $5.08 \mathrm{~mm}$ of rainfall over the area of the flume's watershed.

We discussed above the definition that the time when each TDR reaches its maximum measured value is actually the time when the relevant point of the flume's surface is clogged and can then experience only deterioration. So the peak time measurement in the experiment, which is an equivalent of the rainfall on the structure of the flume, must be plotted against the distance of clogging from the up gradient of the flume.

In order to plot the progression of clogging on a graph, the time when each TDR peaks should be marked with its location, which indicates that clogging has reached a particular point of the flume. The progression of clogging in experiment \#1 vs. the amount of rainfall is plotted on Figure 61. 


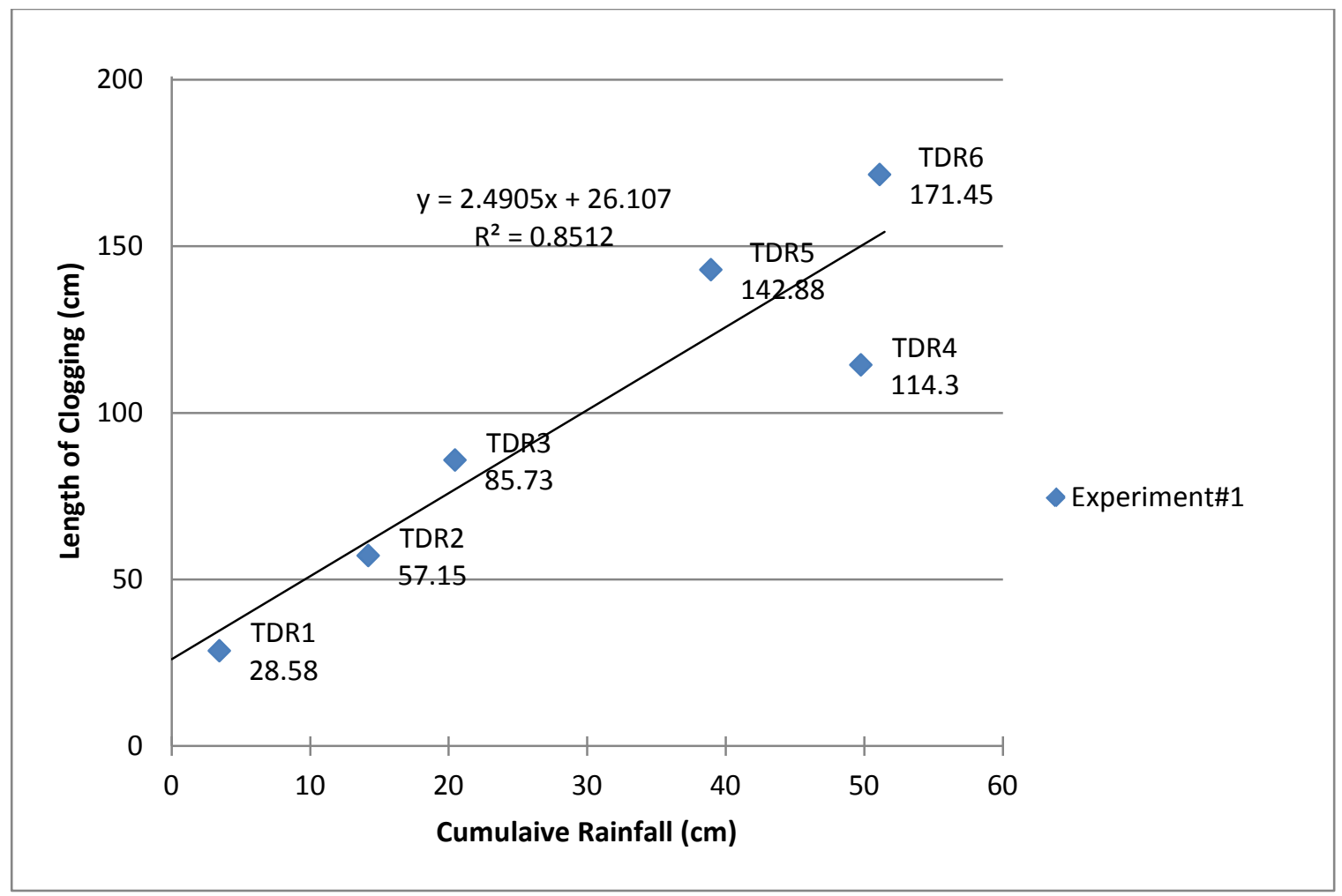

Figure 61: Graph for progression of clogging for experiment \#1

In some of the experiments, a TDR located at a location further down the gradient peaks before a TDR located at a location on an up gradient. As an example of this effect, illustrated in Figure 61, surface clogging has progressed as expected from TDR1 to TDR3; however, the further down gradient TDR5, which is located at $142.88 \mathrm{~cm}$, has reached its maximum measurement before TDR4 at $114.3 \mathrm{~cm}$. In other words, the graph suggests that the clogging has moved backwards at some point in the test. In fact, however, based on the visual observation made during the test, the clogging did not move backwards. Figure 62 is an image of the flume after the first experiment, which shows what happened. 


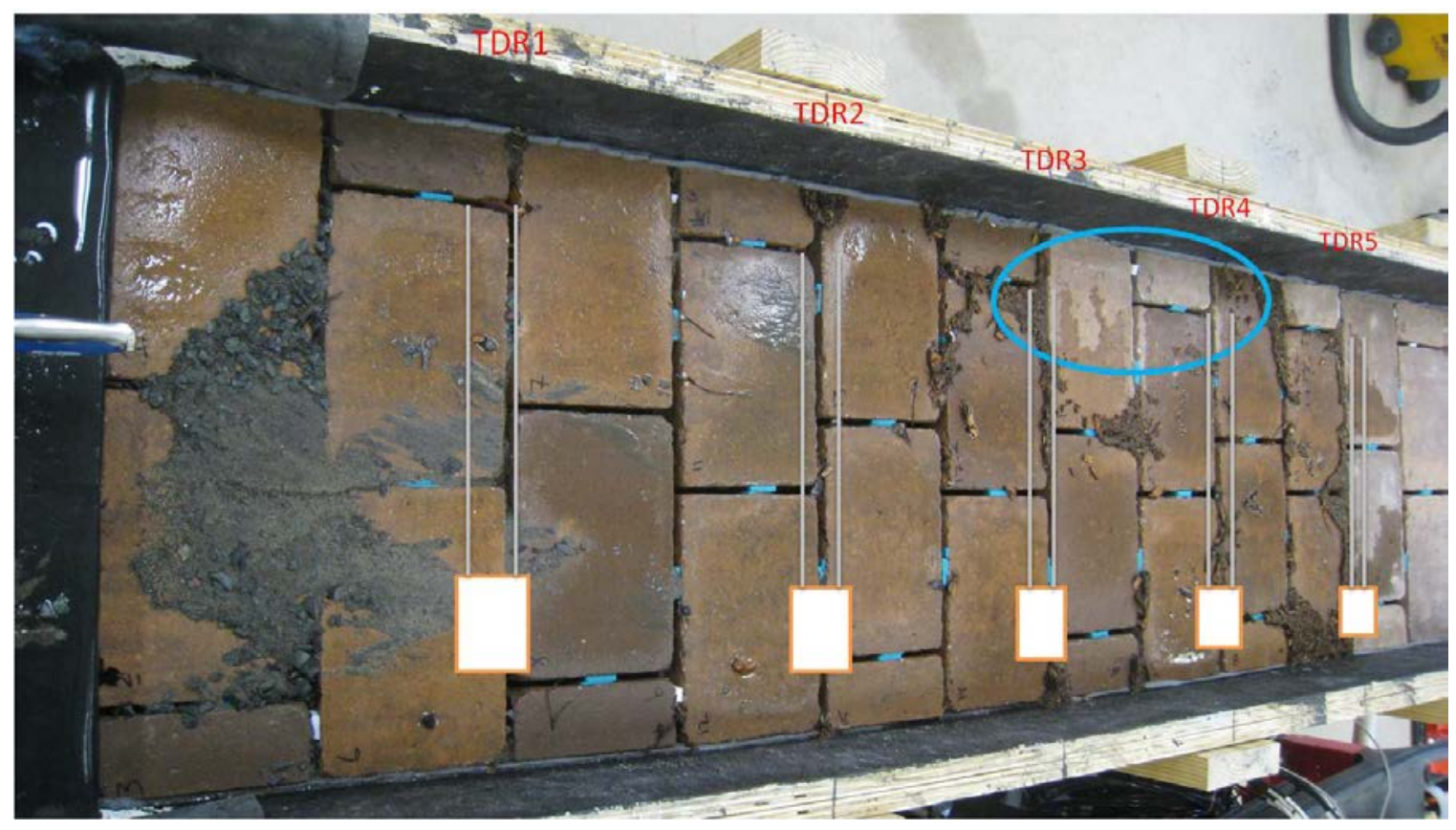

Figure 62: Flume's overview for progression of clogging in experiment \#1

As the clogging progressed during experiment \#1, the blue circle that was over the $4^{\text {th }}$ TDR's rods was blocked by mulch deposited on the surface. However, unlike what the numbers might suggest, the clogging had reached this point. After further progress of surface clogging, the dam created by the deposited mulch eventually broke, letting runoff into the gaps. This behavior is a problem of scaling; however, the visual observations during the test can help to eliminate this problem. By excluding the superficial rainfall associated with the time of peak at the location of TDR4, and plotting the trend using the remaining data points, this graph can be plotted correctly. Figure 63 is the corrected plot for the progression of clogging in experiment \#1. 


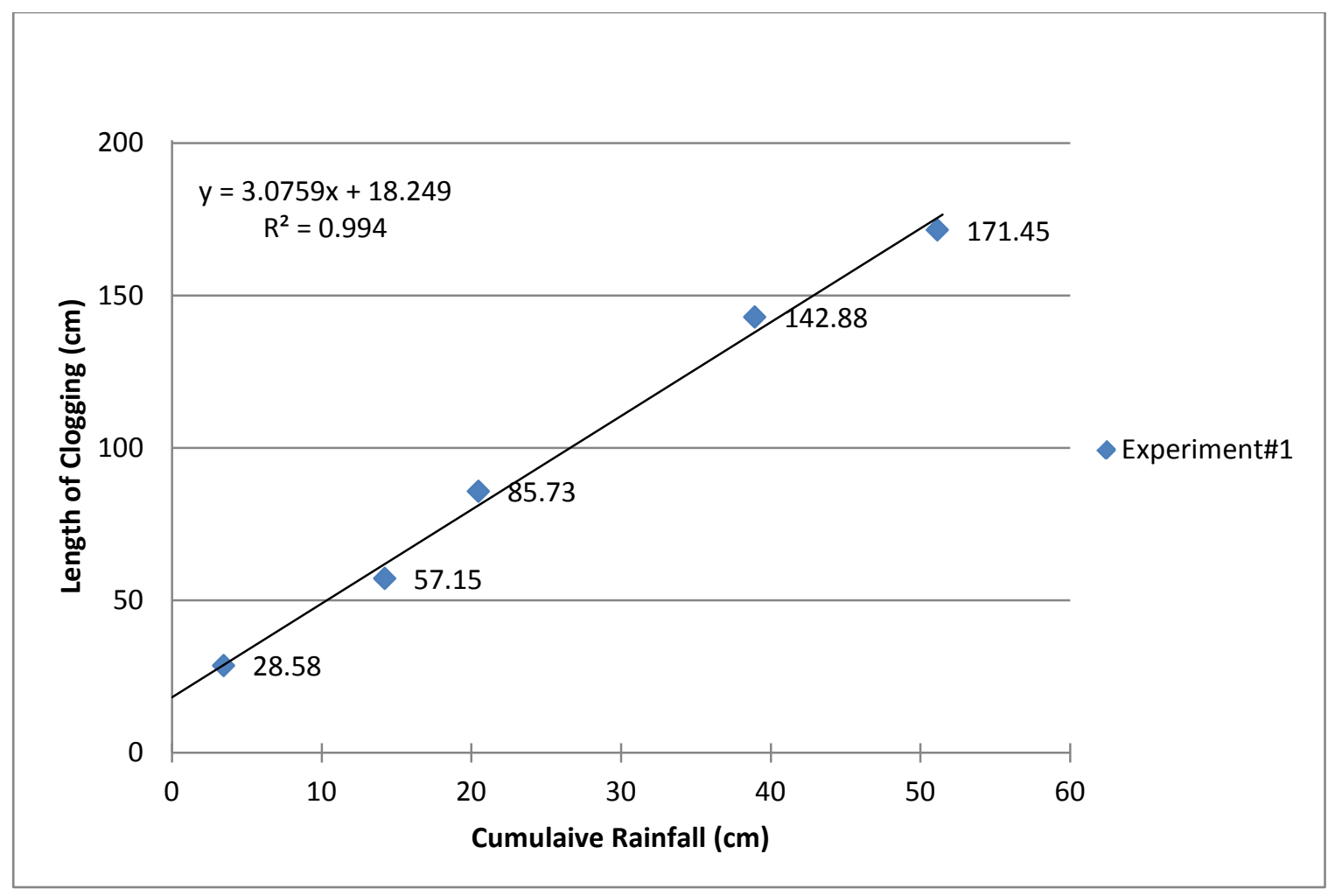

Figure 63: Corrected graph for progression of clogging for experiment \#1

Using the corrected plot which shows the progression of clogging during an experiment, the speed at which the surface clogging moves on the surface can be compared between experiments. This is a valuable tool for assessing the effect of a change in the physical environment on the progression of clogging and for determining the best settings to achieve enhanced GI performance.

The first sets of data compared to each other are experiments \#1, \#5, and\#7, featuring the $1 \%$ longitudinal slope, all three gap sizes, and no permeable joint material in between the pavers. They should be compared because many paver products are available and choosing the best paver type for an installation can significantly affect the performance of the GI and reduce the frequency of maintenance. The progression of clogging in these tests is plotted in Figure 64: 


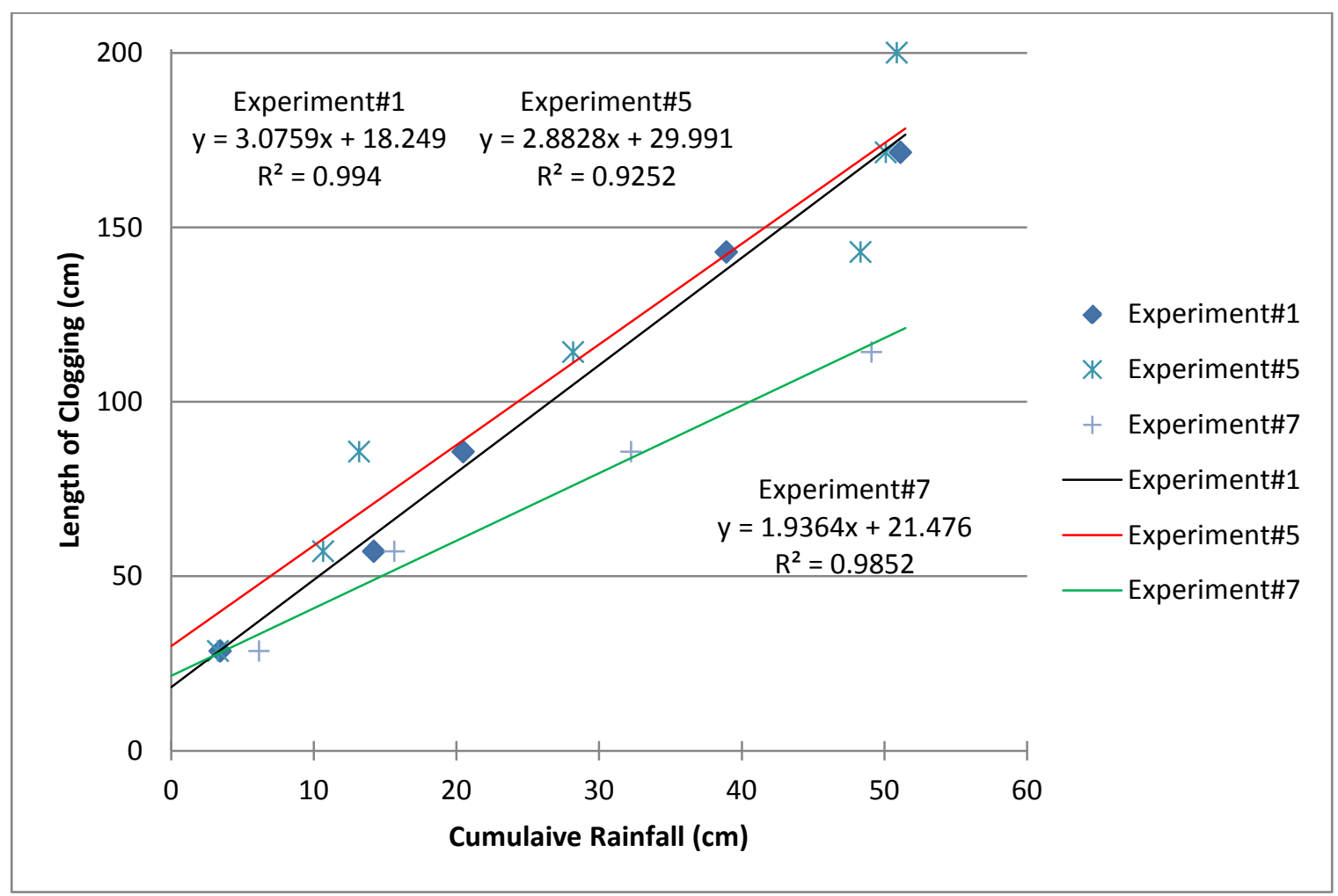

Figure 64: Comparison of progression of clogging in experiments \#1, \#5, and \#7

It is seen that the speed clogging progress, which is the slope of the trend line, decreases with the increase in the size of the paver gap. However, the increase from 6 $\mathrm{mm}$ to $9 \mathrm{~mm}$ is not as significant as the increase from $9 \mathrm{~mm}$ to $12 \mathrm{~mm}$. Appendix $\mathrm{F}$ includes the corrected graph for the progression of clogging for all the individual experiments.

\subsubsection{Progression of the Infiltration Edge}

It was described that during the performance of permeable pavers, two of the defined zones can be used to determine the assessment criteria and help in developing maintenance plans based on the specifications of the GI. The first zone starts with the wetting of a specific location in the flume and ends with the maximum percentage of 
saturation around the TDR, which coincides with the maximum surface infiltration rate. After passing the maximum surface infiltration rate, the GI goes through deteriorating conditions and the surface infiltration decreases until the section is eventually clogged. By tracking the maximum measurement, the progression of clogging can be plotted and the slope of the plotted line can determine how fast a section of the GI will deteriorate.

The time that each TDR records its first increase from the baseline measurement, is actually the time when this point of the flume's surface has started to infiltrate into the runoff and will most probably experience an increase in surface infiltration rates. So the first significant increase from the time of the baseline measurement in the experiment, which is the equivalent of the rainfall on the structure of the flume, must be plotted against the distance from this point to the up gradient of the flume.

In order to plot the progression of an infiltration edge on a graph, we must manually determine and mark, along with the location of a specific TDR, the time when each TDR's measurement experiences a significant increase from the baseline measurement, which indicates that the edge of infiltration has reached a particular point of the flume. This location will experience an increase in the received stormwater runoff flow. The progression of infiltration edge in experiment \#1 vs. the amount of rainfall is been plotted in Figure 65. 


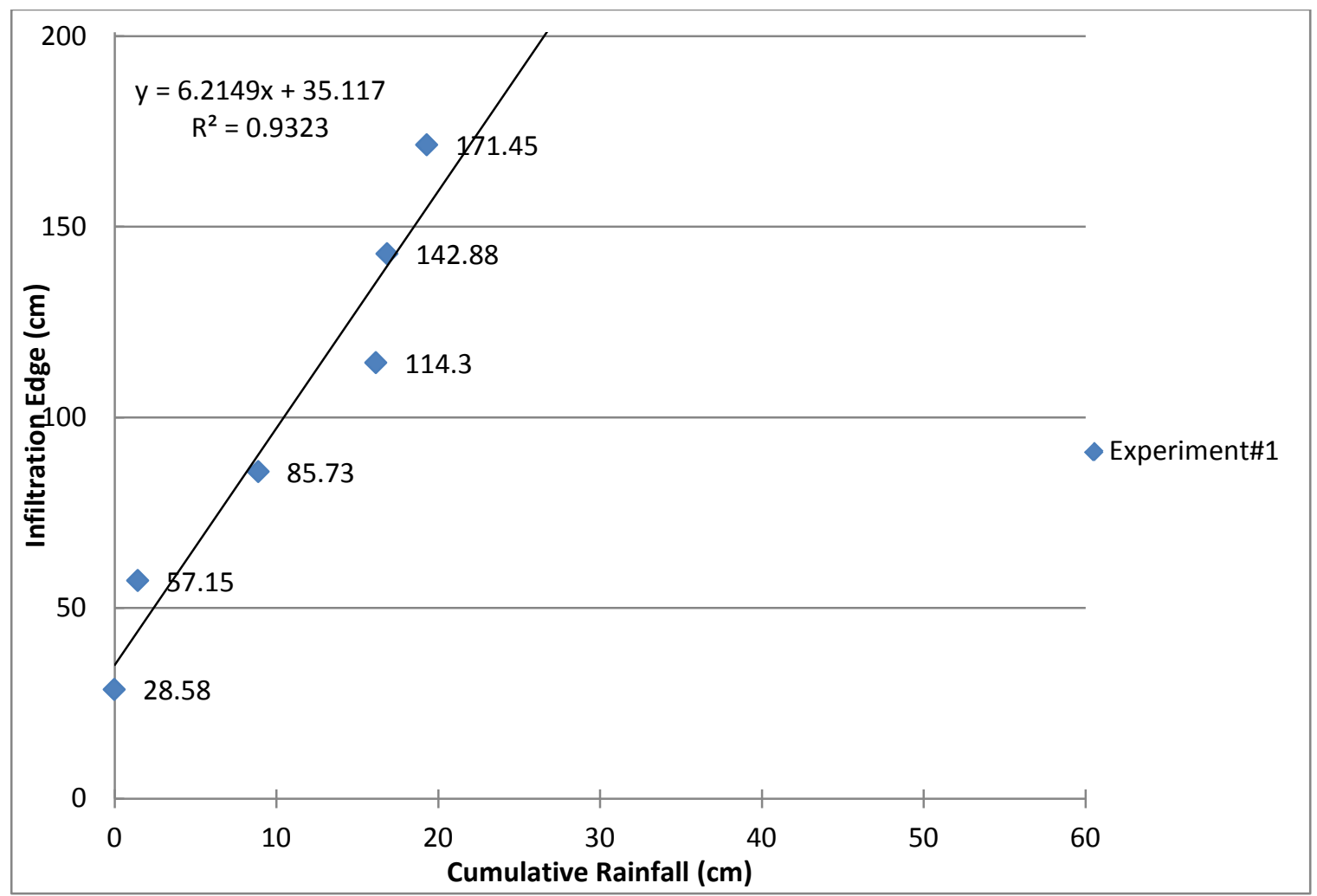

Figure 65: Progression of infiltration edge, experiment \#1, graph

Determining the effects of the physical environment factors, such as the slope, on the progress of infiltration edge or the wetting front can also be a helpful assessment tool and provide valuable information. In all the experiments conducted for this research, the first TDR, which is located at $28.58 \mathrm{~cm}$ from the up gradient, experienced a significant increase from the baseline measurement almost at once; therefore the first data point on the graph is somewhat superficial and is associated with 0 of rainfall. This is due to the location of the first TDR. However the next data points and their location in relation to the first one determine how fast different locations of the flume become useful and infiltrate stormwater runoff. 


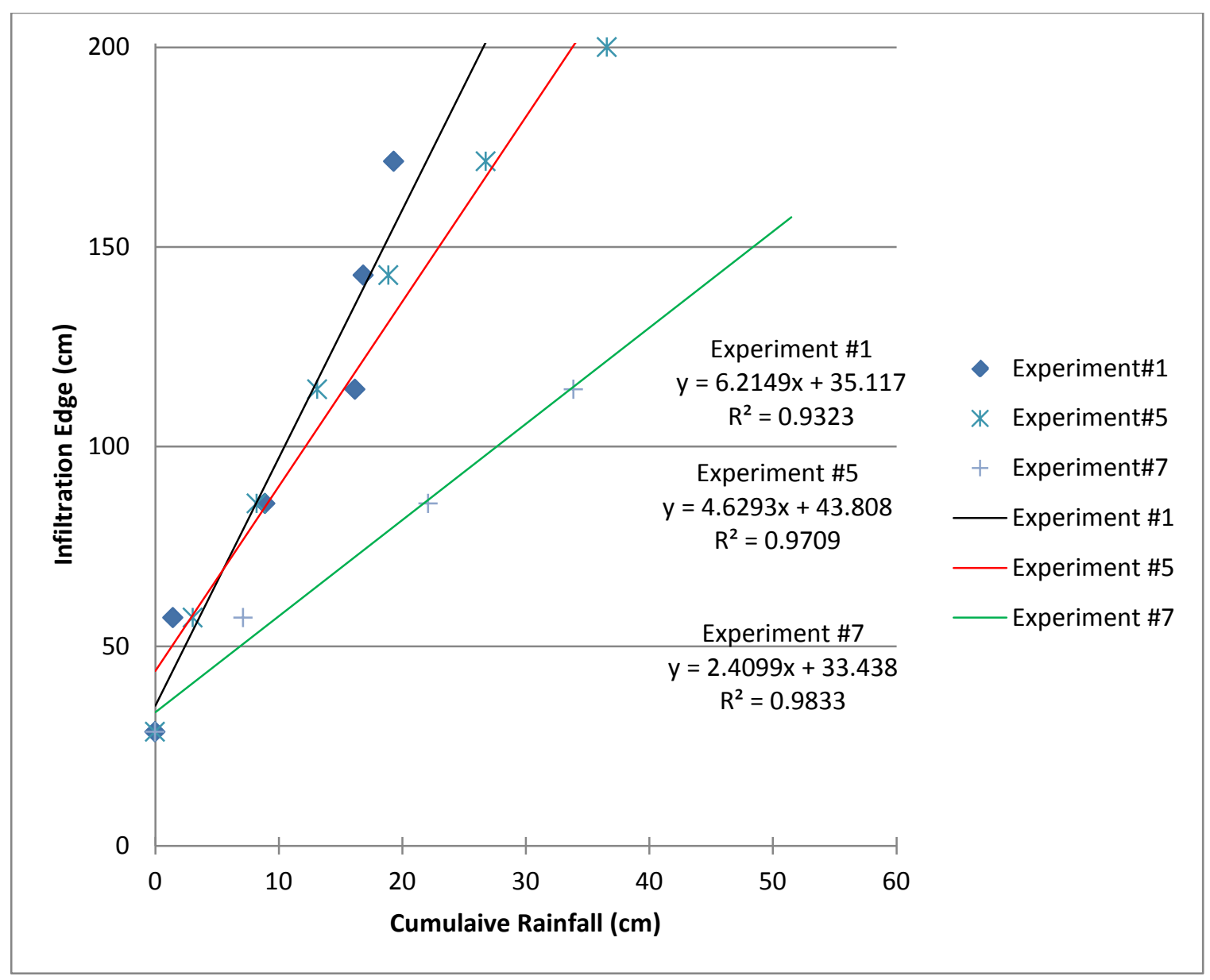

Figure 66: Comparison of progression of infiltration edge in experiments \#1, \#5 and \#7

Similarly, the plotted progression of the infiltration edge can be used to compare the different configurations of interlocking concrete pavers with respect to their physical environment, sediment loading, etc. and determine the lead time for the maintenance requirements of the GI. Figure 66 compares the progression of the infiltration edge in experiments conducted on a 1\% longitudinal slope, different gap sizes and no permeable joint material. The progression of the infiltration edge illustrates that, with the increase in the permeable paver gap size, the speed at which more segments of the permeable surface are being used decreases. 
The graphs reporting the progression of the infiltration edge for each individual experiment are included in Appendix G.

\subsection{Experimental Approach}

An accurate experimental study must include the use of an appropriate measurement process to be reliable. The research must also be conducted in a planned measurement program that is specifically designed to answer the questions of a welldefined problem (Taylor \& Cihon, 2004). The process of experimenting as part of a scientific investigation must include the following steps. Firstly, the scope of the work must be limited to aspects that can be accomplished with reasonable and practical certainty. Secondly, the aspects considered in the study must be judged and only the most appropriate one chosen. Finally, the hypotheses of the research must be tested by experiment, where a successful hypothesis would result in matching the current known facts and all the current knowledge on the topic (Wilson, 2012).

Using an experimental approach, however, has both advantages and disadvantages. Among the advantages of using it is that testing in a controlled environment where there is no unpredicted variable interfering with the experiment can result in a very clean dataset. Limiting the variables in an experiment would help to reveal the effects of change on a specific and limited number of factors in the test results. At the same time, the experimenter must avoid using excessive precision which would result in excessively sanitized data, since this result could be obtained only by the best operators and in conditions where the experimenter took unusual steps to get "good" results (ASTM, 2011). Some disadvantages of conducting an experiment to analyze a 
hypothesis include the need to simplify the hypothesis, testing within practical and physical limits, etc.

There have been many applications of GI around the USA. Many different types of GI, such as permeable pavements, bio-retention cells, rain gardens, tree boxes, and infiltration basins have been used to reduce stormwater runoff and minimize the environmental effects caused by combined sewer overflows. Different types of GI have been designed and constructed to meet the goals and needs of specific projects. With the collaboration of municipalities and academia, there have been numerous cases of GI practices that have been used as a case study to assess performance. However, many of these case studies have concerned pilot projects using a specific design and configuration to achieve a specific set of goals.

Only a few of the installed full scale GI have been instrumented for research and monitoring purposes. These GI have been instrumented with the aim of remotely monitoring their performance. Although the current path is developing very rapidly and progress has been made in different aspects of it, remote data collection still has some pitfalls. The Louisville CSO130 project, where two strips of permeable pavement were installed on Adams Street, was equipped with remote monitoring instruments that have been very useful in understanding the performance of the GI. Considering the advantages of the remote data collection method, many instances have arisen where the recorded data makes no sense. They provoke speculation about what may have happened and the causes, but there is no definite answer. 
Relying on remotely collected data from a GI constructed in an environment with certain characteristics can be misleading; in order to fully understand how a GI works, what affects performance and how it can fail, an experimental approach is often very useful. Taking an experimental approach also helps to observe the working of the system and match the remotely collected data with observation, which can refute or justify some of the data. The experiments conducted in this research provide similar advantages, where the observation of many months' worth of accumulated rainfall on a GI's watershed has been very informative and has revealed many hidden patterns.

The methods chosen for this work are all based on experimental research. Another advantage of conducting such experiments is that numerous systems are created with minimum time and money and tested to determine how some variables in the experiment affect the performance of the system.

\subsubsection{Data Quality Considerations}

Due to the nature of this research and the fact that no work on this scale has ever been done before, the data collection method and the collected data must be carefully observed and evaluated to make sure that it can be used to investigate the hypotheses of the study. There are multiple methods available that can be used to check the quality of the collected data.

One of the common methods used to indicate the quality of the data collected is to compare it with the data collected in similar studies. Since the data collected in this research and the methods used in this research have never been employed before, qualitative comparisons of the collected data with expected patterns and behavior, which 
are based on the experience of working with Louisville CSO130 project, are among the important tools used to evaluate the quality of the collected data.

\subsubsection{Qualitative Accuracy}

The flume experiment is a simulation of the rainfall and the runoff associated with this rainfall over a long period of time over the area of the flume's watershed. Louisville CSO130 project, experiences $50.8 \mathrm{~cm}$ worth of cumulative rainfall, over a period of approximately 6 months. This number is used as the basis to determine the volume of runoff for the flume. It is obvious that over such a long period, the performance of the GI is not only affected by the rainfall on the watershed and the debris carried by the stormwater runoff, but also many other factors related to the local and physical environment of the GI, such as the vehicular traffic movements, seasonal effects, pedestrians, extreme weather, such as heavy rain and wind, street sweeping by the municipal workers or private residents, construction works and/or periods with higher erosion may affect the performance of the GI. The laboratory experiment was designed to determine the effect of factors in the physical environment which were thought to significantly affect the performance; however the unpredicted events that might ensue in a GI in practice were not simulated.

In order to ensure the qualitative accuracy of the collected data, the following patterns were observed and compared to the expectations and observations made in Louisville:

- Expected patterns for the effect of permeable gap size on the performance, where increasing the gap would improve performance 
- Expected patterns for the effects of permeable joint material on the state of clogging, where the presence of aggregate would result in more surface clogging

- Expected effect of longitudinal slope in the performance and progression of clogging, where increasing the installation slope would result in faster deterioration

- Expected patterns for the material deposited on the surface after the experiments, where there would be a clear segregation between organics and inorganics

Any odd and/or unpredicted pattern must be individually analyzed and justified to ensure the accuracy of the data.

\subsubsection{Quantitative Accuracy}

Evaluating the quantitative accuracy of the measured values during the experiments can be an obvious indicator of data quality. Due to the variability of the tests, the collected data will bear some degree of uncertainty, but if all the designed steps of the experiments are properly executed, a quantitative limit of accuracy can be assigned to the data (Taylor \& Cihon, 2004). For the data analysis and comparisons made between the results, a confidence interval of $95 \%$ was chosen as the acceptable variability in the data.

\subsubsection{Completeness}

Completeness of a dataset is a measure of the amount of data obtained, compared with the amount that was expected to determine a meaningful pattern. Having an 
incomplete dataset can easily complicate the analytical process and results based on incomplete datasets may be compromised.

During the process of determining the variables for the experiment, such as the slope, the gap size and the permeable joint material, all possible experiment scenarios were used, which combined all the variables to ensure the completeness of the dataset.

\subsubsection{Repeatability and Reproducibility of Results}

Conducting tests and experiments in identical conditions and using identical materials does not necessarily produce identical results. Unavoidable random errors and factors that may influence the results cannot be completely controlled. However, these uncontrolled factors and the variation they bring to the results do not stop the results from being comparable. Some degree of variation within the results of duplicate tests must be tolerated (ASTM, 2011).

The repeatability of an experiment is tested when the conditions of the test are kept reasonably constant and the test is repeated. Conditions of reproducibility for a test constitute different conditions, such as changing the laboratory or the experiment environment. Repeatability and reproducibility are two practical extremes of precision (ASTM, 2011).

Due to the scope and nature of the planned work for this research, the repeatability of the test results was tested by conducting duplicate experiments of one of the configurations. The first experiment was selected for testing the repeatability of the test results and three duplicate tests were conducted. According to ASTM Standard E961 -12 , the experiments results are expected to be repeated within a probability of 
approximately 0.95.The results of the experiments presented in Chapter 6 were used to test calculate the confidence intervals for the duplicate tests and compare their repeatability. Finally the calculations made to determine the repeatability and reproducibility of the test results are presented in Appendix $\mathrm{H}$.

\subsection{Experiment \#4}

During experiment \#4, conducted with a $1 \%$ longitudinal slope, $6 \mathrm{~mm}$ gap size and \#8 as the permeable joint material, flow into the flume was clogged by a piece of mulch in the funnel of the flow educator. . This event, which occurred approximately 23 minutes into the experiment, was identified due to a decrease in flow rate. After the clog was discovered, the experiment was stopped, and the funnel section of the feeder was dismantled and unclogged. The test was resumed afterwards.

Figure 67 has used a narrow range for the percentage of saturation, between 0 and 14 percent, to illustrate the points where the experiment was interrupted and was resolved, with higher resolution. The vertical black lines parallel to the Y-Axis show the points when the test was stopped and then resumed. It can be seen that, when the experiment was stopped, the measurements suddenly started to decrease, like the measurements seen at the end of each experiment. After resuming the test, which results in a sudden increase in the measurements due to the resumption of the runoff flow, the experiment follows a normal and predicted path.

The unplanned events for this experiment are stored and, with the normal analysis methods that have been used for the other experiments, this experiment has been brought 
into play to compare the effects of the interruption of the flow, which is closer to what happens outside the laboratory.

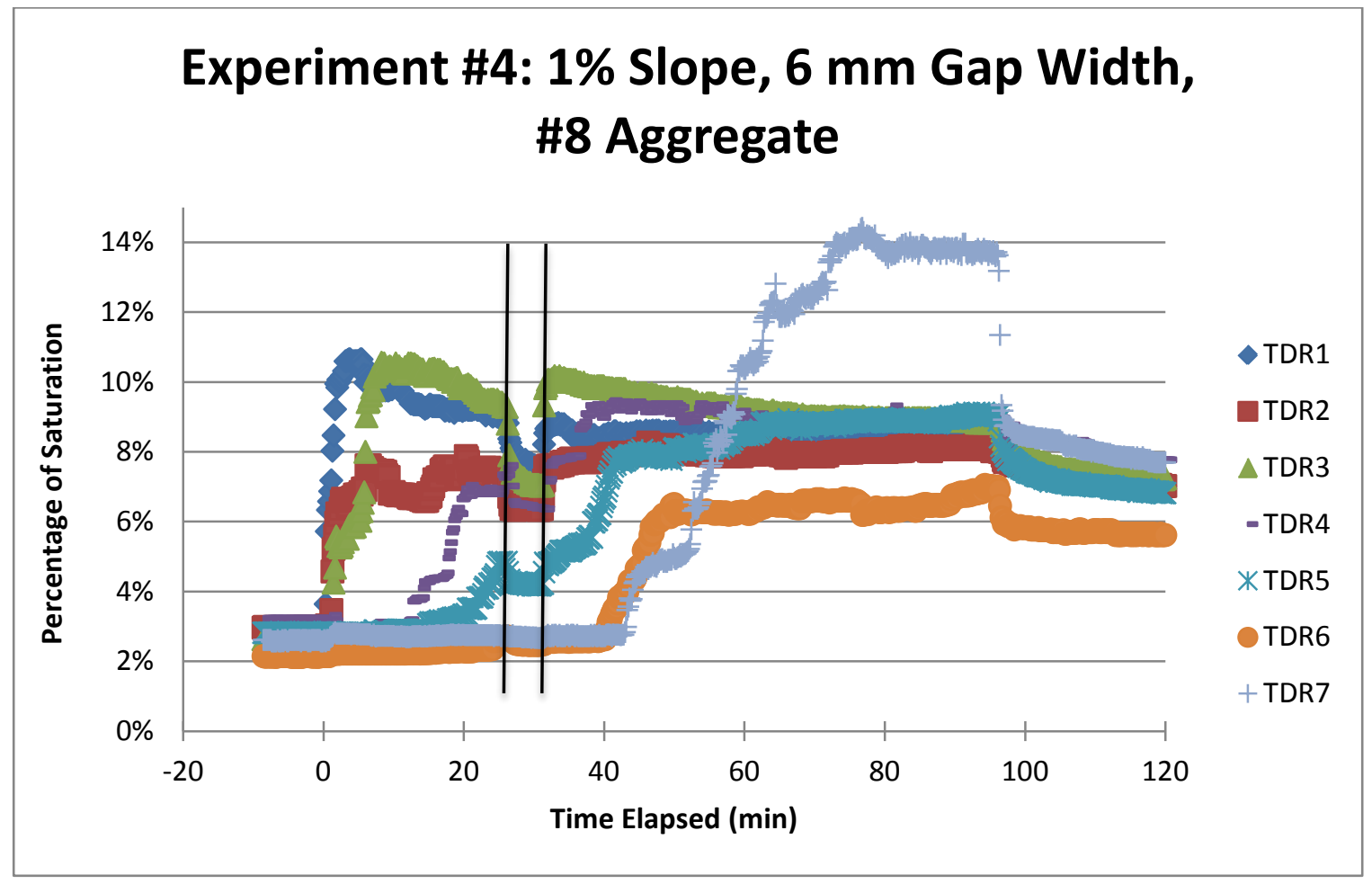

Figure 67: Experiment \#4, illustration of the interruption in the experiment

\subsection{Experiment \#21}

During the 20 completed experiments, it was observed that the organic materials in the clogging sediment had a significant effect on the performance of the GI. Since the characteristics of the clogging material were derived from a series of samples from Louisville's CSO130 project, the results of the study are expected to simulate the effect of different configurations in Louisville. Although a different location may result in a different composition of clogging sediment and it is impossible to simulate every possible situation, a project similar to the Louisville CSO130 project is in place where the same 
configuration is being used in an area where the percentage of organics in the street debris is significantly less.

The behavior of organic debris and their possible effects on surface clogging was used as a basis for conducting an additional flume experiment using inorganic material as the only clogging debris in the stormwater runoff. The effect of those eliminated material was investigated in this experiment. The test was conducted on a $1 \%$ longitudinal slope, with $6 \mathrm{~mm}$ gap size and no permeable joint material. The total sediment load used for the experiment was similar to the other experiments; however $100 \%$ of the sediment was inorganic material. Table 19 lists the weight of each sedimentary component used in the experiment.

Table 19: Sediment Used in Experiment \#21

\begin{tabular}{|c|c|}
\hline Sieve & Inorganic Sediment (gr) \\
\hline $1 / 4 ”$ & 82.2 \\
\hline$\# 4$ & 102.6 \\
\hline$\# 10$ & 195.5 \\
\hline$\# 20$ & 247.9 \\
\hline$\# 40$ & 302.3 \\
\hline$\# 60$ & 252 \\
\hline$\# 100$ & 39.5 \\
\hline$\# 200$ & 70.8 \\
\hline Pan & 23.1 \\
\hline
\end{tabular}

The same steps and methods of analysis were used in experiment \#21. Figure 68 illustrates the percentage of saturation plotted according to the time in the experiment. The analysis shows that, without the organics in the clogging debris, the progression of clogging and the response of GI to the stormwater runoff are significantly different. Comparing Figure 68 and Figure 56 provides an essential tool to compare two GI with 
similar configurations but possibly in different locations or different strategies to trap sediment before reaching the GI.

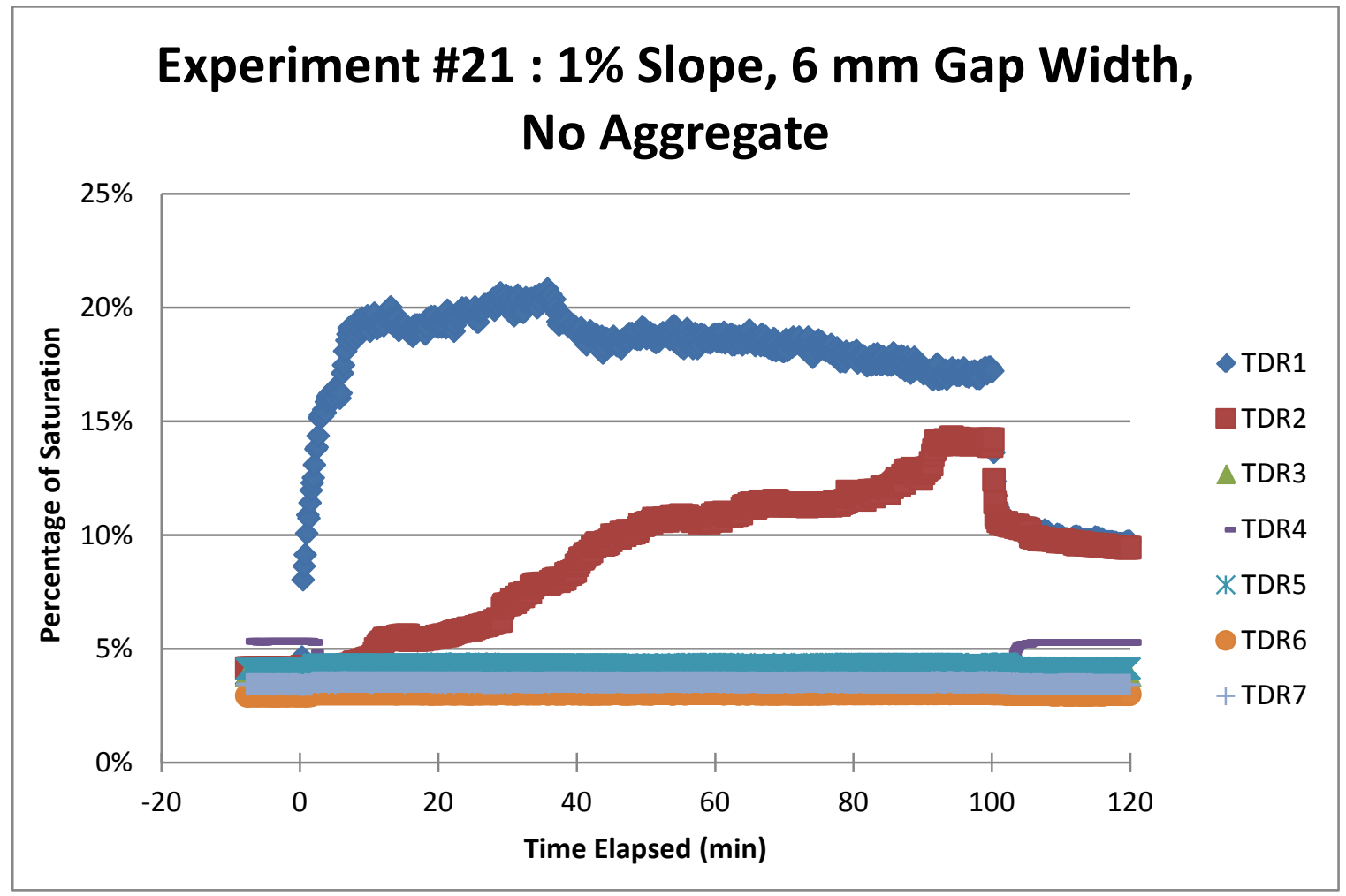

Figure 68: Experiment \#21, 1\% slope, 6 mm gap size, no aggregate between the pavers 


\section{RESULTS AND DISCUSSION}

\subsection{Introduction}

The increasing impervious areas in urban environments and growing CSO related problems has created an enthusiasm for using different types of GI, including permeable pavement systems. However proper siting, design, construction, and maintenance are essential tools to optimize any existing and future GI and to and help achieve stormwater control goals. The CSO130 GI installed in Louisville, KY is an example of how unknown factors can affect the performance of permeable pavement systems.

Lessons learned from the Louisville CSO130 project have shown that the physical environment of the GI can significantly influence the performance, effectiveness, and maintenance needs of the system. The surrounding area, however, is not the only factor affecting the performance of the GI; the characteristics of the paver blocks and the amount and type of sediment carried by stormwater runoff also affect performance. An optimum outcome can be expected only from a full and in depth analysis of the effects on performance of the physical environment and the characteristics of the GI system.

The experiments designed in this study are aimed to determine the effect of some of those neglected factors that are thought to affect the performance of permeable pavement systems. Using remote monitoring instruments, TDRs, as well as observations. 
made during each experiment. Based on the nature of the experiments and methods of data collection the results are divided into three separate subchapters.

At first, the observations made during the experiments are used to interpret the collected data and explain behavior of the experimental module before, during and after each test. Using both the observations and the manual measurements, such as the TSS in the stormwater tank, the discharge flow rates, etc., has enabled some of the unpredicted data patterns to be explained. The observations and the preliminary analysis tools and methods used have been a secondary assessment tool for understanding the performance of the flume.

Using the methods described in Chapter 5, the data collected using TDRs are turned into meaningful information that reveal hidden patterns of performance of the flume. The analysis of the collected data and finding those hidden patterns will help to firstly turn the hypothesis of the study into findings and compare them to those in similar studies. Also these patterns will help to provide useful recommendations for any existing or future GI project in terms of enhancing the location, design, construction and maintenance.

In the following sections observations made in Louisville and in the experiment are used to assess some of the findings of the study and expand the knowledge. Also a series of comparisons of the progression of clogging and infiltration edge between the different experiments are made to determine the effects of changes in those variables of the experiment in performance of the flume. At the end the individual comparisons made here are used to draw a broader range of results, and to determine the effects of change in 
one of the variables across the entire experiments and understand the changes caused by that variable in performance and failure of the flume.

\subsection{Observations and Preliminary Analyses}

Observations and tests made in Louisville where the GI uses a permeable paver product with $6 \mathrm{~mm}$ gap size and no permeable joint material has shown that clogging material deposited on the surface and in the gaps follows consistent patterns. The up gradient of such GI is mostly clogged with inorganic particles. Recovered clogging material suggests that the debris is well graded, meaning that the coarse particles clog first, trapping smaller particles, which creates a layer with significantly lower surface infiltration rates. Down gradient segments have mostly been clogged with organic particles: mulch, leaves, etc. Although the different surface infiltration rates of the segments clogged with inorganics and organics have not been tested in Louisville, it is expected that, if tested, they would be noticeably different. The difference in surface infiltration rates was observed in the experiments conducted at maximum longitudinal slopes, where the surface clogging reached the most down gradient of the flume.

The results of analyzing the collected material in Louisville were provided in Chapter 33. After the first part of this study, two other samplings were conducted in the same GI that confirms the same patterns with minor seasonal differences. The organic content of these events are listed in Table 20. The sections in the $19 \mathrm{G}$ permeable pavements are shown in Figure 6. 
Table 20: Additional Sampling in Louisville CSO130

\begin{tabular}{|c|c|c|}
\hline Sampling Date & Sampling Location & Percent of Organics (\%) \\
\hline $12 / 18 / 2012$ & 19 G-A & 56.77 \\
\hline $12 / 18 / 2012$ & 19 G-D & 8.30 \\
\hline $3 / 19 / 2013$ & 19 G-A & 51.93 \\
\hline $3 / 19 / 2013$ & 19 G-B & 41.92 \\
\hline $3 / 19 / 2013$ & $10 \mathrm{G}-\mathrm{D}$ & 14.41 \\
\hline
\end{tabular}

In the experiments, and in configurations where permeable joint material was not included, the same patterns were observed. The up gradient of the flume was clogged with debris that were visibly sandy and inorganic particles; the down gradient segment of the flume, however, was clogged with visibly organic debris carried by runoff. Although no actual measurements were conducted during the experiment to determine the comparable numbers for this pattern, the observations were helpful in estimating. These observations for experiments \#2, \#5 and \#7 are illustrated in Figure 69.

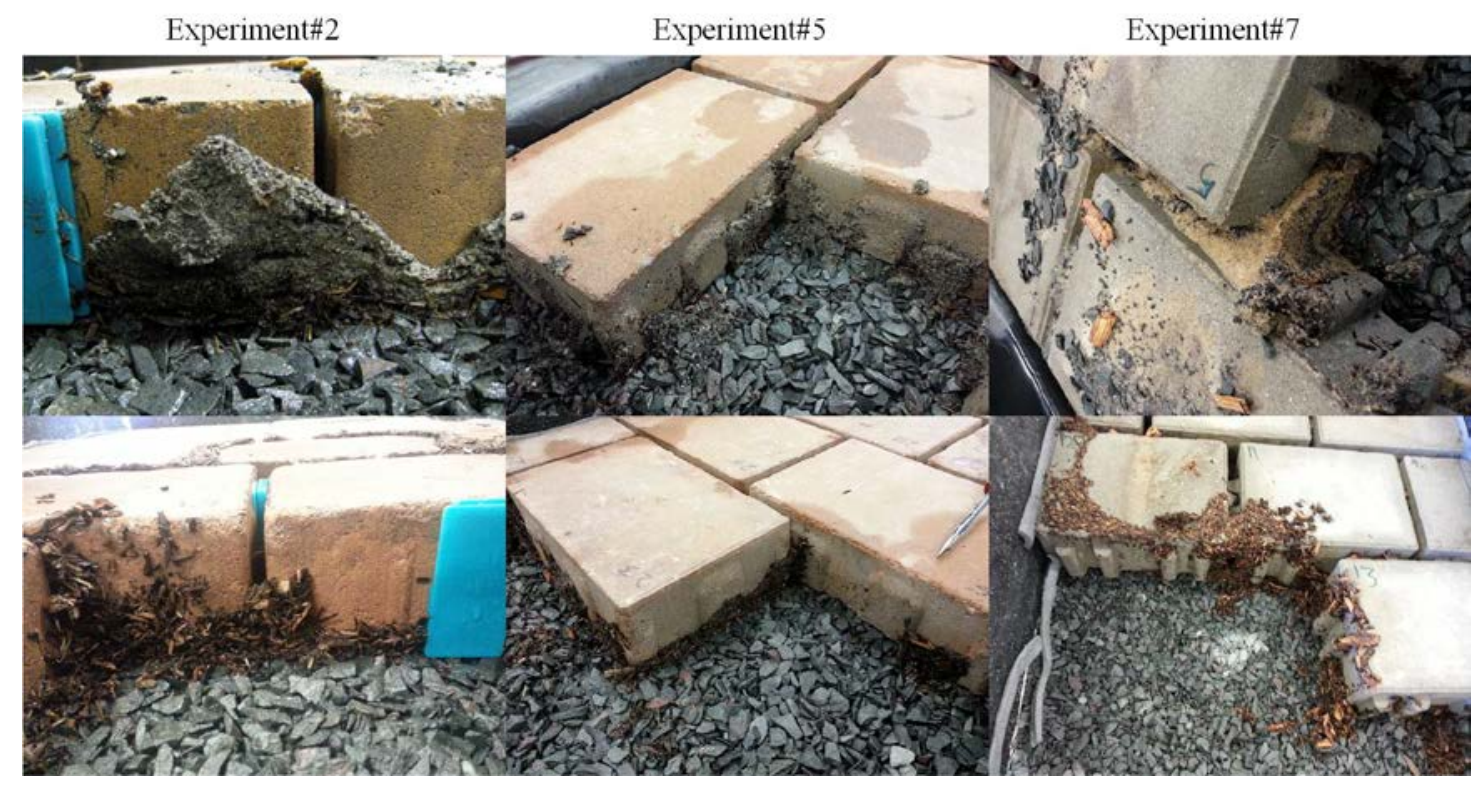

Figure 69: Comparison of clogging debris between the pavers gaps in experiments conducted with no permeable joint material 
In the experiments where the permeable joint material was a part of the permeable surface configuration, a different pattern was observed. The inorganic clogging debris was carried by the surface runoff and was deposited along the length of the flume from the up gradient segments to the furthest down gradient segment. Yet the organic debris followed the same patterns as the experiments, where permeable joint material was not present with a significant amount of the organics being deposited in the down gradient segments.

\subsection{Progression of Clogging and Infiltration Edge}

\section{Introduction}

Clogging progress is measured by using the time when a TDR's measurement reaches the peak measured number, which is then linked to the rainfall associated with such time, and the physical location of that TDR in the flume. Based on the data points of each TDR and their associated rainfall, a graph is plotted. The graphs for the progression of clogging for each individual experiment are included in Appendix F. By comparing the slope of each graph to experiments with one of the variables as a similar configuration, the effect of change in this variable on the progression of clogging is determined.

In order to determine the effect of change in a variable on the progression of clogging, the comparable parameters of each graph are extracted from Appendix F and listed in Table 21. 
Table 21: Characteristics of Progression of Clogging for All Experiments

\begin{tabular}{|l|l|c|c|c|}
\hline Test No. & $\begin{array}{c}\text { Specifications } \\
\text { (Slope, Gap } \\
\text { (mm), } \\
\text { Aggregate) }\end{array}$ & $\begin{array}{c}\text { Slope of trend } \\
\text { line }\end{array}$ & R squared $\left(\mathrm{R}^{2}\right.$ ) & Intercept \\
\hline Experiment \#1 & $1 \%, 6$, None & 3.0759 & 0.994 & 18.249 \\
\hline Experiment \#2 & $1 \%$, , None & 2.5723 & 0.9466 & 27.964 \\
\hline Experiment \#3 & $1 \%, 6$, None & 2.6824 & 0.8679 & 12.881 \\
\hline Experiment \#4 & $1 \%, 6, \# 8$ & 2.2389 & 0.8248 & 53.237 \\
\hline Experiment \#5 & $1 \%, 9$, None & 2.8828 & 0.8252 & 29.991 \\
\hline Experiment \#6 & $1 \%, 9, \# 8$ & 3.5548 & 0.8846 & 33.799 \\
\hline Experiment \#7 & $1 \%, 12$, None & 1.9364 & 0.9852 & 21.476 \\
\hline Experiment \#8 & $1 \%, 12, \# 8$ & 1.8971 & 0.9382 & 20.484 \\
\hline Experiment \#9 & $3 \%, 12, \# 8$ & 4.2221 & 0.8878 & 35.6 \\
\hline Experiment \#10 & $3 \%, 12$, None & 2.4395 & 0.9541 & 28.144 \\
\hline Experiment \#11 & $3 \%, 9$, None & 2.9904 & 0.9926 & 22.329 \\
\hline Experiment \#12 & $3 \%, 9, \# 8$ & 7.6882 & 0.8733 & 30.321 \\
\hline Experiment \#13 & $3 \%, 6$, None & 3.3737 & 0.9812 & 15.487 \\
\hline Experiment \#14 & $3 \%, 6, \# 8$ & 4.7918 & 0.8882 & 42.295 \\
\hline Experiment \#15 & $5 \%, 12$, None & 2.2255 & 0.9871 & 27.581 \\
\hline Experiment \#16 & $5 \%, 12, \# 8$ & 3.9595 & 0.9113 & 47.83 \\
\hline Experiment \#17 & $5 \%, 9$, None & 3.2353 & 0.9778 & 28.566 \\
\hline Experiment \#18 & $5 \%, 9, \# 8$ & 4.485 & 0.8975 & 51.431 \\
\hline Experiment \#19 & $5 \%, 6$, None & 3.7441 & 0.9925 & 21.423 \\
\hline Experiment \#20 & $5 \%, 6, \# 8$ & 7.6779 & 0.9158 & 36.739 \\
\hline Experiment \#21 & $1 \%, 6$, None & 0.9614 & 1.000 & 11.08 \\
\hline
\end{tabular}

The first parameter extracted from the graphs of the progression of clogging is the slope of the trend line. The slope is also a representative of the rate of increase of the progression of clogging in the flume, and the steeper slopes suggest that the clogging progresses faster along the entire length of the flume, while the less steep slopes suggest that the clogging progresses more slowly.

In order to use the progression of the infiltration edge to get results, the slope of the data points' trend line has been extracted and the points listed in Table 22. 
Table 22: Characteristics of Progression of Infiltration Edge for All Experiments

\begin{tabular}{|c|c|c|c|c|}
\hline Test No. & $\begin{array}{l}\text { Specifications } \\
\text { (Slope, Gap } \\
\text { (mm), } \\
\text { Aggregate) }\end{array}$ & $\begin{array}{l}\text { Slope of trend } \\
\text { line }\end{array}$ & $\mathrm{R}$ squared $\left(\mathrm{R}^{2}\right)$ & Intercept \\
\hline Experiment \#1 & $1 \%, 6$, None & 6.2149 & 0.9323 & 35.117 \\
\hline Experiment \#2 & $1 \%, 6$, None & 4.088 & 0.9642 & 44.867 \\
\hline Experiment \#3 & $1 \%, 6$, None & 3.6112 & 0.9181 & 44.318 \\
\hline Experiment \#4 & $1 \%, 6, \# 8$ & 6.1126 & 0.8577 & 64.472 \\
\hline Experiment \#5 & $1 \%, 9$, None & 4.6293 & 0.9709 & 43.808 \\
\hline Experiment \#6 & $1 \%, 9, \# 8$ & 3.9945 & 0.7059 & 73.719 \\
\hline Experiment \#7 & $1 \%, 12$, None & 2.4099 & 0.9833 & 33.438 \\
\hline Experiment \#8 & $1 \%, 12, \# 8$ & 2.1608 & 0.7741 & 52.248 \\
\hline Experiment \#9 & $3 \%, 12, \# 8$ & 5.4235 & 0.8447 & 53.034 \\
\hline Experiment \#10 & $3 \%, 12$, None & 2.8501 & 0.9492 & 41.761 \\
\hline Experiment \#11 & $3 \%, 9$, None & 3.3425 & 0.9754 & 40.822 \\
\hline Experiment \#12 & $3 \%, 9, \# 8$ & 10.757 & 0.9379 & 52.633 \\
\hline Experiment \#13 & $3 \%, 6$, None & 3.2335 & 0.9167 & 57.906 \\
\hline Experiment \#14 & $3 \%, 6, \# 8$ & 13.069 & 0.8712 & 61.192 \\
\hline Experiment \#15 & $5 \%, 12$, None & 2.4938 & 0.9458 & 43.289 \\
\hline Experiment \#16 & $5 \%, 12, \# 8$ & 7.7432 & 0.9538 & 51.928 \\
\hline Experiment \#17 & $5 \%, 9$, None & 3.3554 & 0.9381 & 54.157 \\
\hline Experiment \#18 & $5 \%, 9, \# 8$ & 9.6802 & 0.8615 & 66.298 \\
\hline Experiment \#19 & $5 \%, 6$, None & 3.8508 & 0.9299 & 57.153 \\
\hline Experiment \#20 & $5 \%, 6, \# 8$ & 15.825 & 0.8557 & 63.388 \\
\hline Experiment \#21 & $1 \%, 6$, None & 8.6523 & 1.000 & 27.847 \\
\hline
\end{tabular}

In the following sections, a series of comparisons between different experiments are used to compare the effect of a change in each of the variables of the experiment on progression of both the infiltration edge and the clogging. The numerous comparisons are explained with graphs and their associated equations. In order to optimize and maximize the extraction of hidden patterns and create clearer results, the comparisons made to get results are grouped into categories, which are based on the variables designed for the experiments. 
In the order appeared here, the effects of change in the paver gap size. The permeable joint material and the longitudinal slope have resulted in 6, 9, and 6 different comparison groups, each describing the effect of changes made in only one variable in experiments with similar configurations. The result is 21 comparisons for progression of infiltration edge and clogging on the surface of the flume, which on their own describe the effects of changes in specific scenarios, but when they come together, using analysis of variance, Section 6.4, they create an image that can be used to draw meaningful conclusions of the effect of the changes in slope, paver gap size and permeable joint material in performance of a permeable pavement system.

\subsubsection{Paver Gap Size}

One of the variables thought to have a significant effect on the performance of GI is the size of the gap between the pavers. The gap in the paver blocks provides stormwater runoff with an entrance to the storage gallery. It is obvious that when the entrance has limited capacity, regardless of the storage volume and exfiltration capability of the storage gallery, the system is doomed to fail.

As mentioned before, performance in practice depends on many factors and this research is an attempt to investigate only a few of them and thus requires the real life situations to be simplified.

The first step is to compare the results to understand the effect of the paver gap size on the performance of the permeable surface in the flume. In order to achieve this aim, the progression of clogging and progression of the infiltration edge graphs from the following experiments are compared to each other. 


\subsubsection{Gap Size with 1\% Longitudinal Slope without Aggregate}

The experiments in this category have the following gap sizes: $6 \mathrm{~mm}$ vs. $9 \mathrm{~mm}$ vs. $12 \mathrm{~mm}$, which are tested in experiments \#2, \#5, and \#7. The progression of clogging in these three experiments is plotted in Figure 70:

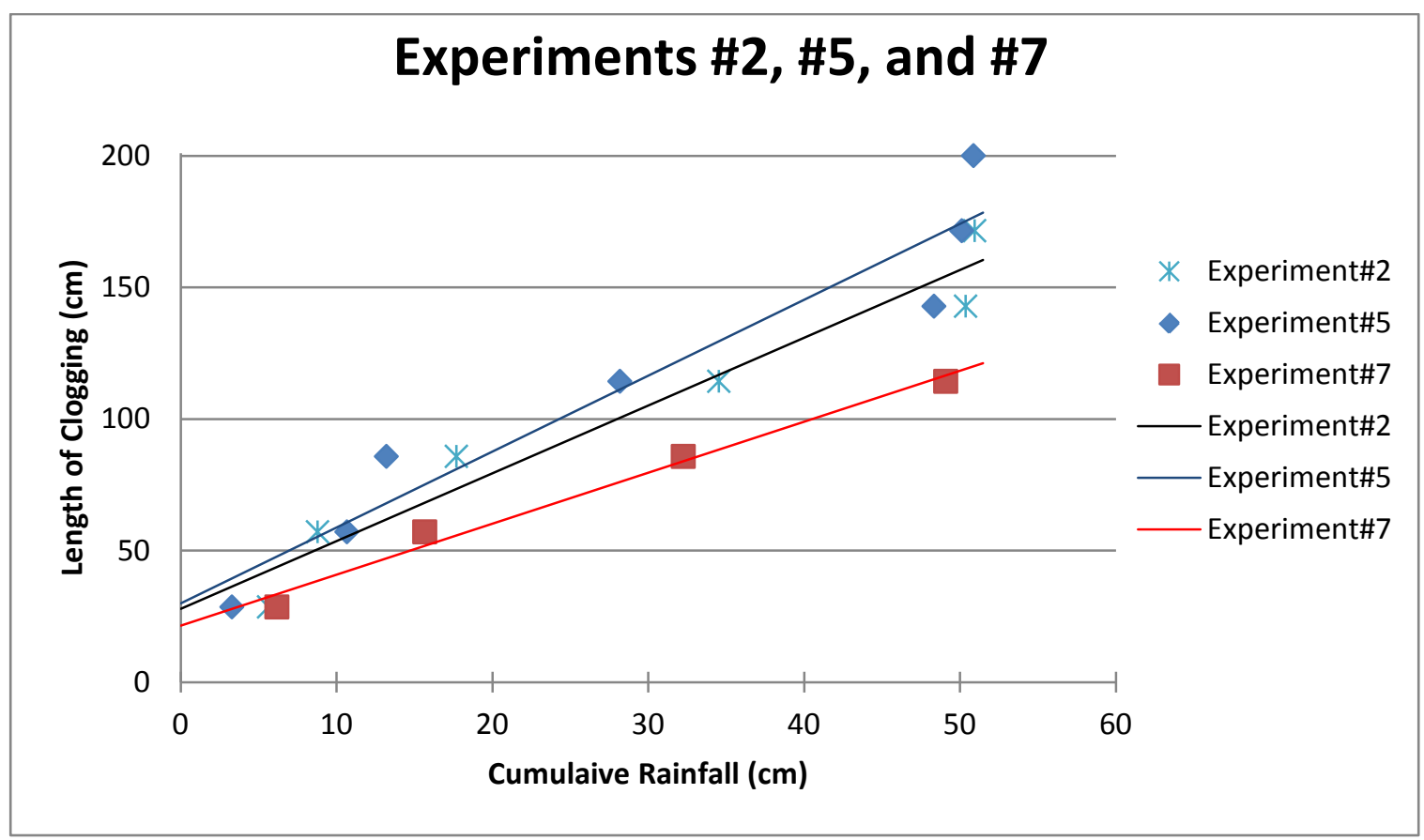

Figure 70: Comparison of progression of clogging in experiments \#2, \#5, and \#7

With a $3 \mathrm{~mm}$ increase in the gap sizes (experiments \#2 and \#5), in a 1\% longitudinal slope, and without any permeable joint material, the slope of progression of clogging increases, meaning that the surface clogging progresses faster. With a further increase in the gap size, the slope of the progression of clogging decreases and is lower than both the previous gaps, meaning that the deterioration of the performance of the permeable surface is the slowest of the three.

Similar patterns are seen in the progression of the infiltration edge, where an increase in the permeable paver gap size, from $6 \mathrm{~mm}$ to $9 \mathrm{~mm}$, results in more rapid 
progression in the infiltration edge, but a further increase, from $9 \mathrm{~mm}$ to $12 \mathrm{~mm}$, results in the slowest increase in infiltration edge in all three experiments. Figure 71 illustrates the progression of the infiltration edge in these three experiments.

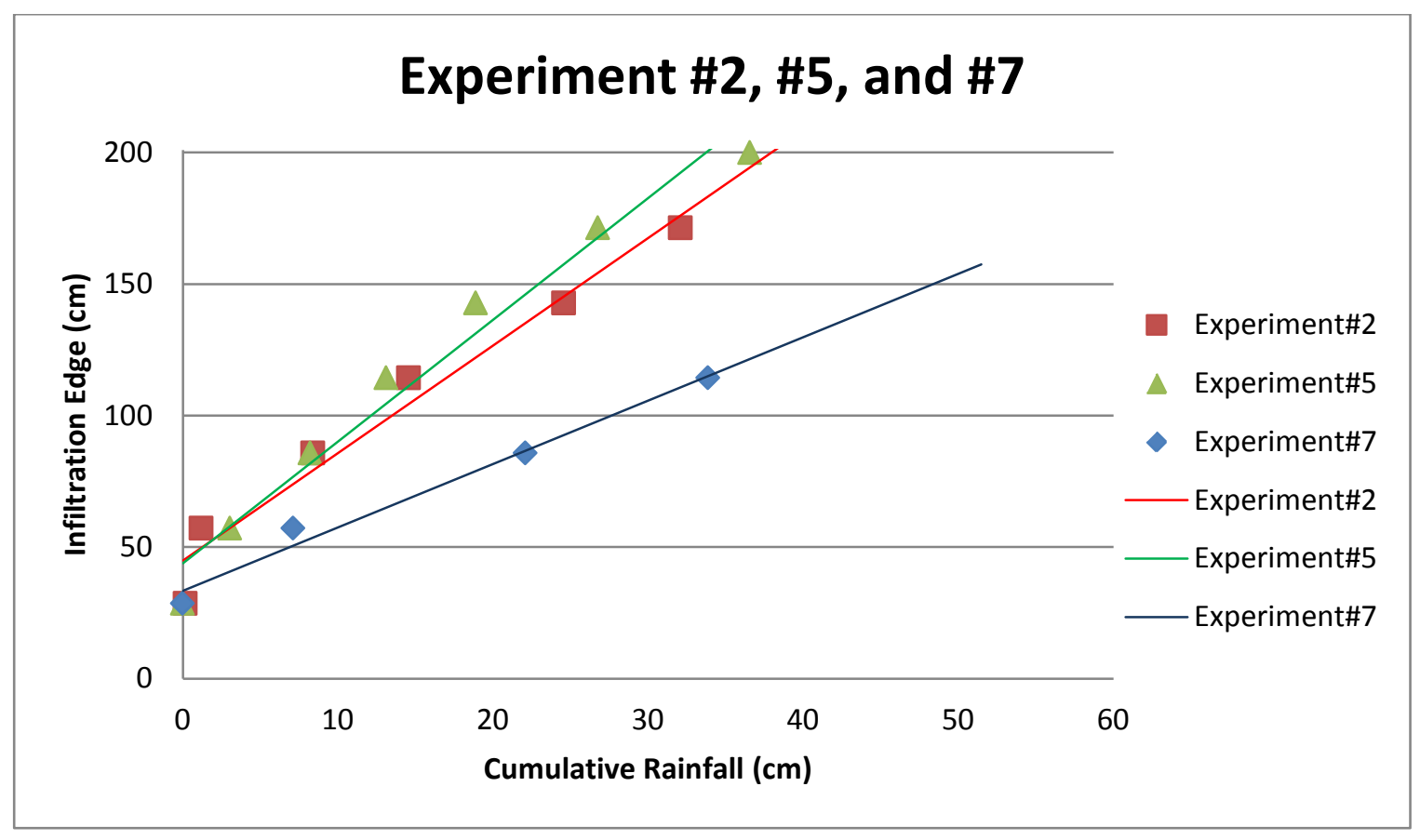

Figure 71: Comparison of progression of infiltration edge in experiments \#2, \#5, and \#7

Using a numerical and simple comparison between these experiments, it is clear that with the first $3 \mathrm{~mm}$ increase in paver gap size, the surface clogging conditions deteriorate by $12 \%$; however, with the second $3 \mathrm{~mm}$ increase in the paver gap size, the conditions experienced a 32\% improvement over the $9 \mathrm{~mm}$ and a $24 \%$ improvement over the $6 \mathrm{~mm}$. 
Table 23: Comparing the Progression of Clogging in Experiments \#2, \#5, And \#7

\begin{tabular}{|l|l|l|l|}
\hline $\begin{array}{l}\text { Gap } \\
\text { Size }\end{array}$ & $\begin{array}{l}6 \\
\mathrm{~mm}\end{array}$ & $\begin{array}{l}9 \\
\mathrm{~mm}\end{array}$ & $\begin{array}{l}12 \\
\mathrm{~mm}\end{array}$ \\
\hline $6 \mathrm{~mm}$ & N/A & $\begin{array}{l}- \\
12 \%\end{array}$ & $+24 \%$ \\
\hline $9 \mathrm{~mm}$ & $\begin{array}{l}+12 \\
\%\end{array}$ & N/A & $+32 \%$ \\
\hline $12 \mathrm{~mm}$ & $-24 \%$ & $\begin{array}{l}- \\
32 \%\end{array}$ & N/A \\
\hline
\end{tabular}

Comparing the experiments conducted on a 1\% longitudinal slope, three different gap sizes and no permeable joint material, using the progression of clogging and progression of infiltration edge shows that, although the common understanding in the role of the permeable paver gap size on the performance of the system is correct, the relationship between gap and performance is not linear, since the experiments show that the first increase is found where the deterioration is quickest.

Table 24: Comparing the Progression of Clogging in Experiments \#2, \#5, And \#7

\begin{tabular}{|l|l|l|l|}
\hline Gap Size & $6 \mathrm{~mm}$ & $9 \mathrm{~mm}$ & $12 \mathrm{~mm}$ \\
\hline $6 \mathrm{~mm}$ & N/A & $-13 \%$ & $+41 \%$ \\
\hline $9 \mathrm{~mm}$ & $-13 \%$ & N/A & $+48 \%$ \\
\hline $12 \mathrm{~mm}$ & $-41 \%$ & $-48 \%$ & N/A \\
\hline
\end{tabular}

Comparing the experiments conducted on 1\% longitudinal slope, three different gap sizes and no permeable joint material, using the progression of clogging and progression of infiltration edge, shows that although the common understanding in the role of the permeable paver gap size on the performance of the system is correct; but the relation between the gap and performance is not linear, as the experiments showed that the first increase resulted in where deterioration happened faster. 


\subsubsection{Gap Size with Base 1\% Longitudinal Slope with \\ Aggregate}

The experiments in this category have the following gap sizes: $6 \mathrm{~mm}$ vs. $9 \mathrm{~mm}$ vs. $12 \mathrm{~mm}$, and they are all conducted on the basis of a 1\% longitudinal slope. Experiments \#4, \#6, and \#8 have they gaps between the permeable pavers filled with permeable joint material, which is \#8 AASHTO aggregate.

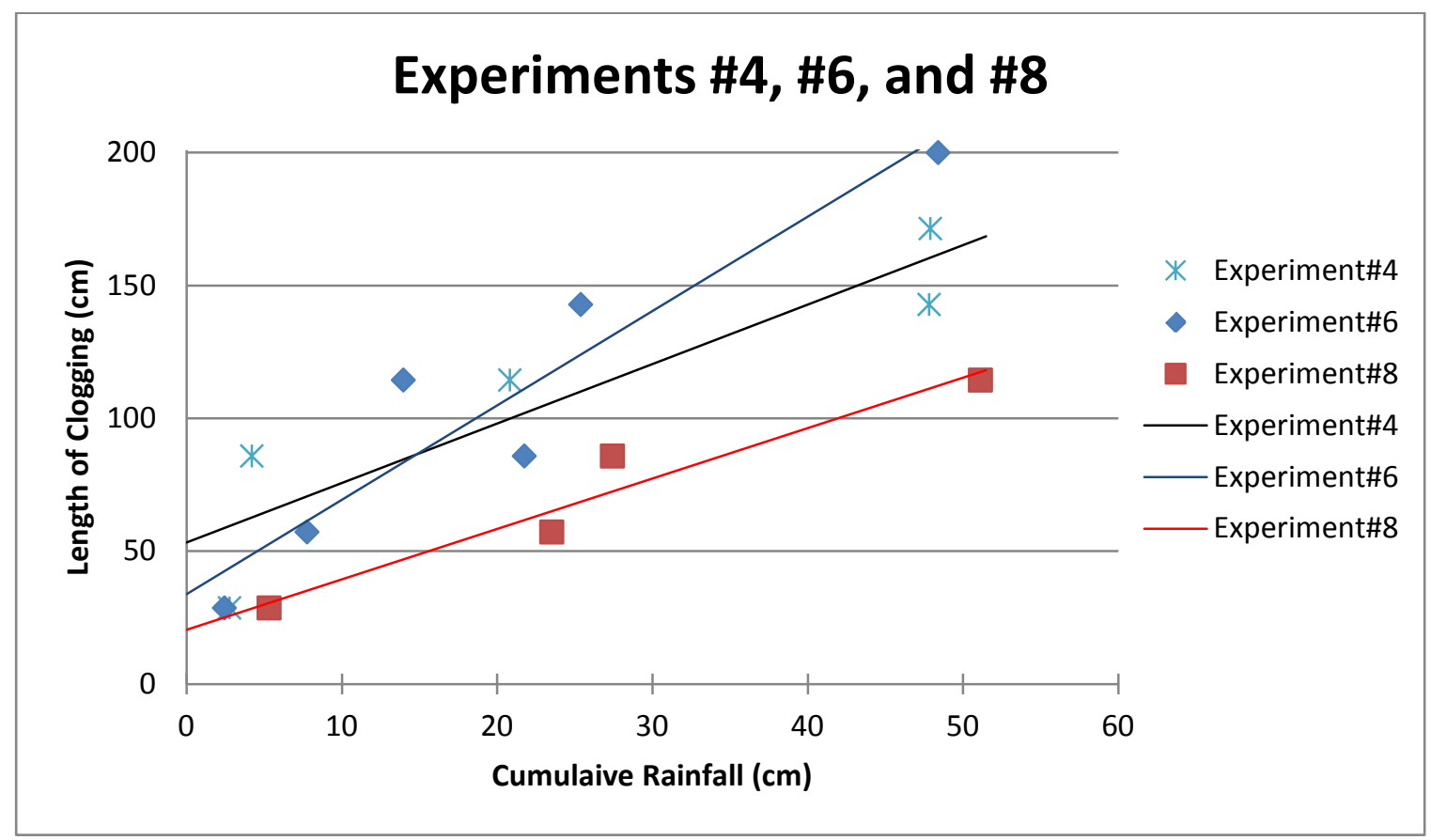

Figure 72: Comparison of progression of clogging in experiments \#4, \#6, and \#8

With a $3 \mathrm{~mm}$ increase in the gap sizes (experiments \#4 and \#6), a 1\% longitudinal slope, and with permeable joint material, the slope of the progression of clogging increases, meaning that the surface clogging progresses faster. With a further increase in the gap size, the slope of the progression of clogging decreases and is lower than both the previous gaps, meaning that the deterioration of the performance of the permeable surface is the slowest of the three. These patterns are illustrated in Figure 72. 


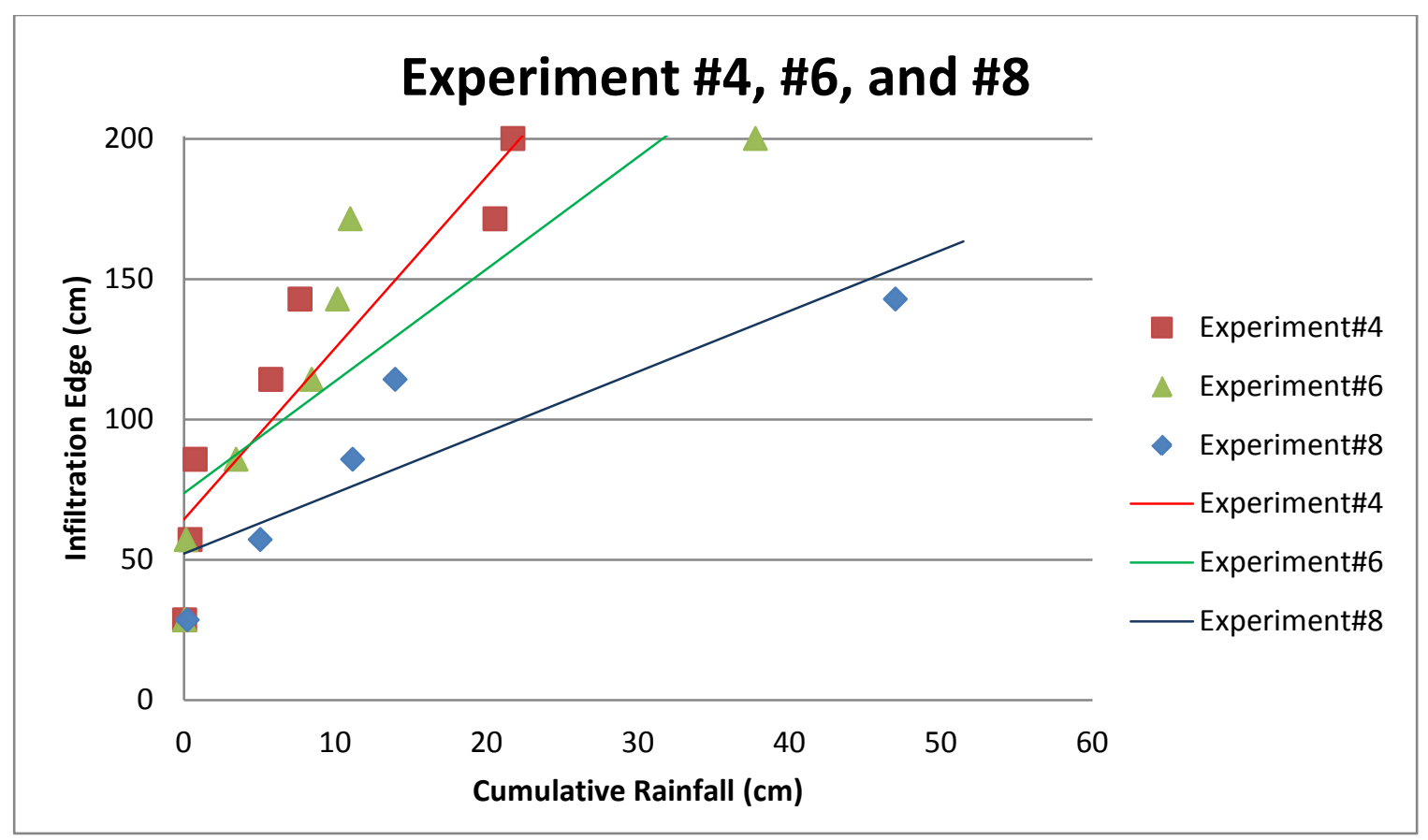

Figure 73: Comparison of progression of infiltration edge in experiments \#4, \#6, and \#8

The comparison of the progression of infiltration edge graphs for these experiments, shown in Figure 73, reveals a different pattern. The presence of the permeable joint material has helped in the progress of the infiltration edge and with increased gap size; there is a consistent decrease in the progression of the wetting front.

Using a simple numerical comparison between these experiments demonstrates that with the first $3 \mathrm{~mm}$ increase in paver gap size, the surface clogging conditions deteriorate by 58\%; however, by the second $3 \mathrm{~mm}$ increase in the paver gap size, the conditions have experienced a 46\% improvement over the $9 \mathrm{~mm}$ and a $9 \%$ improvement over the $6 \mathrm{~mm}$. 
Table 25: Comparing the Progression of Clogging in Experiments \#4, \#6, and \#8

\begin{tabular}{|l|l|l|l|}
\hline $\begin{array}{l}\text { Gap } \\
\text { Size }\end{array}$ & $\begin{array}{l}6 \\
\mathrm{~mm}\end{array}$ & $\begin{array}{l}9 \\
\mathrm{~mm}\end{array}$ & $\begin{array}{l}12 \\
\mathrm{~mm}\end{array}$ \\
\hline $6 \mathrm{~mm}$ & N/A & $\begin{array}{l}- \\
58 \%\end{array}$ & $+9 \%$ \\
\hline $9 \mathrm{~mm}$ & $\begin{array}{l}+58 \\
\%\end{array}$ & N/A & $+46 \%$ \\
\hline $12 \mathrm{~mm}$ & $-9 \%$ & $\begin{array}{l}- \\
46 \%\end{array}$ & N/A \\
\hline
\end{tabular}

Comparing the progression of the infiltration edge between the experiments shows that with a $3 \mathrm{~mm}$ increase in the gap between the pavers and from a $6 \mathrm{~mm}$ original gap size to a $9 \mathrm{~mm}$ gap size, the conditions improve by 34\%. Similarly, by another increase from $9 \mathrm{~mm}$ gap size to $12 \mathrm{~mm}$, the movement of the rate of progression of the infiltration edge on the permeable surface experiences a $45 \%$ decrease. Finally, comparing the $6 \mathrm{~mm}$ gap size to the $12 \mathrm{~mm}$ gap size shows the rate of progress to have decreased by $64 \%$.

Table 26: Comparing the Progression of Infiltration Edge in Experiments \#4, \#6, and \#8

\begin{tabular}{|l|l|l|l|}
\hline Gap Size & $6 \mathrm{~mm}$ & $9 \mathrm{~mm}$ & $12 \mathrm{~mm}$ \\
\hline $6 \mathrm{~mm}$ & N/A & $+34 \%$ & $+64 \%$ \\
\hline $9 \mathrm{~mm}$ & $-34 \%$ & N/A & $+45 \%$ \\
\hline $12 \mathrm{~mm}$ & $-64 \%$ & $-45 \%$ & N/A \\
\hline
\end{tabular}

In the end, comparing the two set of graphs and tables shows that, in the initial response of the flume to the stormwater runoff, with increasing gap size, the flume requires less surface area to infiltrate the same amount of runoff. However, after becoming clogged, the $12 \mathrm{~mm}$ gap size performs the best, the performance of the $6 \mathrm{~mm}$ gap size comes second and the $9 \mathrm{~mm}$ gap size is the worst of the three. 


\subsubsection{Gap Size with Base 3\% Longitudinal Slope without \\ Aggregate}

The experiments in this category have the following gap sizes: $6 \mathrm{~mm}$ vs. $9 \mathrm{~mm}$ vs. $12 \mathrm{~mm}$; they include experiments \#13, \#11, and \#10, with respect to their gap sizes. The longitudinal slope of the experiments has been increased to $3 \%$ and no permeable joint material is present in the gaps between the pavers.

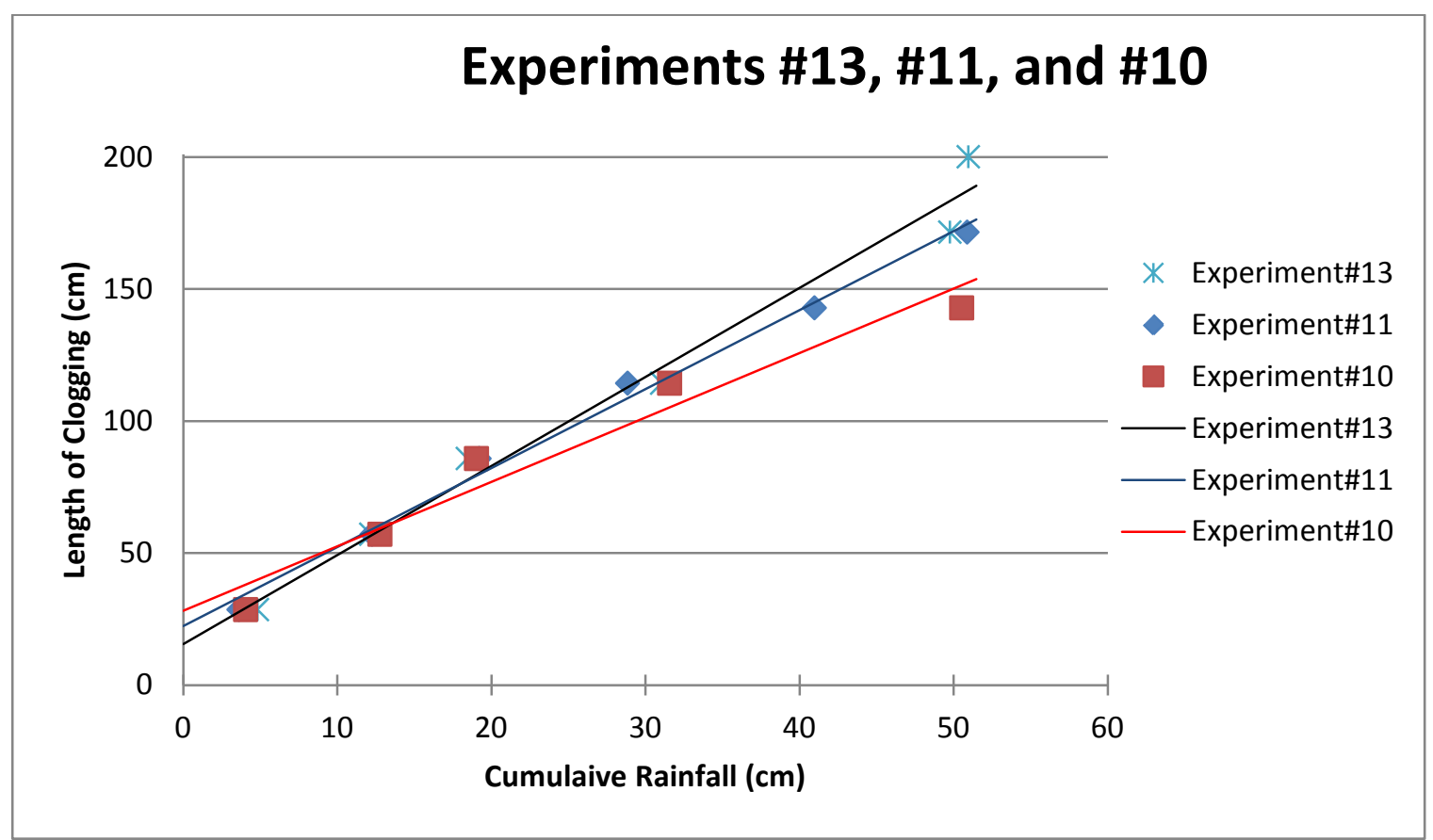

Figure 74: Comparison of progression of clogging in experiments \#13, \#11, and \#10

With a $3 \mathrm{~mm}$ increase in the gap sizes (experiments \#13 and \#11), in a 3\% longitudinal slope, and without the permeable joint material, the slope of the progression of clogging decreases, meaning that the surface clogging progresses more slowly. With a further increase in the gap size, the slope of the progression of clogging decreases even more and is lower than both the previous gaps, meaning that the deterioration of the performance of permeable surface is the slowest of the three. 


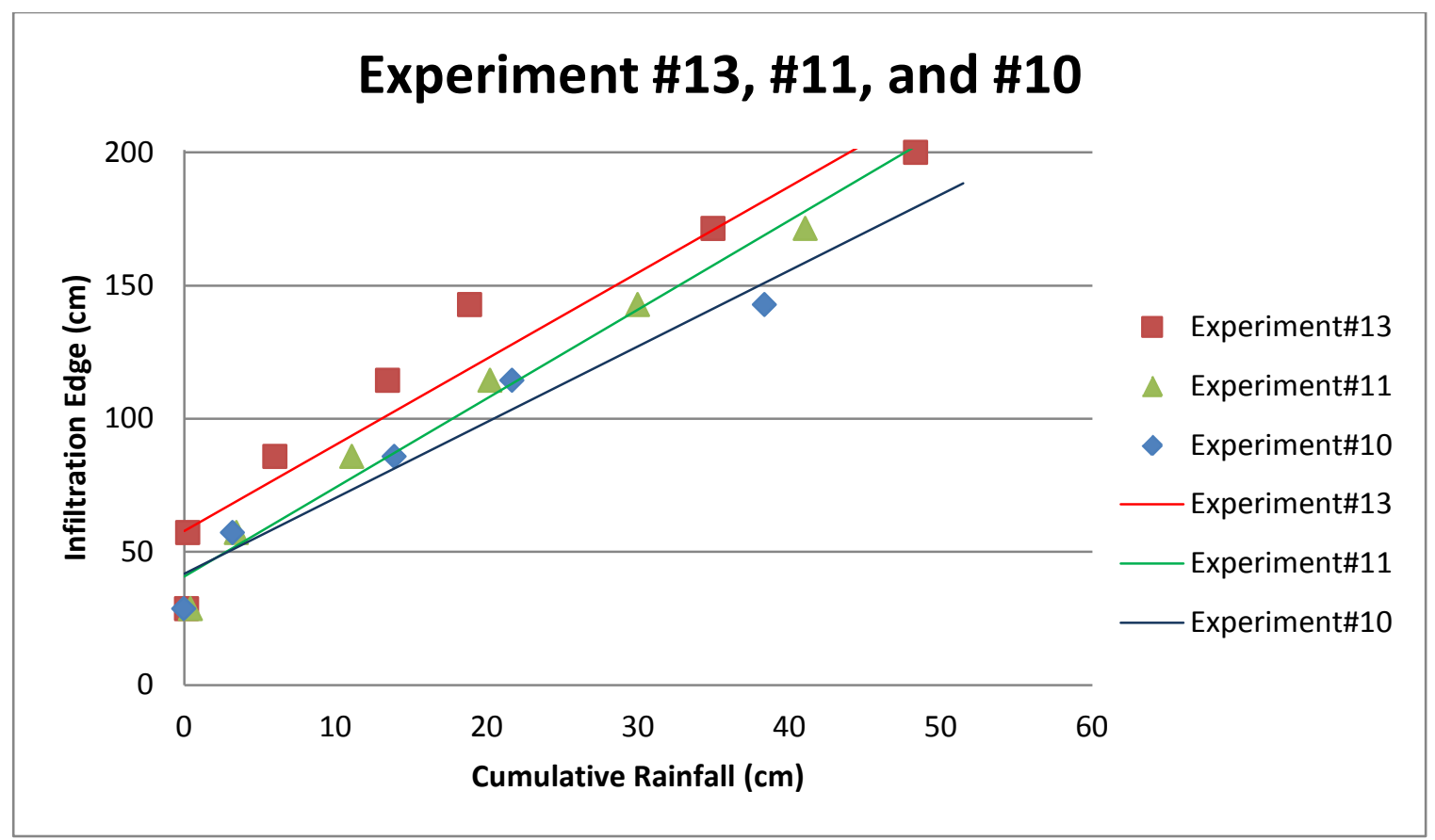

Figure 75: Comparison of progression of infiltration edge in experiments \#13, \#11, and \#10

The progression of the infiltration edge goes at an increased rate with an increase in the gap size from $6 \mathrm{~mm}$ to $9 \mathrm{~mm}$; however, with a further increase in the paver gap size, from $9 \mathrm{~mm}$ to $12 \mathrm{~mm}$, the rate of progress of the wetting front decreases to the lowest of all.

Using the same numerical methods to calculate the percentage of increase and compare the three different configurations, it can be seen that the increase from a $6 \mathrm{~mm}$ gap size to a $9 \mathrm{~mm}$ gap size results in a $11 \%$ improvement in the progression of clogging, while a further gap size increase from $9 \mathrm{~mm}$ to $12 \mathrm{~mm}$ results in another $22 \%$ improvement. Increasing the $6 \mathrm{~mm}$ gap to $12 \mathrm{~mm}$ gap creates a 27\% improvement in terms of the progression seen in the clogging front. 
Table 27: Comparing the Progression of Clogging in Experiments \#13, \#11, and \#10

\begin{tabular}{|l|l|l|l|}
\hline Gap Size & $6 \mathrm{~mm}$ & $9 \mathrm{~mm}$ & $12 \mathrm{~mm}$ \\
\hline $6 \mathrm{~mm}$ & N/A & $+11 \%$ & $+27 \%$ \\
\hline $9 \mathrm{~mm}$ & $-11 \%$ & N/A & $+22 \%$ \\
\hline $12 \mathrm{~mm}$ & $-27 \%$ & $-22 \%$ & N/A \\
\hline
\end{tabular}

Numerical analysis with the progression of the infiltration edge shows that with an increase of gap size from $6 \mathrm{~mm}$ to $9 \mathrm{~mm}$, the rate at which the permeable surface is used to infiltrate runoff decreases by 3\%. A further increase in the paver gap size, from 9 $\mathrm{mm}$ to $12 \mathrm{~mm}$, results in a 14\% improvement. A direct comparison between $6 \mathrm{~mm}$ and 12 mm shows an $11 \%$ improvement in the progression of the infiltration edge.

Table 28: Comparing the Progression of Infiltration Edge in Experiments \#13, \#11, and \#10

\begin{tabular}{|l|l|l|l|}
\hline Gap Size & $6 \mathrm{~mm}$ & $9 \mathrm{~mm}$ & $12 \mathrm{~mm}$ \\
\hline $6 \mathrm{~mm}$ & N/A & $-3 \%$ & $+11 \%$ \\
\hline $9 \mathrm{~mm}$ & $+3 \%$ & N/A & $+14 \%$ \\
\hline $12 \mathrm{~mm}$ & $-11 \%$ & $-14 \%$ & N/A \\
\hline
\end{tabular}

Overall, the comparisons made in this section show that the steep slope of the flume (3\% longitudinal) has resulted in a better performance in terms of the progression of clogging, where the bigger gap sizes have consistently provided more capacity for infiltration and tolerated the surface clogging better.

\subsubsection{Gap Size with Base 3\% Longitudinal Slope with Aggregate}

The experiments in this category have the following gap sizes: $6 \mathrm{~mm}$ vs. $9 \mathrm{~mm}$ vs. 12. Experiments \#14, \#12, \#9 in order of their gap size were set with permeable joint material and 3\% longitudinal slope. 


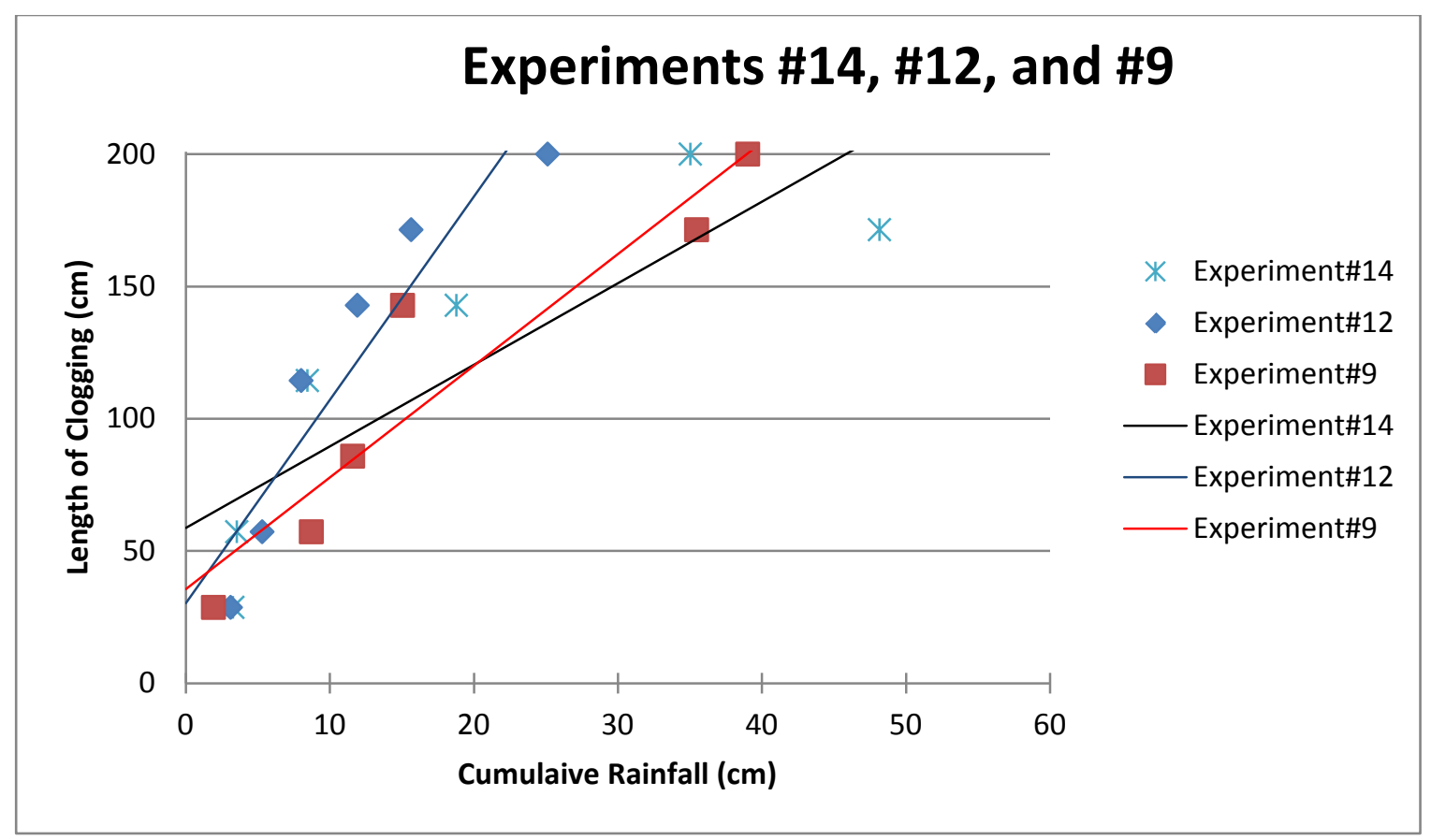

Figure 76: Comparison of progression of clogging in experiments \#14, \#12, and \#9

Figure 76 illustrates a comparison of the progression of clogging, where the $6 \mathrm{~mm}$ gap size is found to be the smallest gap size of all to have resulted in the second best rate of progress of the clogging front. The $9 \mathrm{~mm}$ gap size filled with the permeable joint material has the fastest rate of increase in the progression of clogging and the $12 \mathrm{~mm}$ gap size shows up as best of the three. Note that the smallest gap size in the steep setup for the flume results in runoff ponding towards the end of the experiment in the down gradient segments. 


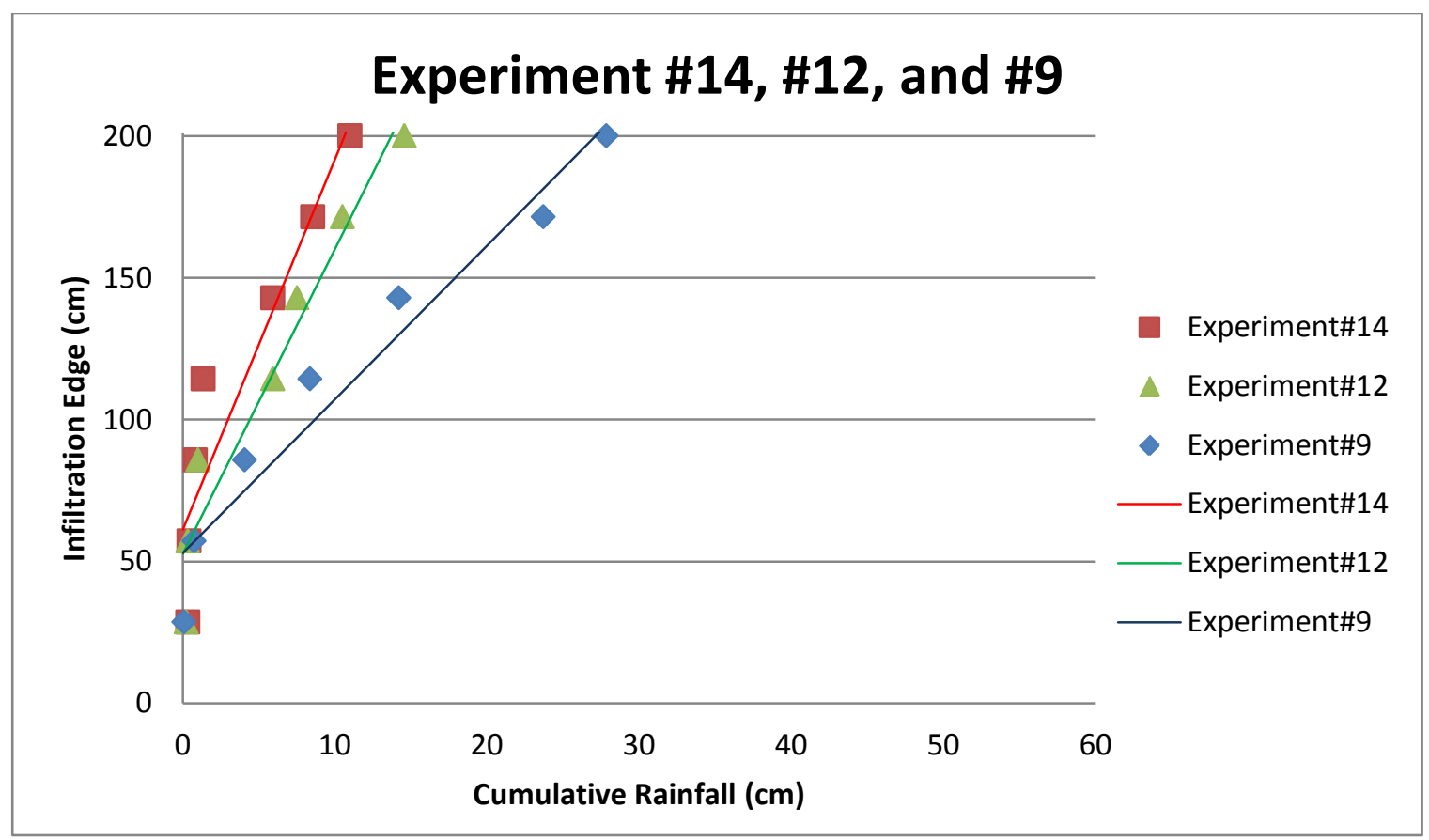

Figure 77: Comparison of progression of infiltration edge in experiments \#14, \#12, and \#9

The progression of the infiltration edge in experiments \#14, \#12 and \#9 are illustrated in Figure 77. As seen on the graph, the steep slope causes rapid progression of the wetting front, meaning that, although the surface may not clog as fast, the slope causes a greater surface area to take partial loads of stormwater runoff and help in the infiltration process. With a constant increase in the gap size, ranging from $6 \mathrm{~mm}$ to 12 $\mathrm{mm}$, the slope of the trend lines shown in the graph decreases, meaning that the wider gaps will enable a smaller unit surface area to infiltrate the same initial flow of runoff.

Comparing the slopes in Figure 76 highlights that the $3 \mathrm{~mm}$ increase in the gap from the $6 \mathrm{~mm}$ original gap size to that of $9 \mathrm{~mm}$, results in a $60 \%$ increase in the rate of progression of surface clogging. Increasing the gap size from $9 \mathrm{~mm}$ to $12 \mathrm{~mm}$ results in a $45 \%$ improvement in the progression of surface clogging. Comparing the $6 \mathrm{~mm}$ gap size with the $12 \mathrm{~mm}$ indicates an $11 \%$ improvement in the progression of surface clogging. 
Table 29: Comparing the Progression of Clogging in Experiments \#14, \#12, and \#9

\begin{tabular}{|l|l|l|l|}
\hline Gap Size & $6 \mathrm{~mm}$ & $9 \mathrm{~mm}$ & $12 \mathrm{~mm}$ \\
\hline $6 \mathrm{~mm}$ & N/A & $-60 \%$ & $+11 \%$ \\
\hline $9 \mathrm{~mm}$ & $+60 \%$ & N/A & $+45 \%$ \\
\hline $12 \mathrm{~mm}$ & $-11 \%$ & $-45 \%$ & N/A \\
\hline
\end{tabular}

Comparing the slopes on Figure 77, the first increase in gap size, $6 \mathrm{~mm}$ to $9 \mathrm{~mm}$, results in a $17 \%$ improvement in the progression of the infiltration edge on the surface, and the second increase in gap size, $9 \mathrm{~mm}$ to $12 \mathrm{~mm}$, and results in a $49 \%$ improvement. The increase from $6 \mathrm{~mm}$ to $12 \mathrm{~mm}$ results in a 58\% improvement in the rate at which the surface infiltration edge progresses on the flume.

Table 30: Comparing the Progression of Infiltration Edge in Experiments \#14, \#12, and \#9

\begin{tabular}{|l|l|l|l|}
\hline Gap Size & $6 \mathrm{~mm}$ & $9 \mathrm{~mm}$ & $12 \mathrm{~mm}$ \\
\hline $6 \mathrm{~mm}$ & N/A & $+17 \%$ & $+58 \%$ \\
\hline $9 \mathrm{~mm}$ & $-17 \%$ & N/A & $+49 \%$ \\
\hline $12 \mathrm{~mm}$ & $-58 \%$ & $-49 \%$ & N/A \\
\hline
\end{tabular}

\subsubsection{5 $\quad$ Gap Size with Base 5\% Longitudinal Slope without}

\section{Aggregate}

This category includes the steepest slope and the following gap sizes: $6 \mathrm{~mm}$ vs. 9 mm vs. 12 mm. Experiments \#19, \#17, and \#15 are all conducted without the permeable joint material. The longitudinal slope of the flume during these three experiments is set at $5 \%$. The progression of clogging and progression of the infiltration edge are reviewed to provide a better understanding of the performance of the flume during these experiments. 


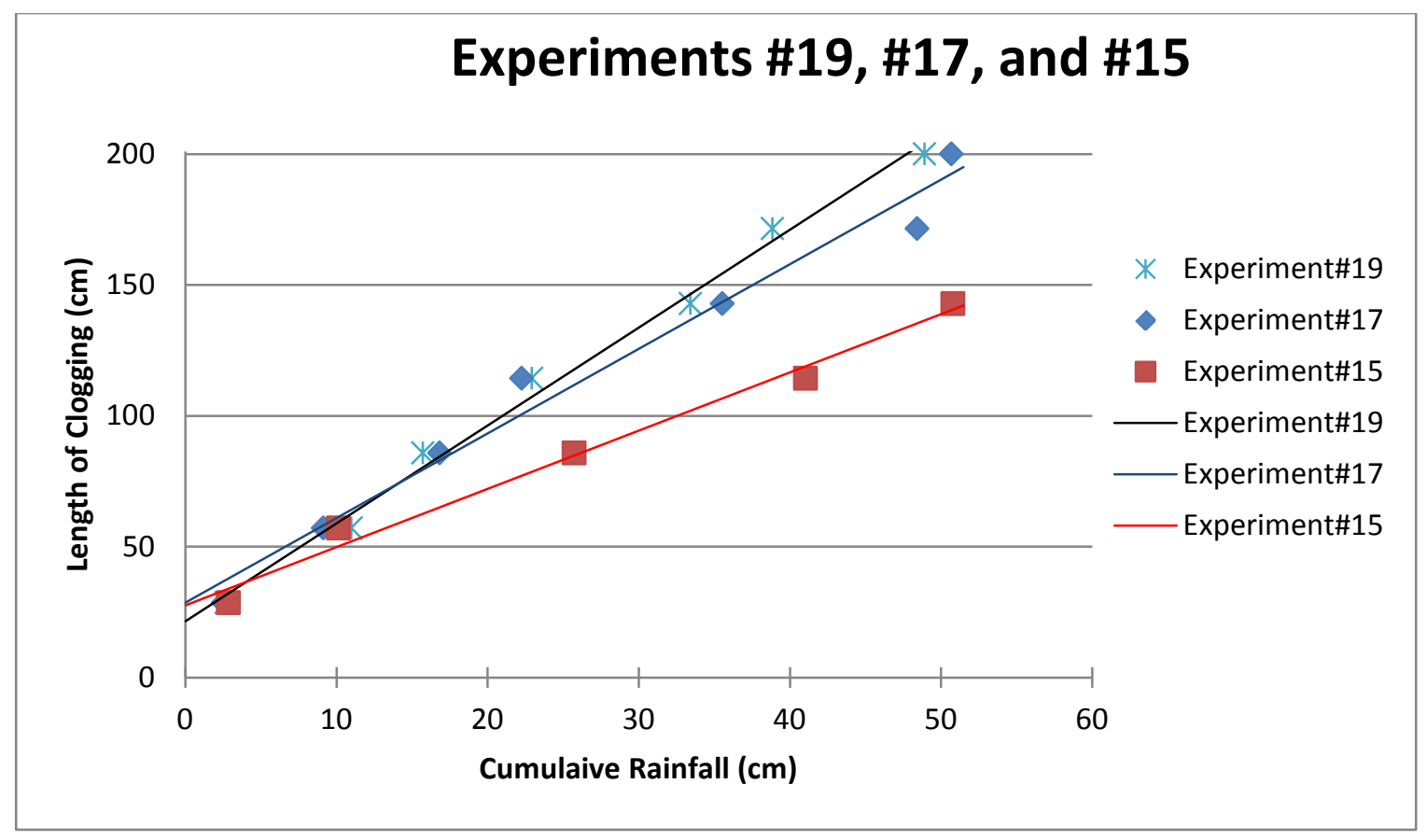

Figure 78: Comparison of progression of clogging in experiments \#19, \#17, and \#15

With an increase in the permeable paver gap size, as illustrated in Figure 78, the rate of progression of clogging experiences a steady decrease, where the $6 \mathrm{~mm}$ gap size has the highest slope and the $12 \mathrm{~mm}$ gap size has the lowest of the three. 


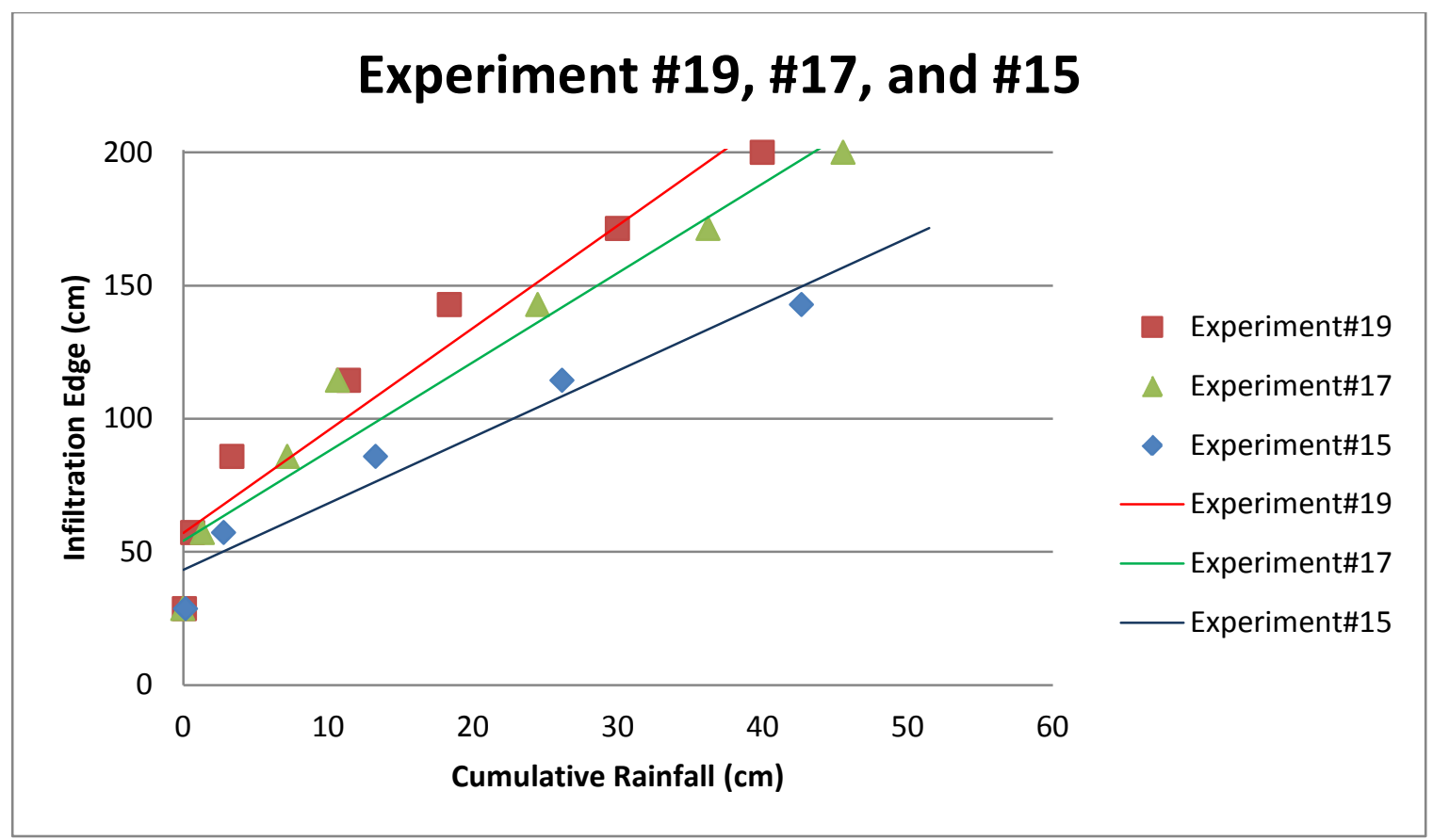

Figure 79: Comparison of progression of infiltration edge in experiments\#19, \#17, and \#15

Like the previous graph, Figure 79 illustrates the progression of the infiltration edge on the surface of the flume at a 5\% longitudinal slope. The increasing pattern in the gap size results in a decreasing pattern in the rate of progression of the wetting front in the flume. In other words, even in the steep setup of the flume, with an increase in the gap size, less surface area is required to infiltrate the same volume of runoff.

With regard to the progression of surface clogging, increasing the permeable paver gap size from $6 \mathrm{~mm}$ to $9 \mathrm{~mm}$ results in a 13\% improvement; this indicates slower progression of clogging. A further increase of the gap size, from $9 \mathrm{~mm}$ to $12 \mathrm{~mm}$, results in a 31\% additional improvement. Comparing the $6 \mathrm{~mm}$ gap size to the $12 \mathrm{~mm}$ gap size, results in a $40 \%$ improvement in the response of the flume to clogging due to polluted stormwater runoff 
Table 31: Comparing the Progression of Clogging in Experiments \#19, \#17, and \#15

\begin{tabular}{|l|l|l|l|}
\hline Gap Size & $6 \mathrm{~mm}$ & $9 \mathrm{~mm}$ & $12 \mathrm{~mm}$ \\
\hline $6 \mathrm{~mm}$ & N/A & $+13 \%$ & $+40 \%$ \\
\hline $9 \mathrm{~mm}$ & $-13 \%$ & N/A & $+31 \%$ \\
\hline $12 \mathrm{~mm}$ & $-40 \%$ & $-31 \%$ & N/A \\
\hline
\end{tabular}

Comparing the progression of the surface infiltration edge indicates that increasing the paver gap size from $6 \mathrm{~mm}$ to $9 \mathrm{~mm}$ results in a $12 \%$ improvement in the progression of the surface infiltration front, while further increasing the paver gap size from $9 \mathrm{~mm}$ to $12 \mathrm{~mm}$ will deliver a further 25\% improvement. Comparing the first gap size, $6 \mathrm{~mm}$, with the last gap size, $12 \mathrm{~mm}$, indicates a 35\% improvement in the rate at which surface clogging progresses in the flume.

Table 32: Comparing the Progression of Infiltration Edge in Experiments \#19, \#17, and \#1

\begin{tabular}{|l|l|l|l|}
\hline Gap Size & $6 \mathrm{~mm}$ & $9 \mathrm{~mm}$ & $12 \mathrm{~mm}$ \\
\hline $6 \mathrm{~mm}$ & $\mathrm{~N} / \mathrm{A}$ & $+12 \%$ & $+35 \%$ \\
\hline $9 \mathrm{~mm}$ & $-12 \%$ & N/A & $+25 \%$ \\
\hline $12 \mathrm{~mm}$ & $-35 \%$ & $-25 \%$ & N/A \\
\hline
\end{tabular}

\subsubsection{Gap Size with Base 5\% Longitudinal Slope with}

\section{Aggregate}

The last group of experiments compared to determine the effect of the paver gap size on the performance of the GI includes the following gap sizes: $6 \mathrm{~mm}$ vs. $9 \mathrm{~mm}$ vs. 12 mm, in experiments \#20, \#18, and \#16. These experiments were conducted with a 5\% longitudinal slope and permeable joint material. 


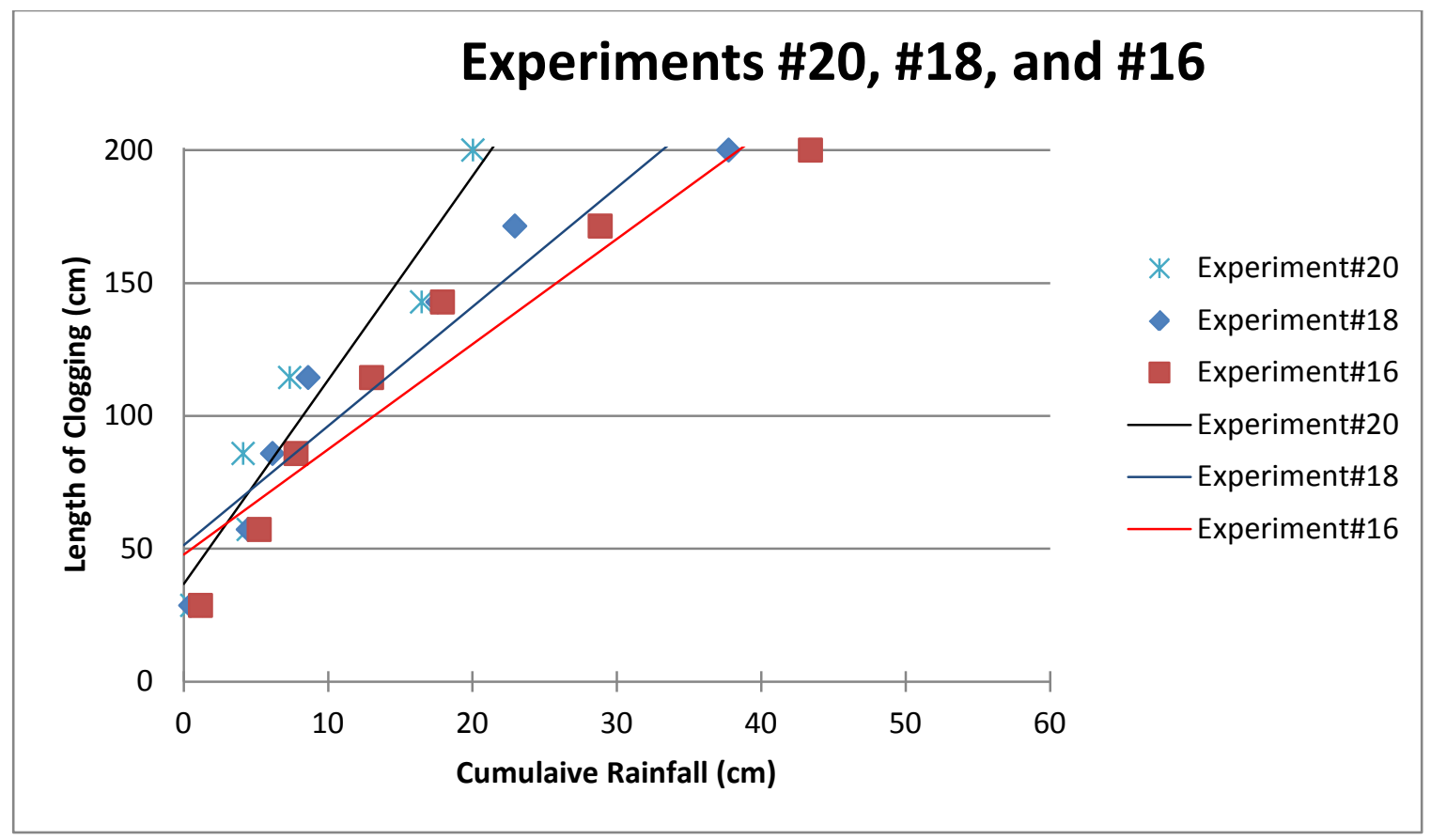

Figure 80: Comparison of progression of clogging in experiments \#20, \#18, and \#16

Figure 80 illustrates the progression of clogging in the experiments compared in this section. Increasing the paver gap size from the initial $6 \mathrm{~mm}$ to $9 \mathrm{~mm}$ and then to 12 $\mathrm{mm}$ results in a steady decrease in the rate at which surface clogging progresses in the flume. Although the pattern may be the same, the graph confirms that the steep slope has caused the clogging to progress and reach the down gradient of the flume much faster than in the similar experiments conducted at longitudinal slopes of $1 \%$ and $3 \%$. 


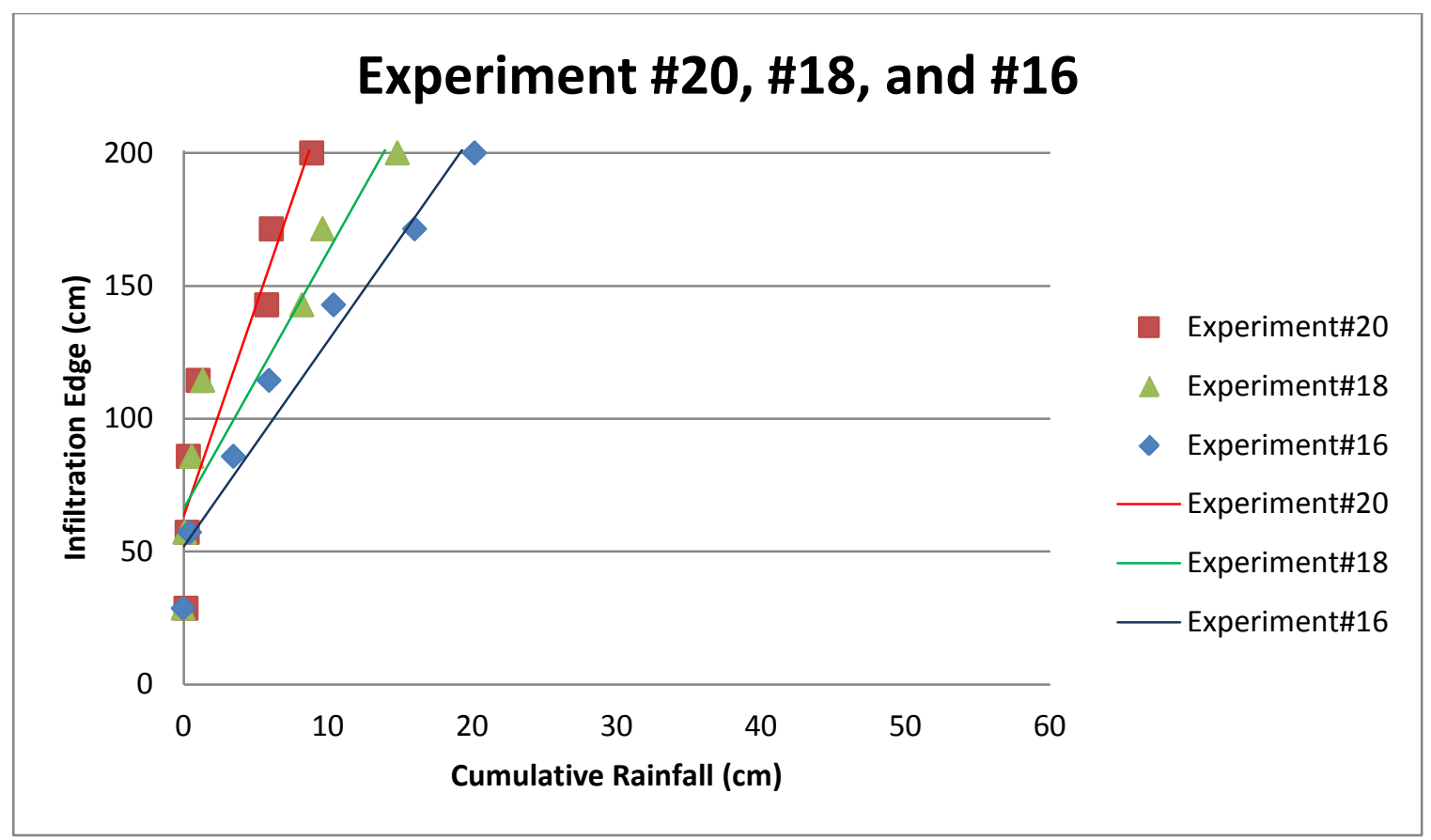

Figure 81: Comparison of progression of infiltration edge in experiments \#20, \#18, and \#16

As expected, the progression of the infiltration edge, which is plotted in Figure 81, illustrates that a certain gap width in the pavers, with only $10 \mathrm{~cm}$ to $20 \mathrm{~cm}$ of the designed rainfall for the area of the flume's watershed, the entire length of the flume contributes in the process of infiltration of the stormwater runoff. In addition, with each increase in the width of the paver gap, the rate at which the wetting front progresses towards the down gradient of the flume decreases.

Comparing the slopes of the trend lines in Figure 80 shows that the increase of paver gap width from $6 \mathrm{~mm}$ to $9 \mathrm{~mm}$ results in a 41\% improvement in the progress of clogging, while a further increase of the gap width, from $9 \mathrm{~mm}$ to $12 \mathrm{~mm}$, results in an additional 11\% improvement of conditions. Increasing the gap width from $6 \mathrm{~mm}$ to 12 mm creates a $48 \%$ improvement in the progression of clogging on the permeable surface. 
Table 33: Comparing the Progression of Clogging in Experiments \#20, \#18, and \#16

\begin{tabular}{|l|l|l|l|}
\hline Gap Size & $6 \mathrm{~mm}$ & $9 \mathrm{~mm}$ & $12 \mathrm{~mm}$ \\
\hline $6 \mathrm{~mm}$ & N/A & $+41 \%$ & $+45 \%$ \\
\hline $9 \mathrm{~mm}$ & $-41 \%$ & N/A & $+11 \%$ \\
\hline $12 \mathrm{~mm}$ & $-45 \%$ & $-11 \%$ & N/A \\
\hline
\end{tabular}

The progress of the surface infiltration edge experiences a 38\% improvement when the gap size increases from $6 \mathrm{~mm}$ to $9 \mathrm{~mm}$. A further increase in the gap size, form $9 \mathrm{~mm}$ to $12 \mathrm{~mm}$, results in another $20 \%$ improvement in the progress of the wetting front. A direct comparison between the $6 \mathrm{~mm}$ gap size and the $12 \mathrm{~mm}$ gap size indicates a 51\% improvement.

Table 34: Comparing the Progression of Infiltration Edge in Experiments \#20, \#18, and \#16

\begin{tabular}{|l|l|l|l|}
\hline Gap Size & $6 \mathrm{~mm}$ & $9 \mathrm{~mm}$ & $12 \mathrm{~mm}$ \\
\hline $6 \mathrm{~mm}$ & N/A & $+38 \%$ & $+51 \%$ \\
\hline $9 \mathrm{~mm}$ & $-35 \%$ & N/A & $+20 \%$ \\
\hline $12 \mathrm{~mm}$ & $-51 \%$ & $-20 \%$ & N/A \\
\hline
\end{tabular}

\subsubsection{Permeable Joint Material}

The aim of this section is to follow a similar path to that of the previous section and use the results extracted from the graphs that are plotted for both the progression of clogging and the progression of the infiltration edge to determine the effect of the permeable joint material on the behavior of the permeable surface. In order to do so, the correlation between experiments conducted with and without the permeable joint material must be determined. This can be done by directly comparing the experiments conducted with the same variables, with and without permeable joint material. Below is a list of comparable experiments for this section: 
- \#8 aggregate vs. no \#8 aggregate:

o 6 mm gap size \& $1 \%$ slope: Experiments 2,4

o 9 mm gap size \& 1\% slope: Experiments 5,6

o $12 \mathrm{~mm}$ gap size \& $1 \%$ slope: Experiments 7,8

o 6 mm gap size \& 3\% slope: Experiments 13,14

o 9 mm gap size \& 3\% slope: Experiments 11,12

o 12 mm gap size \& 3\% slope: Experiments 9,10

o 6 mm gap size \& 5\% slope: Experiments 19,20

o 9 mm gap size \& 5\% slope: Experiments 17,18

o 12 mm gap size \& 5\% slope: Experiments 15,16

\subsubsection{Permeable Joint Material for $6 \mathrm{~mm}$ Gap at 1\% Slope}

The experiments compared for this section are experiments \#2 and \#4, which are conducted respectively without and with permeable joint aggregate. The pavers with the smallest gap size were used in these two experiments. Note that experiment \#4 experienced an interruption during the process. Comparing an experiment lasting 100 minutes without interruption with another which was interrupted can be useful in getting closer to real life conditions where the GI may experience wet and dry weather in no specific sequence for no specified period. 


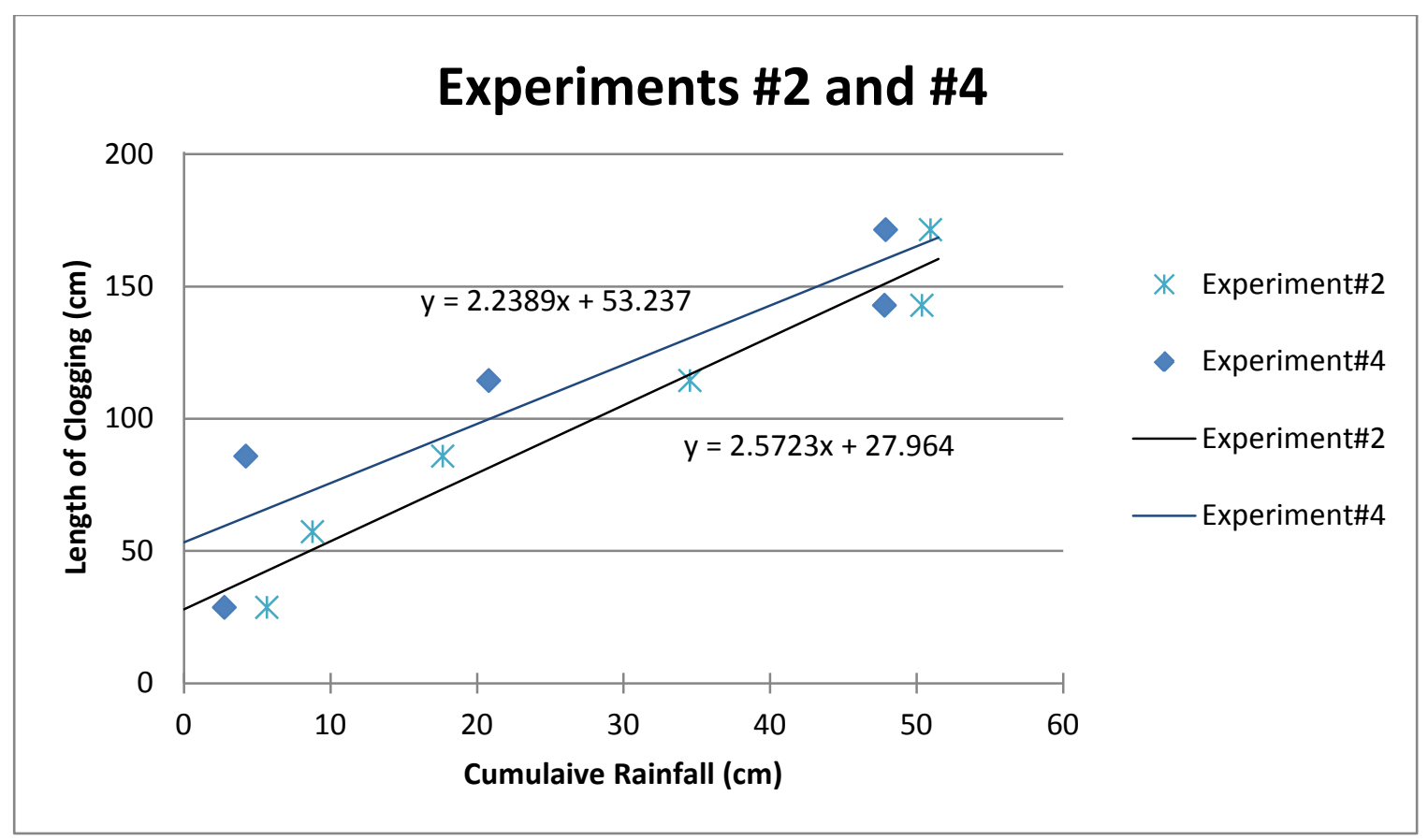

Figure 82: Comparison of progression of clogging in experiments \#2 and \#4

Figure 82 illustrates the progression of clogging between these experiments, and shows that the experiment conducted with the permeable joint material had a slower rate in the progression of clogging on the surface of the flume, while the lack of permeable joint material helped the rate to increase. Comparing the slopes of the lines shown in Figure 82 reveals that the presence of permeable joint material resulted in a $12 \%$ improvement in the rate at which surface clogging progresses. This comparison and the next ones should in turn be compared to determine the effect of the interruption on the experiment. 


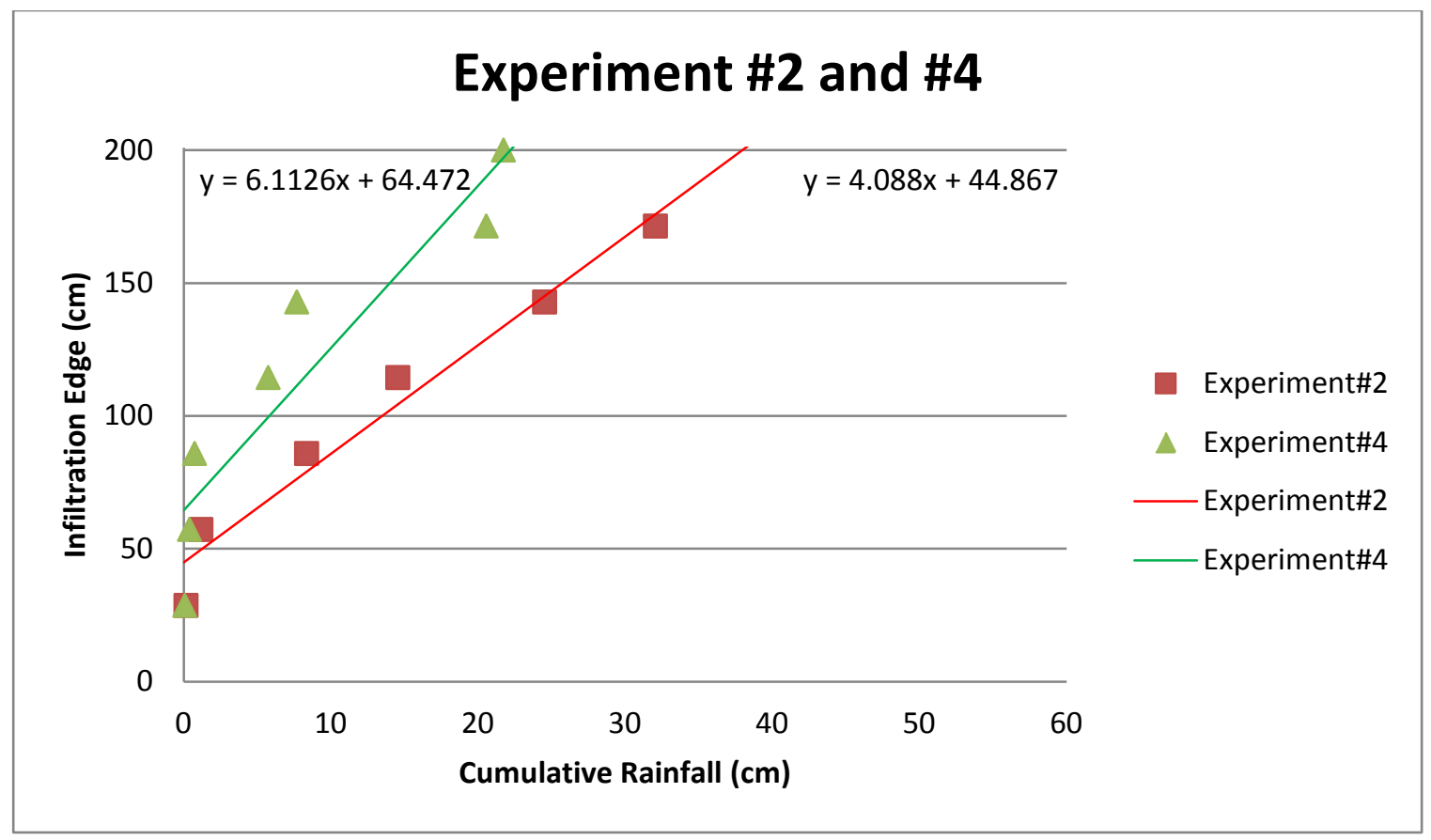

Figure 83: Comparison of progression of infiltration edge in experiments \#2 and \#4

By comparing the plots of experiments \#2 and \#4 for the progression of the infiltration edge, as illustrated in Figure 83, we see the effect of permeable joint material in the speed in the initial performance of the permeable surface. Since the \#8 aggregate fills the gaps, more of the permeable surface could be used to infiltrate the same volume of runoff. Using simple numerical analysis, it is clear that the presence of \#8 AASHTO aggregate as the permeable joint material resulted in a $49 \%$ difference in the rate at which the surface infiltration edge progressed.

\subsubsection{2. $\quad$ Permeable Joint Material for $9 \mathrm{~mm}$ Gap at $1 \%$ Slope}

The experiments compared for this section are experiments \#5 (without permeable joint material) and \#6 (with permeable joint material). Pavers with a $9 \mathrm{~mm}$ gap were used in these two experiments. As in the previous section, the progression of clogging and progression of infiltration edge are used as the points of comparison. 


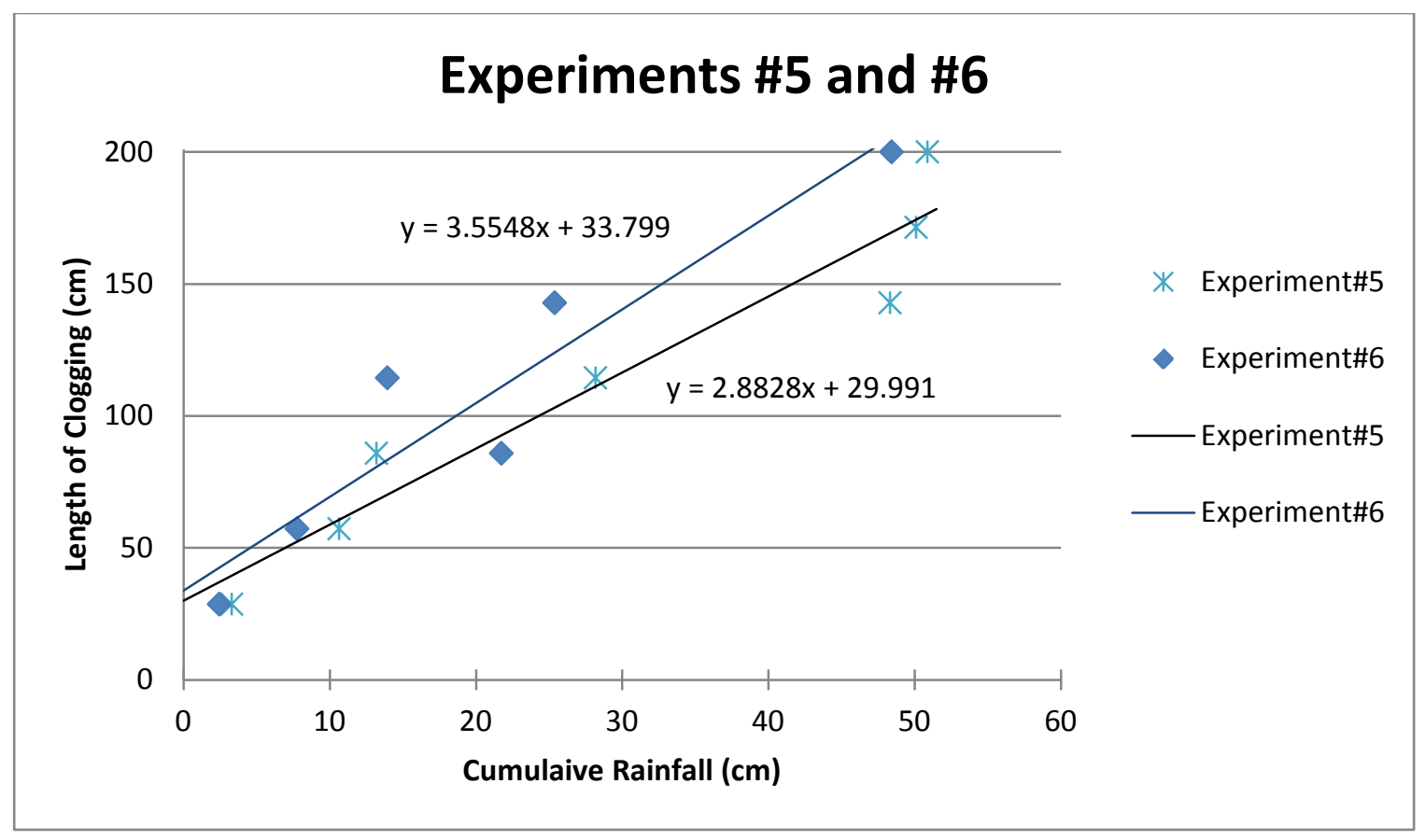

Figure 84: Comparison of progression of clogging in experiments \#5 and \#6

Comparing the progression of clogging in experiments \#5 and \#6, as illustrated in Figure 84, shows that, unlike the comparison of experiments \#2 and \#4, in the experiment where permeable joint material was present, the clogging progressed faster. Numerical analysis of the slopes of the two trend lines shows a $23 \%$ difference in the performance of the two experiments. 


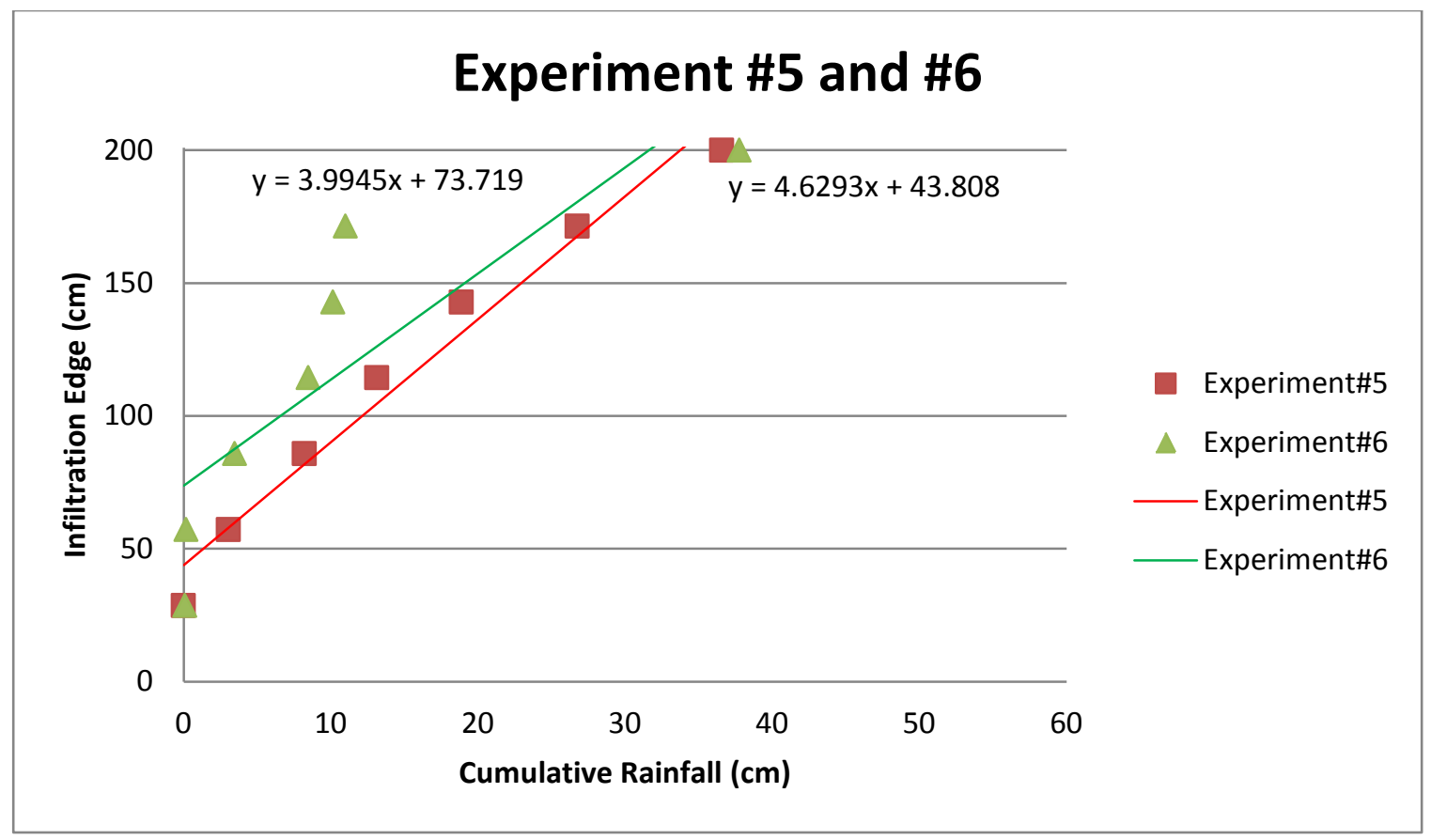

Figure 85: Comparison of progression of infiltration edge in experiments \#5 and \#6

Comparing the progression of the infiltration edge in these two experiments shows a $13 \%$ improvement for the experiment where permeable joint material is present. The experiment conducted with permeable joint material seemed to improve the progression of clogging by slowing it down.

\subsubsection{Permeable Joint Material for $12 \mathrm{~mm}$ Gap at $1 \%$ Slope}

The comparisons made to determine the effect of permeable joint material on the $12 \mathrm{~mm}$ gap size and at 1\% longitudinal slope are of experiments \#7 and \#8. The pavers used for these two experiments have the biggest gap size of the three. 


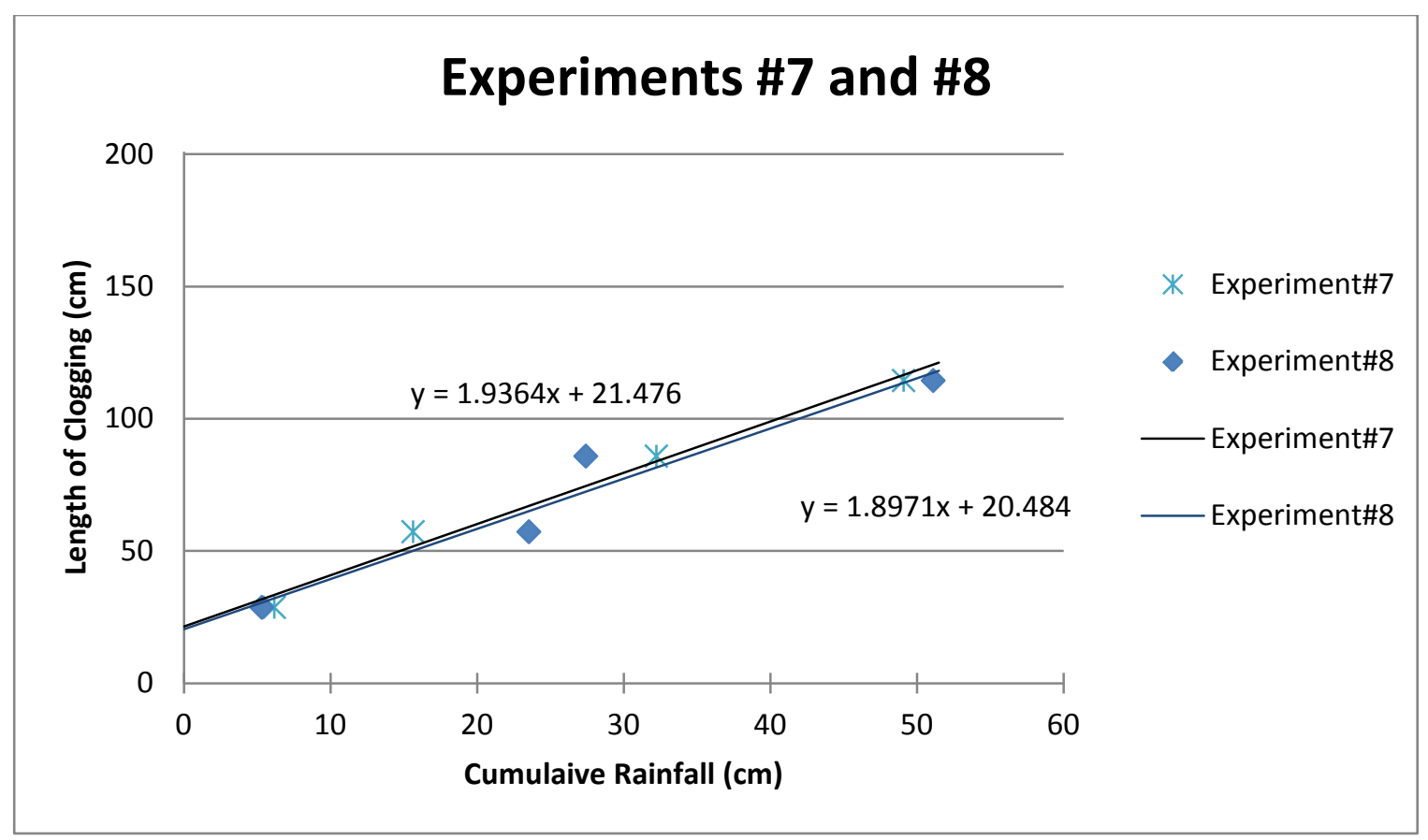

Figure 86: Comparison of progression of clogging in experiments \#7 and \#8

Figure 86 illustrates the progression of clogging for the experiments conducted using paver blocks with the biggest gap size. At a 1\% longitudinal slope, the presence of permeable joint material in the Eco-Pavers resulted in only a $2 \%$ improvement. In other words, the comparison without and with \#8 aggregate showed no significant difference between the two. 


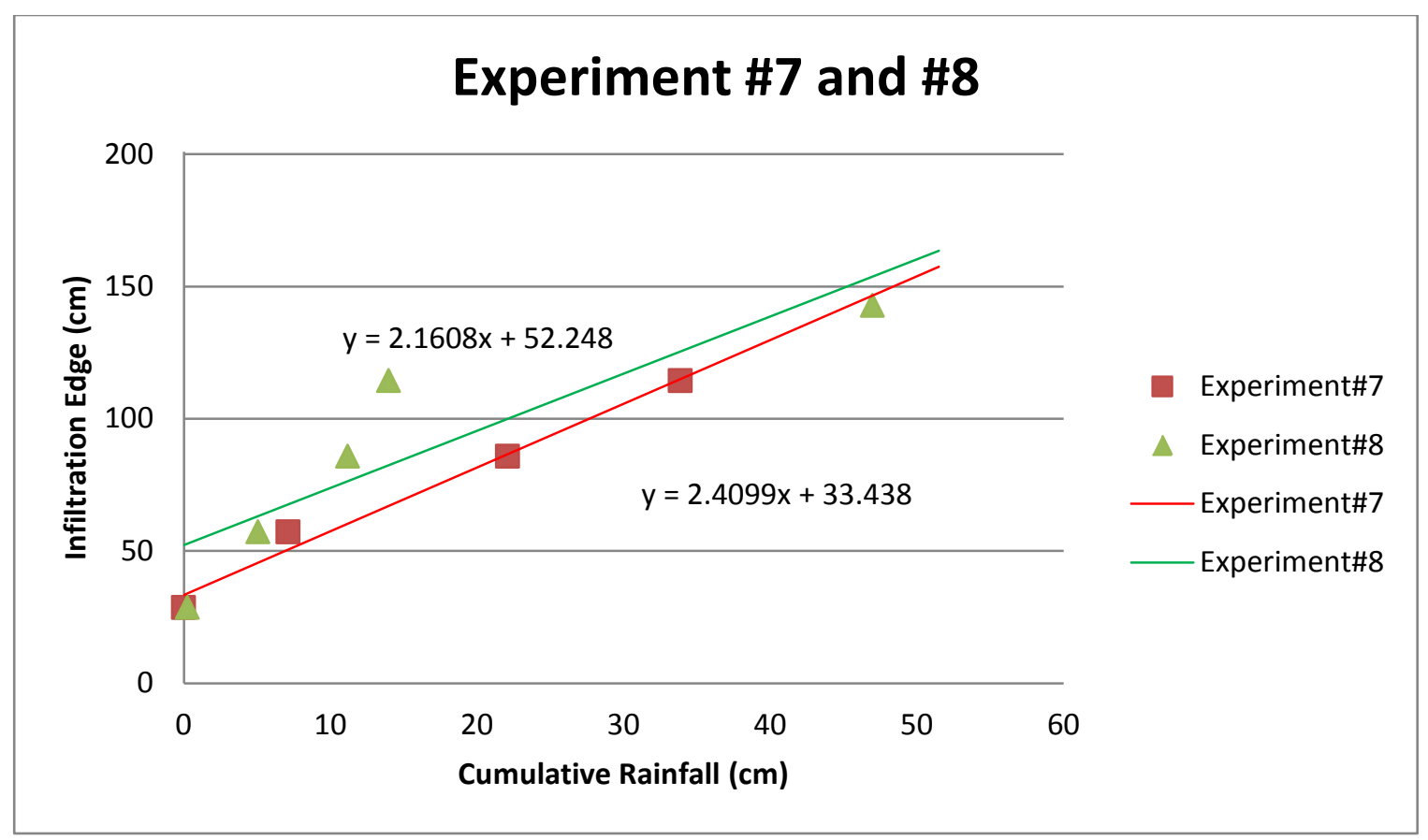

Figure 87: Comparison of progression of infiltration edge in experiments \#7 and \#8

The comparison of the progression of infiltration edge between the experiments conducted without and with the permeable joint material, which is illustrated in Figure 87, shows that the experiment with aggregate in between the pavers could use the same surface area to infiltrate more stormwater runoff during the initial steps of the experiment. This difference is calculated to be about $10 \%$.

\subsubsection{Permeable Joint Material for $6 \mathrm{~mm}$ Gap at 3\% Slope}

The experiments conducted on a 3\% longitudinal slope and with the smallest gap size of the three, experiments \#13 and \#14, were compared by plotting the progression of clogging and progression of infiltration edge for the two experiments. 


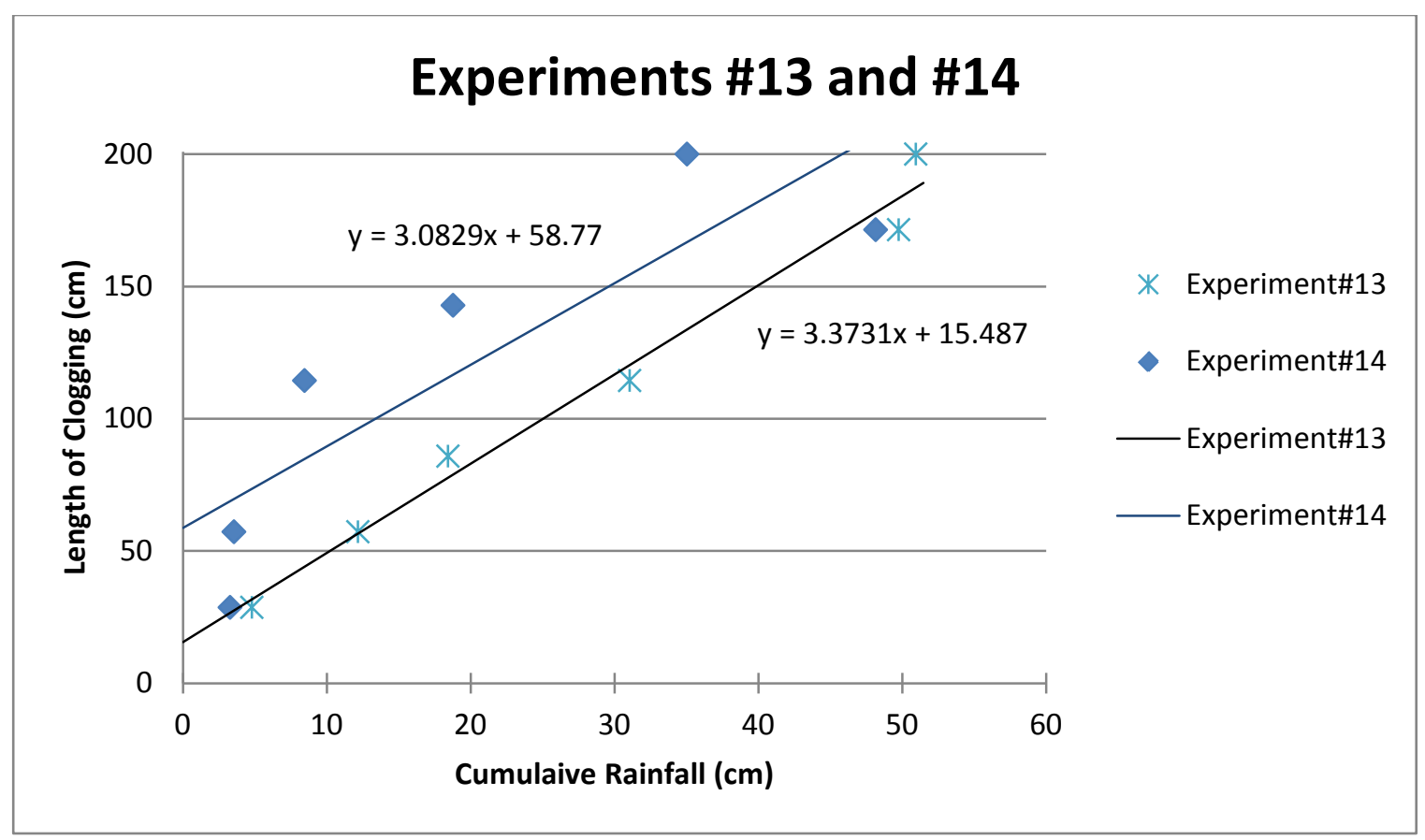

Figure 88: Comparison of progression of clogging in experiments \#13 and \#14

This comparison of the progression of clogging for the experiments plotted in Figure 88, which used pavers with $6 \mathrm{~mm}$ gaps, shows an $8 \%$ improvement over the other in the progression of surface clogging in the experiment with the permeable joint material. 


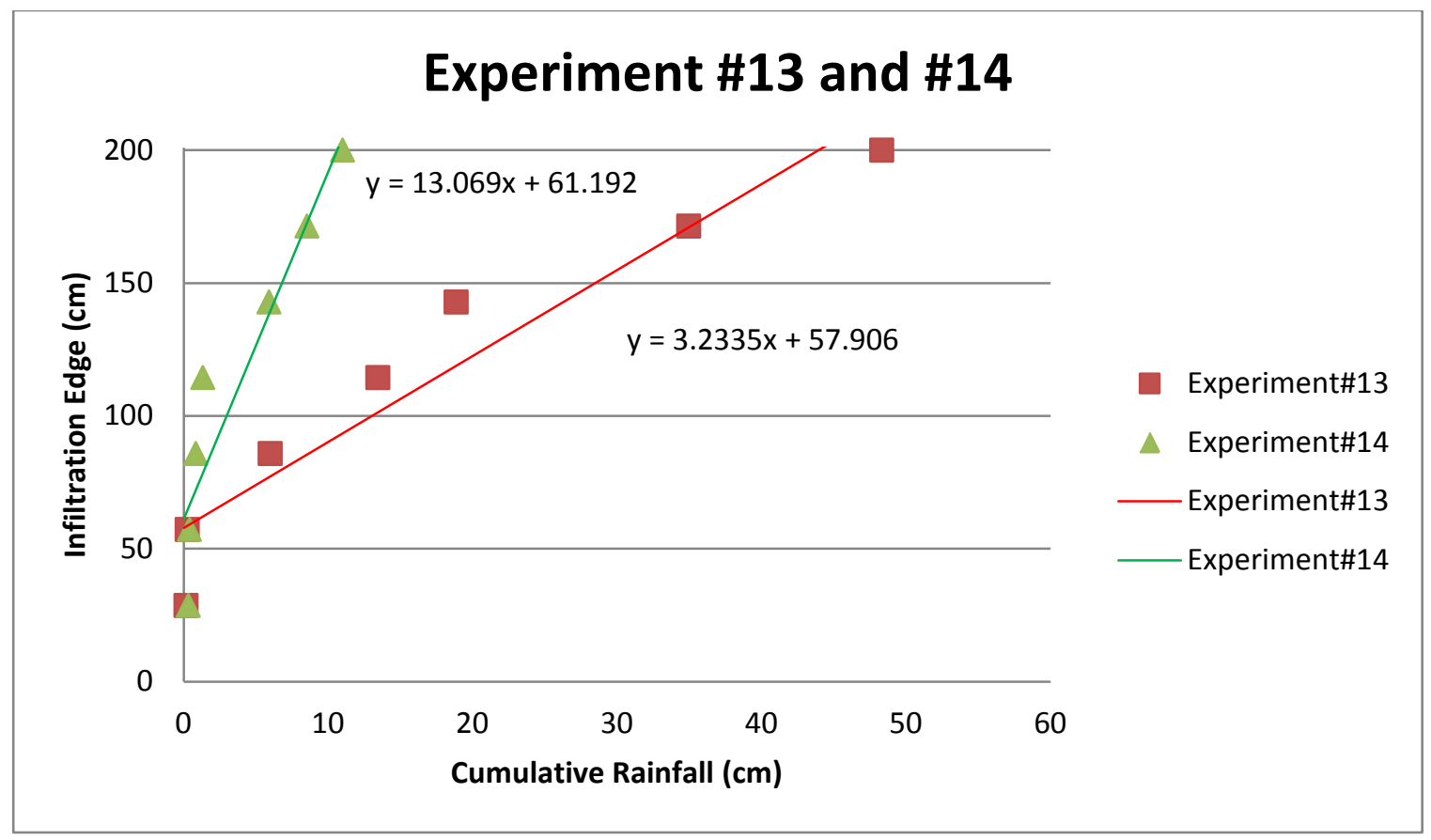

Figure 89: Comparison of progression of infiltration edge in experiments \#13 and \#14

Comparing the progression of the wetting front on the permeable surface of the flume, as illustrated in Figure 89, clearly manifests the difference in the effect of the permeable joint material on the $6 \mathrm{~mm}$ gap size at a 3\% slope. Experiment \#14 was the first test that ended in runoff ponding at the down gradient segment of the flume, and it can be seen that the entire length of the flume was being used to infiltrate runoff before receiving $10 \mathrm{~cm}$ rainfall. The rate at which surface infiltration edge progressed was 300\% slower for the experiment conducted without the permeable joint material.

\subsubsection{Permeable Joint Material for $9 \mathrm{~mm}$ Gap at $3 \%$ Slope}

Experiments \#13 and \#14 are compared in this section to determine the effect of permeable joint material in the performance of pavers with $9 \mathrm{~mm}$ gap and on a 3\% longitudinal slope. 


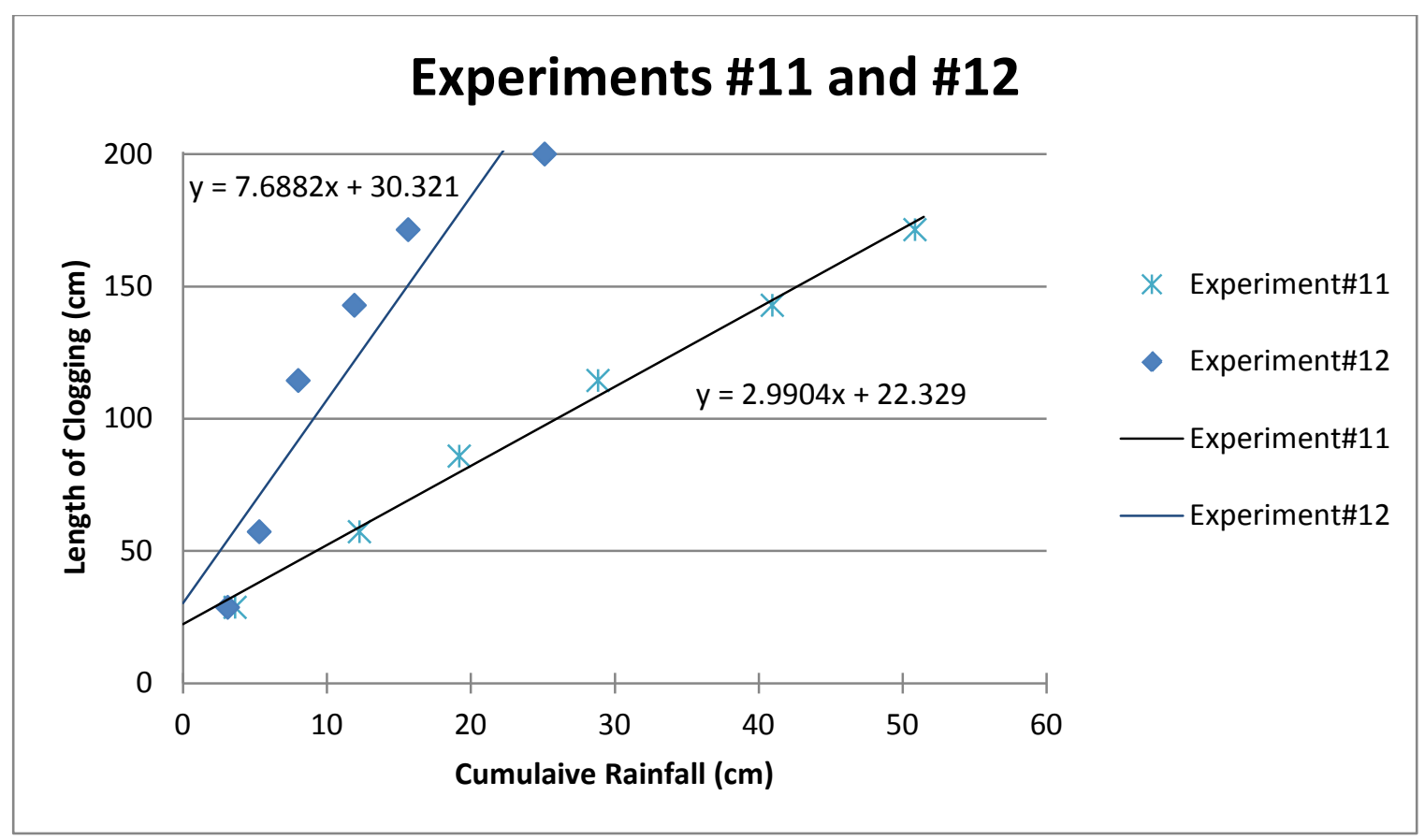

Figure 90: Comparison of progression of clogging in experiments \#11 and \#12

The progression of clogging for the experiments showed a significant difference in performance by the experiments illustrated in Figure 90. By comparing the slopes of trend lines plotted with the graphs of progression of clogging, it can be seen that the presence of the \#8 aggregate resulted in a 156\% faster rate of clogging. The steep slopes in this scenario caused the effect of the permeable joint material to be more significant. 


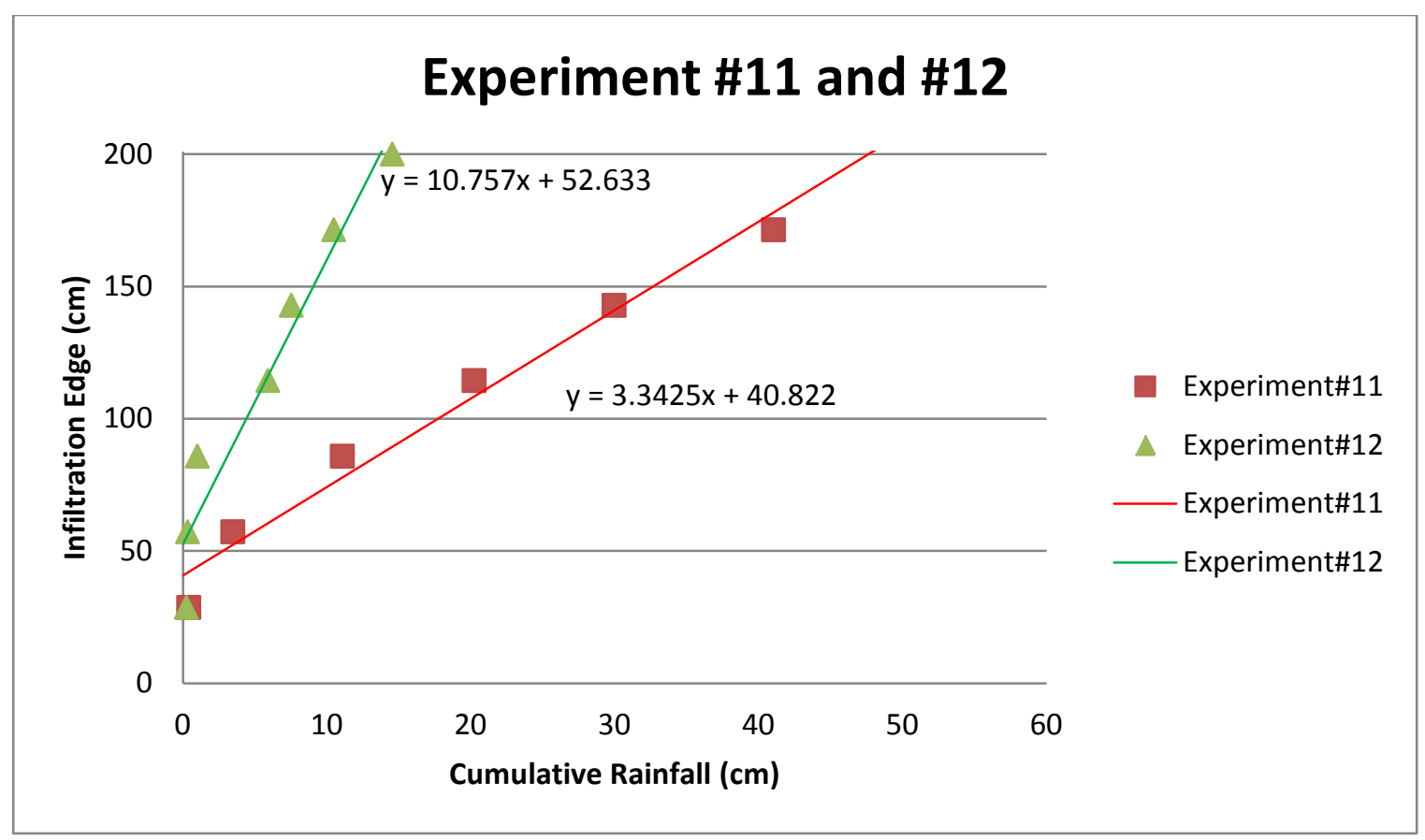

Figure 91: Comparison of progression of infiltration edge in experiments \#11 and \#12

The progression of the infiltration edge follows similar patterns to those of the progression of clogging. As seen in Figure 91, the wetting front in experiment \#12 where the permeable joint material was present progressed at a rapid rate and, when compared to experiment \#1, where progress occurred 221\% more slowly, shows that the steep installation slope could hardly be compensated by increasing the gap size.

\subsubsection{Permeable Joint Material for $12 \mathrm{~mm}$ Gap at 3\% Slope}

Experiments \#9 and \#10 were conducted using paver blocks that provided a 12 mm gap and on a 3\% longitudinal slope. 


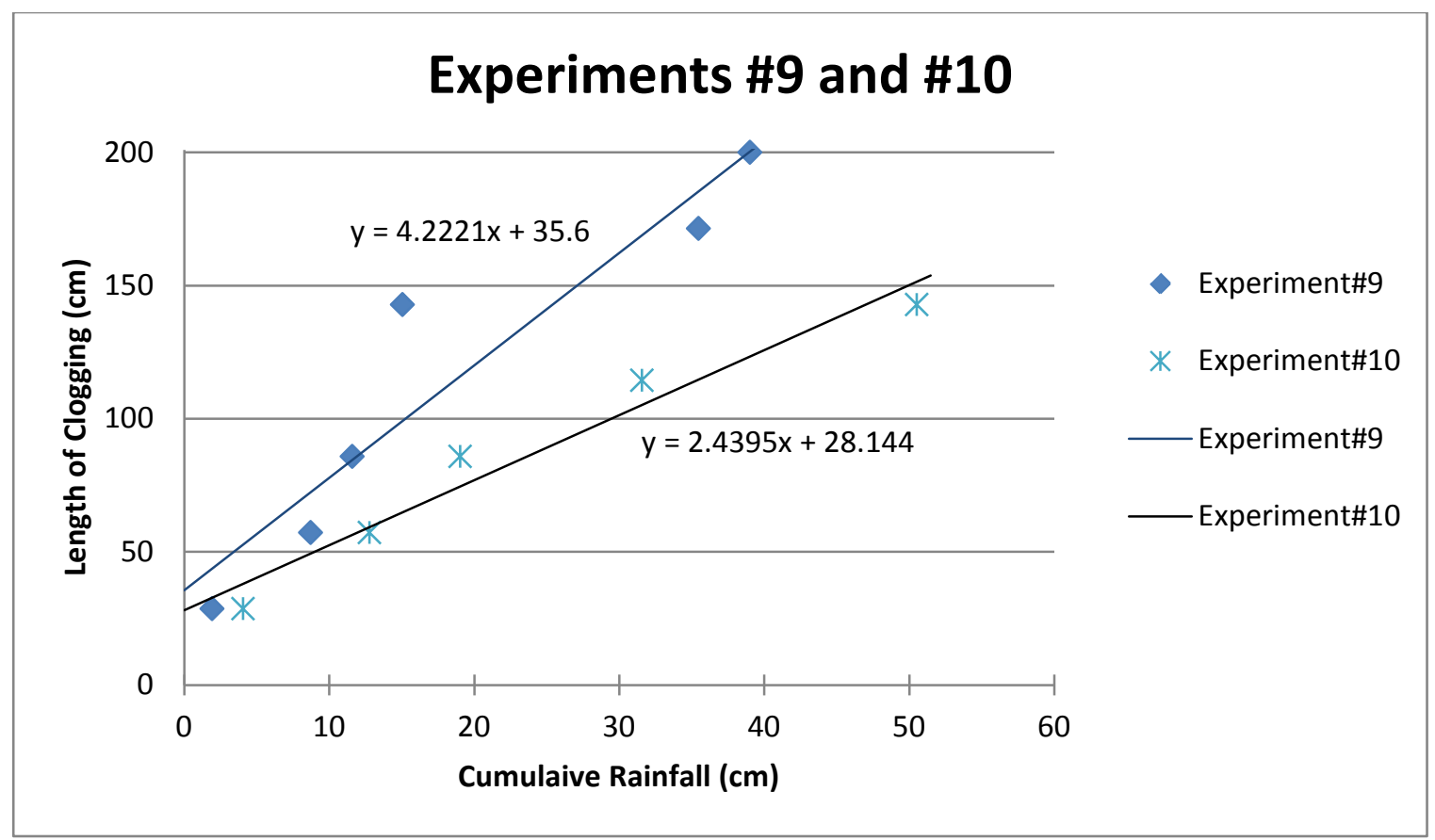

Figure 92: Comparison of progression of clogging in experiments \#9 and \#10

Figure 92 illustrates the progression of clogging of flume in tests that used the biggest paver gap size and a $3 \%$ longitudinal slope, experiments \#9 and \#10. As in the previous graphs for the progression of clogging, the experiment that was conducted with permeable joint material experienced a rapid progression of clogging, while the experiment conducted without the permeable joint material indicated a $72 \%$ improvement in the progression of surface clogging. 


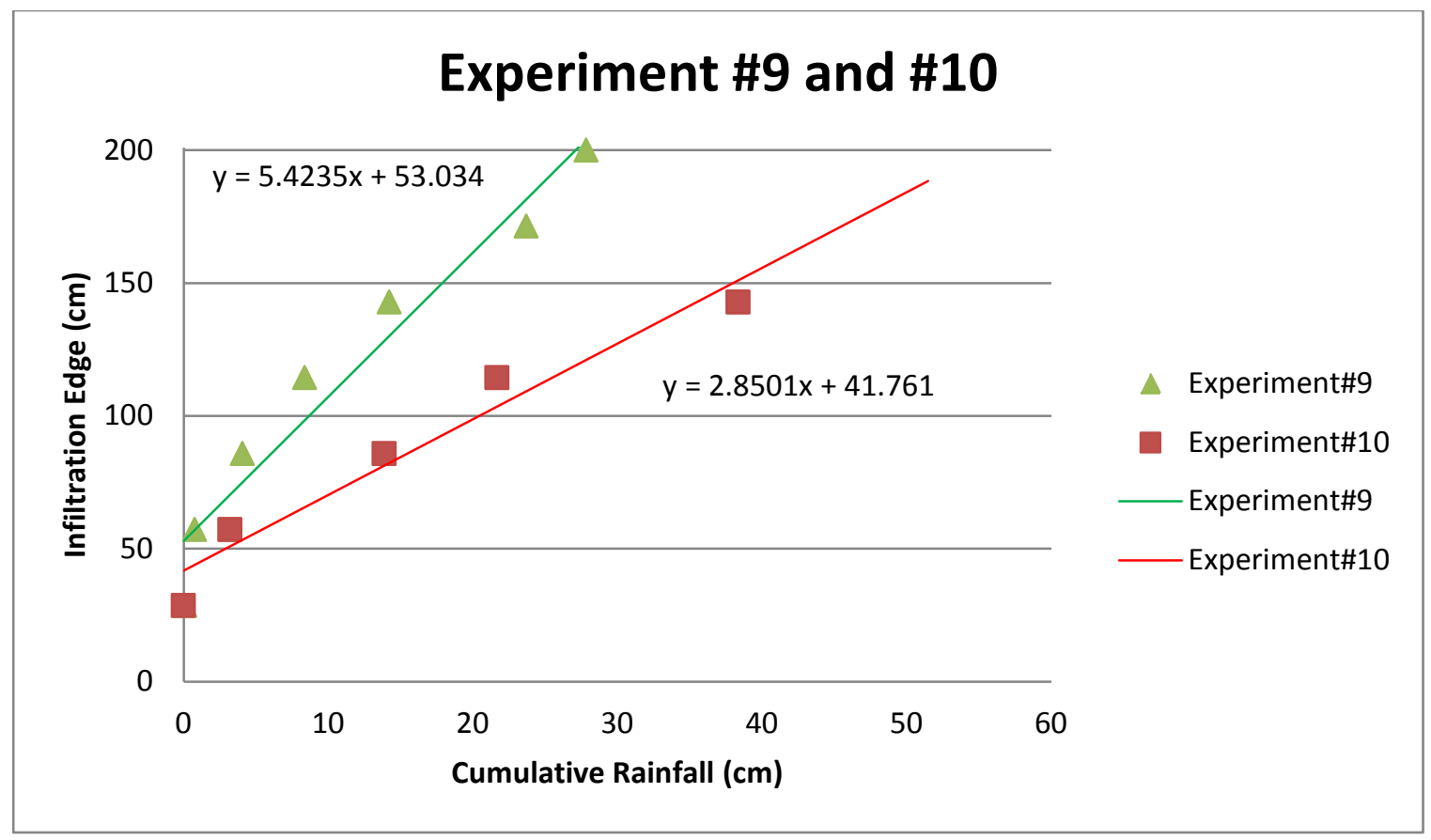

Figure 93: Comparison of progression of infiltration edge in experiments \#9 and \#10

Figure 93 is an illustration of the progression of the infiltration edge on the permeable surface of the flume. Comparing the two sets of data presented in the graph indicates a 90\% improvement in the rate at which the surface infiltration edge progresses, by removing the permeable joint material. Since these experiments were conducted with the pavers that have the biggest gap, the difference of the experiments conducted with and without the \#8 aggregate is not as significant as in similar experiments in which pavers with smaller gaps were used.

\subsubsection{7. $\quad$ Permeable Joint Material for $6 \mathrm{~mm}$ Gap at 5\% Slope}

In the steepest setup of the flume, experiments \#19 and \#20 were conducted using the pavers with a $6 \mathrm{~mm}$ gap. The progression of clogging and progression of the infiltration edge in these two experiments was plotted and compared to determine the 
effect of permeable joint material on the performance of the permeable surface in the flume.

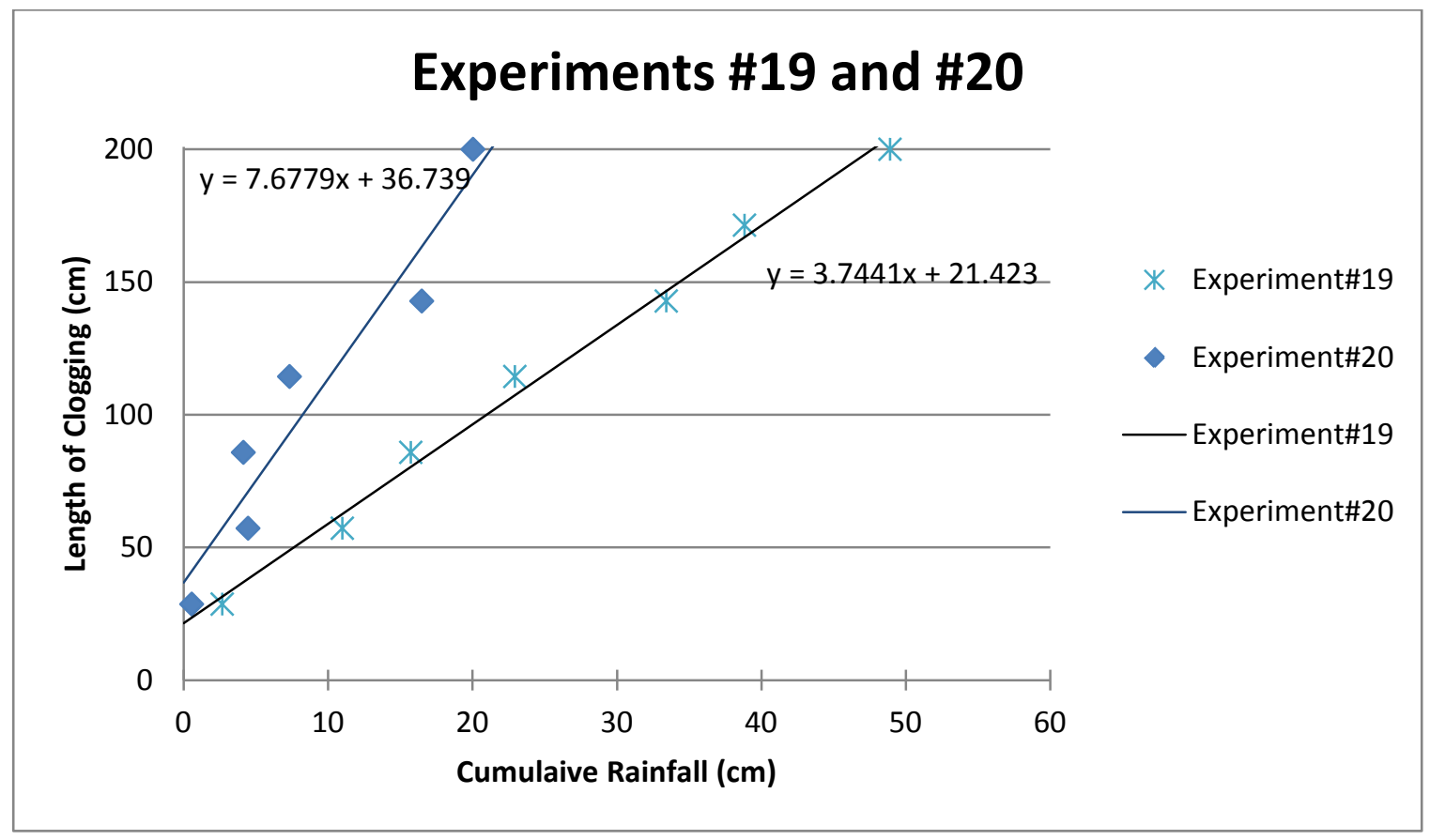

Figure 94: Comparison of progression of clogging in experiments \#19 and \#20

Using the steepest setup for the flume, and pavers with a $6 \mathrm{~mm}$ gap, the progression of clogging in the experiment that introduced the permeable joint material reached the down gradient of the flume very rapidly. Observations made during these experiments showed that in experiment \#20, conducted with permeable joint material, not only was the entire length of the flume clogged, towards the end of the test but half of the entire flume also suffered from runoff ponding. Still, experiment \#19 did not suffer from ponding and clogging did not reach the furthest point in the down gradient section of the flume. As a result of the steep slope and narrow gap size filled with \#8 aggregate, the clogging progressed 105\% faster in experiment \#20 than in experiment \#19 (Figure 94). 


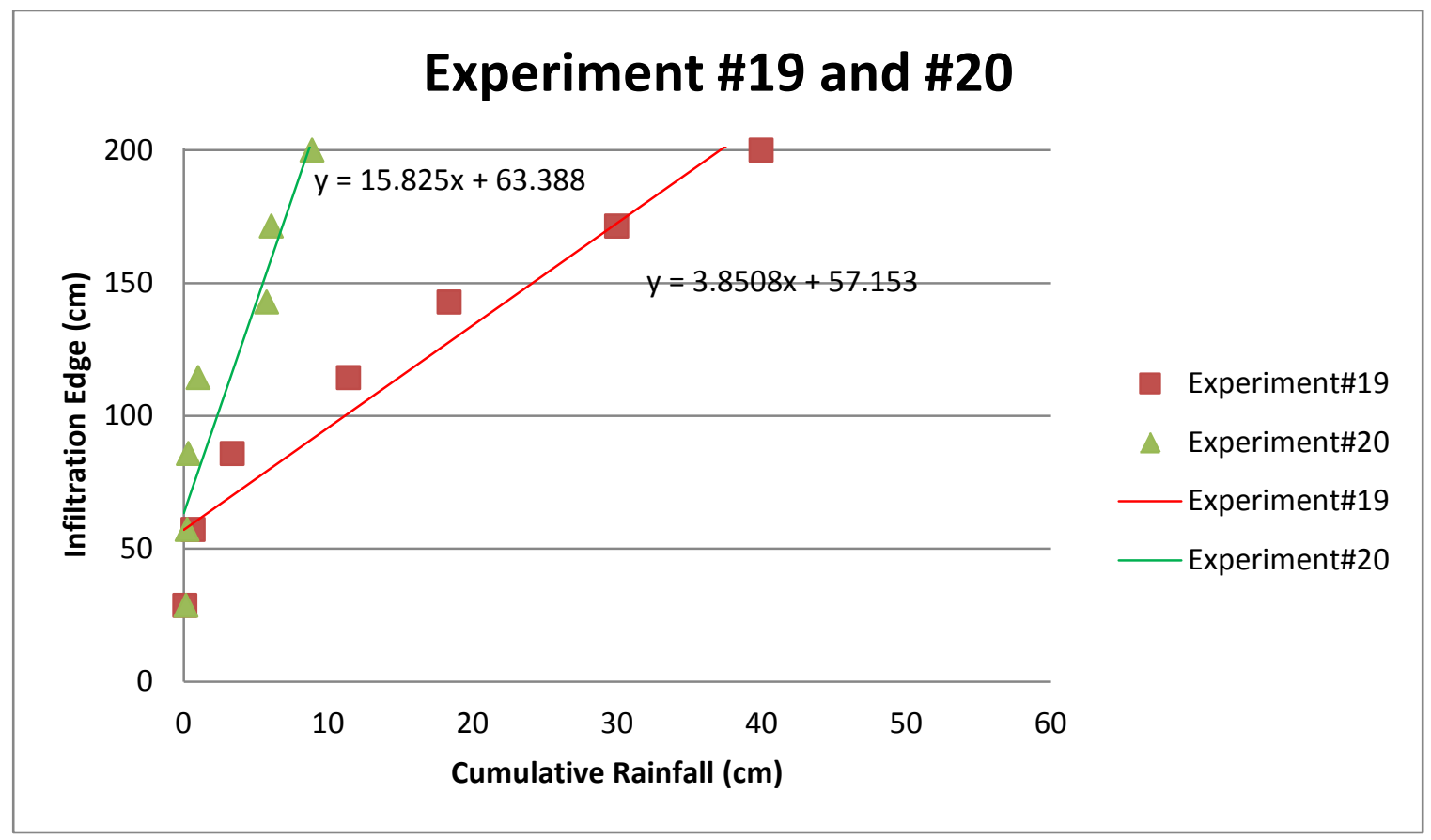

Figure 95: Comparison of progression of infiltration edge in experiments \#19 and \#20

The progression of the surface infiltration edge, which is shown in Figure 95, indicates that the absence of permeable joint material improved the progression of the wetting front by $310 \%$. The steep slope caused a significant difference between the experiments with and without permeable joint material.

\subsubsection{Permeable Joint Material for $9 \mathrm{~mm}$ Gap at 5\% Slope}

Flume experiments \#17 and \#18 were conducted using pavers with 9 mm gap size and on a 5\% longitudinal slope. The relevant graphs (below) are plotted to show the results of the analysis of the progression of clogging and progression of the infiltration edge to provide a clearer tool for assessing the effect of the \#8 ASSHTO aggregate in the paver gaps. 


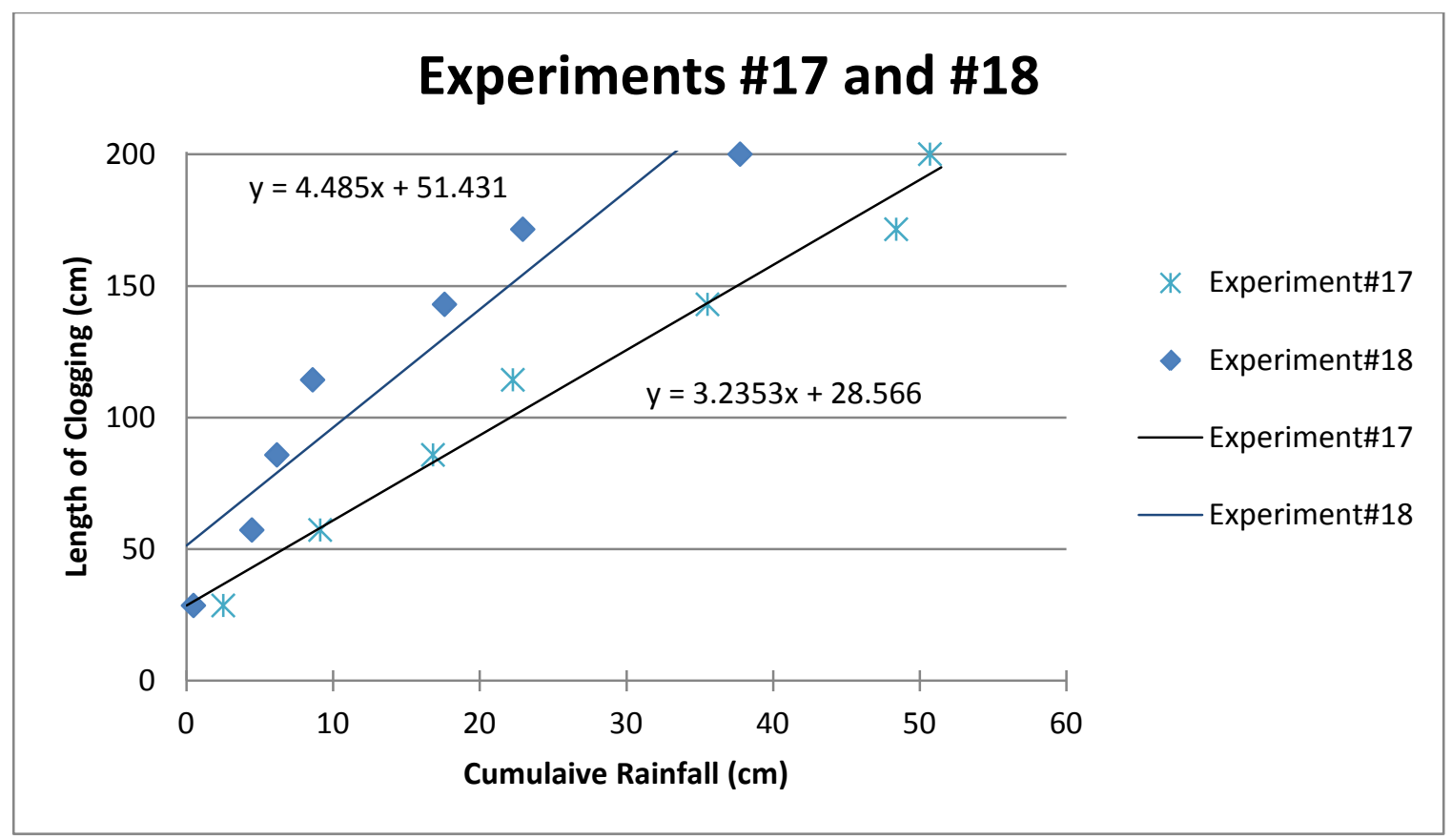

Figure 96: Comparison of progression of clogging in experiments \#17 and \#18

Comparing the progression of clogging for experiments \#17 and \#18, which is plotted in Figure 96, suggests that the presence of the permeable clogging material resulted in the same patterns as seen before, creating a rapid progression of surface clogging. Removing the \#8 aggregate from the paver gaps resulted in a 38\% improvement in the rate at which the clogging on the surface progressed. Figure 96 posits a $9 \mathrm{~mm}$ paver gap size and compares it to illustrate the fact that similar experiments with a $6 \mathrm{~mm}$ paver gap size confirm that the increased gap size offset the radical effects of the progress of surface clogging. 


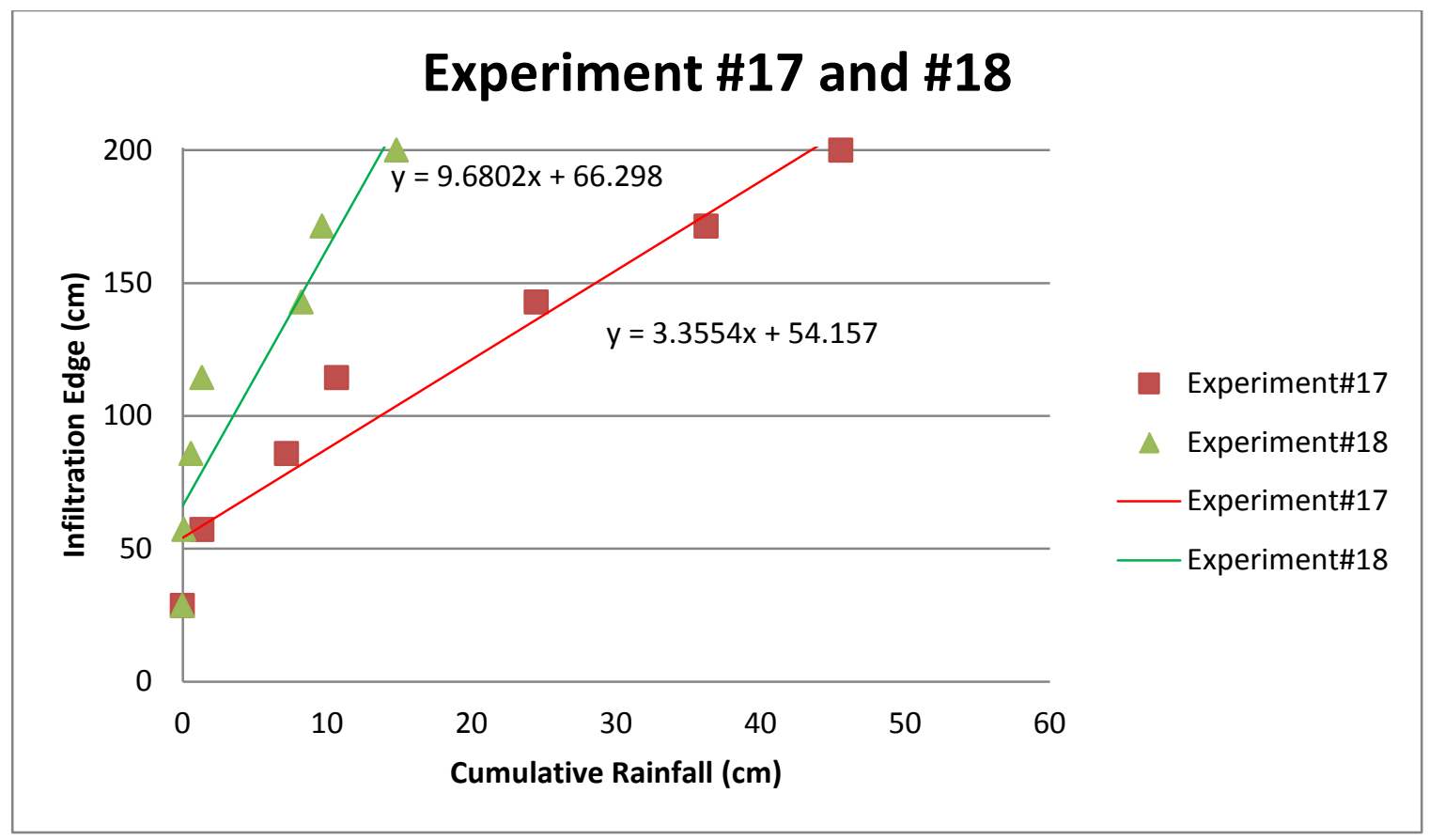

Figure 97: Comparison of progression of infiltration edge in experiments \#17 and \#18

The progression of the infiltration edge on the permeable surface matched the results of the previous experiments. Figure 97 shows that the presence of the permeable joint material increased by $188 \%$ the rate at which the wetting front progressed on the surface. The surface infiltration edge in experiment \#18, which was conducted with \#8 aggregate filling the permeable paver gaps, covered the entire length of the flume after about $10 \mathrm{~cm}$ worth of accumulated rain.

\subsubsection{Permeable Joint Material for $12 \mathrm{~mm}$ Gap at 5\% Slope}

The last experiments compared to determine the effect of permeable joint material on the performance of the permeable surface in the flume were experiments \#15 and \#16, which were conducted using pavers with a $12 \mathrm{~mm}$ gap and on a setup with a $5 \%$ longitudinal slope. 


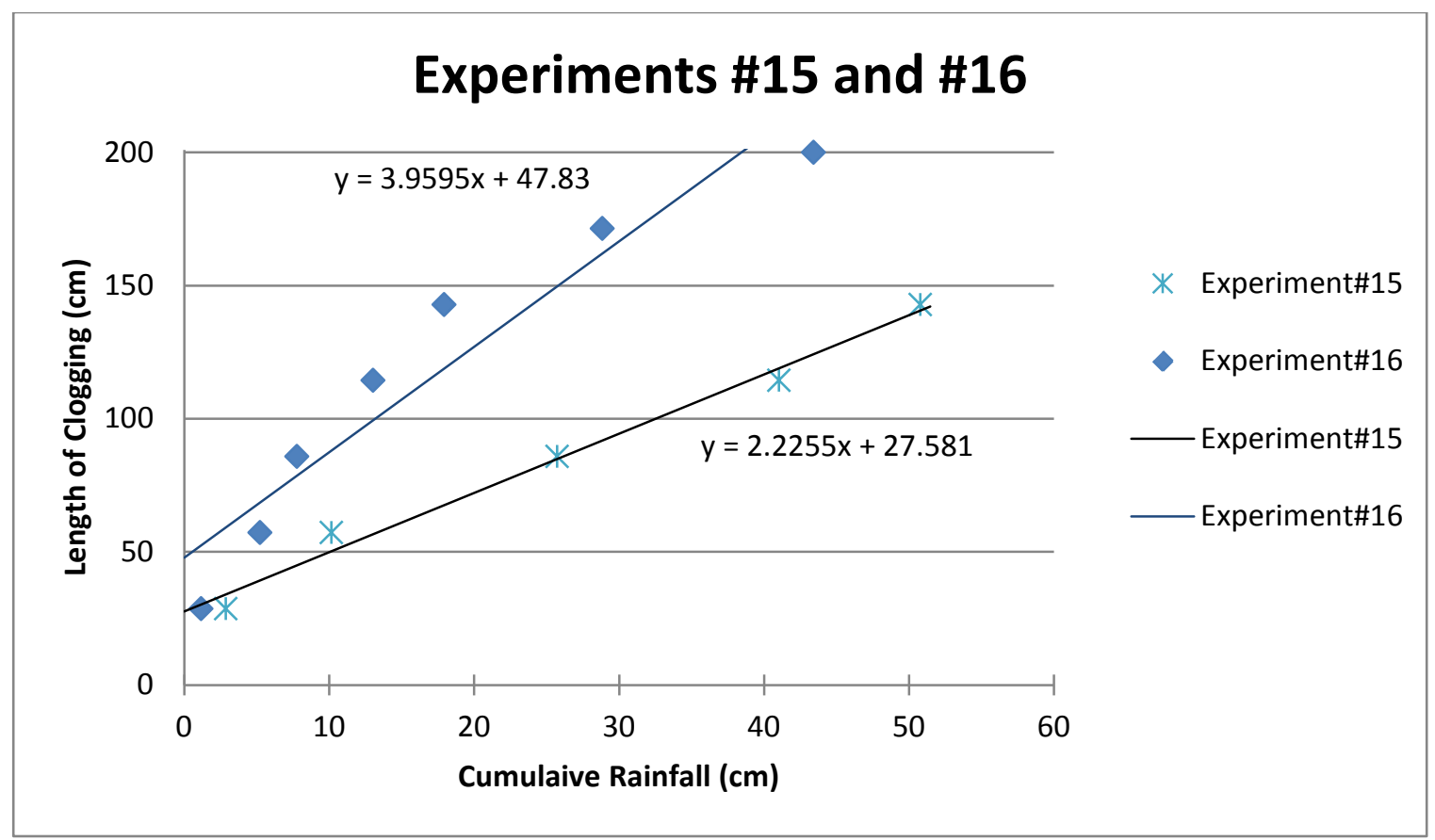

Figure 98: Comparison of progression of clogging in experiments \#15 and \#16

Figure 98 shows the progression of surface clogging in the steepest longitudinal slope and with the biggest gap between pavers As expected, the $12 \mathrm{~mm}$ gap size compensated for the slope and, compared to experiments conducted with smaller gaps, this difference in the progression of surface clogging in the experiments with permeable joint material and those without, was not as significant. The absence of permeable joint material resulted in a 77\% improvement in the progress of surface clogging. 


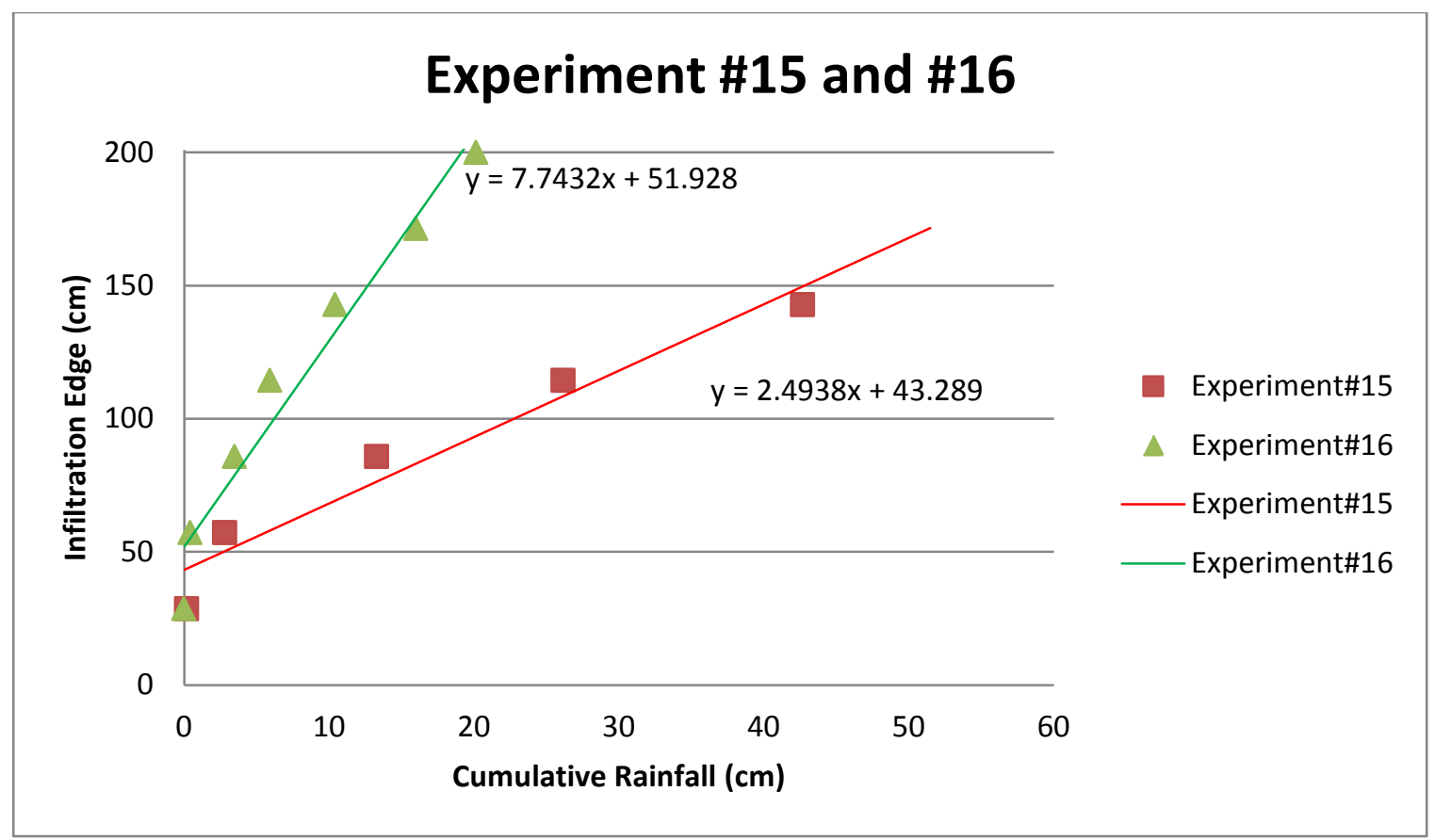

Figure 99: Comparison of progression of infiltration edge in experiments \#15 and \#16

The comparisons in this section were between experiments \#15 and \#16, which used Eco-Pavers separated by $12 \mathrm{~mm}$ gaps. The progression of the infiltration edge in the experiments without permeable joint material indicates a $210 \%$ difference from experiment \# 16.

\subsubsection{Longitudinal Slope}

Finally, the progression of clogging and progression of infiltration edge were used to determine the effect of the longitudinal installation slope on the performance of a permeable pavement GI. The following experiments were compared to each other to fully examine the effect of changes in slope on the outcome of experiments:

- $1 \%$ vs. $3 \%$ vs. $5 \%$

o Different slopes with a $6 \mathrm{~mm}$ gap size and no aggregate

o Different slopes with a $6 \mathrm{~mm}$ gap size and \#8 aggregate 
o Different slopes with a $9 \mathrm{~mm}$ gap size and no aggregate

o Different slopes with a 9 mm gap size and \#8 aggregate

o Different slopes with a $12 \mathrm{~mm}$ gap size and no aggregate

o Different slopes with a $12 \mathrm{~mm}$ gap size and \#8 aggregate

\subsubsection{1. $\quad$ Slope for $6 \mathrm{~mm}$ Gap without Aggregate}

Experiments \#2, \#13, and \#19 were conducted at 1\%, 3\%, and 5\% longitudinal slopes, respectively. The progression of clogging and progression of infiltration edge graphs for the experiments were plotted in Figure 100 and Figure 101.

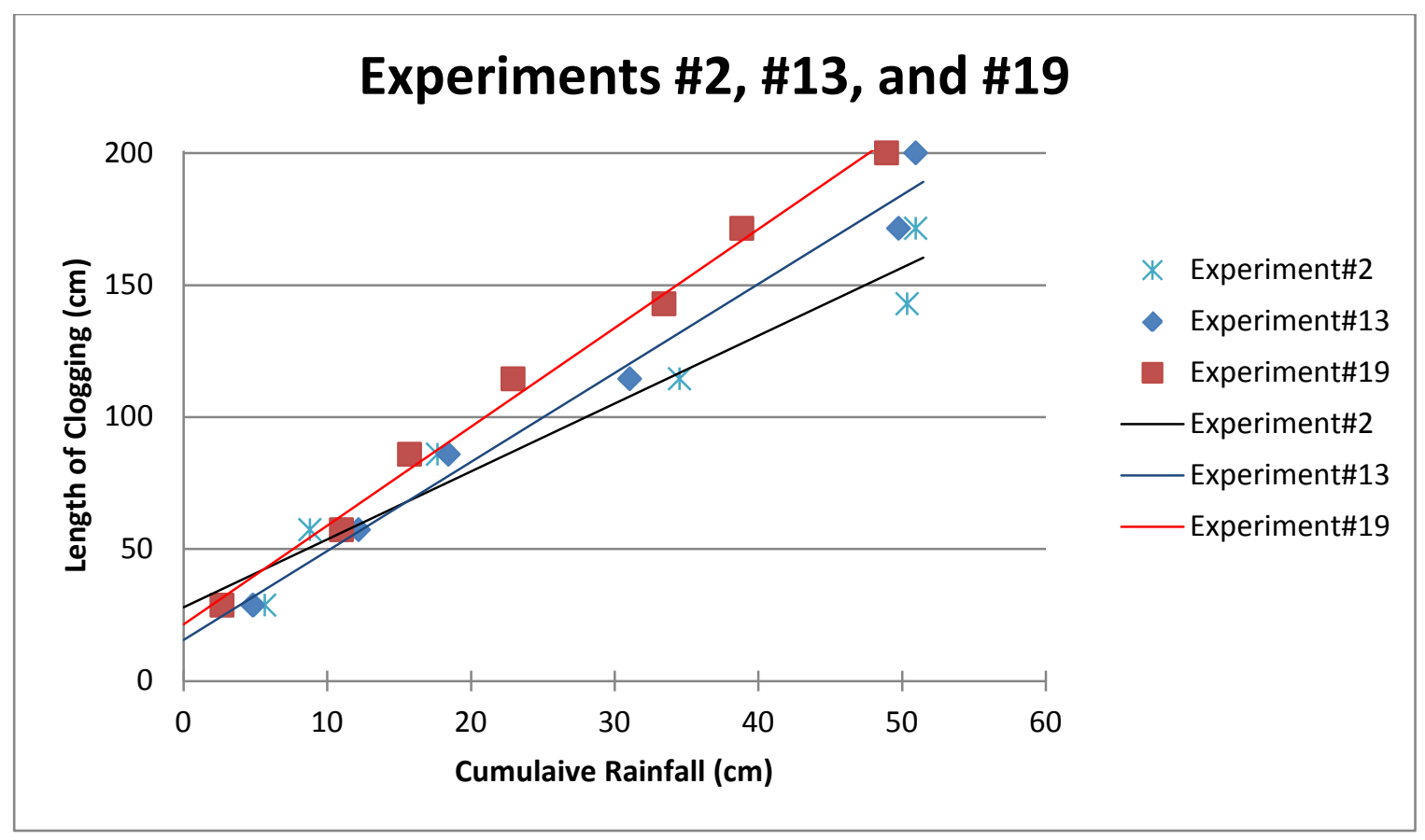

Figure 100: Comparison of progression of clogging in experiments \#2, \#13, and \#19

Comparing the progression of clogging for different slopes, as shown in Figure 100, illustrates that for this specific gap size without the permeable joint material, with an increase in the slope, surface clogging progressed faster. With numerical analysis of the 
graphs, their slopes, which represent the rate of their increase, are calculated and listed in Table 35.

Table 35: Comparison of the Effects of Slope in Progression of Clogging in Experiments with 6 mm Gap Size and No Permeable Joint Material

\begin{tabular}{|l|l|l|l|}
\hline Gap Size & $1 \%$ & $3 \%$ & $5 \%$ \\
\hline $1 \%$ & N/A & $-31 \%$ & $-45 \%$ \\
\hline $3 \%$ & $+31 \%$ & N/A & $-10 \%$ \\
\hline $5 \%$ & $+45 \%$ & $+10 \%$ & N/A \\
\hline
\end{tabular}

The numerical analysis of the effect of slope clearly suggests that working with a $6 \mathrm{~mm}$ gap size, where no permeable joint material is present - like the conditions in Louisville - increasing the slope of the installation would reduce the system's tolerance of sediment, and clogging might ensue up to $45 \%$ faster.

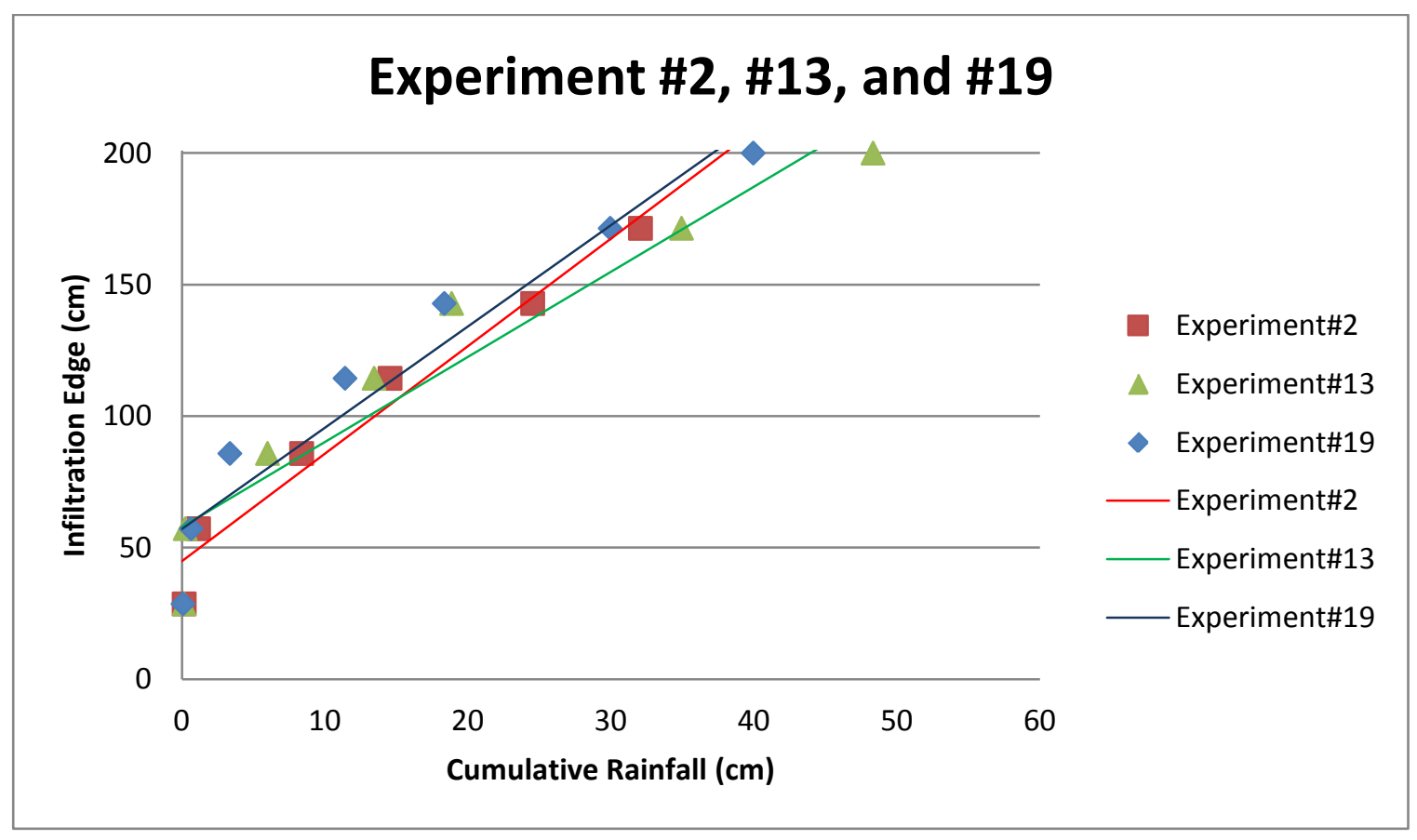

Figure 101: Comparison of progression of infiltration edge in experiments \#2, \#13, and \#19 
Comparing the progression of the infiltration edge for the experiments analyzed here suggests an unexpected pattern, that the increase of slope from $1 \%$ to $3 \%$ resulted in a 20\% improvement in the rate at which the infiltration edge progressed on the surface, while a further increase of the slope, from 3\% to $5 \%$, resulted in a $19 \%$ reduction in this rate. Direct comparison between the slopes, see Figure 101, shows that the changing the longitudinal slope from $1 \%$ to $5 \%$ resulted in an insignificant effect on the progression of the infiltration edge.

Table 36: Comparison of the Effects of Slope in Progression of Infiltration Edge in Experiments with 6 mm Gap Size and No Permeable Joint Material

\begin{tabular}{|l|l|l|l|}
\hline Gap Size & $1 \%$ & $3 \%$ & $5 \%$ \\
\hline $1 \%$ & N/A & $+20 \%$ & $+5 \%$ \\
\hline $3 \%$ & $-20 \%$ & N/A & $-19 \%$ \\
\hline $5 \%$ & $-5 \%$ & $+19 \%$ & N/A \\
\hline
\end{tabular}

\subsubsection{Slope for $6 \mathrm{~mm}$ Gap and \#8 Aggregate}

Experiments \#4, \#14, and \#20 were conducted at longitudinal slopes of 1\%, 3\%, and 5\%, respectively. Using the calculations and comparisons on their TDRs measurements, the progression of clogging and progression on the infiltration edge for the three experiments are plotted in Figure 102 and Figure 103. 


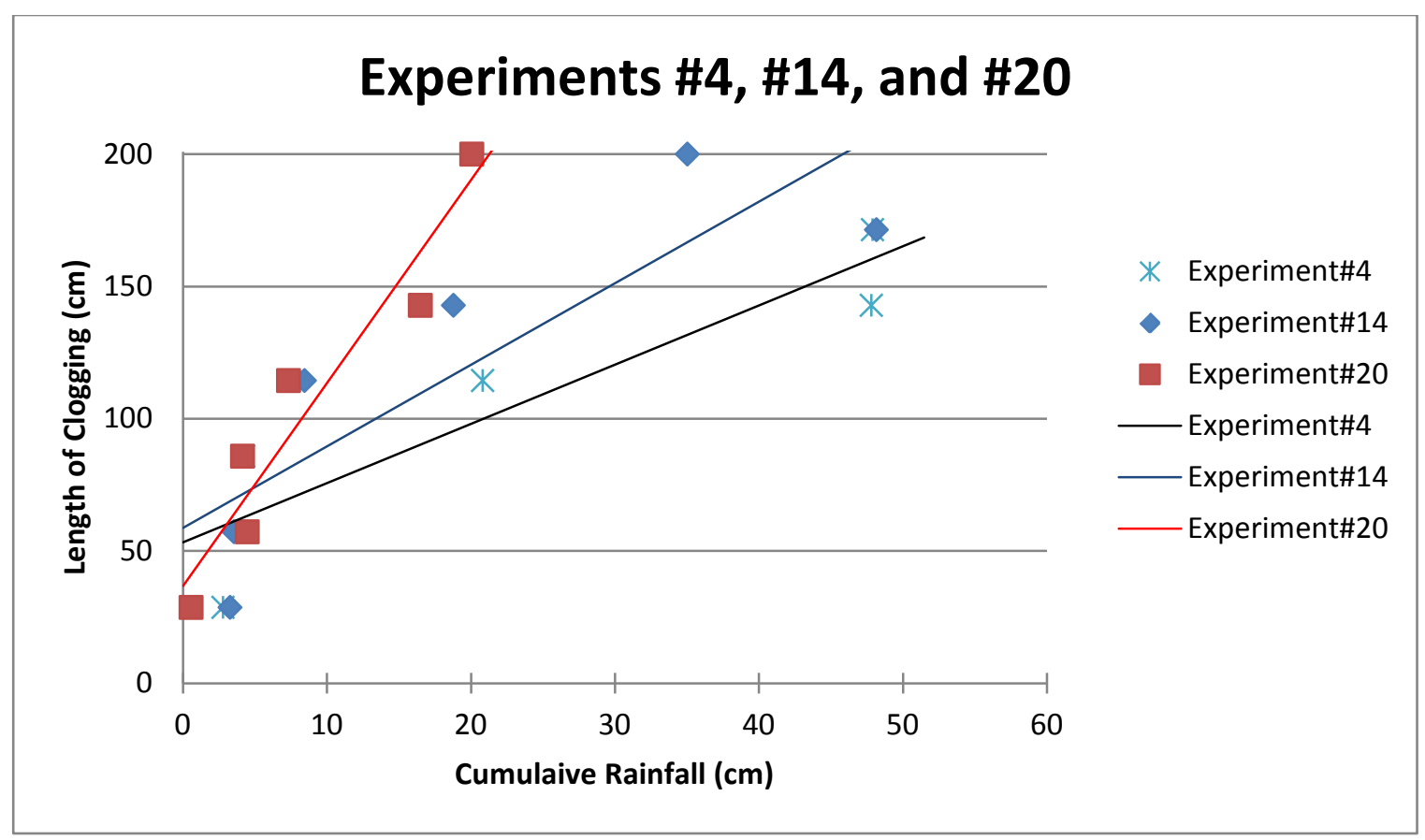

Figure 102: Comparison of progression of clogging in experiments \#4, \#14, and \#20

Comparing the progression of clogging for the experiments shows that when the flume is set up with paver blocks featuring a $6 \mathrm{~mm}$ gap size filled with the permeable joint filling material of \#8 aggregate, the increase in the slope resulted in a consistent increase in the rate at which surface clogging progressed on the surface.

Table 37: Comparison of the Effects of Slope in Progression of Clogging in Experiments with 6 mm Gap Size and Permeable Joint Material

\begin{tabular}{|l|l|l|l|}
\hline Gap Size & $1 \%$ & $3 \%$ & $5 \%$ \\
\hline $1 \%$ & N/A & $+114 \%$ & $+242 \%$ \\
\hline $3 \%$ & $-114 \%$ & N/A & $+60 \%$ \\
\hline $5 \%$ & $-242 \%$ & $-60 \%$ & N/A \\
\hline
\end{tabular}

As shown in Figure 102, changing the longitudinal slope from 1\% to 3\% resulted in a $114 \%$ increase in the rate at which surface clogging progressed. Further changes made to the slope would have resulted in another $60 \%$ increase in this rate. Direct comparison between the initial slope and 5\% indicates that going from the minimum 
vertical slope to the maximum suggested slope would result in a $242 \%$ increase in the rate at which surface clogging progressed (Table 37).

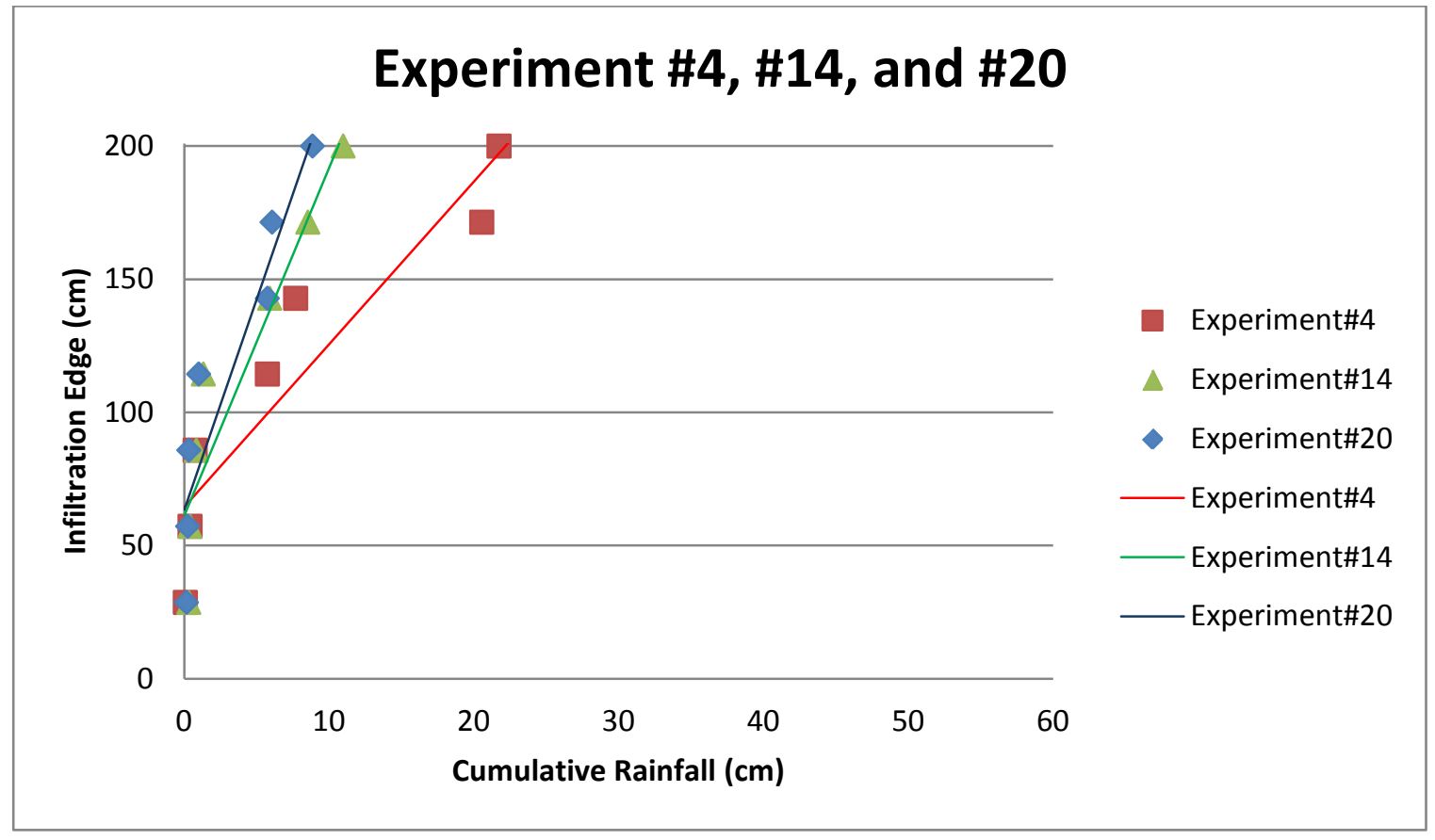

Figure 103: Comparison of progression of infiltration edge in experiments \#4, \#14, and \#20

The presence of permeable joint material in Figure 103 created different patterns from those shown in the previous graph plotted for the progression of the infiltration edge, Figure 101. As the longitudinal slope increased the wetting front progressed more quickly.

Table 38: Comparison of the Effects of Slope in Progression of Infiltration Edge in Experiments with 6 mm Gap Size and Permeable Joint Material

\begin{tabular}{|l|l|l|l|}
\hline Gap Size & $1 \%$ & $3 \%$ & $5 \%$ \\
\hline $1 \%$ & N/A & $+113 \%$ & $+158 \%$ \\
\hline $3 \%$ & $-113 \%$ & N/A & $+21 \%$ \\
\hline $5 \%$ & $-158 \%$ & $-21 \%$ & N/A \\
\hline
\end{tabular}


Increasing the longitudinal slope in this specific configuration from $1 \%$ to $3 \%$ resulted in a more than $110 \%$ increase in the rate at which the wetting front progressed. A further increase, from $3 \%$ to $5 \%$, created another $21 \%$ increase in this rate (Table 38 ).

\subsubsection{Slope for $9 \mathrm{~mm}$ Gap without Aggregate}

Experiments \#5, \#11, and \#17 were set up on longitudinal slopes of 1\%, 3\%, and $5 \%$, respectively. The progression of clogging and progression of infiltration edge were used to determine the effects of the slope in these specific experiments. Figure 104 and Figure 105 were used for these comparisons.

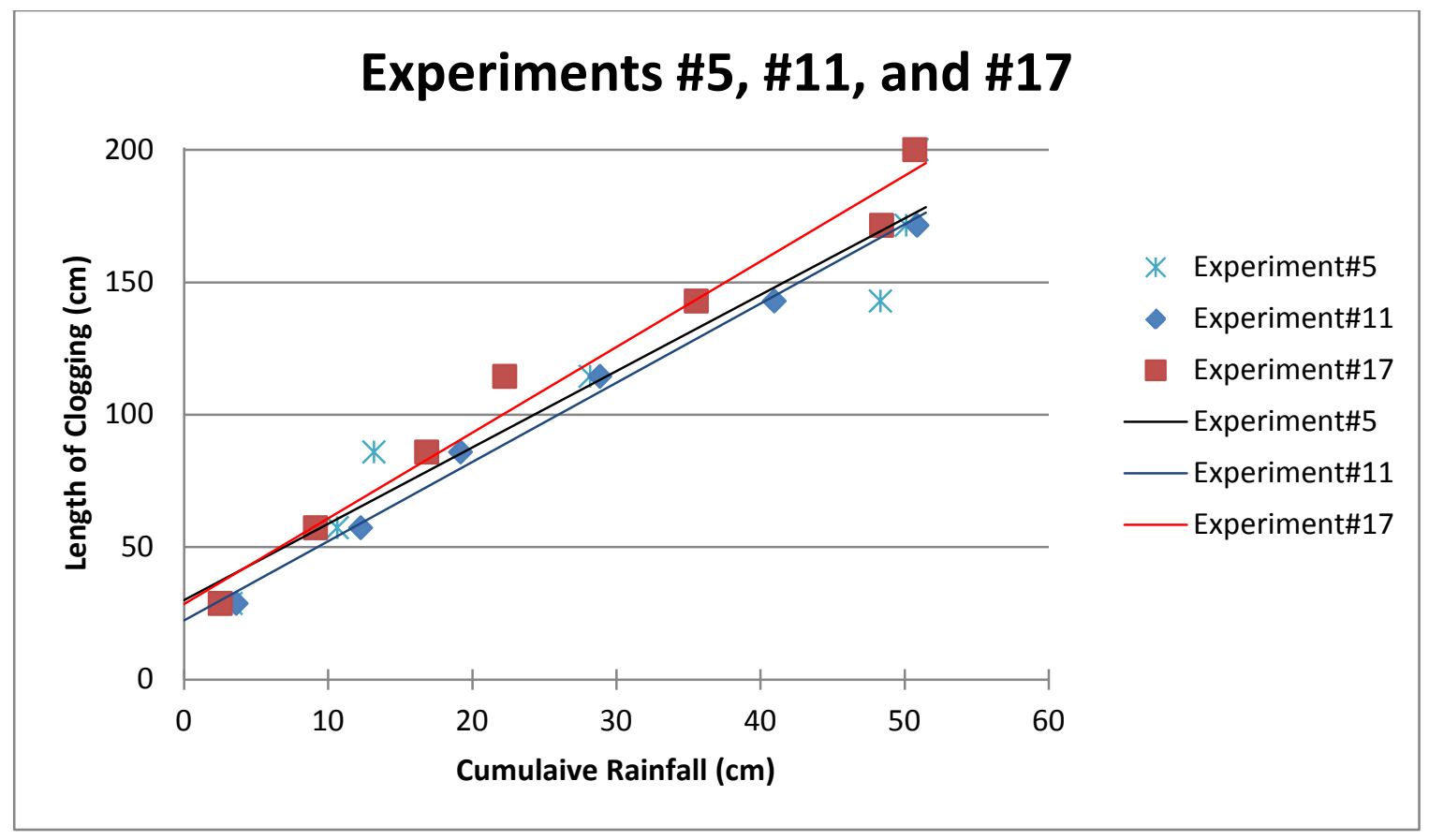

Figure 104: Comparison of progression of clogging in experiments \#5, \#11, and \#17

As illustrated in Figure 104, the progression of surface clogging in the experiments conducted without the permeable joint material was not as significant as in the experiments conducted with it. The increase in the installation slopes caused a steady 
yet insignificant effect on the progression of clogging. The slopes of the trend lines were compared to each other to determine the extent of this effect.

Table 39: Comparison of the Effects of Slope in Progression of Clogging in Experiments with 9 mm Gap Size and no Permeable Joint Material

\begin{tabular}{|l|l|l|l|}
\hline Gap Size & $1 \%$ & $3 \%$ & $5 \%$ \\
\hline $1 \%$ & N/A & $+3 \%$ & $+12 \%$ \\
\hline $3 \%$ & $-3 \%$ & N/A & $+8 \%$ \\
\hline $5 \%$ & $-12 \%$ & $-8 \%$ & N/A \\
\hline
\end{tabular}

Comparing the numbers listed in Table 39 and Table 37 shows that a $3 \mathrm{~mm}$ increase in the gap size offset the significant changes caused by the slope change. In other words, the bigger gap size seems to be more tolerant to changes in slope.

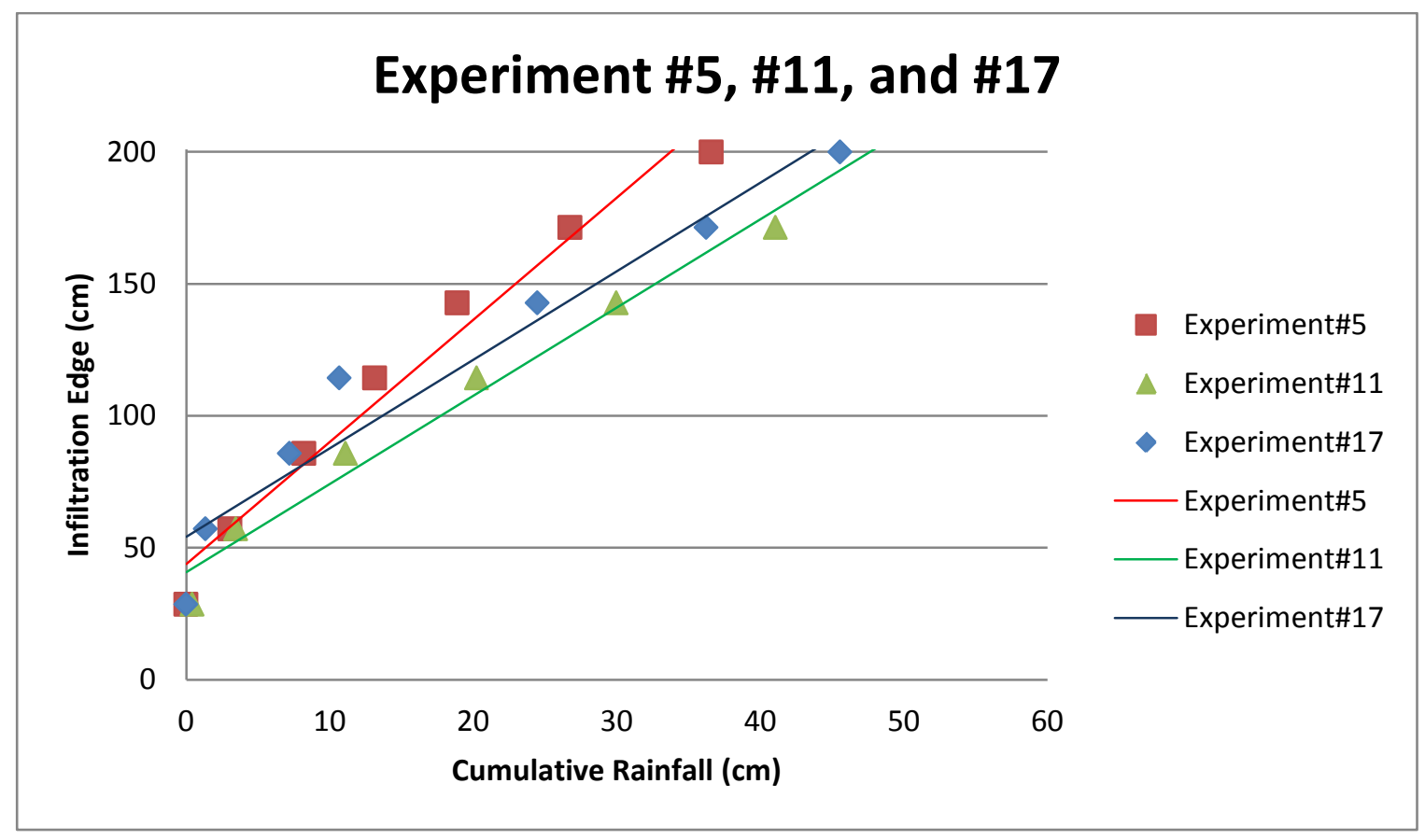

Figure 105: Comparison of progression of infiltration edge in experiments \#5, \#11, and \#17

Comparing the progression of the infiltration edge for the three experiments, as illustrated in Figure 105, shows that the experiment conducted in the lowest slope 
experienced the quickest rate of progression of the infiltration edge. Increasing the slope to $3 \%$ resulted in a $27 \%$ improvement in this rate, but increasing the slope further made no significant difference to the rate (Table 40).

Table 40: Comparison of the Effects of Slope in Progression of Infiltration Edge in Experiments with $9 \mathrm{~mm}$ Gap Size and No Permeable Joint Material

\begin{tabular}{|l|l|l|l|}
\hline Gap Size & $1 \%$ & $3 \%$ & $5 \%$ \\
\hline $1 \%$ & N/A & $+27 \%$ & $+27 \%$ \\
\hline $3 \%$ & $-27 \%$ & N/A & $0 \%$ \\
\hline $5 \%$ & $-27 \%$ & $0 \%$ & N/A \\
\hline
\end{tabular}

\subsubsection{4. $\quad$ Slope for $9 \mathrm{~mm}$ Gap and \#8 Aggregate}

Experiments \#6, \#12, and \#18 were conducted on respectively 1\%, 3\%, and 5\% longitudinal slopes and with similar analysis tools as before, the progression of clogging and progression of infiltration edge are used to assess the effect of the longitudinal slope on the performance of the GI. 


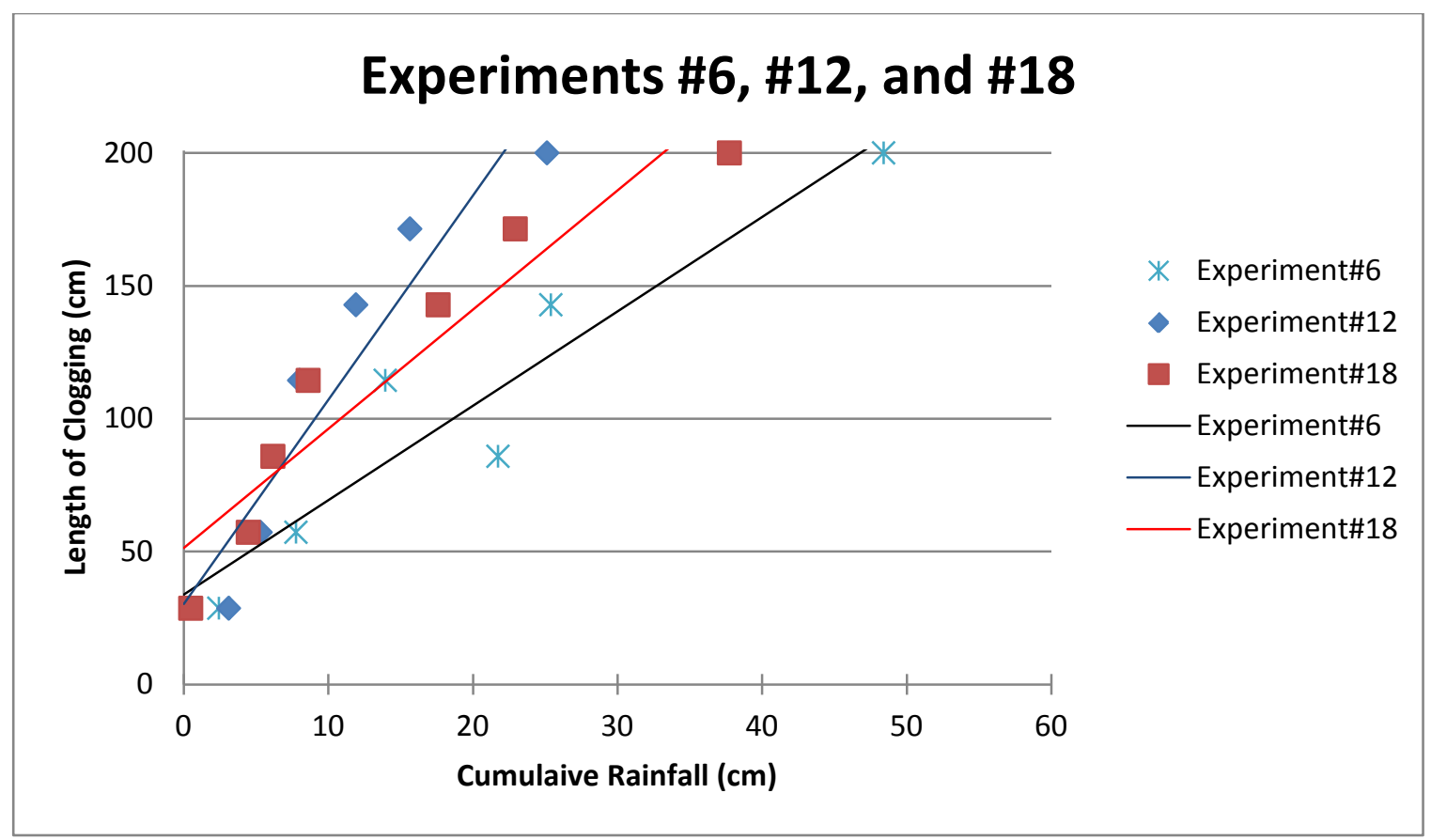

Figure 106: Comparison of progression of clogging in experiments \#6, \#12, and \#18

Figure 107 illustrates the effect of installation slope on the progression of surface clogging, suggesting that with an increase of longitudinal slope from $1 \%$ to $3 \%$, the rate at which surface clogging progresses, increases; however and with further increase of slope from $3 \%$ to $5 \%$ the rate decreases.

Table 41: Comparison of the Effects of Slope in Progression of Clogging in Experiments with 9 mm Gap Size and Permeable Joint Material

\begin{tabular}{|l|l|l|l|}
\hline Gap Size & $1 \%$ & $3 \%$ & $5 \%$ \\
\hline $1 \%$ & N/A & $+162 \%$ & $+26 \%$ \\
\hline $3 \%$ & $-162 \%$ & N/A & $-41 \%$ \\
\hline $5 \%$ & $-26 \%$ & $+41 \%$ & N/A \\
\hline
\end{tabular}




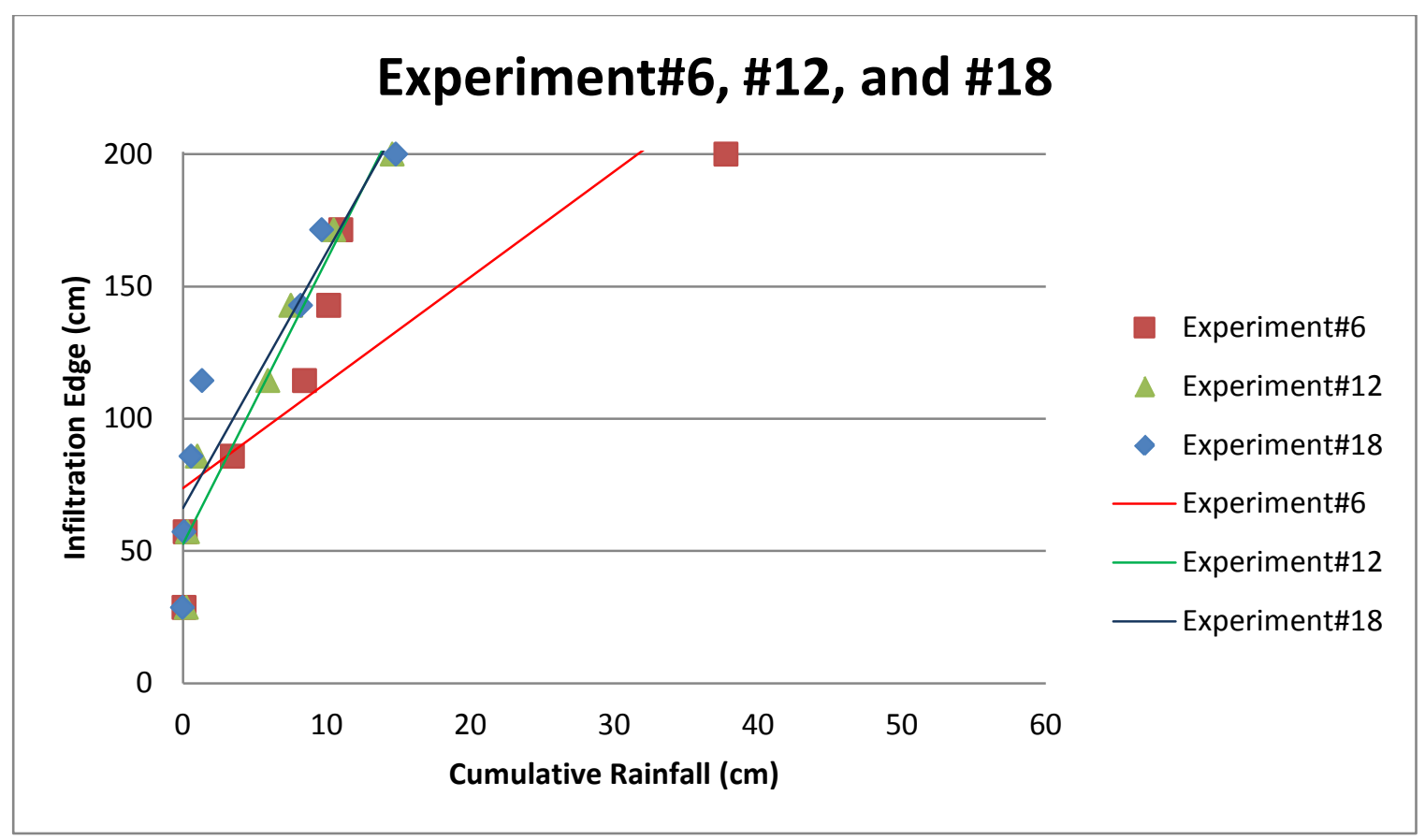

Figure 107: Comparison of progression of infiltration edge in experiments \#6, \#12, and \#18

Similar to progression of infiltration edge, the comparisons made for progression of infiltration edge also suggest that the increase of slope from $1 \%$ to $3 \%$ has resulted in a significant increase in the rate at which infiltration edge progresses, while further increase in the slope has resulted in a slight decrease in this rate. Although an increase has been measured, compared to the first increase, the $10 \%$ decrease seems insignificant.

Table 42: Comparison of the Effects of Slope in Progression of Infiltration Edge in Experiments with 9 mm Gap Size and Permeable Joint Material

\begin{tabular}{|l|l|l|l|}
\hline Gap Size & $1 \%$ & $3 \%$ & $5 \%$ \\
\hline $1 \%$ & N/A & $+169 \%$ & $+142 \%$ \\
\hline $3 \%$ & $-169 \%$ & N/A & $-10 \%$ \\
\hline $5 \%$ & $-142 \%$ & $+10 \%$ & N/A \\
\hline
\end{tabular}

\subsubsection{Slope for $12 \mathrm{~mm}$ Gap without Aggregate}

Experiments \#7, \#10, and \#15 were conducted on respectively 1\%, 3\%, and 5\% longitudinal slopes. These experiments were conducted with the Eco-Pavers, which 
provide the biggest gap size among the three. There is no \#8 aggregate filling in the gaps. Progression of clogging and progression of infiltration edge are plotted in Figure 108 and Figure 109 and are used to assess the performance of the flume in these experiments.

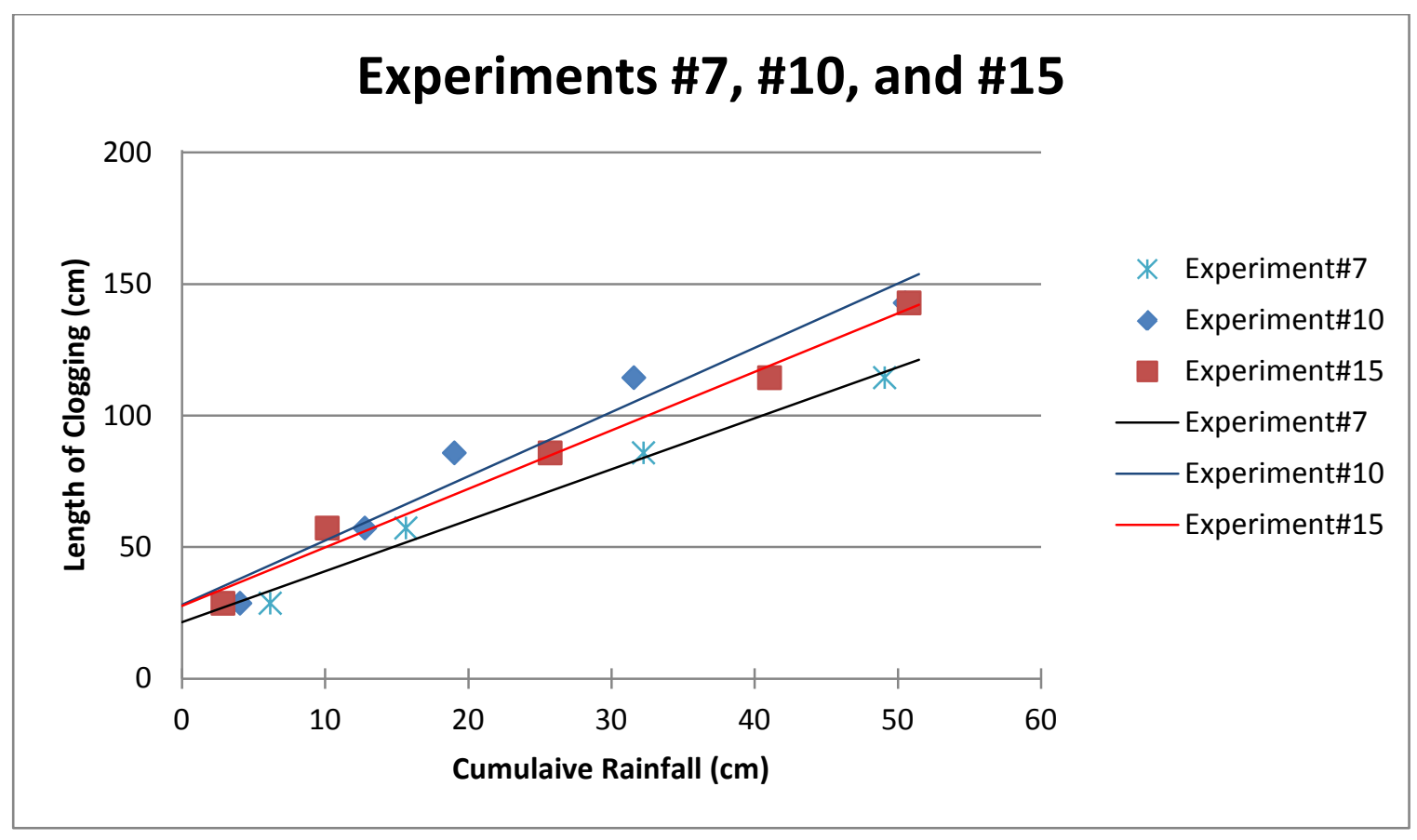

Figure 108: Comparison of progression of clogging in experiments \#7, \#10, and \#15

Comparing the progression of clogging in experiments conducted using pavers with the biggest gap size and those conducted with smaller gap sizes; indicate that the bigger gap size is resulting in change of slope and its effects to be less significant. Figure 108 shows that increasing the installation longitudinal slope from $1 \%$ to $3 \%$ are resulting in a $25 \%$ increase in the rate at which clogging progresses on the surface. Further increase causes this trend to change and results in 8\% improvements. Direct comparison between $1 \%$ and $5 \%$ indicates that increasing the slope is resulting in a $14 \%$ increase in the rate at which surface clogging progresses (Table 43). 
Table 43: Comparison of the Effects of Slope in Progression of Clogging in Experiments with 12 mm Gap Size and No Permeable Joint Material

\begin{tabular}{|l|l|l|l|}
\hline Gap Size & $1 \%$ & $3 \%$ & $5 \%$ \\
\hline $1 \%$ & N/A & $+25 \%$ & $+14 \%$ \\
\hline $3 \%$ & $-25 \%$ & N/A & $-8 \%$ \\
\hline $5 \%$ & $-14 \%$ & $+8 \%$ & N/A \\
\hline
\end{tabular}

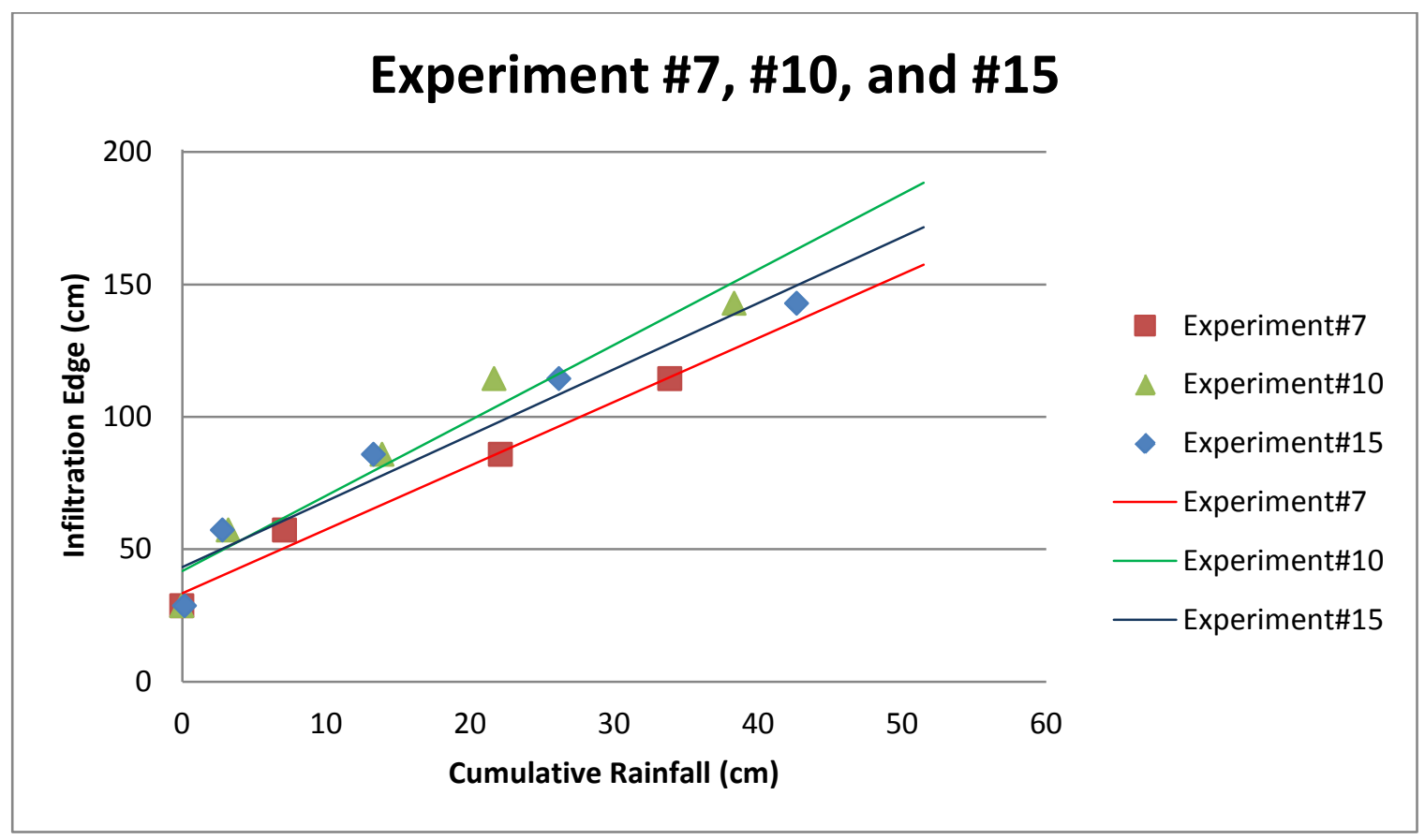

Figure 109: Comparison of progression of infiltration edge in experiments \#7, \#10, and \#15

Progression of infiltration edge shows similar patterns to those seen from progression of clogging. The increase of slope from $1 \%$ to $3 \%$ has resulted in an increase in the rate at which infiltration edge progresses; however further increase has resulted in $12 \%$ decrease in that rate (Table 44).

Table 44: Comparison of the Effects of Slope in Progression of Infiltration Edge in Experiments with $12 \mathrm{~mm}$ Gap Size and No Permeable Joint Material

\begin{tabular}{|l|l|l|l|}
\hline Gap Size & $1 \%$ & $3 \%$ & $5 \%$ \\
\hline $1 \%$ & N/A & $+18 \%$ & $+3 \%$ \\
\hline $3 \%$ & $-18 \%$ & N/A & $-12 \%$ \\
\hline $5 \%$ & $-3 \%$ & $+12 \%$ & N/A \\
\hline
\end{tabular}




\subsubsection{Slope for $12 \mathrm{~mm}$ Gap and \#8 Aggregate}

Experiments \#8, \#9, and \#16 have been set up with respectively 1\%, 3\%, and 5\% longitudinal slopes. Using the progression of clogging and progression of infiltration edge as assessment tools, the performance of the flume in these configurations was examined.

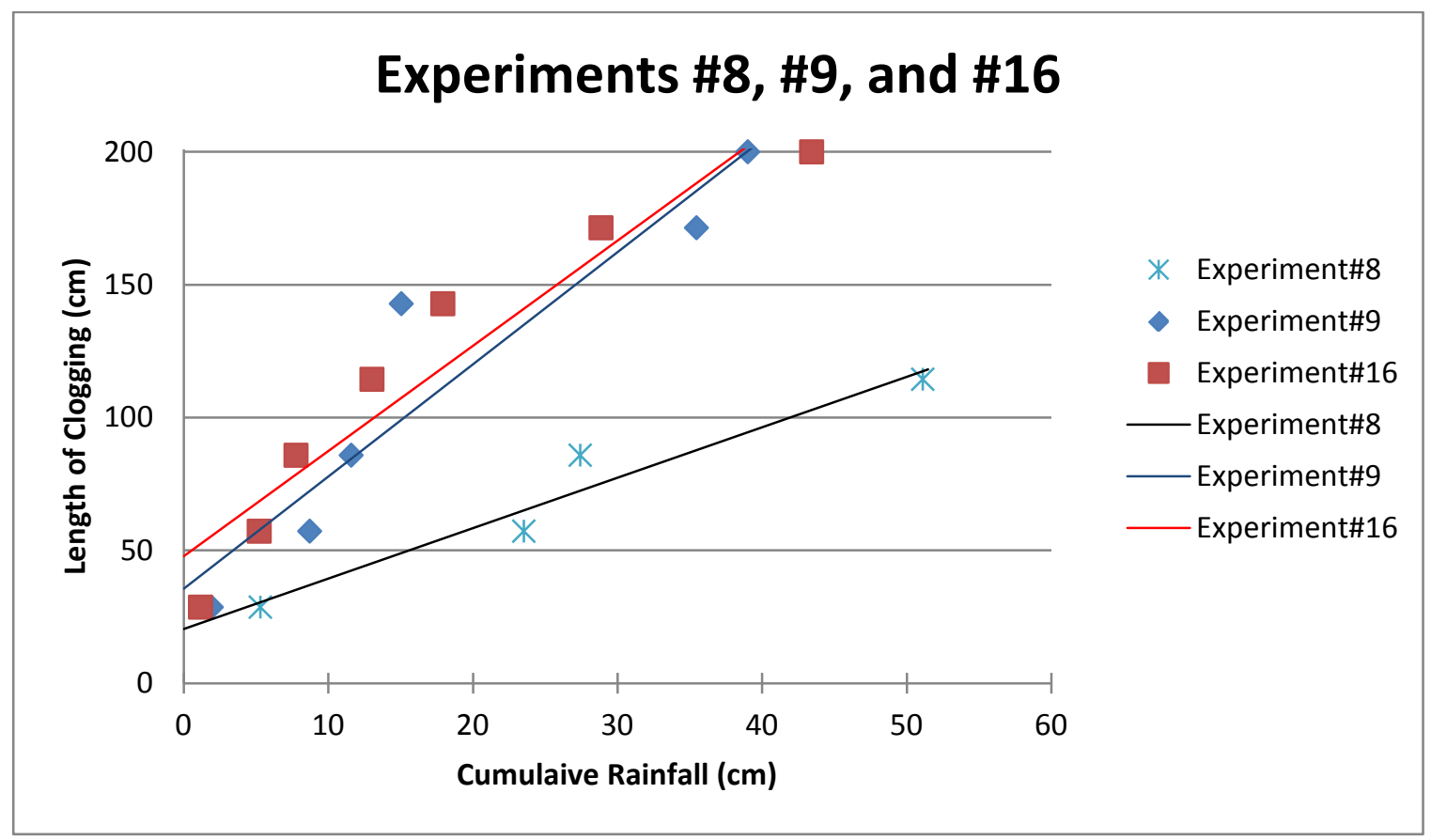

Figure 110: Comparison of progression of clogging in experiments \#8, \#9, and \#16

The progression of clogging (Figure 110) shows that, with the first increase in slope, where a $1 \%$ longitudinal slope changes to $3 \%$, the rate at which the clogging progressed on the permeable surface of the flume increased by $122 \%$. Increasing the slope further, from $3 \%$ to $5 \%$, caused this rate to decrease by $6 \%$. Comparing the initial slope to the final slope, the increase was calculated to be about 108\% (Table 45). 
Table 45: Comparison of the Effects of Slope in Progression of Clogging In Experiments with 12 mm Gap Size and Permeable Joint Material

\begin{tabular}{|l|l|l|l|}
\hline Gap Size & $1 \%$ & $3 \%$ & $5 \%$ \\
\hline $1 \%$ & N/A & $+122 \%$ & $+108 \%$ \\
\hline $3 \%$ & $-122 \%$ & N/A & $-6 \%$ \\
\hline $5 \%$ & $-108 \%$ & $+6 \%$ & N/A \\
\hline
\end{tabular}

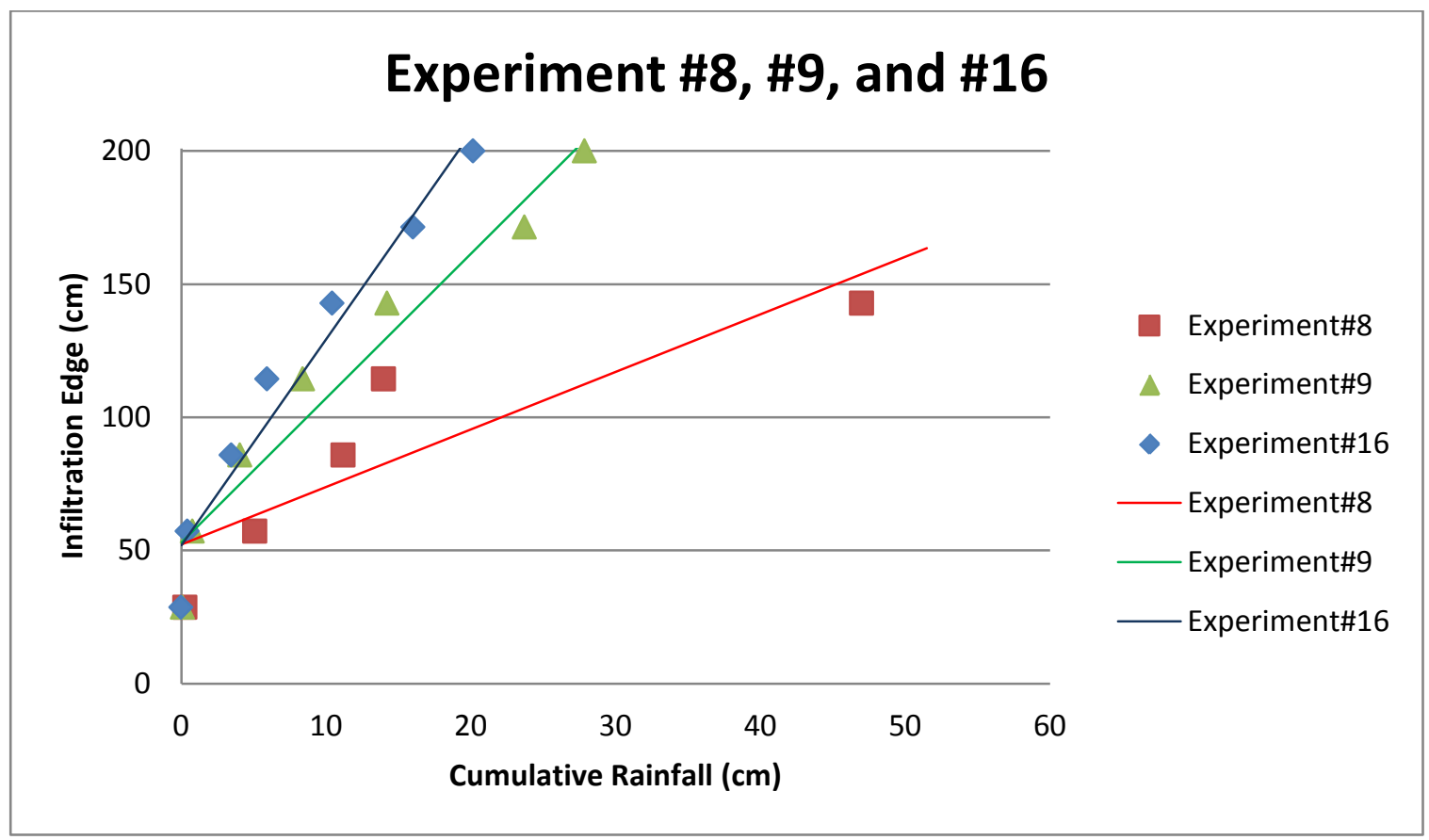

Figure 111: Comparison of progression of infiltration edge in experiments \#8, \#9, and \#16

The progression of the infiltration edge shows that, with the first increase in the slope of the flume, the progression of the infiltration edge increased $150 \%$ faster. Further increases in the slope caused this rate to increase another $42 \%$. This shows that the increase from the minimum slope created a much bigger impact on the performance and progression of the infiltration edge than the second increase from an already steep trend. 
Table 46: Comparison of the Effects of Slope in Progression of Infiltration Edge in Experiments with $12 \mathrm{~mm}$ Gap Size and Permeable Joint Material

\begin{tabular}{|l|l|l|l|}
\hline Gap Size & $1 \%$ & $3 \%$ & $5 \%$ \\
\hline $1 \%$ & N/A & $+150 \%$ & $+258 \%$ \\
\hline $3 \%$ & $-150 \%$ & N/A & $+42 \%$ \\
\hline $5 \%$ & $-258 \%$ & $-42 \%$ & N/A \\
\hline
\end{tabular}

\subsubsection{Clogging Sediment}

In the previous chapter, it was mentioned that an extra experiment was conducted at the end to determine the effects of the characteristics of the clogging sediment. In order to conduct this investigation, the organics were removed from the prepared sediment and the flume was run with $100 \%$ inorganic clogging debris.

A comparison made between experiment \#2, which was conducted on a $1 \%$ longitudinal slope using pavers with $6 \mathrm{~mm}$ gaps and no permeable joint materials and experiment \#21 which used the same configuration, but involved wholly inorganic clogging debris. This showed that the progression of surface clogging in a case where organics were removed proceeds $167 \%$ more slowly. Comparing the slope of the trend line for experiment \#21 also indicates that this was the slowest rate of all the experiments exceeding that of the experiment conducted with pavers set $12 \mathrm{~mm}$ apart on a $1 \%$ slope. 


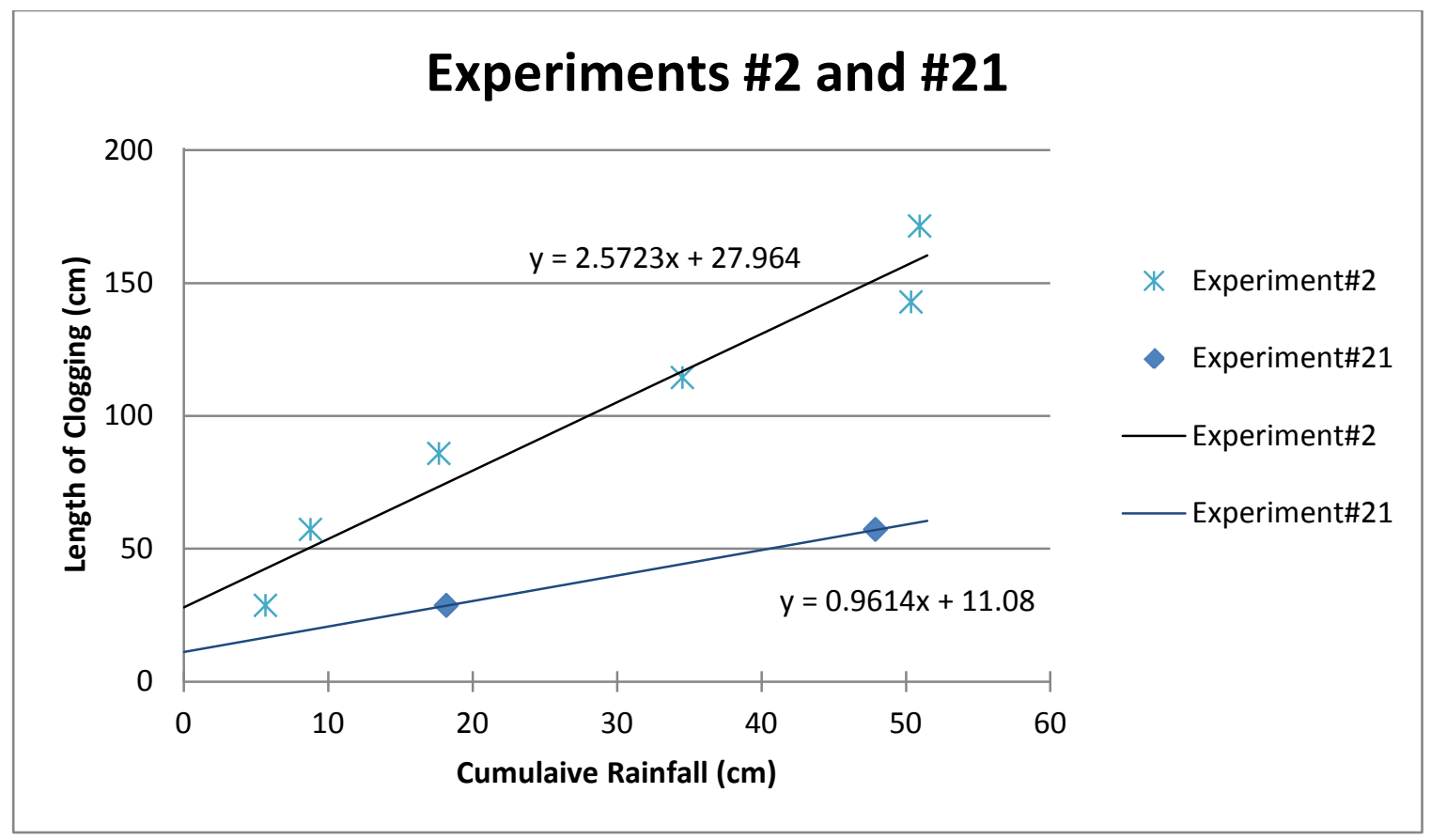

Figure 112: Comparison of progression of clogging in experiments \#2 and \#21

\subsection{Analysis of Variance}

The presented results are based on data that includes several sources of variance. Analysis of variance (ANOVA) is a statistical tool to test hypotheses. In order to determine whether the comparisons based on the slopes of the graphs to show the progression of clogging and progression of the infiltration edge are statistically significant, they should be tested to find whether they meet the significance level. The defined threshold, which is the confidence limit for the experiments, is the 95 percentile.

Using this method, the observed changes in the slope of the plotted graphs were statistically analyzed to determine whether the observed changes were statistically significant or not. Although the statistical analysis uses all the experiments to determine the relationships between the variables, comparisons made between individual experiments can still be used to draw conclusions about these specific experiments. 


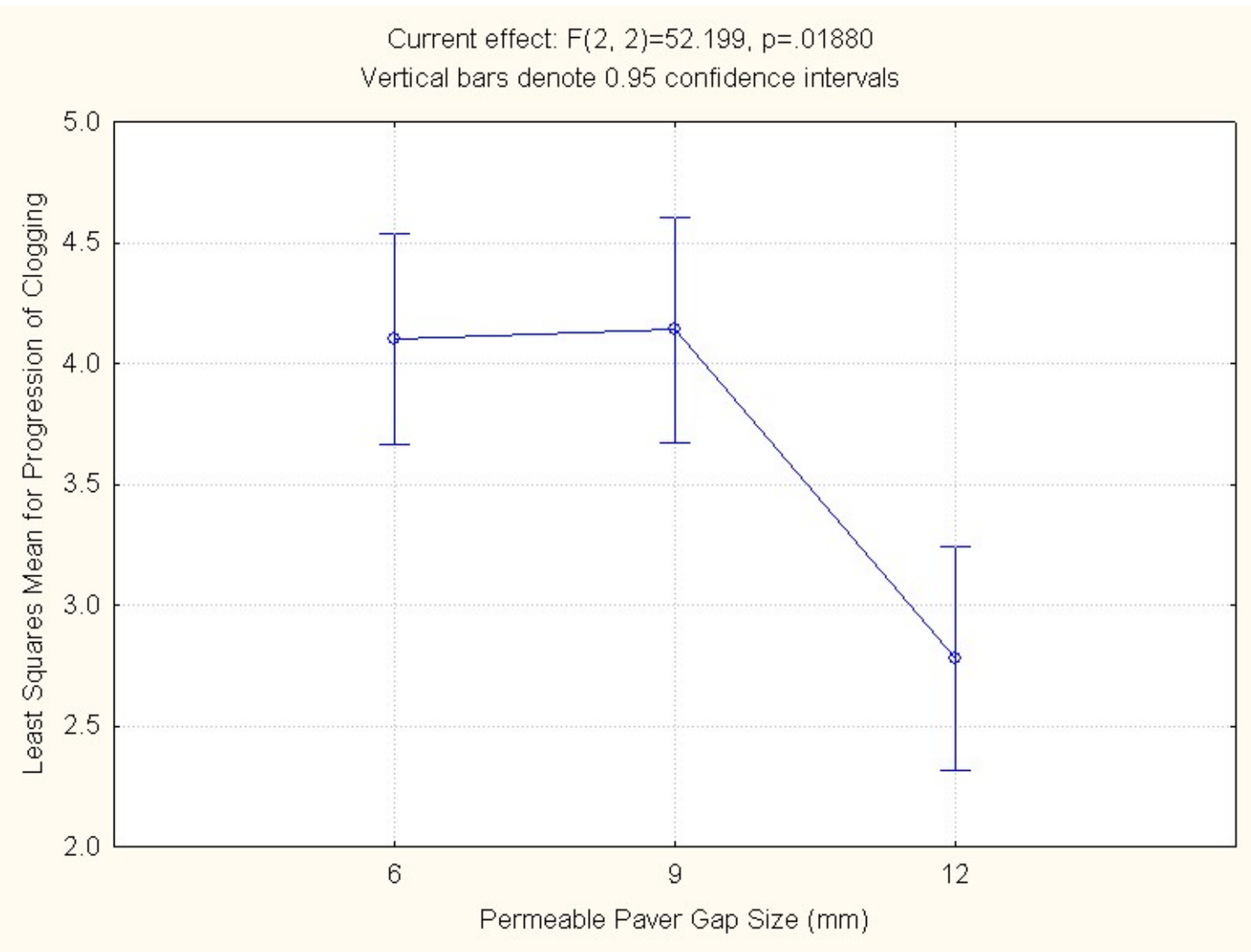

Figure 113: Analysis of variance of paver gap size for progression of clogging

Figure 113 illustrates the ANOVA for the paver gap size in all the experiments, showing that the increase of paver gap size from $6 \mathrm{~mm}$ to $9 \mathrm{~mm}$ did not result in a statistical significance in the rate at which the slope of the progression of clogging changes. In other words, the changes in the slope, by a 95\% confidence interval, may be a result of noise and/or other unknown variables. However, the increase of the gap size to $12 \mathrm{~mm}$ resulted in a significant difference in the rate at which the slope changed. Therefore the $12 \mathrm{~mm}$ gap size resulted in a change in the performance of the system, whereas the $9 \mathrm{~mm}$ did not. This result agrees with the overall patterns seen in the analysis. 


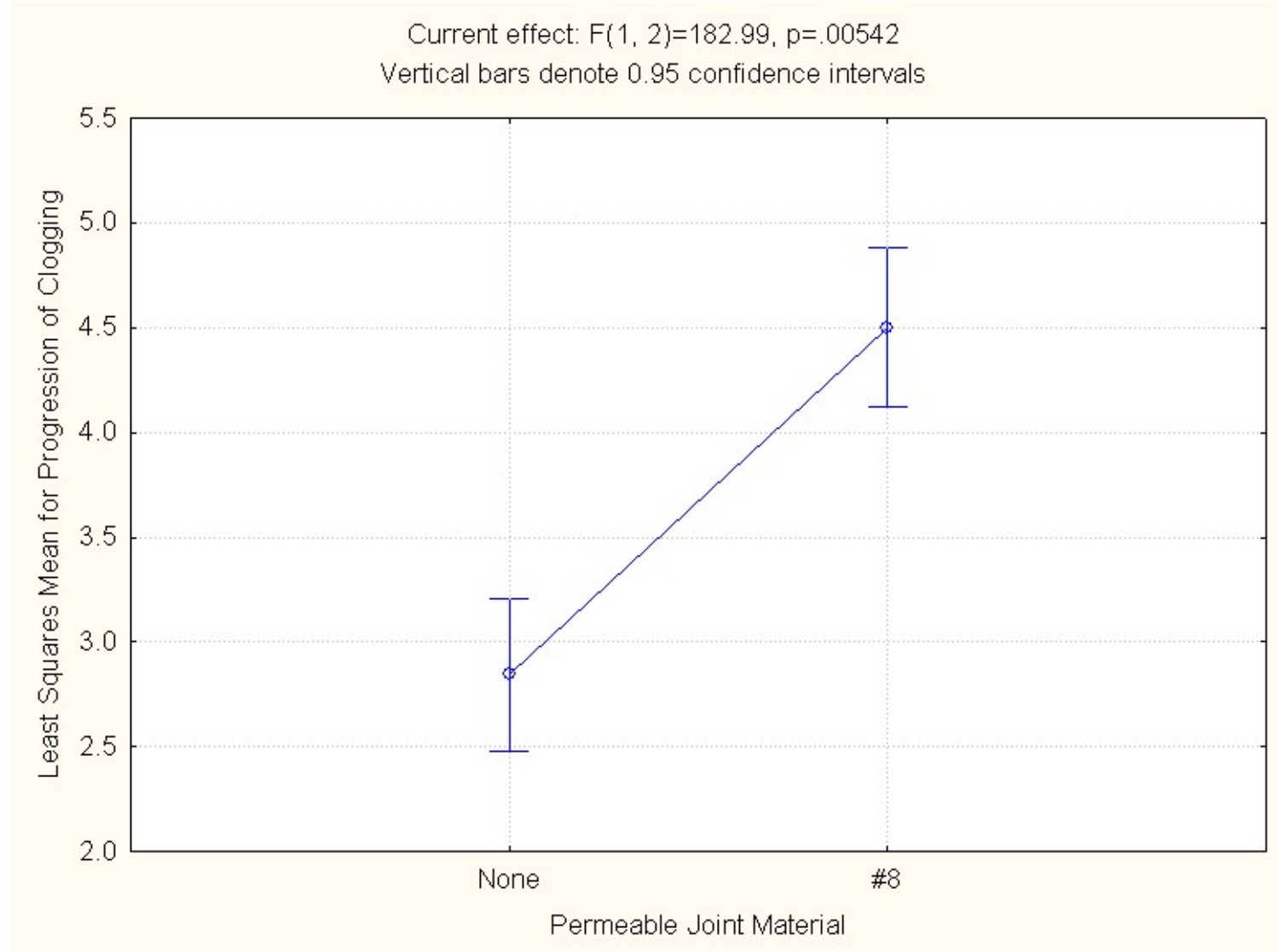

Figure 114: Analysis of variance of permeable joint material for progression of clogging

Figure 114 illustrates that the presence or absence of the permeable joint material does have a statistical significant effect on the slope of progression of clogging. The experiments conducted without the permeable joint material have a less steep slope than those with the \#8 AASHTO aggregate. This confirms the overall trend of the patterns seen in the experiments. In the experiments where the gaps are left empty, the clogging progresses at a slower rate, for the debris can fill up each gap before moving on to the next gap, but in the experiments where the gaps are filled with \#8 aggregate, the rate of clogging is faster. 


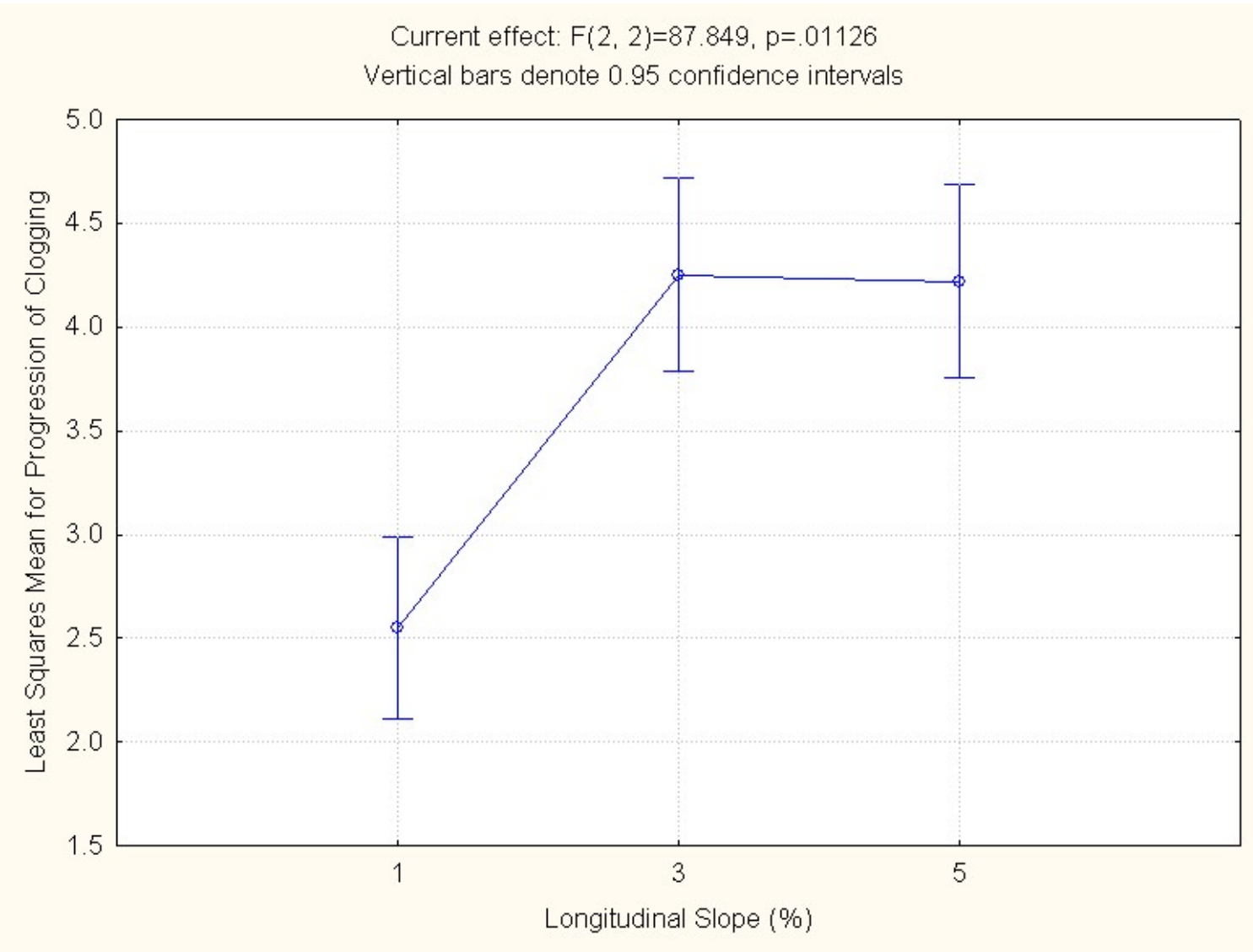

Figure 115: Analysis of variance of longitudinal slope for progression of clogging

Figure 115 illustrates the last analysis of variance for the progression of clogging and shows that the change of the longitudinal slope from $1 \%$ to $3 \%$ makes a statistical difference in the rate at which the surface clogged. This increase in the longitudinal slope was shown to increase the rate of the progression of clogging. The next increase, from $3 \%$ to $5 \%$, was not shown to be statistically significant in creating an effect on the overall trend of changes seen in the rate of the progression of clogging. 
Current effect: $F(2,2)=12.839, p=.07226$

Vertical bars denote 0.95 confidence intervals

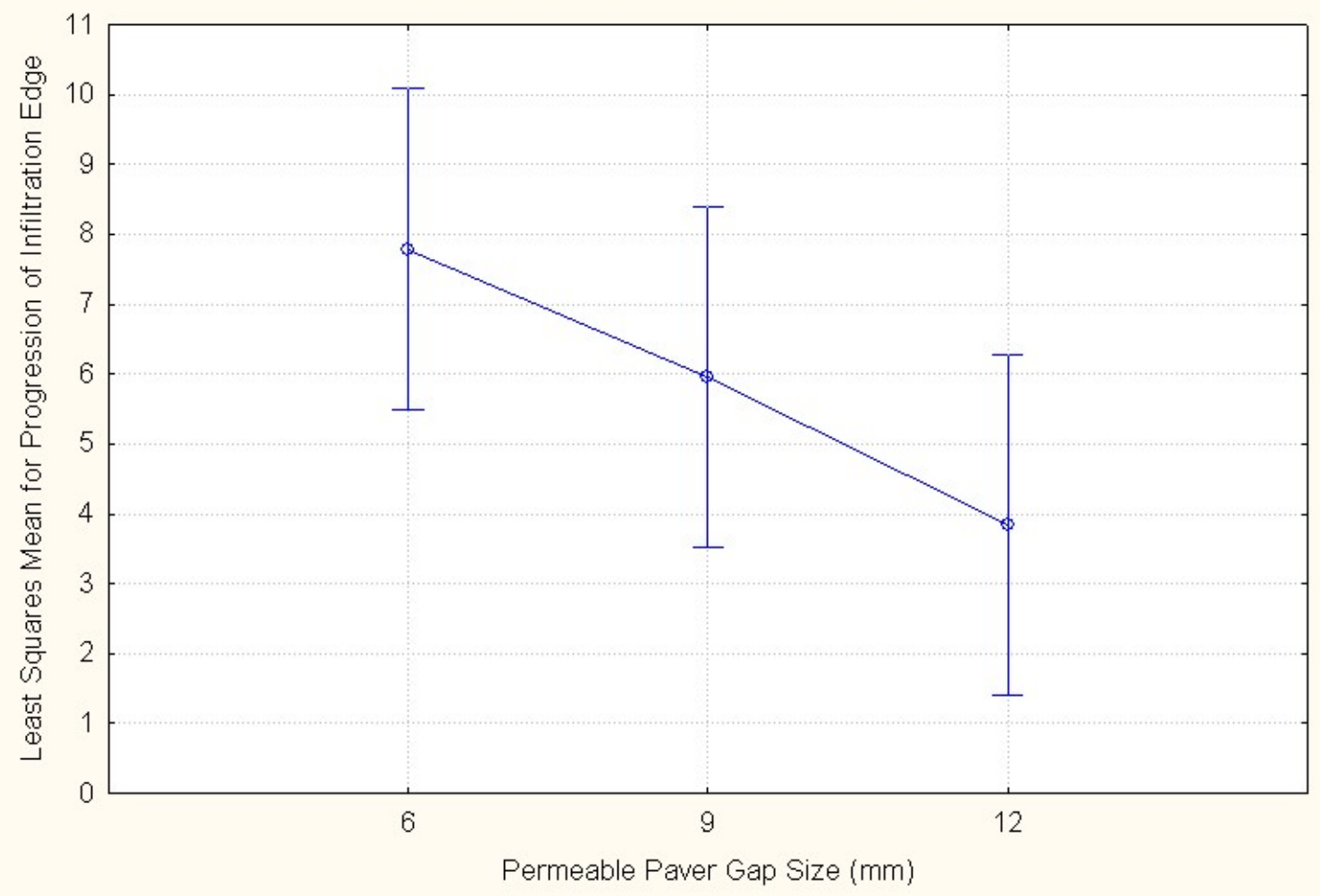

Figure 116: Analysis of variance of paver gap size for progression of infiltration edge

Figure 116 reveals no indication that the paver gap size has a statistically significant effect on the progression of the infiltration edge. In other words, there is a $10 \%$ chance that the decreasing trend seen in the plotted graph is caused by other factors. Although ANOVA's presentation must be acknowledged, comparisons made between two specific experiments can show variations caused by the change in gap size. 
Current effect: $F(1,2)=58.022, p=.01680$

Vertical bars denote 0.95 confidence intervals

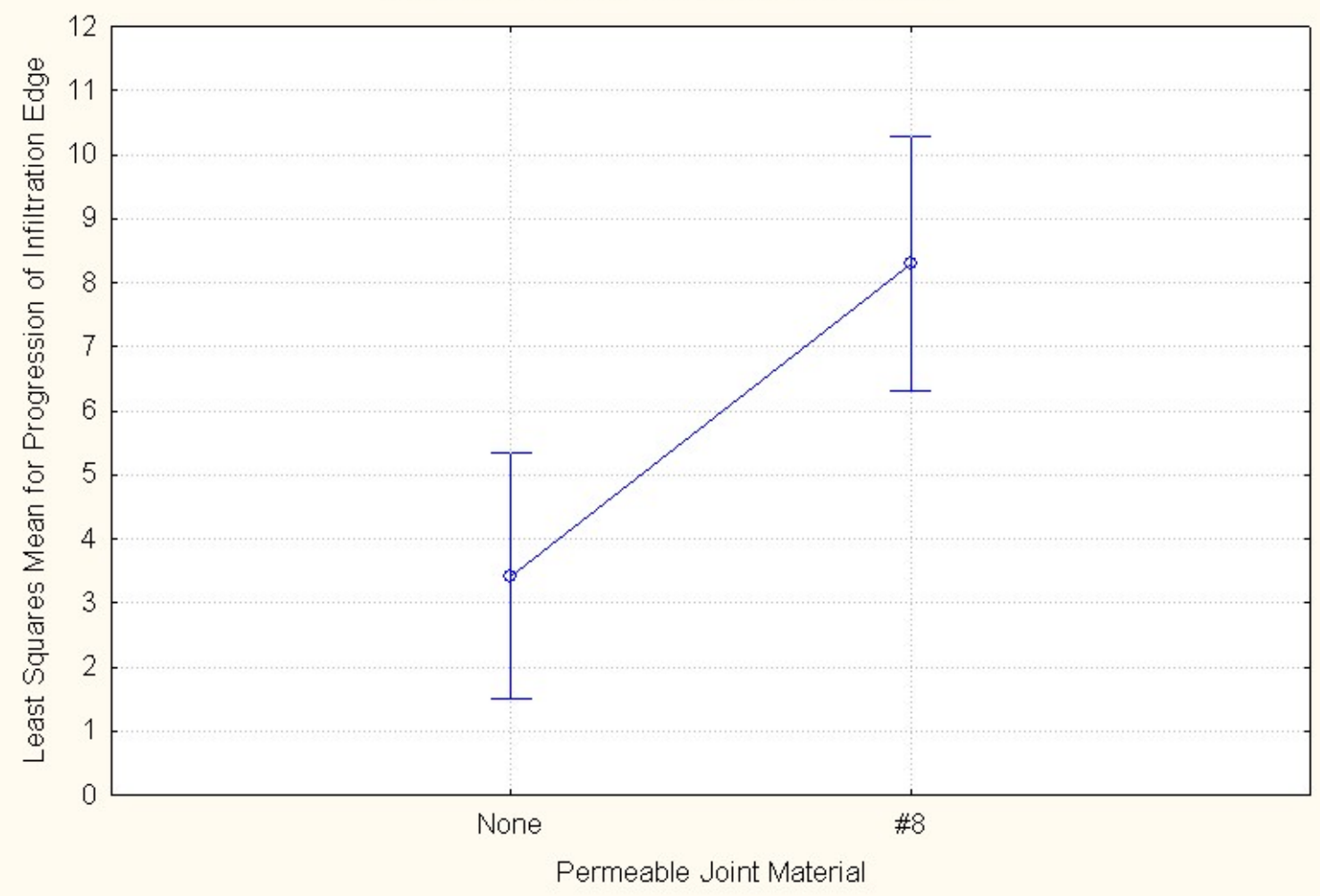

Figure 117: Analysis of variance of permeable joint material for progression of infiltration edge

Figure 117 shows the analysis of variance for the progression of the infiltration edge by permeable joint material. The analysis shows that the rate at which the progression of the infiltration edge changes is significantly different in experiments without the permeable joint material than those with the permeable joint material. As with the progression of clogging and also based on observation, the presence of permeable joint material significantly affects the performance of the system both initially and when the system clogs. 
Current effect: $F(2,2)=9.2783, p=.09729$

Vertical bars denote 0.95 confidence intervals

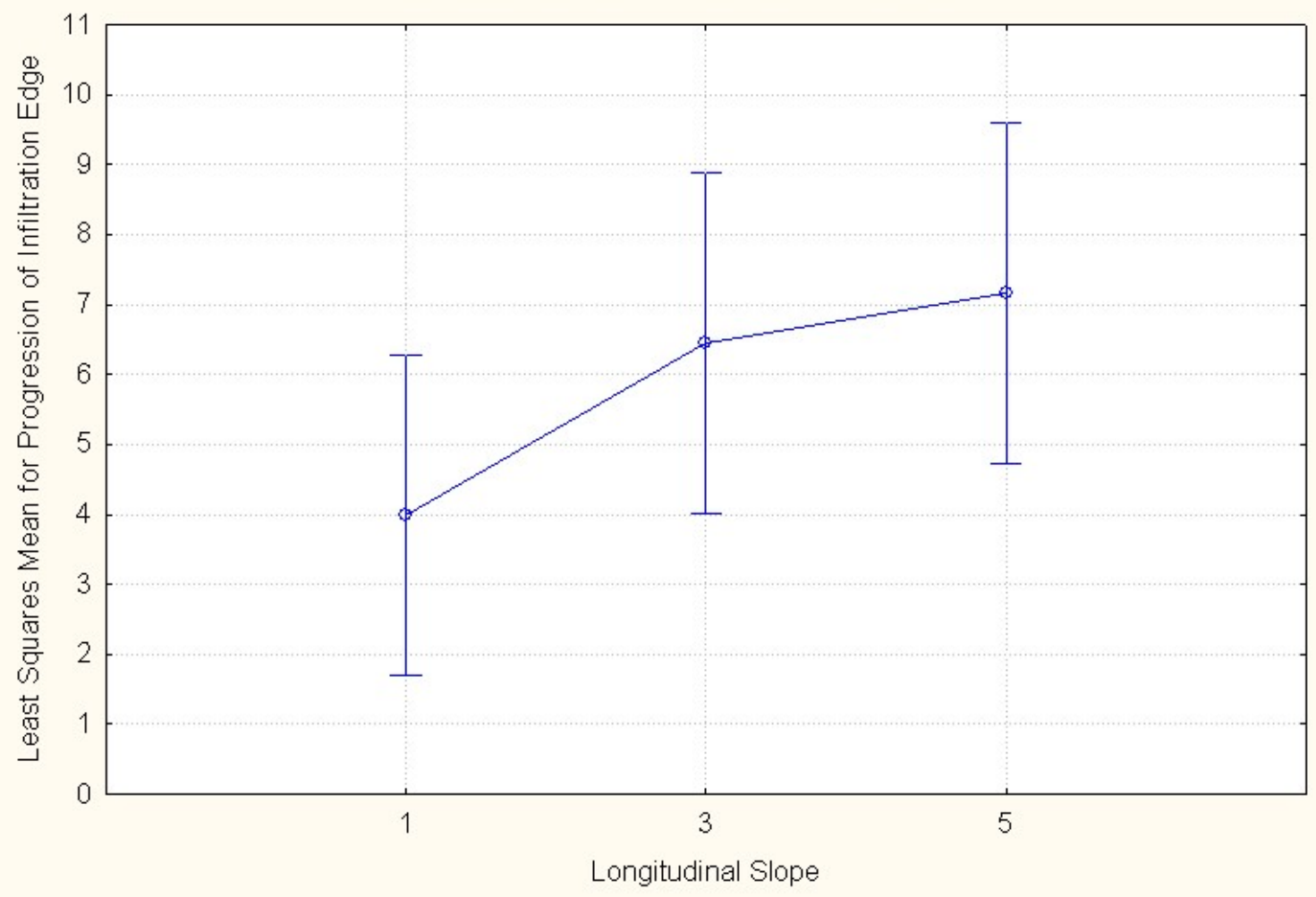

Figure 118: Analysis of variance of longitudinal slope for progression of infiltration edge

Figure 118 indicates that the effects of different longitudinal slopes on the rate at which the infiltration edge progresses are not statistically significant. However, as in previously observed patterns, the increase of longitudinal slope created a constant increase in the rate at which the infiltration edge progressed.

\subsection{Maintenance}

In order to accurately achieve the aims of the research project, the physical experiment module or the flume, had to be maintained and restored after each experiment. Maintenance was meant to restore the surface and the bedding layer of the flume to a pristine condition and would eliminate the undesired effects of ageing in the system. 
Maintenance can be carried out in various ways, using various tools and methods, and a detailed investigation is required to determine the effectiveness of each method and tool on the restoration of the GI's characteristics, which are crucial to keep the system in good working condition. However, this level of investigation for maintenance was not included in the work plan for this research project and the system was restored with methods that are neither practical nor feasible for full scale GI.

The maintenance carried out done during the experiments, including the sequence of events, was to use an industrial vacuum cleaner to remove any debris from the surface and the gaps in the pavers, removing the paver blocks, cleaning the fine particles of the blocks using a brush, removing the \#8 AASHTO aggregate from the bedding layer, replacing the bedding layer with clean aggregate, leveling the bedding layer and putting the pavers back in. Although full and in-depth analysis of the maintenance was not a part of the present research, it should be noted that valuable information was gained on some aspects of GI maintenance by repeating the process of cleaning and restoring the system 20 times in different conditions, including different extents of surface clogging after the experiments, and the use of three different paver products.

During the different maintenance activities on the flume, it was found that the using the industrial vacuum cleaner for cleaning the surface and the gaps is often more effective once the clogging debris had dried. This method also seemed more effective for those experiments where the down gradient segment was clogged with mostly organic debris. A dried clogged surface facilitates maintenance with a vacuum cleaner, but the period required for the GI to dry out depends heavily on the outside temperature. 
During the cleaning process, it was observed that in the experiments where the permeable joint material was not introduced to the gaps, the clogging debris, in particular the fine inorganic particles penetrated the bedding layer, while the presence of a permeable joint material somewhat stopped the penetration. 


\section{CONCLUSION}

\subsection{Introduction}

The rapid growth of cities and increasing urban areas has resulted in an increase in impervious surfaces in urban and suburban areas. This increase has created an imbalance in the natural hydrologic cycle and has caused a significant increase in the urban stormwater runoff as a result of precipitation on impervious surfaces. Stormwater generated in urban areas can carry pollutants, such as hydrocarbons, nutrients, metals, etc. which contaminate down gradient streams. This problem is multiplied in communities with combined sewer systems as their core sewer infrastructure. In many severe weather conditions, the runoff generated by rain causes these systems to overflow and dump untreated sewage in streams and rivers.

In order to solve the issue of CSOs, mimicking natural and undeveloped conditions, which includes creating pervious surfaces, may be a part of a bigger solution. Any solution that includes creating an environment where stormwater runoff can infiltrate to the ground close to the source is referred to as green infrastructure (GI).

GI can include many different practices, such as permeable pavements, bioretention cells, rain gardens and infiltration basins, all designed with the same goal in mind. Two GI stormwater control measures that use interlocking concrete pavers as their permeable surface were installed in the Louisville, KY CSO130 demonstration project in 
December 2011. During the time that they have been performing, and unlike any expectation of their performance and behavior, they have had to be maintained more than either the paver vendor had suggested or researchers had expected. The biggest issue that reduces the performance of this infrastructure and brings up the need for maintenance has been the surface clogging caused by the debris carried by stormwater runoff.

Poorer performance and the need for numerous maintenance treatments per year, in addition to the inefficiency of the maintenance, have caused the cost of green solutions to rise significantly. Using the current inefficient GI practices in Louisville’s CSO130, and studying them to determine the main causes of their defects reveals not only ways to reduce the need for both preventive and remedial maintenances, but also ways to improve the GI planned for other locations.

In Louisville’s CSO130 project, the clogging debris was sampled and analyzed during 5 separate events. Improving our understanding of the characteristics of the clogging debris can help in determining the factors that cause deterioration in performance. In the present study, using common analytical methods, such as testing for particle size distribution and organic content, the characteristics of the clogging debris were determined. It was also found that many other factors derived from the physical environment affected the performance of the permeable pavement system. In the light of this information the following hypothesis was written and used as the core of this research project:

The physical environment of permeable pavement systems, such as the installation slope, the size of the gaps in the interlocking concrete pavers and the 
permeable joint filling material, will affect the performance, maintenance needs and clogging patterns.

It was believed that determining the effect of these factors would help to advance the field in predicting the progression of surface clogging and help design a system to avoid or reduce the failures that it caused.

Achieving the goal and determining the effect of the variables stated in the hypothesis would also produce knowledge that could be used to optimize the criteria for choosing the paver product, optimizing and/or selecting the best locations for the GI, and designing the GI. With all these benefits, the outcome of the research could from the planning stages of a project provide a guideline plan for the frequency of the desired and required maintenance treatments for the GI.

In order to investigate the effects of the physical environment, a series of experiments were designed, the physical experiment module was constructed, and all the different aspects of the experiment needed to simulate a full scale GI in the laboratory environment and within practical limits were prepared. The module in the present study is called the flume; it was used to investigate the effects of change in longitudinal slope, paver gap size and permeable joint material on performance by assessing the progression of surface clogging. The flume was heavily instrumented to collect data, which would then facilitate an accurate assessment.

The collected data were analyzed in several steps, and the results of the analysis, along with the unique observations made during each experiment, were used as the basis from which to compare the different experiments and predict the extent of the effects of 
the factors related to the physical environment. At the end, using the results of the research project, two set of conclusions were drawn: one is a detailed comparison of two stages of the GI performance for each configuration and the other is a platform and a method that can be used to perform other experiments based on one GI's specific goals and with the best configuration for every GI using them.

In order to provide suggestions the effects of the variables in the physical environment were investigated separately. By combining these suggestions, one may be able to draw separate conclusions which are based on combinations of configurations.

\subsection{Gap Size}

The permeability of the interlocking concrete pavers is provided by the gaps formed once they are put together. When they are arranged in a sheet, the spacer lugs built into their molds, or the ones that are placed separately between the paver blocks, form a series of gaps that will allow runoff to infiltrate to the storage gallery and eventually to the native soil. The shape and size of the gaps between the pavers can influence the rate of infiltration and the clogging mechanism.

The upper limit of the gap size in the permeable paver s is bound to ADA requirements which states that the gap between interlocking concrete pavers must not exceed $12 \mathrm{~mm}$ ( $1 \frac{1}{2}$ inch). Hence, this size was used as the biggest gap size for the experiments. The minimum gap size considered for the present study was the gap size between the pavers found in Louisville’s CSO130 project. After consulting with an industry expert, a $9 \mathrm{~mm}$ gap was selected as a suitable gap size between these upper and lower limits (Antunes, 2013). 
With these gap sizes, it was expected that the surface clogging would be affected by changes in the size of the gaps in the permeable pavers; however, the extent of this effect was not known. Using the analysis of variance for progression of clogging, it was found that the rate at which surface clogging progresses, which can be used to assess the susceptibility of a configuration to surface clogging and its ability to tolerate the sediment carried by urban runoff, did not experience a statistically significant change when the gap increased from $6 \mathrm{~mm}$ to $9 \mathrm{~mm}$. The increase of the paver gap size to $12 \mathrm{~mm}$, however, did result in a statistically significant effect in this rate.

This means that the gap between the pavers does have an important effect on the performance of the system, and choosing the best gap size can minimize the need for maintenance. However, increasing the gap size is not necessarily a good solution. The optimum gap size, considering all 21 experiments, is overall the $12 \mathrm{~mm}$ gap size. Using the $12 \mathrm{~mm}$ gap size in the experiment has shown that the surface clogging progresses more slowly than it does with narrower gaps and this eventually provides more operational time before a GI needs to be maintained.

The analysis of variance for the progression of the infiltration edge with the three different gap sizes used in the experiment shows that the rate at which the infiltration edge progresses is not significantly different with a change in gap size. This rate essentially describes the speed at which different locations along the length of the flume become effective in infiltrating stormwater runoff and can be used to determine the lead time for maintenance. Although ANOVA suggests that the rate at which the infiltration edge progresses on the surface is not significantly different for different gap sizes, as the gap size increases a constant decrease is observed in the rate. 
Therefore the biggest gap size can not only tolerate surface clogging, but also takes more time to become operational further down the gradient location. This can be particularly useful for GI that are relatively long.

\subsection{Joint Filling Material}

Sediments that are smaller in size have a higher ratio of surface area to mass, and can therefore provide greater capacity for transporting heavy metals and nonpolar organics (Krein \& Schorer, 2000; Roesner \& Kidner, 2007). It has been proven that smaller particles in urban stormwater runoff (smaller than 100 micrometers) carry about $70 \%$ of the metal pollution and therefore impose a greater risk to the health of underground waters(Ellis \& Revitt, 1982).

Joint filling material is described as trapping the clogging material in the top 20 to $25 \mathrm{~mm}$ layer of the surface of the pavement (PICP, 2007), and therefore it will slow down the migration of polluted fines to the lower layers of the storage gallery. It is thus beneficial to have joint filling material, which helps to retain pollution.

Based on the results produced by ANOVA, in the presence of the permeable joint material, the rate of progression of clogging and the progression of infiltration edge changed significantly, compared to those experiments without the permeable joint material. Having a gap filled with \#8 AASHTO aggregate resulted in a significant increase of both rates. Therefore if improving water quality is among the goals of the GI project, capturing the debris carrying the pollutants at the surface can cost the GI its improved performance rates. This information can be used by those responsible in the planning and design of GI. 


\subsection{Longitudinal Slope}

The longitudinal slope of the GI installation can affect the performance of the GI. Analysis of variance in the rates of progression of clogging for the experiments has shown that the base slope, which is $1 \%$, experienced a significantly lower rate the progression of surface clogging. Increasing the installation slope from $1 \%$ to $3 \%$ was shown to significantly affect this rate, while further increasing the slope, from 3\% to $5 \%$ was shown not to significantly affect the rate at which surface clogging progressed.

With regard to the progression of infiltration edge, the analysis of variance shows that none of the changes in the slope resulted in a significant change in the rate. Although none of the changes was significant, the overall trend of the changes matched that of the individual experiments, and, with an increase in the slope, the rate at which the wetting front progresses on the surface also increased.

With the observed effect of the installation's longitudinal slope on the performance of the GI, the optimum location for any proposed GI must have a relatively flat surface. But if a steep location is unavoidable, the negative effect of the slope can be compensated for by increasing the gap size and eliminating the \#8 aggregate in the paver's gaps. Other methods such as creating speed bumps on the surface of the GI, which would essentially reduce the velocity of the runoff on the surface, may also become effective; but further investigation is required to determine the full effects of such remedies. 


\subsection{Maintenance}

The maintenance requirements for the flume were to restore the system to pre-test conditions. This was necessary to assure that the accuracy of the collected data would not deteriorate as the experiments proceeded. The maintenance requirements of a full scale stormwater control measure, however, are designed to improve the system's performance by reducing some of the effects on it of ageing. Another difference between the maintenance carried out on the flume and a full scale GI is that, due to the size of the flume, and the limitations of lab work, some options such as air pressure and sweeping with a truck were not practicable.

With the methods used to maintain the flume between the experiments, it was found that the power of the vacuum device can greatly influence the efficiency of the maintenance. This was concluded by the increased efficiency of the maintenance after a clogged filter was removed from the vacuum cleaner, which had reduced the suction power.

Using sediment traps has been suggested by MSD as an alternative to trap some of the sediment before it reaches the GI. Commonly, sediment traps work best for capturing inorganic fines that are heavier than water and would sink in the trap. However, the observations made during the experiments suggest that organics contribute greatly to the surface clogging, and therefore the use of a sediment trap at an up gradient location may not be very effective.

One of the other concerns for a permeable pavement GI is that the migration of fine particles to the lower layers of the storage gallery would decrease the exfiltration rate 
of the system to the native soil. The particles that are migrating to the lower layers cannot be removed using common maintenance methods and removing them from the system may be costly; however, with the use of permeable joint material fines were trapped close to the surface. One solution may be to remove the \#8 AASHTO aggregate as a part of maintenance, which would result in removing the fines as well.

Since the maintenance of a GI is an unavoidable and ongoing process, all aspects of the maintenance such as its cost must be considered prior to construction. Given the available long term operation and maintenance budget of the GI project, a configuration may be chosen that requires a very expensive maintenance exercise every 15 years or a very cheap one every year.

Choosing a location or developing an already chosen location to the specifics of a GI can significantly reduce the required long term maintenance. Erosion control, using proper separation for any onsite planting, the appropriate use of salting and sanding in the winter, a tree canopy over the GI, the traffic loading over the GI, tailoring the configuration to meet or limit the maintenance needs of a project, among other factors, can significantly affect the maintenance requirements of GI.

The best places for installing permeable pavement systems are parking lots, alleys, and remote locations where stormwater runoff is an issue but other factors either are absent or minimal.

\subsection{Recommendations}

Finally, it may be concluded that prior to the design and construction of a permeable pavement GI, many aspects that are not currently investigated must be 
included for study in the pre-design work. Tasks such as a thorough examination of the proposed site to determine items and events that might damage the GI must be noted and preventive measures based on these observations must be included.

After further developing the understanding of the aspects of the physical environment that affect the performance of GI, a points based system can be developed according to the extent of the effect/of damage from these factors, and a full list of these factors may be used as an itemized checklist to assess the priorities of the preconstruction work needed for each site.

\subsection{Future Research}

The flume experiment has been an investigation of the effects of the physical environment on surface clogging and the performance of various interlocking concrete pavements. Although this research has focused on the physical environment of the ICP GI systems, not all their aspects have been investigated and many other aspects and their effects still remain unknown. Using the methods, results, and conclusions of this research as a platform to continue and develop other research projects, many other unknown aspects of the work might be determined.

A summary of the factors that did not fit the scope of this study, or were not investigated due to the limitations of the study, is provided as an example and can be used to continue the path and contribute further to the field:

Some of the unknowns that can be investigated using the flume platform is the effects on performance of different shapes and laying methods of pavers. This can be as general as the overall shape of the paver block or as detailed as the effect of the shape of 
the spacer lugs on performance. Such investigations may also be useful for making recommendations for paver design and also as a tool in choosing the most suitable paver product.

Using sediment traps has been generally recommended; however, with the observed patterns for the sediment deposited on the GI's surface, a need was felt to determine the criteria for choosing the best sediment trap and in a similar study to the present one these criteria could be determined. The effectiveness of sediment traps in capturing organic debris, inorganic debris and a mix of both could be investigated with different mixtures of sediment to find the best methods of preventive maintenance.

Another topic that could be investigated using the same experimental approach is the effect of different rainfall rates and volumes of stormwater runoff. Construction of a limiting structure that would allow only a specific flow rate to the GI SCM of stormwater runoff might be helpful to maximize the performance and minimize the need for maintenance (Haselbach et al., 2006).

In the end, much is still needed to determine the best methods of maintaining a GI SCM. There is no maintenance plan that can be used for all GI, but a best maintenance plan surely exists for each GI, based upon its specific configuration and physical environment. The path for determining the best maintenance plan is yet unknown and may require many experiments.

This list of unknowns comprises only the factors that were discovered during and after the research and are based on the perception of one individual; many other aspects of the work may be determined for future research. 


\section{REFERENCES}

Abbott, C. L., \& Comino-Mateos, L. (2003). In-situ hydraulic performance of a permeable pavement sustainable urban drainage system. Water and Environment Journal, 17(3), 187-190. doi: 10.1111/j.1747-6593.2003.tb00460.x

AGF. (2000). Drainage Factsheet: Drain Filters and Envelopes. British Columbia: Ministery of Agriculture and Food.

Amirjani, Mahsa. (2010). Clogging of permeable pavements in semi-arid areas. (Master of Science), Delft University of Technology.

Antunes, Mark (2013, 01/09/2013). [Consulting with Industry Expert from EPHenry on Interlocking Concrete Pavers].

Aravena, J., \& Dussaillant, A. (2009). Storm-Water Infiltration and Focused Recharge Modeling with Finite-Volume Two-Dimensional Richards Equation: Application to an Experimental Rain Garden. Journal of Hydraulic Engineering, 135(12), 1073-1080. doi: doi:10.1061/(ASCE)HY.1943-7900.0000111

Arnold, Chester L., \& Gibbons, C. James. (1996). Impervious Surface Coverage: The Emergence of a Key Environmental Indicator. Journal of the American Planning Association, 62(2), 243-258. doi: 10.1080/01944369608975688

ASCE. (1992). Design and Construction of Urban Stormwater Management Systems ASCE manuals and reports engineering practices: no. 77: ASCE.

ASTM. (2007a). Standard Practices for Preserving and Transporting Soil Samples $D$ 4220 - 95. West Conshohocken, PA: ASTM International.

ASTM. (2007b). Standard Test Methods for Moisture, Ash, and Organic Matter of Peat and Other Organic Soils D 2974 - 07a. West Conshohocken, PA: ASTM International. 
ASTM. (2009). Standard Test Methods for Particle-Size Distribution (Gradation) of Soils Using Sieve Analysis D6913-04. West Conshohocken, PA: ASTM International.

ASTM. (2011). Standard Practice for Conducting and Interlaboratory Study to Determine the Precision of a Test Method E691 - 12. West Conshohocken, PA: ASTM International.

Balades, JD, Legret, M, \& Madiec, H. (1995). Permeable pavements: pollution management tools. Water Science and Technology, 32(1), 49-56.

Bean, E., Hunt, W., \& Bidelspach, D. (2007). Field Survey of Permeable Pavement Surface Infiltration Rates. Journal of Irrigation and Drainage Engineering, 133(3), 249-255. doi: doi:10.1061/(ASCE)0733-9437(2007)133:3(249)

Bean, Eban Z., Hunt, William F., \& Bidelspach, David A. (2007). Field survey of permeable pavement surface infiltration rates. Journal of Irrigation and Drainage Engineering, 133(3), 249-255. doi: 10.1061/(asce)0733-9437(2007)133:3(249)

Bean, Eban Zachary, Hunt, William Frederick, \& Bidelspach, David Alan. (2007). Evaluation of four permeable pavement sites in Easter North Carolina for runoff reduction and water quality impacts. Journal of Irrigation and Drainage Engineering, 133(6).

Berndtsson, Justyna Czemiel, Bengtsson, Lars, \& Jinno, Kenji. (2009). Runoff water quality from intensive and extensive vegetated roofs. Ecological Engineering, 35(3), 369-380. doi: 10.1016/j.ecoleng.2008.09.020

Booth, Derek B., \& Leavitt, Jennifer. (1999). Field Evaluation of Permeable Pavement Systems for Improved Stormwater Management. Journal of the American Planning Association, 65(3), 314.

Borst, Michael, Rowe, Amy A., Stander, Emilie K., \& O'Connor, Thomas P. (2010). Surface Infiltration Rates of Permeable Surfaces: Six Month Update (November 2009 through April 2010). EPA/600/R-10/083: Office of Research and Development.

Boucher, A. B., Tremwel, Terry K., \& Campbell, Kenneth L. (1995). Best management practices for water quality improvement in the Lake Okeechobee watershed. Ecological Engineering, 5(2-3), 341-356. doi: 10.1016/0925-8574(95)00031-3 
Brattebo, Benjamin O., \& Booth, Derek B. (2003). Long-term stormwater quantity and quality performance of permeable pavement systems. Water Research, 37(18), 4369-4376. doi: Doi: 10.1016/s0043-1354(03)00410-x

Brown, Daniel C. (2003). Managing stormwater with porous asphalt. Public Works, 134(8).

Brown, R., \& Borst, M. (2013). Assessment of Clogging Dynamics in Permeable Pavement Systems with Time Domain Reflectometers (TDRs). Journal of Environmental Engineering. doi: doi:10.1061/(ASCE)EE.1943-7870.0000734

Campbell-Scientific. (2012). CS616 and CS626 Water Content Reflectometers (pp. 142). Logan, Utah: Campbell Scientific Inc.

Campbell-Scientific. (2013a). CR1000 Measurement and Control System Operation's Manual $b$. Logan, Utah: Campbell Scientific.

Campbell-Scientific. (2013b). L107 and L108 Temperature Sensors $a$. Logan, Utah: Campbell Scientific.

Chopra, M., Kakuturu, S., Ballock, C., Spence, J., \& Wanielista, M. (2010). Effect of Rejuvenation Methods on the Infiltration Rates of Pervious Concrete Pavements. Journal of Hydrologic Engineering, 15(6), 426-433. doi: doi:10.1061/(ASCE)HE.1943-5584.0000117

Clark, Corrie, Adriaens, Peter, \& Talbot, F. Brian. (2008). Green Roof Valuation: A Probabilistic Economic Analysis of Environmental Benefits. Environmental Science \& Technology, 42(6), 2155-2161. doi: 10.1021/es0706652

Collins, Kelly A., Hunt, William F., \& Hathaway, Jon M. (2008). Evaluation of various types of permeable pavement with respect to water quality improvement and flood control. Paper presented at the 2nd National Low Impact Development Conference 2007: New and Continuing Applications, March 12, 2007 - March 14, 2007, Wilmington, NC, United states.

CWP. (2003). Impacts of Impervious Cover on Aquatic Systems. In C. f. W. Protection (Ed.), Watershed Protection Research Monograph No.1. Ellicott City, MD. 
Davis, Allen P, Shokouhian, Mohammad, Sharma, Himanshu, Minami, Christie, \& Winogradoff, Derek. (2003). Water quality improvement through bioretention: Lead, copper, and zinc removal. Water Environment Research, 73-82.

DECNY. (2012). Combined Sewer Overflow (CSO) Impact on Receiving Water and Recreational Activities. Retrieved 06.08.2012, from http://www.dec.ny.gov/chemical/48595.html

Deo, O., Sumanasooriya, M., \& Neithalath, N. (2010). Permeability Reduction in Pervious Concretes due to Clogging: Experiments and Modeling. Journal of Materials in Civil Engineering, 22(7), 741-751. doi: doi:10.1061/(ASCE)MT.1943-5533.0000079

Dietz, Michael. (2007). Low Impact Development Practices: A Review of Current Research and Recommendations for Future Directions. Water, Air, \& Soil Pollution, 186(1), 351-363. doi: 10.1007/s11270-007-9484-z

Dietz, Michael. (2011). Permeable Pavements for Stormwater Control.

Dietz, Michael E., \& Clausen, John C. (2005a). Saturation to Improve Pollutant Retention in a Rain Garden. Environmental Science \& Technology, 40(4), 1335-1340. doi: 10.1021/es051644f

Dietz, MichaelE, \& Clausen, JohnC. (2005b). A Field Evaluation of Rain Garden Flow and Pollutant Treatment. Water, Air, and Soil Pollution, 167(1-4), 123-138. doi: $10.1007 / \mathrm{s} 11270-005-8266-8$

DOJ. (1994). Nondiscrimination on the Basis of Disability in the State and Local Government Services; Public Accommodations and Commercial Facilities; Accessibility Standards. Washington, DC.

Dreelin, Erin A., Fowler, Laurie, \& Ronald Carroll, C. (2006). A test of porous pavement effectiveness on clay soils during natural storm events. Water Research, 40(4), 799-805. doi: 10.1016/j.watres.2005.12.002

Dunne, Thomas, \& Leopold, Luna. (1978). Water in environmental planning New York: W. H. Freeman \& Co. 
Ehsaei, Amirhossein, \& Rockaway, Thomas D. (under review). Examination of empirical evidence and refining maintenance techniques for GI. Journal of Environmental Engineering.

El Samrani, A. G., Lartiges, B. S., Ghanbaja, J., Yvon, J., \& Kohler, A. (2004). Trace element carriers in combined sewer during dry and wet weather: an electron microscope investigation. Water Research, 38(8), 2063-2076. doi:

10.1016/j.watres.2004.01.029

El Samrani, A. G., Lartiges, B. S., \& Villiéras, F. (2008). Chemical coagulation of combined sewer overflow: Heavy metal removal and treatment optimization. Water Research, 42(4-5), 951-960. doi: 10.1016/j.watres.2007.09.009

Ellis, J., \& Revitt, D. M. (1982). Incidence of Heavy Metals in Street Surface Sediments: Solubility and Grain Size Studies. Water, Air, \& Soil Pollution, 17(1), 87-100.

Fassman, Elizabeth A, \& Blackbourn, Samuel. (2010). Urban runoff mitigation by a permeable pavement system over impermeable soils. Journal of Hydrologic Engineering, 15(6), 475-485.

Fassman, Elizabeth A., \& Blackbourn, Samuel D. (2011). Road Runoff Water-Quality Mitigation by Permeable Modular Concrete Pavers. Journal of Irrigation and Drainage Engineering, 137(11), 720-729. doi: 10.1061/(asce)ir.19434774.0000339

Field, R., Sullivan, D., \& Tafuri, A.N. (2003). Management of Combined Sewer Overflows: Taylor \& Francis.

Gerrits, Christopher, \& James, William. (2002). Restoration of infiltration capacity of permeable pavers. Paper presented at the 9th International Conference on Urban Drainage, Portland, Oregon.

González-Angullo, N., Castro, D., Rodríguez-Hernández, J., \& Davies, J. W. (2008). Runoff infiltration to permeable paving in clogged conditions. Urban Water Journal, 5(2), 117-124. doi: 10.1080/15730620701723538

Grote, K., Hubbard, S., Harvey, J., \& Rubin, Y. (2005). Evaluation of infiltration in layered pavements using surface GPR reflection techniques. Journal of Applied Geophysics, 57(2), 129-153. doi: 10.1016/j.jappgeo.2004.10.002 
Grout, Hélène, Wiesner, Mark R., \& Bottero, Jean-Yves. (1999). Analysis of Colloidal Phases in Urban Stormwater Runoff. Environmental Science \& Technology, 33(6), 831-839. doi: 10.1021/es980195z

Gunderson, Jeff, Roseen, Robert, Janeski, Todd, Houle, Jamie, \& Simpson, Michael. (2011). Economical CSO Management. Stormwater, April 2011.

Hamilton, R. S., Revitt, D. M., \& Warren, R. S. (1984). Levels and physico-chemical associations of $\mathrm{Cd}, \mathrm{Cu}, \mathrm{Pb}$ and $\mathrm{Zn}$ in road sediments. Science of The Total Environment, 33(1-4), 59-74. doi: 10.1016/0048-9697(84)90381-4

Harwood, Robert, \& Saul, Adrian J. (2001). Combined Sewer Overflows. Retrieved 03/09/2011, from http://www.fluidcodes.com/pdf/Sewer\%20Overflows.pdf

Haselbach, Liv M., Valavala, Srinivas, \& Montes, Felipe. (2006). Permeability predictions for sand-clogged Portland cement pervious concrete pavement systems. Journal of Environmental Management, 81(1), 42-49. doi: 10.1016/j.jenvman.2005.09.019

Haster, Thomas W, \& James, Wesley P. (1994). Predicting sediment yield in storm-water runoff from urban areas. Journal of Water Resources Planning and Management, 120(5), 630-650.

Houle, Kristopher M. (2008). Winter performance assessment of permeable pavements. University of New Hampshire.

Hsieh, Chi-hsu, \& Davis, Allen P. (2005). Evaluation and optimization of bioretention media for treatment of urban storm water runoff. Journal of Environmental Engineering, 131(11), 1521-1531.

Hunt, W., Smith, J., Jadlocki, S., Hathaway, J., \& Eubanks, P. (2008). Pollutant Removal and Peak Flow Mitigation by a Bioretention Cell in Urban Charlotte, N.C. Journal of Environmental Engineering, 134(5), 403-408. doi: doi:10.1061/(ASCE)0733-9372(2008)134:5(403)

Hvitved-Jacobsen, Thorkild. (1982). The impact of combined sewer overflows on the dissolved oxygen concentration of a river. Water Research, 16(7), 1099-1105. doi: 10.1016/0043-1354(82)90125-7 
Jaffe, Martin, Zellner, Moira, Minor, Emily, Gonzales-Meler, Miquel, Cotner, Lisa, Massey, Dean, . . Miller, Brian. (2010). A Review of Selected Practices and State Programs, A draft report to The Illinois Environmental Protection Agency: University of Illinois at Chicago, Chicago Metropolitan Agency for Planning, Center for Neighborhood Technology,University of Illinois at UrbanaChampaign.

Kayhanian, Masoud, Rasa, Ehsan, Vichare, Akshay, \& Leatherbarrow, Jon E. (2008). Utility of suspended solid measurements for storm-water runoff treatment. Journal of Environmental Engineering, 134(9), 712-721.

Kevern, John. (2010). Maintenance and repair options for pervious concrete. Paper presented at the 2010 International Low Impact Development ConferenceRedefining Water in the City.

Krein, Andreas, \& Schorer, Marcel. (2000). Road runoff pollution by polycyclic aromatic hydrocarbons and its contribution to river sediments. Water Research, 34(16), 4110-4115. doi: http://dx.doi.org/10.1016/S0043-1354(00)00156-1

Legret, M., Colandini, V., \& Le Marc, C. (1996). Effects of a porous pavement with reservoir structure on the quality of runoff water and soil. Science of The Total Environment, 189-190(0), 335-340. doi: http://dx.doi.org/10.1016/0048$\underline{9697(96) 05228-X}$

Leisenring, Marc, Clary, Jane, Lawler, Ken, \& Hobson, Paul. (2011). International Stormwater Best Management Practices (BMP) Database Pollutant Category Summary: Solids (TSS, TDS and Turbidity): BMPDatabase.org.

Mackisack, Margaret, \& Pywell, C. (1994). Managing Block Paver Gap Performance: A Modelling Solution. Paper presented at the Proceedings, Second International Workshop on Concrete Block Paving, Oslo, Norway.

Mackisack, MS. (1996). Effect of shape and laying strategies on gap performance of segmental block pavers. Pave Israel.

Marsalek, J., Rochfort, Q., Mayer, T., Servos, M., Dutka, B., \& Brownlee, B. (1999). Toxicity testing for controlling urban wet-weather pollution: advantages and limitations. Urban Water, 1(1), 91-103. doi: 10.1016/s1462-0758(99)00006-0 
Mata, Luis Alexander. (2008). Sedimentation of pervious concrete pavement systems. North Carolina State University, Releigh, NC.

Moffa, P.E. (1997). The Control and Treatment of Combined Sewer Overflows: Wiley.

MSD. (2010a). Federal Concent Decree for Prject WIN. Retrieved 11.20.2012, from http://msdprojectwin.org/About-Us/Federal-Consent-Decree.aspx

MSD. (2010b). Green Infrastructure Incentives and Savings. Louisville, KY.

MSD. (2011). Crosscurrents: MSD shifting gray to green. Retrieved 03/08/2011, from http://www.msdlouky.org/aboutmsd/cross/cc_winter11.pdf

MSD. (2012). Project WIN (Waterway Improvements Now). Retrieved 6/5/2012, from http://www.msdprojectwin.org/Projects.aspx

NJ-DEP. (2004). New Jersey Stormwater BMP Manual Standards for Pervious Paving Systems: New Jerseu Department of Environmental Protection.

Osei, K., Andoh, R. Y. G., Brown, L., \& Gwinn, A. An Innovative and Rapid Method of Assessing Particle Shape and Size in Stormwater Runoff World Environmental and Water Resources Congress 2010 (pp. 3737-3744).

PaveDrain. (2013). Installation \& Maintenance. Retrieved 05/30/2013, from http://pavedrain.com/installation-maintenance/

PICP. (2007). The role of joint filling materials in permeable interlocking concrete pavements. Retrieved 05/21/2013, from http://www.icpi.org/sites/default/files/07_May_RoleofJointFill.pdf

Pitt, Robert. (2004). The National Stormwater Qaulity Database (NSQD, Version 1.1). Retrieved from: http://rpitt.eng.ua.edu/Research/ms4/Paper/Mainms4paper.html

Pitt, Robert, Maestre, Alex, \& Morquecho, Renee. (2011). The National Stormwater Qaulity Database (NSQD, Version 3.1).

Pratt, C. J., Mantle, J. D. G., \& Schofield, P. A. (1995). UK research into the performance of permeable pavement, reservoir structures in controlling 
stormwater discharge quantity and quality. Water Science and Technology, 32(1), 63-69. doi: http://dx.doi.org/10.1016/0273-1223(95)00539-Y

Rigid. (2013). 3000 PSI/ 2.6 GPM Premium Pressure Washer. Retrieved 05/21/2013, from http://www.ridgid.com/Tools/rd80746-Pressure-Washer/en/index.htm

Roesner, Larry A., \& Kidner, Elizabeth M. (2007). Improved protocol for classification and analysis of stormwater-borne solids. Proceedings of the Water Environment Federation, 2007(13), 5539-5566. doi: 10.2175/193864707787969324

Roseen, Robert M., Janeski, Todd V., Simpson, Michael, Houle, James J., Gunderson, Jeff, \& Ballestero, Thomas P. (2012). Economic and Adaptation Benefits of Low Impact Development. Paper presented at the 2011 Low Impact Development Symposium, Philadelphia.

Sansalone, J, Kuang, X, Ying, G, \& Ranieri, V. (2011). Filtration and clogging of permeable pavement loaded by urban drainage. Water research.

Scholz, Miklas, \& Grabowiecki, Piotr. (2007). Review of permeable pavement systems. Building and Environment, 42(11), 3830-3836. doi:

10.1016/j.buildenv.2006.11.016

Shammaa, Y., Zhu, D. Z., \& Labatiuk, C. W. (2002). Effectiveness of dry ponds for stormwater total suspended solids removal. Canadian Journal of Civil Engineering, 29(2), 316-324.

Shuster, W. D., Bonta, J., Thurston, H., Warnemuende, E., \& Smith, D. R. (2005). Impacts of impervious surface on watershed hydrology: A review. Urban Water Journal, 2(4), 263-275. doi: 10.1080/15730620500386529

Siriwardene, N. R., Deletic, A., \& Fletcher, T. D. (2007). Clogging of stormwater gravel infiltration systems and filters: Insights from a laboratory study. Water Research, 41(7), 1433-1440. doi: 10.1016/j.watres.2006.12.040

Smith, David R. (2011). Permeable Interlocking Concrete Pavements (Fourth Edition ed.): Interlocking Concrete Pavement Institute (ICPI).

Strecker, E., Quigley, M., Urbonas, B., Jones, J., \& Clary, J. (2001). Determining Urban Storm Water BMP Effectiveness. Journal of water resources planning and 
management, 127(3), 144-149. doi: doi:10.1061/(ASCE)0733-

9496(2001)127:3(144)

Taylor, J.K., \& Cihon, C. (2004). Statistical Techniques for Data Analysis, Second Edition: Taylor \& Francis.

Tennis, Paul D, Leming, Michael L, \& Akers, David J. (2004). Pervious concrete pavements: Portland Cement Association Skokie, IL.

Topp, G. C., Davis, J. L., \& Annan, A. P. (1980). Electromagnetic determination of soil water content: Measurements in coaxial transmission lines. Water Resources Research, 16(3), 574-582. doi: 10.1029/WR016i003p00574

Tyner, JS, Wright, WC, \& Dobbs, PA. (2009). Increasing exfiltration from pervious concrete and temperature monitoring. Journal of environmental management, 90(8), 2636-2641.

Urbonas, Ben R. (2003). Effectiveness of Urban Stormwater BMPs in Semi-Arid Climates. Paper presented at the Experience with Best Management Practices in Colorado, Colorado.

US-Government. (2000). National pollutant discharge elimination system. Retrieved 03/08/2011, from http://www.law.cornell.edu/uscode/33/1342(q).html

Title 40--Protection of Environment (2012).

USEPA. (1983). Results of the National Urban Runoff Program: Volume 1 - Final Report. In W. P. Division (Ed.). Washington, DC: U.S. Environmental Protection Agency.

Combined Sewer Overflow (CSO) Control Policy; Notice (1994).

USEPA. (1998). National Water Quality Inventory: 1998 Report to Congress. In U. S. E. P. Agency (Ed.): Office of Water.

USEPA. (2003a). Protecting Water Quality from Urban Runoff: U.S. Environmental Protection Agency. 
USEPA. (2003b). Urban Nonpoint Source Fact Sheet. Retrieved 11.15.2012, from http://water.epa.gov/polwaste/nps/urban_facts.cfm

USEPA. (2004). National Water Quality Inventory: Report to Congress. In U. S. E. P. Agency (Ed.): Office of Water.

USEPA. (2007). Reducing Stormwater Costs through Low Impact Development (LID) Strategies and Practices. (EPA 841-F-07-006). Washington DC.

USEPA. (2008). Combined Sewer Overflows. Retrieved 03/08/2011, from http://cfpub.epa.gov/npdes/home.cfm?program_id=5

USEPA. (2010). Permeable Interlocking Concrete Pavement Fact Sheet. from http://cfpub.epa.gov/npdes/stormwater/menuofbmps/index.cfm?action=browse\& $\underline{\text { Rbutton }=\text { detail } \& b m p=136}$

USEPA. (2012). National Enforcement Initiatives for Fiscal Years 2008-2010: Clean Water Act: Municipal Sewer Overflows (CSOs and SSOs). Retrieved 11.20.2012, from http://www.epa.gov/oecaerth/data/planning/priorities/cwamso.html

USGS. (2012). The Water Cycle. Retrieved 11.15.2012, from http://ga.water.usgs.gov/edu/watercycle.html

Van Heystraeten, G, \& Moraux, C. (1990). Ten years'experience of porous asphalt in belgium. Transportation Research Record(1265).

Vancura, Mary E, MacDonald, Kevin, \& Khazanovich, Lev. (2012). Location and depth of pervious concrete clogging material before and after void maintenance with common municipal utility vehicles. Journal of Transportation Engineering, 138(3), 332-338.

Vaze, J., \& Chiew, F. (2004). Nutrient Loads Associated with Different Sediment Sizes in Urban Stormwater and Surface Pollutants. Journal of Environmental Engineering, 130(4), 391-396. doi: doi:10.1061/(ASCE)07339372(2004)130:4(391) 
Viklander, . (1998). Particle Size Distribution and Metal Content in Street Sediments. Journal of Environmental Engineering, 124(8), 761-766. doi: doi:10.1061/(ASCE)0733-9372(1998)124:8(761)

Virginia-DCR. (2011). Permeable Pavement, Version 1.8. Virginia, USA.

Wastewater-Treatment-Division. (2011). Green stormwater infrastructure for combined sewer overflow control. Retrieved 6.9.2012, from http://www.kingcounty.gov/environment/wastewater/CSO/Controlling/Reducing/ GSI.aspx

Welker, A., Jenkins, J., McCarthy, L., \& Nemirovsky, E. (2012). Examination of the Material Found in the Pore Spaces of Two Permeable Pavements. Journal of Irrigation and Drainage Engineering. doi: doi:10.1061/(ASCE)IR.19434774.0000543

Wild, T., \& Davis, A. (2009). Simulation of the Performance of a Storm-Water BMP. Journal of Environmental Engineering, 135(12), 1257-1267. doi: doi:10.1061/(ASCE)EE.1943-7870.0000106

Wilson, E.B. (2012). An Introduction to Scientific Research: Dover Publications, Incorporated.

Wossink, GAA, \& Hunt, Bill. (2003). The economics of structural stormwater BMPs in North Carolina: Water Resources Research Institute of the University of North Carolina.

Yang, Jing, \& Jiang, Guoliang. (2003). Experimental study on properties of pervious concrete pavement materials. Cement and Concrete Research, 33(3), 381-386. doi: 10.1016/s0008-8846(02)00966-3 


\section{APPENDIX A}

\section{Data logger programming}

The program written for CR1000 data logger (reference to Campbell Scientific, reference to Campbell Scientific CR1000 online manual) is set out below:

'CR1000

'Created by Short Cut (2.8)

'Declare Variables and Units

Public BattV

Public VW_1

Public PA_uS_1

Public VW_2

Public PA_uS_2

Public T107_C

Public VW_3

Public PA_uS_3

Public VW_4

Public PA_uS_4

Public VW_5

Public PA_uS_5

Public VW_6

Public PA_uS_6

Public VW_7

Public PA_uS_7 


$$
\begin{aligned}
& \text { Units BattV=Volts } \\
& \text { Units PA_uS_1=uSec } \\
& \text { Units PA_uS_2=uSec } \\
& \text { Units T107_C=Deg C } \\
& \text { Units PA_uS_3=uSec } \\
& \text { Units PA_uS_4=uSec } \\
& \text { Units PA_uS_5=uSec } \\
& \text { Units PA_uS_6=uSec } \\
& \text { Units PA_uS_7=uSec }
\end{aligned}
$$

\section{'Define Data Tables}

DataTable(Flume,True,-1)

DataInterval $(0,10$, Sec,10)

Sample(1,VW_1,FP2)

Sample(1,PA_uS_1,FP2)

Sample(1,VW_2,FP2)

Sample(1,PA_uS_2,FP2)

Sample(1,VW_3,FP2)

Sample(1,PA_uS_3,FP2)

Sample(1,VW_4,FP2)

Sample(1,PA_uS_4,FP2)

Sample(1,VW_5,FP2)

Sample(1,PA_uS_5,FP2)

Sample(1,VW_6,FP2)

Sample(1,PA_uS_6,FP2)

Sample(1,VW_7,FP2)

Sample(1,PA_uS_7,FP2) 
Sample(1,T107_C,FP2)

EndTable

'Main Program
BeginProg

$\operatorname{Scan}(10, \operatorname{Sec}, 1,0)$

'Default Datalogger Battery Voltage measurement BattV

Battery(BattV)

'CS616 Water Content Reflectometer measurements VW_1 and PA_uS_1

CS616(PA_uS_1,1,1,1,1,1,0)

$V W \_1=-0.0663+\left(-0.0063 * P A \_u S \_1\right)+\left(0.0007 * P A \_u S \_1 \wedge 2\right)$

'CS616 Water Content Reflectometer measurements VW_2 and PA_uS_2

CS616(PA_uS_2,1,2,1,1,1,0)

$V W \_2=-0.0663+\left(-0.0063 * P A \_u S \_2\right)+\left(0.0007 * P A \_u S \_2 \wedge 2\right)$

'107 Temperature Probe measurement T107_C

Therm107(T107_C,1,8,1,0,_60Hz,1,0)

'CS616 Water Content Reflectometer measurements VW_3 and PA_uS_3

CS616(PA_uS_3,1,3,1,1,1,0)

$V W \_3=-0.0663+\left(-0.0063 * P A \_u S \_3\right)+\left(0.0007 * P A \_u S \_3 \wedge 2\right)$

'CS616 Water Content Reflectometer measurements VW_4 and PA_uS_4

CS616(PA_uS_4,1,4,3,1,1,0)

$V W \_4=-0.0663+\left(-0.0063 * P A \_u S \_4\right)+\left(0.0007 * P A \_u S \_4 \wedge 2\right)$

'CS616 Water Content Reflectometer measurements VW_5 and PA_uS_5

CS616(PA_uS_5,1,5,3,1,1,0)

$V W \_5=-0.0663+\left(-0.0063 * P A \_u S \_5\right)+\left(0.0007 * P A \_u S \_5 \wedge 2\right)$ 
'CS616 Water Content Reflectometer measurements VW_6 and PA_uS_6 CS616(PA_uS_6,1,6,3,1,1,0)

$V W \_6=-0.0663+\left(-0.0063 * P A \_u S \_6\right)+\left(0.0007 * P A \_u S \_6 \wedge 2\right)$

'CS616 Water Content Reflectometer measurements VW_7 and PA_uS_7

CS616(PA_uS_7,1,7,3,1,1,0)

$V W \_7=-0.0663+\left(-0.0063 * P A \_u S \_7\right)+\left(0.0007 * P A \_u S \_7 \wedge 2\right)$

'Call Data Tables and Store Data

CallTable(Flume)

NextScan

EndProg 
APPENDIX B

\section{Test Day ‘To do’ list}

This section is intended to guide researchers and experimenter in reproducing the conditions used in the present study and make similar arrangements to investigate the same or some other aspects of the permeable pavement systems used in GI. It is essential for this research to create a path that others can follow by which the work can continue. To this end, a complete list is provided below of the tasks carried out during the experiments and the materials used to create the experimental module, which can also be used as a step by step guide. The tasks are split into groups, based on the defined milestones of the experiment. The guidance provided in this section can be used along with the text in all chapters.

\section{Construction of the flume}

The following materials were used to construct the testing module:

- Pressure treated plywood is the main material for constructing the flume: $19.05 \mathrm{~mm}$ ( $3 / 4 \mathrm{inch}$ ) thick, $122 \mathrm{~cm}$ by $244 \mathrm{~cm}$ ( 4 ft. by $8 \mathrm{ft}$.) sheets. Each side of the flume is constructed by attaching two of these sheets together to provide additional support and prevent bending.

- Additional wooden beams, small sections $5 \mathrm{~cm}$ by $15 \mathrm{~cm}$ (2 in. by 6 in.) are used on the outside of the side walls to prevent bending by the lateral forces.

- In addition to the additional wooden beams, two long clamps are used to support the flume laterally. 
- The flume's bottom panel has check dams, which are $10 \mathrm{~cm}$ high. Three walls in total are equally spaced from each other and both ends of the flume. With a check valve installed directly in front of the check dams, water samples can be drawn from the flume.

- Steel L brackets are used to attach the side walls to the bottom section of the flume.

- Truck bed liner is used to cover the interior of the flume and water proof the joints, to minimize the damage done by letting water stand inside the flume.

- High quality aquarium sealant is used to seal the joints of the flume and prevent leaks.

- Wood screws and wood glue are used for assembling the flume.

- Cinderblocks are used as the staging; 5-6 saw horses can replace the cinderblocks. The cinderblocks can be replaced with sawhorses; however, the weight rating must be checked and used to determine the number of sawhorses required.

- A pallet jack is used to lift the flume and adjust the slope of the installation.

- Power tools are necessary for cutting the plywood, etc.

- The number of pavers is based on the available surface area, the dimensions of the flume, and the dimensions of the paver blocks.

- A masonry saw is needed for cutting the pavers for the sides in half.

- Sieves, mulch, sediment, weights, and balances are used to prepare the sediment. 
- TDRs and a thermistor are used as the primary instruments to monitor the performance of the flume and collect performance data.

- A data logger that is compatible with the instruments is used to collect and record the monitoring data of the performance

- A computer is used to monitor the data in real time.

- A tank is used to store the stormwater runoff used for the test.

- A stirrer is installed in the stormwater tank to keep the initial level of TSS suspended for sampling purposes.

- A sewage pump is used to transfer the water from the tank to the feeder section of the flume. The flow rate of the pump is adjusted with a valve. The valve is then glued to avoid any accidental changes in the flow rate.

- A feeder structure is needed to equally distribute the polluted water after adding the sediment along the width of the flume.

- An effluent structure must be designed to transfer the water from the flume and discharge it into a nearby trench.

The first milestone in the experiment is going through the pre test checklist for the first time, after which the experiment can be run for the first time. Prior to the first running of the flume, some tests should be conducted to collect performance and failure data, which will then be used in the analysis. 


\section{Schedule and order of work for the test day}

\section{Pre-test steps}

After completing the construction of the experiment module, the flume must be filled up with AASHTO aggregates \#57and \#8. Aggregate \#57 will form the storage gallery and aggregate \#8 will be the bedding layer. In order to minimize the effect of the solids attached to the aggregate, all the stones used in both the storage gallery and the bedding layer must be washed thoroughly in advance with a 3000 psi pressure washer.

Using aggregate \#57, the first $25.4 \mathrm{~cm}$ of the flume is filled up with stone. The monitoring instruments are all placed at this level in locations as set out in Chapter 5. The storage gallery is then topped up with another $10.2 \mathrm{~cm}$ of \#57. Then a $5.1 \mathrm{~cm}$ bedding layer is created using aggregate \#8. Finally the surface of the bedding layer is compacted using a dead blow hammer and leveled. Pavers are placed on the bedding layer and their slope is checked.

Before the first run, the flume is tested for leaks. The first test is uses tap water. This test is also used to flood the flume and create complete failure data. Prior to the first run and with the same configurations as those used in the first run, water is pumped to the flume started at a rate of 35 liters per minute. As the test continues, the flow rate is slowly increased to the maximum, at which the flume experiences a rapid increase in the ponding of stormwater runoff, followed, by complete failure of the system. With the same flow rate the experiment module is filled with water and the entire length of the flume is submerged. The TDRs record the relative volumetric water content at the time of the failure. The measurements recorded at this point are used in the analysis. Another 
purpose served by having a test run is that it tests the stability of the flume and its support under its own weight plus the additional water.

The preparation of the sediments used in the experiment includes:

- Preparing a mix of mulch and leaf shreds which has an $80 \%$ content smaller than size of the paver gap and 20\% content bigger than the size of the paver gap. (The percentages are based on numbers derived from Louisville’s samples)

- Preparing a well graded mix of inorganic sediment.

- Mixing $20 \%$ by mass of organic sediment and $80 \%$ of inorganic well-graded sediment.

- The sediments are mixed thoroughly and put into 20 smaller cups, used for direct dumping into the stormwater.

Runoff Flow to the flume:

- The stormwater runoff used for the experiment is then pumped in and stored in a 5000 liter tank located at the up gradient of the flume.

- The water is pumped from the tank and the flow rate is adjusted using a valve.

\section{Test steps}

1. Prior to each experiment, the following information is recorded in a sheet: the water level in the stormwater tank, the stormwater runoff temperature, the configurations of the experiment and the date.

2. A sample of the stormwater in the tank is taken for TSS analysis. The stirrer must last half an hour at least before taking the TSS sample.

3. The test begins by starting the timer and plugging in the pump. 
4. The first cup of sediment is poured in after 30 seconds.

5. After the first sediment dump, the flow rate of the effluent is checked using a graduated cylinder and a timer.

6. The sediment dumps continue every 5 minutes after the first dump.

7. Two more flow rate measurements, after the $10^{\text {th }}$ and $20^{\text {th }}$ sediment cups, are taken using the same method.

8. After the last dump, the test is stopped by unplugging the pump 100 minutes into the test.

9. The level of stormwater runoff remaining in the tank is measured.

\section{After the test steps}

After the end of each test except the last, the flume must be prepared for the next run. The first step in maintaining the system and preparing it for the next run is to remove the pavers. Prior to removing the pavers, the sediment left on the surface and in the gaps is cleaned off, using a Shop Vac. After removing the pavers, the bedding layer of aggregate is removed using the Shop Vac. and replaced with previously washed and stored \#8 aggregate. At this point, the steps are similar to those taken at the beginning of test 1 , and the flume is prepared by compaction, leveling the stone and putting the pavers back in. 


\section{APPENDIX C}

\section{Experiment guide}

The following table provides an overview of the test date of each experiment and the specific configuration used for each experiment.

\begin{tabular}{|c|c|c|c|c|}
\hline Experiment No. & Test Date & Slope (\%) & Paver Gap (mm) & Gap Filling \\
\hline 1 & 2/26/2013 & 1 & 6 & None \\
\hline 2 & $2 / 27 / 2013$ & 1 & 6 & None \\
\hline 3 & 2/28/2013 & 1 & 6 & None \\
\hline 4 & $4 / 2 / 2013$ & 1 & 6 & $\# 8$ \\
\hline 5 & 4/3/2013 & 1 & 9 & None \\
\hline 6 & $4 / 4 / 2013$ & 1 & 9 & $\# 8$ \\
\hline 7 & 4/8/2013 & 1 & 12 & None \\
\hline 8 & $4 / 8 / 2013$ & 1 & 12 & $\# 8$ \\
\hline 9 & 4/9/2013 & 3 & 12 & \#8 \\
\hline 10 & $4 / 10 / 2013$ & 3 & 12 & None \\
\hline 11 & $4 / 11 / 2013$ & 3 & 9 & None \\
\hline 12 & $4 / 12 / 2013$ & 3 & 9 & \#8 \\
\hline 13 & 4/15/2013 & 3 & 6 & None \\
\hline 14 & $4 / 16 / 2013$ & 3 & 6 & \#8 \\
\hline 15 & 4/17/2013 & 5 & 12 & None \\
\hline 16 & $4 / 18 / 2013$ & 5 & 12 & \#8 \\
\hline 17 & $4 / 22 / 2013$ & 5 & 9 & None \\
\hline 18 & 4/23/2013 & 5 & 9 & \#8 \\
\hline 19 & $4 / 24 / 2013$ & 5 & 6 & None \\
\hline 20 & $4 / 25 / 2013$ & 5 & 6 & \#8 \\
\hline 21 & $5 / 6 / 2013$ & 1 & 6 & \#8 \\
\hline
\end{tabular}




\section{APPENDIX D}

\section{Rain and runoff calculations}

The dimensions of the flume and the total area of both the flume and the watershed as follows:

\begin{tabular}{|c|c|c|c|c|}
\hline Length $(\mathrm{cm})$ & Width $(\mathrm{cm})$ & Depth $(\mathrm{cm})$ & Area of flume $\left(\mathrm{m}^{2}\right)$ & $\begin{array}{l}\text { Area of watershed }\left(\mathrm{m}^{2}\right) \\
(21 \text { times })\end{array}$ \\
\hline 228.6 & 55.8 & 60.9 & 1.2 & 26.8 \\
\hline
\end{tabular}

The amount of projected cumulative rainfall over the area of the flume is $50.8 \mathrm{~cm}$.

This amount of rainfall over the total area of the water shed will result in 13627.303 liters of stormwater runoff.

The amount of rainfall over the area of the watershed is simulated during a period of 100 minutes. This means that every minute 136.27 liters of stormwater runoff is generated over the area of the watershed.

With the total of $50.8 \mathrm{~cm}$ of cumulative rainfall simulated over 100 minutes, every minute of the experiment is equal to $5.08 \mathrm{~mm}$ of rainfall over the area of the flume’s watershed.

Since is impractical to use so great a volume of water as posited above in the experiment, the total volume of water is reduced to approximately $26 \%$ of the generated runoff; however the sediment is equal to the amount carried by the full volume of runoff generated by $50.8 \mathrm{~cm}$ of cumulative rainfall. 


\section{APPENDIX E}

“Percentage of saturation vs. Time” graphs

Experiment \#0

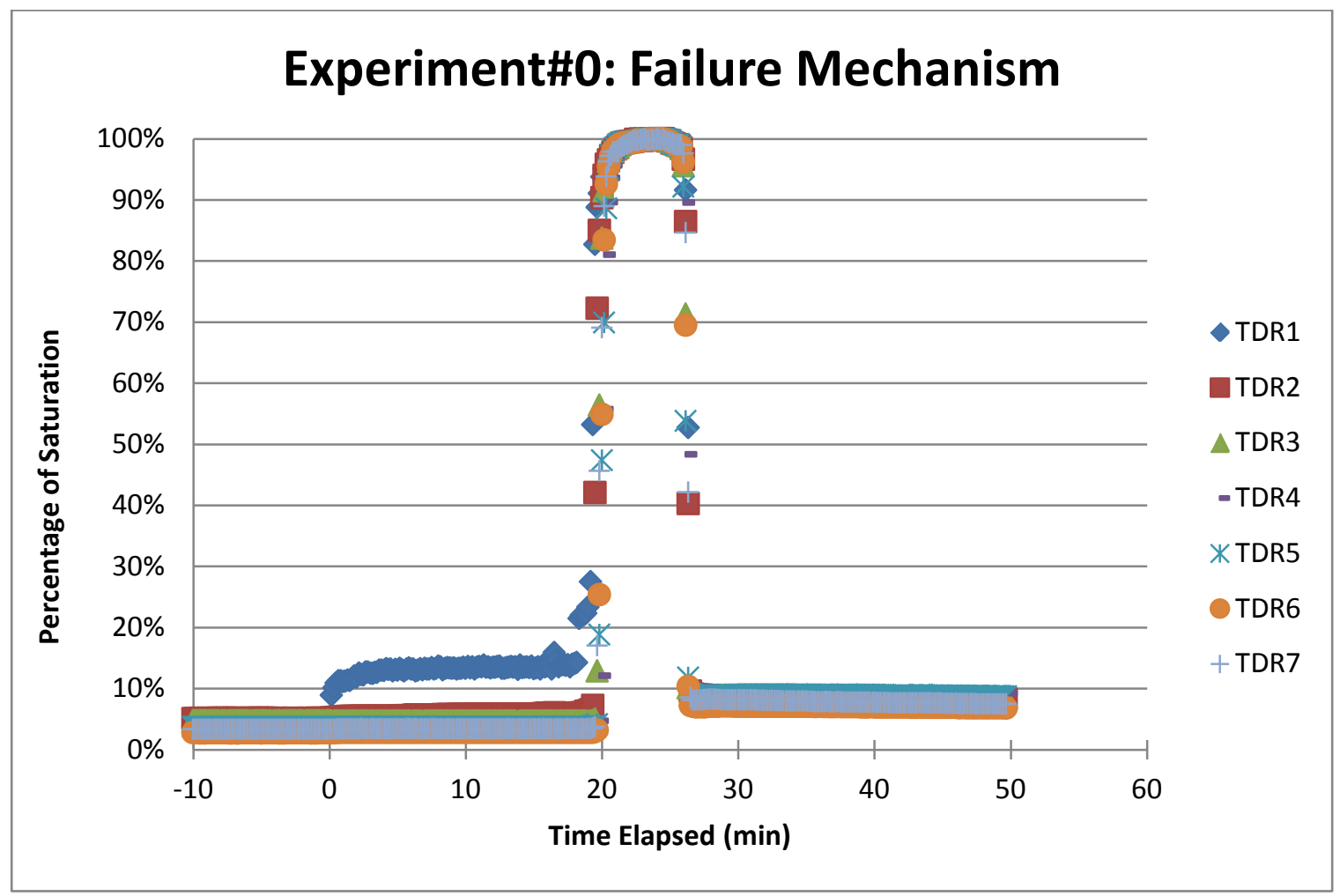


Experiment \#1

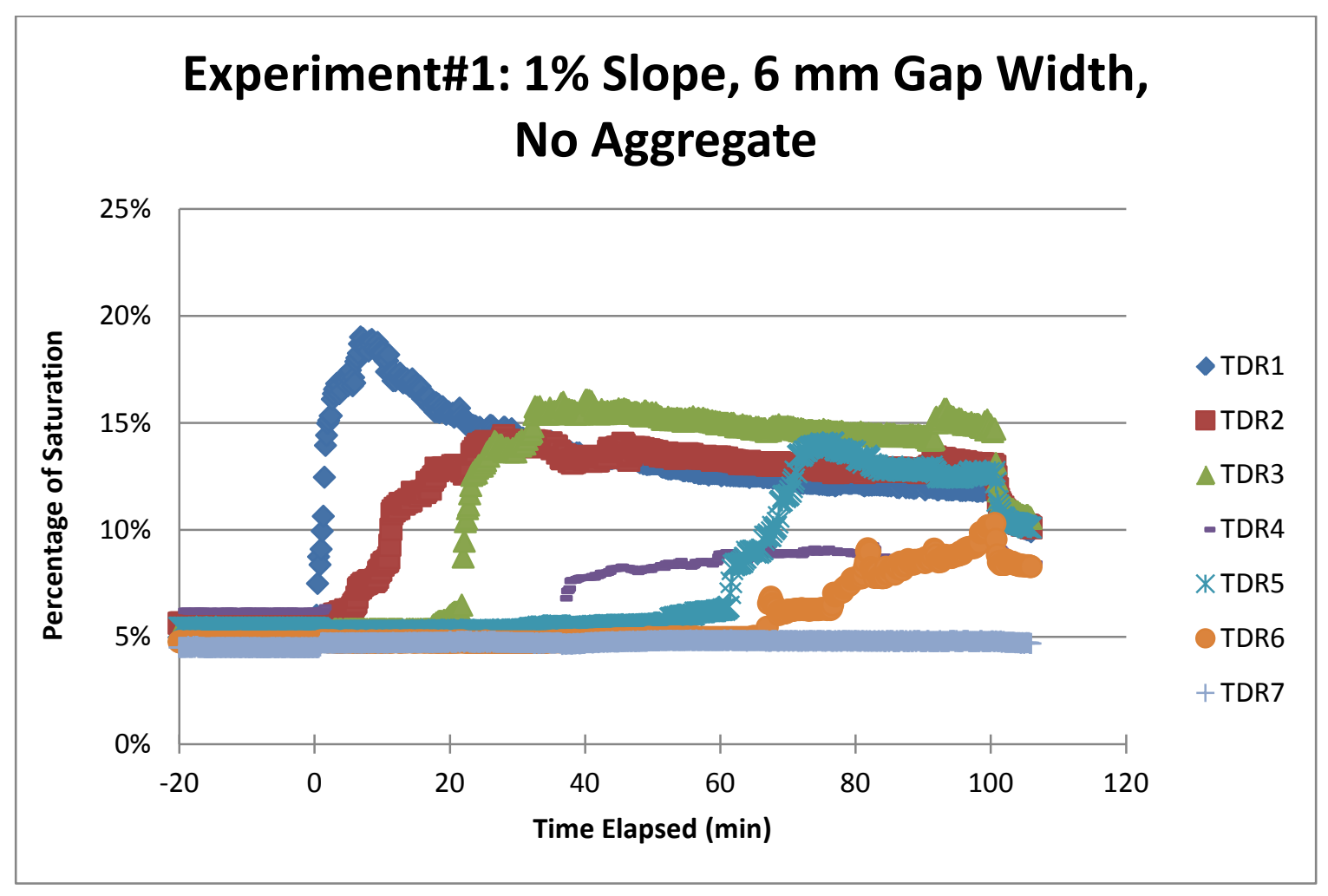

\begin{tabular}{|l|l|l|l|l|l|l|l|}
\hline Rate of increase & TDR1 & TDR2 & TDR3 & TDR4 & TDR5 & TDR6 & TDR7 \\
\hline Slope per minute & 0.01656 & 0.00365 & 0.00471 & 0.00033 & 0.00201 & 0.00089 & N/A \\
\hline $\begin{array}{l}\text { Slope per 100 } \\
\text { minutes }\end{array}$ & 1.656 & 0.365 & 0.471 & 0.033 & 0.201 & 0.089 & N/A \\
\hline
\end{tabular}


Experiment \#2

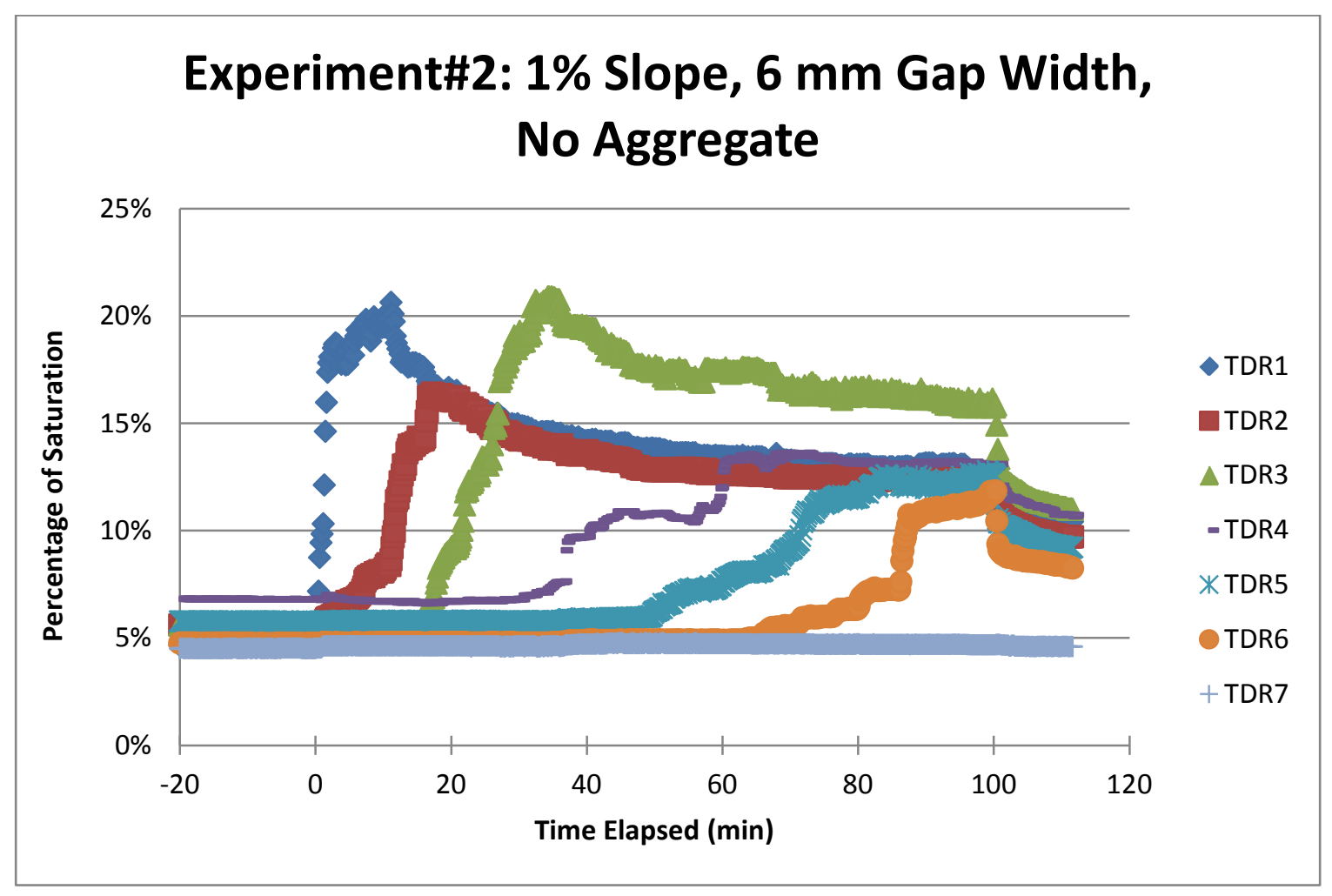

\begin{tabular}{|l|l|l|l|l|l|l|l|}
\hline Rate of increase & TDR1 & TDR2 & TDR3 & TDR4 & TDR5 & TDR6 & TDR7 \\
\hline Slope per minute & 0.00858 & 0.00729 & 0.00877 & 0.00164 & 0.00148 & 0.00217 & N/A \\
\hline $\begin{array}{l}\text { Slope per 100 } \\
\text { minutes }\end{array}$ & 0.858 & 0.729 & 0.877 & 0.164 & 0.148 & 0.217 & N/A \\
\hline
\end{tabular}


Experiment \#3

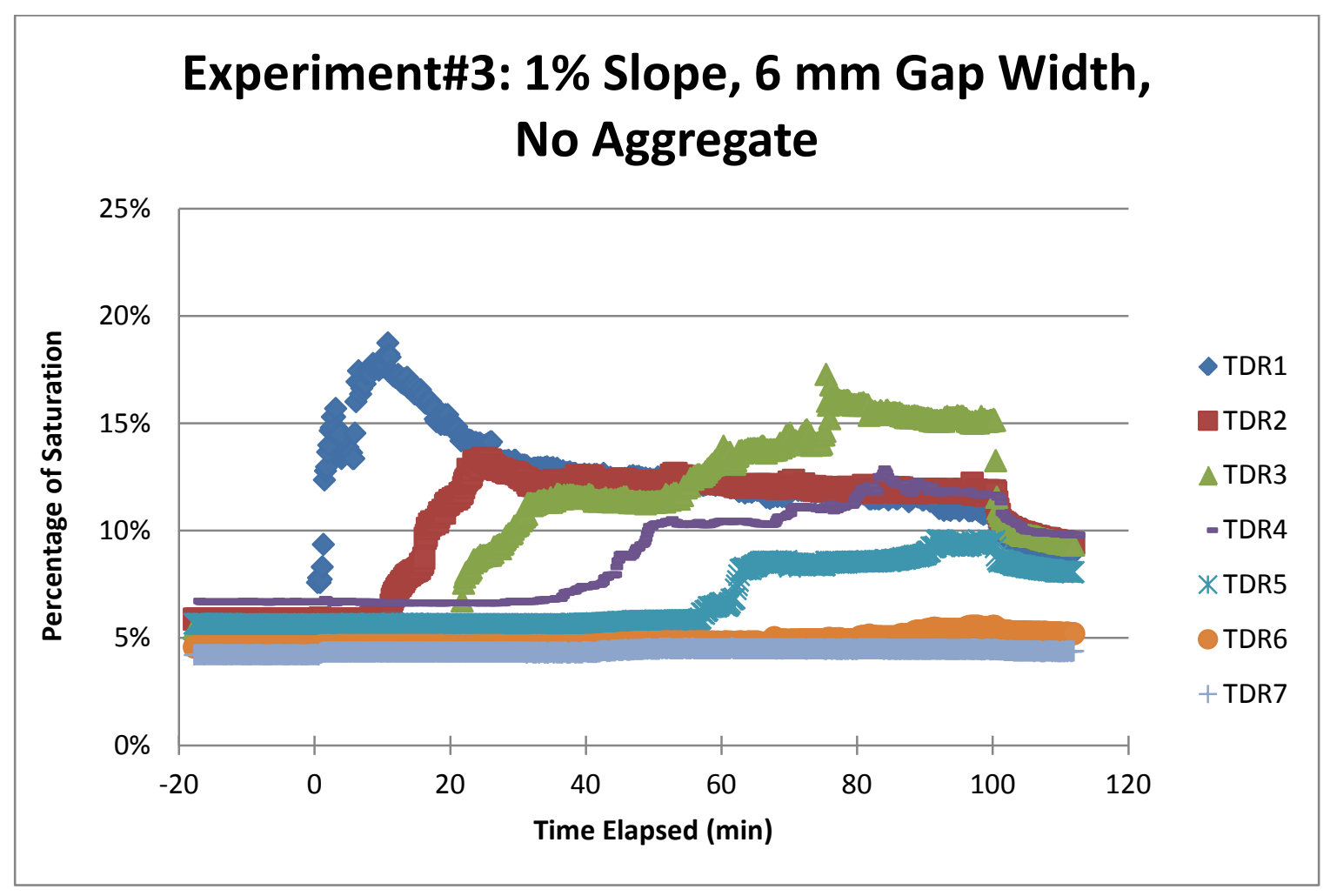

\begin{tabular}{|l|l|l|l|l|l|l|l|}
\hline Rate of increase & TDR1 & TDR2 & TDR3 & TDR4 & TDR5 & TDR6 & TDR7 \\
\hline Slope per minute & 0.00801 & 0.00494 & 0.00104 & 0.00099 & 0.00082 & 0.00032 & N/A \\
\hline $\begin{array}{l}\text { Slope per 100 } \\
\text { minutes }\end{array}$ & 0.801 & 0.494 & 0.104 & 0.099 & 0.082 & 0.032 & N/A \\
\hline
\end{tabular}


Experiment \#4

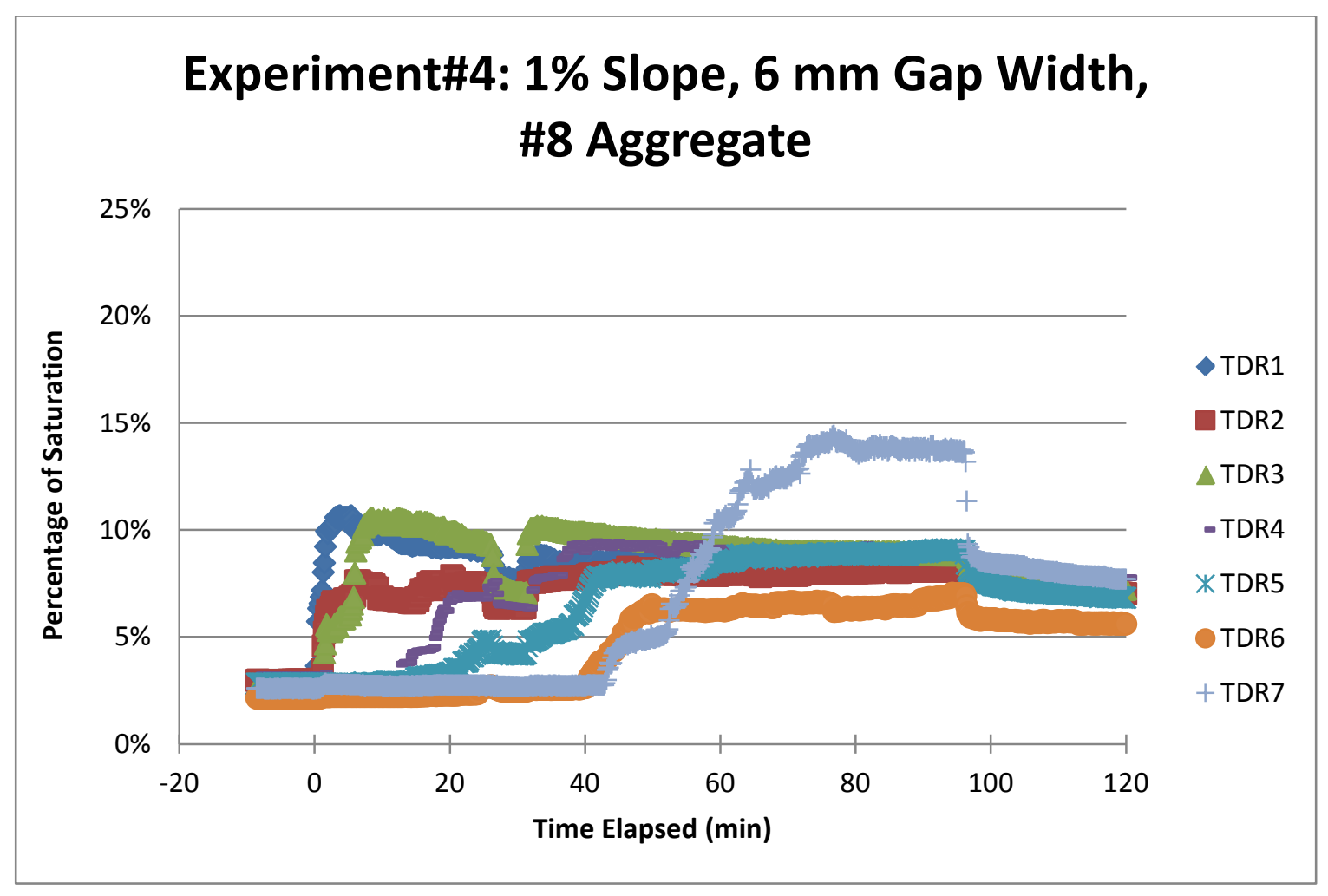

\begin{tabular}{|l|l|l|l|l|l|l|l|}
\hline Rate of increase & TDR1 & TDR2 & TDR3 & TDR4 & TDR5 & TDR6 & TDR7 \\
\hline Slope per minute & 0.01084 & 0.00033 & 0.00941 & 0.00177 & 0.00081 & 0.00033 & 0.00355 \\
\hline $\begin{array}{l}\text { Slope per 100 } \\
\text { minutes }\end{array}$ & 1.084 & 0.033 & 0.941 & 0.177 & 0.081 & 0.033 & 0.355 \\
\hline
\end{tabular}


Experiment \#5

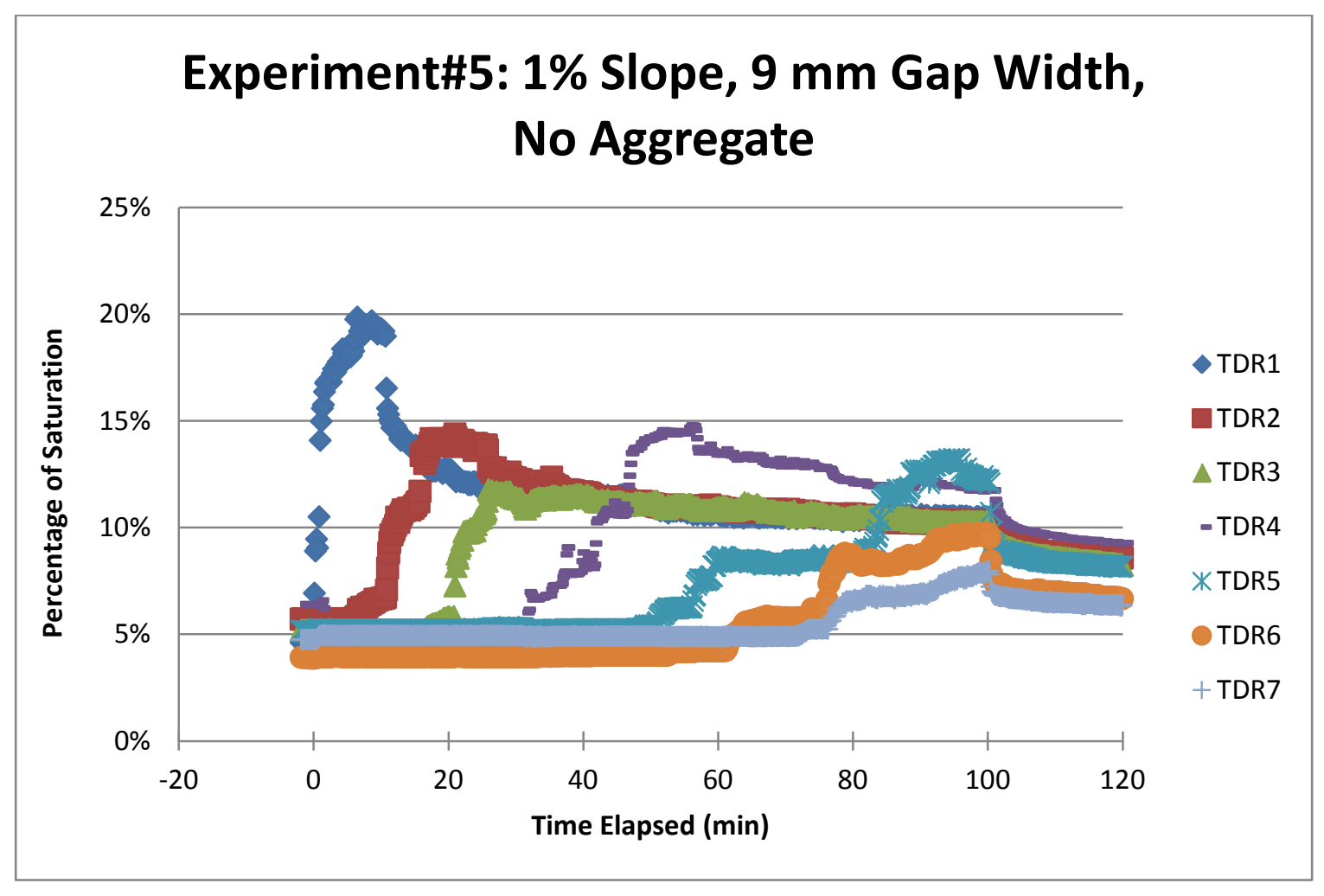

\begin{tabular}{|l|l|l|l|l|l|l|l|}
\hline Rate of increase & TDR1 & TDR2 & TDR3 & TDR4 & TDR5 & TDR6 & TDR7 \\
\hline Slope per minute & 0.01480 & 0.00691 & 0.00742 & 0.00388 & 0.00136 & 0.00140 & 0.00095 \\
\hline $\begin{array}{l}\text { Slope per 100 } \\
\text { minutes }\end{array}$ & 1.480 & 0.691 & 0.742 & 0.388 & 0.136 & 0.140 & 0.095 \\
\hline
\end{tabular}


Experiment \#6

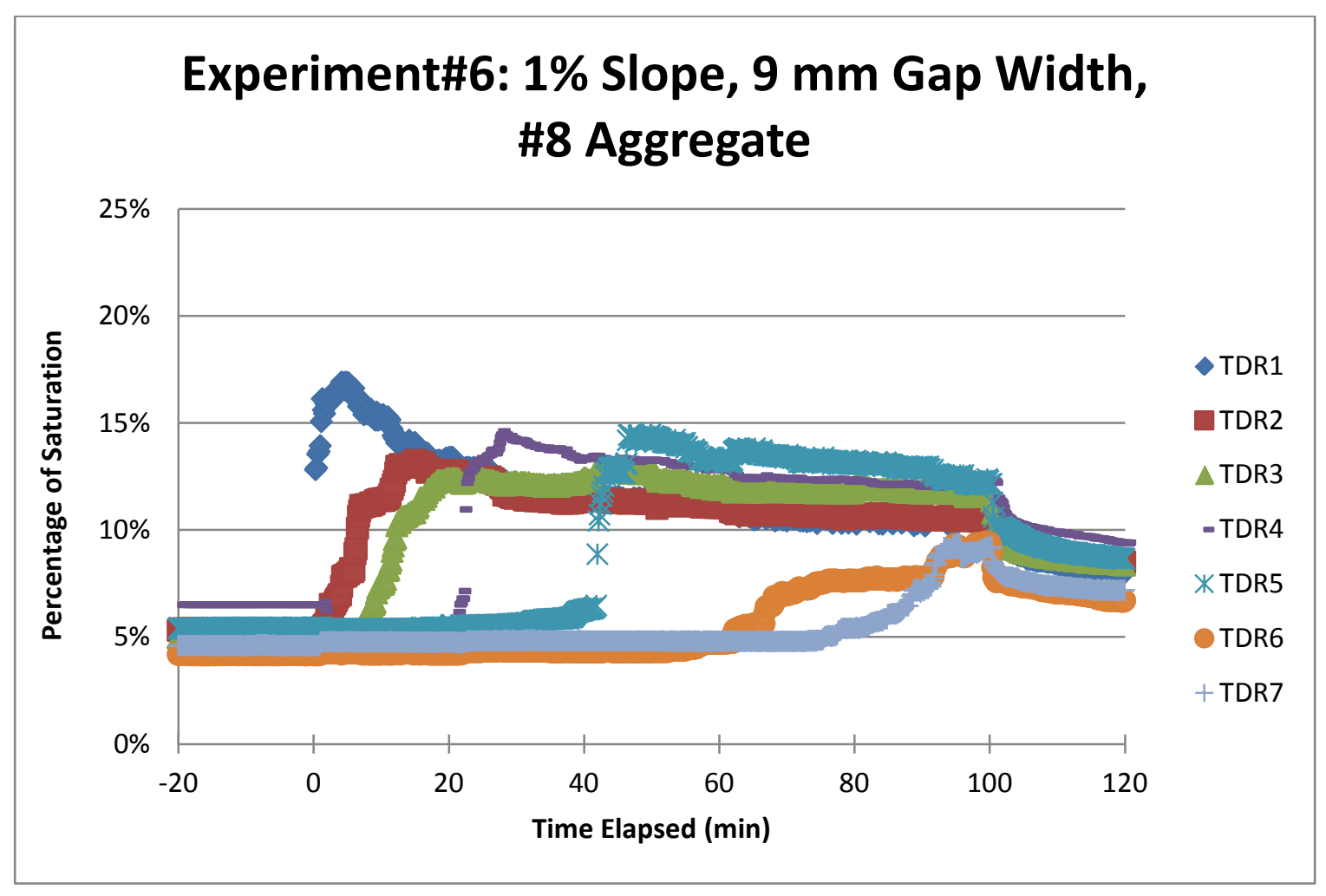

\begin{tabular}{|l|l|l|l|l|l|l|l|}
\hline Rate of increase & TDR1 & TDR2 & TDR3 & TDR4 & TDR5 & TDR6 & TDR7 \\
\hline $\begin{array}{l}\text { Slope per } \\
\text { minute }\end{array}$ & 0.01143 & 0.00600 & 0.00132 & 0.01109 & 0.00317 & 0.00071 & 0.002104 \\
\hline $\begin{array}{l}\text { Slope per } 100 \\
\text { minutes }\end{array}$ & 1.143 & 0.600 & 0.132 & 1.109 & 0.317 & 0.071 & 0.210 \\
\hline
\end{tabular}


Experiment \#7

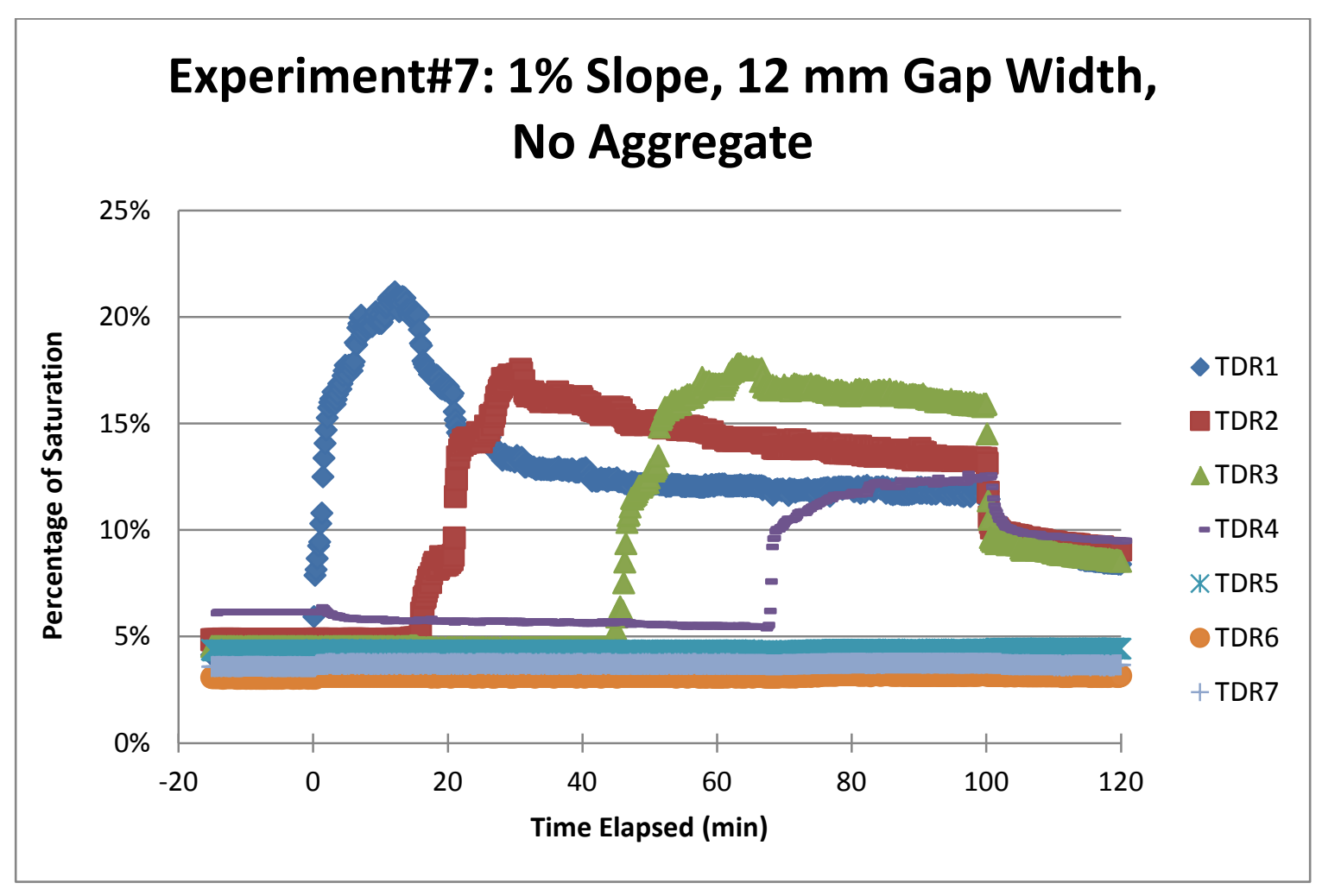

\begin{tabular}{|l|l|l|l|l|l|l|l|}
\hline Rate of increase & TDR1 & TDR2 & TDR3 & TDR4 & TDR5 & TDR6 & TDR7 \\
\hline Slope per minute & 0.00949 & 0.00853 & 0.00619 & 0.00101 & N/A & N/A & N/A \\
\hline Slope per 100 minutes & 0.949 & 0.853 & 0.619 & 0.101 & N/A & N/A & N/A \\
\hline
\end{tabular}


Experiment \#8

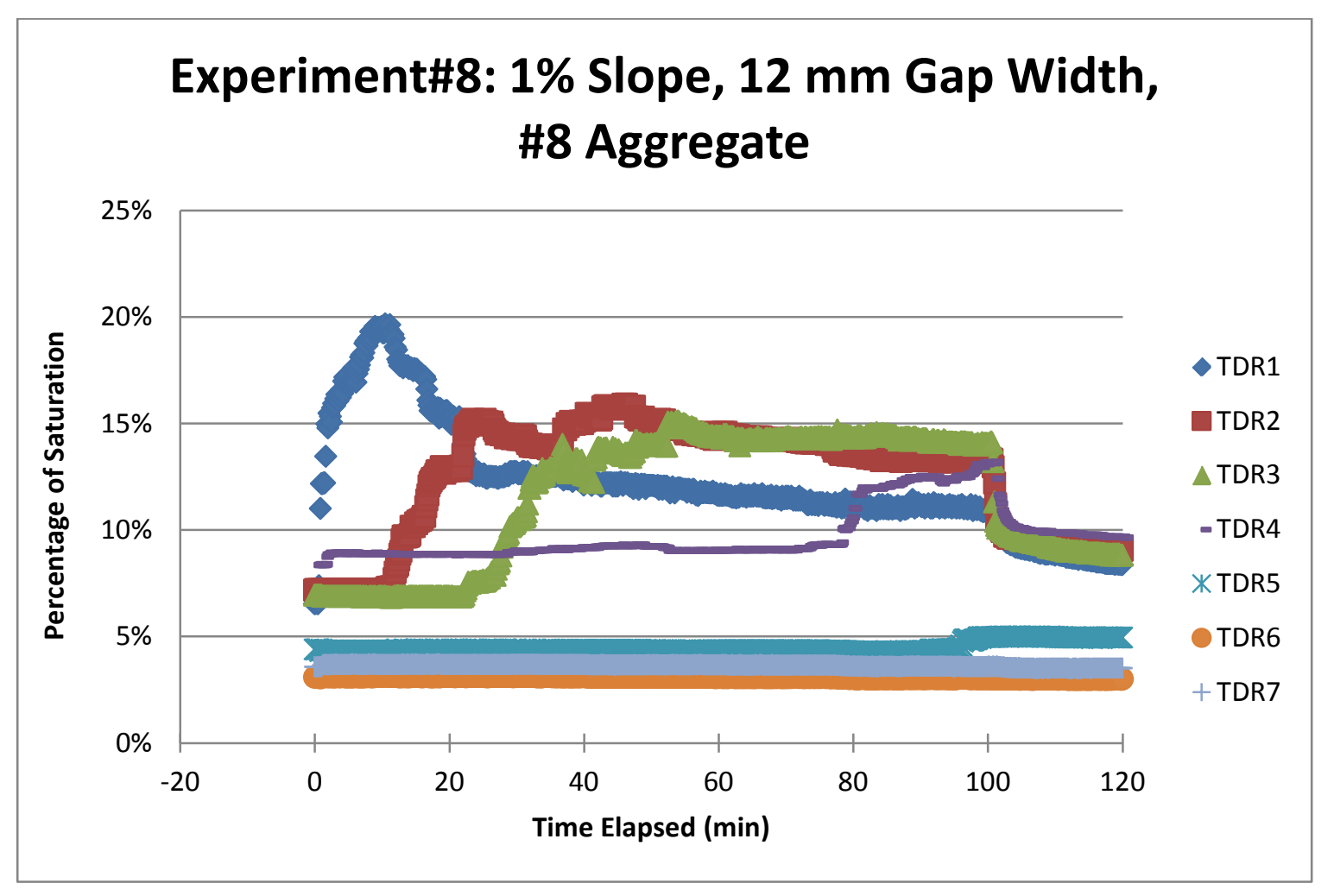

\begin{tabular}{|l|l|l|l|l|l|l|l|}
\hline Rate of increase & TDR1 & TDR2 & TDR3 & TDR4 & TDR5 & TDR6 & TDR7 \\
\hline Slope per minute & 0.00828 & 0.00183 & 0.00232 & 0.00058 & 0.00049 & N/A & N/A \\
\hline $\begin{array}{l}\text { Slope per } 100 \\
\text { minutes }\end{array}$ & 0.828 & 0.183 & 0.232 & 0.058 & 0.049 & N/A & N/A \\
\hline
\end{tabular}


Experiment \#9

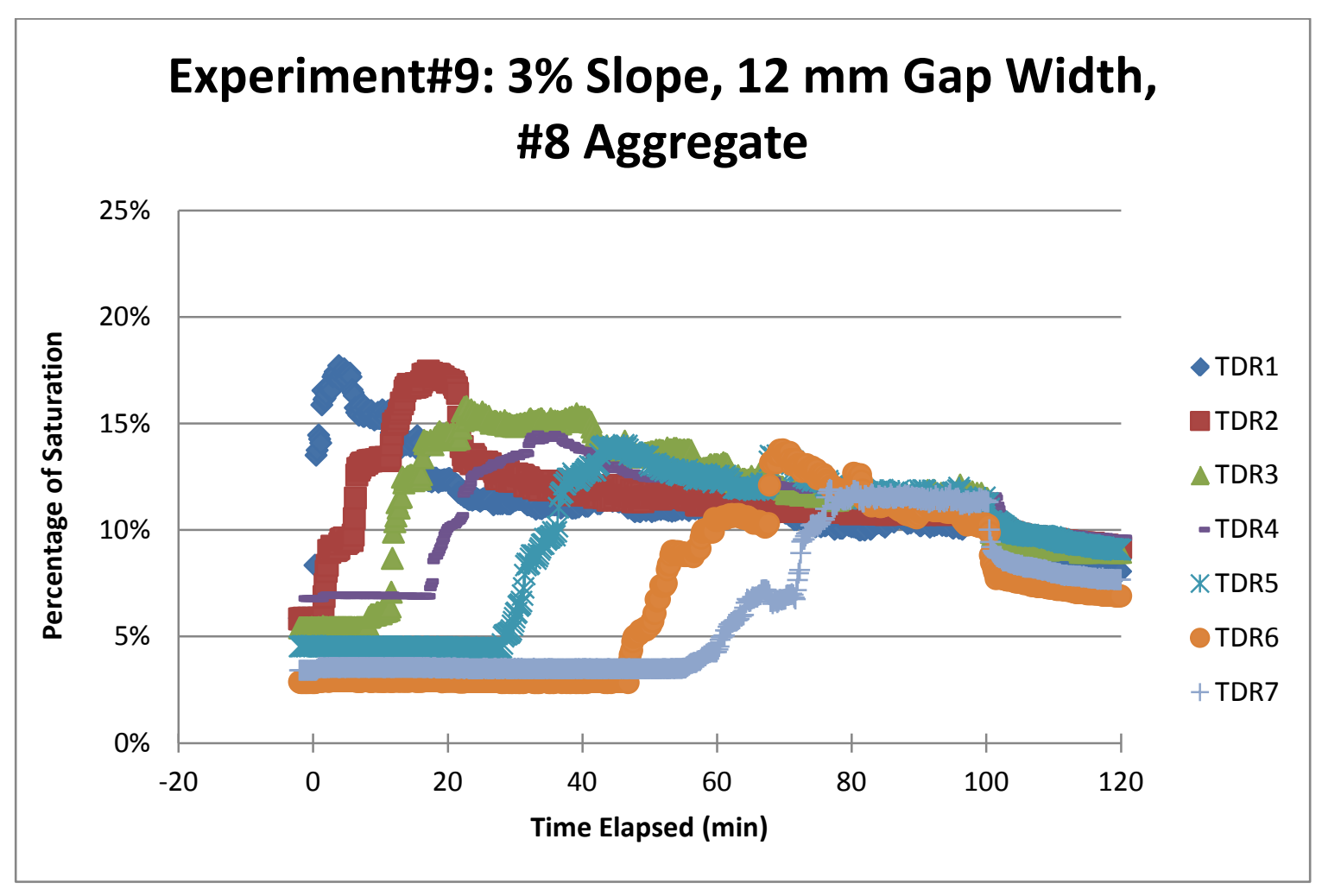

\begin{tabular}{|l|l|l|l|l|l|l|l|}
\hline Rate of increase & TDR1 & TDR2 & TDR3 & TDR4 & TDR5 & TDR6 & TDR7 \\
\hline Slope per minute & 0.02003 & 0.00652 & 0.00733 & 0.00377 & 0.00482 & 0.00337 & 0.00333 \\
\hline $\begin{array}{l}\text { Slope per 100 } \\
\text { minutes }\end{array}$ & 2.003 & 0.652 & 0.733 & 0.377 & 0.482 & 0.337 & 0.333 \\
\hline
\end{tabular}


Experiment \#10

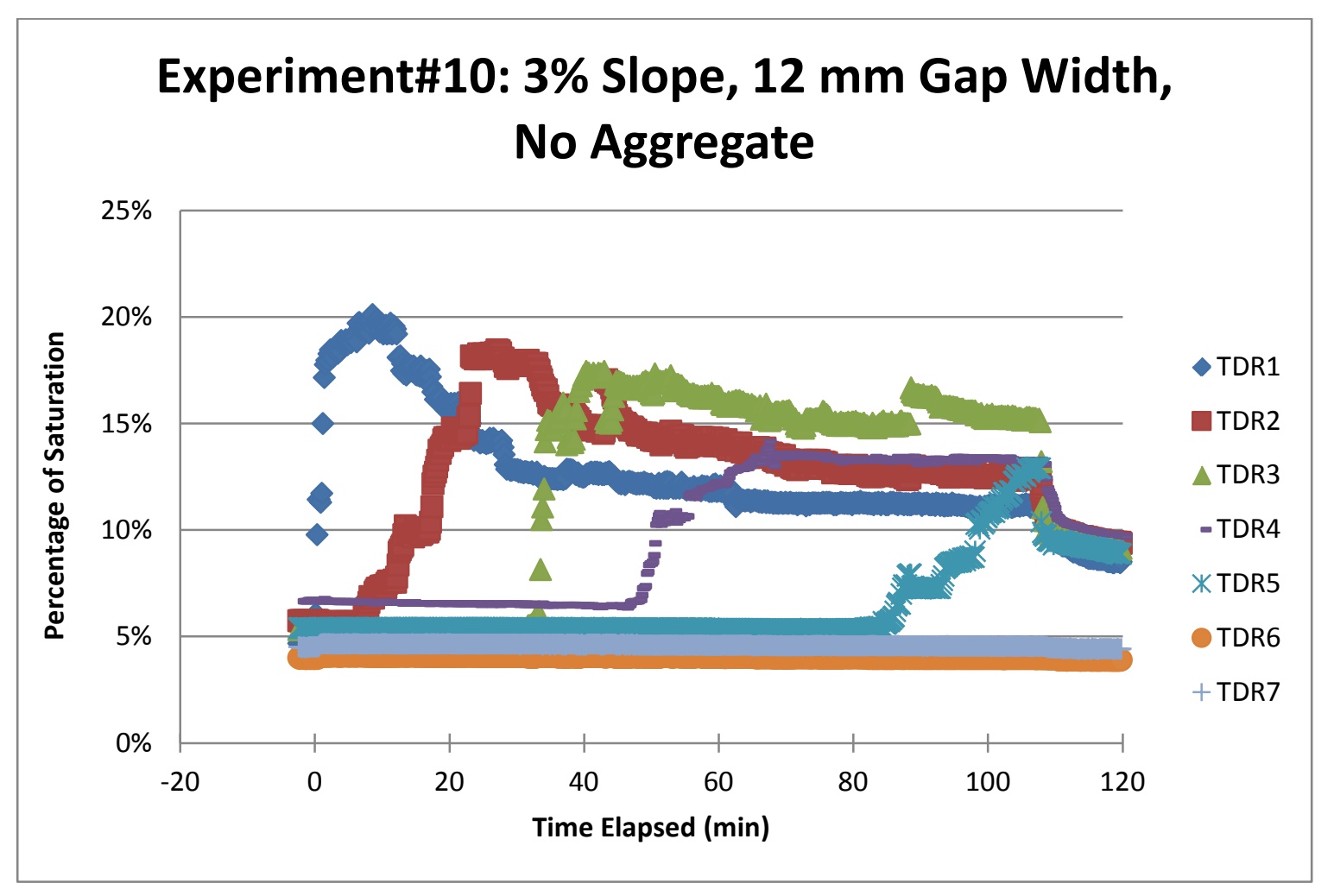

\begin{tabular}{|l|l|l|l|l|l|l|l|}
\hline Rate of increase & TDR1 & TDR2 & TDR3 & TDR4 & TDR5 & TDR6 & TDR7 \\
\hline Slope per minute & 0.01005 & 0.00688 & 0.01348 & 0.00321 & 0.00314 & N/A & N/A \\
\hline $\begin{array}{l}\text { Slope per } 100 \\
\text { minutes }\end{array}$ & 1.005 & 0.688 & 1.348 & 0.321 & 0.314 & N/A & N/A \\
\hline
\end{tabular}


Experiment \#11

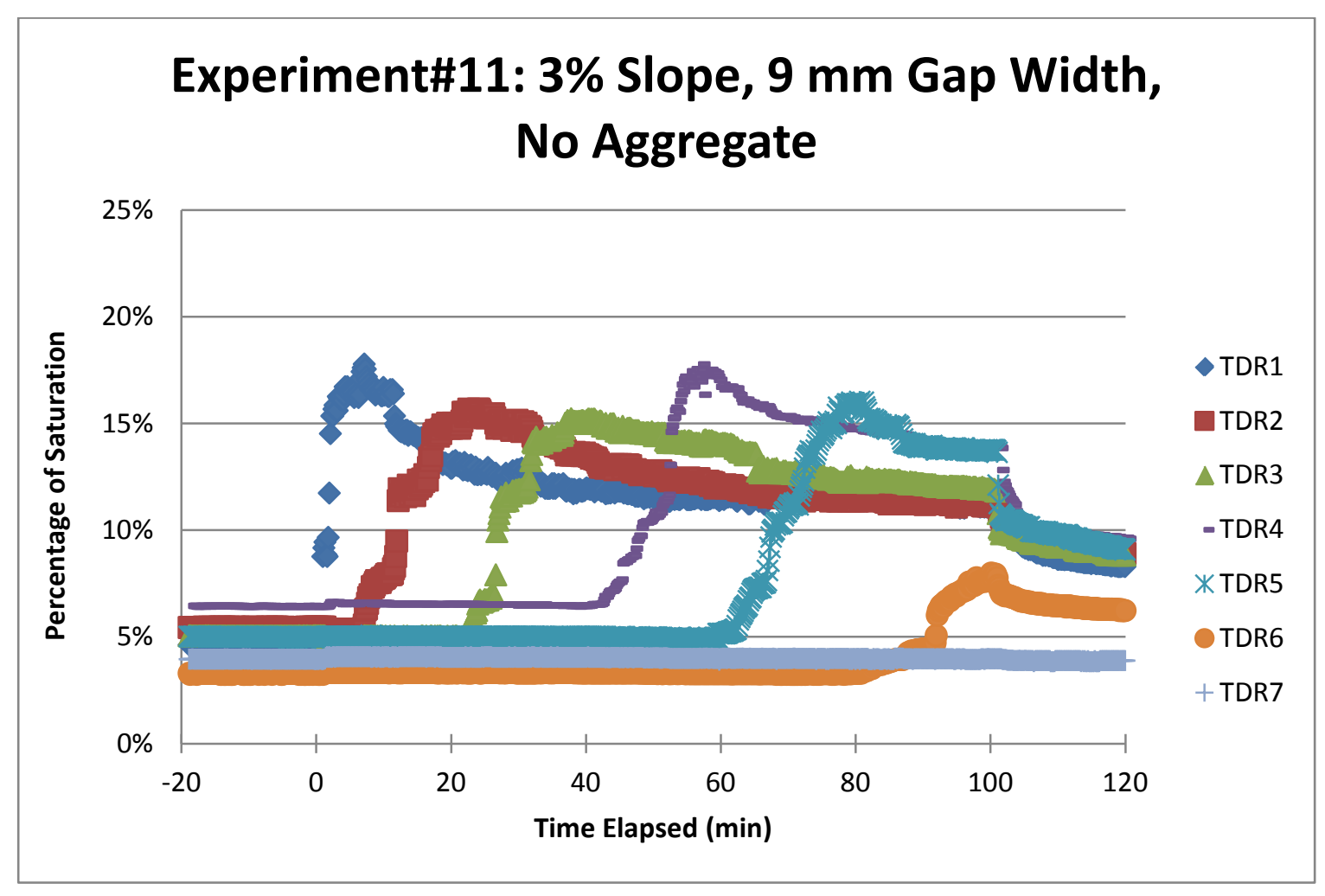

\begin{tabular}{|l|l|l|l|l|l|l|l|}
\hline Rate of increase & TDR1 & TDR2 & TDR3 & TDR4 & TDR5 & TDR6 & TDR7 \\
\hline Slope per minute & 0.01332 & 0.00617 & 0.00704 & 0.00756 & 0.00614 & 0.00273 & N/A \\
\hline $\begin{array}{l}\text { Slope per } 100 \\
\text { minutes }\end{array}$ & 1.332 & 0.617 & 0.704 & 0.756 & 0.614 & 0.273 & N/A \\
\hline
\end{tabular}


Experiment \#12

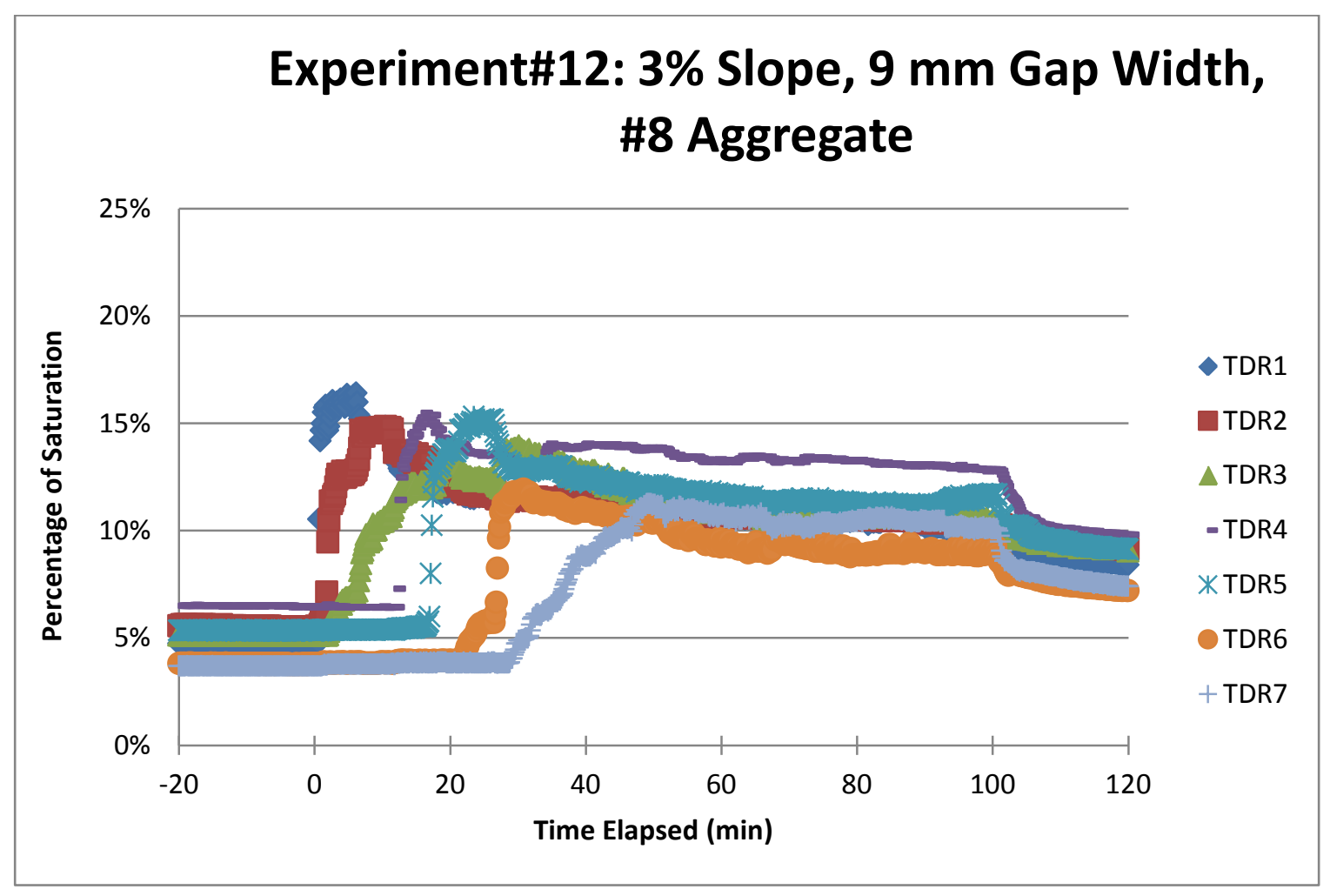

\begin{tabular}{|l|l|l|l|l|l|l|l|}
\hline Rate of increase & TDR1 & TDR2 & TDR3 & TDR4 & TDR5 & TDR6 & TDR7 \\
\hline Slope per minute & 0.00659 & 0.00864 & 0.00249 & 0.01345 & 0.01311 & 0.00982 & 0.00348 \\
\hline $\begin{array}{l}\text { Slope per 100 } \\
\text { minutes }\end{array}$ & 0.659 & 0.864 & 0.249 & 1.345 & 1.311 & 0.982 & 0.348 \\
\hline
\end{tabular}


Experiment \#13

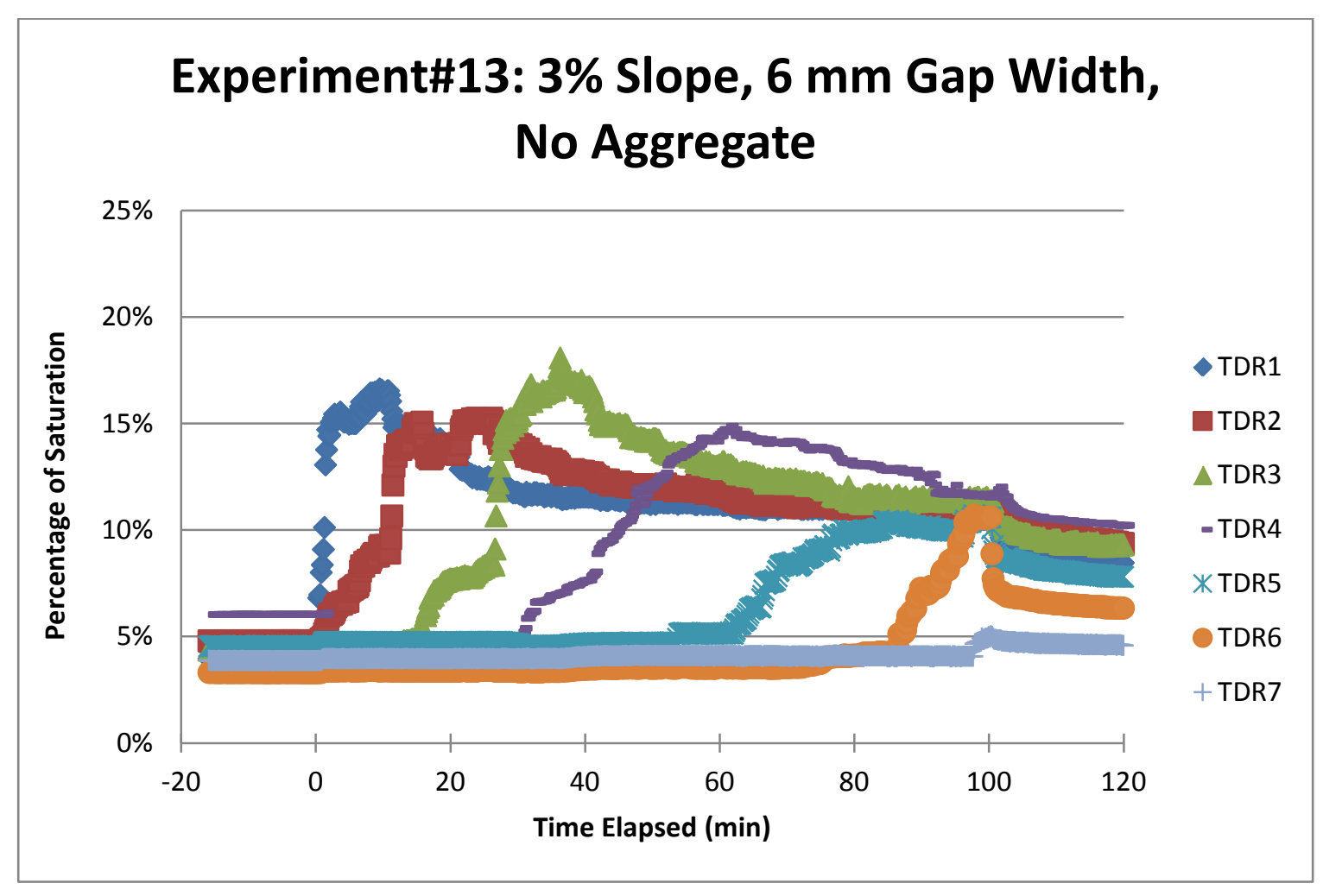

\begin{tabular}{|l|l|l|l|l|l|l|l|}
\hline Rate of increase & TDR1 & TDR2 & TDR3 & TDR4 & TDR5 & TDR6 & TDR7 \\
\hline Slope per minute & 0.00710 & 0.00479 & 0.00607 & 0.00324 & 0.00128 & 0.00225 & 0.00236 \\
\hline $\begin{array}{l}\text { Slope per 100 } \\
\text { minutes }\end{array}$ & 0.710 & 0.479 & 0.607 & 0.324 & 0.128 & 0.225 & 0.236 \\
\hline
\end{tabular}


Experiment \#14

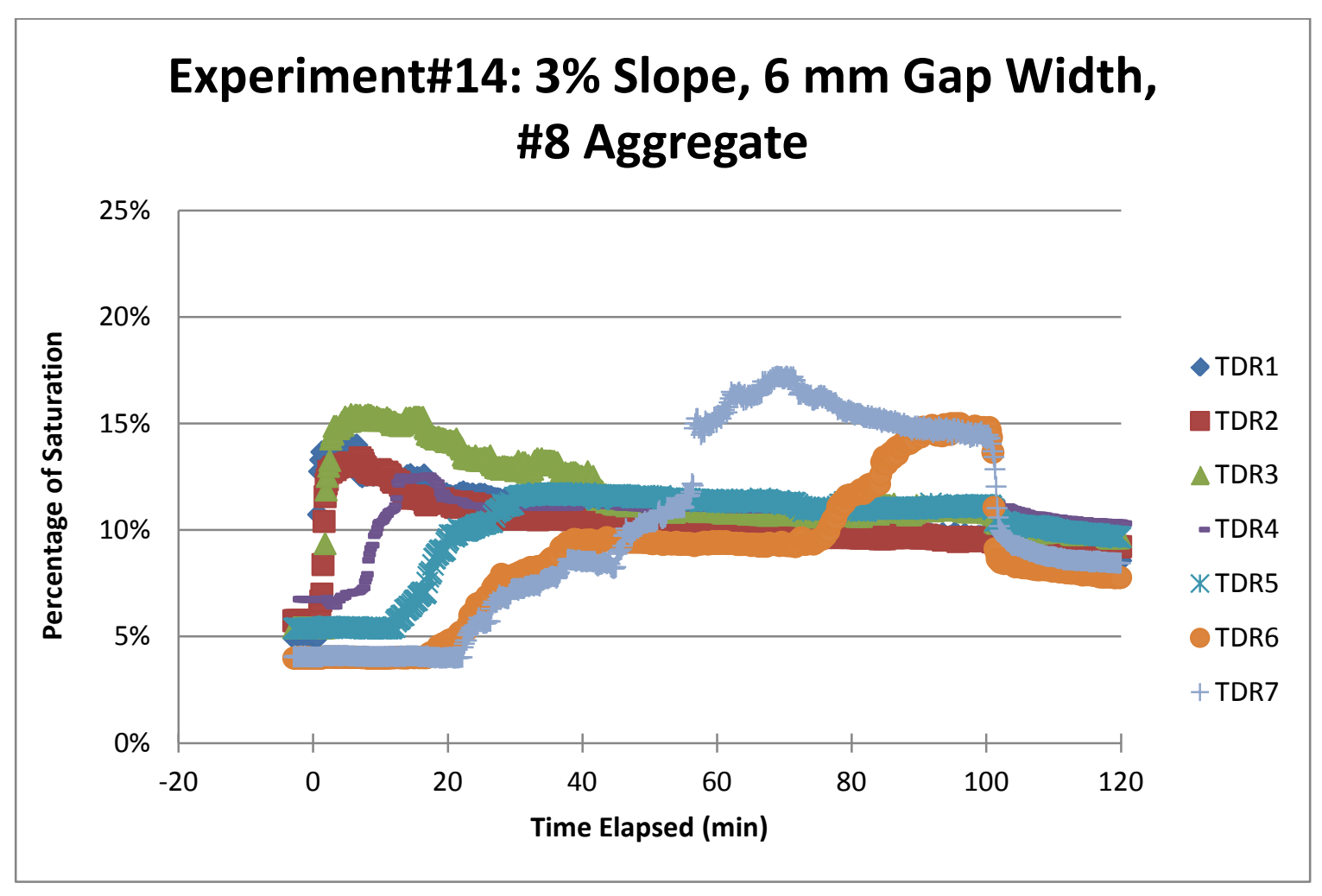

\begin{tabular}{|l|l|l|l|l|l|l|l|}
\hline Rate of increase & TDR1 & TDR2 & TDR3 & TDR4 & TDR5 & TDR6 & TDR7 \\
\hline Slope per minute & 0.00424 & 0.00895 & 0.01302 & 0.00541 & 0.00256 & 0.00097 & 0.00263 \\
\hline $\begin{array}{l}\text { Slope per 100 } \\
\text { minutes }\end{array}$ & 0.424 & 0.895 & 1.302 & 0.541 & 0.256 & 0.097 & 0.263 \\
\hline
\end{tabular}


Experiment \#15

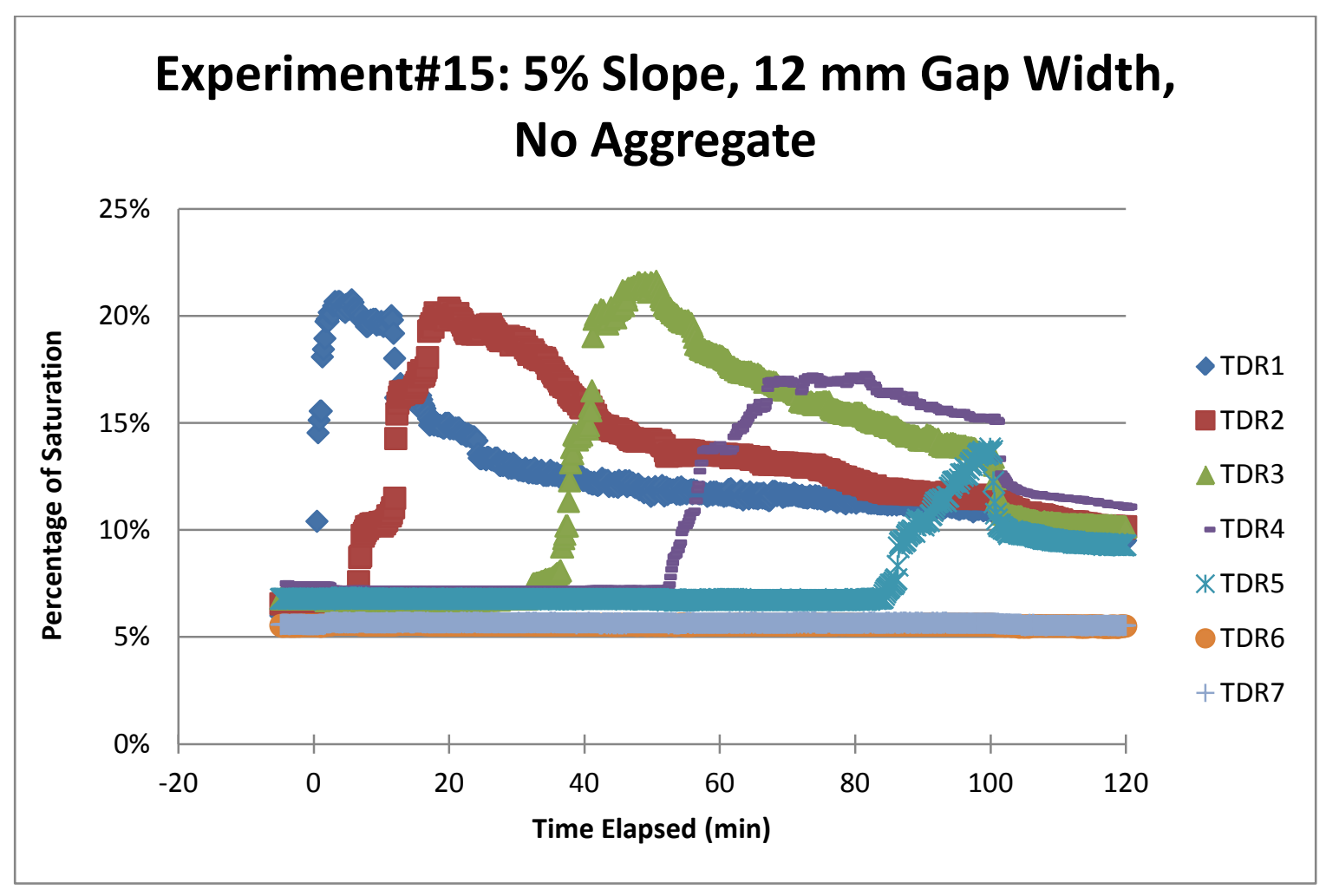

\begin{tabular}{|l|l|l|l|l|l|l|l|}
\hline Rate of increase & TDR1 & TDR2 & TDR3 & TDR4 & TDR5 & TDR6 & TDR7 \\
\hline Slope per minute & 0.01445 & 0.01020 & 0.00827 & 0.00276 & 0.00418 & N/A & N/A \\
\hline $\begin{array}{l}\text { Slope per } 100 \\
\text { minutes }\end{array}$ & 1.445 & 1.020 & 0.827 & 0.276 & 0.418 & N/A & N/A \\
\hline
\end{tabular}


Experiment \#16

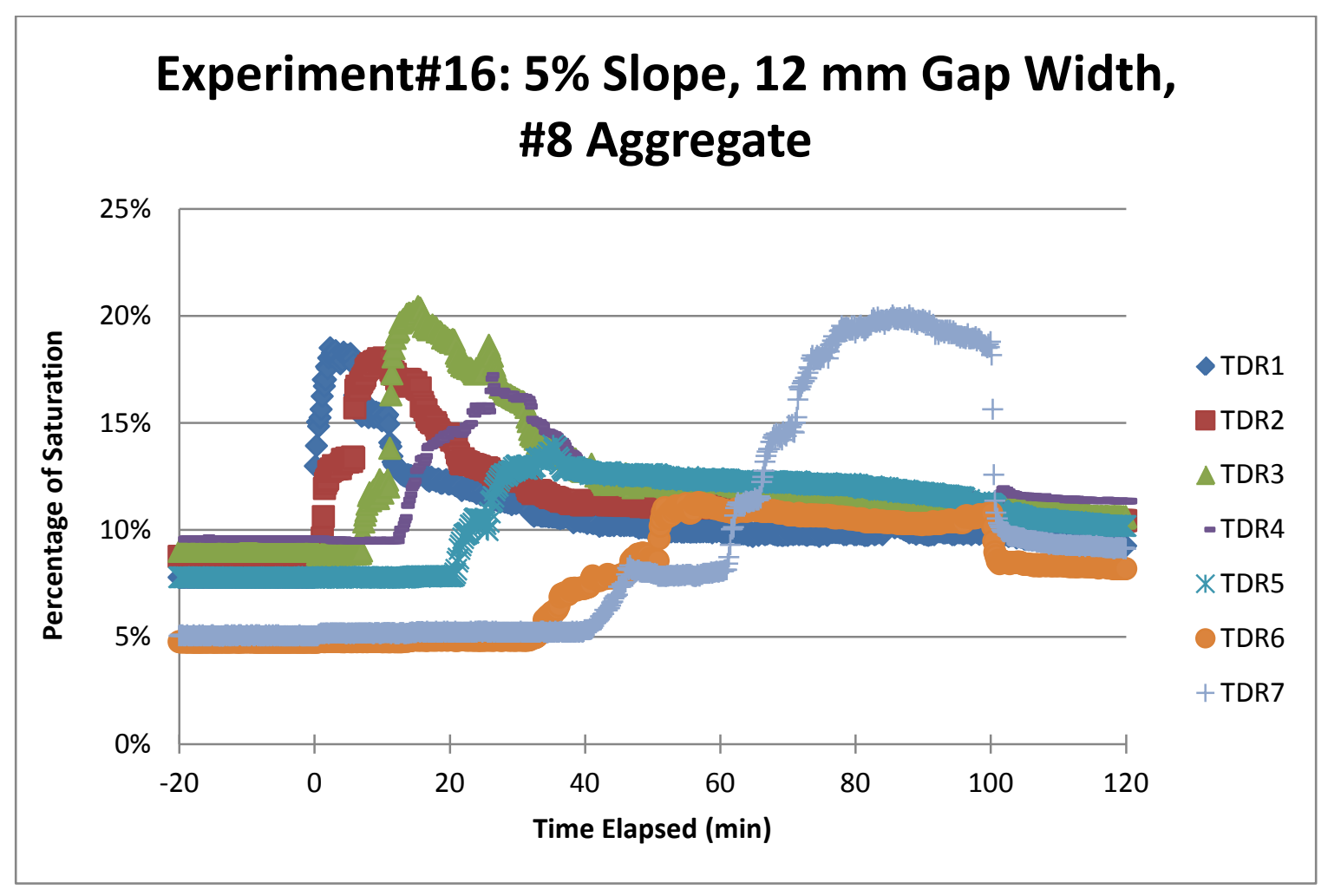

\begin{tabular}{|l|l|l|l|l|l|l|l|}
\hline Rate of increase & TDR1 & TDR2 & TDR3 & TDR4 & TDR5 & TDR6 & TDR7 \\
\hline Slope per minute & 0.03095 & 0.00895 & 0.01547 & 0.00395 & 0.00356 & 0.00242 & 0.00366 \\
\hline $\begin{array}{l}\text { Slope per 100 } \\
\text { minutes }\end{array}$ & 3.095 & 0.895 & 1.547 & 0.395 & 0.356 & 0.242 & 0.366 \\
\hline
\end{tabular}




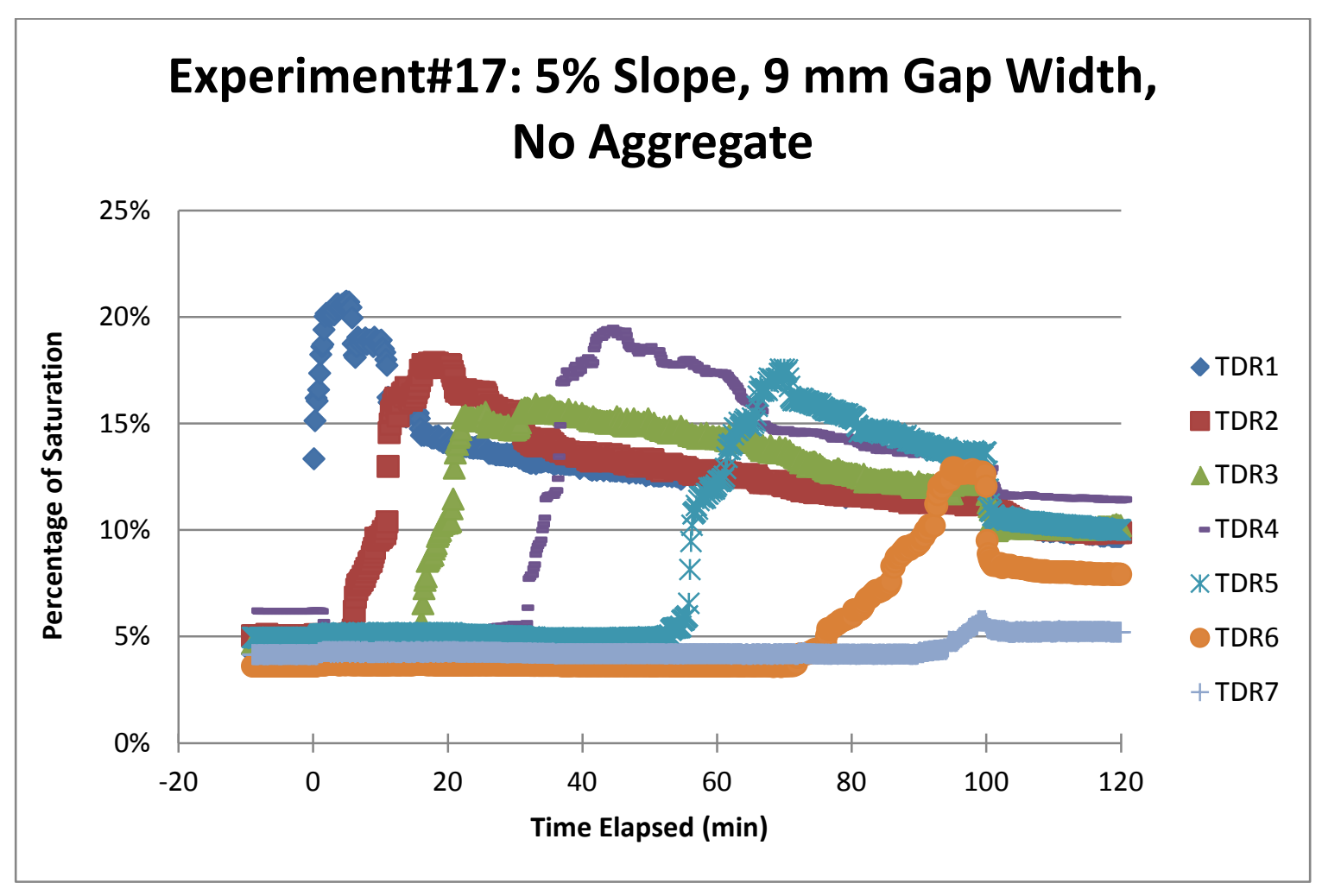

\begin{tabular}{|l|l|l|l|l|l|l|l|}
\hline Rate of increase & TDR1 & TDR2 & TDR3 & TDR4 & TDR5 & TDR6 & TDR7 \\
\hline Slope per minute & 0.01542 & 0.01065 & 0.00576 & 0.00802 & 0.00713 & 0.00365 & 0.00169 \\
\hline $\begin{array}{l}\text { Slope per 100 } \\
\text { minutes }\end{array}$ & 1.542 & 1.065 & 0.576 & 0.802 & 0.713 & 0.365 & 0.169 \\
\hline
\end{tabular}


Experiment \#18

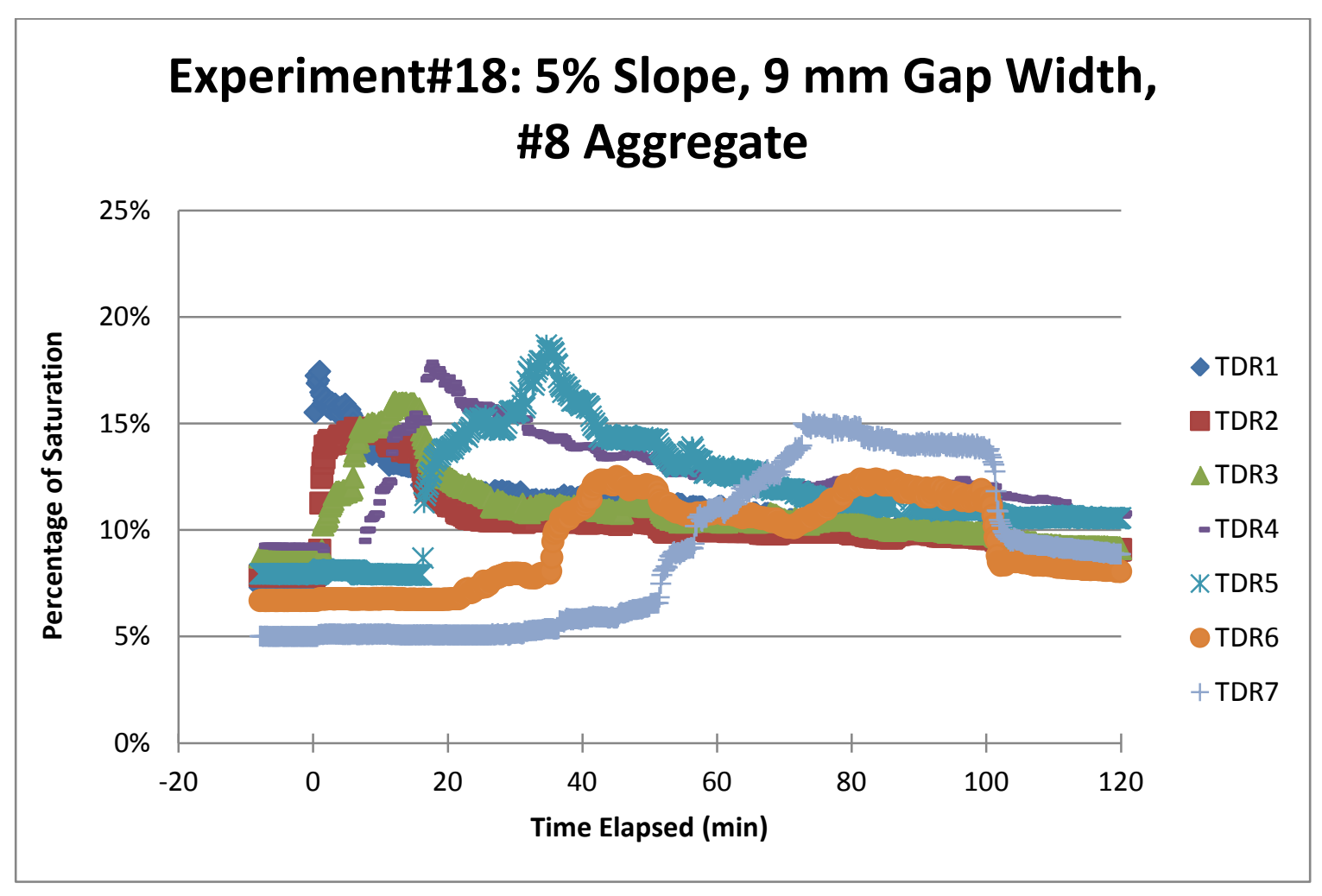

\begin{tabular}{|l|l|l|l|l|l|l|l|}
\hline Rate of increase & TDR1 & TDR2 & TDR3 & TDR4 & TDR5 & TDR6 & TDR7 \\
\hline Slope per minute & 0.10955 & 0.00537 & 0.00568 & 0.00786 & 0.00288 & 0.00239 & 0.00232 \\
\hline $\begin{array}{l}\text { Slope per 100 } \\
\text { minutes }\end{array}$ & 10.955 & 0.537 & 0.568 & 0.786 & 0.288 & 0.239 & 0.232 \\
\hline
\end{tabular}


Experiment \#19

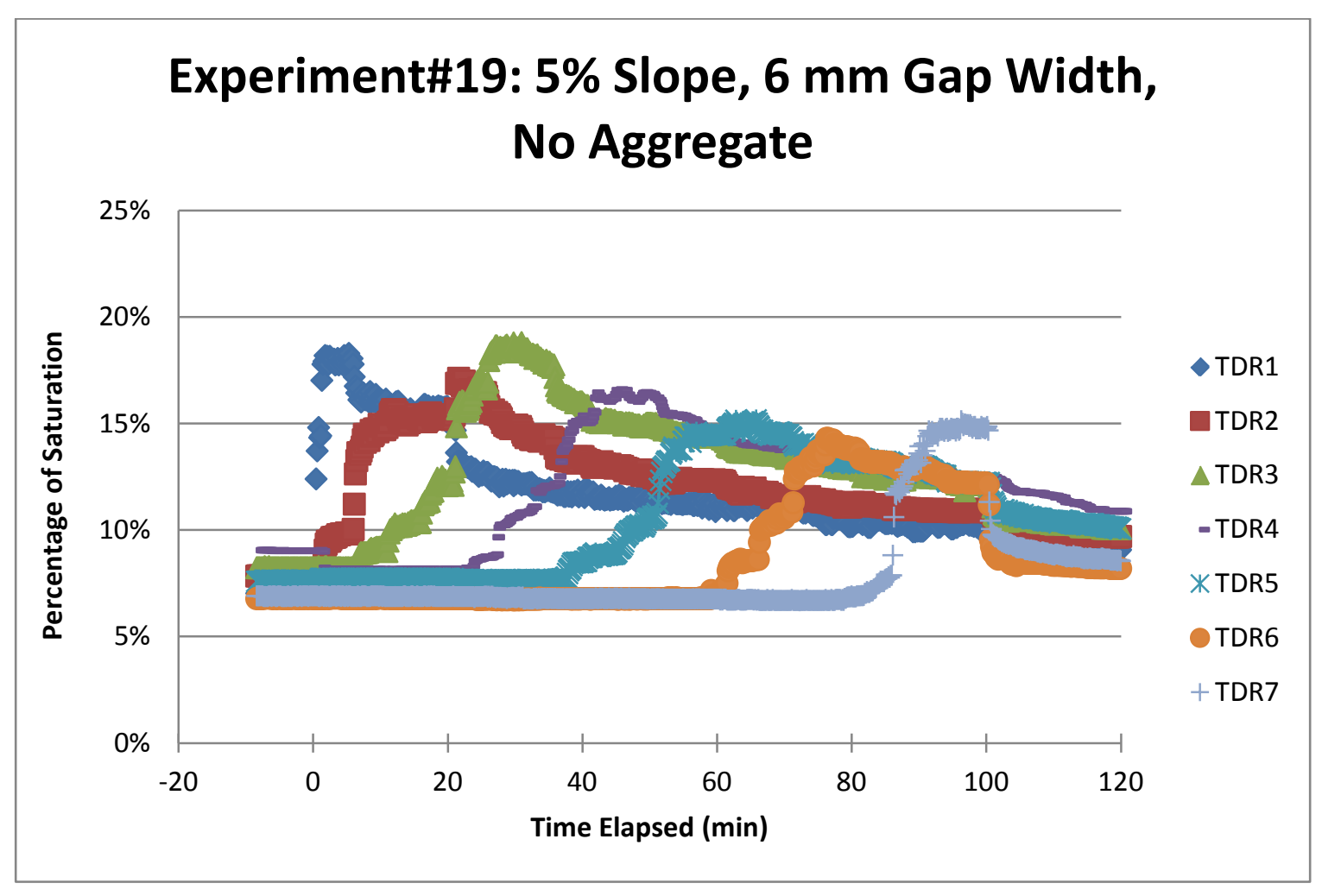

\begin{tabular}{|l|l|l|l|l|l|l|l|}
\hline Rate of increase & TDR1 & TDR2 & TDR3 & TDR4 & TDR5 & TDR6 & TDR7 \\
\hline Slope per minute & 0.01259 & 0.00348 & 0.00497 & 0.00411 & 0.00303 & 0.00435 & 0.00617 \\
\hline $\begin{array}{l}\text { Slope per 100 } \\
\text { minutes }\end{array}$ & 1.259 & 0.348 & 0.497 & 0.411 & 0.303 & 0.435 & 0.617 \\
\hline
\end{tabular}


Experiment \#20

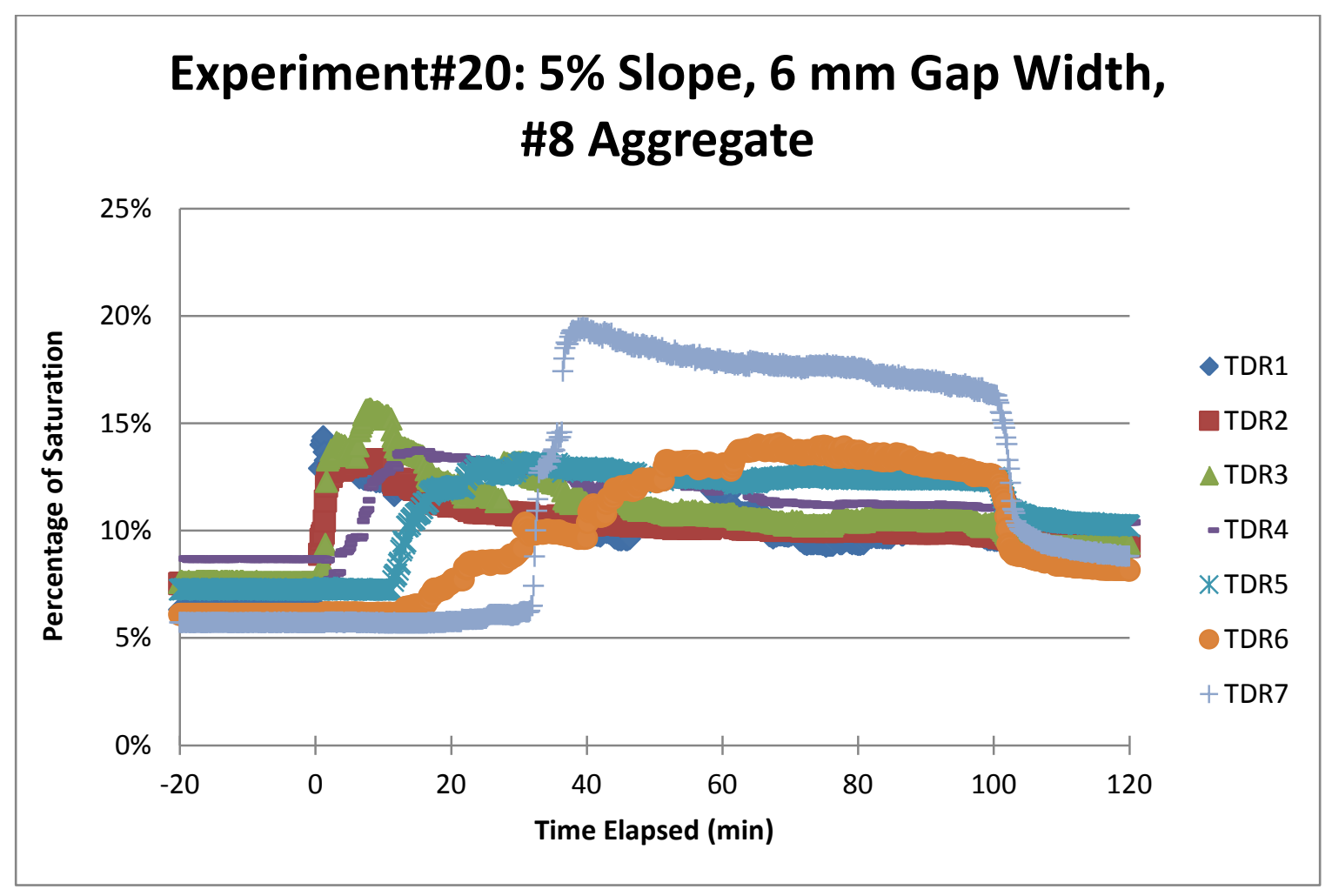

\begin{tabular}{|l|l|l|l|l|l|l|l|}
\hline Rate of increase & TDR1 & TDR2 & TDR3 & TDR4 & TDR5 & TDR6 & TDR7 \\
\hline Slope per minute & 0.11105 & 0.00355 & 0.00789 & 0.00516 & 0.00193 & 0.00145 & 0.00642 \\
\hline $\begin{array}{l}\text { Slope per 100 } \\
\text { minutes }\end{array}$ & 11.105 & 0.355 & 0.789 & 0.516 & 0.193 & 0.145 & 0.642 \\
\hline
\end{tabular}


Experiment \#21

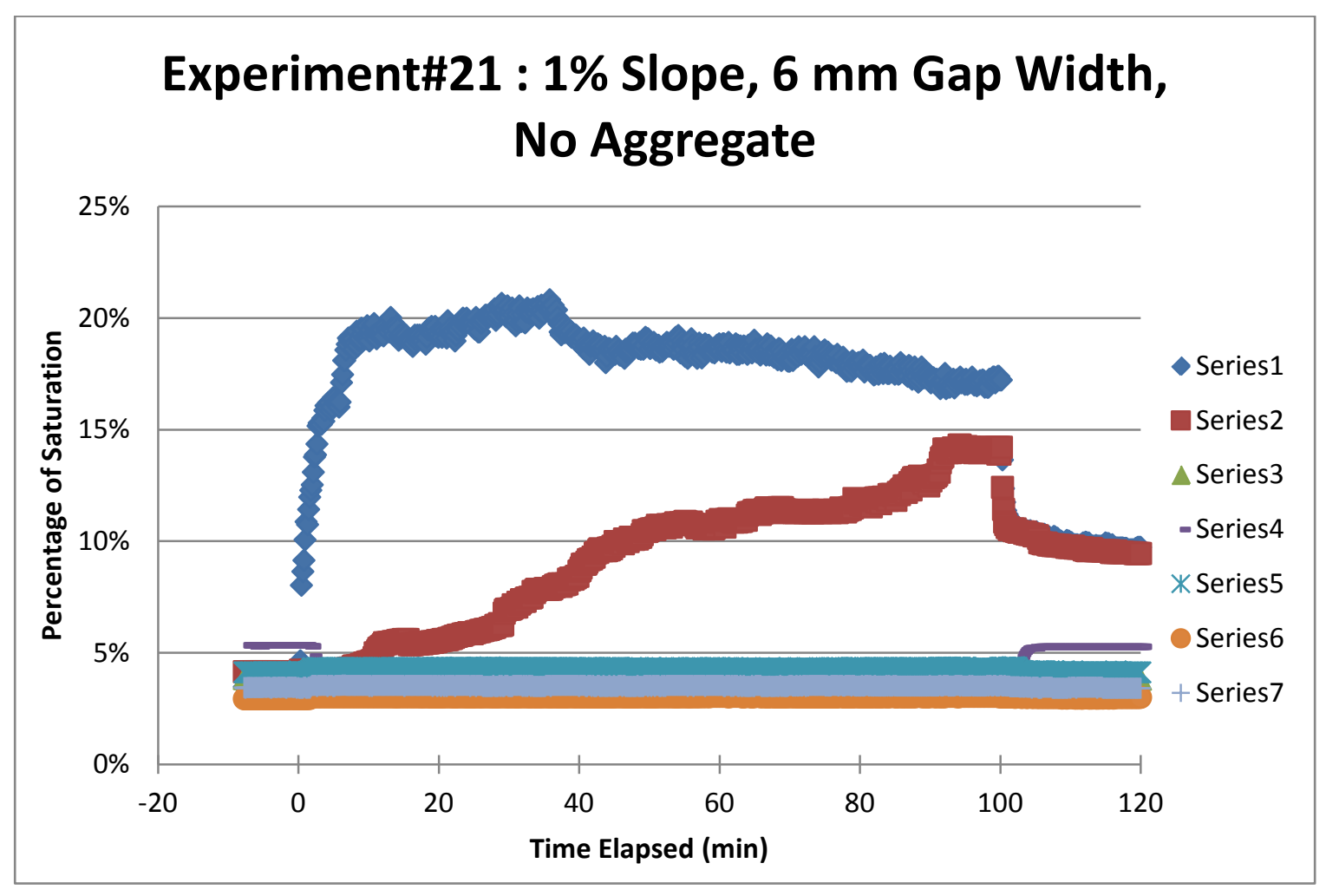

\begin{tabular}{|l|l|l|l|l|l|l|l|}
\hline Rate of increase & \multicolumn{1}{|c|}{ TDR1 } & \multicolumn{1}{|c|}{ TDR2 } & TDR3 & TDR4 & TDR5 & TDR6 & TDR7 \\
\hline Slope per minute & 0.00173 & 0.00104 & N/A & N/A & N/A & N/A & N/A \\
\hline Slope per 100 minutes & 0.173 & 0.104 & N/A & N/A & N/A & N/A & N/A \\
\hline
\end{tabular}




\section{APPENDIX F}

\section{Progression of clogging graphs}

In this appendix, the graph for the progression of clogging for each individual experiment is plotted. Each graph has a trend line with its equation and the R squared value for the data points presented in the graph.

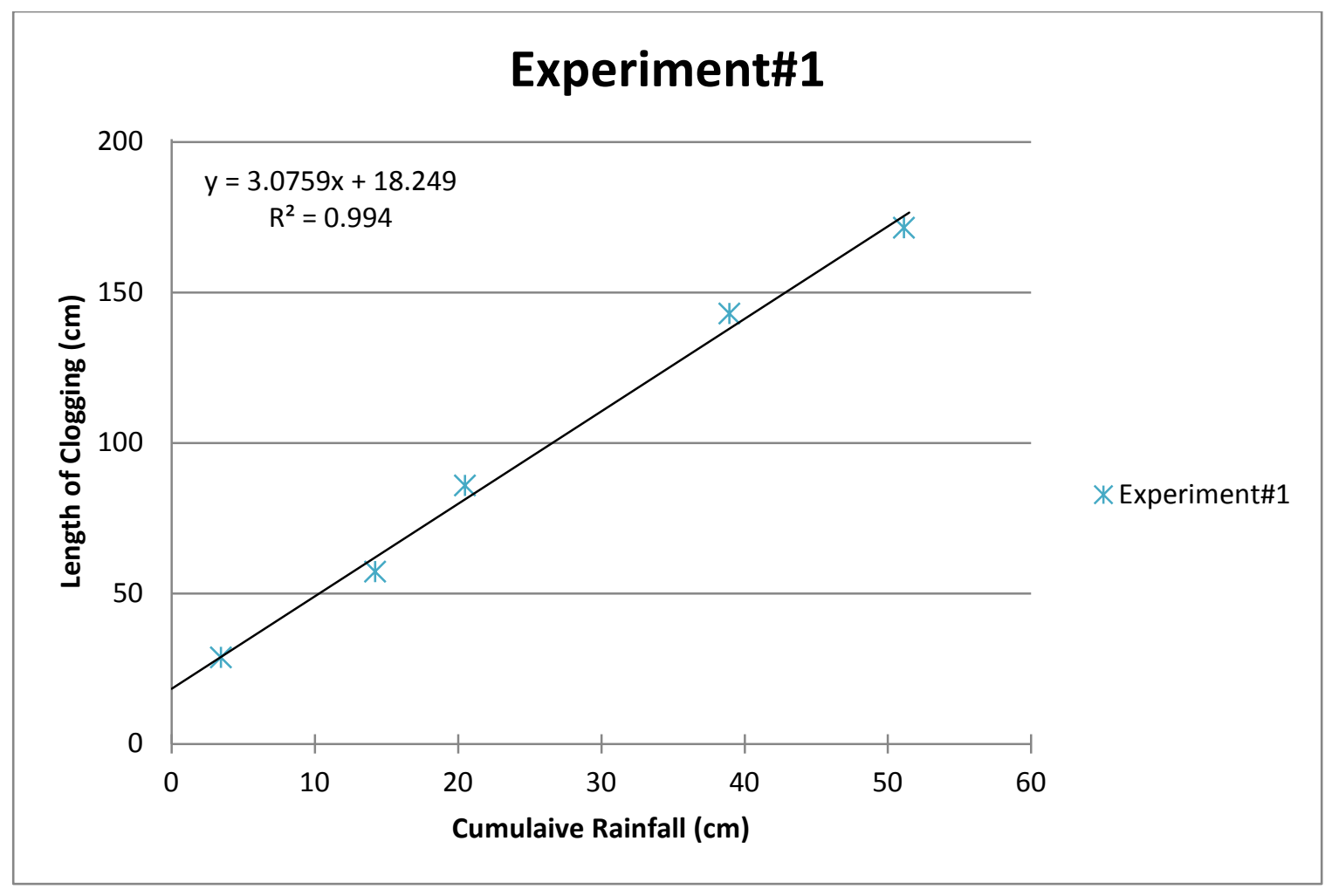

The correction in this graph is that the $4^{\text {th }}$ TDR at 114.30 reaches the maximum later than the $5^{\text {th }}$ TDR; therefore the data point for the $4^{\text {th }}$ TDR has been excluded from the graph. 


\section{Experiment\#2}

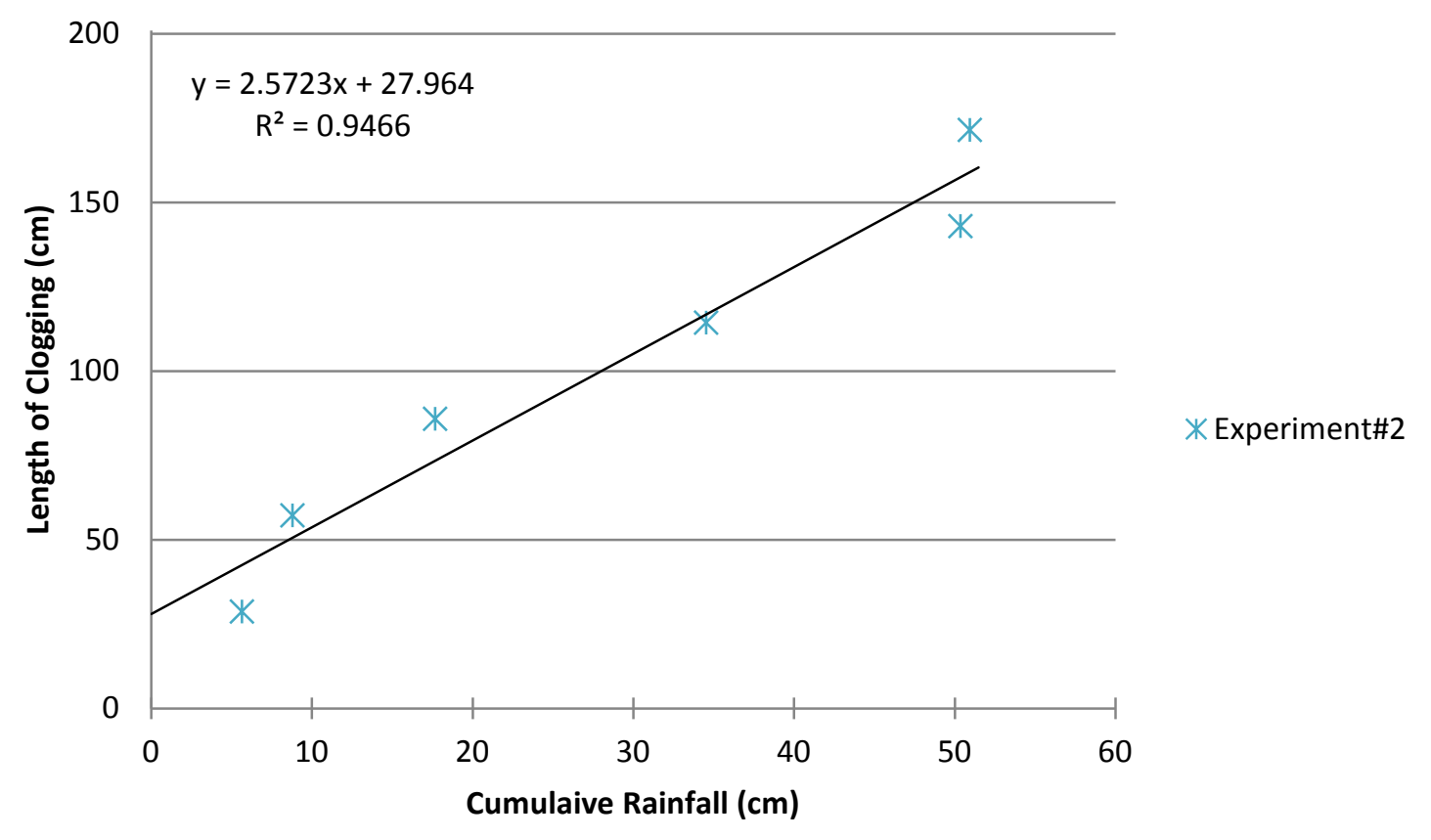




\section{Experiment\#3}

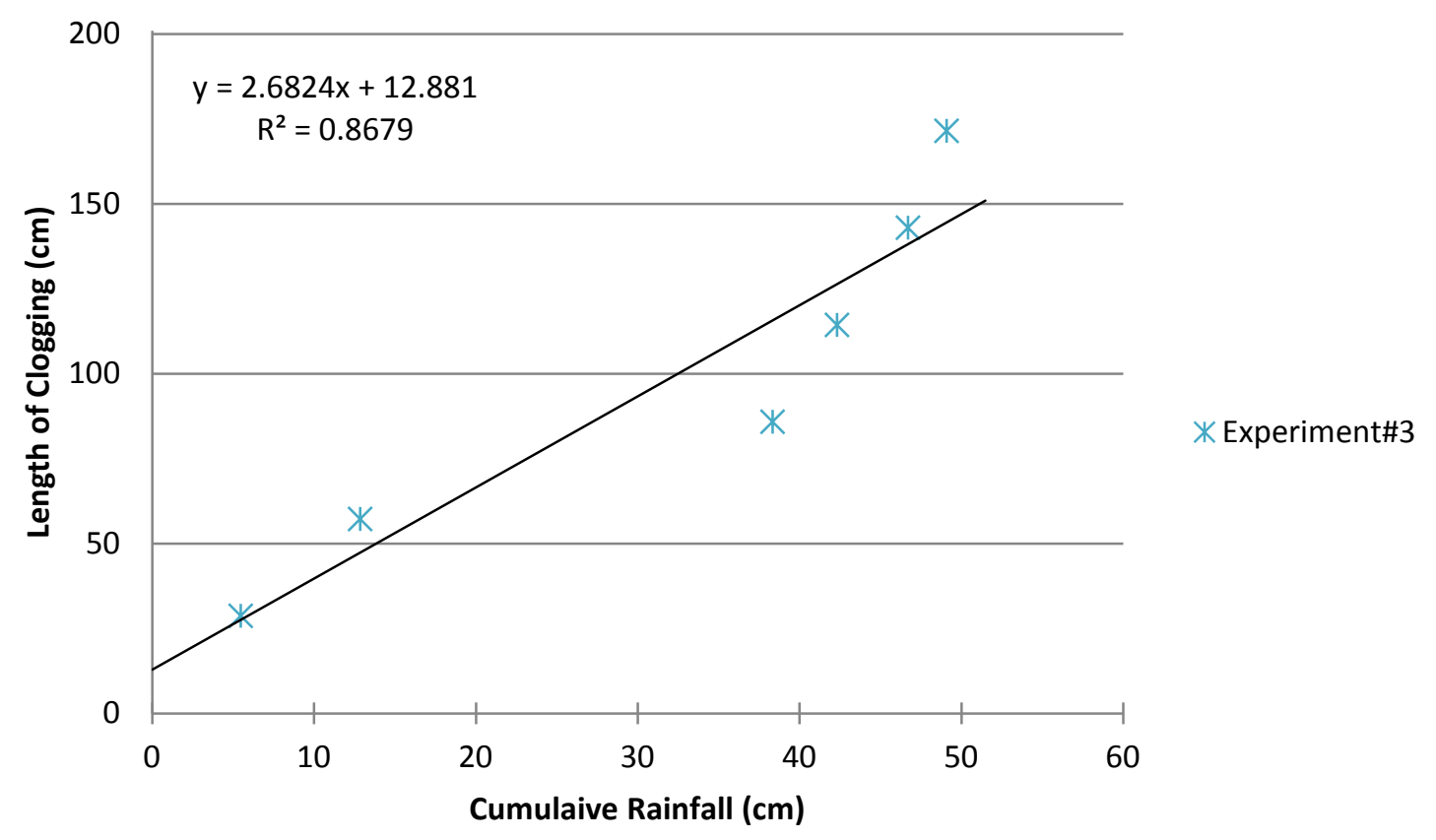




\section{Experiment\#4}

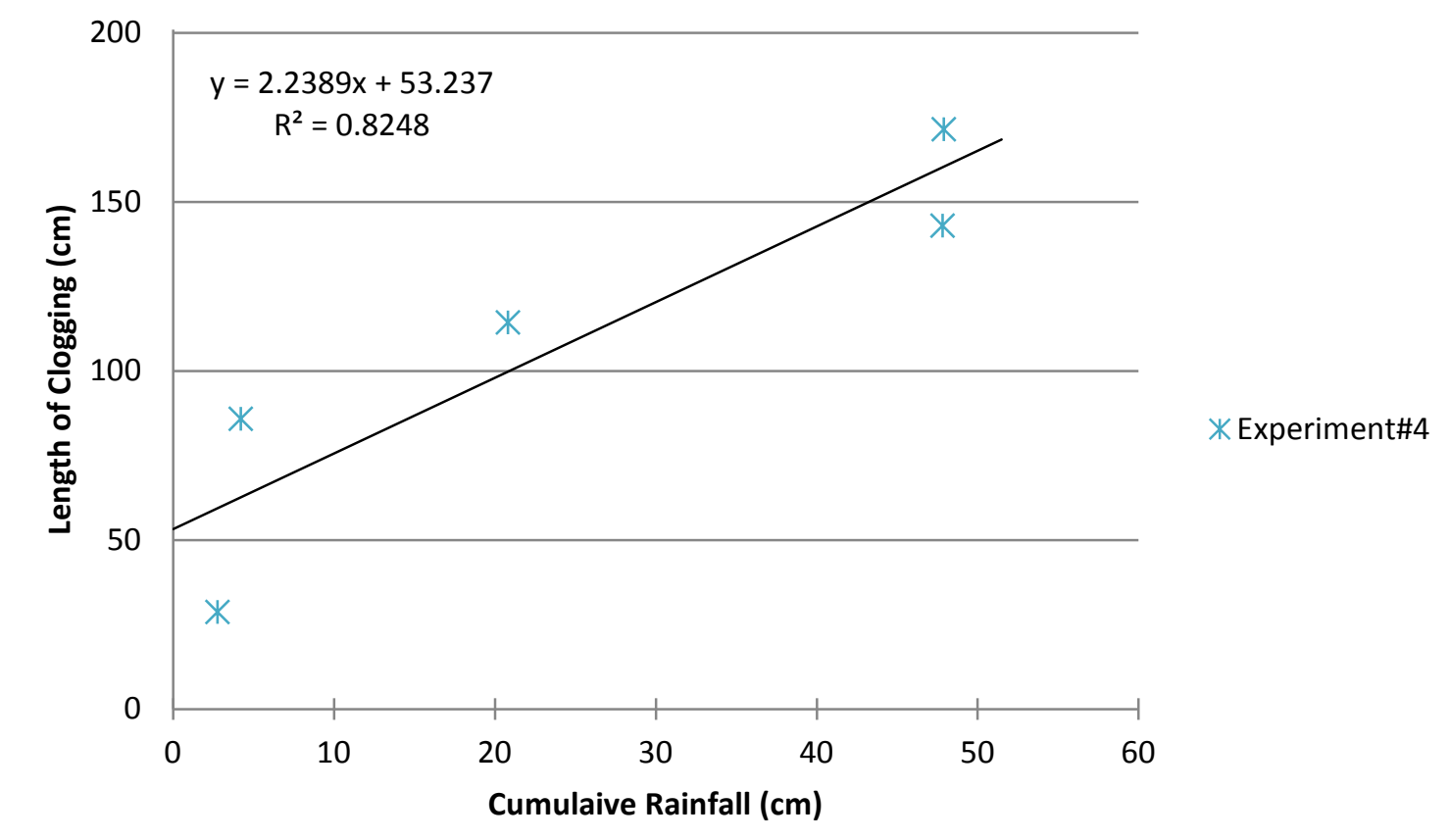

In order to plot this experiment, two of the points which appeared out of order were excluded. The first point excluded is the maximum number measured by the $2^{\text {nd }}$ TDR, which occurs later than the $3^{\text {rd }}$ and $4^{\text {th }}$ TDRs. This is mainly due to the interruption in the test. The last TDR measurement was also excluded, since the interruption resulted in a reduced, yet steady surface infiltration rate on the segments over the $5^{\text {th }}$ and $6^{\text {th }}$ TDRs and this caused the measurements to reach maximum at a very late stage in the tests. 


\section{Experiment\#5}

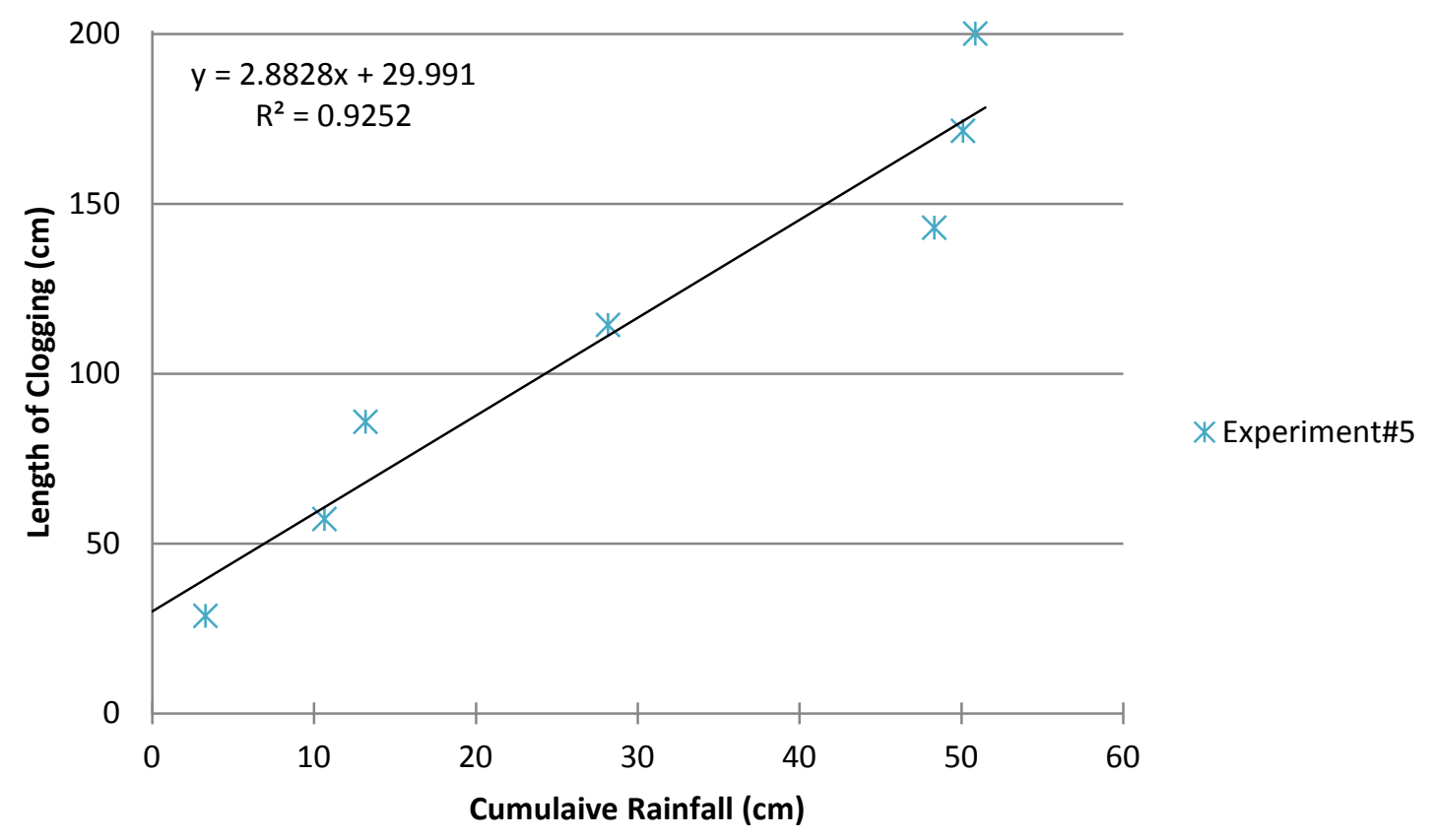




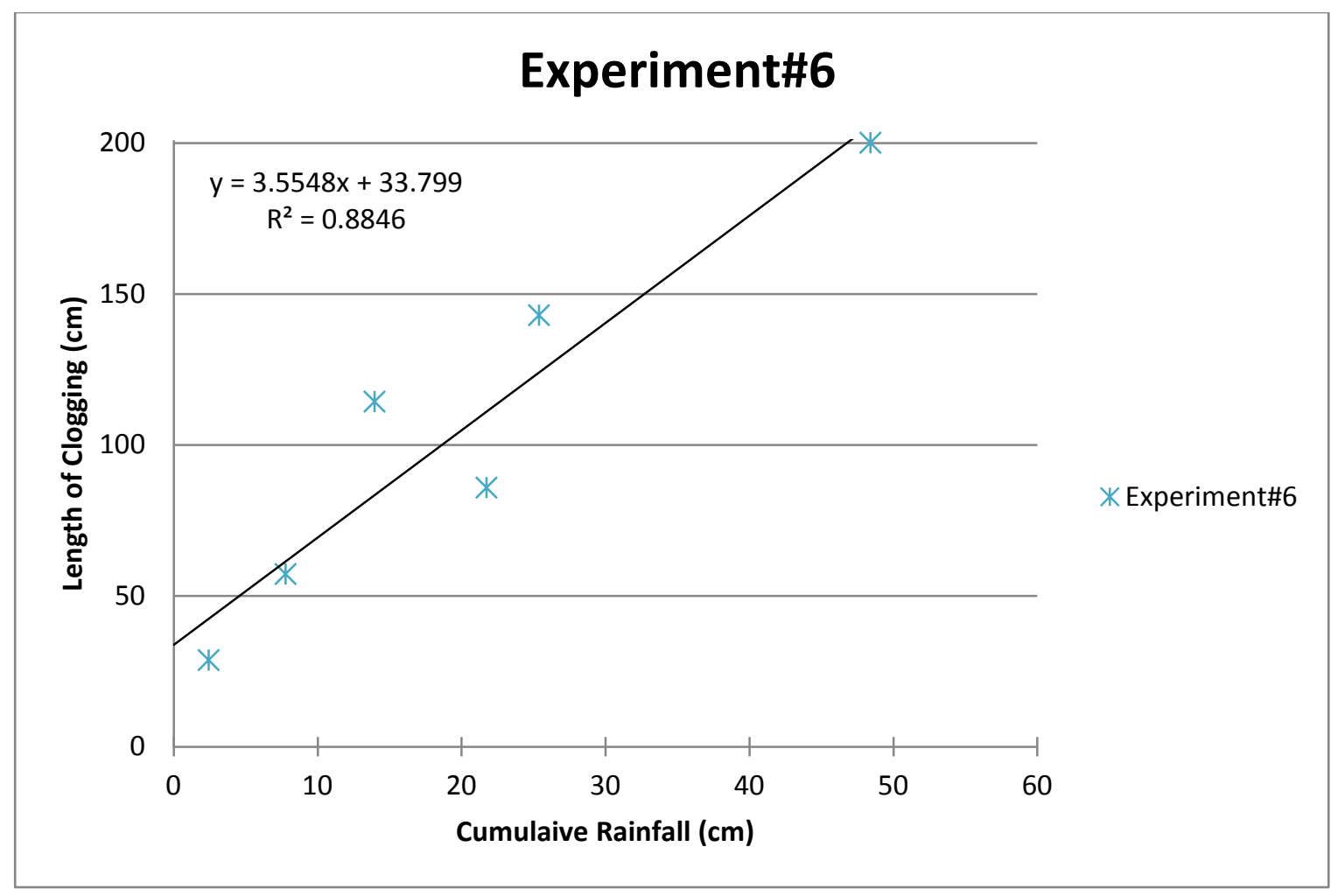

One data point was excluded from this experiment. The $6^{\text {th }}$ TDR measurement was left out because the TDR reaches the maximum measured value later than the last TDR, TDR 7, in the flume. 


\section{Experiment\#7}

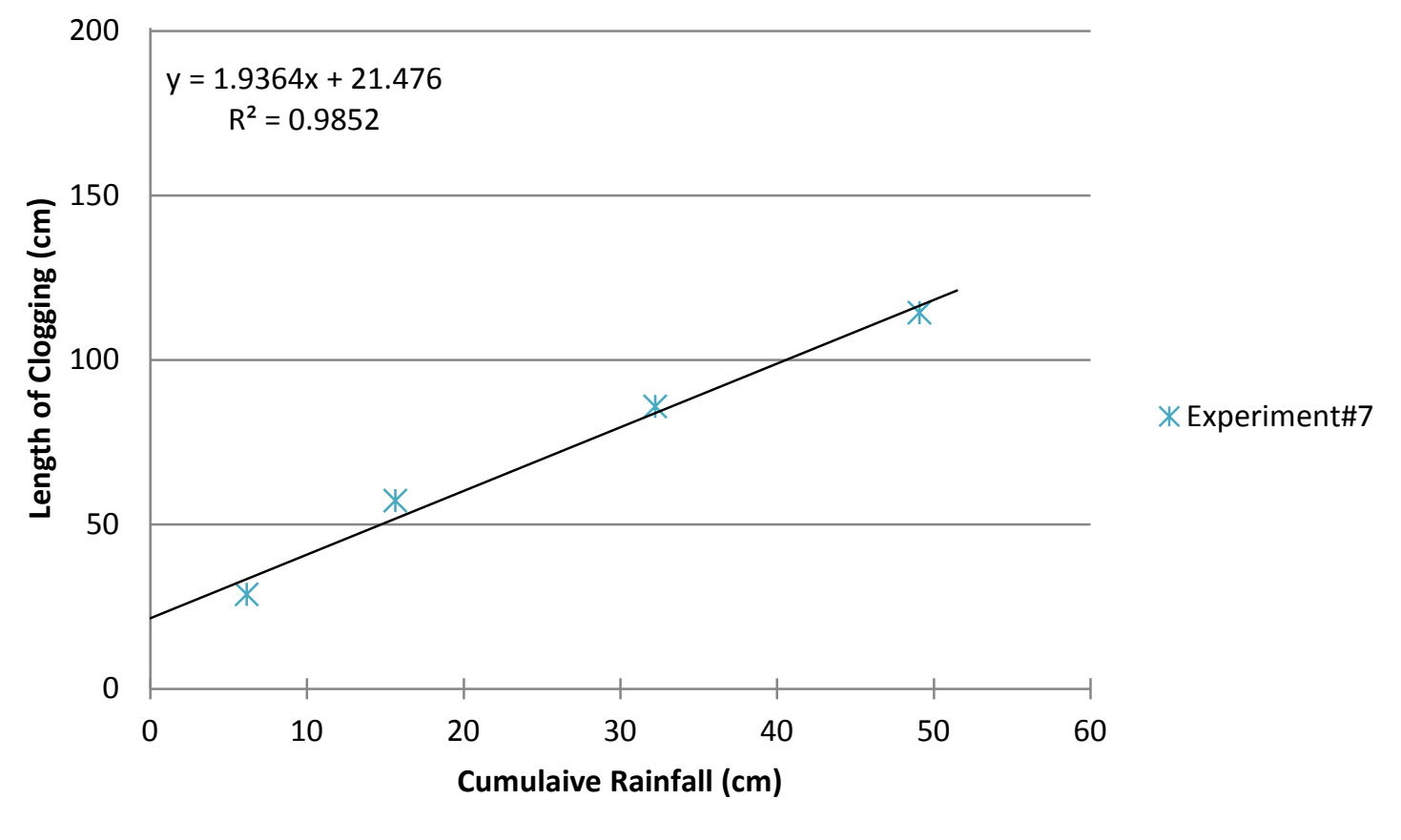




\section{Experiment\#8}

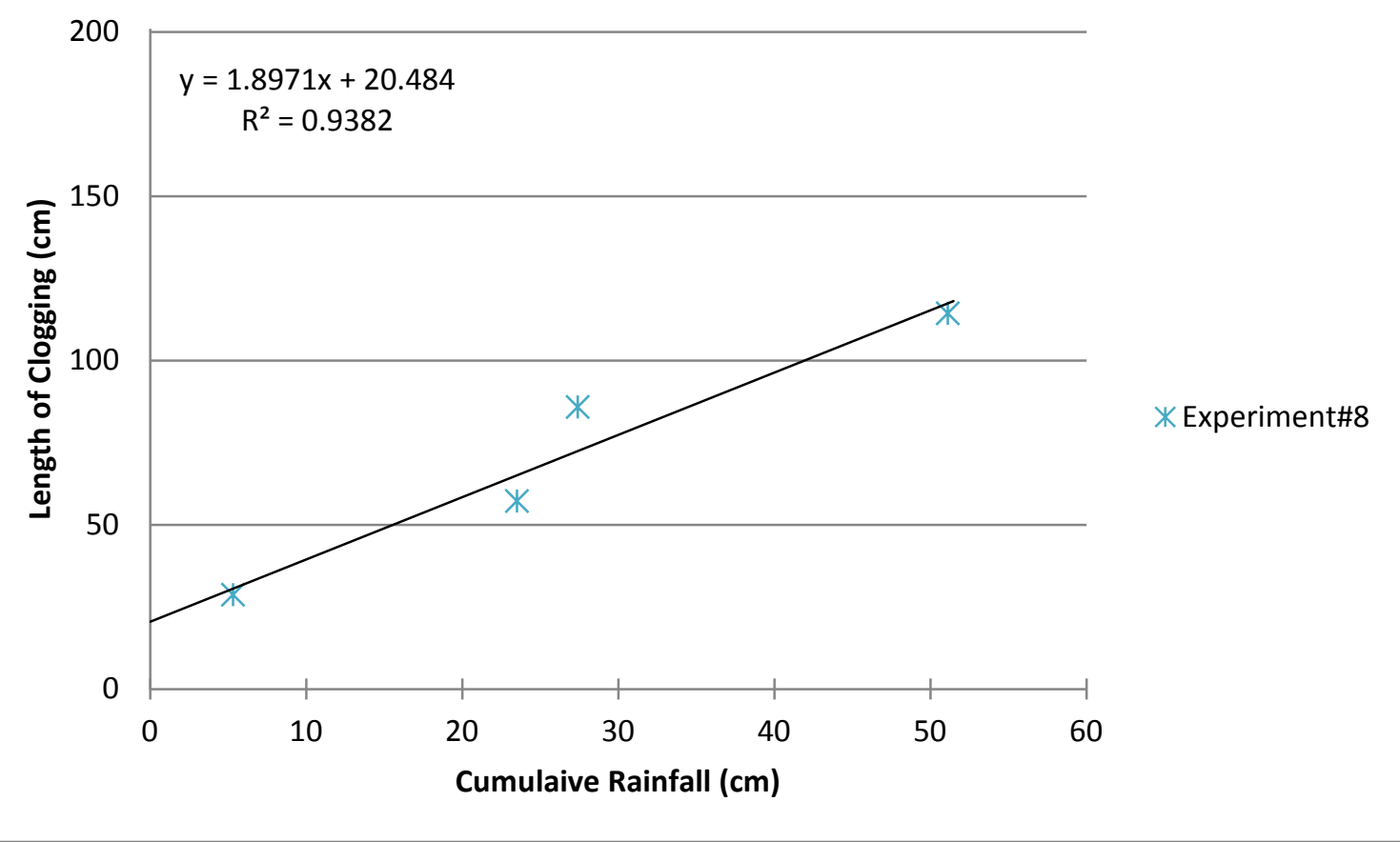




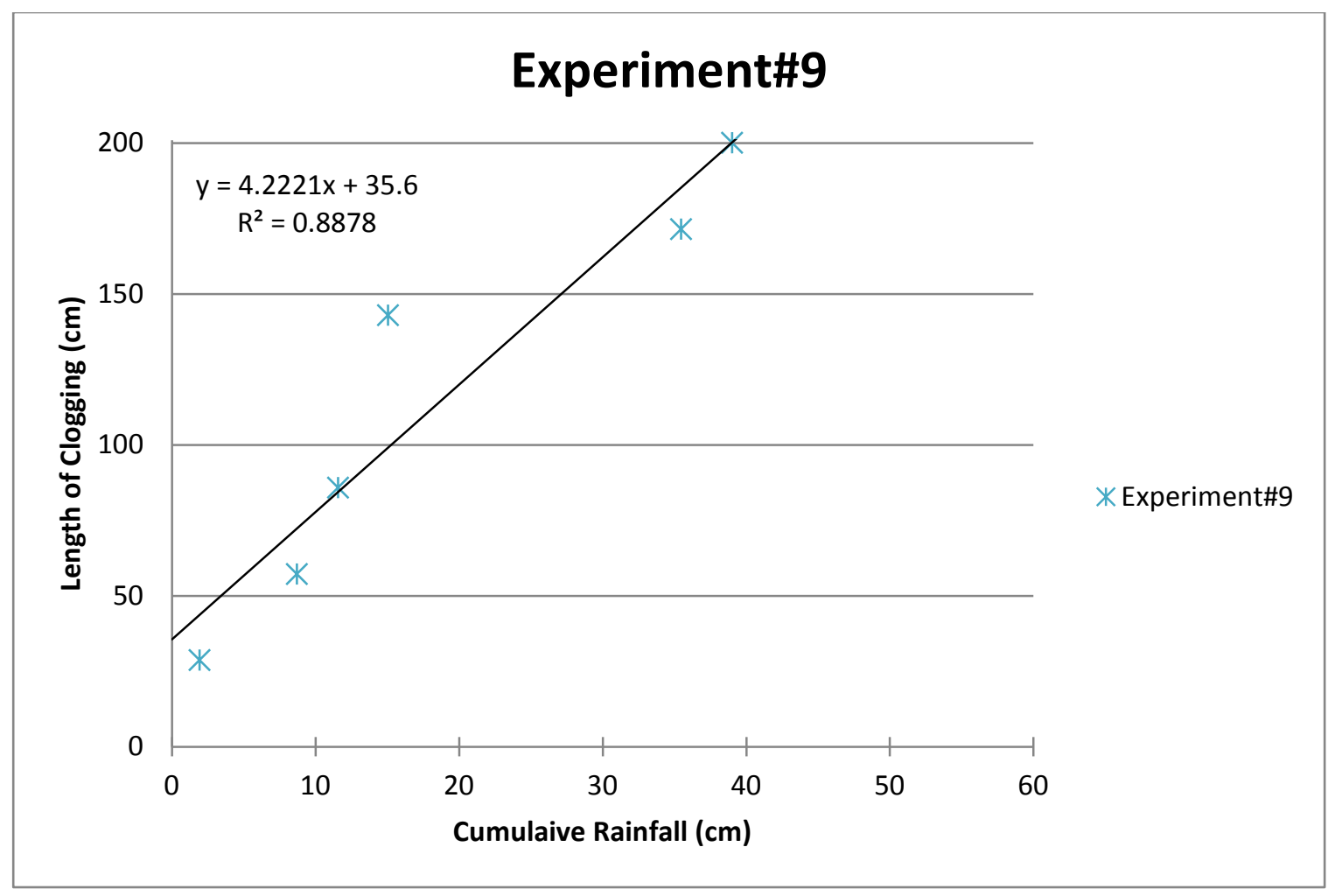

The $4^{\text {th }}$ TDR measurement has been excluded from the above graph because it reaches the maximum measured value 3 minutes after the $5^{\text {th }}$ TDR. 


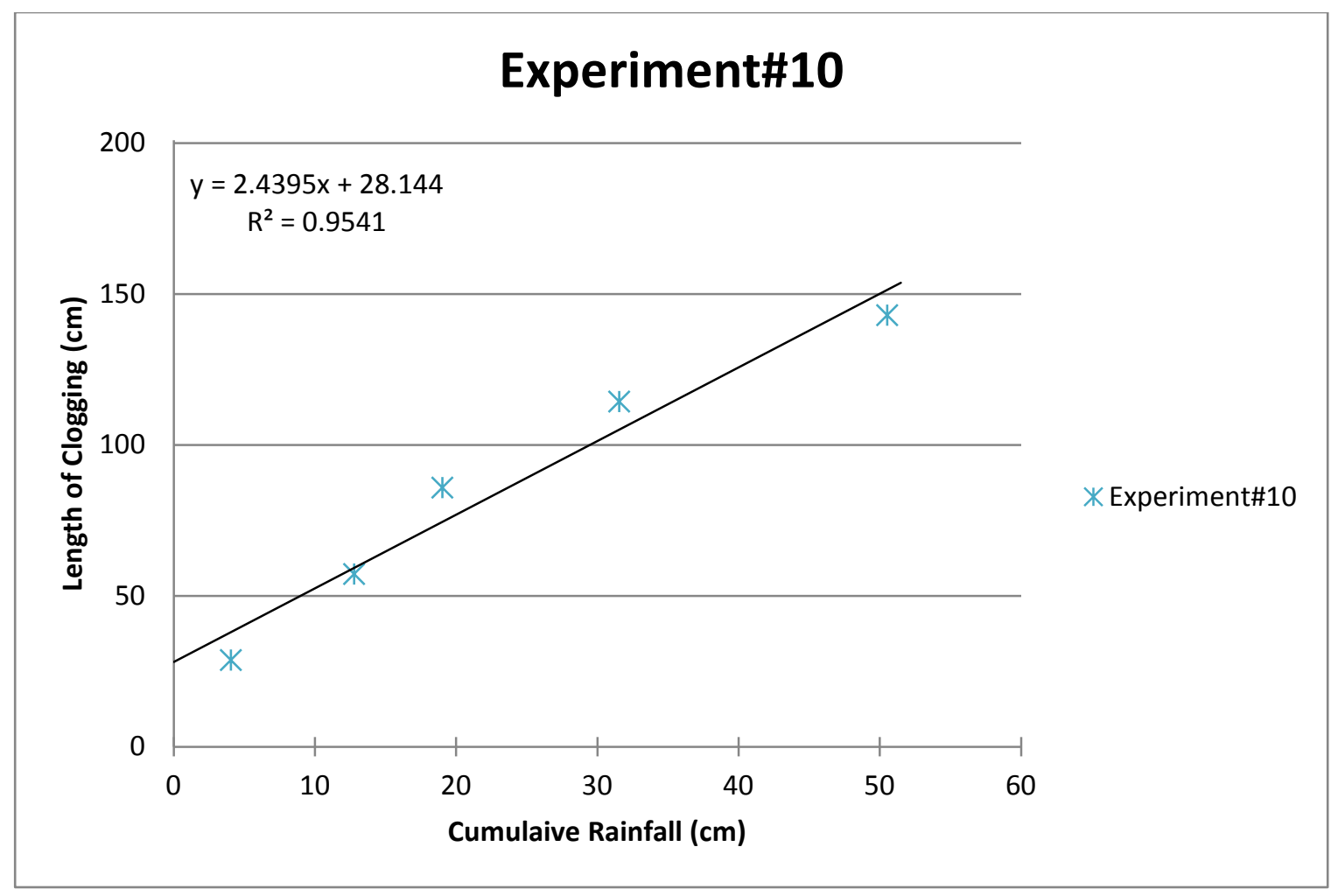




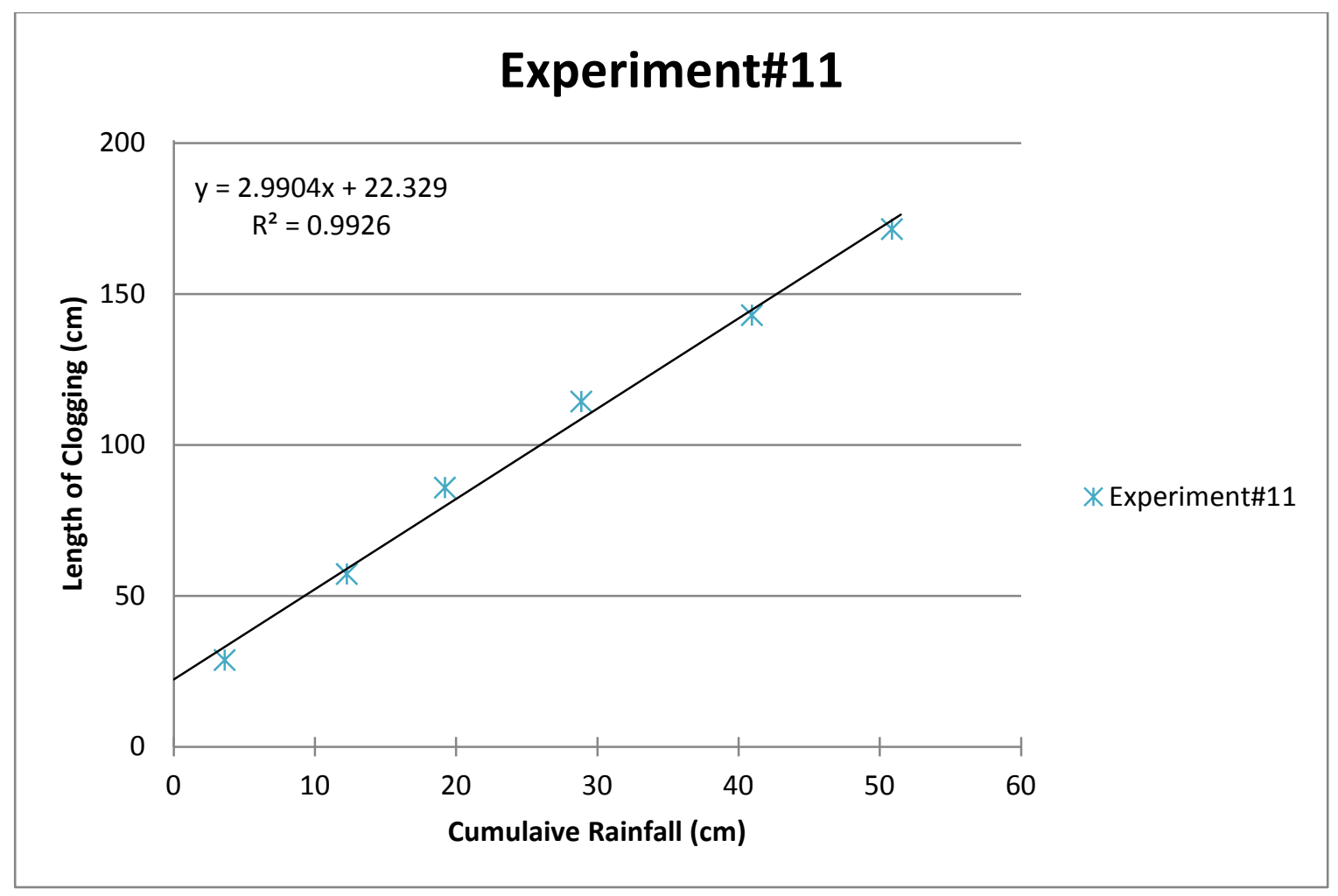




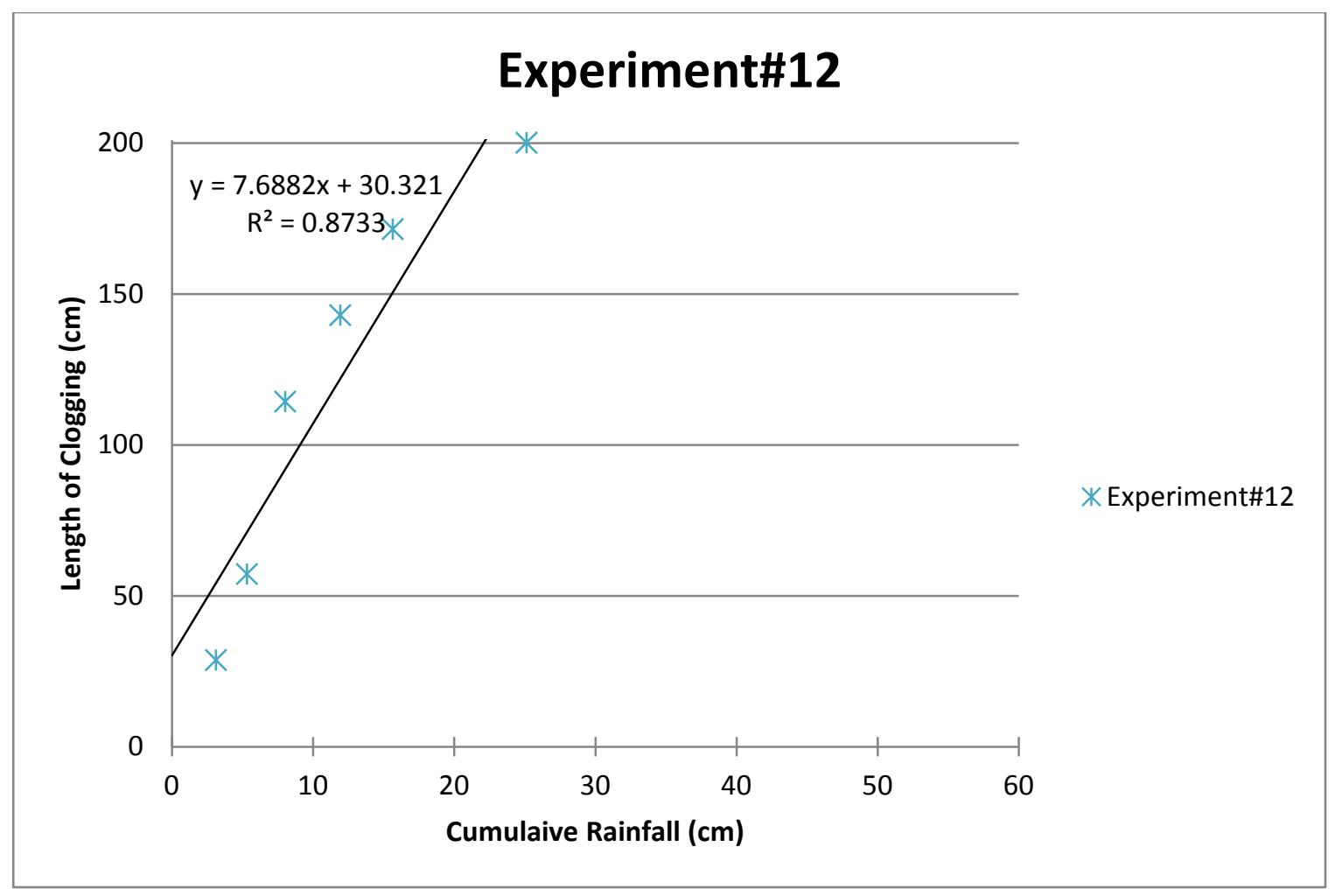

The $3^{\text {rd }}$ TDR measurement has been excluded because it reaches the maximum measured value after the $4^{\text {th }}$ and $5^{\text {th }}$ TDRs. 


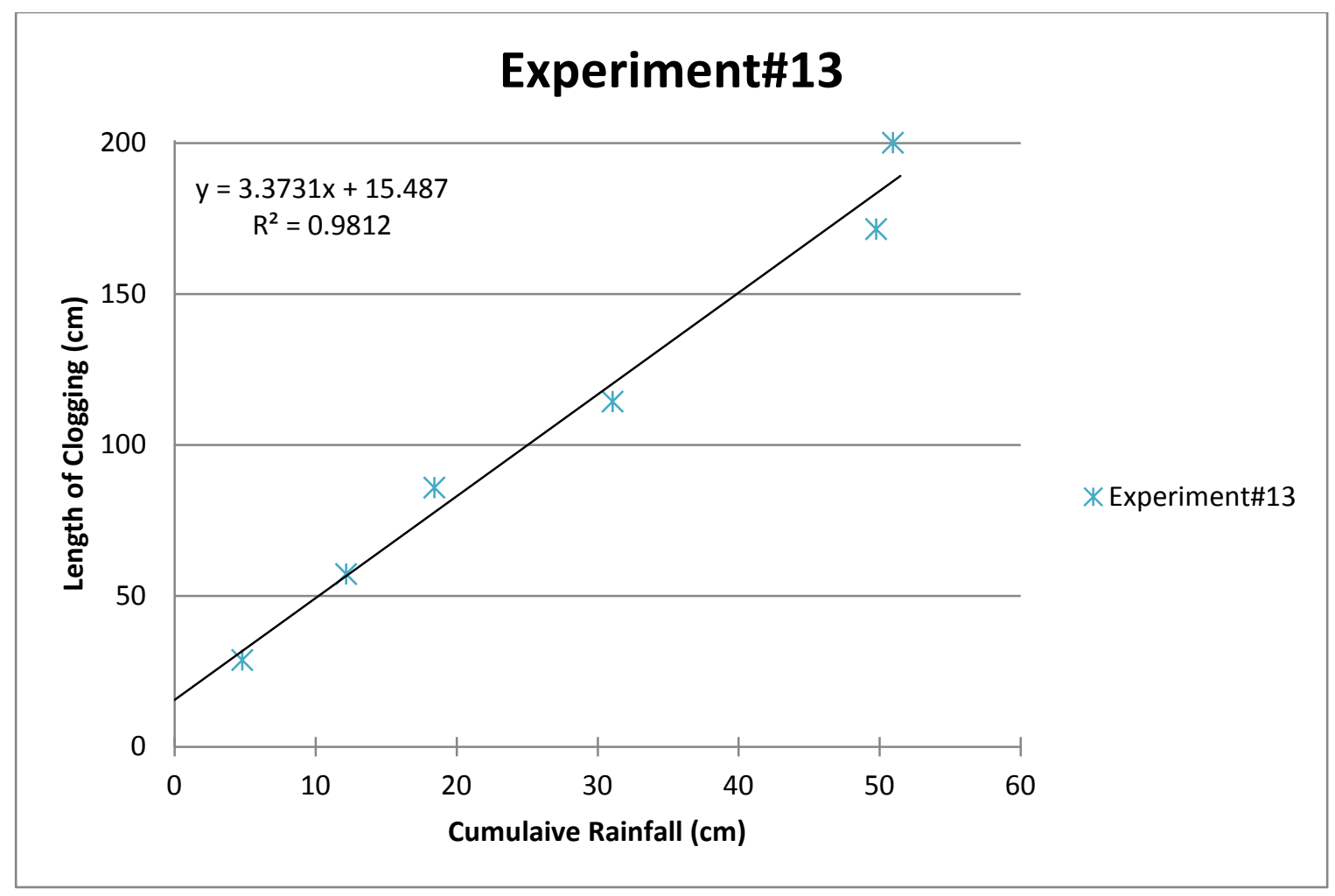




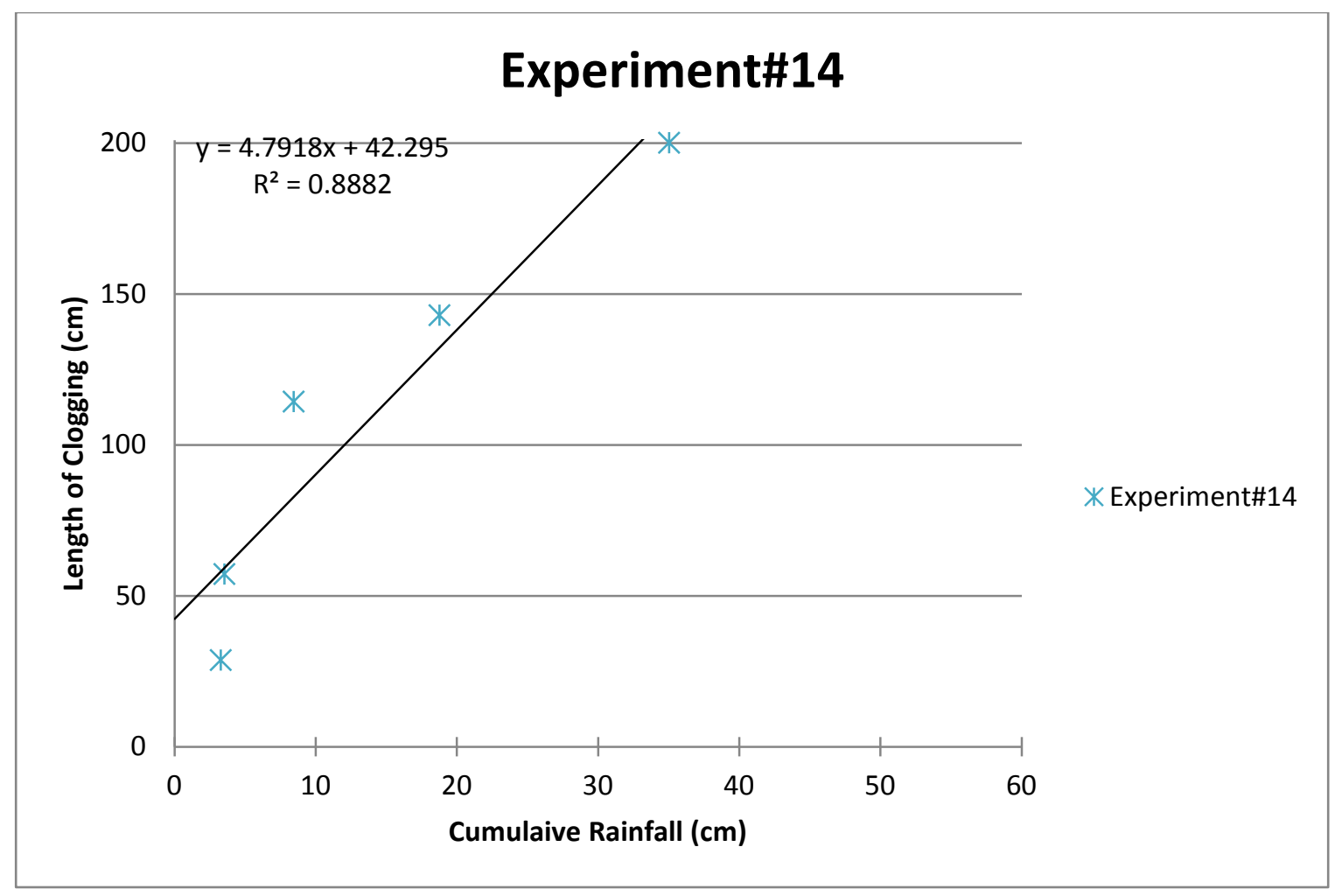

The $3^{\text {rd }}$ TDR measurement has been excluded from this graph, since it reaches the maximum measured value sooner than either the $1^{\text {st }}$ or $2^{\text {nd }}$ TDRs. Moreover, the maximum measurement recorded by the $5^{\text {th }}$ TDR comes towards the end of the test when the ponding of runoff pushes the ponding backwards, which is excluded from the graph. 


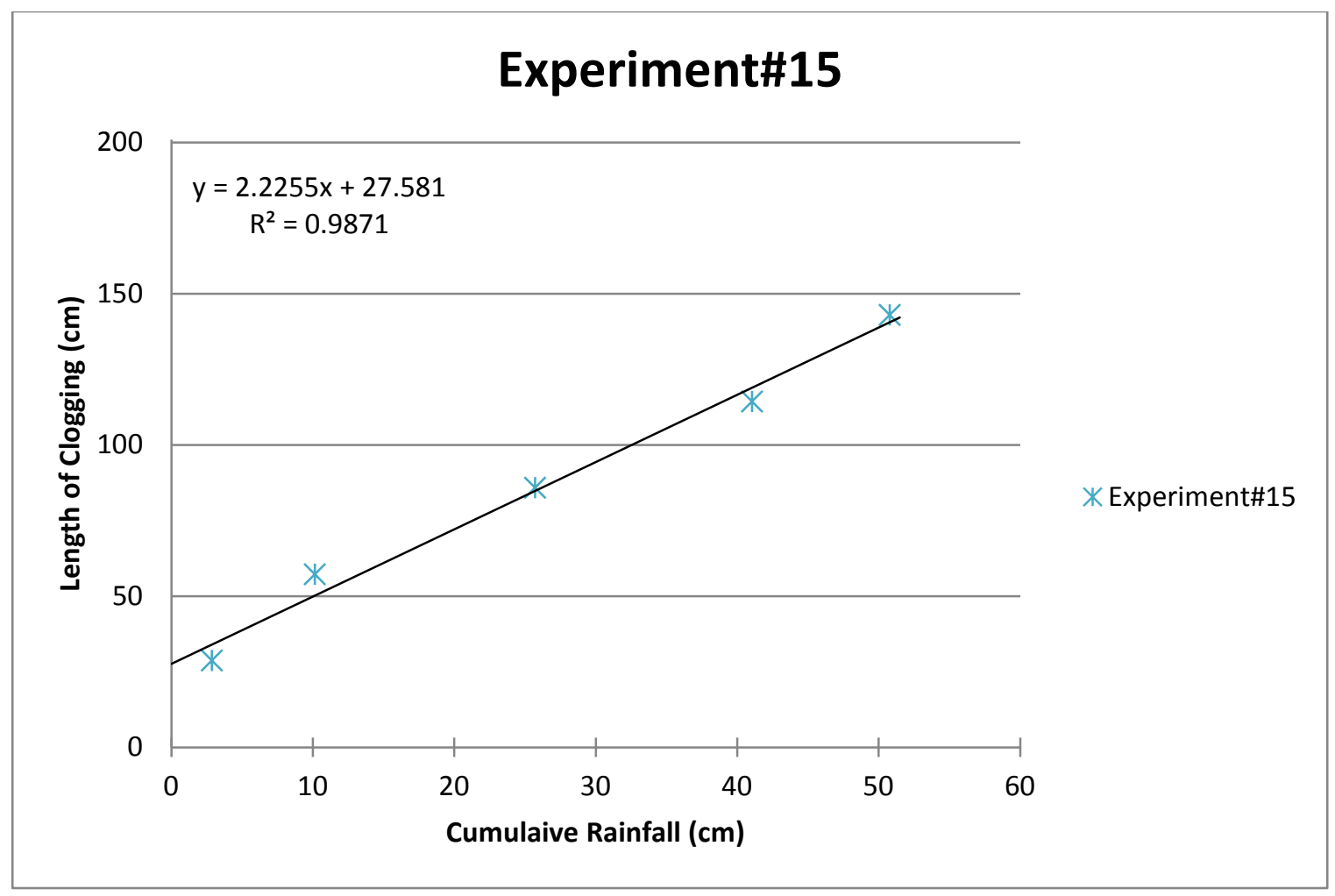




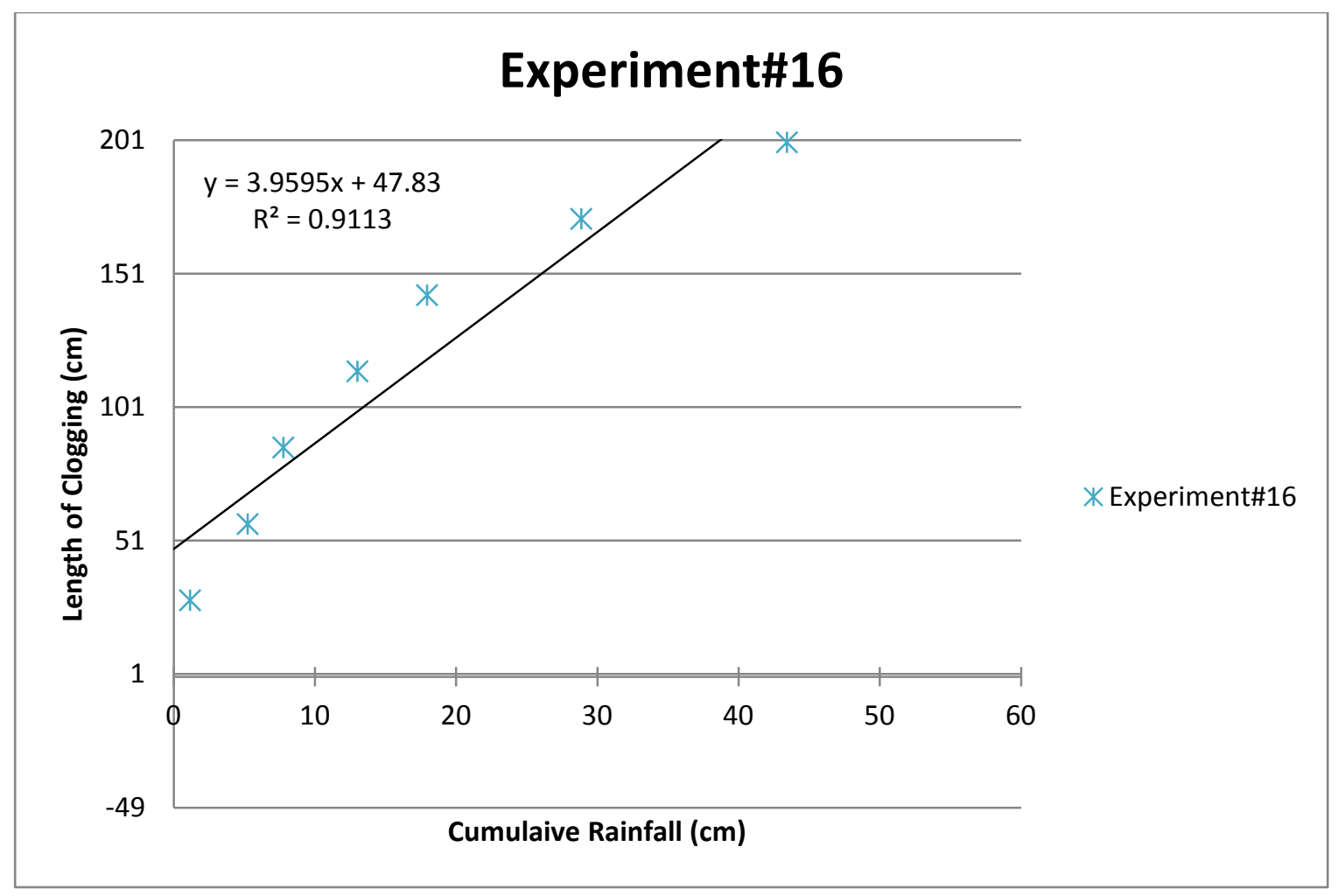




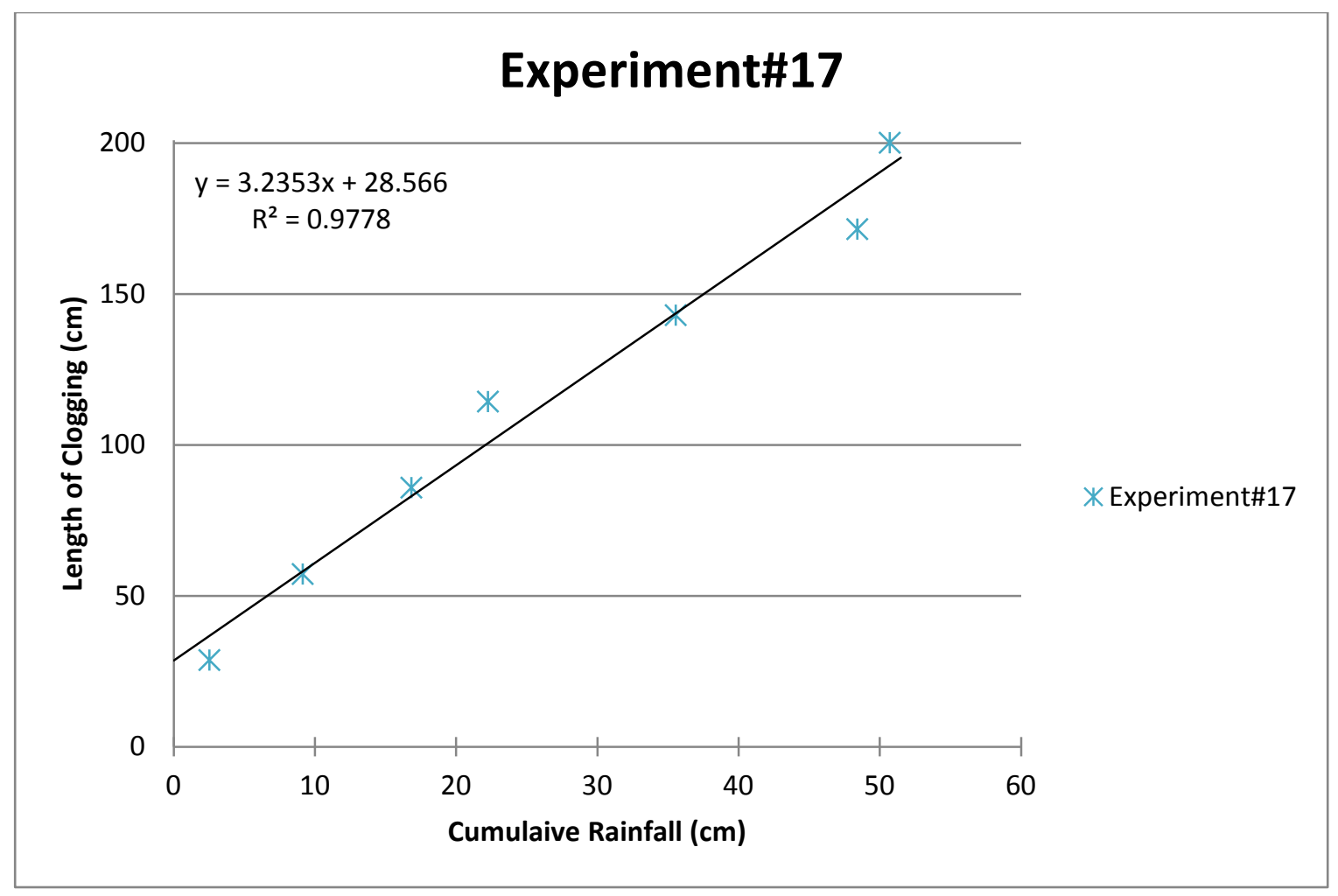




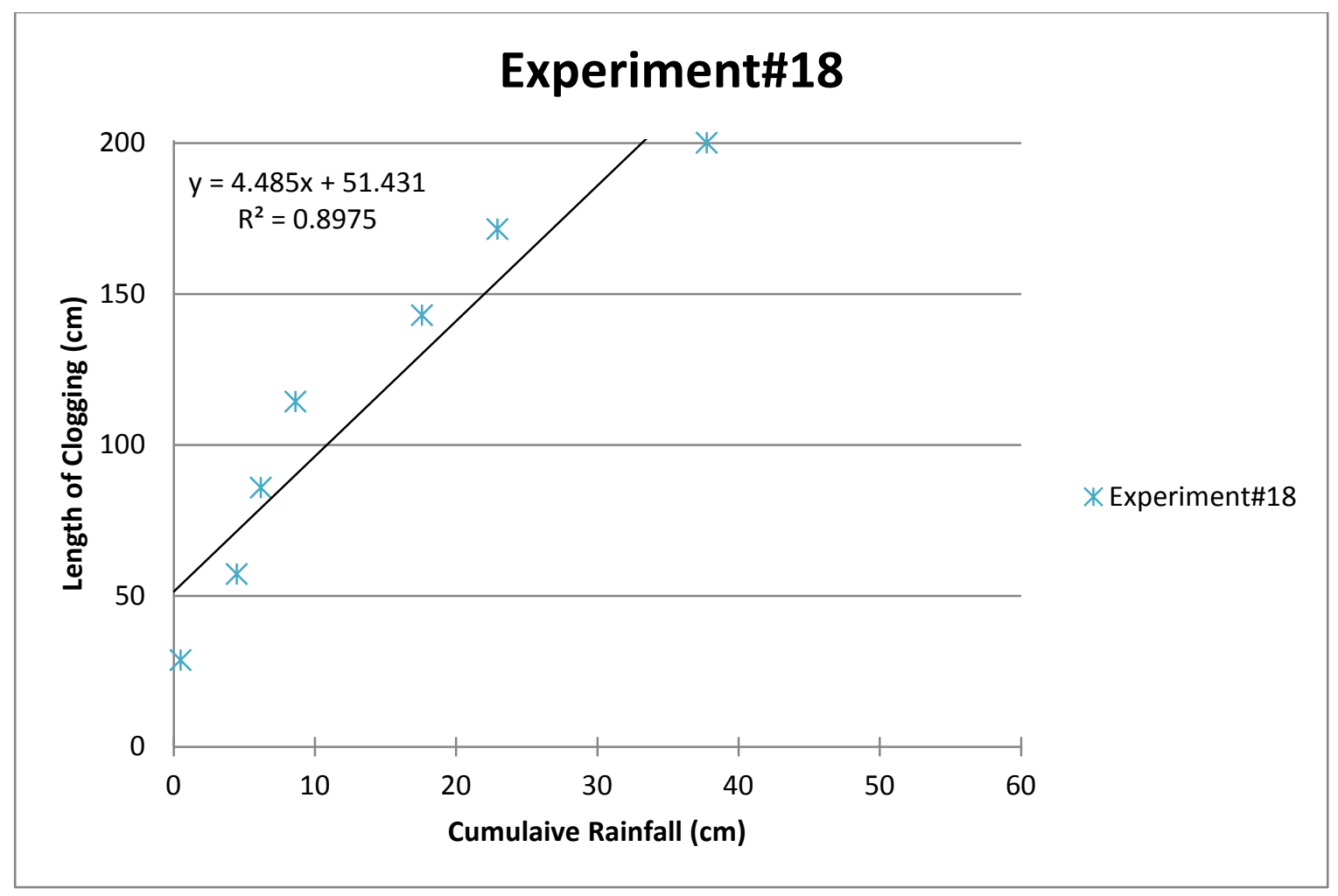




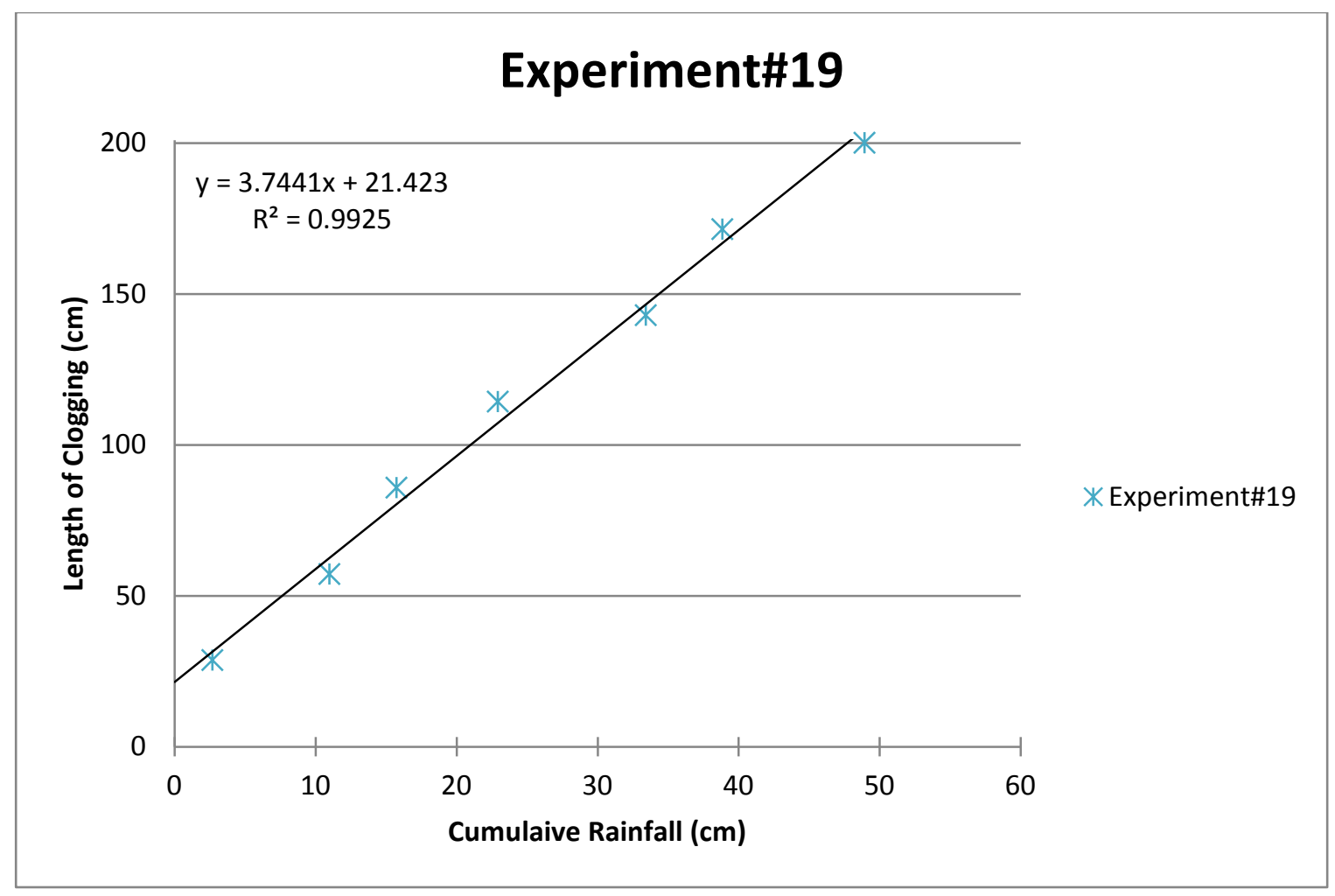




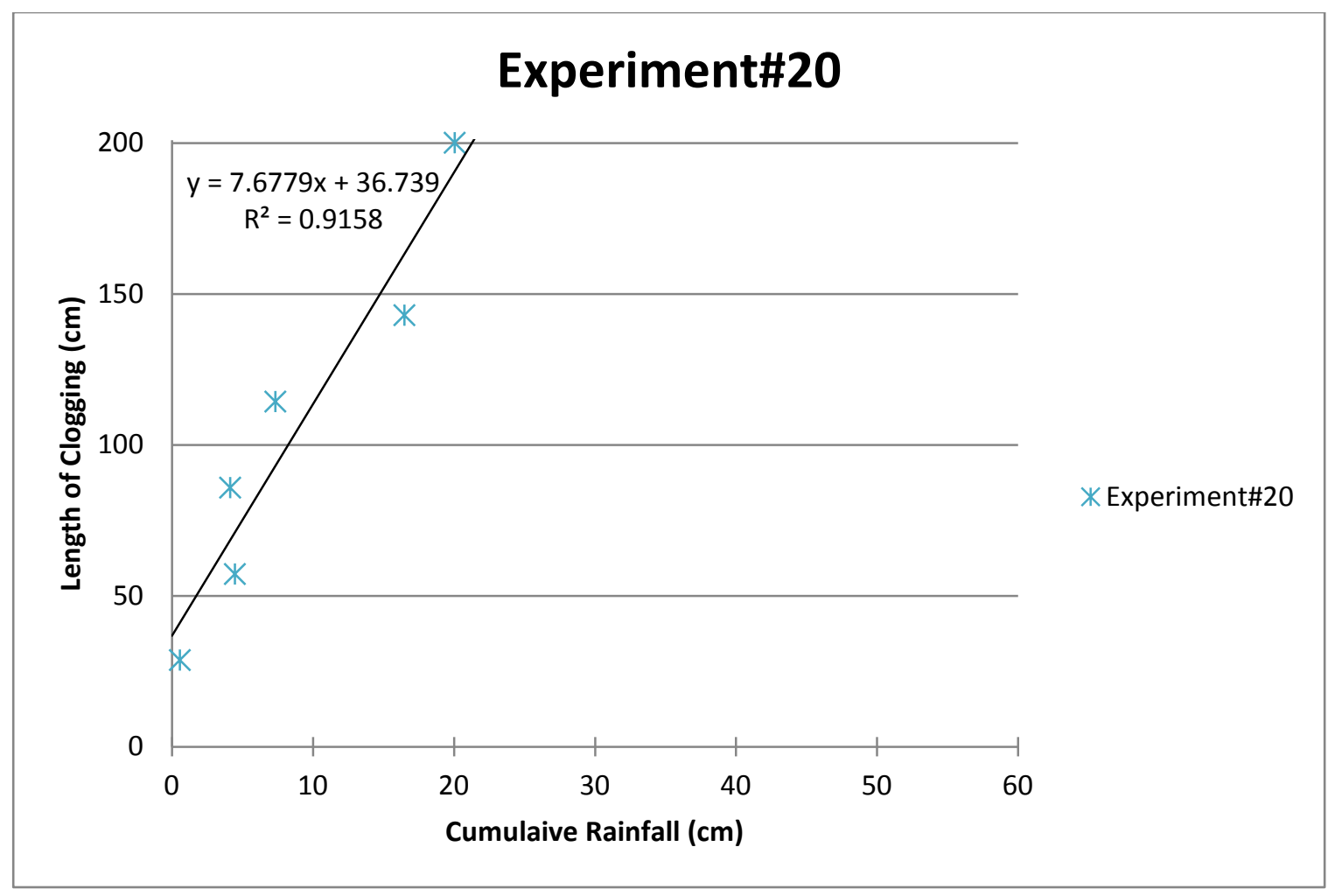

The $6^{\text {th }}$ TDR measurement reaches the maximum measured value only after the entire length of the flume is clogged and ponding has backed up and covered the gaps over this TDR; therefore this particular measurement has been excluded from the graph. 


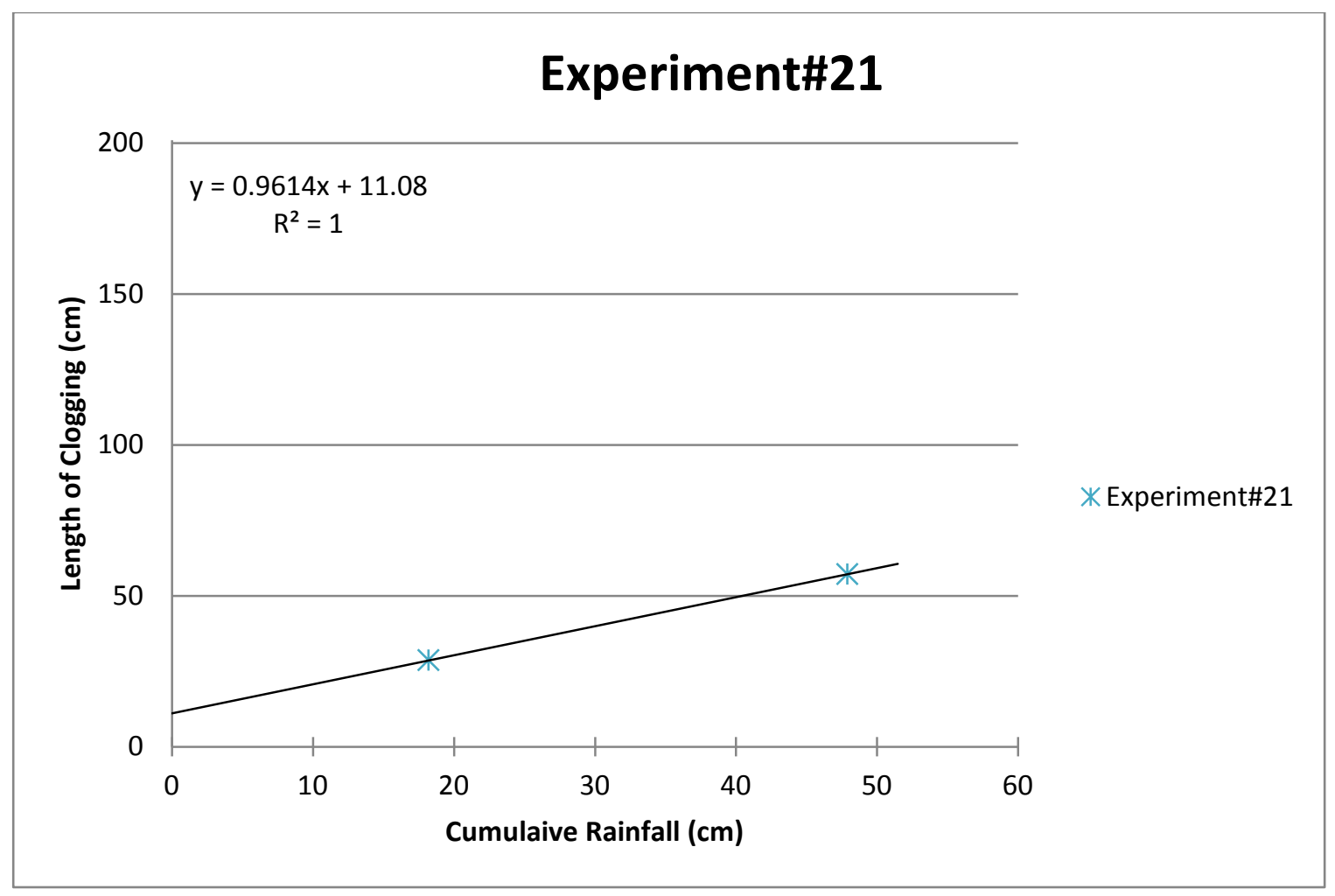




\section{APPENDIX G}

\section{Progression of infiltration edge}

The graphs showing the infiltration edge for all experiments are illustrated in this appendix.

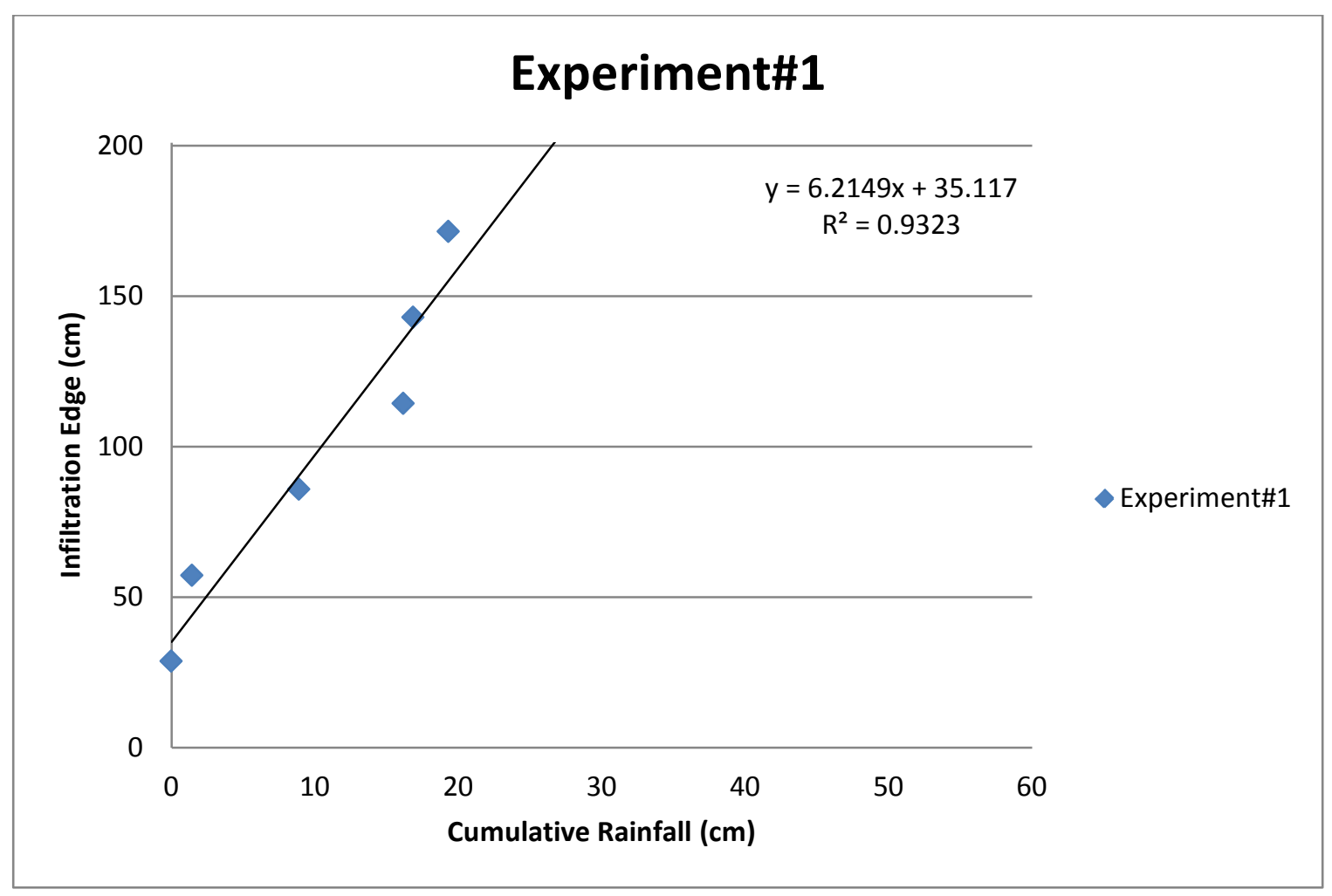




\section{Experiment\#2}

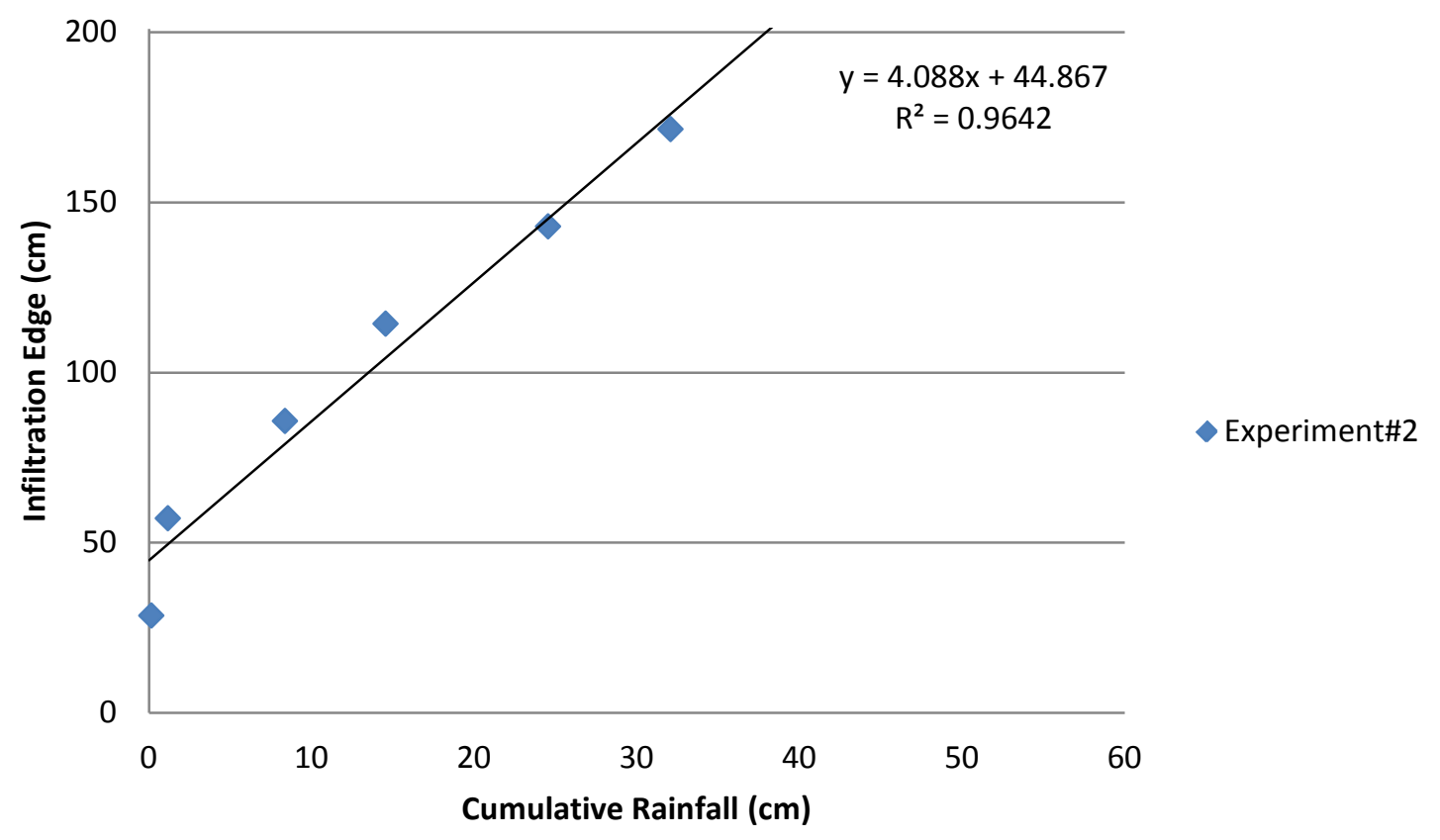




\section{Experiment\#3}

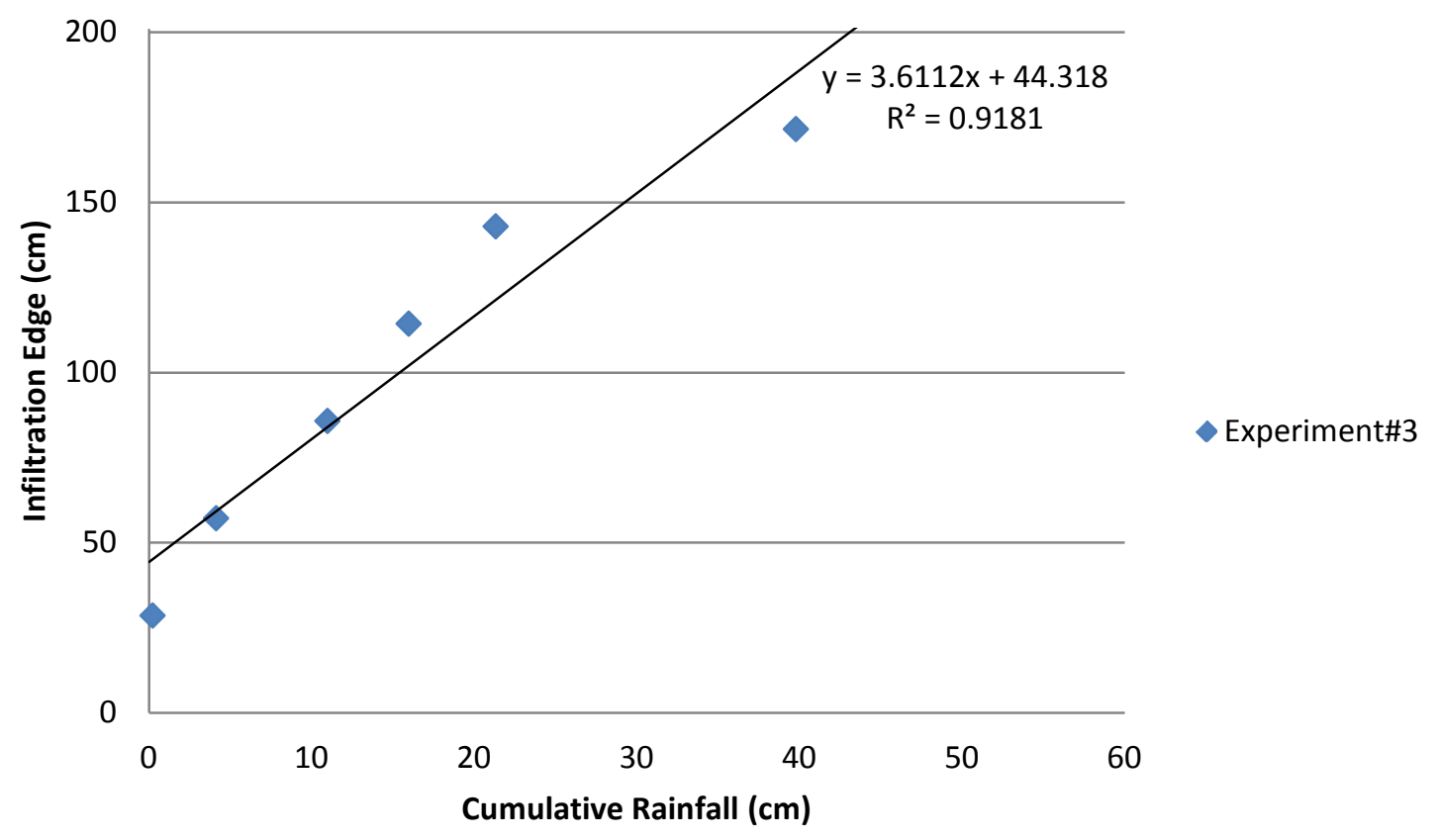




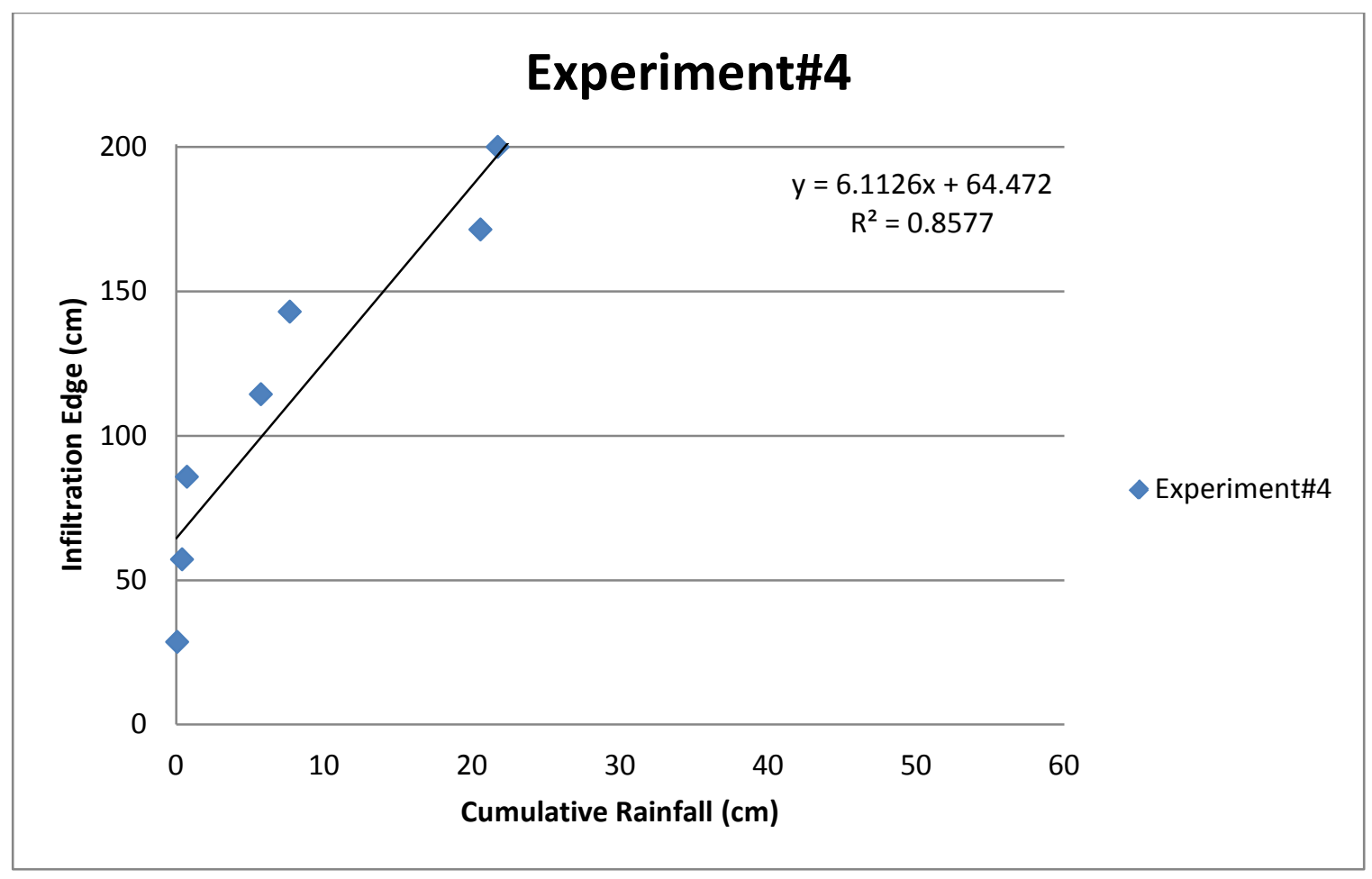




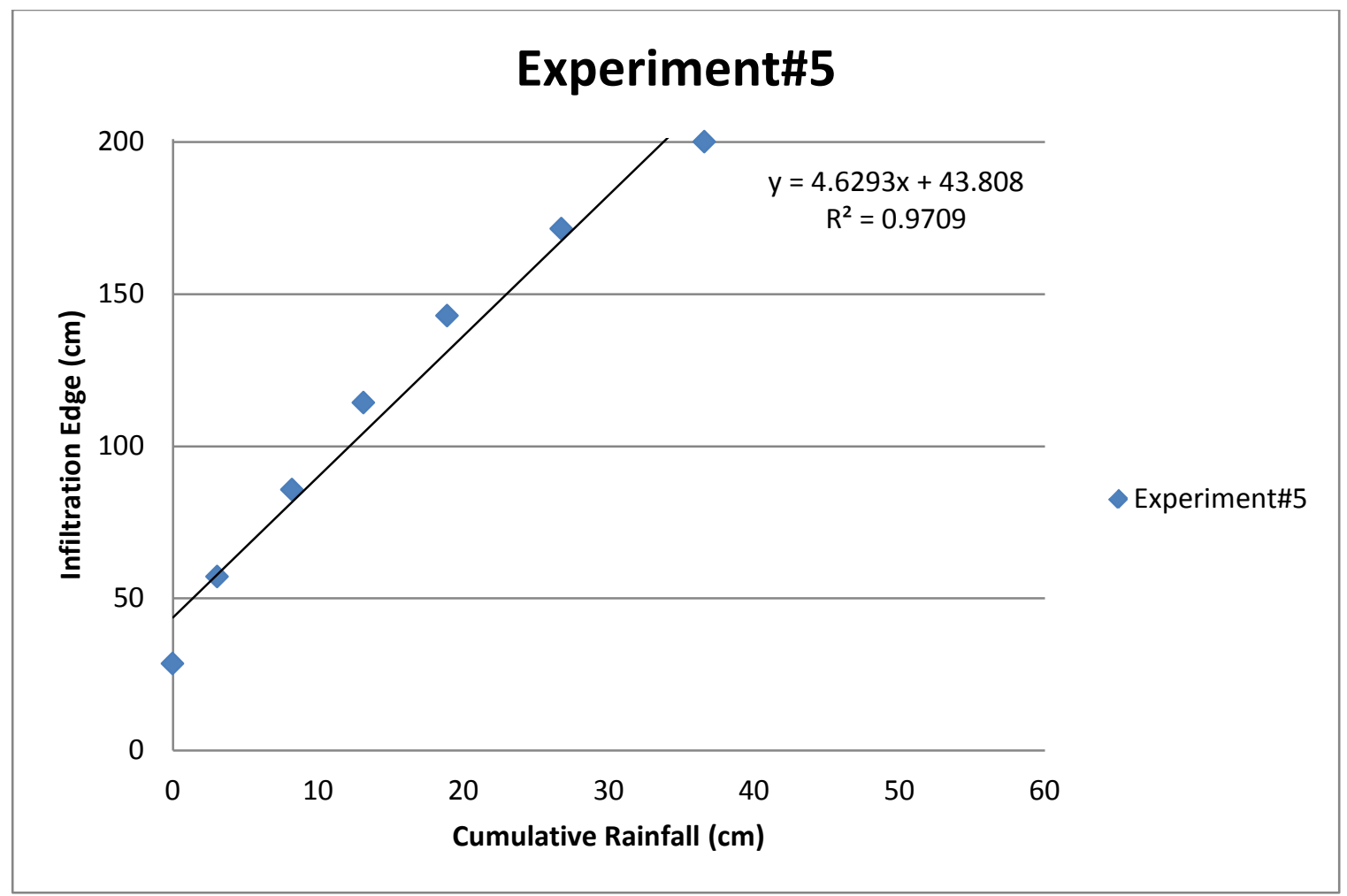




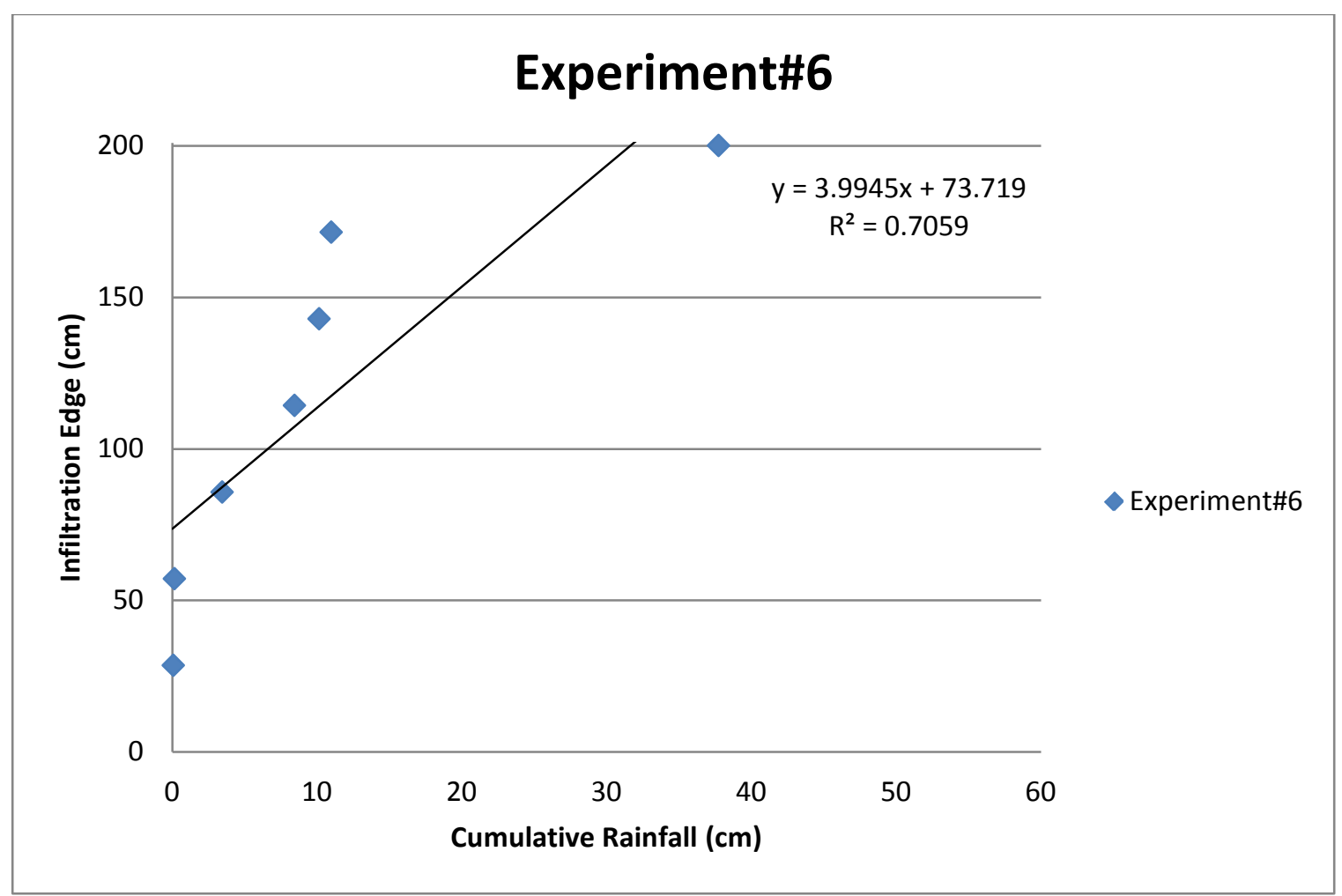




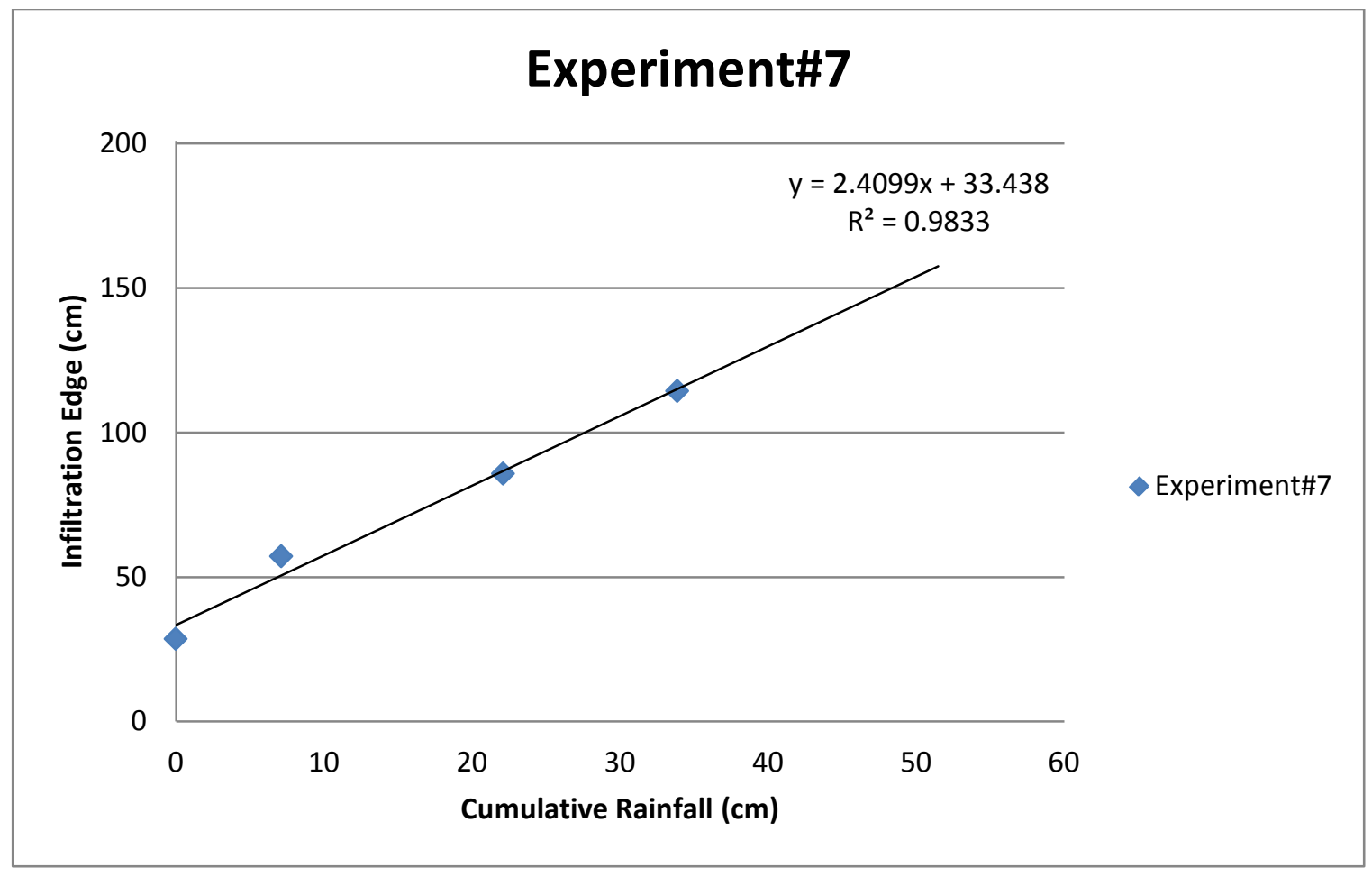




\section{Experiment\#8}

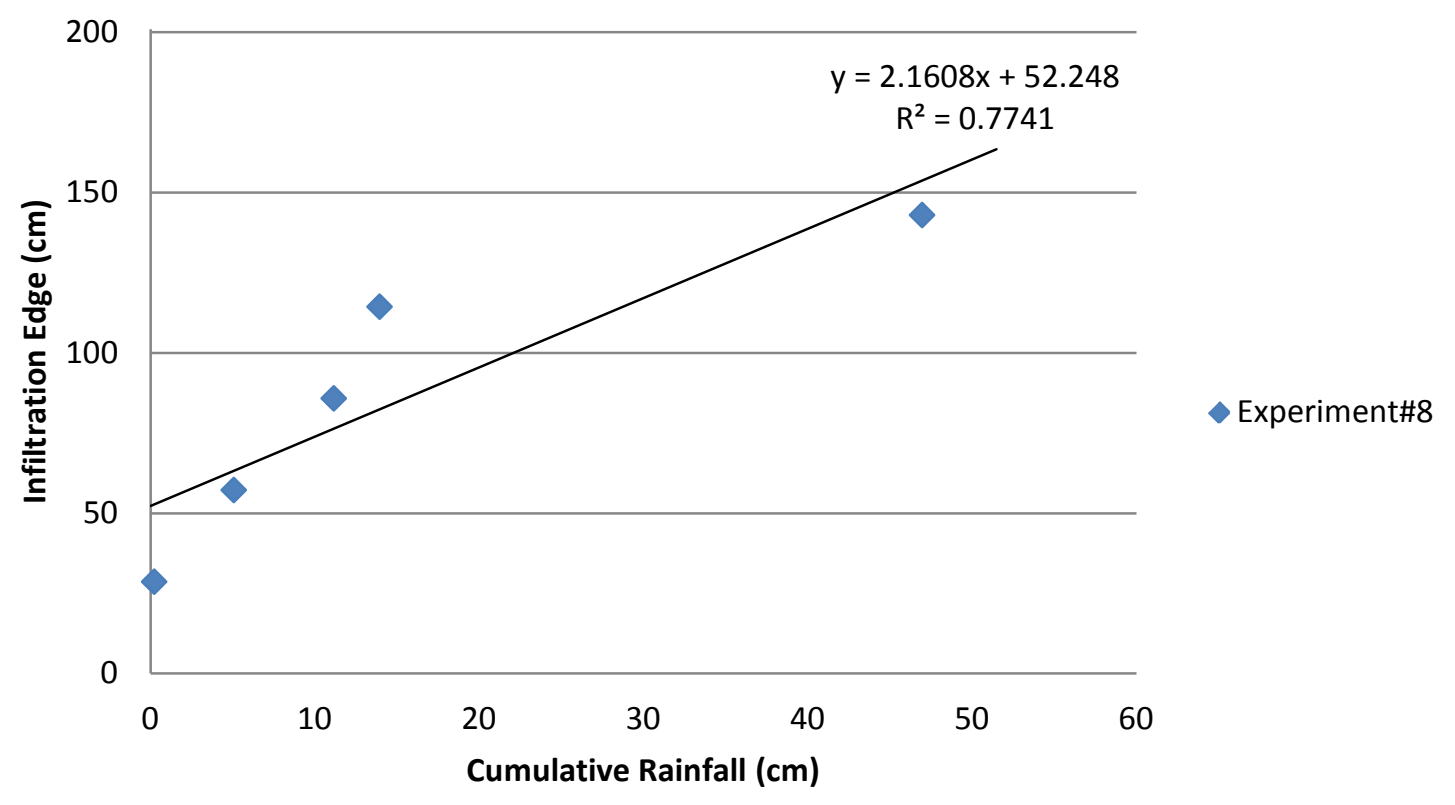




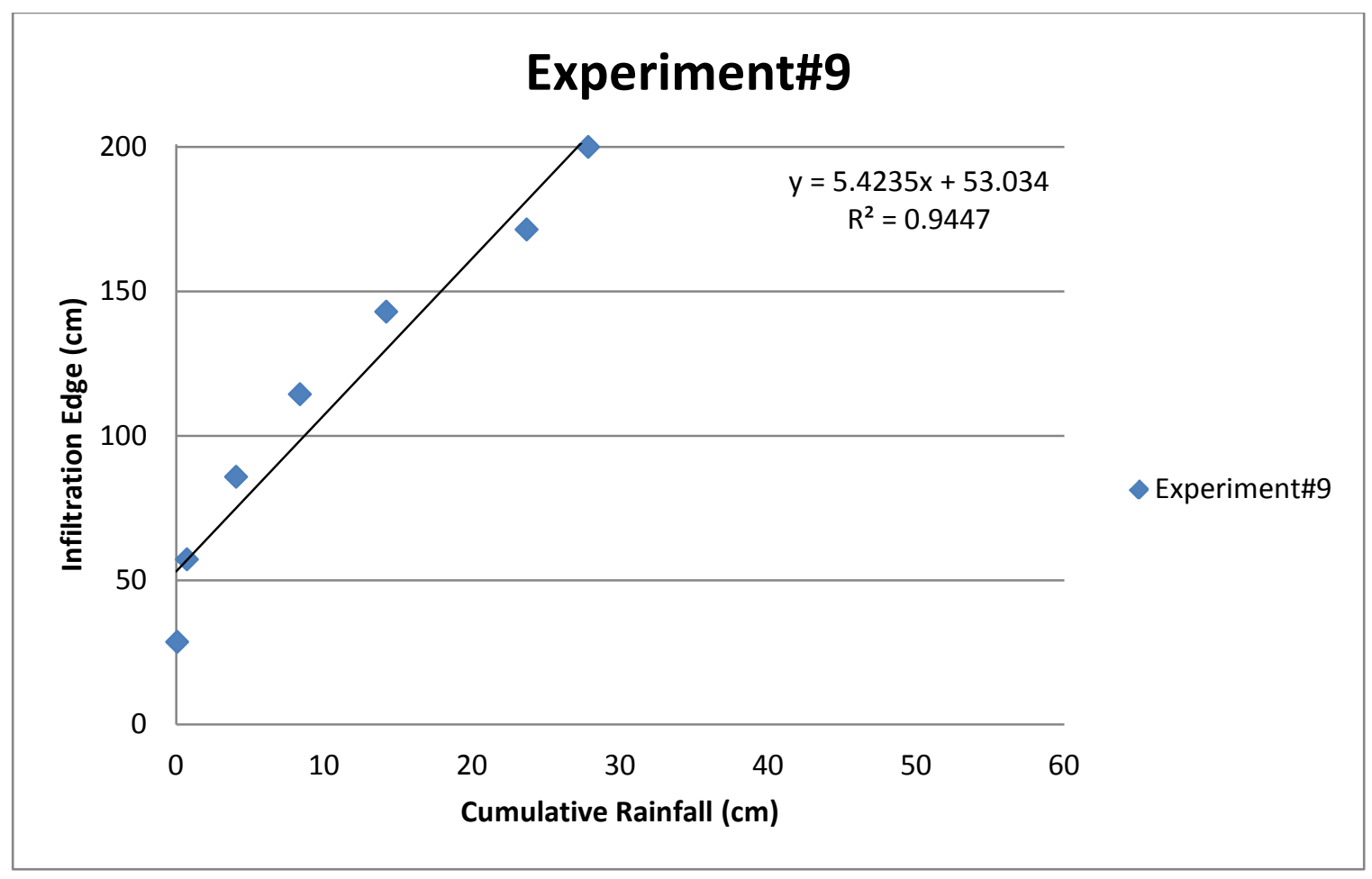




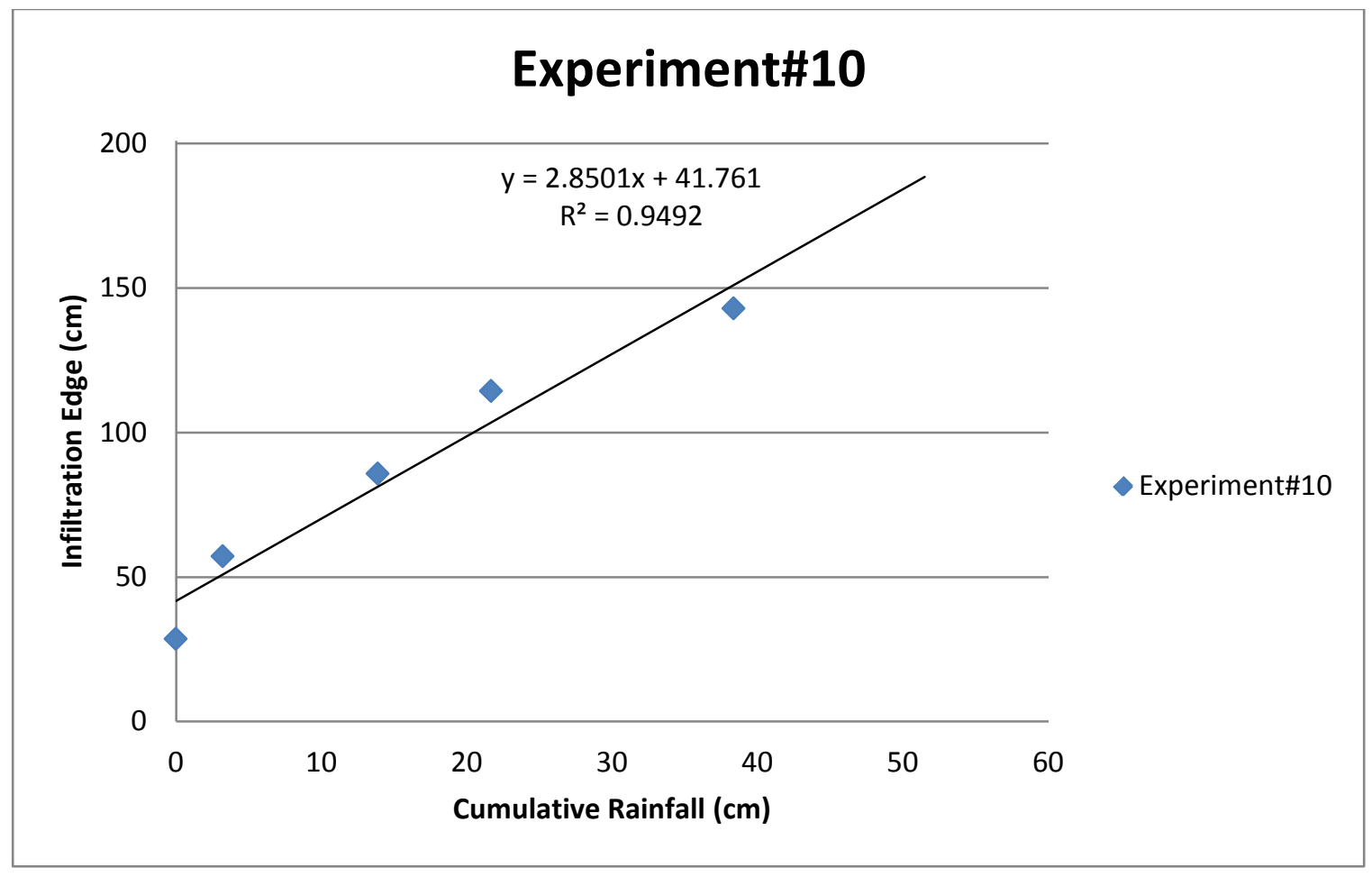




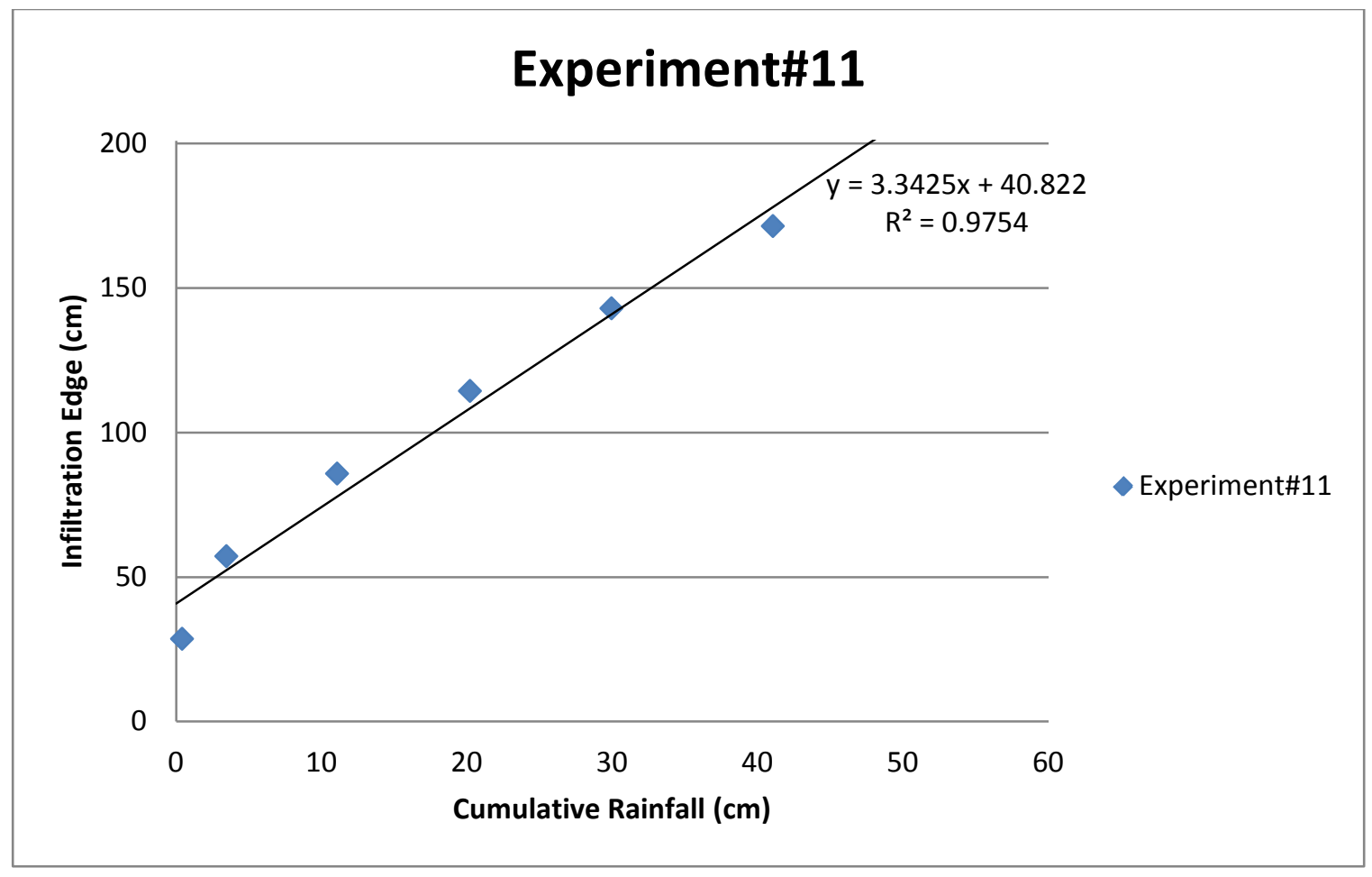




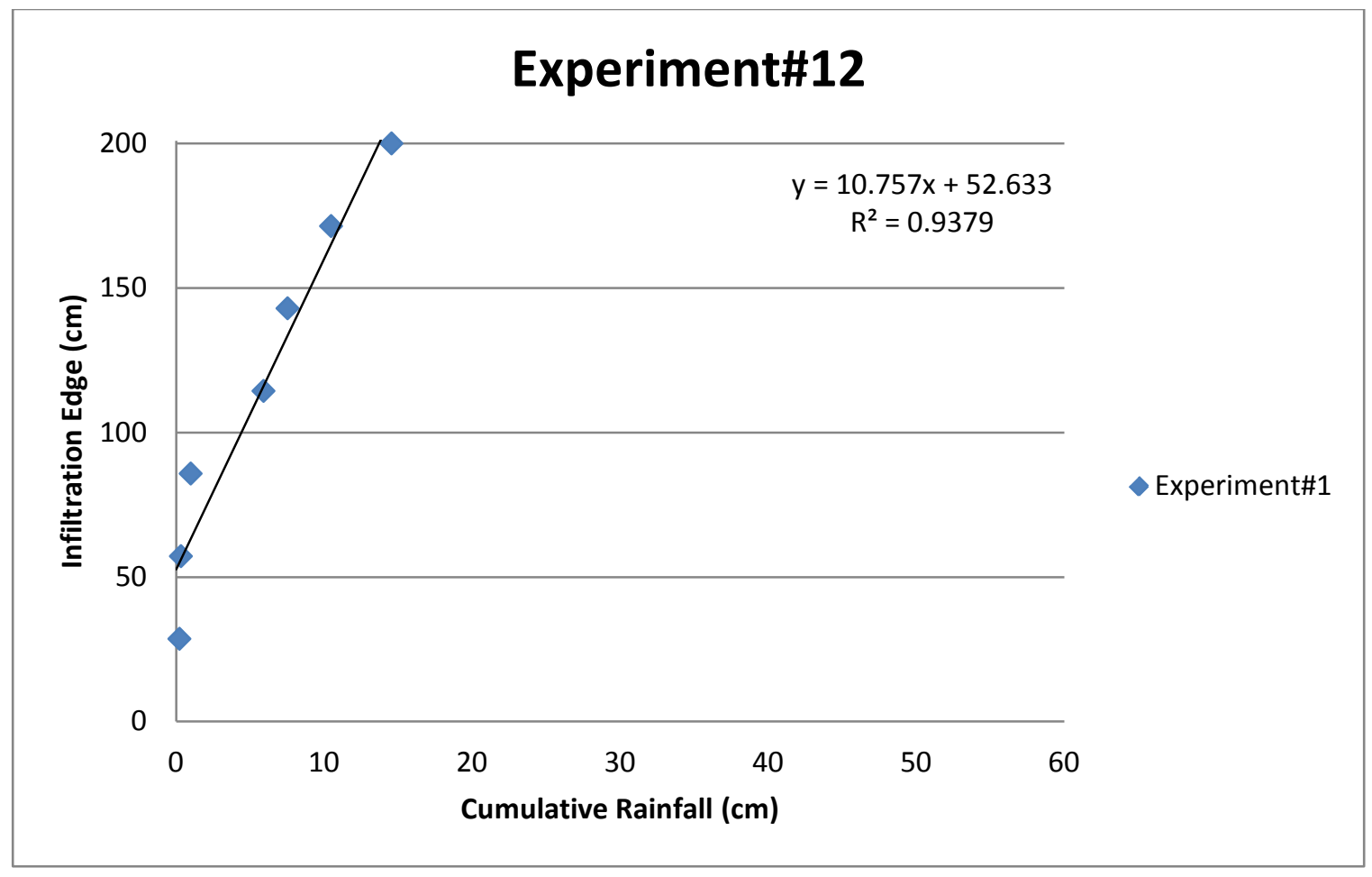




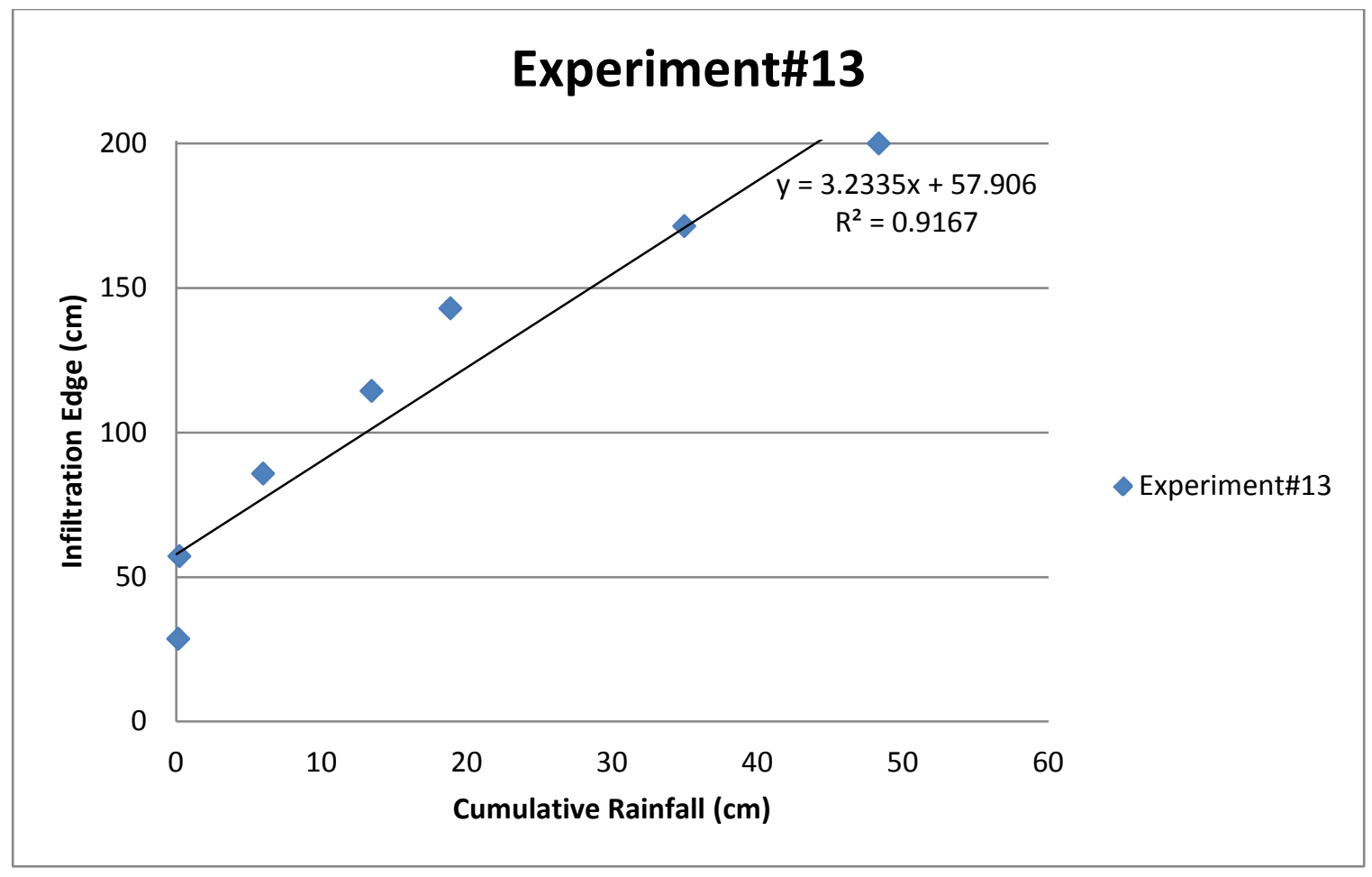




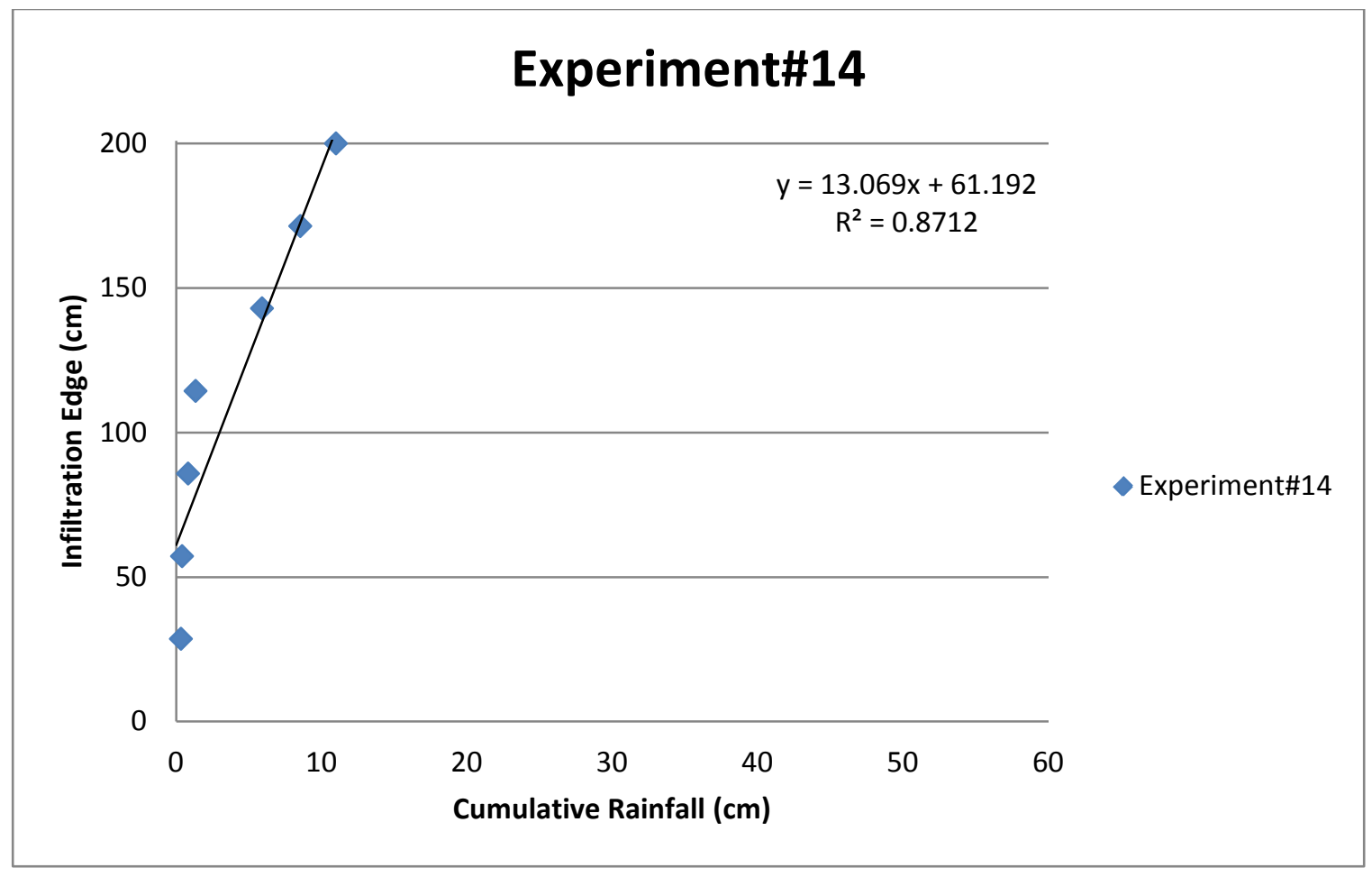




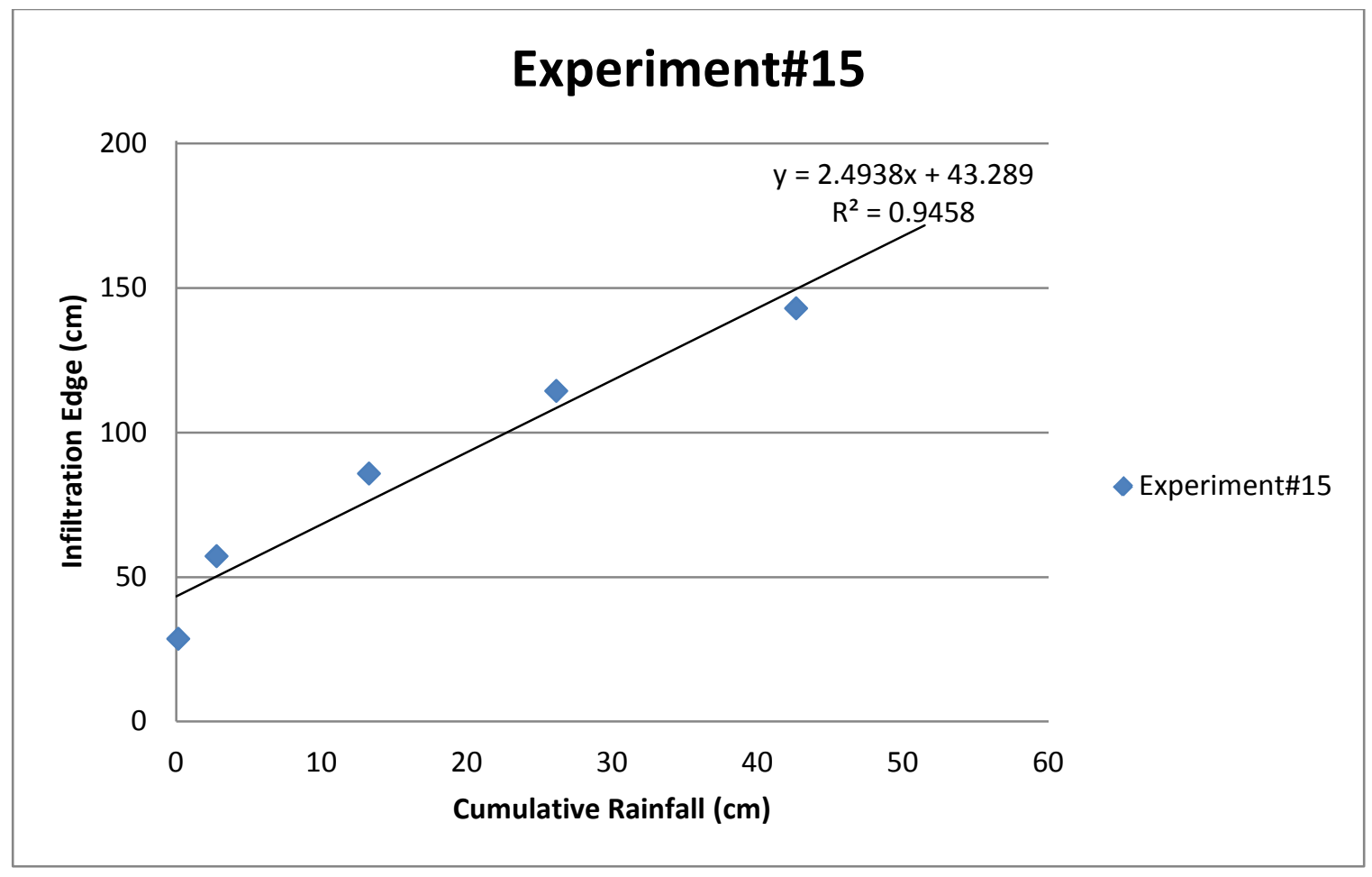




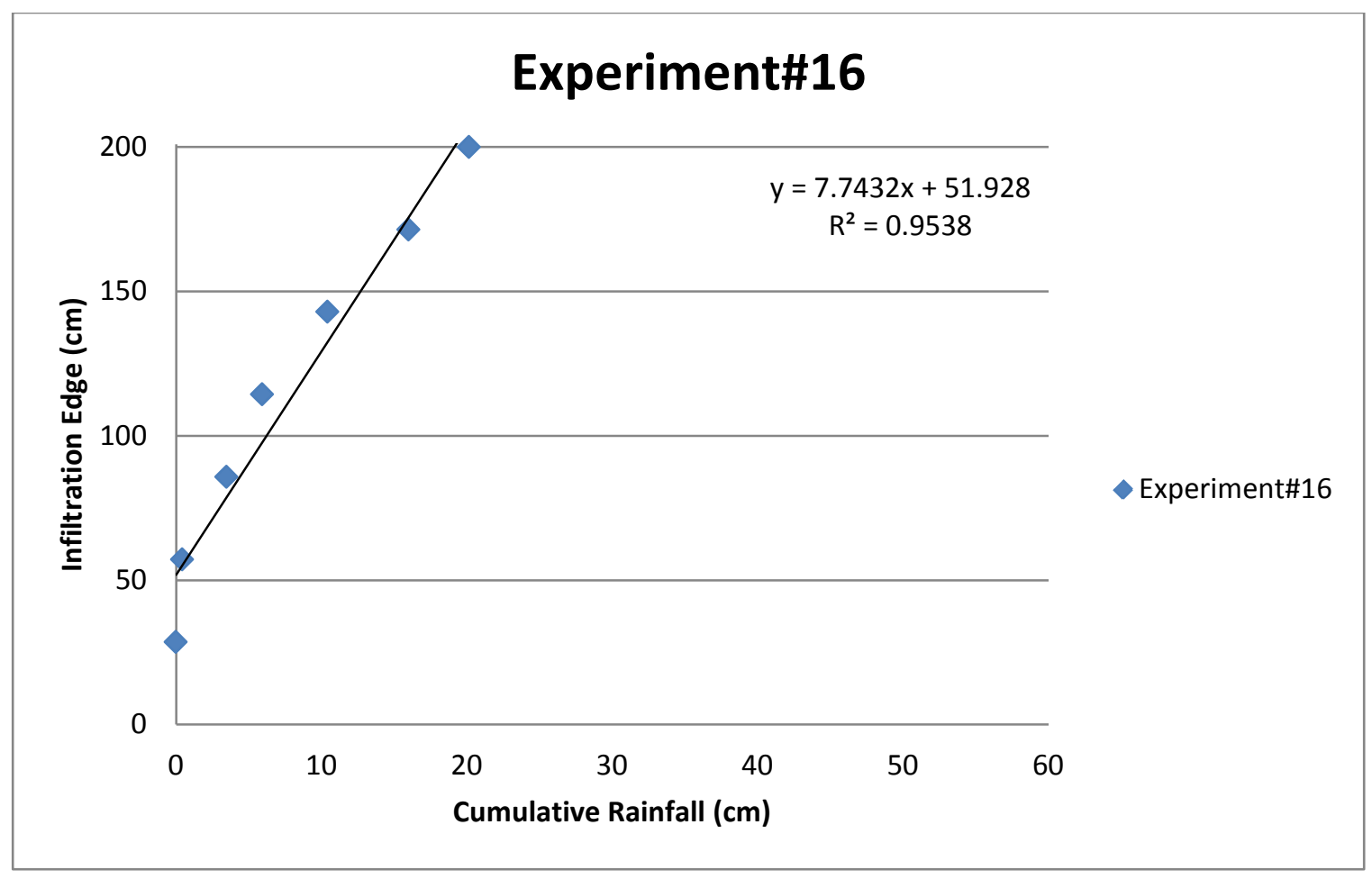




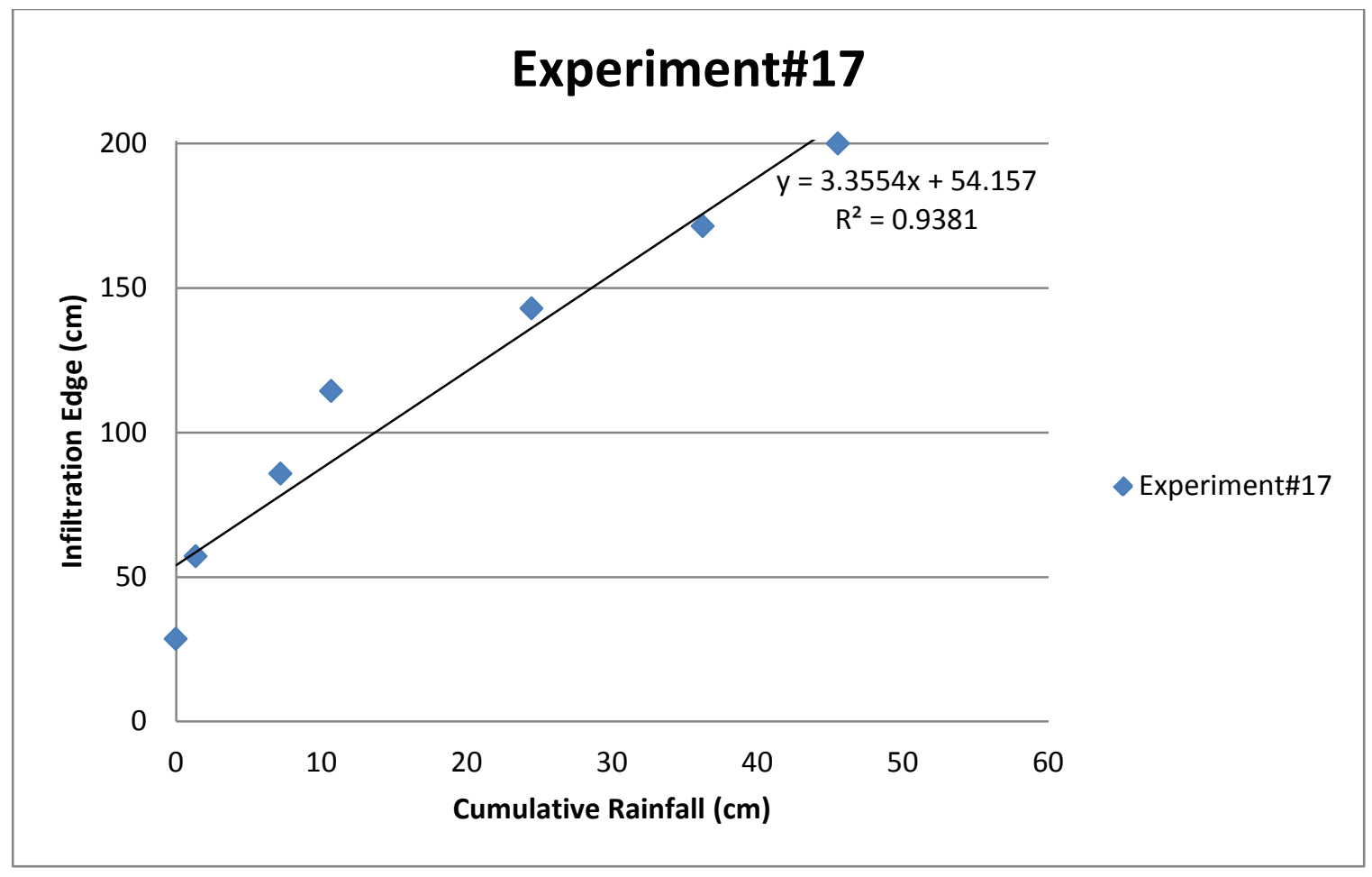




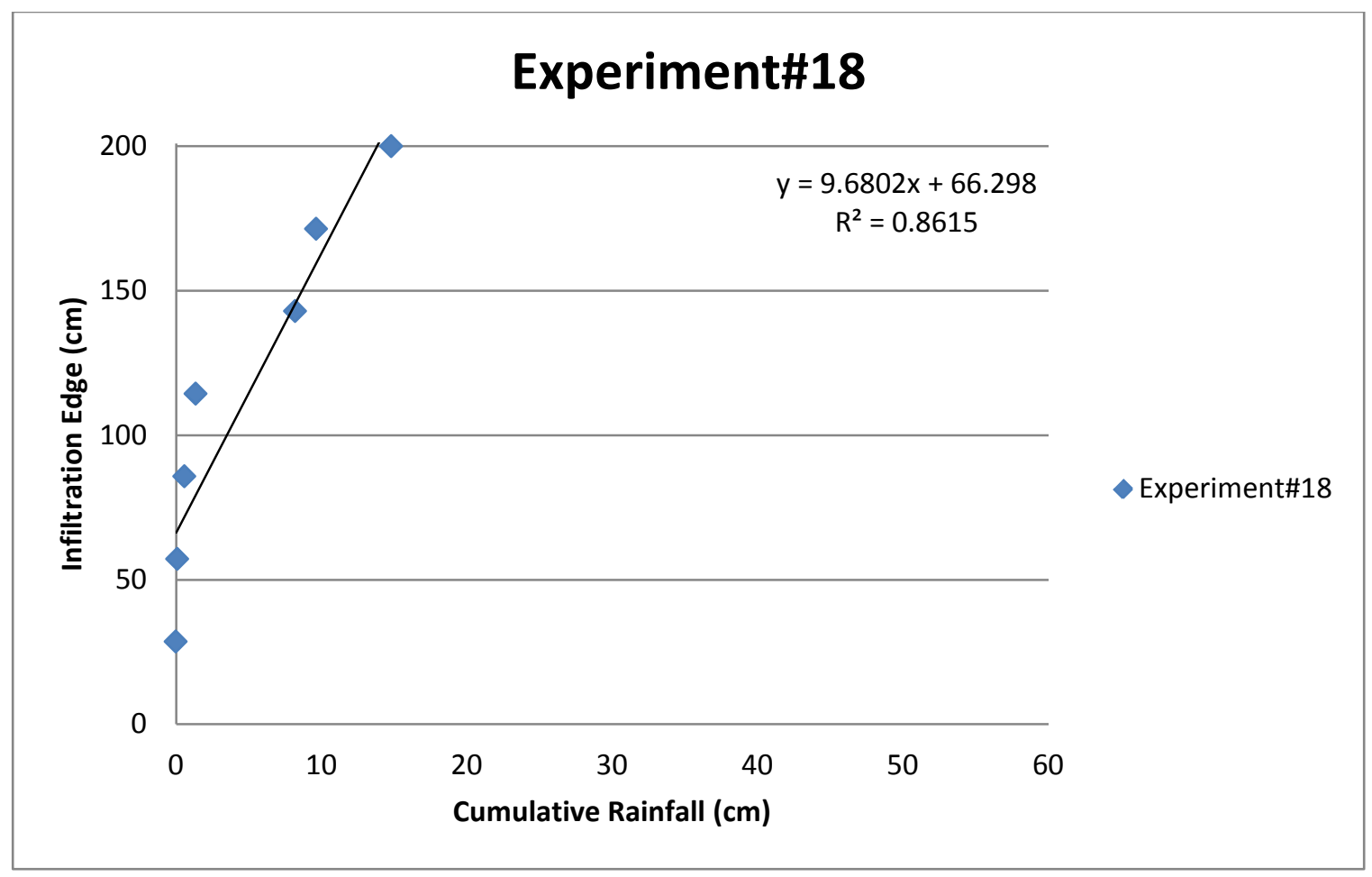




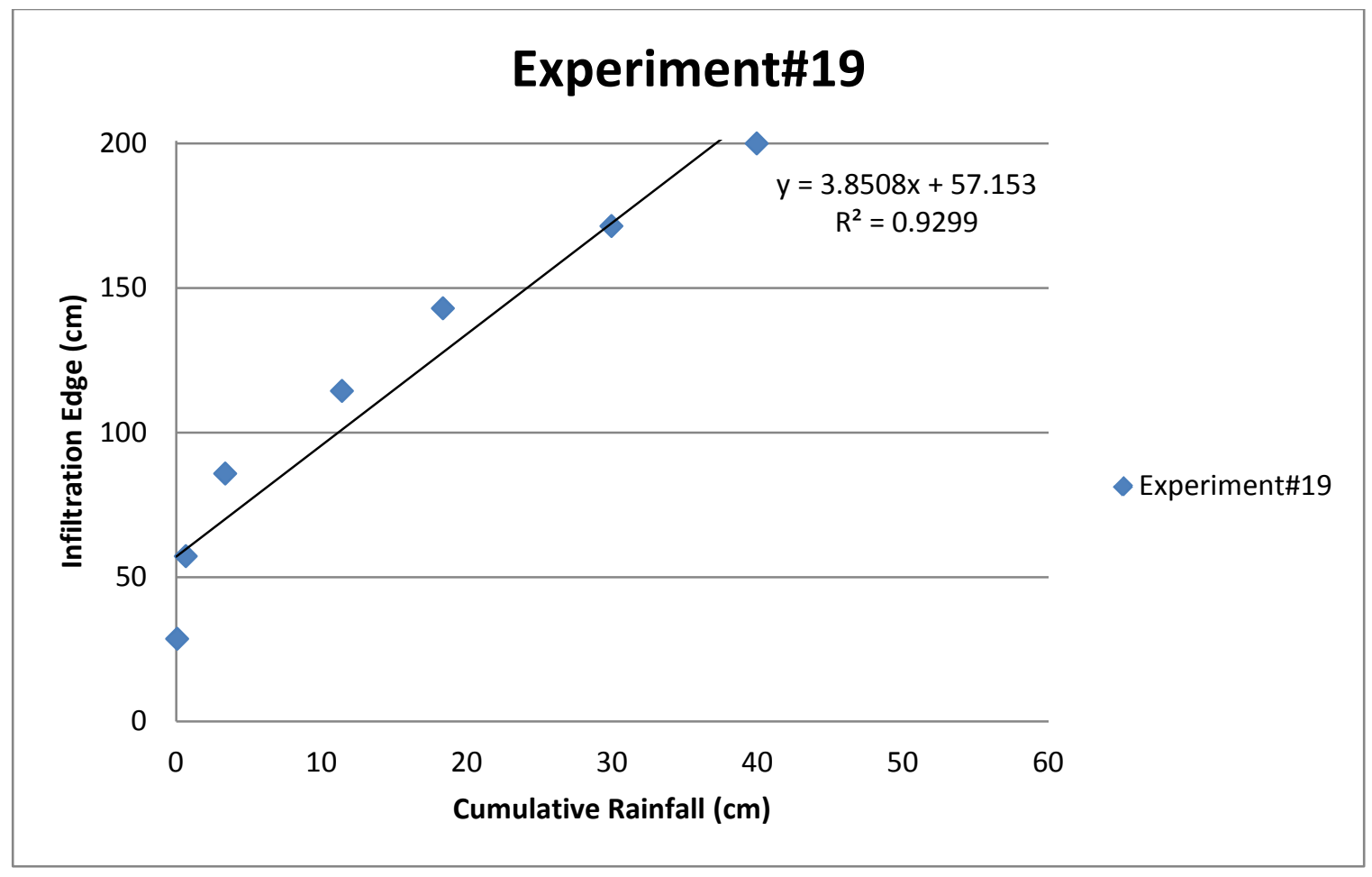




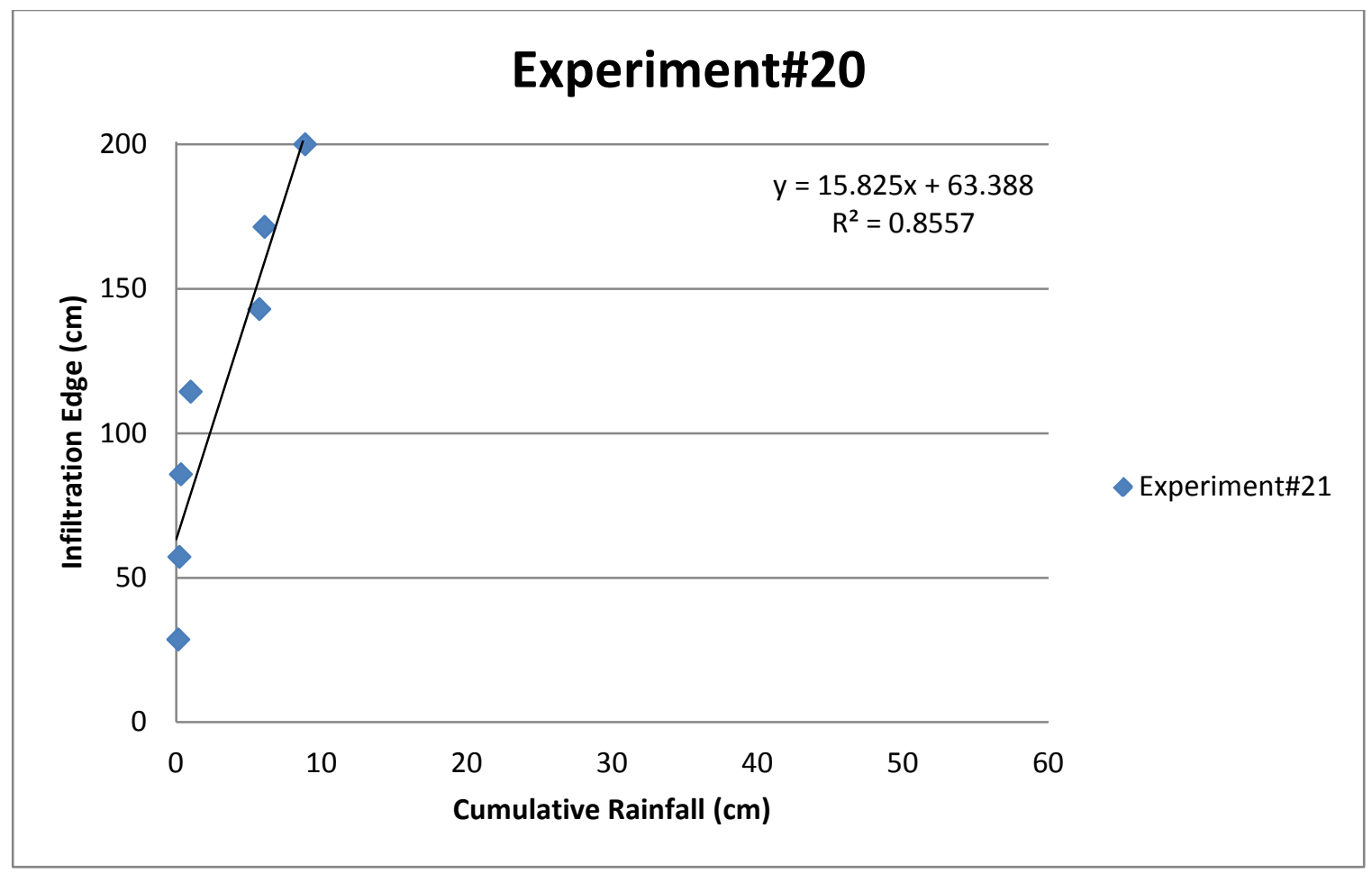




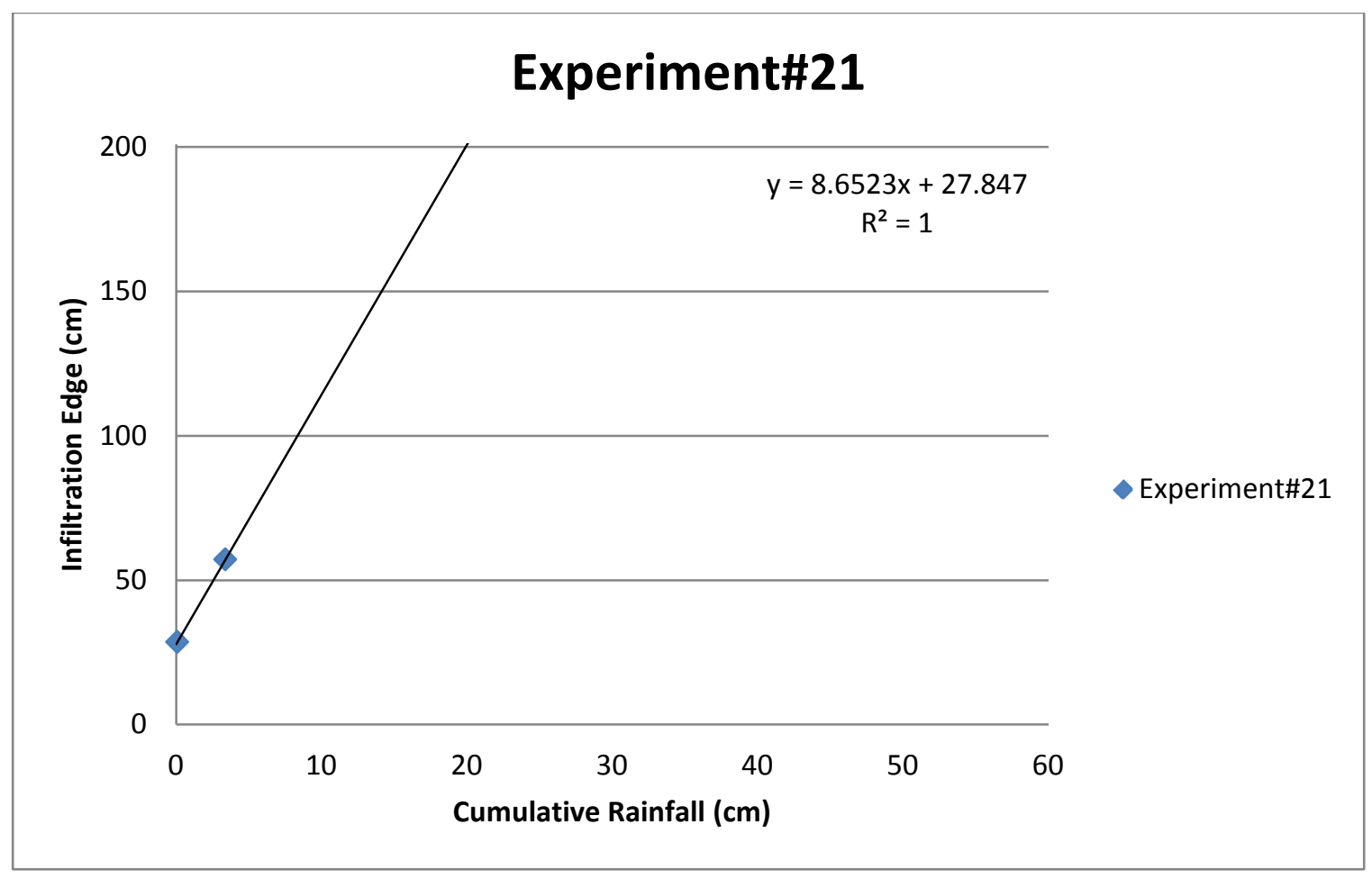




\begin{abstract}
APPENDIX H
As explained in the chapter 5, the first experiment was selected and three duplicates of the same experiment were conducted to test on repeatability of the results. The reason for doing so was to determine whether or not the test conditions were kept reasonably constant during the experiments. Due to the scope of the planned work for this research and the nature of this work, the first experiment was selected for this purpose using ASTM Standard E961 - 12. The experiment results are expected to be repeated within a probability of approximately 0.95 .
\end{abstract}

By comparing the first three experiments, the progression of clogging was selected as a tool to investigate the repeatability of the test results. The reason for this choice is that the progression of clogging is an indication of the performance of the test results and answers one of the main and early hypotheses of the experiment, which concerns the performance of the permeable surface.

Plotting all the data points obtained by the TDRs installed in the flume to ascertain the progression of clogging in the first three experiments, and comparing the equations of the trend lines indicates that the rate at which the clogging progresses in these duplicate experiments matches the repeatability criteria and happens with equal or more than 0.95 probability every time. This comparison is illustrated in Figure below.

In ideal conditions, having more experiments as points to investigate the repeatability of the test results would result in higher accuracy for the statement made in the previous paragraph. However, due to practical limits, such as the durability of the 
module in the physical experiment and the time limits, conducting further investigations on this matter was not feasible.

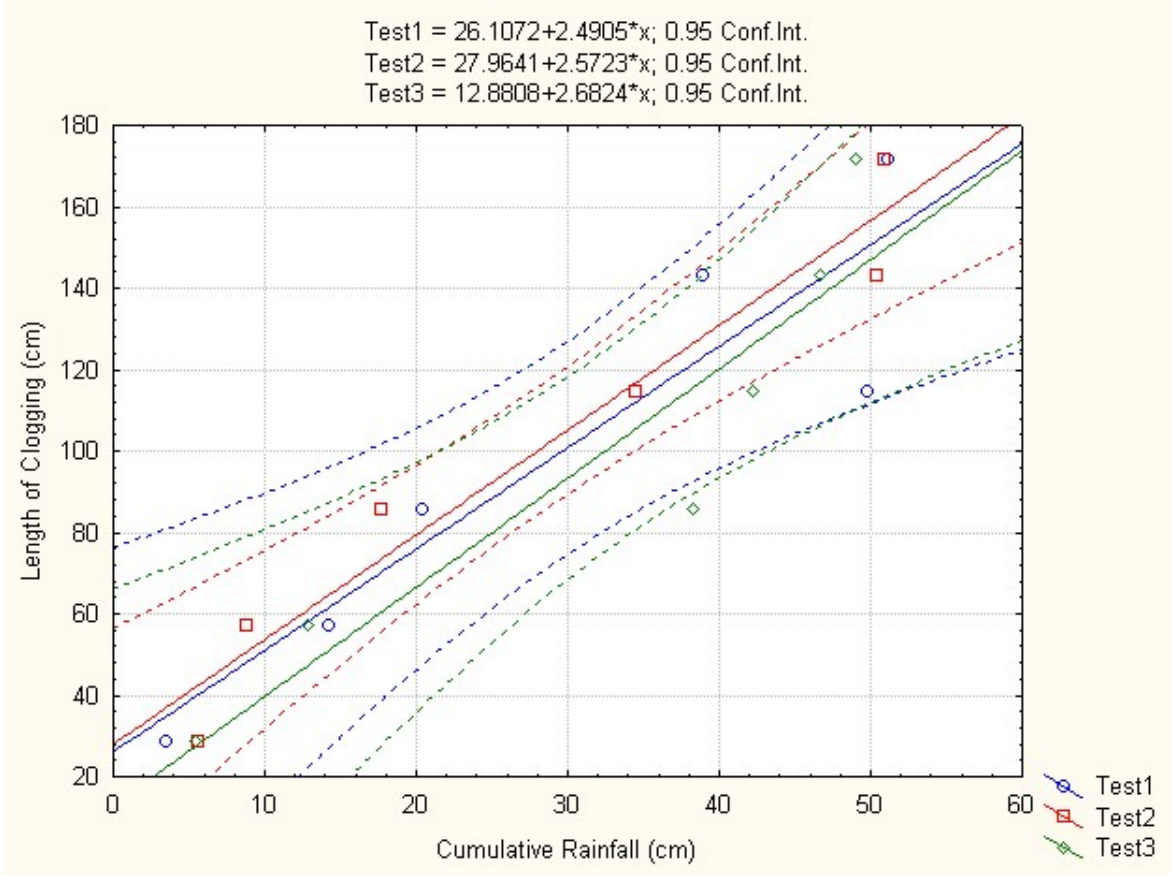

Comparison of progression of clogging for the first three experiments 


\section{CURRICULUM VITAE}

NAME:

ADDRESS:

PLACE \& DATE

OF BIRTH:

EDUCATION

\& TRAINING:
Amirhossein Ehsaei

Department of Civil and Environmental Engineering

W. S. Speed Hall

University of Louisville

Louisville, KY 40292

Mashhad, Iran - March 22, 1986
B.S. Civil Engineering

Ferdowsi University of Mashhad, Iran

2004-2009

M.S. Road Engineering and Management University of Birmingham, United Kingdom 2009-2010

Ph.D. Candidate

Civil and Environmental Engineering

University of Louisville

2011-2013

PUBLICATIONS

\& PRESENTATIONS:

Ehsaei, Amirhossein; Rockaway, T. (under review), "EXAMINATION OF EMPIRICAL EVIDENCE AND REFINING MAINTENANCE TECHNIQUES FOR GI”, Journal of Irrigation and Drainage Engineering

Ehsaei, Amirhossein; Abdollahian, Sam; Rockaway, T; Low Impact Development (LID) 2013 Conference, "REFINING THE MAINTENANCE TECHNIQUES FOR INTERLOCKING CONCRETE PAVER GI”, Minneapolis, MN 
Borst, Michael J.; Robert A. Brown; Joong G. Lee; Amirhossein Ehsaei, Michelle A. Simon, "QUANTIFICATION OF THE PERFORMANCE OF GI IN LOUISVILLE, KY.”

KY-TN Waterworks Association 2012, oral presentation: "EFFECTIVE MONITORING INSTRUMENTATION OF GREEN STORMWATER INFRASTRUCTURE”

Road Safety Simulation (RSS) 2011 Conference, paper on "TEMPORAL VARIATION OF ROAD ACCIDENT DATA CAUSED BY ROAD INFRASTRUCTURE”

KY-TN Waterworks Association 2011, Second Prize Award for poster presentation on "EFFECTIVE MONITORING INSTRUMENTATION OF BEST MANAGEMENT PRACTICES”

Ehsaei, Amirhossein, "TEMPORAL VARIATION OF ROAD ACCIDENT DATA CAUSED BY ROAD INFRASTRUCTURE”, MS thesis, University of Birmingham, Birmingham, United Kingdom 